\title{
Prenatal exposure to polyunsaturated fatty acids and child health
}

Citation for published version (APA):

Stratakis, N. (2018). Prenatal exposure to polyunsaturated fatty acids and child health: a population-based approach. [Doctoral Thesis, Maastricht University]. Maastricht University. https://doi.org/10.26481/dis.20180302ns

Document status and date:

Published: 01/01/2018

DOI:

10.26481/dis.20180302ns

Document Version:

Publisher's PDF, also known as Version of record

\section{Please check the document version of this publication:}

- A submitted manuscript is the version of the article upon submission and before peer-review. There can be important differences between the submitted version and the official published version of record.

People interested in the research are advised to contact the author for the final version of the publication, or visit the DOI to the publisher's website.

- The final author version and the galley proof are versions of the publication after peer review.

- The final published version features the final layout of the paper including the volume, issue and page numbers.

Link to publication

\footnotetext{
General rights rights.

- You may freely distribute the URL identifying the publication in the public portal. please follow below link for the End User Agreement:

www.umlib.nl/taverne-license

Take down policy

If you believe that this document breaches copyright please contact us at:

repository@maastrichtuniversity.nl

providing details and we will investigate your claim.
}

Copyright and moral rights for the publications made accessible in the public portal are retained by the authors and/or other copyright owners and it is a condition of accessing publications that users recognise and abide by the legal requirements associated with these

- Users may download and print one copy of any publication from the public portal for the purpose of private study or research.

- You may not further distribute the material or use it for any profit-making activity or commercial gain

If the publication is distributed under the terms of Article $25 \mathrm{fa}$ of the Dutch Copyright Act, indicated by the "Taverne" license above, 
Prenatal exposure to polyunsaturated fatty acids and child health:

a population-based approach

Nikos Stratakis 


\section{ACKNOWLEDGMENTS}

The MEFAB cohort study was financially supported by the Dutch Organization for Scientific Research (NWO, Grant number 90462 186) and the University Hospital of Maastricht (Profilerings Fonds). The 23-year follow-up of the MEFAB cohort was financially supported by the Nutricia Research Foundation (2015-24), and Universiteitsfonds Limburg/SWOL.

The Mother-Child "RHEA" cohort was financially supported by European projects [EU FP6-003-Food-3-NewGeneris - contract no. 16320, EU FP6 STREP Hiwate - contract no. 36224, EU FP7 ENV.2007.1.2.2.2. Project No. 211250 Escape, EU FP7-2008-ENV-1.2.1.4 Envirogenomarkers contract no. 226756, EU FP7-HEALTH-2009-single stage CHICOS contract no. 241604, EU FP7 ENV.2008.1.2.1.6. proposal no. 226285 ENRIECO, EU-FP7, proposal no. 264357 MeDALL, EU- FP7- HEALTH-2012 proposal no. 308333 HELIX], and the Greek Ministry of Health (Program of Prevention of obesity and neurodevelopmental disorders in preschool children, in Heraklion district, Crete, Greece: 2011-2014; "Rhea Plus": Prevention Program of Environmental Risk Factors for Reproductive Health, and Child Health: 2012-2015.

The work presented in this thesis was conducted at the Section of Complex Genetics and Epidemiology, Department of Genetics and Cell Biology, School for Nutrition and Translational Research in Metabolism (NUTRIM), Faculty of Health, Medicine and Life Sciences in Maastricht University, and at the Department of Social Medicine, Faculty of Medicine in the University of Crete.

We are grateful to the participants of the MEFAB and RHEA cohorts for their time and commitment.

ISBN: 978-94-6295-839-5

Cover and logo design: Nikos Chrysanthakopoulos

Layout and printed by: ProefschriftMaken // www.proefschriftmaken.nl

Copyright (C) 2018 N. Stratakis, Maastricht, The Netherlands

All rights reserved. No part of this thesis may be reproduced or transmitted in any form or by any means, electronic or mechanical, including photocopying, recording or any information storage or retrieval system, without permission in writing from the author, or, when appropriate, from the publishers of the publications. 


\section{Prenatal exposure to polyunsaturated fatty acids and child health:}

a population-based approach

\section{DISSERTATION}

to obtain the degree of Doctor at Maastricht University, on the authority of the Rector Magnificus,

Prof.dr. Rianne M. Letschert

in accordance with the decision of the Board of Deans, to be defended in public

on Friday March 2 2018, at 12:00 hours

by

Nikos Stratakis 


\section{Promotiecommissie}

Promotoren: $\quad$ Prof. dr. M.P. Zeegers

Prof. dr. R.H.M de Groot (Open University, Heerlen)

Co-promotoren: $\quad$ Dr. L. Chatzi (University of Crete, Greece)

Dr. M. Gielen

\section{Beoordelingscommissie:}

Prof. dr. P.C. Dagnelie (chair)

Prof. dr. A. Schols

Prof. dr. L. Zimmermann

Dr. L. Lauritzen (University of Copenhagen, Denmark)

Dr. V. Benetou (National and Kapodistrian University of Athens, Greece) 
$\sum \tau O \nu \alpha \delta \varepsilon \varrho \varphi \dot{~} \mu O \nu$ 



\section{Contents}

$\begin{array}{lll}\text { Chapter } 1 \text { General introduction } & 13\end{array}$

Chapter 2 Effect of maternal n-3 long-chain polyunsaturated fatty acid supplementation during pregnancy and/or lactation on adiposity in childhood: a systematic review and metaanalysis of randomized controlled trials

Chapter 3 Polyunsaturated fatty acid status at birth, childhood growth and cardiometabolic risk: a pooled analysis of the MEFAB and RHEA cohorts

Chapter 4 Polyunsaturated fatty acid levels at birth and child-to-adult growth: results from the MEFAB cohort

Chapter 5 Polyunsaturated fatty acid status at birth and allergyrelated phenotypes in childhood: a pooled analysis of the MEFAB and RHEA birth cohorts

Chapter 6 Maternal fish intake during pregnancy and effects on the offspring

Chapter 7 Fish intake in pregnancy and child growth: a pooled analysis of 15 European and US birth cohorts

Chapter 8 Fish and seafood consumption during pregnancy and the risk of asthma and allergic rhinitis in childhood: a pooled analysis of 18 European and US birth cohorts.

Chapter 9 General discussion

Chapter 10 Summary

Samenvatting

Valorization addendum

Chapter 11 List of publications-Presentations

About the author

Dankwoord 



\section{Manuscripts that form the basis of this thesis}

\section{Chapter 2}

Stratakis N, Gielen M, Chatzi L, Zeegers MP. Effect of maternal n-3 long-chain polyunsaturated fatty acid supplementation during pregnancy and/or lactation on adiposity in childhood: a systematic review and meta-analysis of randomized controlled trials. Eur J Clin Nutr. 2014 Dec;68(12):1277-87.

\section{Chapter 3}

Stratakis N, Gielen M, Margetaki K, de Groot RHM, Apostolaki M, Chalkiadaki G, Vafeiadi M, Leventakou V, Karachaliou M, Godschalk RW, Kogevinas M, Stephanou EG, MP Zeegers*, L Chatzi*. Polyunsaturated fatty acid status at birth, childhood growth and cardiometabolic risk: a pooled analysis of the MEFAB and RHEA cohorts. Provisionally accepted Eur J Clin Nutr.

* shared senior co-authorship

\section{Chapter 4}

Stratakis N, Gielen M, Margetaki K, Godschalk RW, van der Wurff I, Rouschop S, Ibrahim A, Antoniou E, Chatzi L, de Groot RHM, MP Zeegers MP. Polyunsaturated fatty acid levels at birth and child-to-adult growth: results from the MEFAB cohort. Prostaglandins Leukot Essent Fatty Acids. 2017 Nov;126:72-78.

\section{Chapter 5}

Stratakis N, Gielen M, Margetaki K, de Groot RHM, Apostolaki M, Chalkiadaki G, Vafeiadi M, Leventakou V, Godschalk RW, Kogevinas M, Stephanou EG, MP Zeegers*, L Chatzi*. Polyunsaturated fatty acid status at birth and allergy-related phenotypes in childhood: a pooled analysis of the MEFAB and RHEA birth cohorts. Br J Nutr (in press).

* shared senior co-authorship

\section{Chapter 6}

L Chatzi, N Stratakis. Maternal fish intake during pregnancy and effects on the offspring. R Rajendram, VR Preedy, VB Patel (Eds.). Diet, Nutrition, and Fetal Programming. Humana Press. 2017. 


\section{Chapter 7}

Stratakis N, Roumeliotaki T, Oken E, Barros H, Basterrechea M, Charles MA, Eggesbø M, Forastiere F, Gaillard R, Gehring U, Govarts E, Hanke W, Heude B, Iszatt N, Jaddoe VW, Kelleher C, Mommers M, Murcia M, Oliveira A, Pizzi C, Polańska K, Porta D, Richiardi L, Rifas-Shiman SL, Schoeters G, Sunyer J, Thijs C, Viljoen K, Vrijheid M, Vrijkotte TG, Wijga AH, Zeegers MP, Kogevinas M, Chatzi L. Fish Intake in Pregnancy and Child Growth: A Pooled Analysis of 15 European and US Birth Cohorts. JAMA Pediatr. 2016 Apr;170(4):381-90.

Stratakis N, Zeegers MP, Chatzi L. Fish Intake During Pregnancy and Offspring Adiposity-Reply. JAMA Pediatr. 2016 Aug 1;170(8):809-10.

\section{Chapter 8}

Stratakis N, Roumeliotaki T, Oken E, Ballester F, Barros H, Basterrechea M, Cordier S, de Groot R, den Dekker HT, Duijts L, Eggesbø M, Pia Fantini M, Forastiere F, Gehring U, Gielen M, Gori D, Govarts E, Inskip HM, Iszatt N, Jansen M, Kelleher C, Mehegan J, Moltó-Puigmartí C, Mommers M, Oliveira A, Olsen SF, Pelé F, Pizzi C, Porta D, Richiardi L, Rifas-Shiman SL, Robinson SM, Schoeters G, Strøm M, Sunyer J, Thijs C, Vrijheid M, Vrijkotte TG, Wijga AH, Kogevinas M, Zeegers MP, Chatzi L. Fish and seafood consumption during pregnancy and the risk of asthma and allergic rhinitis in childhood: a pooled analysis of 18 European and US birth cohorts. Int J Epidemiol. 2017 Oct 1;46(5):14651477. 



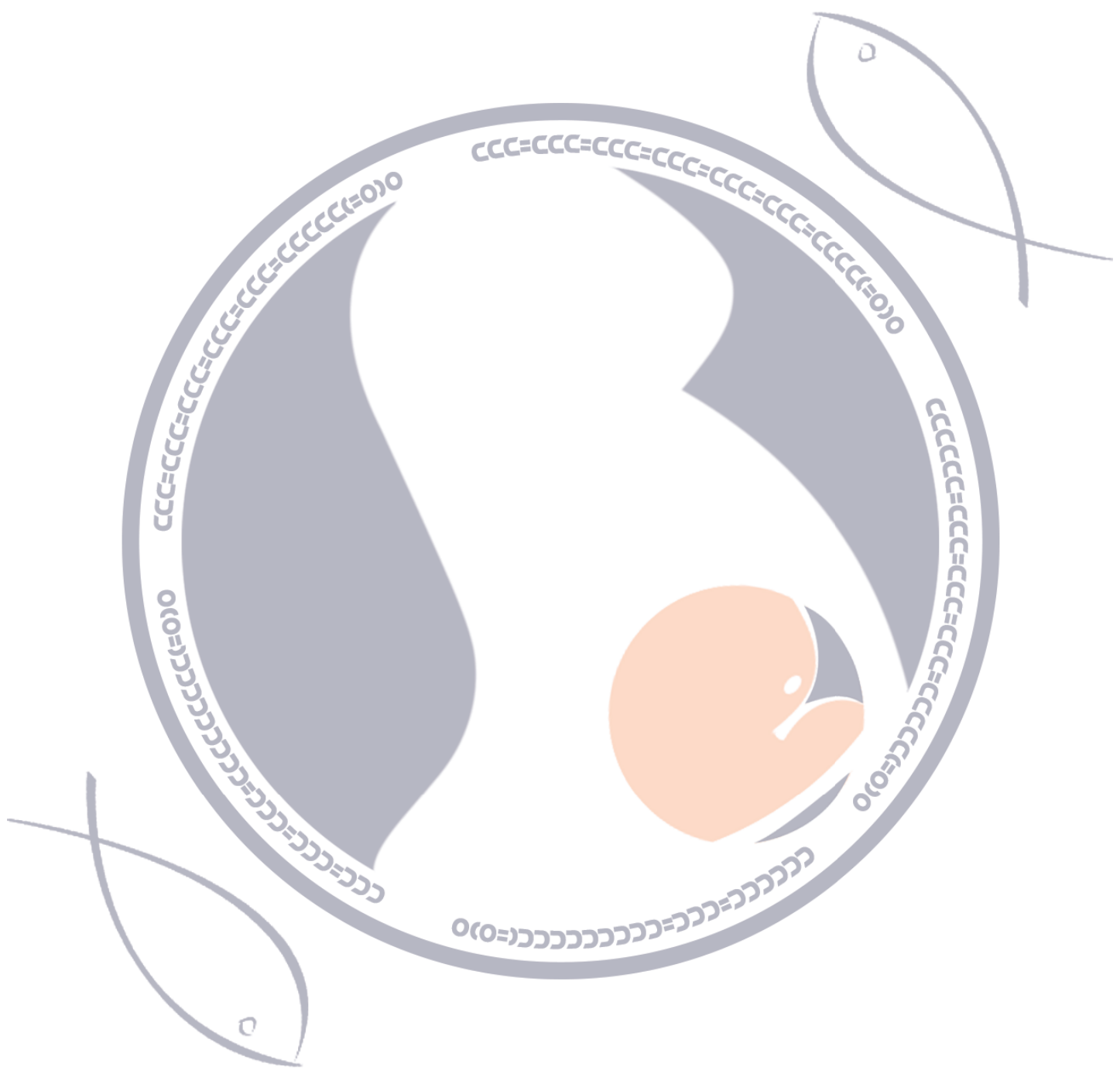




\section{CHAPTER 1}

General Introduction 



\section{INTRODUCTION}

Improving child health and development is likely to bring substantial benefits over the whole lifespan ${ }^{1}$ and ensure a sustainable future. ${ }^{2}$ Among the health issues that the youth of today is facing in developed countries, obesity and allergic disease are two of the most serious ones, with life-long consequences. ${ }^{3-5}$ Indeed, the European Commission classifies childhood obesity and allergy as two chronic conditions of major public health importance. ${ }^{6}$ Over the last years, efforts to improve population health have focused to a large extent on the earliest stage of human development (the intrauterine period), during which the individual is highly vulnerable to environmental influences. In this context, prenatal exposure to polyunsaturated fatty acids (PUFAs) has attracted a lot of attention as a modifiable factor affecting chronic disease risk.

This chapter briefly describes the rationale for focusing on childhood obesity and allergy-like symptoms, the importance of the prenatal environment, and relevant background information on PUFAs. Lastly, the aim, general design, and outline of this thesis are presented.

\section{Rationale for studying childhood obesity}

Childhood obesity has reached epidemic proportions. It is estimated that approximately $30 \%$ of children and adolescents in the United States and about 15-30\% of those in Europe can be classified as overweight or obese, according to the body mass index (BMI) cutoffs proposed by the International Obesity Taskforce. ${ }^{7,8}$

Obesity is difficult to treat, and once established in childhood, tends to persist into adulthood. Studies have shown that even children aged less that 2 years with BMI higher than the $90^{\text {th }}$ percentile are likely to become obese as adults. Childhood obesity can adversely affect almost every organ system in the human body. ${ }^{3}$ The obesity-associated cardiometabolic disturbances including impaired glucose tolerance, adverse lipidemic profile and high blood pressure can already be seen at young age. ${ }^{3}$ Notably, it has been shown that not only did these cardiometabolic disturbances tend to coexist with obesity in childhood, ${ }^{10}$ but that their rates of change also cluster over time. ${ }^{11}$ Obese children experience an accelerated atherosclerotic process. A large body of epidemiologic evidence suggests that high childhood BMI predicts adult cardiovascular morbidity and mortality. ${ }^{12}$ Autopsy and imaging studies have also shown that the extent of early atherosclerosis is accelerated with increasing number of concurrent cardiometabolic risk factors. ${ }^{13,14}$ Given the recent obesity epidemic in the youth and its long-term cardiometabolic complications, it has been advocated that the gains in health and longevity achieved by modern advances in health care may be quickly reversed, unless effective interventions to reduce obesity are developed. ${ }^{15}$ 


\section{Rationale for studying childhood allergic disease}

The prevalence of asthma and allergy has markedly increased over the last decades. Asthma symptoms are estimated to affect $10-14 \%$ of children aged less than 14 years worldwide, with the prevalence rising to more than $20 \%$ in many European countries. ${ }^{4}$ Likewise, eczema and allergic rhinoconjunctivitis are common in developed countries, affecting up to $16 \%$ and $20 \%$ of school children, respectively. ${ }^{16}$ Asthma and allergy are characterized by inflammatory processes, with $\mathrm{CD}^{+} \mathrm{T}$ helper $\left(\mathrm{T}_{\mathrm{H}}\right)$-cell responses of the $\mathrm{T}_{\mathrm{H}} 2$-cell phenotype being considered to be important for the initiation and maintenance of related inflammation. ${ }^{17}$ Allergic diseases result in significant disruption of life and physical capability, and constitute a major cause of hospitalizations among children. ${ }^{18,19}$ Indeed, asthma is currently considered one of the top 20 chronic conditions for global ranking of disability-adjusted life years in children. ${ }^{20}$ Moreover, childhood symptoms of asthma have been shown to strongly correlate with chronic asthma up to the fifth decade of life, ${ }^{21}$ and severe asthma in children has been associated with an up to 32 -fold increased risk of chronic obstructive pulmonary disease in later life. ${ }^{22}$ Hence, prevention of asthma and other allergy-related diseases will have a significant impact on population's morbidity levels, and currently constitutes one of the priorities of the European Union's public health policy. ${ }^{23}$

\section{Importance of the early-life environment in health and disease}

Genes play an important role in obesity and allergy occurrence, though the increase in the prevalence of these diseases has occurred too rapidly in order for genetic changes to explain the current epidemics. Thus, environmental factors are likely to be important contributors. ${ }^{24,25}$ Advances in our knowledge on specific time windows during which environmental factors may exert their major influence have led to the "Developmental Origins of Health and Disease" concept. According to this concept, the environment encountered in utero exerts significant echoes in disease risk throughout the lifecourse. ${ }^{26}$ Fetal life is a critical period of developmental plasticity. An environmental stressor or stimulus applied during this period can permanently alter body's structure, physiology, and metabolism, the consequences of which are often observed much later in life. ${ }^{27}$ Hence, pregnancy constitutes a time period of both great opportunity and considerable risk.

Maternal diet impacts energy fuels, hormones and inflammation with considerable effects on fetal metabolic, endocrine and immune systems, thus, largely influencing fetal developmental plasticity. ${ }^{28}$ Seminal studies by Barker and colleagues underlined the importance of maternal nutrition by suggesting that the propensity to develop hypertension, type II diabetes and coronary heart disease were consequences of fetal adaptations to maternal malnourishment. ${ }^{29,30}$ Results from the 
famous Dutch Famine studies also provided strong support to the concept of fetal imprinting by maternal nutritional status by showing that undernutrition during gestation was associated with adverse adult health outcomes including obesity and obstructive airways disease. . $^{31,32}$

The maternal diet in developed countries has changed considerably over the last decades. Birth cohort studies have been trying to elucidate which key nutritional exposures in the prenatal period could have implications in the obesity and asthma epidemics. In this context, there has been considerable interest in the potential effect of the qualitative composition of fatty acids in the diet of pregnant women, and particularly the intake of n-3 PUFAs relative to that of n-6 PUFAs. ${ }^{33}$

\section{Classification and metabolism of polyunsaturated fatty acids}

Fatty acids are molecules composed of a hydrocarbon chain with a carboxylic acid $(-\mathrm{COOH})$ at one end and a methyl group $\left(-\mathrm{CH}_{3}\right)$ at the other end. The length and degree of saturation of the hydrocarbon chain varies among fatty-acid families; these characteristics determine the physical and physiological properties of fatty acids. Fatty acids may have multiple, but usually no more than six, double bonds (PUFAs), one double bond (monounsaturated fatty acids), or no double bonds (saturated fatty acids). Fatty acids have common names but also are identified by a shorthand designation indicating their number of carbon atoms, their number of double bonds, and the site of the first double bond from the terminal methyl group of the molecule. For example, arachidonic acid (AA), designated as 20:4n6, has 20 carbons, four double bonds, and the first double bond is at the sixth carbon from the methyl terminal. The present thesis will follow this shorthand designation.

Fatty acids with double bonds at the n-3 and n-6 positions are of particular importance, as they constitute the only fatty acids that cannot be synthesized de novo by the human species; humans lack the enzyme required for the insertion of a double bond at the third and sixth carbon from the terminal methyl group of the molecule. ${ }^{34,35}$ Alpha-linolenic (ALA, 18:3n-3) and linoleic (LA, 18:2n-6) acids constitute the parent fatty acids of the $n-3$ and n- 6 families, respectively, and must be derived from the diet. ${ }^{34,35}$ Hence, they are termed "essential fatty acids". The essentiality of ALA and LA has been illustrated by studies as early as the late 1920s showing symptoms of fatty acid deficiency that are similar to other nutrient deficiencies, including poor growth, and scaly skin lessions. ${ }^{36,37}$

Once consumed, ALA and LA can be further metabolized within mammalian cells by the same series of desaturase- and elongase-enzymes to longer-chain, more unsaturated fatty acids (Figure 1). LA is converted to $\gamma$-linolenic acid (GLA, 18:3n-6), and dihomo- $\gamma$-linolenic acid (DGLA, 20:3n-6). DGLA is metabolized 
to the eicosanoids 1-series prostaglandins or arachidonic acid (AA, 20:4n-6). AA can be further metabolized to Osbond acid (22:5n-6) or the eicosanoids 2-series prostaglandins and thromboxanes, 4-series leukotrienes, hydroxyl and hydroperoxy derivatives, and lipoxins. The $n-3$ parent fatty acid ALA is converted to eicosapentaenoic acid (EPA, 20:5n-3), which is further metabolized to docosahexaenoic acid (DHA, 22:6n-3) or eicosanoids including 3-series prostaglandins and thromboxanes, and 5-series leukotrienes, as well as E-series resolvins. Moreover, DHA can be further metabolized to the docosanoids D-series resolvins, maresins, and protectins. PUFAs are important building blocks of cell membranes, and exert a critical role in maintaining membrane fluidity and permeability. Moreover, the terminal metabolites of specific long-chain fatty acids (Figure 1.1) possess diverse biological functions, including regulation of inflammation, immune reactivity, and cell differentiation. ${ }^{38,39}$

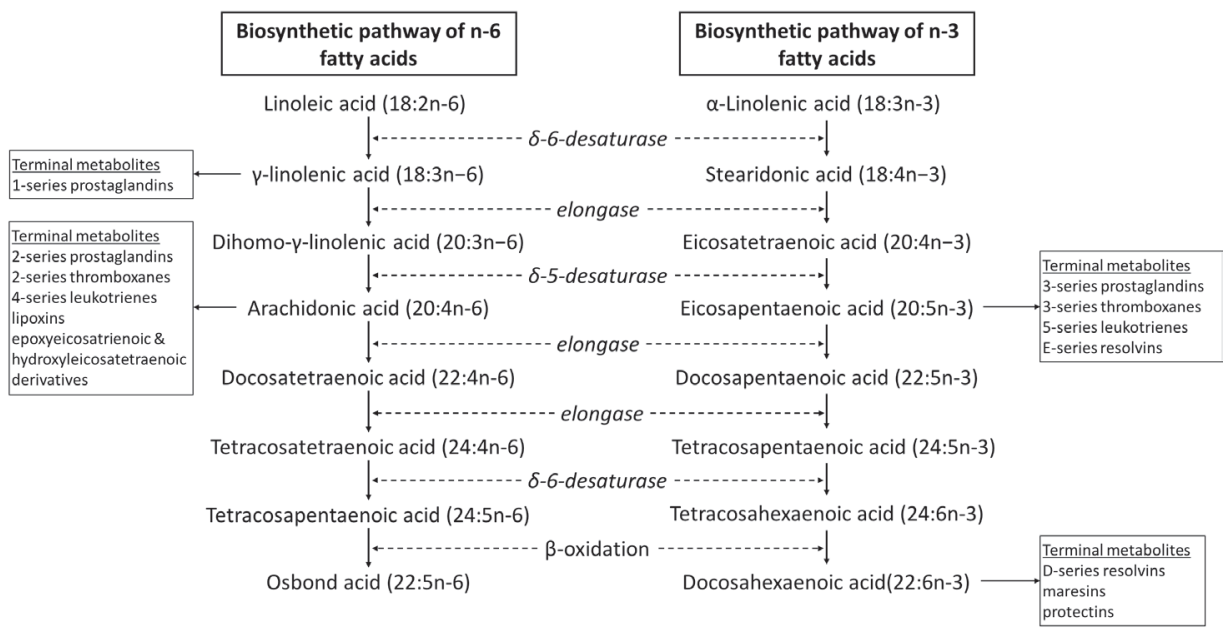

Figure 1.1. Metabolism of $n-6$ and n-3 fatty acids. Source: $:^{34,35,43}$

Enzymatic conversion of LA to AA is much more efficient than the conversion of ALA to DHA; the conversion rate of ALA to EPA is less than $5-10 \%$ and to DHA is $1-5 \%{ }^{40,41}$ Hence, it has been proposed that individuals should rely mainly on dietary consumption of preformed n-3 long-chain PUFAs to maintain adequate levels. ${ }^{42}$ Major dietary sources of the parent n-6 LA are corn, safflower, and sunflower oils, while for the n-3 ALA, walnuts, and flaxseed, rapeseed and soy oils are major sources. The main dietary source of the n-6 AA is meat, meat products, and eggs. For the longer-chain n-3 fatty acids, fish is considered the primary dietary source. Over the last years, the composition of fatty acids in the western diets has shifted toward an increasing dominance of n- 6 relative to $n-3$ 
PUFAs; it is estimated that the current ratio of n-3 to n-6 PUFAs in most diets in developed countries is approximately $1: 20-30 .{ }^{33}$ This has important implications for population's fatty acid status, given the competition between $n-3$ and n-6 fatty acids for the same series of enzymes involved in elongation and desaturation. ${ }^{34}$ Consequently, the excess of $n-6$ fatty acids causes a significant decrease in the metabolism of those of the $n-3$ family.

Maternal metabolism and availability of PUFAs during pregnancy determine fetal supply. The fetus has limited desaturase activity, and the amount of long-chain PUFAs produced from the parent fatty acids is insufficient to match its needs. ${ }^{44}$ Therefore, the primary source of PUFAs for the fetus is placental transfer from the mother. Studies using cord blood PUFA biomarkers to assess fetal exposure have been shown that percentages of the parent PUFAs are lower in lipid classes of cord than of maternal plasma, while proportions of the longer chain PUFAs are higher in cord than in maternal lipids. ${ }^{44}$ This selective increase in the concentration of long-chain PUFAs in the fetal circulation has been termed biomagnification, and seems to coincide with the period of maximum fetal demand, at around 20 weeks of gestation onwards, when the vast majority of the fetal fat deposition occurs. ${ }^{45}$ Biomagnification of PUFAs in the fetal circulation is thought to be facilitated by preferential delivery through the placenta to support the maximum accretion rates. ${ }^{45}$ Several proteins located on the microvillous and basal membranes (fatty acid translocase, fatty acid transport protein, plasma membrane fatty acid-binding protein) and within the cytoplasm (fatty acid-binding protein) of the syncytiotrophoblast have been suggested to mediate this preferential placental transfer of PUFAs to the fetus. ${ }^{45,46}$

\section{Prenatal PUFAs, childhood growth, and cardiometabolic risk}

PUFAs of the n- 6 and n-3 families are readily transferred across the placenta, and have been shown in animal and in vitro studies to exert pleiotropic effects, often in the opposite direction. ${ }^{47-50}$ For instance, n-6 fatty acids have been suggested to increase adipocyte formation -a process that is proposed to occur at the expense of osteoblastogenesis- and adipose tissue deposition, and promote inflammation, whereas n-3 fatty acids seem to exhibit opposite effects. ${ }^{47-50}$ Several mechanisms have been proposed to underline the effects of PUFAs including modulation of cytokine production, alterations in the activation of the transcription factors peroxisome proliferator-activated receptors and sterol regulatory element-binding protein-1, and regulation of steroid hormones and adipokines. ${ }^{47-50}$ Hence, there has been a growing interest in the potential role of prenatal PUFA exposure in influencing later growth, obesity development and related cardiometabolic risk factors. 
A few studies have assessed childhood height and have mostly focused on $n-3$ long-chain PUFA supplementation, with reports of null ${ }^{51-53}$ or positive (ie, higher height $)^{54}$ effects. A number of cohort studies have examined the association of prenatal PUFA exposure with measures of adiposity in childhood. The Dutch Generation R cohort study showed that lower maternal n-3 PUFA concentrations and higher n-6 PUFA concentrations in mid-pregnancy were associated with higher body fat measured by dual-energy X-ray absorptiometry (DXA) and ultrasound-measured abdominal fat in children aged 6 years. ${ }^{55}$ In a previous analysis of the Dutch MEFAB cohort, de Vries et $\mathrm{a}^{56}$ reported that n-6 DGLA levels in maternal plasma throughout pregnancy were associated with higher BMI at age 7 years, but no associations for other fatty acids were found. An analysis from the British Southampton Women's Survey also found a positive association between plasma concentrations of n-6 PUFAs, but no other fatty acids, in the last trimester of pregnancy with DXA-assessed body composition at 4 years and 6 years. ${ }^{57}$ By way of contrast, the US Project Viva reported that higher concentrations of the n-3 EPA and DHA in umbilical cord plasma, reflecting fetal exposure in late pregnancy, were associated with lower skinfold thicknesses and lower odds of obesity among children aged 3 years. ${ }^{58}$ However, maternal mid-pregnancy plasma concentrations of DHA and EPA were not associated with adiposityrelated outcomes. Likewise, the authors found no significant associations for $\mathrm{n}-6$ fatty acids neither in maternal nor in cord blood. ${ }^{58} \mathrm{~A}$ recent analysis from the German LISAplus birth cohort showed no associations of n- 6 and n-3 long-chain PUFA levels in cord blood with later BMI up to the age of 10 years. ${ }^{59}$ However, in this study, the authors reported a significant interaction between the n-6:n-3 long-chain PUFA ratio and childhood BMI over time: there was a negative effect at 2 years, no effect at 6 years, and a positive effect at 10 years. ${ }^{59}$ Altogether, the results from previous cohort studies have provided little evidence to substantiate that an increase in n-3 PUFA status, a decrease in n-6 PUFA status, or both in pregnancy is an effective strategy to prevent offspring obesity. Trials have also not found a clear and consistent effect of prenatal n-3 long-chain PUFA supplementation on adiposity status later in life (reviewed in Chapter 2). Likewise, studies assessing maternal intake of fish, which constitutes the main dietary source of $n-3$ long-chain PUFAs, have yielded discrepant results (reviewed in Chapter 6).

A few studies have examined the association of prenatal PUFA exposure with other childhood cardiometabolic risk factors, and have reported mixed results. In a previous analysis of the MEFAB cohort, Rump et $\mathrm{al}^{60}$ reported that cord blood levels of the n-6 GLA, but not of other fatty acids, were associated with insulin resistance at 7 years. The Generation $\mathrm{R}$ study showed higher maternal plasma n-3 PUFA levels in mid-pregnancy to be associated with lower systolic blood 
pressure and higher total cholesterol, HDL-cholesterol, and insulin levels at age 6. ${ }^{61,62}$ In contrast, the Aarhus birth cohort study reported no association between marine n-3 PUFA intake in mid-pregnancy and offspring lipid profile, blood pressure and glucose metabolism in early adulthood ${ }^{63}$. Two large trials in Mexico and Denmark also showed no effect of n-3 long-chain PUFA supplementation in late pregnancy on offspring cardiometabolic factors, including lipid levels ${ }^{64,65}$ and blood pressure ${ }^{66}$ up to 19 years.

Inconsistencies in findings may at least partly be explained by differences in sample sizes exposure profile heterogeneity (i.e. definition and timing of exposure), or differences in adjustment for covariates. Moreover, findings from any prior study have not been replicated across populations with different characteristics and behaviors. Hence, to date, there is no clear answer regarding the potential programming effect of prenatal PUFAs on later growth, obesity and related cardiometabolic outcomes.

\section{Prenatal PUFAs and allergy phenotypes in childhood}

It has been hypothesized that a shift in PUFA composition of Western diets towards an increased intake of vegetable oils containing n-6 fatty acids, and lower n-3 PUFA and fish consumption is implicated in the observed increase in the prevalence of allergic disease over the last years. ${ }^{67}$ Animal and in vitro studies have suggested that n-6 and n-3 fatty acids can exert immune-regulatory effects through involvement in several pathways. n-6 PUFAs, as substrate for eicosanoid synthesis, can promote inflammation, favor T-helper cell type 2-driven immune responses, and enhance the synthesis of immunoglobulin E antibodies ${ }^{68}$. On the contrary, n-3 PUFAs might exhibit a protective effect by competing with the n-6 fatty acids for metabolism, reducing inflammation, and inhibiting $T$ cell signalling through alteration of membrane lipid rafts. ${ }^{69}$ Fetal life is a critical time period of susceptibility during which a nutritional stressor can affect the development and maturation of respiratory and immune systems, and thus, have irreversible influences on the development of allergy. ${ }^{25}$ Hence, there has been a lot of interest in the potential role of prenatal PUFA exposure in the etiology of allergy-related diseases.

Many cohort studies have assessed the association of PUFA levels in pregnancy with the occurrence of asthma symptoms in childhood. The British Avon Longitudinal Study of Parents and Children (ALSPAC) and Southampton Women's Survey showed lower rates of childhood wheeze up to 6 years of age with higher $n-3$ PUFA levels ${ }^{70}$ or a lower n-6:n-3 fatty acid ratio in late pregnancy. ${ }^{71}$ In contrast, the Generation R study found maternal levels of n-6 PUFAs, but no other PUFAs, to be associated with a decreased asthma risk at age $6 .^{72}$ The Dutch KOALA birth 
cohort study found no association of maternal PUFA levels in late pregnancy with asthma symptoms up to the age of 6-7 years. ${ }^{73}$ Similarly, the LISAplus and the Singaporean GUSTO studies showed no associations of prenatal exposure to PUFAs with child asthma symptoms. ${ }^{74,75}$

A number of studies have also examined eczema occurrence in relation to PUFAs. The Generation R study found a higher eczema risk with higher maternal $n-6$ PUFA levels ${ }^{72}$, while the KOALA study reported a lower risk with increasing n6:n-3 fatty acid ratio, but showed no associations with individual PUFAs ${ }^{73}$. The Spanish INMA birth cohort study showed a lower risk of childhood eczema at the age of 14 months with increasing cord blood n-3 PUFAs levels. ${ }^{76}$ Other studies from the UK, Germany, and Singapore showed null associations of prenatal PUFA levels with rates of childhood eczema. ${ }^{71,74,75}$ Only a few studies have examined childhood rhinitis, and found no associations with prenatal PUFA levels. ${ }^{73-75}$

Overall, previous longitudinal studies on prenatal PUFA status cannot unequivocally confirm or reject an association with childhood allergic disease symptoms. Likewise, prior cohort studies on maternal fish intake (reviewed in Chapter 6), and trials of fish oil supplementation in pregnancy ${ }^{77-79}$ have yielded discrepant results. Reasons for the divergent results may be differences in sample sizes, exposure heterogeneity, inconsistencies in outcome definition, or differences in statistical analysis. Given the substantial health burden of childhood allergy, it is important to better characterize the potential role that PUFAs may play in the development of asthma and other allergy-related diseases.

\section{AIM AND HYPOTHESIS OF THE THESIS}

The major aim of the thesis was to identify the association of PUFA status in the prenatal period with childhood growth and health. We also examined prenatal fish intake as an exposure of interest, given that fish is the primary source of $n-3$ long-chain PUFAs in the human diet. We focused on major child health outcomes including obesity and associated cardiometabolic risk, and allergy-like symptoms. Our hypothesis was that n-3 PUFA status and fish intake may exert a beneficial effect on later growth and development, and thus, would be inversely associated with obesity development and cardiometabolic risk traits and with the occurrence of allergy-related phenotypes. We also hypothesized that higher prenatal exposure to n-6 fatty acids would exhibit detrimental effects, and be directly related to childhood obesity and excess cardiometabolic risk, as well as to allergy-related symptoms. 


\section{GENERAL DESIGN}

The studies presented in this thesis were embedded in two population-based prospective cohort studies, the Dutch Maastricht Essential Fatty Acid Birth (MEFAB) and the Greek Mother-Child "RHEA" cohort studies (Figure 1.2A), as well as in an international collaboration project (Figure 1.2B).

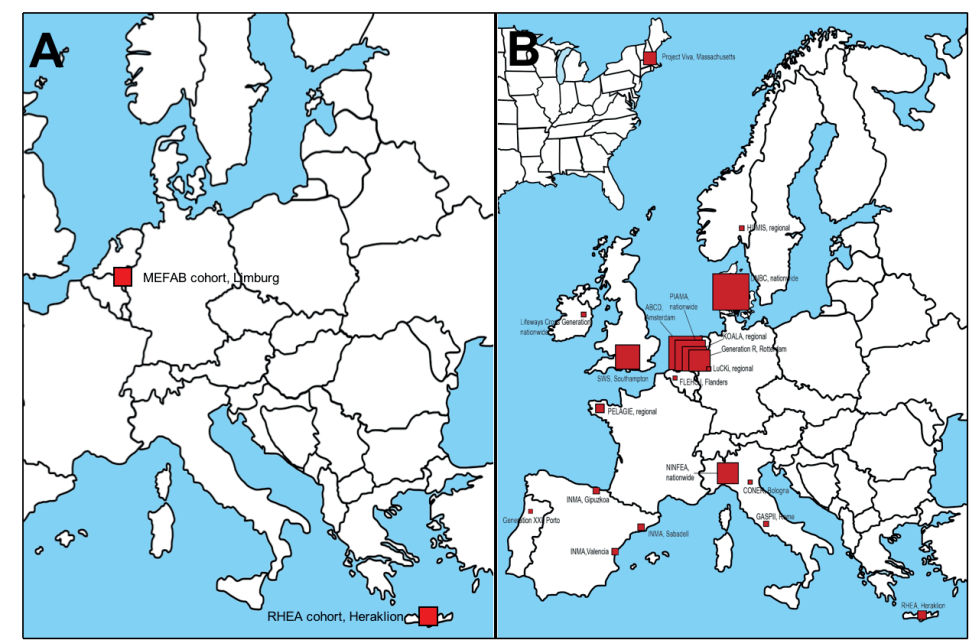

Figure 1.2. Geographic location of A) the MEFAB and RHEA cohorts, and B) the cohorts participating in the international collaboration project.

\section{MEFAB cohort}

The MEFAB study (www.mefab.org) is a population-based prospective cohort study following pregnant women and their children at the province of Limburg, the Netherlands. Between 1989 and 1995, pregnant women attending three maternity clinics in Limburg for their first antenatal visit were invited to participate in the study. Eligibility criteria included having a gestational age of less than 16 weeks, having a diastolic blood pressure of less than $90 \mathrm{mmHg}$, and not suffering from any cardiovascular, neurological, renal, or metabolic disorder. At baseline, 1334 women were enrolled, and a total of 1203 women with singleton pregnancies were followed till delivery. Umbilical cord blood samples were collected from 1008 singletons, and assayed for PUFA concentrations using standard methodology. For the offspring's follow-up, mother-offspring pairs were followed at three waves: wave I, median age 7.2 years (mid-childhood); wave II, median 12.8 years (adolescence); and wave III, median 23.2 years (young adulthood). 
Figure 1.3 demonstrates the timing, and available data in MEFAB that were used for the present thesis. At follow-up wave I, data on length/height and weight measurements were collected during the preschool years (from 6 months until the age of 4 years) from paediatrician records, which form part of standard child care in the Netherlands. At wave I, trained investigators also measured children's current weight, height, waist circumference, systolic and diastolic blood pressure, and collected blood samples. Additionally, information on allergy-related phenotypes was obtained at age 7 with the use of questionnaires adapted from the International Study on Asthma and Allergy in Childhood (ISAAC). At age 12 (wave II), adolescent weight and height was measured following standard methodology, while at the 23-year follow-up evaluation (wave III), self-reported information on weight and height was collected using questionnaires. Information on birth outcomes and several prenatal and postnatal sociodemographic characteristics was collected using face-to-face structured along with self-administered questionnaires and medical records.

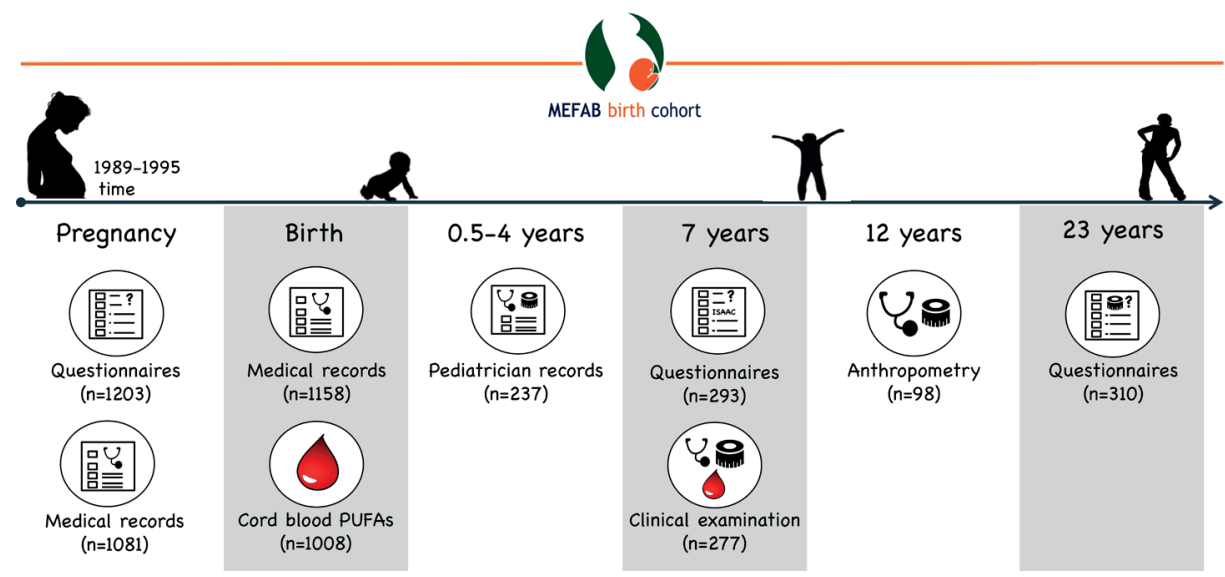

Figure 1.3. Measurement timing and availability of MEFAB data used in the present thesis

\section{Mother-Child "RHEA" cohort}

The Mother-Child "RHEA" cohort study (www.rhea.gr) prospectively examines a population-based sample of pregnant women and their children at the prefecture of Heraklion, Crete, Greece. Pregnant women (Greek and immigrants) residents that became pregnant during 1 year starting in February 2007 were contacted at the time of their first comprehensive ultrasound examination (around week 12 of gestation) at four antenatal clinics, and asked to participate in the study. The inclusion criteria were: residents in the study area; pregnant women older than 16 years; first antenatal visit: hospitals or private clinics in Heraklion district; and no 
communication handicap. A total of 1610 agreed to participate, and 1363 singletons were followed up until delivery. During the birth admission, cord blood samples were collected, and PUFA concentrations were measured for a random sub-sample of 500 singletons. For the child's follow-up, in-person clinical visits with mothers and their children were contacted during infancy (median ages 9 and 18 months), early childhood (median 4.2 years) and mid-childhood (median 6.5 years).

Figure 1.4 demonstrates the timing, and available data in RHEA that were used for the present thesis. Trained research assistants measured weight and height at each follow-up examination using standard procedures. At the 6-year examination, trained research assistants also measured waist circumference waist circumference, systolic and diastolic blood pressure, and collected blood samples. Additionally, information on allergy-related phenotypes was obtained at age 2, 4, and 6 years with the use of questionnaires adapted from ISAAC. Face-to-face questionnaires along with self-administered questionnaires and medical records were used to obtain information on pregnancy outcomes, several sociodemographic characteristics, and dietary habits.

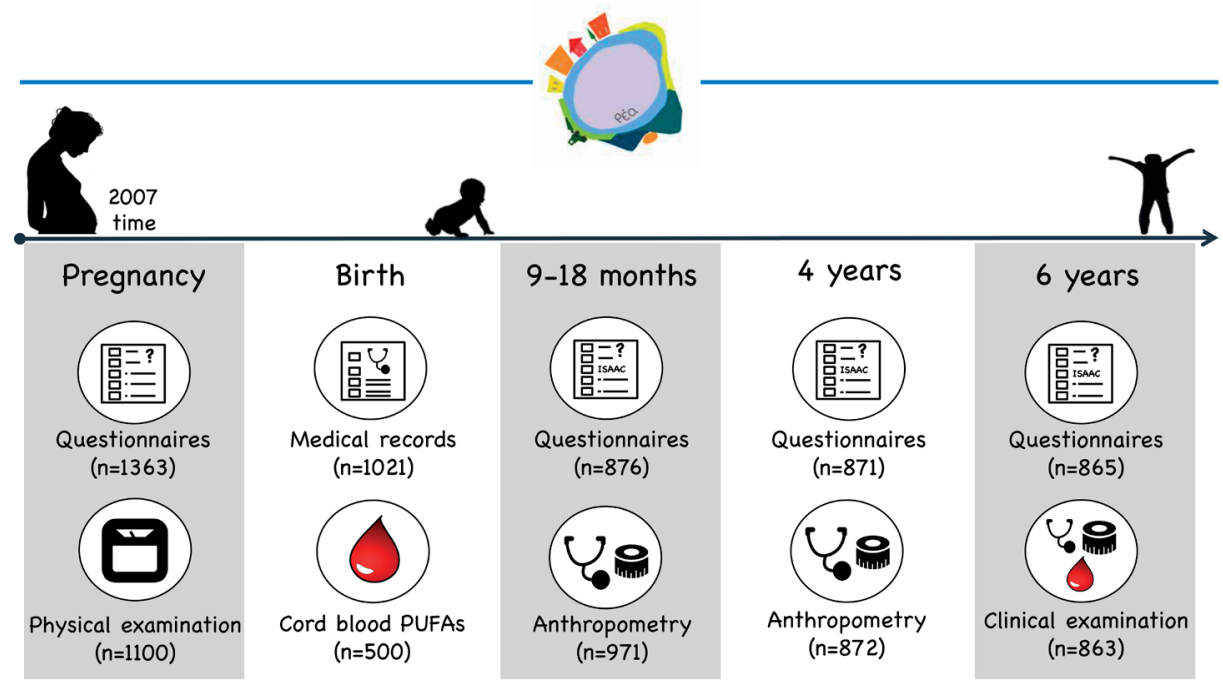

Figure 1.4. Measurement timing and availability of RHEA data used in the present thesis.

\section{International Consortium}

We invited European and US birth cohorts to participate in pooled analyses on the association of maternal fish intake during pregnancy with child growth and respiratory symptoms. Cohorts were able to participate if they included children 
born from 1990 onwards, assessed maternal fish consumption during pregnancy, and had information available on growth measurements or symptoms of asthma and allergic rhinitis during childhood. We selected population-based cohorts from the European Consortium CHICOS (Child Cohort Research Strategy for Europe, www.chicosproject.eu), and inventory of birth cohorts (www.birthcohorts.net), as well as from other existing collaborations.

\section{OUTLINE OF THE THESIS}

In Chapters 2 to 5, we present our studies on PUFA status. Specifically, in Chapter 2, we present a systematic review and meta-analysis of trials on the impact of n-3 long-chain PUFA supplementation in pregnancy and/or lactation on offspring adiposity. In Chapter 3, we harmonized and pooled individual data of the MEFAB and RHEA birth cohorts to assess whether n-3 and n-6 PUFA concentrations in cord blood phospholipids, reflecting fetal exposure in late pregnancy, are associated with rapid growth in infancy, childhood BMI, and cardiometabolic risk phenotype at 6-7 years of age. In Chapter 4, we used long-term longitudinal data of the MEFAB birth cohort and investigated the associations of cord blood phospholipid PUFAs with child-to-adult height and BMI. In Chapter 5, we studied childhood symptoms of allergic disease in relation to cord blood PUFA levels in a pooled analysis of the MEFAB and RHEA cohorts.

Chapters 6 to 8 shift the focus of the thesis to fish intake during pregnancy. In Chapter 6, we present results of prior cohort studies on maternal fish intake during pregnancy and child health outcomes. Next, we present our analyses from the international consortium of cohort studies assessing the strength and consistency of the associations of fish intake during pregnancy with childhood growth and obesity (Chapter 7), and the risk of asthma and allergic rhinitis (Chapter 8). Finally, Chapter 9 provides a general overview and interpretation of the main findings of this thesis, considers general methodological issues applied in our studies, and suggests directions for future research. 


\section{REFERENCES}

1. European Commision. Commission staff working document: Investing in health 2013.

2. Daelmans B, Darmstadt GL, Lombardi J, et al. Early childhood development: the foundation of sustainable development. Lancet 2017;389:9-11.

3. Han JC, Lawlor DA, Kimm SY. Childhood obesity. Lancet 2010;375:1737-48.

4. The Global Asthma Report 2014. Auckland, New Zealand: Global Asthma Network.

5. Meltzer EO. Quality of life in adults and children with allergic rhinitis. J Allergy Clin Immunol 2001;108:S45-53.

6. Cattaneo A, Cogoy L, Macaluso A, Tamburlini G (eds). Child Health in the European Union. 2012. European Comission.

7. $\mathrm{Ng} \mathrm{M}$, Fleming $\mathrm{T}$, Robinson $\mathrm{M}$, et al. Global, regional, and national prevalence of overweight and obesity in children and adults during 1980-2013: a systematic analysis for the Global Burden of Disease Study 2013. Lancet 2014;384:766-81.

8. Lobstein T, Baur L, Uauy R, et al. Obesity in children and young people: a crisis in public health. Obes Rev 2004;5 Suppl 1:4-104.

9. Baird J, Fisher D, Lucas P, et al. Being big or growing fast: systematic review of size and growth in infancy and later obesity. BMJ 2005;331:929.

10. Weiss R, Dziura J, Burgert TS, et al. Obesity and the metabolic syndrome in children and adolescents. N Engl J Med 2004;350:2362-74.

11. Chen W, Srinivasan SR, Li S, et al. Clustering of long-term trends in metabolic syndrome variables from childhood to adulthood in Blacks and Whites: the Bogalusa Heart Study. Am J Epidemiol 2007;166:52733.

12. Reilly JJ, Kelly J. Long-term impact of overweight and obesity in childhood and adolescence on morbidity and premature mortality in adulthood: systematic review. Int J Obes (Lond) 2011;35:891-8.

13. Berenson GS, Srinivasan SR, Bao W, et al. Association between multiple cardiovascular risk factors and atherosclerosis in children and young adults. The Bogalusa Heart Study. N Engl J Med 1998;338:16506.

14. Juonala M, Jarvisalo MJ, Maki-Torkko N, et al. Risk factors identified in childhood and decreased carotid artery elasticity in adulthood: the Cardiovascular Risk in Young Finns Study. Circulation 2005;112:1486-93.

15. Olshansky SJ, Passaro DJ, Hershow RC, et al. A potential decline in life expectancy in the United States in the 21st century. N Engl J Med 2005;352:1138-45.

16. Asher MI, Montefort S, Bjorksten B, et al. Worldwide time trends in the prevalence of symptoms of asthma, allergic rhinoconjunctivitis, and eczema in childhood: ISAAC Phases One and Three repeat multicountry cross-sectional surveys. Lancet 2006;368:733-43.

17. Georas SN, Guo J, De Fanis U, et al. T-helper cell type-2 regulation in allergic disease. Eur Respir J 2005;26:1119-37.

18. Bousquet J, Nikolai Khaltaev N. Global surveillance, prevention and control of chronic respiratory diseases : a comprehensive approach. Geneva, Switzerland: World Health Organization 2007.

19. Weiss KB, Sullivan SD. The health economics of asthma and rhinitis. I. Assessing the economic impact. J Allergy Clin Immunol 2001;107:3-8.

20. Asher I, Pearce N. Global burden of asthma among children. Int J Tuberc Lung Dis 2014;18:1269-78.

21. Tai A, Tran H, Roberts M, et al. Outcomes of childhood asthma to the age of 50 years. J Allergy Clin Immunol 2014;133:1572-8 e3. 
22. Tai A, Tran H, Roberts M, et al. The association between childhood asthma and adult chronic obstructive pulmonary disease. Thorax 2014;69:805-10.

23. Samolinski B, Fronczak A, Wlodarczyk A, et al. Council of the European Union conclusions on chronic respiratory diseases in children. Lancet 2012;379:e45-6.

24. Kopelman PG. Obesity as a medical problem. Nature 2000;404:635-43.

25. Devereux G. The increase in the prevalence of asthma and allergy: food for thought. Nat Rev Immunol 2006;6:869-74.

26. Gluckman PD, Hanson MA, Cooper C, et al. Effect of in utero and early-life conditions on adult health and disease. N Engl J Med 2008;359:61-73.

27. Hanson MA, Gluckman PD. Early developmental conditioning of later health and disease: physiology or pathophysiology? Physiol Rev 2014;94:1027-76.

28. Friedman JE. Obesity and Gestational Diabetes Mellitus Pathways for Programming in Mouse, Monkey, and Man-Where Do We Go Next? The 2014 Norbert Freinkel Award Lecture. Diabetes Care 2015;38:1402-11.

29. Barker DJ. Fetal origins of coronary heart disease. BMJ 1995;311:171-4.

30. Barker DJ. Fetal programming of coronary heart disease. Trends Endocrinol Metab 2002;13:364-8.

31. Roseboom TJ, van der Meulen JH, Ravelli AC, et al. Effects of prenatal exposure to the Dutch famine on adult disease in later life: an overview. Mol Cell Endocrinol 2001;185:93-8.

32. Lopuhaa CE, Roseboom TJ, Osmond C, et al. Atopy, lung function, and obstructive airways disease after prenatal exposure to famine. Thorax 2000;55:555-61.

33. Simopoulos AP. Essential fatty acids in health and chronic disease. Am J Clin Nutr 1999;70:560S-9S.

34. Schmitz G, Ecker J. The opposing effects of n-3 and n-6 fatty acids. Prog Lipid Res 2008;47:147-55.

35. Heird WC, Lapillonne A. The role of essential fatty acids in development. Annu Rev Nutr 2005;25:54971.

36. Burr GO, Burr MM. Nutrition classics from The Journal of Biological Chemistry 82:345-67, 1929. A new deficiency disease produced by the rigid exclusion of fat from the diet. Nutr Rev 1973;31:248-9.

37. Hansen AE, Stewart RA, Hughes G, et al. The relation of linoleic acid to infant feeding. Acta Paediatr Suppl 1962;137:1-41.

38. Calder PC. n-3 polyunsaturated fatty acids, inflammation, and inflammatory diseases. Am J Clin Nutr 2006;83:1505S-19S.

39. Ailhaud G, Guesnet P, Cunnane SC. An emerging risk factor for obesity: does disequilibrium of polyunsaturated fatty acid metabolism contribute to excessive adipose tissue development? Br J Nutr 2008;100:461-70.

40. Brenna JT, Salem N, Jr., Sinclair AJ, et al. alpha-Linolenic acid supplementation and conversion to n-3 long-chain polyunsaturated fatty acids in humans. Prostaglandins Leukot Essent Fatty Acids 2009;80:85-91.

41. Wijendran V, Hayes KC. Dietary n-6 and n-3 fatty acid balance and cardiovascular health. Annu Rev Nutr 2004;24:597-615.

42. Brenna JT, Salem N, Jr. Workshop proceedings: DHA as a required nutrient. Prostaglandins Leukot Essent Fatty Acids 2009;81:97.

43. Wang X, Lin H, Gu Y. Multiple roles of dihomo-gamma-linolenic acid against proliferation diseases. Lipids Health Dis 2012;11:25.

44. Larque E, Demmelmair H, Gil-Sanchez A, et al. Placental transfer of fatty acids and fetal implications. Am J Clin Nutr 2011;94:1908S-13S.

45. Haggarty P. Fatty acid supply to the human fetus. Annu Rev Nutr 2010;30:237-55. 
46. Duttaroy AK. Transport of fatty acids across the human placenta: a review. Prog Lipid Res 2009;48:5261.

47. Ailhaud G, Massiera F, Weill P, et al. Temporal changes in dietary fats: role of n-6 polyunsaturated fatty acids in excessive adipose tissue development and relationship to obesity. Prog Lipid Res 2006;45:20336.

48. Kalupahana NS, Claycombe KJ, Moustaid-Moussa N. (n-3) Fatty acids alleviate adipose tissue inflammation and insulin resistance: mechanistic insights. Adv Nutr 2011;2:304-16.

49. Calder PC. Long-chain fatty acids and inflammation. Proc Nutr Soc 2012;71:284-9.

50. Kruger MC, Coetzee M, Haag M, et al. Long-chain polyunsaturated fatty acids: selected mechanisms of action on bone. Prog Lipid Res 2010;49:438-49.

51. Much D, Brunner S, Vollhardt C, et al. Effect of dietary intervention to reduce the n-6/n-3 fatty acid ratio on maternal and fetal fatty acid profile and its relation to offspring growth and body composition at 1 year of age. Eur J Clin Nutr 2013;67:282-8.

52. Innis SM, Friesen RW. Essential n-3 fatty acids in pregnant women and early visual acuity maturation in term infants. Am J Clin Nutr 2008;87:548-57.

53. Dunstan JA, Simmer K, Dixon G, et al. Cognitive assessment of children at age 2(1/2) years after maternal fish oil supplementation in pregnancy: a randomised controlled trial. Arch Dis Child Fetal Neonatal Ed 2008;93:F45-50.

54. Stein AD, Wang M, Martorell R, et al. Growth to age 18 months following prenatal supplementation with docosahexaenoic acid differs by maternal gravidity in Mexico. J Nutr 2011;141:316-20.

55. Vidakovic AJ, Gishti O, Voortman T, et al. Maternal plasma PUFA concentrations during pregnancy and childhood adiposity: the Generation R Study. Am J Clin Nutr 2016;103:1017-25.

56. de Vries PS, Gielen M, Rizopoulos D, et al. Association between polyunsaturated fatty acid concentrations in maternal plasma phospholipids during pregnancy and offspring adiposity at age 7: the MEFAB cohort. Prostaglandins Leukot Essent Fatty Acids 2014;91:81-5.

57. Moon RJ, Harvey NC, Robinson SM, et al. Maternal plasma polyunsaturated fatty acid status in late pregnancy is associated with offspring body composition in childhood. J Clin Endocrinol Metab 2013;98:299-307.

58. Donahue SM, Rifas-Shiman SL, Gold DR, et al. Prenatal fatty acid status and child adiposity at age 3 y: results from a US pregnancy cohort. Am J Clin Nutr 2011;93:780-8.

59. Standl M, Thiering E, Demmelmair H, et al. Age-dependent effects of cord blood long-chain PUFA composition on BMI during the first 10 years of life. Br J Nutr 2014;111:2024-31.

60. Rump P, Popp-Snijders C, Heine RJ, et al. Components of the insulin resistance syndrome in seven-yearold children: relations with birth weight and the polyunsaturated fatty acid content of umbilical cord plasma phospholipids. Diabetologia 2002;45:349-55.

61. Vidakovic AJ, Jaddoe VW, Voortman T, et al. Maternal plasma polyunsaturated fatty acid levels during pregnancy and childhood lipid and insulin levels. Nutr Metab Cardiovasc Dis 2017;27:78-85.

62. Vidakovic AJ, Gishti O, Steenweg-de Graaff J, et al. Higher Maternal Plasma n-3 PUFA and Lower n-6 PUFA Concentrations in Pregnancy Are Associated with Lower Childhood Systolic Blood Pressure. $J$ Nutr 2015;145:2362-8.

63. Rytter D, Bech BH, Halldorsson T, et al. No association between the intake of marine n-3 PUFA during the second trimester of pregnancy and factors associated with cardiometabolic risk in the 20-year-old offspring. BrJ Nutr 2013;110:2037-46.

64. Gutierrez-Gomez Y, Stein AD, Ramakrishnan U, et al. Prenatal Docosahexaenoic Acid Supplementation Does Not Affect Nonfasting Serum Lipid and Glucose Concentrations of Offspring at 4 y of Age in a Follow-Up of a Randomized Controlled Clinical Trial in Mexico. J Nutr 2016. 
65. Rytter D, Schmidt EB, Bech BH, et al. Fish Oil Supplementation During Late Pregnancy Does Not Influence Plasma Lipids or Lipoprotein Levels in Young Adult Offspring. Lipids 2011;46:1091-9.

66. Rytter D, Christensen JH, Bech BH, et al. The effect of maternal fish oil supplementation during the last trimester of pregnancy on blood pressure, heart rate and heart rate variability in the 19-year-old offspring. Br J Nutr 2012;108:1475-83.

67. Black PN, Sharpe S. Dietary fat and asthma: is there a connection? Eur Respir J 1997;10:6-12.

68. Calder PC, Kremmyda LS, Vlachava M, et al. Is there a role for fatty acids in early life programming of the immune system? Proc Nutr Soc 2010;69:373-80.

69. Calder PC, Krauss-Etschmann S, de Jong EC, et al. Early nutrition and immunity - progress and perspectives. Br J Nutr 2006;96:774-90.

70. Pike KC, Calder PC, Inskip HM, et al. Maternal Plasma Phosphatidylcholine Fatty Acids and Atopy and Wheeze in the Offspring at Age of 6 Years. Clin Dev Immunol 2012;2012:474613.

71. Newson RB, Shaheen SO, Henderson AJ, et al. Umbilical cord and maternal blood red cell fatty acids and early childhood wheezing and eczema. J Allergy Clin Immunol 2004;114:531-7.

72. Rucci E, den Dekker HT, de Jongste JC, et al. Maternal fatty acid levels during pregnancy, childhood lung function and atopic diseases. The Generation R Study. Clin Exp Allergy 2016;46:461-71.

73. Notenboom ML, Mommers M, Jansen EH, et al. Maternal fatty acid status in pregnancy and childhood atopic manifestations: KOALA Birth Cohort Study. Clin Exp Allergy 2011;41:407-16.

74. Standl M, Demmelmair H, Koletzko B, et al. Cord blood LC-PUFA composition and allergic diseases during the first 10 yr. Results from the LISAplus study. Pediatr Allergy Immunol 2014;25:344-50.

75. Yu YM, Chan YH, Calder PC, et al. Maternal PUFA status and offspring allergic diseases up to the age of 18 months. Br J Nutr 2015;113:975-83.

76. Montes R, Chisaguano AM, Castellote AI, et al. Fatty-acid composition of maternal and umbilical cord plasma and early childhood atopic eczema in a Spanish cohort. Eur J Clin Nutr 2013;67:658-63.

77. Best KP, Gold M, Kennedy D, et al. Omega-3 long-chain PUFA intake during pregnancy and allergic disease outcomes in the offspring: a systematic review and meta-analysis of observational studies and randomized controlled trials. Am J Clin Nutr 2016;103:128-43.

78. Hansen S, Strom M, Maslova E, et al. Fish oil supplementation during pregnancy and allergic respiratory disease in the adult offspring. J Allergy Clin Immunol 2017;139:104-111.e4.

79. Best KP, Sullivan T, Palmer D, et al. Prenatal Fish Oil Supplementation and Allergy: 6-Year Follow-up of a Randomized Controlled Trial. Pediatrics 2016;137. 



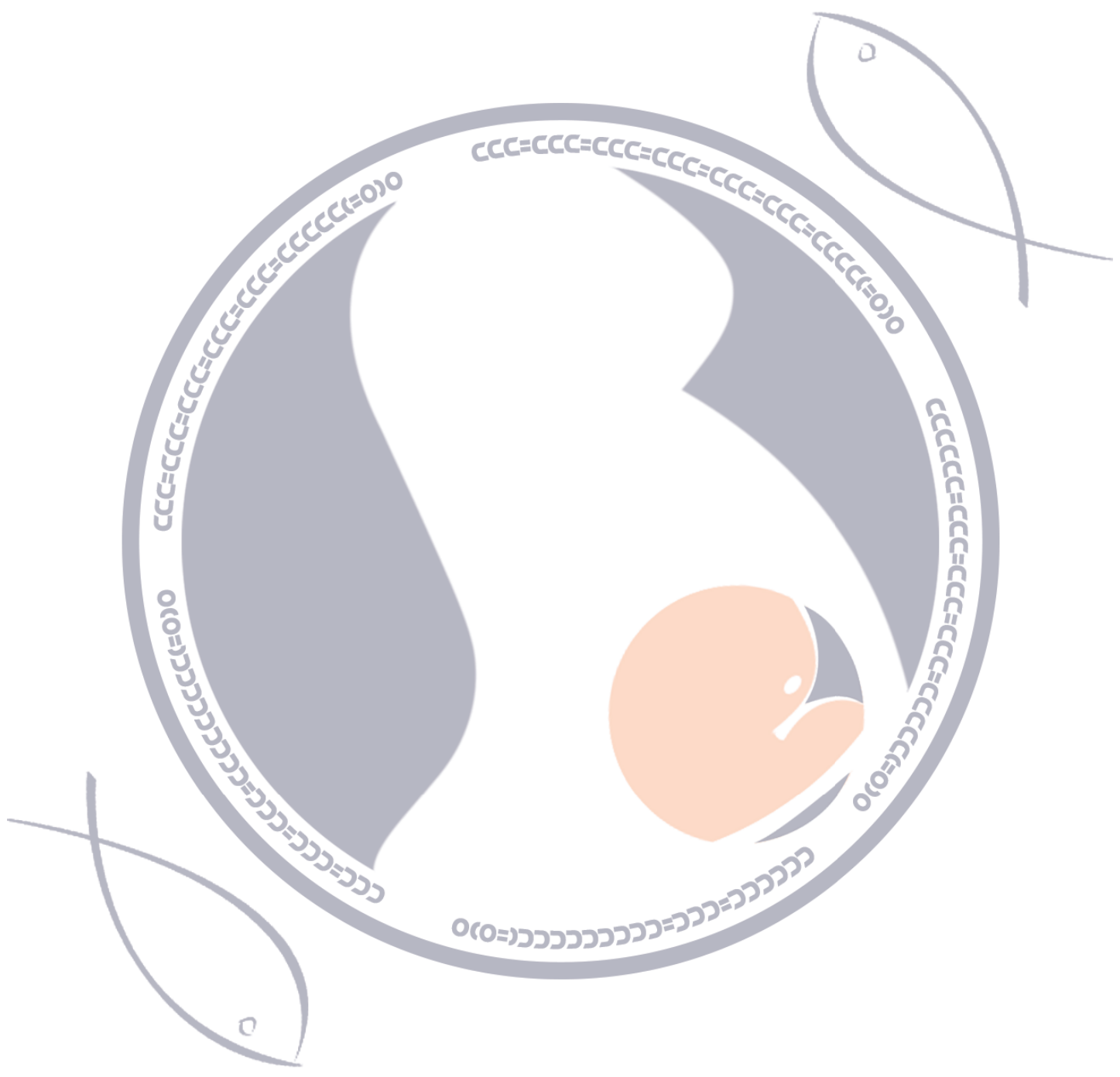




\section{CHAPTER 2}

Effect of maternal n-3 long-chain polyunsaturated fatty acid supplementation during pregnancy and/or lactation on adiposity in childhood: a systematic review and metaanalysis of randomized controlled trials

N Stratakis, M Gielen, L Chatzi, MP Zeegers

Adapted from Eur J Clin Nutr 2014 Dec;68(12):1277-1287 


\section{ABSTRACT}

Background: It is hypothesized that prenatal and early postnatal exposure to $n-3$ long-chain polyunsaturated fatty acids (LCPUFAs) is negatively associated with adiposity later in life.

Objective: We conducted a systematic review and meta-analysis to evaluate whether maternal n-3 LCPUFA supplementation in pregnancy and/or lactation exerts a beneficial effect on adiposity status in childhood.

Methods: We searched six electronic databases till May 20, 2014 for randomized controlled trials (RCTs) of n-3 LCPUFA supplementation to pregnant and/or lactating women that reported data on body mass index (BMI), waist circumference, sum of skinfold thicknesses, or body fat mass in children. Adiposity measures were grouped into three age categories: preschool children ( $<5$ years), school-aged children (6-12 years), and adolescents ( $>13$ years). Trial quality was assessed. We conducted fixed-effect and random-effects meta-analyses to combine studyspecific estimates of differences between the supplemented and control groups.

Results: A total of six RCTs (9 publications) involving 2847 participants were included. Summary estimates showed no effect of maternal supplementation on BMI in preschool (standardized mean difference $[S M D]=0.07[95 \% \mathrm{CI}-0.22$ to $0.36] ; \mathrm{P}=0.65)$ and school-aged children $(\mathrm{SMD}=0.12$ [95\% CI -0.06 to 0.30$]$; $\mathrm{P}=0.20$ ). Due to sparse data, it was not possible to pool study results relating to other adiposity measures.

Conclusions: There is currently no evidence to support that n-3 LCPUFA supplementation during pregnancy and/or lactation favourably affects child adiposity. Further high-quality trials are needed. 


\section{INTRODUCTION}

Excess adiposity in childhood represents a major health concern in the Western world. It is estimated that approximately $30 \%$ of children and adolescents in the US and about $15-30 \%$ of those in Europe can be classified as overweight or obese, according to the body mass index (BMI) cut-offs proposed by the International Obesity Taskforce. ${ }^{1,2}$ The vascular and metabolic disturbances associated obesity can be already seen at young ages, ${ }^{3}$ and accumulating evidence suggests that childhood obesity strongly predicts adult morbidity, particularly cardiometabolic morbidity, and premature mortality. ${ }^{4}$

Multiple factors are likely to contribute to obesity development. ${ }^{5,6}$ An increasing body of evidence now suggests that the nutritional environment encountered in utero and the early postnatal life may elicit permanent alterations in adipose tissue structure or function and, thereby, programme the individual's propensity to later obesity. ${ }^{7,8}$ In this context, there has been considerable interest in the potential programming effect of fatty acid composition of the diet of pregnant and breastfeeding women, and particularly the ratio of n-6 to n-3 long-chain polyunsaturated fatty acids (LCPUFAs). The composition of fatty acids in the Western diets has shifted toward an increasing dominance of n-6 relative to $n-3$ LCPUFAs over the past decades. ${ }^{9,10}$ This shift is also reflected in the fatty acid composition of breast milk. ${ }^{9}$ Evidence from animal studies suggests that the n- 6 LCPUFA arachidonic acid (AA) promotes adipose tissue deposition, whereas the n-3 LCPUFAs eicosapentaenoic acid (EPA) and docosahexaenoic acid (DHA) seem to exert an opposite effect. Hence, it has been hypothesized that increasing the intake of n-3 LCPUFAs during the early development of adipose tissue may help prevent childhood obesity. ${ }^{11}$

Some prospective cohort studies have shown a beneficial effect of prenatal or early postnatal exposure to n-3 LCPUFAs on later adiposity, ${ }^{12,13}$ whereas others have failed to find such an effect. ${ }^{14,15}$ Randomized controlled trials (RCTs) of maternal n-3 LCPUFA supplementation during pregnancy and/or lactation have also yielded conflicting results, and most of them had only modest sample sizes. Two systematic reviews have attempted to evaluate this body of literature. ${ }^{16,17}$ However, these reviews have carried out only a qualitative assessment of trial results. Besides that, the reviews have not included many recently published trials. ${ }^{18-20} \mathrm{~A}$ comprehensive systematic review of RCTs for which results are quantified in a meta-analysis is needed to enhance the precision of the estimated supplementation effect.

Thus, we conducted a systematic review in accordance with the Cochrane methodology $^{21}$ to evaluate whether $\mathrm{n}-3$ LCPUFA supplementation, as compared with a control regimen, in pregnancy and/or lactation exerts a beneficial effect on adipos- 
ity status in childhood. The primary outcome of interest was BMI. Secondary outcomes of interest included body fat mass, waist circumference, and sum of skinfold thicknesses.

\section{METHODS}

We followed the PRISMA (Preferred Reporting Items for Systematic Reviews and Meta-Analyses) guidelines ${ }^{22}$ for this review (Supplementary Material of Chapter 2). The protocol of this review has not been published.

\section{Search strategy}

The Pubmed (http://www.ncbi.nlm.nih.gov/pubmed; since 1966), EMBASE (http://www.ovid.com; since 1974), Cumulative Index to Nursing and Allied Health Literature (CINAHL; http://www.ebscohost.com/academic/cinahlplus-with-full-text; since 1981), Cochrane Central Register of Controlled Trials (CENTRAL; Issue 3, 2014), Google Scholar (http://www.scholar.google.com), and Clinicaltrials.gov (http://www.clinicaltrials.gov) databases were searched till May 20, 2014 for relevant articles evaluating the association between n-3 LCPUFA supplementation in pregnancy and/or lactation with child adiposity. Searches were tailored to each database and combined various terms related to n-3 LCPUFAs (eg, fish oil, omega-3 fatty acids, essential fatty acids, docosahexaenoic acid, eicosapentaenoic acid), pregnancy or lactation (eg, intrauterine, fetus, antenatal, postnatal, prenatal, perinatal, breastfeeding), adiposity status (eg, fat mass, anthropometry, body mass index, skinfold thickness, waist, obesity) and study design (eg, randomized, placebo, clinical trial) by using controlled vocabulary and text words. See Supplementary Material of Chapter 2 for the complete search strategy. Furthermore, Google Scholar and the Science Citation Index were searched in order to find highly cited publications relevant to the hypothesis under study. Reference lists of eligible articles and previous reviews identified from this search were also scanned to reveal other potentially relevant trials. No language or publication restrictions were applied, although searches were limited to human studies.

\section{Study selection}

Studies were eligible for inclusion if they were RCTs of supplementation with n-3 LCPUFAs, as compared with a control regimen, to pregnant and/or lactating women and reported data on at least one of the outcomes of interest in healthy children. The primary outcome of interest was BMI. Secondary outcomes of interest included body fat mass, waist circumference, and sum of skinfold thicknessess. 
Trials including co-interventions were considered only if co-interventions were balanced between the study groups. Titles, abstracts, and then full-text reports of potentially relevant articles were assessed for inclusion independently by 2 review authors (NS and MG), and any discrepancies were resolved through discussion.

\section{Data extraction and assessment of trial quality}

For each eligible study, two review authors (NS and MG) independently extracted the following data: general information (published/unpublished, title, authors, journal citation, contact address, country of origin, language, year of publication, notable conflicts of interest of trial authors); trial characteristics (design, methods used for sequence generation and allocation concealment, blinding); participant characteristics (inclusion/exclusion criteria, total number in intervention and control groups, age, withdrawals/losses to follow-up, comparability of groups, intention-to-treat analysis); intervention and control regimen (type, dose, timing of delivery, duration); and adiposity outcomes (means and SDs at the end of follow-up for the intervention and control groups). Inconsistencies were checked and resolved through discussion. Additional information was requested, ${ }^{23-25}$ and provided only from Asserhoj et al. ${ }^{24}$

Two authors (NS and MG) independently assessed the quality of included trials by using the following criteria: randomization technique, concealment of allocation, blinding, and loss to follow-up for each class of outcomes. ${ }^{22,26,27} \mathrm{We}$ categorized each criterion as adequate, inadequate, or unclear. Randomization was considered adequate when a random process was used to generate the sequences (eg, computer-generated random numbers), and inadequate when any nonrandom process was used (eg, case record numbers). Allocation concealment was considered adequate when the concealment process did not allow investigators and participants to identify the upcoming assignments (eg, sequentially number, opaque, sealed envelopes), and inadequate when inappropriate methods were used (eg, unsealed or non-opaque envelopes). Blinding was considered adequate when participants and outcome assessors were not aware of the group allocation, and inadequate when the group allocation was known to participants or outcome assessors. Loss to follow-up was considered adequate when missing outcome data were less than $20 \%$ or balanced between groups, and inadequate when missing outcome data were imbalanced between groups. Any disagreements were resolved through discussion, or when necessary, through consultation with a third reviewer (MPZ). 


\section{Data synthesis and analysis}

To take into account growth and maturation during childhood, each adiposity measure (BMI, body fat mass, waist circumference, and sum of skinfold thicknesses) was assessed according to the following age categories in children: preschool children ( $<5$ years), school-aged children (6-12 years), and adolescents ( $>13$ years). The effect size of maternal n-3 LCPUFA supplementation was defined as the difference in each adiposity measure at the end of follow-up between children in the supplemented group and those in the control group. We present effect estimates as mean difference (MD) and as standardized mean difference (SMD) when BMI was reported with different metrics between studies, with their corresponding $95 \%$ CIs. Owing to the paucity of available data, we pooled study results relating to BMI only for preschool ( $<5$ years) and school-aged (6-12 y) children. Additionally, it was not possible to combine study results relating to the other adiposity measures, and a summary of the effect sizes in individual studies was presented.

Heterogeneity of the effect sizes for BMI between studies was assessed by using the $\mathrm{Q}$ test and $\mathrm{I}^{2}$ statistic, which indicates the proportion of variability in the pooled estimate that is attributable to heterogeneity. ${ }^{28,29}$ If the $\mathrm{P}$ value of $\mathrm{Q}$ test was below 0.10 , or $\mathrm{I}^{2}$ exceeded $50 \%$, we considered heterogeneity to be substantial and used a random-effects meta-analysis to model it; otherwise, a fixed-effect meta-analysis was used. We planned to investigate whether there were differential effects of maternal n-3 LCPUFA supplementation by the timing of intervention (pregnancy, pregnancy and lactation, or lactation), maternal pre-pregnancy or booking BMI (underweight, normal weight, overweight, or obese), birth weight (low, normal, or high) or maturity (preterm-born or term-born children). Because of the limited available data, subgroup analyses were based only on the timing of intervention. Interaction tests were used to assess subgroup differences. ${ }^{30} \mathrm{To}$ examine the potential effects of n-3 LCPUFA dose and age on BMI, we performed meta-regression analysis. ${ }^{31}$ We planned to carry out sensitivity analysis on the basis of trial quality by excluding the trials rated as inadequate or unclear for randomization, allocation concealment, blinding or loss to follow-up; however, all trials were judged to be inadequate or unclear for at least one of these criteria. We examined whether any single study exerted an undue influence on the overall pooled estimate by recalculating the pooled estimates while omitting one study at a time (influence analysis). The potential for publication bias was assessed with the use of Egger's test ${ }^{32}$ and the Duval and Tweedie nonparametric "trim and fill" method. ${ }^{33}$ The "trim and fill" method estimates the number of hypothetical missing studies in a meta-analysis and the effect that these studies might have had on the summary estimate. ${ }^{33}$ Statistical analyses were performed by using STATA, 
version 13.0 (StataCorp, College Station, TX, USA). Statistical significance was set at $\mathrm{P}<0.05$, unless otherwise stated.

\section{RESULTS}

\section{Study selection}

Figure 2.1 shows the study selection process. The literature search yielded of a total of 2371 citations, 2347 of which were excluded after titles and abstracts were screened using general criteria. The full text of 24 articles was reviewed. One additional citation was identified from reference lists of previously published systematic reviews. Sixteen articles were further excluded for being a duplicate report from the same study population $(n=1)$, not reporting any of the outcomes of interest $(n=7)$, applying an additional treatment only in the experimental group $(n=5)$, or being a review article $(n=3)$. Further details of the excluded studies are presented in Table 2.1. A total of nine reports from six RCTs were included in this review. $^{18-20,23-25,34-36}$

Table 2.1. Characteristics of excluded studies

\begin{tabular}{|c|c|}
\hline Study, reference & Reason for exclusion \\
\hline Helland et al., ${ }^{39,58}$ & Did not report any of our defined outcomes \\
\hline Dunstan et al., ${ }^{59}$ & Did not report any of our defined outcomes \\
\hline Hauner et al., ${ }^{53,60}$ & Applied an additional treatment only in the intervention group \\
\hline Muhlhausler et al., ${ }^{17}$ & Review article \\
\hline Ramakrishnan et al., ${ }^{61}$ & Conference abstract; reported the same population as in another study ${ }^{19}$ \\
\hline Courville et al., ${ }^{62}$ & Did not report any of our defined outcomes \\
\hline Rodríguez et al., ${ }^{16}$ & Review article \\
\hline Much et al., ${ }^{63,64}$ & $\begin{array}{l}\text { Applied an additional treatment only in the intervention group; reported the } \\
\text { same population as in another trial }\end{array}$ \\
\hline Hauner et al., ${ }^{65}$ & Review article \\
\hline Escolano-Margarit et al., ${ }^{66}$ & Did not report any of our defined outcomes \\
\hline Brunner et al., ${ }^{67}$ & $\begin{array}{l}\text { Applied an additional treatment only in the intervention group; reported the } \\
\text { same population as in another trial }{ }^{60}\end{array}$ \\
\hline Catalano et al., ${ }^{68}$ & Did not report any of our defined outcomes; measured fetal adiposity \\
\hline Parisi et al., ${ }^{69}$ & Did not report any of our defined outcomes; measured fetal adiposity \\
\hline
\end{tabular}




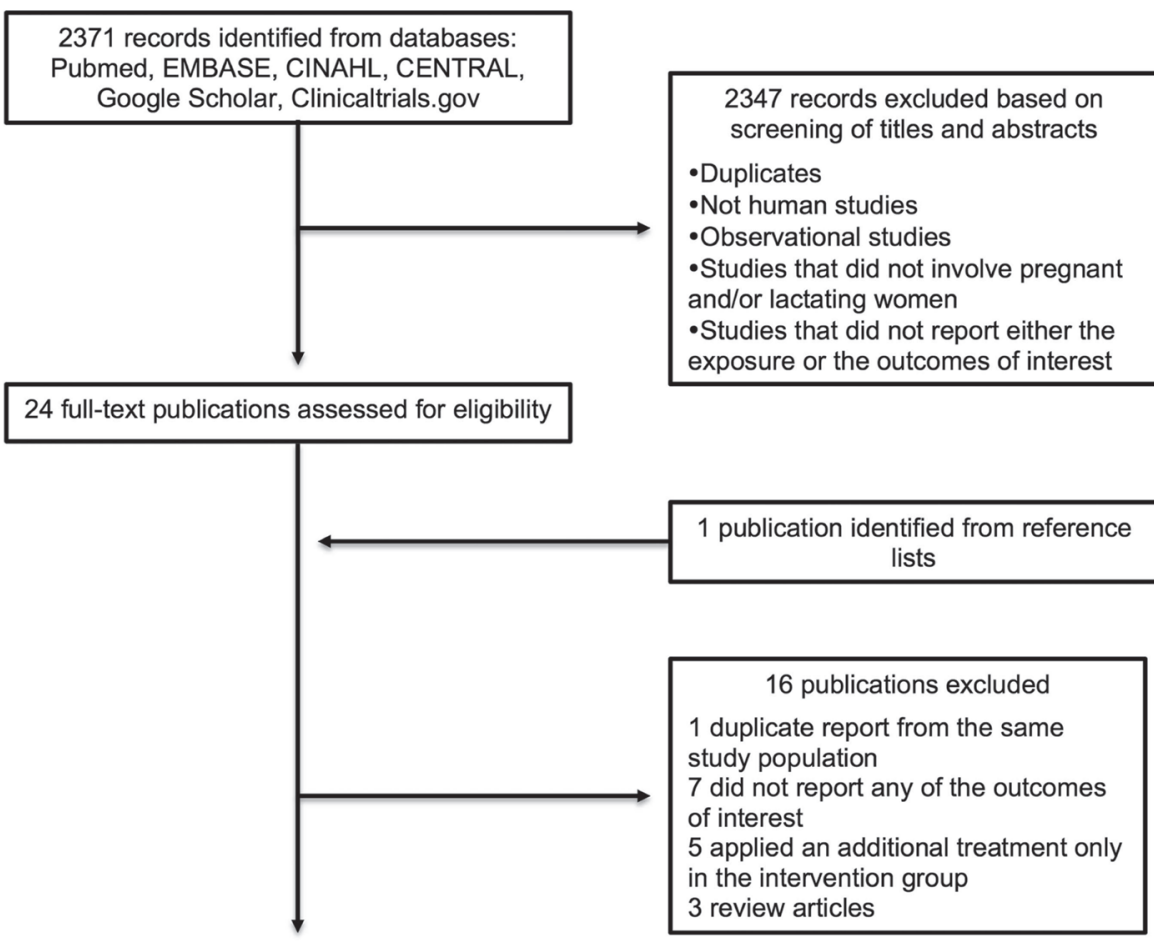

9 publications from 6 RCTs included in qualitative synthesis

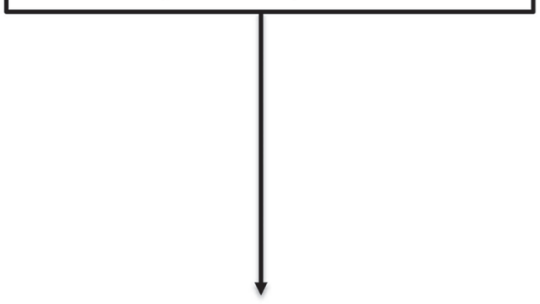

9 studies measured BM

2 studies measured waist circumference

2 studies measured the sum of skinfold

thicknesses

1 study measured body fat mass

Figure 2.1. Flow chart describing the process of study selection in the systematic review

\section{Description of included trials}

A summary of the characteristics of included trials is presented in Table 2.2. The six trials involved a total of 2847 participants. In all trials, randomization was performed at the individual level. Five of the six trials were carried out in Europe. Of 
these, two were conducted in Denmark, ${ }^{37,38}$ one each in Germany ${ }^{23}$ and Norway, ${ }^{39}$ and another was a multicentre trial conducted in Germany, Spain, and Hungary. ${ }^{40}$ The remaining trial ${ }^{41}$ was conducted in Mexico. The main inclusion criteria were women with a healthy singleton pregnancy, while three trials excluded infants born premature or with complications requiring special attention. ${ }^{23,37,39}$ All studies performed a per-protocol analysis.

The trials differed in the intervention period. The intervention was provided during pregnancy in three trials, beginning in either week $20^{40,41}$ or week $30^{38}$ of gestation. In two trials, the intervention period extended from 18 weeks ${ }^{39}$ or 21 weeks $^{23}$ of pregnancy to three months postpartum. Investigators in the remaining trial supplemented women during lactation only, and particularly during the first four months after delivery. ${ }^{37}$

The experimental groups were supplemented with DHA alone ${ }^{41}$ or in combination with EPA. ${ }^{23,37-40}$ The dose of $n-3$ LCPUFAs used in trials ranged from $260^{23}$ to $2200 \mathrm{mg} /$ day ${ }^{38}$ One trial had a $2 \times 2$ factorial design $;^{40}$ the efficacy of $\mathrm{n}-3$ LCPUFAs in the meta-analysis was examined by comparing adiposity measures in children of women supplemented with n-3 LCPUFAs (with or without folate) with those in children of women not supplemented with these fatty acids (folate only or placebo). In the remainder of trials, the control group received a vegetable oil (olive or corn oil) ${ }^{37-39,41}$ or a basic multivitamin/multi-mineral supplement with or without probiotics. ${ }^{23}$

\section{Study quality}

An overview of the quality of each included trial is shown in Table 2.3. All trials used independent processes for generating the randomization sequence. The method used to conceal allocation was adequate in three trials ${ }^{37,38,41}$ and unclear in the remaining trials. ${ }^{23,39,40}$ All trials adequately blinded the participants and outcome assessors, except for one, in which it was unclear whether outcome assessors were unaware of group allocation. ${ }^{24}$ Loss to follow-up for the intervention and control groups was described in all but one study. ${ }^{23}$ One study had a follow-up rate of $80 \% .{ }^{18}$ In the remainder of studies, attrition ranged from 32.5 to $75.8 \%{ }^{19,20,24,25,34-36}$ This resulted in selective loss from the intervention groups in four studies. ${ }^{20,24,25,34}$ Dropout rates were similar between the intervention and control groups in three studies; however, there was evidence of differences in sociodemographic characteristics between the groups or between participants who were followed successfully and those who were lost to follow-up. ${ }^{19,35,36}$ 


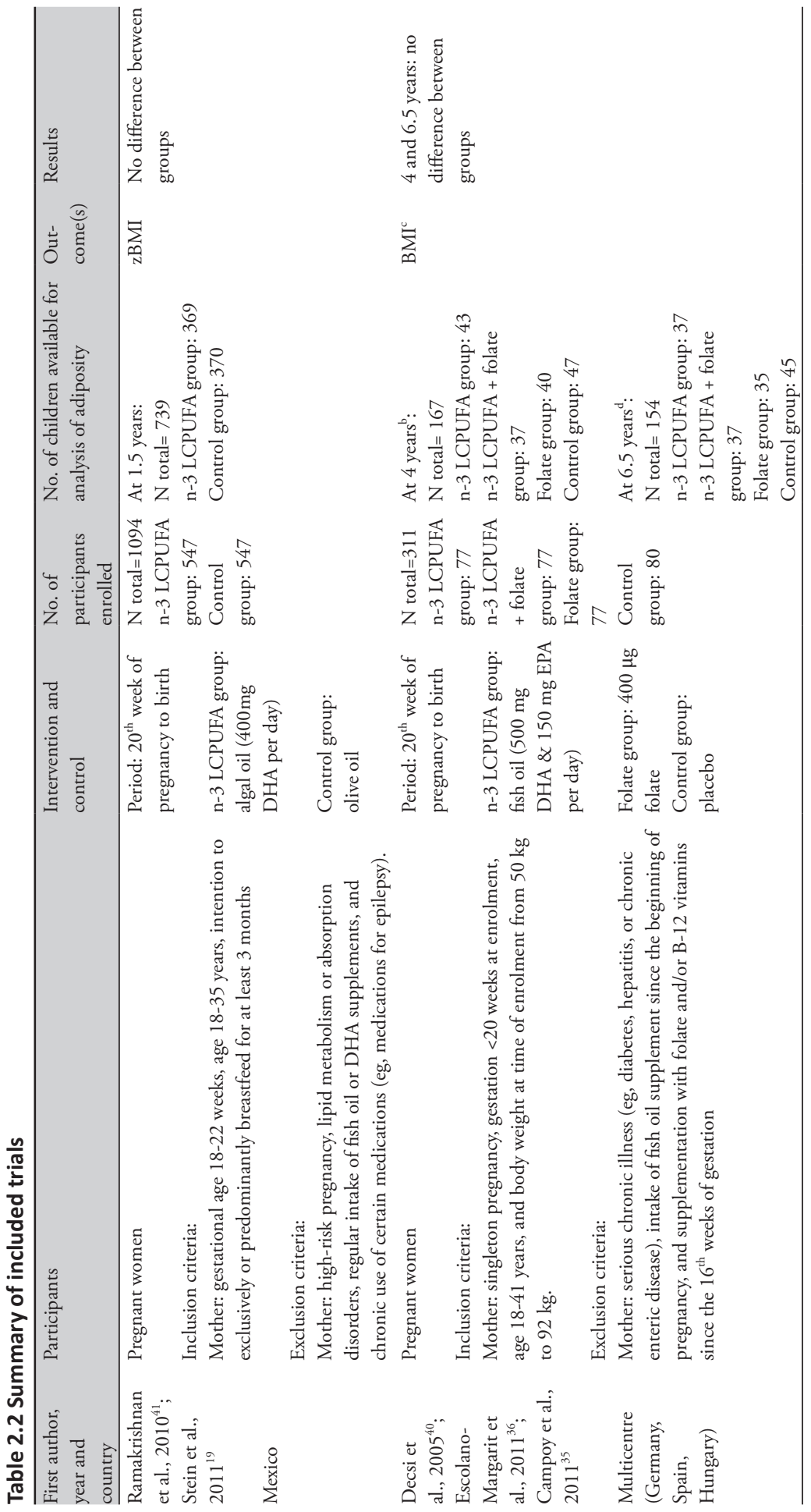




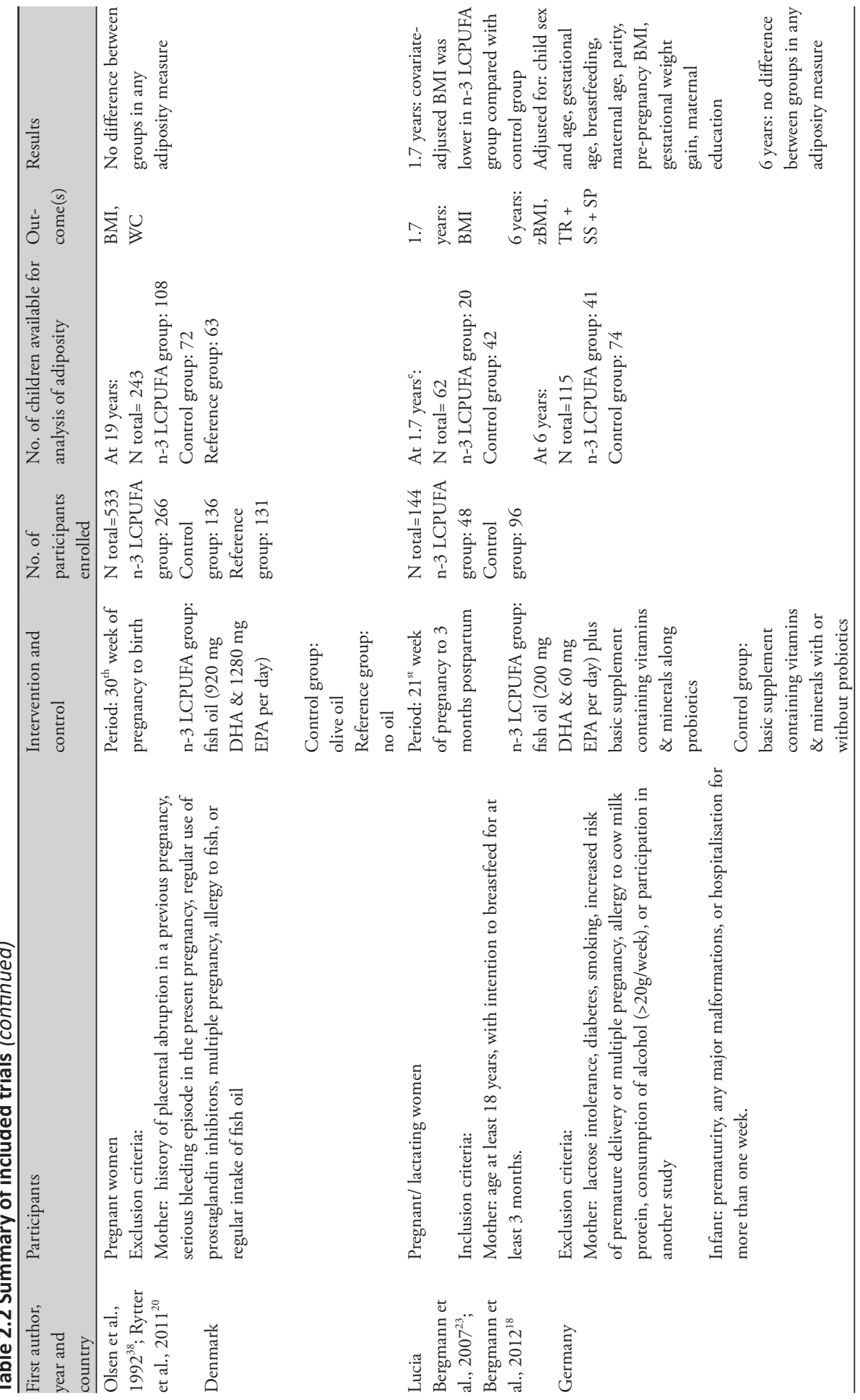




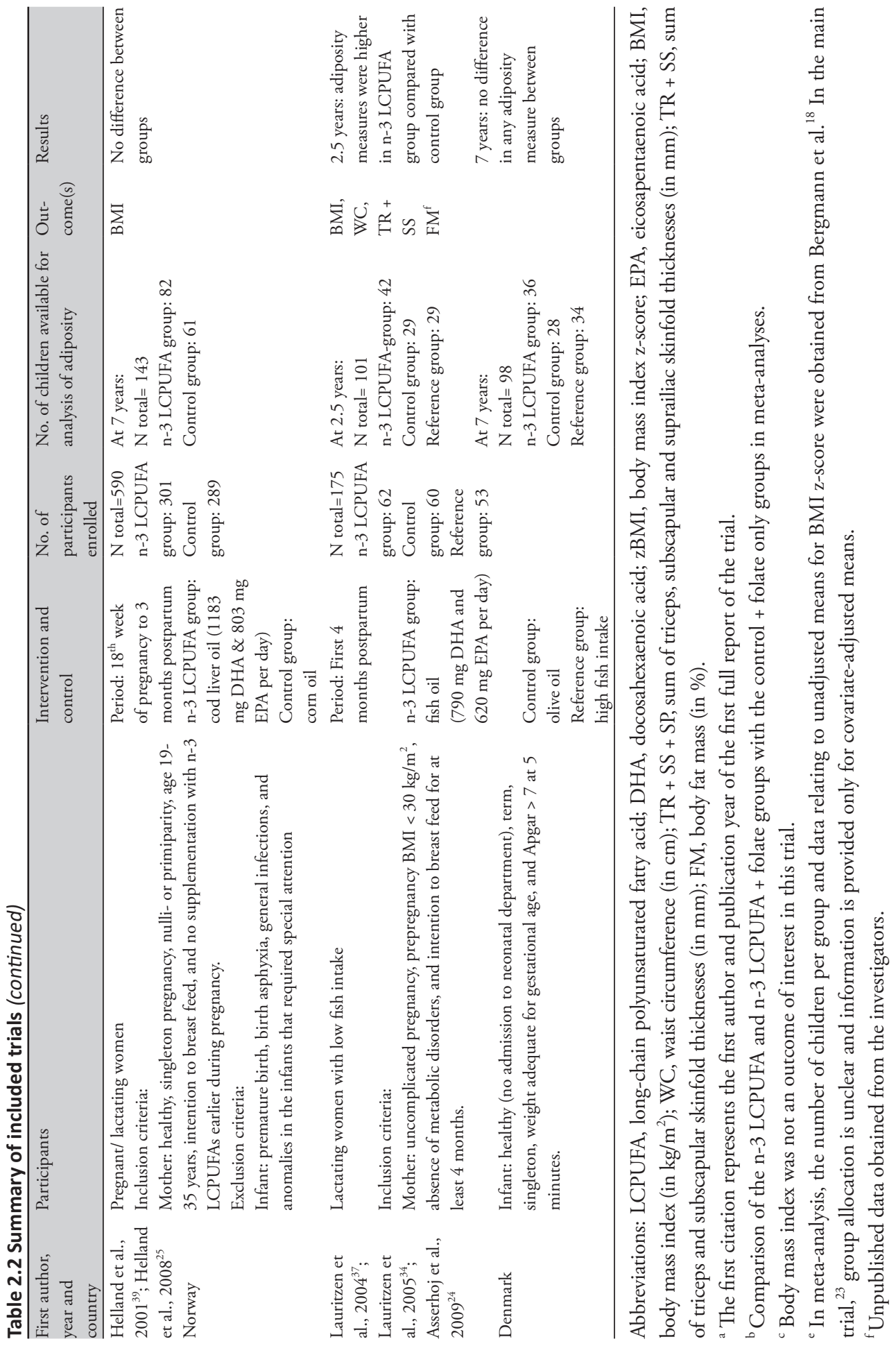




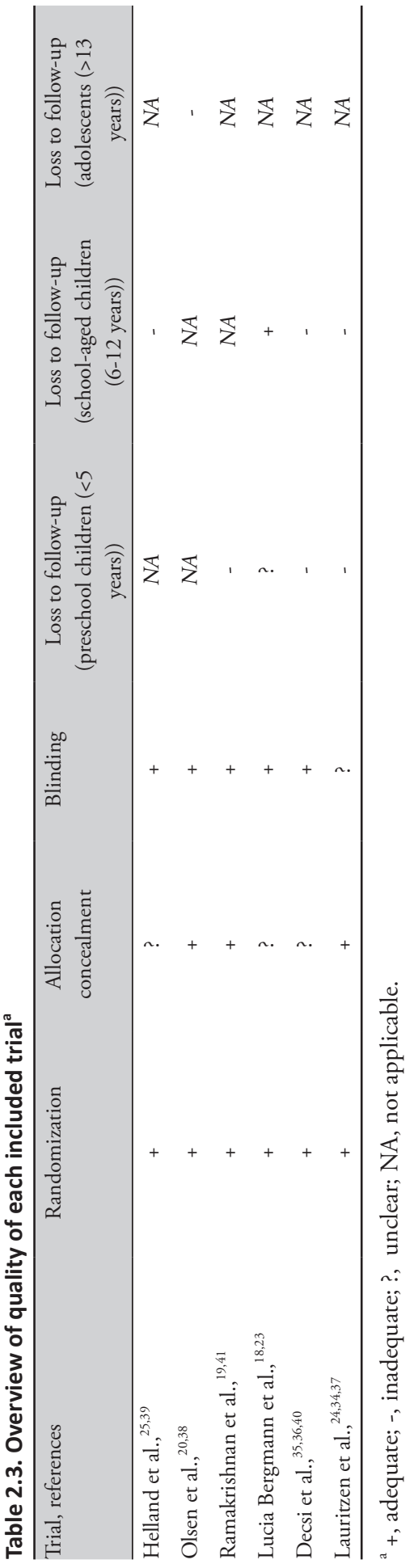




\section{Body mass index}

Four trials reported data on BMI, expressed as $\mathrm{kg} / \mathrm{m}^{2}$ or $\mathrm{z}$-score, in preschool children ( $<5$ years). ${ }^{19,23,34,36}$ Our analysis showed no significant difference between the $\mathrm{n}-3$ LCPUFA and control groups ( $\mathrm{SMD}=0.07$ [95\% CI -0.22 to 0.36 ]; $\mathrm{P}=$ $0.65 ; \mathrm{n}=1038$; random-effects) (Figure 2.2). There was evidence of significant heterogeneity (P-heterogeneity $=0.02, \mathrm{I}^{2}=67.9 \%$ ). In subgroup analysis based on the timing of intervention, the supplementation effect varied between subgroups (P-interaction $=0.02)$. No difference in BMI was shown between the intervention and control groups for women supplemented either in pregnancy (SMD $=0.06$ [95\% CI -0.15 to 0.26 ]; $\mathrm{P}=0.57$; two trials, $\mathrm{n}=906$; random-effects; P-heterogeneity $\left.=0.19, \mathrm{I}^{2}=41.4 \%\right)$ or in pregnancy and lactation $(\mathrm{SMD}=-0.50[95 \% \mathrm{CI}$ -1.04 to 0.04]; $\mathrm{P}=0.07$; one trial, $\mathrm{n}=62$ ), whereas $\mathrm{n}-3$ LCPUFA supplementation in lactation was associated with an increase in BMI (SMD $=0.52$ [95\% CI 0.03 to 1.01]; $\mathrm{P}=0.04$; one trial, $\mathrm{n}=70$ ) (Figure 2.2). Meta-regression showed no association of the n-3 LCPUFA dose and age with BMI in preschool children $(\mathrm{P}=0.12$ and 0.50 , respectively

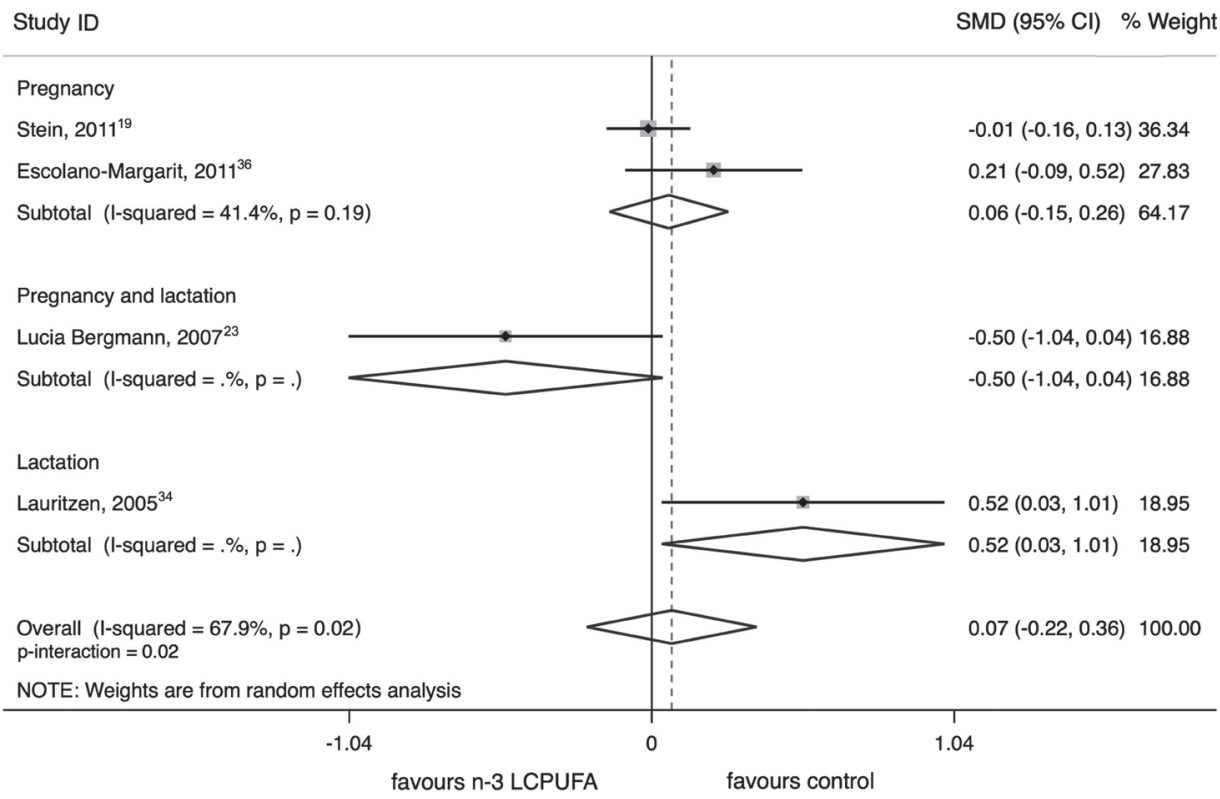

Figure 2.2. Meta-analysis forest plot for the effect of maternal n-3 LCPUFA supplementation on BMI (in $\mathrm{kg} / \mathrm{m}^{2}$ or as z-score) in preschool children ( $<5$ years). Subgroup analysis was based on the timing of intervention. Horizontal lines denote $95 \% \mathrm{Cls}$; solid diamonds represent the point estimate of each study. Open diamonds represent pooled estimates and the dashed line denotes the point estimate of the overall result from a random-effects meta-analysis. The $I^{2}$ and $P$ values for heterogeneity are shown. 
Four trials involving school-aged children (6-12 years) provided data on BMI, expressed as $\mathrm{kg} / \mathrm{m}^{2}$ or z-score. ${ }^{18,24,25,35}$ We found no significant difference between children from the $\mathrm{n}-3$ LCPUFA group and those from the control group (SMD= 0.12 [95\% CI -0.06 to 0.30 ]; $\mathrm{P}=0.20 ; \mathrm{n}=476$ ) (Figure 2.3). Heterogeneity was not significant $\left(\mathrm{P}\right.$-heterogeneity $\left.=0.97, \mathrm{I}^{2}=0 \%\right)$. The supplementation effect did not differ between subgroups according to the timing of intervention (Pinteraction $=0.92$ ). No difference in BMI between the intervention and control groups was seen, regardless of whether the intervention took place in pregnancy $(\mathrm{SMD}=0.14$ [95\% CI -0.18 to 0.46 ]; $\mathrm{P}=0.38$; one trial, $\mathrm{n}=154)$, pregnancy and lactation $(\mathrm{SMD}=0.09$ [95\% CI -0.16 to 0.34$]$; $\mathrm{P}=0.50$; two trials, $\mathrm{n}=258$; $\mathrm{P}$ heterogeneity $\left.=0.81, \mathrm{I}^{2}=0 \%\right)$, or lactation $(\mathrm{SMD}=0.19$ [95\% CI -0.30 to 0.69]; $\mathrm{P}=0.45$; one trial, $\mathrm{n}=64$ ) (Figure 2.3). In meta-regression, we also found no association of the n-3 LCPUFA dose and age with BMI in school-aged children $(\mathrm{P}=0.46$ and 0.83 , respectively).

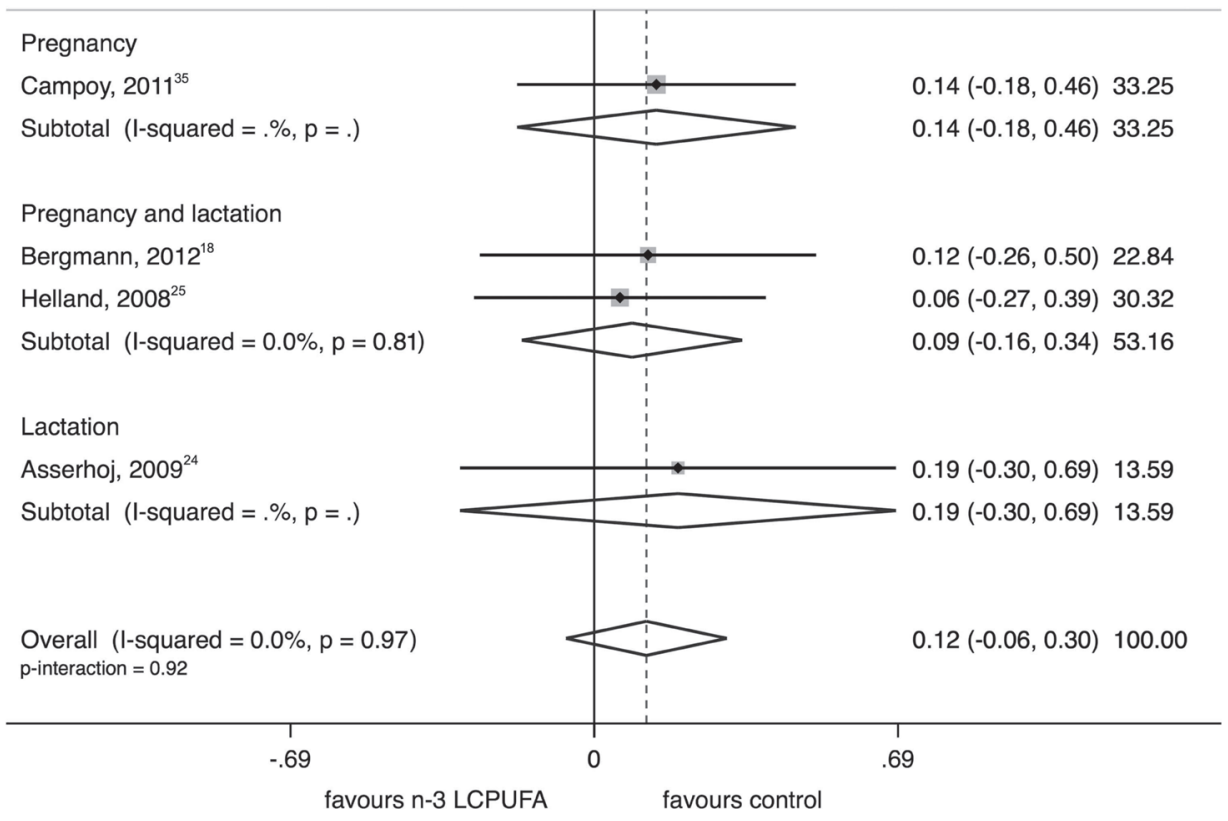

Figure 2.3. Meta-analysis forest plot for the effect of maternal n-3 LCPUFA supplementation on BMI (in $\mathrm{kg} / \mathrm{m}^{2}$ or as z-score) in school-aged children (6-12 years). Subgroup analysis was based on the timing of intervention. Horizontal lines denote $95 \% \mathrm{Cls}$; solid diamonds represent the point estimate of each study. Open diamonds represent pooled estimates and the dashed line denotes the point estimate of the overall result from a fixed-effect meta-analysis. The $I^{2}$ and $P$ values for heterogeneity are shown. 
The summary estimates for BMI in preschool ( $<5$ years) and school-aged (6-12 years) children were not significantly influenced by individual studies (Figure 2.4). No publication bias was detected for either outcome by using Egger's test $(\mathrm{P}=0.82$ for $\mathrm{BMI}$ in preschool children, and $\mathrm{P}=0.42$ for $\mathrm{BMI}$ in school-aged children), although the small number of studies was a limitation. The "trim and fill" method added one estimate in the meta-analysis of studies of school-aged children; however, the recalculated summary estimate for BMI did not change significantly (SMD $=0.11[95 \% \mathrm{CI}-0.06$ to 0.28$] ; \mathrm{P}=0.22$ ).
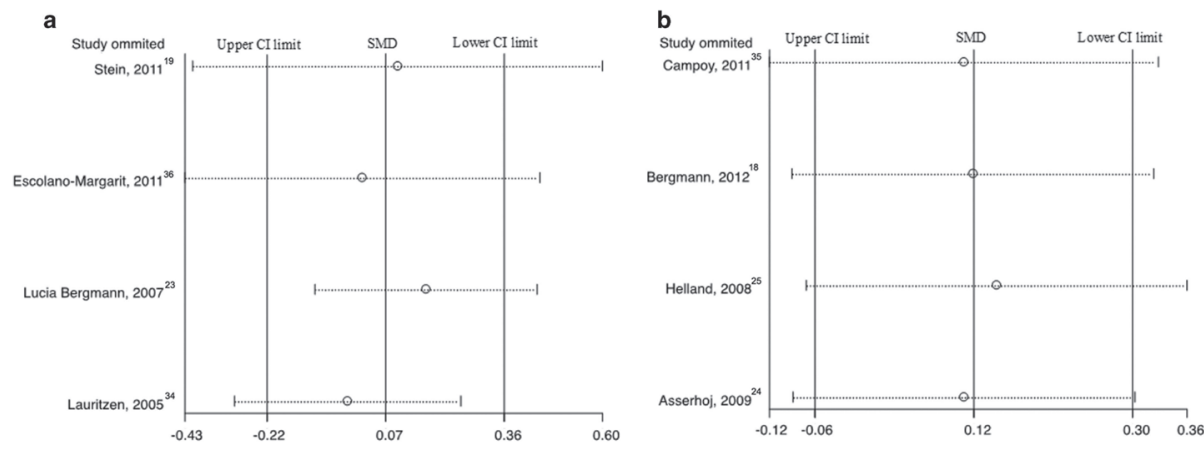

Figure 2.4 Pooled SMDs with their $95 \%$ Cls for BMI (in $\mathrm{kg} / \mathrm{m} 2$ or as z-score) in preschool children ( $<5$ years) (a) and school-aged children (6-12 years) (b) by omitting one study at a time.

In one trial involving adolescents aged 19 years, n-3 LCPUFA supplementation during pregnancy had also no effect on BMI compared with the control regimen $\left(\mathrm{MD}-0.10 \mathrm{~kg} / \mathrm{m}^{2}(95 \% \mathrm{CI}-1.20\right.$ to 1.00$\left.) ; \mathrm{P}=0.86 ; \mathrm{n}=180\right) .^{20}$

\section{Other adiposity measures}

In one trial of supplementation during lactation, preschool children in the $\mathrm{n}-3$ LCPUFA group had increased waist circumference $(\mathrm{MD}=1.60 \mathrm{~cm}[95 \% \mathrm{CI} 0.35$ to 2.85]; $\mathrm{P}=0.01 ; \mathrm{n}=68)$, sum of triceps and subscapular skinfold thicknesses $(\mathrm{MD}=1.50 \mathrm{~mm}[95 \% \mathrm{CI} 0.05$ to 2.95$] ; \mathrm{P}=0.04 ; \mathrm{n}=64)$ and body fat mass $(\mathrm{MD}=$ $1.40 \%$ [95\% CI 0.08 to 2.72 ]; $\mathrm{P}=0.04 ; \mathrm{n}=64$ ) at 2.5 years of age compared to those in the control group. ${ }^{34} \mathrm{~A}$ further assessment at 7 years of age showed no difference between the groups $(\mathrm{MD}=0.55 \mathrm{~cm}[95 \% \mathrm{CI}-1.47$ to 2.57$]$; $\mathrm{P}=0.59$; $\mathrm{n}=64$ for waist circumference, $-0.08 \mathrm{~mm}$ [95\% CI -2.59 to 2.43$]$; $\mathrm{P}=0.95 ; \mathrm{n}=$ 64 for the sum of 2 skinfold thicknesses, and $-0.14 \%$ [95\% CI -2.22 to 1.94$]$; $\mathrm{P}=$ $0.89 ; \mathrm{n}=64$ for body fat mass). ${ }^{24}$ 
In another trial, there was no difference between children of women supplemented with n-3 LCPUFAs during pregnancy and lactation and those of women from the control group in the sum of triceps, subscapular and suprailiac skinfold thicknesses at 6 years of age $(\mathrm{MD}=2.10 \mathrm{~mm}[95 \% \mathrm{CI}-0.48$ to 4.68$] ; \mathrm{P}=0.11 ; \mathrm{n}=115){ }^{18}$

No significant effect of n-3 LCPUFAs supplementation during pregnancy was also found on waist circumference in 19-year-old adolescents $(M D=-0.30 \mathrm{~cm}$ [95\% CI -3.23 to 2.63 ]; $\mathrm{P}=0.84 ; \mathrm{n}=180$ ) in one trial. ${ }^{20}$

\section{DISCUSSION}

In the present review, we summarized finding of RCTs to evaluate whether maternal n-3 LCPUFA supplementation during pregnancy and/or lactation favourably affects child adiposity. To the best of our knowledge, this is the first meta-analysis to combine results from individual studies on the impact of n-3 LCPUFA administration in pregnancy and/or lactation on childhood BMI. Overall, no effect of supplementation was found on BMI in preschool ( $<5$ years) and school-aged (6-12 years) children. In subgroup analyses, supplementation during lactation was associated with increased BMI in preschool children in one small trial with methodological limitations. There was also no evidence to support a benefit of $n-3$ LCPUFA supplementation in the other adiposity measures in individual studies. A large body of evidence demonstrates that increased adiposity, once established in childhood, tends to track into adulthood. ${ }^{42}$ Many studies have shown that even children younger than 2 years with a high BMI are at increased risk of developing obesity later in life. ${ }^{43}$ It has been argued that efforts to prevent obesity should begin in the early years and even before birth. ${ }^{44,45}$ Adipocyte development and fat deposition increases with gestational age; body fat is estimated to contribute approximately $2 \%$ of body weight in a 1000 -g preterm infant to upwards of $20 \%$ of body weight in a 3500 -g term infant. ${ }^{46}$ In the postnatal period, there is a marked increase in the number and size of adipocytes during the first year of life. ${ }^{47,48}$ The acquisition of fat cells early in life appears to be an irreversible process. ${ }^{49}$ Evidence from cell culture and animal studies suggests that early exposure to n-3 LCPUFAs has the potential to limit adipose tissue deposition mainly by attenuating the production of the AA metabolite prostacyclin, which has been shown to enhance adipogenesis. $^{50}$

Human evidence on the effects of maternal intake of n-3 LCPUFAs on somatic growth is limited. Few prospective birth cohort studies have examined the potential programming effect of $n-3$ LCPUFAs and yielded discrepant findings. ${ }^{12-15,51}$ Our findings extend and expand those of a recent Cochrane review demonstrating no effect of maternal n-3 LCPUFA supplementation on child growth. ${ }^{52}$ 
Given the antagonistic roles of n-3 and n-6 LCPUFAs, it can be argued that maternal intake of $\mathrm{n}-6$ LCPUFAs should also be taken into consideration and that a high intake of $n-3$ relative to $n-6$ LCPUFAs may have a more substantial effect on adipose tissue development. A US cohort study reported that a higher ratio of n-6: n-3 LCPUFAs in the prenatal period was associated with a higher sum of 2 skinfold thicknesses and increased odds of obesity in 3-year-old children, whereas no association was found between prenatal n-6 LCPUFA status and child adiposity. ${ }^{12}$ A German cohort study also demonstrated no association between n-6 LC-PUFA levels in cord blood and later BMI, but reported a significant interaction between the n-6: n-3 LCPUFA ratio and BMI over time; there was a negative effect at 2 years, no effect at 6 years, and a positive effect at 10 years. ${ }^{51} \mathrm{~A}$ cohort study conducted in the UK showed the n-6 LCPUFA concentration, but not the $\mathrm{n}-3$ : $\mathrm{n}-6$ ratio, in maternal plasma during pregnancy to predict child adiposity at 4 and 6 years of age. ${ }^{14}$ In another study, no association was shown between the n-6: n-3 ratio in breast milk and BMI between the age of 2 and 7 years of age. ${ }^{13}$ Hauner et al. ${ }^{53}$ randomized women to n-3 LCPUFA supplementation with an concomitant advice on reducing dietary AA intake during pregnancy and lactation in a small open label trial, and found no significant differences in body fat mass, as assessed by skinfold thickness and abdominal ultrasonography, in the offspring during the first year of life. Hence, no firm conclusions can be drawn from the existing data regarding the potential role of $n-3: n-6$ ratio in the perinatal period in influencing adipose tissue growth.

We found a high degree of heterogeneity between studies assessing BMI in preschool children. However, the timing of intervention may partially account for the observed heterogeneity. Owing to the limited available information, we were not able to carry out other pre-specified subgroup analyses based on maternal pre-pregnancy or booking BMI, birth weight, or maturity (ie, term birth vs. preterm birth) to further explore other potential sources of heterogeneity or effect modification. ${ }^{54-56}$ Most trials used fish oil as a source of $n-3$ LCPUFAs. The dose of n-3 LCPUFAs administered was high enough to cover the recommendations for DHA intake in pregnancy and lactation $(\geq 200 \mathrm{mg} / \text { day })^{57}$ but varied considerably between trials (up to $2200 \mathrm{mg} /$ day). There was no evidence for an association between n-3 LCPUFA dose and childhood BMI.

Compared to previous qualitative reviews, ${ }^{16,17}$ our review was conducted in accordance with well-established methods for performing and reporting systematic reviews, ${ }^{21,22}$ evaluated updated long-term findings from many trials ${ }^{18-20}$ and added results from 2 studies $(n=321)$ that were not primarily focused on adiposity, but nevertheless reported relevant outcomes. ${ }^{35,36}$ Additional information was also obtained from the investigators of one trial. ${ }^{34}$ Thus, our review provides the most 
comprehensive view of the literature to date regarding the impact of n-3 LCPUFA supplementation in the perinatal period on childhood adiposity.

Several limitations should be taken into account during interpretation of the findings of this review. There were a few available studies and most of them had relatively small sample sizes ( $\mathrm{n}<90$ per group), which might have resulted to loss of statistical power. Consequently, the results, especially those of sub-analyses, should be viewed with caution. Furthermore, the majority of studies suffered from selective attrition. This is likely to have affected the initial randomization schedule, decreasing the possibility that familial -ie, environmental and genetic- influences on adiposity are equally distributed between groups and do not confound trial outcomes. It should also be noted that the control regimen used in most of the trials might not be appropriate. Olive and corn oils have been shown to influence fatty acid profile and metabolism in healthy adults and, thus, might have induced physiologic responses exerting an effect on the outcome measures. ${ }^{17}$ We found no influence of publication bias, but this bias is difficult to ascertain with a small number of studies.

In conclusion, there is currently no evidence to support that maternal n-3 LCPUFA supplementation during pregnancy and/or lactation exerts a favourable programming effect on adiposity status in childhood. However, our systematic review highlights that most of the trials reviewed were prone to methodological limitations. Further evidence from high-quality trials is needed to establish whether n-3 LCPUFA supplementation in the perinatal period affects adiposity in children. The results from such trials will provide valuable information on the potential of a modification of dietary fat intake in pregnancy and lactation to assist in reducing the burden of childhood obesity. 


\section{REFERENCES}

1. International Association for the Study of Obesity. Global childhood overweight. 2011.http://www.iaso. org/site_media/uploads/Global_Childhood_Overweight_April_2011.pdf (January 23 2014, date last accessed)

2. Lobstein T, Baur L, Uauy R. Obesity in children and young people:a crisis in public health. Obes Rev 2004;5 Suppl 1:4-104.

3. Daniels SR. Complications of obesity in children and adolescents. Int J Obes (Lond) 2009;33 Suppl $1:$ S60-65.

4. Reilly JJ, Kelly J. Long-term impact of overweight and obesity in childhood and adolescence on morbidity and premature mortality in adulthood:systematic review. Int J Obes (Lond) 2011;35:891-898.

5. Swinburn BA, Sacks G, Hall KD, McPherson K, Finegood DT, Moodie ML, et al. The global obesity pandemic:shaped by global drivers and local environments. Lancet 2011;378:804-814.

6. Ebbeling CB, Pawlak DB, Ludwig DS. Childhood obesity:public-health crisis, common sense cure. Lancet 2002;360:473-482.

7. Mostyn A, Symonds ME. Early programming of adipose tissue function:a large-animal perspective. Proc Nutr Soc 2009;68:393-400.

8. Lukaszewski MA, Eberle D, Vieau D, Breton C. Nutritional manipulations in the perinatal period program adipose tissue in offspring. Am J Physiol Endocrinol Metab 2013;305:E1195-1207.

9. Sanders TA. Polyunsaturated fatty acids in the food chain in Europe. Am J Clin Nutr 2000;71:176S178 S.

10. Meyer BJ, Mann NJ, Lewis JL, Milligan GC, Sinclair AJ, Howe PR. Dietary intakes and food sources of omega-6 and omega-3 polyunsaturated fatty acids. Lipids 2003;38:391-398.

11. Ailhaud G, Guesnet P, Cunnane SC. An emerging risk factor for obesity:does disequilibrium of polyunsaturated fatty acid metabolism contribute to excessive adipose tissue development? Br J Nutr 2008;100:461-470

12. Donahue SM, Rifas-Shiman SL, Gold DR, Jouni ZE, Gillman MW, Oken E. Prenatal fatty acid status and child adiposity at age 3 y:results from a US pregnancy cohort. Am J Clin Nutr 2011;93:780-788.

13. Pedersen L, Lauritzen L, Brasholt M, Buhl T, Bisgaard H. Polyunsaturated fatty acid content of mother's milk is associated with childhood body composition. Pediatr Res 2012;72:631-636.

14. Moon RJ, Harvey NC, Robinson SM, Ntani G, Davies JH, Inskip HM, et al. Maternal plasma polyunsaturated fatty acid status in late pregnancy is associated with offspring body composition in childhood. J Clin Endocrinol Metab 2013;98:299-307.

15. Rytter D, Bech BH, Halldorsson T, Christensen JH, Schmidt EB, Danielsen I, et al. No association between the intake of marine n-3 PUFA during the second trimester of pregnancy and factors associated with cardiometabolic risk in the 20-year-old offspring. Br J Nutr 2013;110:2037-2046.

16. Rodríguez G, Iglesia I, Bel-Serrat S, Moreno LA. Effect of n-3 long chain polyunsaturated fatty acids during the perinatal period on later body composition. Br J Nutr 2012;107 Suppl 2:S117-128.

17. Muhlhausler BS, Gibson RA, Makrides M. Effect of long-chain polyunsaturated fatty acid supplementation during pregnancy or lactation on infant and child body composition: a systematic review. Am J Clin Nutr 2010;92:857-863.

18. Bergmann RL, Bergmann KE, Richter R, Haschke-Becher E, Henrich W, Dudenhausen JW. Does docosahexaenoic acid (DHA) status in pregnancy have any impact on postnatal growth? Six-year follow-up of a prospective randomized double-blind monocenter study on low-dose DHA supplements. J Perinat Med 2012;40(6):677-84 
19. Stein AD, Wang M, Martorell R, Neufeld LM, Flores-Ayala R, Rivera JA, et al. Growth to age 18 months following prenatal supplementation with docosahexaenoic acid differs by maternal gravidity in Mexico. J Nutr 2011;141:316-320.

20. Rytter D, Bech BH, Christensen JH, Schmidt EB, Henriksen TB, Olsen SF. Intake of fish oil during pregnancy and adiposity in 19-y-old offspring: follow-up on a randomized controlled trial. Am J Clin Nutr 2011;94:701-708.

21. Higgins JPT, Green S. Cochrane Handbook for Systematic Reviews of Interventions Version 5.1.0 [updated March 2011]. The Cochrane Collaboration;2011.

22. Liberati A, Altman DG, Tetzlaff J, Mulrow C, Gotzsche PC, Ioannidis JP, et al. The PRISMA statement for reporting systematic reviews and meta-analyses of studies that evaluate healthcare interventions:explanation and elaboration. BMJ 2009;339:b2700.

23. Lucia Bergmann R, Bergmann KE, Haschke-Becher E, Richter R, Dudenhausen JW, Barclay D, et al. Does maternal docosahexaenoic acid supplementation during pregnancy and lactation lower BMI in late infancy? J Perinat Med 2007;35:295-300.

24. Asserhoj M, Nehammer S, Matthiessen J, Michaelsen KF, Lauritzen L. Maternal fish oil supplementation during lactation may adversely affect long-term blood pressure, energy intake, and physical activity of 7-year-old boys. J Nutr 2009;139:298-304.

25. Helland IB, Smith L, Blomen B, Saarem K, Saugstad OD, Drevon CA. Effect of supplementing pregnant and lactating mothers with n-3 very-long-chain fatty acids on children's IQ and body mass index at 7 years of age. Pediatrics 2008;122:e472-479.

26. Schulz KF, Chalmers I, Hayes RJ, Altman DG. Empirical-Evidence of Bias - Dimensions of Methodological Quality Associated with Estimates of Treatment Effects in Controlled Trials. JAMA 1995;273:408-412.

27. Moher D, Pham B, Jones A, Cook DJ, Jadad AR, Moher M, Tugwell P, Klassen TP. Does quality of reports of randomised trials affect estimates of intervention efficacy reported in meta-analyses? Lancet 1998;352:609-613.

28. Higgins JP, Thompson SG. Quantifying heterogeneity in a meta-analysis. Stat Med 2002;21:1539-1558.

29. Higgins JP, Thompson SG, Deeks JJ, Altman DG. Measuring inconsistency in meta-analyses. BMJ 2003;327:557-560.

30. Borenstein M, Hedges LV, Higgins JPT, Rothstein HR (eds). Subgroup analyses. In:Introduction to Meta-analysis. John Wiley \& Sons, Ltd; Chichester, UK, 2009, pp. 149-186.

31. Higgins JP, Thompson SG. Controlling the risk of spurious findings from meta-regression. Stat Med 2004;23:1663-82.

32. Egger M, Davey Smith G, Schneider M, Minder C. Bias in meta-analysis detected by a simple, graphical test. BMJ 1997;315:629-634.

33. Duval S, Tweedie R. Trim and fill: A simple funnel-plot-based method of testing and adjusting for publication bias in meta-analysis. Biometrics 2000;56:455-63.

34. Lauritzen L, Hoppe C, Straarup EM, Michaelsen KF. Maternal fish oil supplementation in lactation and growth during the first 2.5 years of life. Pediatr Res 2005;58:235-242.

35. Campoy C, Escolano-Margarit MV, Ramos R, Parrilla-Roure M, Csabi G, Beyer J, et al. Effects of prenatal fish-oil and 5-methyltetrahydrofolate supplementation on cognitive development of children at 6.5 y of age. Am J Clin Nutr 2011;94:1880S-1888S.

36. Escolano-Margarit MV, Ramos R, Beyer J, Csabi G, Parrilla-Roure M, Cruz F, et al. Prenatal DHA status and neurological outcome in children at age 5.5 years are positively associated. J Nutr 2011;141:12161223. 
37. Lauritzen L, Jorgensen MH, Mikkelsen TB, Skovgaard 1 M, Straarup EM, Olsen SF, et al. Maternal fish oil supplementation in lactation:effect on visual acuity and n-3 fatty acid content of infant erythrocytes. Lipids 2004;39:195-206.

38. Olsen SF, Sorensen JD, Secher NJ, Hedegaard M, Henriksen TB, Hansen HS, et al. Randomised controlled trial of effect of fish-oil supplementation on pregnancy duration. Lancet 1992;339:1003-1007.

39. Helland IB, Saugstad OD, Smith L, Saarem K, Solvoll K, Ganes T, et al. Similar effects on infants of n-3 and n-6 fatty acids supplementation to pregnant and lactating women. Pediatrics 2001;108:E82.

40. Decsi T, Campoy C, Koletzko B. Effect of N-3 polyunsaturated fatty acid supplementation in pregnancy:the Nuheal trial. Adv Exp Med Biol 2005;569:109-113.

41. Ramakrishnan U, Stein AD, Parra-Cabrera S, Wang M, Imhoff-Kunsch B, Juárez-Márquez $S$, et al. Effects of docosahexaenoic acid supplementation during pregnancy on gestational age and size at birth:randomized, double-blind, placebo-controlled trial in Mexico. Food Nutr Bull 2010;31:S108-116.

42. Singh AS, Mulder C, Twisk JW, van Mechelen W, Chinapaw MJ. Tracking of childhood overweight into adulthood:a systematic review of the literature. Obes Rev 2008;9:474-88.

43. Baird J, Fisher D, Lucas P, Kleijnen J, Roberts H, Law C. Being big or growing fast:systematic review of size and growth in infancy and later obesity. BMJ 2005;331:929.

44. Gillman MW, Ludwig DS. How early should obesity prevention start? N Engl J Med 2013;369:2173-5.

45. Taveras EM, Gillman MW, Kleinman K, Rich-Edwards JW, Rifas-Shiman SL. Racial/ethnic differences in early-life risk factors for childhood obesity. Pediatrics 2010;125:686-95.

46. Ziegler EE, O'Donnell AM, Nelson SE, Fomon SJ. Body composition of the reference fetus. Growth 1976;40:329-41.

47. Baum D, Beck RQ, Hammer LD, Brasel JA, Greenwood MR. Adipose tissue thymidine kinase activity in man. Pediatr Res 1986;20:118-21.

48. Salans LB, Cushman SW, Weismann RE. Studies of human adipose tissue. Adipose cell size and number in nonobese and obese patients. J Clin Invest 1973;52:929-41.

49. Spalding KL, Arner E, Westermark PO, Bernard S, Buchholz BA, Bergmann O, Blomqvist L, Hoffstedt J, Näslund E, Britton T, Concha H, Hassan M, Rydén M, Frisén J, Arner P. Dynamics of fat cell turnover in humans. Nature 2008;453:783-7.

50. Ailhaud G, Massiera F, Weill P, Legrand P, Alessandri JM, Guesnet P. Temporal changes in dietary fats:role of n-6 polyunsaturated fatty acids in excessive adipose tissue development and relationship to obesity. Prog Lipid Res 2006;45:203-236.

51. Standl M, Thiering E, Demmelmair H, Koletzko B, Heinrich J. Age-dependent effects of cord blood long-chain PUFA composition on BMI during the first 10 years of life. Br J Nutr 2014;13:1-8.

52. Delgado-Noguera MF, Calvache JA, Bonfill Cosp X. Supplementation with long chain polyunsaturated fatty acids (LCPUFA) to breastfeeding mothers for improving child growth and development. Cochrane Database Syst Rev 2010:CD007901.

53. Hauner H, Much D, Vollhardt C, Brunner S, Schmid D, Sedlmeier EM, et al. Effect of reducing the n-6:n-3 long-chain PUFA ratio during pregnancy and lactation on infant adipose tissue growth within the first year of life:an open-label randomized controlled trial. Am J Clin Nutr 2012;95:383-394.

54. Kerkhof GF, Hokken-Koelega ACS. Rate of neonatal weight gain and effects on adult metabolic health. Nat Rev Endocrinol 2012;8:689-692.

55. Lawrence GM, Shulman S, Friedlander Y, Sitlani CM, Burger A, Savitsky B, et al. Associations of maternal pre-pregnancy and gestational body size with offspring longitudinal change in BMI. Obesity (Silver Spring) 2014;22:1165-71. 
56. Schellong K, Schulz S, Harder T, Plagemann A. Birth weight and long-term overweight risk:systematic review and a meta-analysis including 643,902 persons from 66 studies and 26 countries globally. PLoS One 2012;7:e47776.

57. Koletzko B, Cetin I, Brenna JT; Perinatal Lipid Intake Working Group; Child Health Foundation; Diabetic Pregnancy Study Group; European Association of Perinatal Medicine; European Association of Perinatal Medicine; European Society for Clinical Nutrition and Metabolism; European Society for Paediatric Gastroenterology, Hepatology and Nutrition, Committee on Nutrition; International Federation of Placenta Associations; International Society for the Study of Fatty Acids and Lipids. Dietary fat intakes for pregnant and lactating women. Br J Nutr 2007;98:873-7.

58. Helland IB, Smith L, Saarem K, Saugstad OD, Drevon CA. Maternal supplementation with very-longchain n-3 fatty acids during pregnancy and lactation augments children's IQ at 4 years of age. Pediatrics 2003;111:e39-44.

59. Dunstan JA, Simmer K, Dixon G, Prescott SL. Cognitive assessment of children at age 2(1/2) years after maternal fish oil supplementation in pregnancy:a randomised controlled trial. Arch Dis Child Fetal Neonatal Ed 2008;93:F45-50.

60. Hauner H, Vollhardt C, Schneider K, Zimmermann A, Schuster T, Amann-Gassner U. The impact of nutritional fatty acids during pregnancy and lactation on early human adipose tissue development. Rationale and design of the INFAT study. Ann Nutr Metab 2009;54:97-103.

61. Ramakrishnan U, Martorell R, Stein AD, Wang M, DiGirolamo A, Schnaas L, et al. Effect of prenatal supplementation with docosahexaenoic acid on child size and development at 18 mo:randomized placebo-controlled trial in Mexico. ISSFAL Maastricht, the Netherlands 2010;CS9.2 (abstract).

62. Courville AB, Harel O, Lammi-Keefe CJ. Consumption of a DHA-containing functional food during pregnancy is associated with lower infant ponderal index and cord plasma insulin concentration. Br J Nutr 2011;106:208-212.

63. Much D, Brunner S, Vollhardt C, Schmid D, Sedlmeier EM, Bruderl M, et al. Effect of dietary intervention to reduce the n-6/n-3 fatty acid ratio on maternal and fetal fatty acid profile and its relation to offspring growth and body composition at 1 year of age. Eur J Clin Nutr 2013;67:282-288.

64. Much D, Brunner S, Vollhardt C, Schmid D, Sedlmeier EM, Bruderl M, et al. Breast milk fatty acid profile in relation to infant growth and body composition:results from the INFAT study. Pediatr Res 2013;74:230-237.

65. Hauner H, Brunner S, Amann-Gassner U. The role of dietary fatty acids for early human adipose tissue growth. Am J Clin Nutr 2013;98:549S-55S.

66. Escolano-Margarit MV, Campoy C, Ramírez-Tortosa MC, Demmelmair H, Miranda MT, Gil A, et al. Effects of fish oil supplementation on the fatty acid profile in erythrocyte membrane and plasma phospholipids of pregnant women and their offspring: a randomised controlled trial. Br J Nutr 2013;109:1647-1656.

67. Brunner S, Schmid D, Huttinger K, Much D, Bruderl M, Sedlmeier EM, et al. Effect of reducing the n-6/n-3 fatty acid ratio on the maternal and fetal leptin axis in relation to infant body composition. Obesity (Silver Spring) 2014;22:217-224.

68. Catalano P, Haghiac M, Smith S, Dettlebach S, Gunzler D, Groh-Wargo S, et al. $\omega$-3 poly-unsaturated fatty acid supplementation in overweight and obese women:a pilot RCT to improve inflammation, insulin sensitivity and decrease fetal adiposity. Am J Obstet Gynecol 2014;210:S43 (abstract 65).

69. Parisi F, Brunetti M, Berti C, Capriata I, Mazzocco M, Cetin I. Effects of DHA supplementation during pregnancy on fetal body composition. Reprod Sci 2013;20:244A (abstract 178). 
Chapter 2

Supplementary Material of Chapter 2 


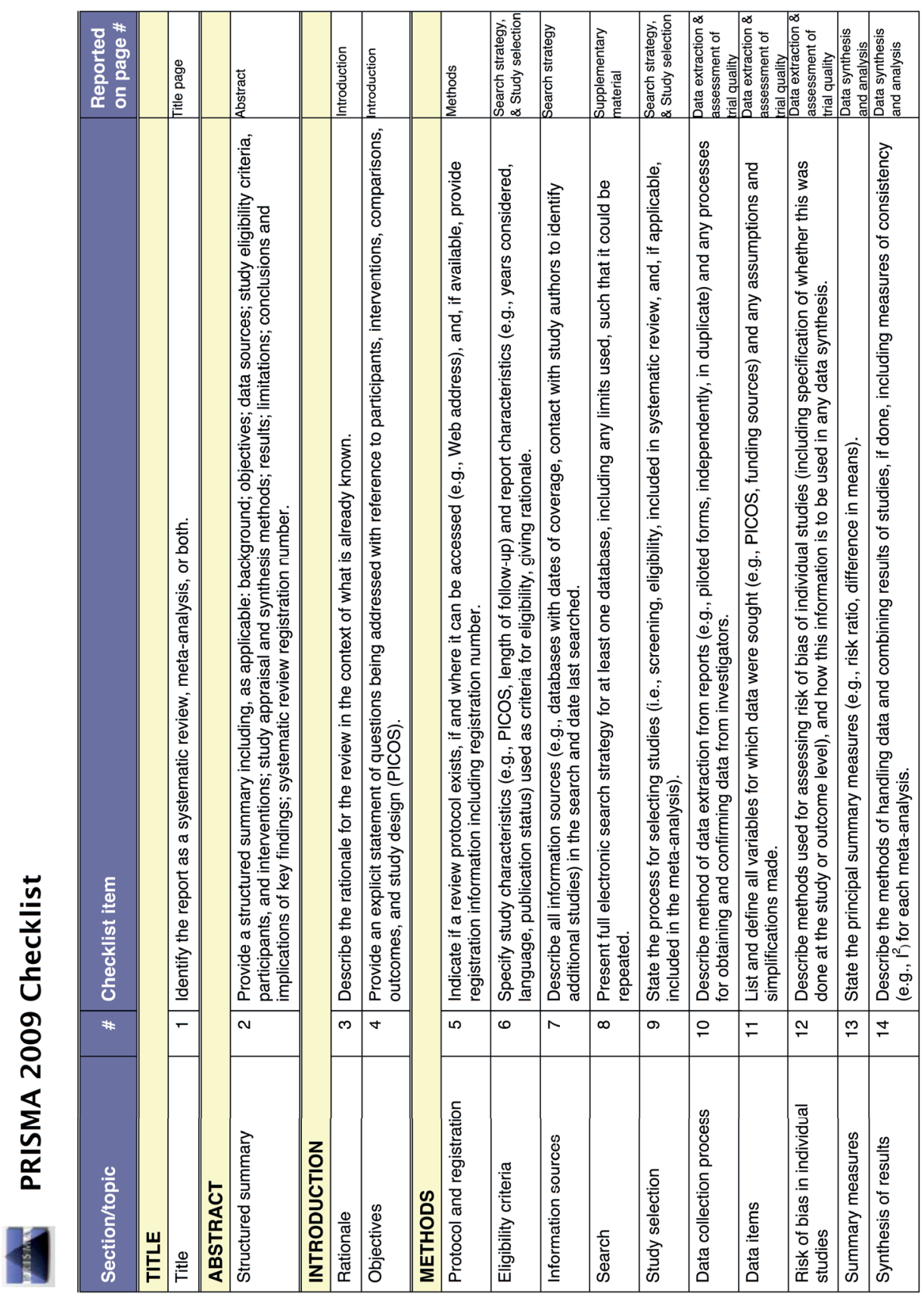




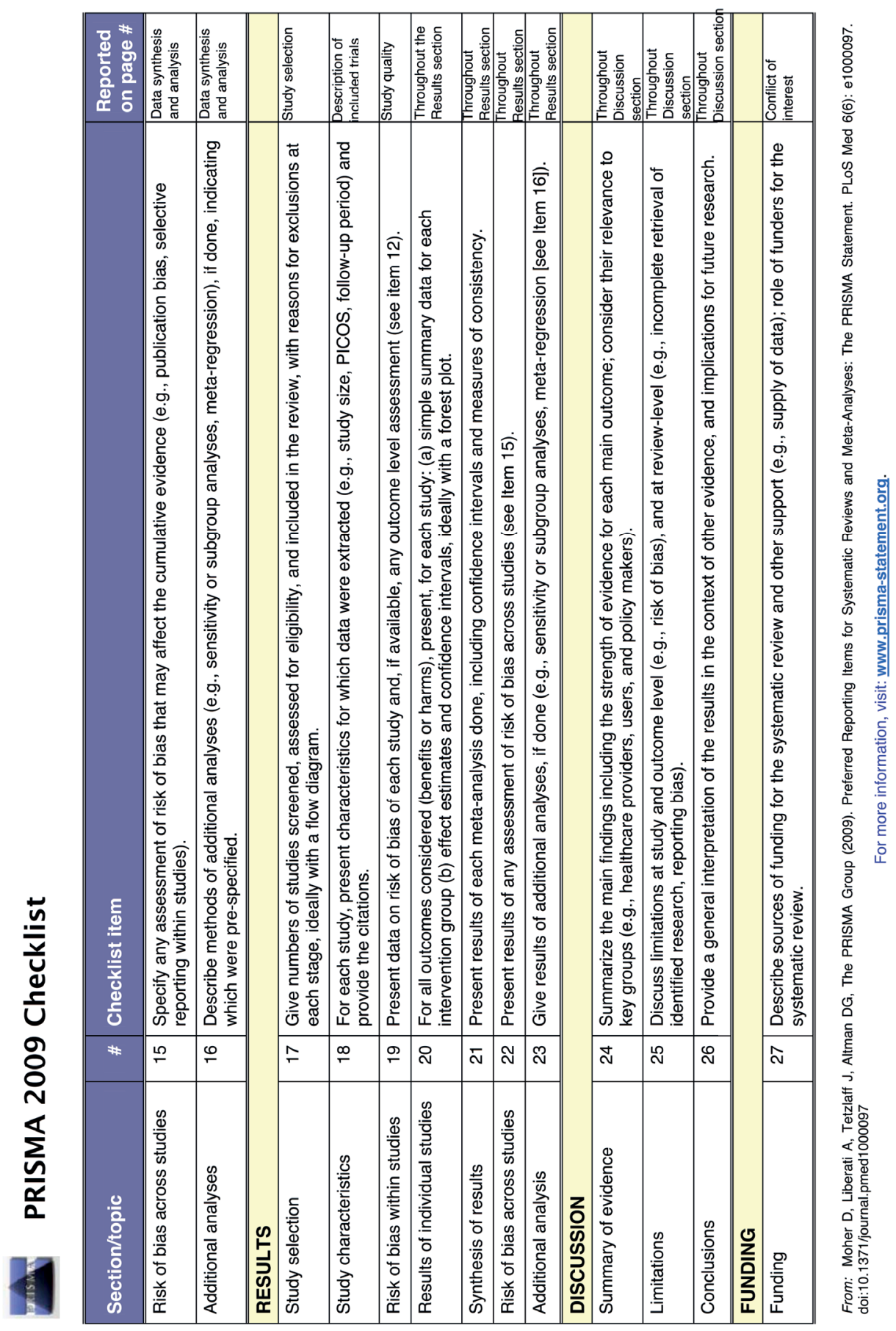




\section{Search strategy}

\section{Pubmed}

1.Fish oils $[\mathrm{MeSH}]$

2.fish oil* [tw] OR algal oil [tw] OR marine oil [tw] OR cod liver oil [tw]

3. Fatty acids [MeSH]

4.fatty acid* $[\mathrm{tw}]$

5. Fatty acids, essential $[\mathrm{MeSH}]$

6.essential fatty acid* [tw]

7.Fatty acids, omega-3 [MeSH]

8.omega-3 fatty acid ${ }^{*}[\mathrm{tw}]$

9.n-3 fatty acid* [tw]

10.Docosahexaenoic acids [MeSH]

11.Eicosapentaenoic acid [MeSH]

12.docosahex* acid [tw]

13.eicosapent* acid [tw]

14.DHA [tiab] OR EPA [tiab]

15.n-3 PUFA* [tw]

16.n-3 polyunsaturated [tw]

17.PUFA* [tw] OR LCPUFA* [tw]

18.1 OR 2 OR 3 OR 4 OR 5 OR 6 OR 7 OR 8 OR 9 OR 10 OR 11 OR 12 OR 13 OR 14 OR

OR 16 OR 17

19.Pregnancy [MeSH]

20.Pregnant women $[\mathrm{MeSH}]$

21.pregnan* [tw] OR maternal [tw] OR prenatal [tw] OR antenatal [tw] OR gestation* $[\mathrm{tw}]$

22.intrauterine [tw] OR intra-uterine [tw] OR in utero [tw] OR in-utero [tw]

23. Fetus [MeSH]

24.fetus [tw] OR foetus [tw] OR fetal [tw] OR foetal [tw]

25.Breastfeeding $[\mathrm{MeSH}]$

26.breast-fe* [tw] or breastfe* [tw]

27.Lactation [MeSH]

28.lactation [tw] OR postnatal [tw] OR postpartum [tw]

29.lactating mother* [tw] OR lactating woman [tw] OR lactating women [tw]

30.perinatal [tw]

31.19 OR 20 OR 21 OR 22 OR 23 OR 24 OR 25 OR 26 OR 27 OR 28 OR 29 OR 30

32.Adiposity [MeSH] 
33.adiposity [tw] OR adipose [tw]

34.Body mass index $[\mathrm{MeSH}]$

35.body mass index [tw] OR BMI [tw]

36.fat mass [tw] OR body composition [tw]

37. Waist circumference $[\mathrm{MeSH}]$

38.waist circumference [tw]

39.Anthropometry $[\mathrm{MeSH}]$

40. antropometr* [tw]

41.Skinfold thickness [MeSH]

42.skinfold [tw] OR skin-fold [tw]

43.obesity [tw] OR obese [tw] OR overweight [tw]

44.32 OR 33 OR 34 OR 35 OR 36 OR 37 OR 38 OR 39 OR 40 OR 41 OR 42 OR 43

45.18 AND 31 AND 44

46.randomized controlled trial [pt]

47.controlled clinical trial [pt]

48.randomized [tiab]

49.placebo [tiab]

50.drug therapy [sh]

51.randomly [tiab]

52.trial [tiab]

53.groups [tiab]

54.46 OR 47 OR 48 OR 49 OR 50 OR 51 OR 52 OR 53

55.animals [mh] NOT (humans [mh] AND animals [mh])

56.54 NOT 55

57.45 AND 56

\section{EMBASE via Ovid}

1. fish.mp.

2. ((marine or algal) and oil\$).mp.

3. exp fish oil/

4. fish oil\$.mp.

5. exp fatty acid/

6. exp essential fatty acid/

7. essential fatty acid\$.mp.

8. exp unsaturated fatty acid/

9. unsaturated fatty acid\$.mp.

10. exp omega 3 fatty acid/

11. (omega3 or omega-3).mp. 
12. ((n-3 or $n 3)$ and fatty acid $\$) . m p$.

13. exp docosahexaenoic acid/

14. (docosahexaenoic acid or docosahexanoic acid or docosahexenoic acid or DHA).mp.

15. exp icosapentaenoic acid/

16. (eicosapentaenoic acid or eicosapentanoic acid or eicosapentenoic acid or EPA).mp.

17. (polyunsaturated fatty acid $\$$ or poly-unsaturated fatty acid $\$$ or PUFA $\$$ or LC-PUFA\$).mp.

18. or/1-17

19. exp pregnant woman/

20. exp pregnancy/

21. (pregnan $\$$ or maternal or prenatal or pre-natal or antenatal or gestation\$).mp.

22. (intrauterine or intra-uterine or (in adj utero) or in-utero).mp.

23. (fetus or foetus or fetal or foetal).mp.

24. exp lactation/

25. lactation.mp.

26. (lactating and (mother\$ or woman or women)).mp.

27. exp breast feeding/

28. (breast-fe $\$$ or breastfe $\$$ ).mp.

29. (breast-milk or breastmilk).mp.

30. or/19-29

31. exp obesity/

32. (adiposity or adipose).mp.

33. exp body mass/

34. (body mass index or BMI).mp.

35. fat mass.mp.

36. exp waist circumference/

37. waist.mp.

38. exp adipose tissue/

39. exp anthropometry/

40. anthropometr\$.mp. [

41. exp skinfold thickness/

42. (skinfold or skin-fold).mp.

43. overweight.mp.

44. (cardiovascular or CVD).mp.

45. exp metabolic syndrome X/

46. ((metabolic or insulin resistance) and syndrome).mp.

47. or/31-46 
48. 18 and 30 and 47

49. exp Clinical trial/

50. Randomized controlled trial/

51. Randomization/

52. double blind procedure/

53. single blind procedure/

54. Crossover procedure/

55. Placebo/

56. randomi\#ed.tw.

57. RCT.tw.

58. Randomi\#ed controlled trial\$.tw.

59. (random $\$$ adj3 (allocat $\$$ or assign $\$)$ ).tw.

60. Prospective Studies/

61. randomly.ab.

62. ( (singl\$ or doubl\$ or trebl\$ or tripl\$) adj3 (blind $\$$ or mask $\$)$ ).tw.

63. Placebo\$.tw.

64. (crossover or cross-over).tw.

65. prospective.tw.

66. or/49-65

67. animal/

68. human/

69. 67 and 68

70.67 not 69

72. 66 not 70

73.48 and 72

\section{CINAHL via EBSCOhost}

S59. S18 AND S43 AND S58

S58. S44 OR S 45 OR S 46 OR S47 OR S 48 OR S 49 OR S50 OR S51 OR S52

OR S53 OR

S54 OR S55 OR S56 OR S57

S57. (MH "Quantitative Studies")

S56. (MH "Placebos")

S55. TX placebo*

S54. TX random* allocat*

S53. (MH "Random Assignment")

S52. TX randomi* control* $^{*}$ trial $^{*}$

S51. TX ((singl* n1 blind*) or (singl* n1 mask $\left.\left.{ }^{*}\right)\right)$

S50. TX ((doubl* n1 blind*) or (doubl* n1 mask $\left.\left.{ }^{*}\right)\right)$ 
S49. TX ((tripl* $\mathrm{n} 1$ blind $\left.^{*}\right)$ or $\left(\right.$ tripl$^{*} \mathrm{n} 1$ mask $\left.\left.^{*}\right)\right)$

S48. TX ((trebl* n1 blind*) or (trebl* ${ }^{*} 1$ mask $\left.\left.^{*}\right)\right)$

S47. PT Clinical trial

S46. TX clinic* $^{*} 1$ trial $^{*}$

S45. TX allocat* random*

S44. MH "Clinical Trials+")

S43. S31 OR S32 OR S33 OR S34 OR S35 OR S36 OR S37 OR S38 OR S39 OR S40 OR

S41 OR S42

S42. obesity OR obese OR overweight

S41. skinfold OR skin-fold

S40. (MH "Skinfold Thickness")

S39. antropometr*

S38. (MH "Anthropometry")

S36. waist circumference

S35. (MH "Waist Circumference")

S34. fat mass OR body composition

S33. body mass index OR BMI

S32. (MH "Body Mass Index")

$\mathrm{S} 31$. adiposity $\mathrm{OR}$ adipose

S30. S20 OR S21 OR S22 OR S 23 OR S24 OR S25 OR S26 OR S27 OR S 28 OR S29

S29. perinatal

S28. lactating mother* OR lactating wom?n

S27. lactation OR postnatal OR postpartum

S26. (MH "Lactation")

S25. breastfe* OR breast-fe*

S24. (MH "Breast Feeding+")

S23. Foetus OR fetus OR foetal OR fetal

S22. (MH "Fetus+")

S21. intrauterine OR intra-uterine OR in utero

S20. pregnan* OR maternal OR prenatal OR antenatal OR gestation*

S19. (MH "Pregnancy+")

S18. S1 OR S2 OR S3 OR S4 OR S5 OR S6 OR S7 OR S8 OR S9 OR S10 OR S11 OR

S12 OR S13 OR S14 OR S15 OR S16 OR S17

S17. LCPUFA*

S16. LC-PUFA*

S15. n-3 polyunsaturated 
S14. n-3 PUFA*

S13. TI EPA OR AB EPA

S12. eicosapent* AND acid

S11. TI DHA OR AB DHA

S10. docosahex* AND acid

S9. (MH "Eicosapentaenoic Acid")

S8. (MH "Docosahexaenoic Acids")

S7. n-3 fatty acid*

S6. omega-3fatty acid*

S5. (MH "Fatty Acids, Omega-3+")

S4. essential fatty acid*

S3. (MH "Fatty Acids, Essential+")

S2. fish oil* OR algal oil OR marine oil OR cod liver oil

S1. (MH "Fish Oils+")

\section{CENTRAL}

\#1MeSH descriptor: [Fish Oils] explode all trees

\#2fish oil ${ }^{*}$

\#3MeSH descriptor: [Fatty Acids, Essential] explode all trees

\#4fatty acid*

\#5omega 3

\#6docosahex*noic acid

\#7eicosapent*noic acid

\#8polyunsaturated or PUFA* or LCPUFA*

$\# 9 \# 1$ or $\# 2$ or $\# 3$ or $\# 4$ or \#5 or \#6 or \#7 or \#8

$\# 10 \mathrm{MeSH}$ descriptor: [Pregnancy] explode all trees

\#11 pregnant wom?n

\#12pregnancy or maternal or prenatal or antenatal or gestation

\#13intrauterine or fetus or fetal

\#14MeSH descriptor: [Lactation] explode all trees

\#15MeSH descriptor: [Breast Feeding] explode all trees

\#16lactation or postnatal or postpartum or breast feeding or breast milk

\#17lactating wom?n or lactating mother*

$\# 18 \# 10$ or $\# 11$ or \#12 or \#13 or \#13 or \#14 or \#15 or \#16 or \#17

$\# 19 \# 9$ and \#18 


\section{Clinicaltrials.gov}

("fish oil" OR "fatty acid" OR docosahexaenoicOR eicosapentaenoicOR "omega 3 fatty acid"

OR "n 3 fatty acid") AND (pregnancy OR pregnant OR postpartum OR prenatalOR fetal OR

gestation OR postnatal OR breastfeeding OR lactating OR lactation)

\section{Google Scholar}

(maternal OR lactation OR breastfeeding OR pregnancy OR prenatal) (docosahexaenoic OR

eicosapentaenoic) "fatty acids" (adiposity OR "fat mass") (trial OR randomised OR

randomized) -animals 


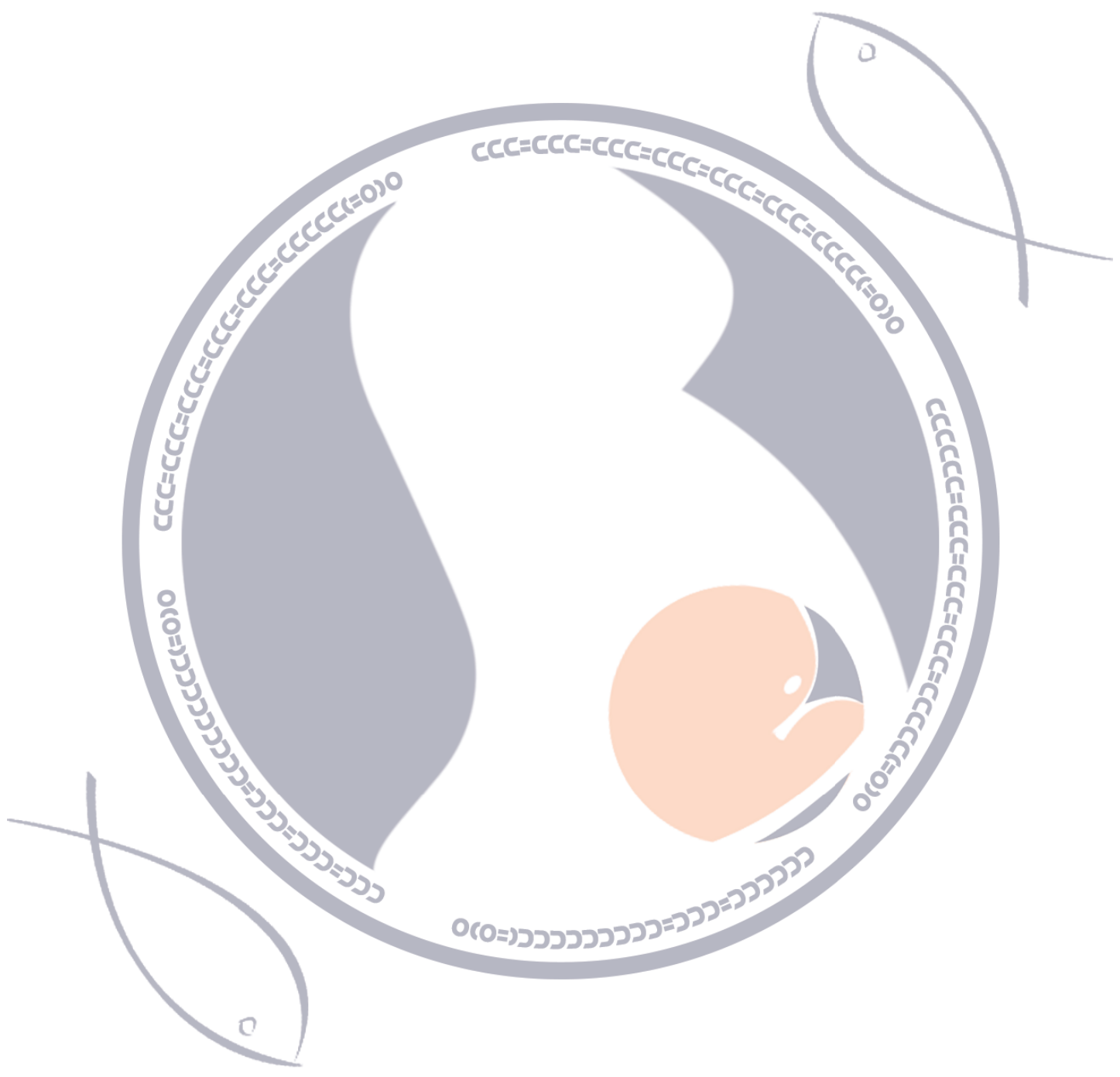




\section{CHAPTER 3}

\section{Polyunsaturated fatty acid status at birth, childhood growth and cardiometabolic risk: a pooled analysis of the MEFAB and RHEA cohorts}

N Stratakis, M Gielen, K Margetaki, RHM de Groot, M Apostolaki,

G Chalkiadaki, M Vafeiadi, V Leventakou, M Karachaliou, RW Godschalk, M Kogevinas, EG Stephanou, MP Zeegers*, L Chatzi*

${ }^{*}$ shared senior co-authorship

Provisionally accepted Eur J Clin Nutr 


\section{ABSTRACT}

Background: Polyunsaturated fatty acid (PUFA) status during pregnancy has been suggested to influence offspring obesity and cardiometabolic health.

Objective: To assess whether prenatal PUFA exposure is associated with rapid infant growth, childhood obesity and cardiometabolic risk.

Methods: In the Dutch MEFAB ( $n=266)$ and Greek RHEA ( $n=263)$ cohorts, we measured n-3 and n-6 PUFA concentrations in cord blood phospholipids, which reflect fetal exposure in late pregnancy. We defined rapid infant growth from birth to 6 months of age as an increase in weight z-score $>0.67$. We analysed body mass index (BMI) as continuous and in categories of overweight/obesity at 4 and 6 years. We computed a cardiometabolic risk score at 6-7 years as the sum of waist circumference, non-high-density lipoprotein cholesterol and blood pressure z-scores. Associations of PUFAs with child health outcomes were assessed using generalized linear models for binary outcomes and linear regression models for continuous ones after adjusting for important covariates, and for the pooled estimates, a cohort indicator.

Results: In pooled analyses, we found no association of PUFA levels with rapid infant growth, childhood BMI ( $\beta$ per 1 -unit increase in the total n-3:n-6 PUFA ratio $=-0.04$ SD $[95 \% \mathrm{CI}-0.12$ to 0.04$] ; \mathrm{P}=0.31$ at 4 years, and $-0.05 \mathrm{SD}[95 \%$ CI -0.15 to 0.05 ]; $\mathrm{P}=0.35$ at 6 years), and overweight/obesity. We also found no associations for clustered cardiometabolic risk and its individual components. The results were similar across cohorts.

Conclusions: Our findings do not support an association of fetal PUFA exposure in late gestation with obesity development and cardiometabolic risk in childhood. 


\section{INTRODUCTION}

Childhood obesity constitutes a major public health concern. Currently, it is estimated that around 50 million girls and 74 million boys aged over 5 years worldwide are obese. ${ }^{1}$ Obesity and associated risk factors in childhood, such as high blood pressure (BP) and dyslipidemia, can induce metabolic changes and contribute to atherosclerosis in adulthood. ${ }^{2}$ Therefore, it is critical to identify early determinants of obesity and cardiometabolic risk that can be targeted for preventive interventions.

Intrauterine life is an important period of developmental plasticity during which a nutritional stressor can alter body metabolism and physiology, and thus, affect child health and development. ${ }^{3}$ Animal and in vitro studies have shown that $\mathrm{n}-6$ PUFAs can increase adipose tissue deposition and promote inflammation, while n-3 PUFAs appear to exert opposite effects. ${ }^{4,5}$

Human studies have provided some evidence for a relation of prenatal PUFA levels with childhood BMI, waist circumference, and fat mass measures, but the lack of consistent findings precludes any conclusions. ${ }^{6-12}$ Few studies have assessed the association of prenatal PUFA status with childhood cardiovascular traits other than adiposity, and have reported mixed results. ${ }^{13}$ A recent systematic review underlined the importance of further studies to clarify the association of prenatal PUFA status with cardiometabolic health in childhood. ${ }^{13}$

A limitation of prior research not allowing firm conclusions is the lack of replicated findings across populations with different characteristics and behaviors. Moreover, to the best of our knowledge, no study has previously examined the association of prenatal PUFAs with aggregate constellations of cardiometabolic risk. Cardiometabolic risk factors including central adiposity, dyslipidemia, and BP may share common pathophysiological mechanisms, and their clustering is considered a better marker of cardiovascular health in children than single factors. ${ }^{14,15}$ Hence, in the present study, we pooled individual data of two birth cohorts from West and South Europe to assess whether n-3 and n-6 PUFA concentrations in cord blood phospholipids are associated with rapid infant growth, childhood BMI, and cardiometabolic risk profile.

\section{METHODS}

\section{Study population}

The Maastricht Essential Fatty Acid Birth (MEFAB) cohort (www.mefab.org) recruited pregnant women at the time of their first antenatal visit in the area of Limburg, The Netherlands, between 1989 and 1995. Of 1203 singleton deliveries, umbilical cord blood samples were assayed for 1008. Of these, 750 children 
were eligible for the follow-up evaluation at 7 years, and 306 attended the clinical examination, during which we measured anthropometry for 266 children, BP for 261, and collected blood samples for 235. A flow chart of the MEFAB participants is shown in Supplementary Figure S3.1.

The RHEA Mother-Child Cohort in Crete, Greece (www.rhea.gr) recruited pregnant women at the time of their first comprehensive ultrasound examination in Heraklion city during 2007-2008. Of 1363 singleton live births, umbilical cord blood samples were essayed for fatty acid levels in a random sample of 500 children. Of these, 263 children had anthropometry measurements up to 6 years of age, 146 provided blood lipid samples, and 156 had BP measurements at 6 years. A flow chart of the RHEA participants is shown in Supplementary Figure S3.2.

Written informed consent was obtained from all participants. The MEFAB study was approved by the ethics committee of Maastricht University/University Hospital of Maastricht, and the RHEA study was approved by the ethics committee of the University Hospital in Heraklion.

Comparison of the participants included in the present analysis and those excluded showed that children with follow-up data had mothers who were more likely to be older at delivery in MEFAB (mean age 29.9 vs. 29.1 years) and less likely to be smokers in RHEA (12.9\% vs. $20.1 \%$ ). Nevertheless, no substantial differences in maternal BMI at study entry, gestational weight gain, birth weight, gestational age, or fatty acid exposure levels were observed (Supplementary Table S3.1).

\section{Polyunsaturated fatty acid analysis}

In each cohort, PUFA concentrations were assessed in cord blood phospholipids using standard procedures (Supplementary methods) and expressed as proportion of total fatty acids measured (weight percentage, wt $\%$ ). Exposures of primary interest were $\alpha$-linolenic acid (ALA, C18:3n-3), the sum of eicosapentaenoic acid (EPA, C20:5n-3) and docosahexaenoic acid (DHA, C22:6n-3), the sum of total n-3 PUFAs measured in each cohort, linoleic acid (LA, C18:2n-6), arachidonic acid (AA, C20:4n-6), and the sum of total n-6 PUFAs measured. We also calculated the ratio of total n-3 to n-6 PUFAs.

Secondary exposures of interest included docosapentaenoic acid (DPA, C22:5n-3), $\gamma$-linolenic acid (GLA, C18:3n-6), dihomo- $\gamma$-linolenic acid (DGLA, C20:3n-6), and osbond acid (C22:5n-6).

\section{Infant growth and child BMI}

We had available information on weight and height from birth up to the age of 6-7 years (Supplementary methods). Because of variation in the number of children 
and age at follow-ups, we estimated weight and height values at the exact age of 6 months, 4 years and 6 years using cohort-, age- and sex-specific growth curves fitted with multilevel models with fractional polynomial of age, and random effects for the child and age terms (Supplementary methods). We chose these time points so as to reflect different developmental periods (infancy, preschool period, and school-age period). We estimated age- and sex-specific weight and BMI z-scores at the time points of interest according to the World Health Organization (WHO) growth standards. ${ }^{16,17}$

We defined rapid infant growth during the first 6 months of life as an increase in weight z-score $>0.67,{ }^{18}$ and overweight/obesity at 4 and 6 years based on the International Obesity Task Force definition. ${ }^{19}$

\section{Child cardiometabolic risk}

We assessed waist circumference (WC) and BP using standard procedures in both cohorts (Supplementary methods). We also measured blood lipids following standard methods (Supplementary methods).

We derived a mid-childhood cardiometabolic risk score as the sum of the following components in each cohort: sex-and age-specific z scores of WC and nonhigh-density lipoprotein (non-HDL) cholesterol, ${ }^{20}$ and the average of sex-, age-, and height-specific z-scores for systolic BP (SBP) and diastolic BP (DBP). Child glucose and insulin levels were not available in RHEA; hence, we constructed the metabolic risk score without including glucose metabolism measurements, but we performed a sensitivity analysis including this type of measures that were available in the MEFAB cohort.

Recent research has utilized similar methods to define cardiometabolic risk in children. ${ }^{14}$ This approach increases statistical power and is used to estimate an individual's cardiometabolic risk profile. A higher score is indicative of a less favorable profile. In our study sample, we observed a graded relationship between the continuous metabolic risk score and the number of dichotomous metabolic syndrome criteria according to the National Cholesterol Education Program definition modified for age ${ }^{21}$ (Supplementary Table S3.2). We also examined the raw individual components of the cardiometabolic risk score as outcomes.

\section{Statistical analysis}

We used multivariable generalized linear models for binary outcomes to assess associations of each PUFA exposure with rapid infant growth and childhood overweight/obesity, and multivariable linear regression models for associations with childhood BMI z-scores and cardiometabolic risk factors. We detected no departures from linearity in the exposure-outcome associations using scatter plots and a Wald test with the STATA command nlcheck. ${ }^{22}$ 
To enable the comparison and pooling of cohort-specific effect estimates, we calculated z-scores for PUFA concentrations in each cohort. We conducted pooled analyses by including a cohort indicator variable in the models and tested for heterogeneity in the associations across cohorts by examining the interaction between PUFA exposures and cohort. We followed a Directed Acyclic Graph approach (Supplementary Figure S3) to select variables for model adjustment. The following covariates were included in the models: maternal age at birth (years), maternal BMI at study entry $\left(\mathrm{kg} / \mathrm{m}^{2}\right)$, gestational weight gain $(\mathrm{kg})$, maternal smoking in pregnancy (never, ever), delivery type (vaginal; caesarian), parity (primiparous; multiparous), parental education (low, medium, or high according to the highest completed education level of either parent), ${ }^{23,24}$ and breastfeeding (months). We also included child sex and age in the models for raw cardiometabolic risk factors. Models of WC and BP were additionally adjusted for child height. In our models, we did not adjust for birthweight for gestational age that is a measure of fetal growth, as it might be in the causal pathway between prenatal PUFA concentrations and child health outcomes. ${ }^{25-27}$

To further assess our research hypothesis, we conducted additional analyses utilizing different levels of available information within each cohort. First, in MEFAB models, we repeated the analyses for the cardiometabolic risk score including z-scores for homeostasis model assessment of insulin resistance (HOMA-IR). Second, we conducted further adjustment for child plasma PUFA levels measured at the age of outcome assessment in MEFAB. Third, in RHEA, we conducted further adjustment for Mediterranean diet adherence in pregnancy (a priori defined score), child fast food intake (times/week) and television viewing (hours per day) available at 6 years $^{28}$ in models of childhood BMI and metabolic risk score at the respective time period.

In a sensitivity analysis, we examined associations of our primary PUFA exposures with rapid BMI growth from birth to 6 months of age, defined as a z-score BMI gain $>0.67$. We also examined overweight/obesity using the WHO definition. ${ }^{16,17}$ Moreover, given that our cardiometabolic score resulted in each included variable having equivalent weights, we also applied factor analysis (varimax rotation) to normalized WC, non-HDL cholesterol and BP measures to derive a new composite score with differential loadings of each measure. ${ }^{29}$ Finally, we assessed whether the effect estimates varied by maternal weight status at study entry (BMI $<25$ vs $\geq 25 \mathrm{~kg} / \mathrm{m}^{2}$ ) and child sex (girl vs boy) by introducing interaction terms between the potential effect modifier and the primary exposure variables. Owing to the large number of analyses, statistical significance was set at $\mathrm{P}<0.05$ for effect measure modification and at $\mathrm{P}<0.01$ for all other effect estimates in order to reduce the likelihood of change findings. 
Our study including 520 children for examining the association of cord blood PUFAs with childhood BMI had $80 \%$ power at the $1 \%$ significance level to detect effect sizes of as small as 0.15 for BMI z-score, and of as small as $61 \%$ for risk estimates of childhood overweight/obesity at 4 years (the outcome with the lowest prevalence in the pooled dataset; $9.4 \%)$ per unit change in the $n-3: n-6$ ratio. Power analysis was conducted using GPower version 3.1. ${ }^{30}$ All other analyses were conducted with STATA version 13.0 (StataCorp); code is available upon request.

\section{RESULTS}

\section{Participant characteristics}

Mothers had a mean $( \pm S D)$ age at delivery of $29.7 \pm 4.6$ years, and were predominantly non-smokers. Participants' characteristics of the separate cohorts are presented in Table 3.1. Mean $( \pm S D)$ levels of $n-3$ and $n-6$ PUFAs in MEFAB were $6.85 \pm 1.60$ and $32.15 \pm 1.68 \mathrm{wt} \%$, respectively; the corresponding values for RHEA were $5.45 \pm 1.18$ and $32.26 \pm 2.38$ wt \% (Table 3.2).

Table 3.1. Parental and child characteristics in the MEFAB and RHEA birth cohorts

\begin{tabular}{|c|c|c|c|c|}
\hline & \multicolumn{2}{|r|}{ MEFAB } & \multicolumn{2}{|r|}{ RHEA } \\
\hline & $\mathrm{n}$ & Percent or Mean (SD) & $\mathrm{n}$ & Percent or Mean (SD) \\
\hline \multicolumn{5}{|l|}{ Parental characteristics } \\
\hline Maternal age at birth (years) & 266 & $29.9(4.1)$ & 263 & $29.4(4.9)$ \\
\hline Maternal BMI at study entry $(\mathrm{kg} / \mathrm{m} 2)$ & 266 & $23.7(4.0)$ & 263 & $25.4(5.4)$ \\
\hline Gestational weight gain (kg) & 266 & $11.2(4.0)$ & 263 & $13.7(6.2)$ \\
\hline \multicolumn{5}{|l|}{ Maternal smoking in pregnancy (\%) } \\
\hline Never & 200 & 75.2 & 229 & 87.1 \\
\hline Ever & 66 & 24.8 & 34 & 12.9 \\
\hline \multicolumn{5}{|l|}{ Mode of delivery (\%) } \\
\hline Vaginal & 248 & 93.2 & 141 & 53.6 \\
\hline Caesarian & 18 & 6.8 & 122 & 46.4 \\
\hline \multicolumn{5}{|l|}{ Parity (\%) } \\
\hline Primiparous & 190 & 71.4 & 115 & 43.7 \\
\hline Multiparous & 76 & 28.6 & 148 & 56.3 \\
\hline \multicolumn{5}{|l|}{ Parental education $(\%)^{\mathrm{a}}$} \\
\hline Low & 68 & 25.5 & 30 & 11.4 \\
\hline Medium & 105 & 39.5 & 132 & 50.2 \\
\hline
\end{tabular}


Table 3.1. Parental and child characteristics in the MEFAB and RHEA birth cohorts (continued)

\begin{tabular}{|c|c|c|c|c|}
\hline & \multicolumn{2}{|r|}{ MEFAB } & \multicolumn{2}{|r|}{ RHEA } \\
\hline & $\mathrm{n}$ & Percent or Mean (SD) & $\mathrm{n}$ & Percent or Mean (SD) \\
\hline High & 93 & 35.0 & 101 & 38.4 \\
\hline \multicolumn{5}{|l|}{ Child characteristics } \\
\hline \multicolumn{5}{|l|}{ Sex $(\%)$} \\
\hline Boys & 147 & 55.3 & 143 & 54.4 \\
\hline Girls & 119 & 44.7 & 120 & 45.6 \\
\hline Birth weight (grams) & 266 & $3300(496)$ & 263 & $3213(438)$ \\
\hline Gestational age (weeks) & 266 & $39.9(1.6)$ & 263 & $38.3(1.5)$ \\
\hline Breastfeeding duration (months) & 266 & $2.2(4.1)$ & 263 & $4.4(4.5)$ \\
\hline Age at 6-7 year-follow-up (years) & 266 & $7.3(0.3)$ & 263 & $6.6(0.3)$ \\
\hline \multicolumn{5}{|l|}{ Child outcomes } \\
\hline \multicolumn{5}{|l|}{ Rapid growth from birth to 6 months $(\%)^{\text {b }}$} \\
\hline No & 203 & 79.0 & 172 & 65.4 \\
\hline Yes & 54 & 21.0 & 91 & 34.6 \\
\hline BMI z-score at 4 years ${ }^{\mathrm{c}}$ & 257 & $0.08(0.83)$ & 263 & $0.53(1.00)$ \\
\hline \multicolumn{5}{|l|}{ Weight status at 4 years $(\%)^{d}$} \\
\hline Normal weight & 245 & 95.3 & 226 & 85.9 \\
\hline Overweight/obese & 12 & 4.7 & 37 & 14.1 \\
\hline BMI z-score at 6 years ${ }^{c}$ & 257 & $0.01(0.93)$ & 263 & $0.74(1.30)$ \\
\hline \multicolumn{5}{|l|}{ Weight status at 6 years $(\%)^{d}$} \\
\hline Normal weight & 236 & 91.8 & 190 & 72.2 \\
\hline Overweight/obese & 21 & 8.2 & 73 & 27.8 \\
\hline Cardiometabolic risk score at $6-7$ years $^{\mathrm{e}}$ & 230 & $-0.01(1.89)$ & 145 & $-0.02(1.94)$ \\
\hline Waist circumference at 6-7 years $(\mathrm{cm})$ & 266 & $55.9(4.8)$ & 161 & $58.8(6.5)$ \\
\hline Non-HDL cholesterol at 6-7 years $(\mathrm{mg} / \mathrm{dl})$ & 235 & $108.6(22.9)$ & 146 & $101.8(22.7)$ \\
\hline Systolic blood pressure at 6-7 years $(\mathrm{mm} \mathrm{Hg})$ & 261 & $100.9(6.7)$ & 156 & $94.4(9.1)$ \\
\hline Diastolic blood pressure at 6-7 years $(\mathrm{mm} \mathrm{Hg})$ & 261 & $61.7(5.6)$ & 156 & $54.4(6.1)$ \\
\hline
\end{tabular}

a Parental education was defined according to the highest completed education level of either parent; for RHEA: low was defined as $\leq 9$ years of mandatory schooling, medium as $>9$ years of schooling up to attending postsecondary school education, and high as attending university or having a university/ technical college degree); for MEFAB, low was defined as attending primary school, medium as attending secondary education, and high as attending tertiary education.

${ }^{\mathrm{b}}$ Rapid growth was defined as a z-score weight gain> $0.67 \mathrm{SD}$.

${ }^{c}$ A BMI z score represents the difference from the mean sex- and age-specific BMI value for the World Health Organization reference population and is expressed in standard deviations.

${ }^{\mathrm{d}}$ Weight status was defined based on the International Obesity Task Force definition.

${ }^{\mathrm{e}}$ The cardiometabolic risk score is expressed in standard deviations was derived as the sum of the following components in each cohort: sex- and age-specific z-scores of waist circumference and non-HDL cholesterol, and the average of age-, sex- and height-specific z-scores for systolic and diastolic blood pressure. 
Table 3.2. Distribution of cord blood phospholipid PUFA levels in the MEFAB $(n=266)$ and RHEA $(n=263)$ birth cohorts

\begin{tabular}{lccc}
\hline & MEFAB & RHEA \\
\cline { 2 - 3 } Total n-3 PUFAs (wt \%) & Mean (SD) & Mean (SD) \\
ALA (wt \%) & $6.85(1.60)$ & $5.45(1.18)$ \\
DPA (wt\%) & $0.05(0.11)$ & $0.07(0.05)$ \\
EPA + DHA (wt \%) & $0.46(0.18)$ & - \\
Total n-6 PUFAs (wt \%) & $6.25(1.45)$ & $5.35(1.17)$ \\
LA (wt \%) & $32.15(1.68)$ & $32.26(2.38)$ \\
GLA (wt \%) & $7.70(1.30)$ & $10.27(1.70)$ \\
DGLA (wt \%) & $0.05(0.04)$ & $0.37(0.11)$ \\
AA (wt \%) & $5.15(0.88)$ & $3.93(0.70)$ \\
Osbond acid (wt\%) & $16.58(1.58)$ & $17.37(2.26)$ \\
Total n-3:n-6 PUFA ratio & $0.83(0.28)$ & - \\
\hline
\end{tabular}

AA, arachidonic acid (C20:4n-6); ALA, a-linolenic acid (C18:3n-3); DGLA, dihomo- $\gamma$-linolenic acid (C20:3n-6); DHA, docosahexaenoic acid (C22:6n-3); DPA, docosapentaenoic acid (C22:5n-3); EPA, eicosapentaenoic acid (C20:5n-3); DHA, docosahexaenoic acid (C22:6n-3); DPA, docosapentaenoic acid (C22:5n-3); GLA, $\gamma$-Linolenic acid (C18:3n-6); LA, linoleic acid (C18:2n-6); Osbond acid, C22:5n-6; PUFA, polyunsaturated fatty acid; total n-3 (n-6) PUFAs, the sum of n-3 (n-6) PUFAs present in the chromatogram; wt \%, weight percentage of total fatty acids measured.

\section{Cord blood PUFAs, rapid infant growth, and child BMI}

Table 3.3 presents the results of the pooled analyses on rapid weight gain in infancy and childhood BMI. Cohort-specific estimates are given in Supplementary Tables S3.3 and S3.4.

In pooled analyses on the risk of rapid infant weight gain, we found weak evidence for an inverse association with $\mathrm{n}-3$ PUFA concentrations $(\mathrm{P}=0.06)$ and a positive association with total n-6 PUFAs $(\mathrm{P}=0.06)$ (Table 3.3). There was some evidence suggesting that a higher ratio of total n-3 to n- 6 PUFAs was associated with lower risk of rapid infant growth (pooled RR per 1-unit increase $=0.85$ [95\% CI 0.730.99]; $\mathrm{P}=0.04$; $\mathrm{P}$ for $\mathrm{n}-3$ :n6 PUFA ratio-cohort interaction $=0.30$ ), though this was not significant after taking into account multiple comparisons $(\mathrm{P}>0.01)$.

In individual fatty acid analyses, there was an inverse association between n-6 LA concentrations and the risk of rapid infant growth (pooled RR per 1-SD increase in ALA concentration=1.17 [95\% CI 1.04-1.33]; $\mathrm{P}=0.01$ ), but this was driven by the MEFAB cohort $(\mathrm{RR}=1.56$ [95\% CI 1.21-2.01]; $\mathrm{P}<0.01$ and 1.09 [95\% CI 0.93-1.28]; $\mathrm{P}=0.29$ for MEFAB and RHEA, respectively; P for LA-cohort interaction $=0.03)$. We did not find any evidence that ALA, EPA+DHA and the 
n-6 AA were associated with rapid infant weight gain in both pooled (Table 3.3) and cohort-specific analyses (Supplementary Tables S3.3 and S3.4).

When we examined child BMI at 4 and 6 years in the pooled dataset, we observed no evidence for an association with cord blood PUFA levels. Similarly, no associations were found for the risk of overweight including obesity in any age group (Table 3.3). Effect estimates for child BMI and the risk of overweight including obesity were similar between cohorts (Supplementary Tables S3.3 and S3.4).

\section{Cord blood PUFAs and child cardiometabolic risk}

Table 3.4 presents the results of the pooled analyses on the clustered cardiometabolic risk score and its individual components at 6-7 years of age. Cohort-specific estimates are given in Supplementary Tables S3.5 and S3.6.

In pooled analyses, we observed no evidence for an association of cord blood PUFA levels with cardiometabolic risk score (Table 3.4). Similarly, no relationships were found for individual cardiometabolic risk factors including WC, non-HDL cholesterol, SBP and DBP in the pooled dataset (Table 3.4). Associations of PUFA levels with cardiometabolic risk were broadly similar in the cohorts (Supplementary Tables S3.5 and S3.6). There was only some evidence for between-cohort heterogeneity for effect estimates of n-3 ALA with SBP $(\beta$ per $1-S D$ increase in ALA $=0.14 \mathrm{mmHg}$ [95\% CI -0.50 to 0.78 ]; $\mathrm{P}=0.67$ for MEFAB, and $2.48 \mathrm{mmHg}$ [95\% CI 0.85 to 4.12]; $\mathrm{P}<0.01$ for RHEA; $\mathrm{P}$ for ALA-cohort interaction< 0.01 ).

\section{Secondary analyses}

We found no associations between the n-3 DPA and n-6 GLA, DGLA and osbond acid with rapid infant growth, childhood BMI and cardiometabolic risk (Supplementary Tables S3.7 and S3.8). Inclusion of child plasma PUFA levels at the age of outcome assessment in MEFAB models or further adjustment for Mediterranean diet adherence in pregnancy, child fast food intake and television viewing in RHEA models did not modify the direction and size of the effect estimates (data not shown). Additionally, no associations were observed between PUFAs and cardiometabolic risk score including HOMA-IR in MEFAB models (Supplementary Table S3.9).

\section{Sensitivity analyses}

We did not find any evidence that cord blood PUFAs were associated with rapid BMI growth in infancy (Supplementary Table S3.10) or childhood overweight/ obesity defined using the WHO classification (Supplementary Table S3.11). Results also did not materially change when we used the score derived from factor analysis (data not shown). There was no evidence of effect measure modification by maternal weight status at study entry and child sex (all $\mathrm{P}$ for interaction> 0.10 ). 


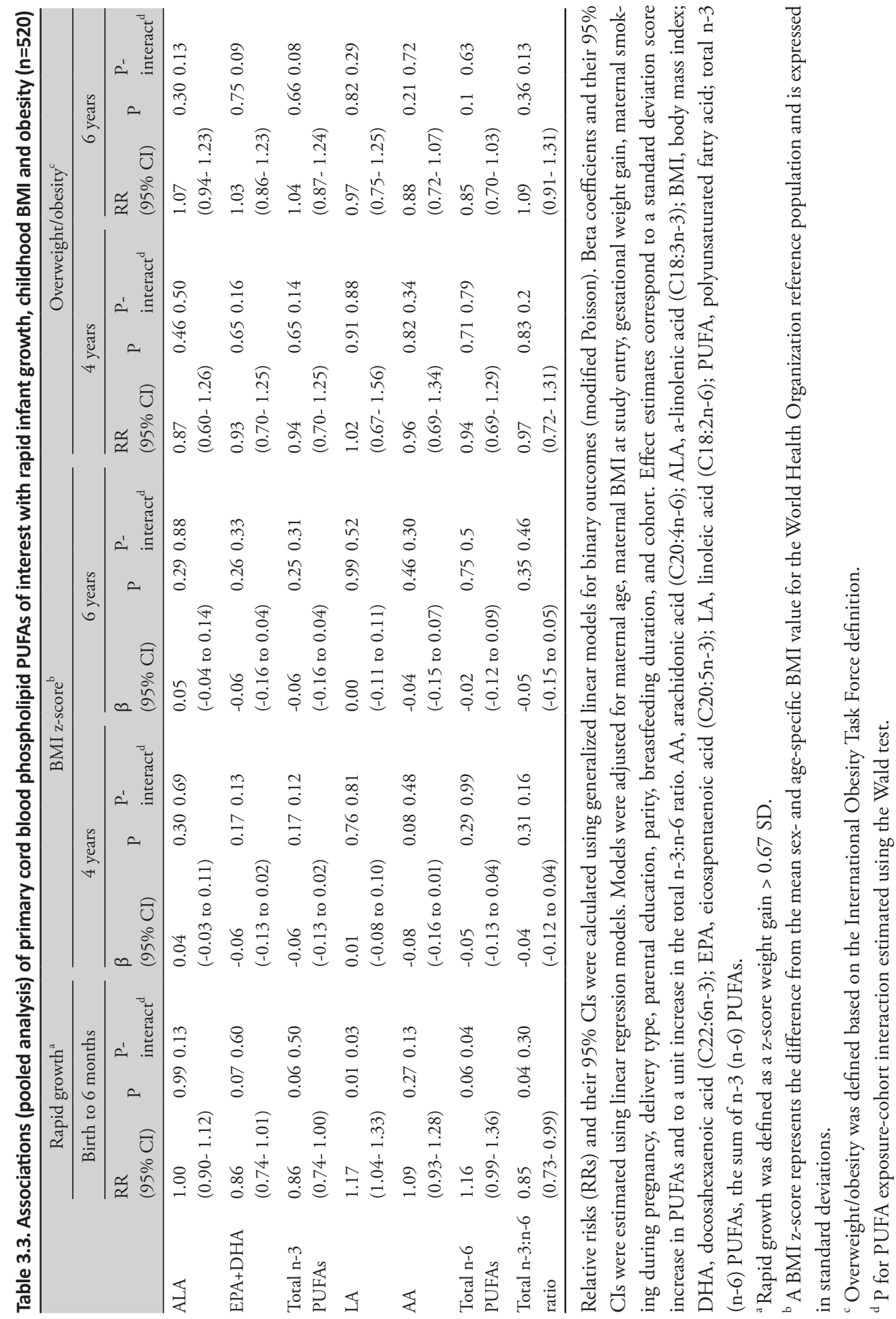




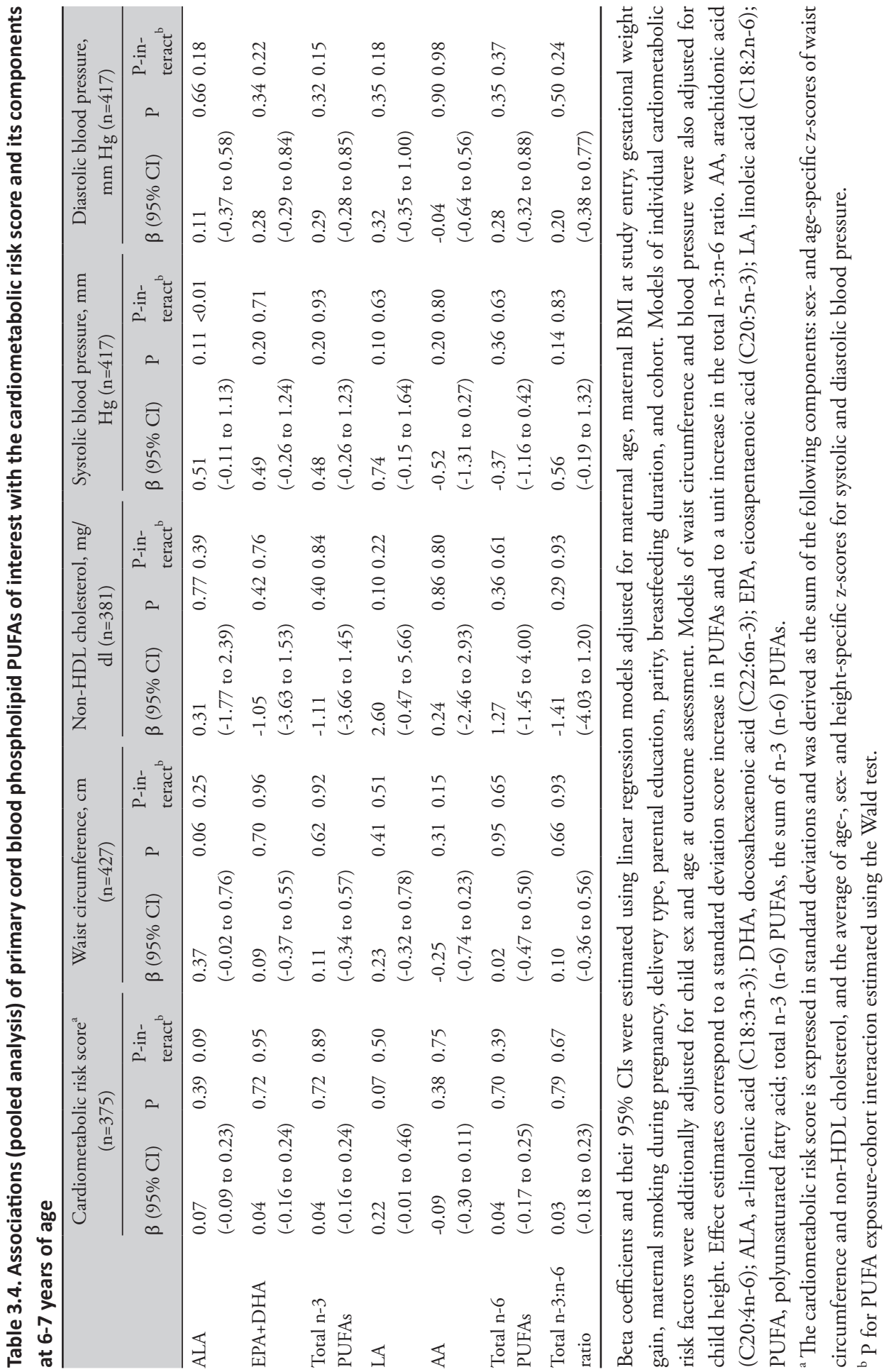




\section{DISCUSSION}

In this analysis of two cohorts from West and South Europe, we found no evidence supporting an association of cord blood PUFA levels with rapid infant growth, childhood BMI, overweight including obesity, and cardiometabolic risk profile. To our knowledge, this is the first study examining the association of prenatal PUFAs with an aggregate constellation of cardiometabolic risk. The results were broadly similar when each of the two cohorts were studied separately. The EPA+DHA status in the Netherlands and Greece is characterized as very low-to-low, which is similar to most European and US populations following a Westernized diet. ${ }^{31}$ PUFA concentrations in cord blood phospholipids are good surrogates of fetal exposure in late pregnancy, as they reflect not only maternal dietary intake of the previous 2-4 weeks, ${ }^{32}$ but also the efficiency of placental transfer. ${ }^{33}$ The timing and biomarker used for assessing fetal exposure in our study are of high relevance as they coincide with the period during which there is an exponential increase in the rate of fetal fat accretion. ${ }^{34}$

Rapid growth in the first few months of life is characterized mainly by a gain in fat, and has been to shown to predict obesity and cardiometabolic complications later in life. ${ }^{35}$ A few previous studies have assessed the effect of prenatal PUFA status on infant somatic growth. The Dutch Generation R study ( $\mathrm{n}=908$ mother-child pairs) examined PUFA levels in mid-pregnancy, and showed no consistent associations of maternal n-6 PUFA levels with infant subcutaneous fat mass measures. ${ }^{36}$ The authors also reported transient effects of maternal n-3 PUFA levels on infant central subcutaneous fat mass development. ${ }^{36}$ In the German INFAT study ( $\mathrm{n}=$ 208), Much et al, reported no association of cord blood PUFA concentrations with adiposity measures during the first year of life. ${ }^{37}$ Likewise, in our pooled analysis, we found no association between cord blood PUFAs and rapid weight gain from birth to 6 months.

Our findings also do not support a role of PUFA exposure in late gestation in the programming of adiposity status in mid-childhood. In line with our findings, a number of trials have showed no effect of n-3 long-chain PUFA supplementation during pregnancy on adiposity status in preschool and school-age children. ${ }^{6,38}$ Previous results from cohort studies in Europe and the US with samples sizes varying from 234 to 4830 mother-child pairs have also provided little evidence to support that an increase in n-3 PUFA status, a decrease in n-6 PUFA status, or both in pregnancy is an effective strategy to prevent offspring obesity. ${ }^{7-12}$

We observed no associations of PUFA levels at birth with an overall cardiometabolic risk profile in mid-childhood. Similar to our findings, two trials in Mexico $(n=524)$ and Denmark $(n=243)$ assessing $n-3$ long-chain PUFA supplementation in late pregnancy did not find any effect on offspring lipid profile ${ }^{39,40}$ and $\mathrm{BP}^{41}$ 
up to 19 years. The Aarhus birth cohort study $(n=443)$ also found no association between n-3 PUFA intake in mid-pregnancy and offspring lipid profile, BP, and glucose metabolism in early adulthood. ${ }^{10}$ By way of contrast, the Generation $\mathrm{R}$ study showed higher maternal plasma n-3 PUFA levels in mid-pregnancy to be associated with lower SBP and higher total cholesterol, HDL cholesterol, and insulin levels at age $6 .^{42,43}$

Differences in exposure definition, outcomes studied, and covariate adjustment may at least party account for inconsistencies in previous findings. In our study, we used two cohorts from different parts of Europe for replication, and followed a centralized statistical analysis following a consensus protocol with harmonized information on exposure variables, potential confounders, and child outcomes. In our analysis, we saw differential associations of the n-6 LA and rapid infant growth, and for n-3 ALA with mid-childhood SBP between cohorts, resulting in heterogeneity in the effect estimates. There was a positive association between LA and rapid infant growth only in MEFAB, while a positive association was observed for ALA and SBP only in RHEA. Compared to MEFAB children, those in RHEA had both higher LA concentrations (25-75\% range: 9.28-12.92 vs. $6.78-8.55 \mathrm{wt} \%)$ and prevalence estimates of rapid infant growth $(34.6 \%$ vs. $21.0 \%$ ), but largely overlapping ALA concentrations (25-75\% range: $0.04-0.10$ vs. $0.00-0.08$ wt $\%$ ) and lower mid-childhood SBP levels (25-75\% range: 88.2 99.7 vs. $96.7-105.3 \mathrm{~mm} \mathrm{Hg}$ ). Hence, we treat these associations with caution, as they might not reflect a biological effect, but rather they might have occurred due to chance or due to a specific confounder pattern in one cohort relative to the other (eg,differences in diet or lifestyle).

As in any observational study, there is still the possibility of unmeasured residual confounding. Although we considered television viewing in our analysis, other differences in sedentariness or physical activity patterns might have influenced the observed findings. Our study had adequate power to detect relative large effect sizes in risk estimates for binary outcomes. It is possible that some smaller but still meaningful effects were missed, especially given the small variability in the fatty acid concentrations. Nevertheless, our study was adequately powered to detect effect sizes that were similar to those previously reported. ${ }^{7}$ Similar to most cohort studies, a limitation of the present study is loss to follow up, which raises the likelihood of attrition bias. It may be possible that the sample of children who participated in the follow-up evaluations had a lower BMI and a healthier cardiometabolic profile than those who did not, thus resulting in reduced variability in the distribution of BMI and other cardiometabolic risk factors. This might have limited our ability to detect an association. Nevertheless, our study population did not substantially differ from excluded mother-child pairs in main 
baseline sociodemographic characteristics. BMI, one of our main outcomes, is a measure that incorporates both lean and fat mass. However, childhood BMI has been shown to strongly correlate with dual emission X-ray absorptiometry (DXA) fat mass. ${ }^{44}$

To conclude, this pooled analysis of two population-based cohort studies from West and South Europe suggests that fetal exposure to PUFAs in late pregnancy is not associated with rapid infant growth, childhood adiposity status, and cardiometabolic risk. 


\section{REFERENCES}

1. NCD Risk Factor Collaboration. Worldwide trends in body-mass index, underweight, overweight, and obesity from 1975 to 2016: a pooled analysis of 2416 population-based measurement studies in 128.9 million children, adolescents, and adults. Lancet 2017. doi: 10.1016/S0140-6736(17)32129-3. [Epub ahead of print]

2. Raitakari OT, Juonala M, Kahonen M, et al. Cardiovascular risk factors in childhood and carotid artery intima-media thickness in adulthood: the Cardiovascular Risk in Young Finns Study. JAMA 2003;290:2277-83.

3. Symonds ME, Sebert SP, Hyatt MA, Budge H. Nutritional programming of the metabolic syndrome. Nat Rev Endocrinol 2009;5:604-10.

4. Ailhaud G, Guesnet P, Cunnane SC. An emerging risk factor for obesity: does disequilibrium of polyunsaturated fatty acid metabolism contribute to excessive adipose tissue development? Brit J Nutr 2008;100:461-70.

5. Kalupahana NS, Claycombe KJ, Moustaid-Moussa N. (n-3) Fatty acids alleviate adipose tissue inflammation and insulin resistance: mechanistic insights. Adv Nutr 2011;2:304-16.

6. Stratakis N, Gielen M, Chatzi L, Zeegers MP. Effect of maternal n-3 long-chain polyunsaturated fatty acid supplementation during pregnancy and/or lactation on adiposity in childhood: a systematic review and meta-analysis of randomized controlled trials. Eur J Clin Nutr 2014;68:1277-87.

7. Donahue SM, Rifas-Shiman SL, Gold DR, et al. Prenatal fatty acid status and child adiposity at age 3 y: results from a US pregnancy cohort. Am J Clin Nutr 2011;93:780-8.

8. Standl M, Thiering E, Demmelmair H, Koletzko B, Heinrich J. Age-dependent effects of cord blood long-chain PUFA composition on BMI during the first 10 years of life. Brit J Nutr 2014;111:2024-31.

9. Vidakovic AJ, Gishti O, Voortman T, et al. Maternal plasma PUFA concentrations during pregnancy and childhood adiposity: the Generation R Study. Am J Clin Nutr 2016;103:1017-25.

10. Rytter D, Bech BH, Halldorsson T, et al. No association between the intake of marine n-3 PUFA during the second trimester of pregnancy and factors associated with cardiometabolic risk in the 20 -year-old offspring. Brit J Nutr 2013;110:2037-46.

11. de Vries PS, Gielen M, Rizopoulos D, et al. Association between polyunsaturated fatty acid concentrations in maternal plasma phospholipids during pregnancy and offspring adiposity at age 7: the MEFAB cohort. Prostaglandins Leukot Essent Fatty Acids 2014;91:81-5.

12. Moon RJ, Harvey NC, Robinson SM, et al. Maternal Plasma Polyunsaturated Fatty Acid Status in Late Pregnancy Is Associated with Offspring Body Composition in Childhood. J Clin Endocr Metab 2013;98:299-307.

13. Voortman T, van den Hooven EH, Braun KV, et al. Effects of polyunsaturated fatty acid intake and status during pregnancy, lactation, and early childhood on cardiometabolic health: A systematic review. Prog Lipid Res 2015;59:67-87.

14. Ahrens W, Moreno LA, Marild S, et al. Metabolic syndrome in young children: definitions and results of the IDEFICS study. Int J Obes (Lond) 2014;38 Suppl 2:S4-14.

15. Berenson GS. Childhood risk factors predict adult risk associated with subclinical cardiovascular disease. The Bogalusa Heart Study. Am J Cardiol 2002;90:3L-7L.

16. WHO Multicentre Growth Reference Study Group. WHO Child Growth Standards: Length/heightfor-age, weight-for-age, weight-for-length, weight-for-height and body mass index-for-age: Methods and development. 2006. Geneva: World Health Organization.

17. de Onis M, Onyango AW, Borghi E, et al. Development of a WHO growth reference for school-aged children and adolescents. Bull World Health Organ 2007;85:660-7. 
18. Monteiro PO, Victora CG. Rapid growth in infancy and childhood and obesity in later life--a systematic review. Obes Rev 2005;6:143-54.

19. Cole TJ, Lobstein T. Extended international (IOTF) body mass index cut-offs for thinness, overweight and obesity. Pediatr Obes 2012;7:284-94.

20. Expert Panel on Integrated Guidelines for Cardiovascular Health and Risk Reduction in Children and Adolescents; National Heart Lung and Blood Institute. Expert panel on integrated guidelines for cardiovascular health and risk reduction in children and adolescents: summary report. Pediatrics 2011;128 Suppl 5:S213-S56.

21. Cook S, Weitzman M, Auinger P, Nguyen M, Dietz WH. Prevalence of a metabolic syndrome phenotype in adolescents: findings from the third National Health and Nutrition Examination Survey, 1988-1994. Arch Pediatr Adolesc Med 2003;157:821-7.

22. Ben J. NLCHECK: Stata module to check linearity assumption after model estimation. Statistical Software Components S456968. Boston College Department of Economics 2008.

23. Netherlands Institute for Public Health and the Environment. (RIVM). Indeling opleidingsniveau. 2008.

24. Vafeiadi M, Roumeliotaki T, Myridakis A, et al. Association of early life exposure to bisphenol A with obesity and cardiometabolic traits in childhood. Environ Res 2016;146:379-87.

25. Koletzko B. Fatty acids and early human growth. Am J Clin Nutr 2001;73:671-2.

26. Grootendorst-van Mil NH, Tiemeier H, Steenweg-de Graaff J, et al. Maternal plasma n-3 and n-6 polyunsaturated fatty acids during pregnancy and features of fetal health: Fetal growth velocity, birth weight and duration of pregnancy. Clin Nutr 2017. doi: 10.1016/j.clnu.2017.06.010. [Epub ahead of print]

27. McMillen IC, Robinson JS. Developmental origins of the metabolic syndrome: prediction, plasticity, and programming. Physiol Rev 2005;85:571-633.

28. Chatzi L, Rifas-Shiman SL, Georgiou V, et al. Adherence to the Mediterranean diet during pregnancy and offspring adiposity and cardiometabolic traits in childhood. Pediatr Obes 2017;12 Suppl 1:47-56.

29. Wijndaele K, Beunen G, Duvigneaud N, et al. A continuous metabolic syndrome risk score: utility for epidemiological analyses. Diabetes Care 2006;29:2329.

30. Faul F, Erdfelder E, Buchner A, Lang AG. Statistical power analyses using G*Power 3.1: tests for correlation and regression analyses. Behav Res Methods 2009;41:1149-60.

31. Stark KD, Van Elswyk ME, Higgins MR, Weatherford CA, Salem N, Jr. Global survey of the omega-3 fatty acids, docosahexaenoic acid and eicosapentaenoic acid in the blood stream of healthy adults. Prog Lipid Res 2016;63:132-52.

32. Hodson L, Skeaff CM, Fielding BA. Fatty acid composition of adipose tissue and blood in humans and its use as a biomarker of dietary intake. Prog Lipid Res 2008;47:348-80.

33. Hornstra G. Essential fatty acids in mothers and their neonates. Am J Clin Nutr 2000;71:1262S-9S.

34. Haggarty P. Fatty acid supply to the human fetus. Annu Rev Nutr 2010;30:237-55.

35. Gillman MW. The first months of life: a critical period for development of obesity. Am J Clin Nutr 2008;87:1587-9.

36. Jelena Vidakovic A, Santos S, Williams MA, et al. Maternal plasma n-3 and n-6 polyunsaturated fatty acid concentrations during pregnancy and subcutaneous fat mass in infancy. Obesity (Silver Spring) 2016;24:1759-66.

37. Much D, Brunner S, Vollhardt C, et al. Effect of dietary intervention to reduce the n-6/n-3 fatty acid ratio on maternal and fetal fatty acid profile and its relation to offspring growth and body composition at 1 year of age. Eur J Clin Nutr 2013;67:282-8. 
38. Blumfield ML. Can long-chain PUFA supplementation during pregnancy influence later obesity risk? Am J Clin Nutr 2016;103:1387-8.

39. Gutierrez-Gomez Y, Stein AD, Ramakrishnan U, et al. Prenatal Docosahexaenoic Acid Supplementation Does Not Affect Nonfasting Serum Lipid and Glucose Concentrations of Offspring at 4 y of Age in a Follow-Up of a Randomized Controlled Clinical Trial in Mexico. J Nutr 2017; 147: 242-247.

40. Rytter D, Schmidt EB, Bech BH, et al. Fish Oil Supplementation During Late Pregnancy Does Not Influence Plasma Lipids or Lipoprotein Levels in Young Adult Offspring. Lipids 2011;46:1091-9.

41. Rytter D, Christensen JH, Bech BH, et al. The effect of maternal fish oil supplementation during the last trimester of pregnancy on blood pressure, heart rate and heart rate variability in the 19-year-old offspring. Brit J Nutr 2012;108:1475-83.

42. Vidakovic AJ, Jaddoe VW, Voortman T, et al. Maternal plasma polyunsaturated fatty acid levels during pregnancy and childhood lipid and insulin levels. Nutr Metab Cardiovasc Dis 2017;27:78-85.

43. Vidakovic AJ, Gishti O, Steenweg-de Graaff J, et al. Higher Maternal Plasma n-3 PUFA and Lower n-6 PUFA Concentrations in Pregnancy Are Associated with Lower Childhood Systolic Blood Pressure. J Nutr 2015;145:2362-8.

44. Boeke CE, Oken E, Kleinman KP, et al. Correlations among adiposity measures in school-aged children. BMC Pediatr 2013;13:99. 


\section{Supplementary Material of Chapter 3}

Text 3A: Supplementary methods

3A.1 Polyunsaturated fatty acid analysis

3A.2 Child anthropometry

3A.3 Modeling of weight and height growth trajectories

3A.4 Child cardiometabolic risk factors

Supplementary Table S3.1. Comparison of subject characteristics in MEFAB and RHEA between those included and not included in the main analyses

Supplementary Table S3.2. Relationship between the continuous cardiometabolic risk score and the number of dichotomous of metabolic syndrome criteria based on the National Cholesterol Education Program definition modified for age. Supplementary Table S3.3. Associations of primary PUFAs of interest with rapid infant growth, childhood body mass index, and overweight/obesity in the MEFAB cohort $(\mathrm{n}=257)$

Supplementary Table S3.4. Associations of primary PUFAs of interest with rapid infant growth, childhood body mass index, and overweight/obesity in the RHEA cohort $(n=263)$

Supplementary Table S3.5. Associations of primary PUFAs of interest with the cardiometabolic risk score and its components at 7 years of age in the MEFAB cohort

Supplementary Table S3.6. Associations of primary PUFAs of interest with the cardiometabolic risk score and its components at 6 years of age in the RHEA cohort

Supplementary Table S3.7. Associations (pooled analysis) of secondary PUFAs of interest with rapid growth in infancy, childhood body mass index, and cardiometabolic risk score

Supplementary Table S3.8. Associations of secondary PUFAs of interest, measured only in MEFAB, with rapid growth in infancy, childhood BMI and cardiometabolic risk score

Supplementary Table S3.9. Associations of primary PUFAs of interest with cardiometabolic risk score including HOMA-IR at 7 years of age in the MEFAB birth cohort $(\mathrm{n}=226)$ 
Supplementary Table S3.10. Associations (pooled analysis) of primary cord blood phospholipid PUFAs of interest with rapid body mass index growth in infancy $(\mathrm{n}=520)$

Supplementary Table S3.11. Associations (pooled analysis) of primary cord blood phospholipid PUFAs of interest with childhood overweight/obesity defined based on the WHO definition $(\mathrm{n}=520)$

Supplementary Figure S3.1. Flow chart of the MEFAB participants.

Supplementary Figure S3.2. Flow chart of the RHEA participants.

Supplementary Figure S3.3. Directed acyclic graph for the association of fatty acid status at birth with childhood growth.

Supplementary References 


\section{Text 3A: Supplementary methods}

\section{A.1 Polyunsaturated fatty acid analysis}

In the MEFAB birth cohort, umbilical cord blood samples were collected in EDTA-containing tubes. Plasma was separated from blood cells by centrifugation and stored in aliquots at $-80^{\circ} \mathrm{C}$ until analysis. Methods of fatty acid analysis and the phospholipid PUFAs identified in MEFAB have been described in detail elsewhere. ${ }^{1}$ In short, after the addition of 1,2-dinonadecanoyl phosphatidylcholine (internal standard), total lipid extracts from $100 \mu \mathrm{L}$ plasma were prepared by using a modified Folch method. ${ }^{2}$ Phospholipids were isolated by solid-phase extraction of total lipid extracts on aminopropyl-silica columns. To check for carryover of other lipid fractions during this procedure, heptadecaenoic acid (17:1) was added to the samples. After saponification of the isolated phospholipids, fatty acids were converted to the corresponding fatty acid methyl esters. The fatty acids were determined by capillary gas chromatography with a polar capillary column (50 m $\times 0.25$ mm CPSil 88; Chrompack, Middelburg, Netherlands) and helium as the carrier gas.

In the RHEA birth cohort, umbilical cord blood samples were collected in 10-mL $\mathrm{BD}$ gel separator vacutainers. Serum was separated within $2 \mathrm{~h}$ after collection using centrifugation and stored in $0.5-\mathrm{mL}$ aliquots at $-80^{\circ} \mathrm{C}$ until assayed. The analysis of polyunsaturated fatty acids was conducted following a standard methodology, as described by Glaser et al. ${ }^{3}$ Briefly, a fatty acid methyl ester (FAME) sample was obtained using Direct In Situ Transesterification. The individual FAMEs were quantified by gas chromatography with flame ionization detection. Gas chromatography analysis was carried out on a Shimadzu-2010 chromatograph equipped with AOC-20i auto-injector; Shimadzu Europe, Duisburg, Germany. Separation of serum phospholipid FAMEs was performed on a SP-2560 fused silica capillary column $(100 \mathrm{~m} \times 0.25 \mathrm{~mm}$ i.d., $0.2 \mu \mathrm{m}$ film thickness; Supelco, Bellefonte, PA, USA) using helium as the carrier gas at a constant flow of $1.1 \mathrm{ml} / \mathrm{min}$. Individual FAME peaks were identified by comparison of their retention times with SUPELCO 37 Component FAME mix; Supelco Bellefonte, PA, USA.

PUFA composition of plasma phospholipids is similar to that of serum phospholipids, and slight differences in the analytical methods used do not appear to affect the way that PUFA composition can be interpreted. ${ }^{4,5}$

\section{A.2 Child anthropometry}

In the MEFAB cohort, information on weight and height up to 4 years was obtained through medical records. At the 7-year examination, a single investigator 
measured children's weight, height, and waist circumference. Body weight was determined to the nearest $0.1 \mathrm{~kg}$ on a SECA electronic digital scale (Seca Corporation, Hanover, $\mathrm{MD}$ ) while bare footed in light underwear. Height was measured to the nearest $0.1 \mathrm{~cm}$ using a wall-mounted stadiometer (Holtain Ltd, Crymych, UK). Waist circumference was measured at the age of 7 years using a tape measure to the nearest $0.1 \mathrm{~cm}$ at the site of the smallest circumference between the rib cage and the ileac crest while the subjects were standing.

In RHEA, trained research assistants measured weight to the nearest $0.1 \mathrm{~kg}$ and height to the nearest $0.1 \mathrm{~cm}$ up to the age of 6 years in children standing without shoes and in light clothing by using validated scales (Seca 354 baby scale, Seca Bellisima 841; Seca Corporation) and stadiometers (Seca 210 measuring mat, and Seca 213). At the 6-year examination, waist circumference was measured in duplicate to the nearest $0.1 \mathrm{~cm}$ in standing position, at the high point of the iliac crest at the end of a gentle expiration, using a flexible tape measure (Seca 201).

\section{A.3 Modeling of weight and height growth trajectories}

Patterns of growth across childhood follow a complex pattern (growth is non-linear). We used a two-step approach to estimate growth curves for the participating cohorts. First we identified for each cohort the best fitting fractional polynomials of age (in days) and constructed sex-and age- specific weight and height growth curves. ${ }^{6}$ Briefly, a series of models were run for each cohort in which age was raised to a large number of combinations of powers (each of the following single powers, plus each combination of two powers: $-2,-1,-0.5,0,0.5,1,2,3$, where a power of zero is the log function), resulting in a wide range of possible weight and height curves. ${ }^{7}$ For each growth measure, we chose the model with the lowest deviance. Then we used mixed-effects linear regression models with the previously identified fractional polynomials of age, including a random intercept for child and random age slopes. Such models allow for individual variation in growth curves within each cohort, and use all available data from all the eligible children under a missing at random assumption. ${ }^{8}$ Only subjects with at least two growth measurements from birth to 6-7 years were included.

The tables below (3A.3.1 and 3A.3.2) show that our multilevel fractional polynomial models fit the data well, as there were high levels of agreement between observed growth measurements and those predicted by the models.

\section{A.4 Child cardiometabolic risk factors}

In the MEFAB birth cohort, information on cardiometabolic risk factors was obtained at 7 years. A physician measured systolic (SBP) and diastolic blood pressure (DBP) in children while seated at the right arm by using an upper arm 
blood pressure monitor. Three measurements were made, and the average of all measurements was used for analysis. Fasting blood samples were obtained from children in EDTA-containing tubes, and plasma was separated by centrifugation and stored at $-80^{\circ} \mathrm{C}$. Total and HDL cholesterol concentrations were determined as described in ". Plasma cholesterol values were transformed to serum values using appropriate formulas ${ }^{10}$. Glucose was determined enzymatically (Glucose HK-method, Hoffmann-La Roch, Basel Switzerland) and insulin was measured as described in ${ }^{11}$. Insulin resistance was estimated according to the homoeostasis model assessment (HOMA) as the product of fasting glucose ( $\mathrm{mmol} / \mathrm{L})$ and insulin $(\mu \mathrm{U} / \mathrm{mL})$ divided by the constant $22.5 .^{12}$

In the RHEA cohort, we used information on cardiometabolic risk factors obtained at 6 years. Trained research assistants measured SBP and DBP in children after 5 minutes rest in the seated position, at the right arm using an automatic oscillometric device (Pro Care 400, Dinamap) with a cuff of appropriate size for arm circumference. Five measurements were made at 1-minute intervals, and the average of all measurements was used for analysis. Non-fasting blood samples were collected from the RHEA children in $10-\mathrm{mL}$ vacutainer tubes with the use of standard procedures, with samples immediately spun, separated, and frozen at $-80^{\circ} \mathrm{C}$. Analysis of serum total cholesterol and high-density lipoprotein (HDL) cholesterol levels was performed by standard enzymatic methods (Medicon) on an automatic analyzer (AU5400 high-volume chemistry analyzer; Olympus America Inc).

In the present analysis, we considered non-high-density lipoprotein (non-HDL) cholesterol (calculated by subtracting HDL cholesterol from total cholesterol), since it includes both cholesterol-rich and triglycerides-rich atherogenic apolipoprotein B- containing lipoproteins (very-low-density lipoprotein [VLDL], intermediate- density lipoproteins, LDL, and lipoprotein [a]). Non-HDL cholesterol levels have been suggested to be more predictive of persistent dyslipidemia and atherosclerosis than total cholesterol, LDL cholesterol or HDL-cholesterol levels. ${ }^{13}$ 
Supplementary Table S3.1. Comparison of subject characteristics in MEFAB and RHEA between those included and not included in the main analyses

\begin{tabular}{|c|c|c|c|c|c|}
\hline \multicolumn{3}{|c|}{ MEFAB } & \multicolumn{3}{|c|}{ RHEA } \\
\hline 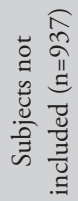 & 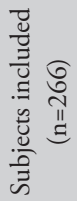 & 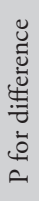 & 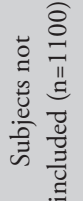 & 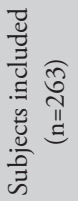 & 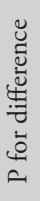 \\
\hline
\end{tabular}

Parental characteristics

Maternal age at birth (years)

$\begin{array}{cccccc}29.1(4.3) & 29.9(4.1) & 0.01 & 29.7(5.0) & 29.4(4.9) & 0.34 \\ 24.3(4.7) & 23.7(4.0) & 0.08 & 25.3(4.7) & 25.4(5.4) & 0.71 \\ 11.7(4.6) & 11.2(4.0) & 0.12 & 14.1(5.9) & 13.7(6.2) & 0.37 \\ 227(25.8) & 66(24.8) & 0.75 & 146(20.1) & 34(12.9) & 0.01 \\ & & & & & \\ 680(76.1) & 190(71.4) & 0.12 & 338(43.6) & 115(43.7) & 0.97 \\ 265(38.6) & 82(35.0) & 0.10 & - & - & \\ & & & & & \\ - & - & & 272(37.5) & 101(38.4) & 0.96 \\ 422(45.0) & 119(44.7) & 0.94 & 549(49.9) & 120(45.6) & 0.21 \\ 3271(517) & 3300(496) & 0.41 & 3201(453) & 3213(438) & 0.70 \\ 39.8(1.8) & 39.9(1.6) & 0.98 & 38.2(1.5) & 38.3(1.5) & 0.71 \\ - & - & & 602(84.4) & 232(88.2) & 0.14 \\ 6.92(1.58) & 6.85(1.60) & 0.54 & 5.15(1.25) & 5.45(1.18) & 0.05 \\ 32.09(1.69) & 32.15(1.68) & 0.63 & 32.30(3.15) & 32.26(2.38) & 0.88\end{array}$

Maternal BMI at study entry $(\mathrm{kg} / \mathrm{m} 2)$

Gestational weight gain $(\mathrm{kg})$

Maternal smoking in pregnancy (yes,

$\%)$

Parity (primiparous, \%)

Area-based socio-economic status at birth (high, \%) $)^{\mathrm{a}}$

Parental education (high, \%)

Child characteristics

Sex (female)

Birth weight (grams)

Gestational age (weeks)

Breastfeeding status (yes, \%)

Cord blood n-3 PUFAs (wt \%)

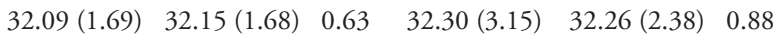

Values are means (SD) or absolute numbers (percentages). P values for difference were estimated using Student's t test for continuous variables and Pearson's $\chi 2$ test for categorical variables.

${ }^{a}$ Area-based socio-economic status at birth was determined based on the postal code of the place of residence. 
Supplementary Table S3.2. Relationship between the continuous cardiometabolic risk score and the number of dichotomous of metabolic syndrome criteria based on the National Cholesterol Education Program definition modified for age.

\begin{tabular}{|c|c|c|c|c|c|c|c|c|c|c|c|}
\hline \multirow{3}{*}{$\begin{array}{l}\text { No. of metabolic } \\
\text { syndrome } \\
\text { criteria }^{b}\end{array}$} & \multicolumn{11}{|c|}{ Cardiometabolic risk score $^{a}$} \\
\hline & \multicolumn{4}{|c|}{ MEFAB } & \multicolumn{4}{|c|}{ RHEA } & \multicolumn{3}{|c|}{ Pooled } \\
\hline & $\mathrm{N}$ & Mean & $\mathrm{SD}$ & P-value ${ }^{c}$ & $\mathrm{~N}$ & Mean & $\mathrm{SD}$ & $P$-value ${ }^{c}$ & Mean & $\mathrm{SD}$ & $P$-value ${ }^{c}$ \\
\hline 0 & 154 & -0.52 & 1.43 & $<0.0001$ & 104 & -0.86 & 1.41 & $<0.0001$ & -0.66 & 1.43 & $<0.0001$ \\
\hline 1 & 65 & 0.74 & 1.88 & & 30 & 0.89 & 1.18 & & 0.79 & 1.69 & \\
\hline 2 & 8 & 3.31 & 2.20 & & 10 & 2.79 & 1.61 & & 3.02 & 1.85 & \\
\hline $3+$ & 3 & 4.99 & 1.34 & & 1 & 5.27 & - & & 5.06 & 1.10 & \\
\hline
\end{tabular}

a The cardiometabolic risk score is expressed in standard deviations and was derived as the sum of the following components: sex- and age-specific z-scores of waist circumference and non-HDL cholesterol, and the average of age-, sex- and height-specific z-scores for systolic and diastolic blood pressure.

b The metabolic syndrome risk criteria were based on to the National Cholesterol Education Program definition modified for age ${ }^{21}$ : waist circumference $\geq 90$ th percentile for age and sex; systolic or diastolic blood pressure $\geq 90$ th percentile for age, sex, and height; glucose $\geq 110 \mathrm{mg} / \mathrm{dl}$ (for MEFAB only); HDL-cholesterol $\leq 40 \mathrm{mg} / \mathrm{dl}$; and triglycerides $\geq 110 \mathrm{mg} / \mathrm{dl}$.

${ }^{\mathrm{c}} \mathrm{P}$-value was calculated by using analysis of variance (ANOVA). 


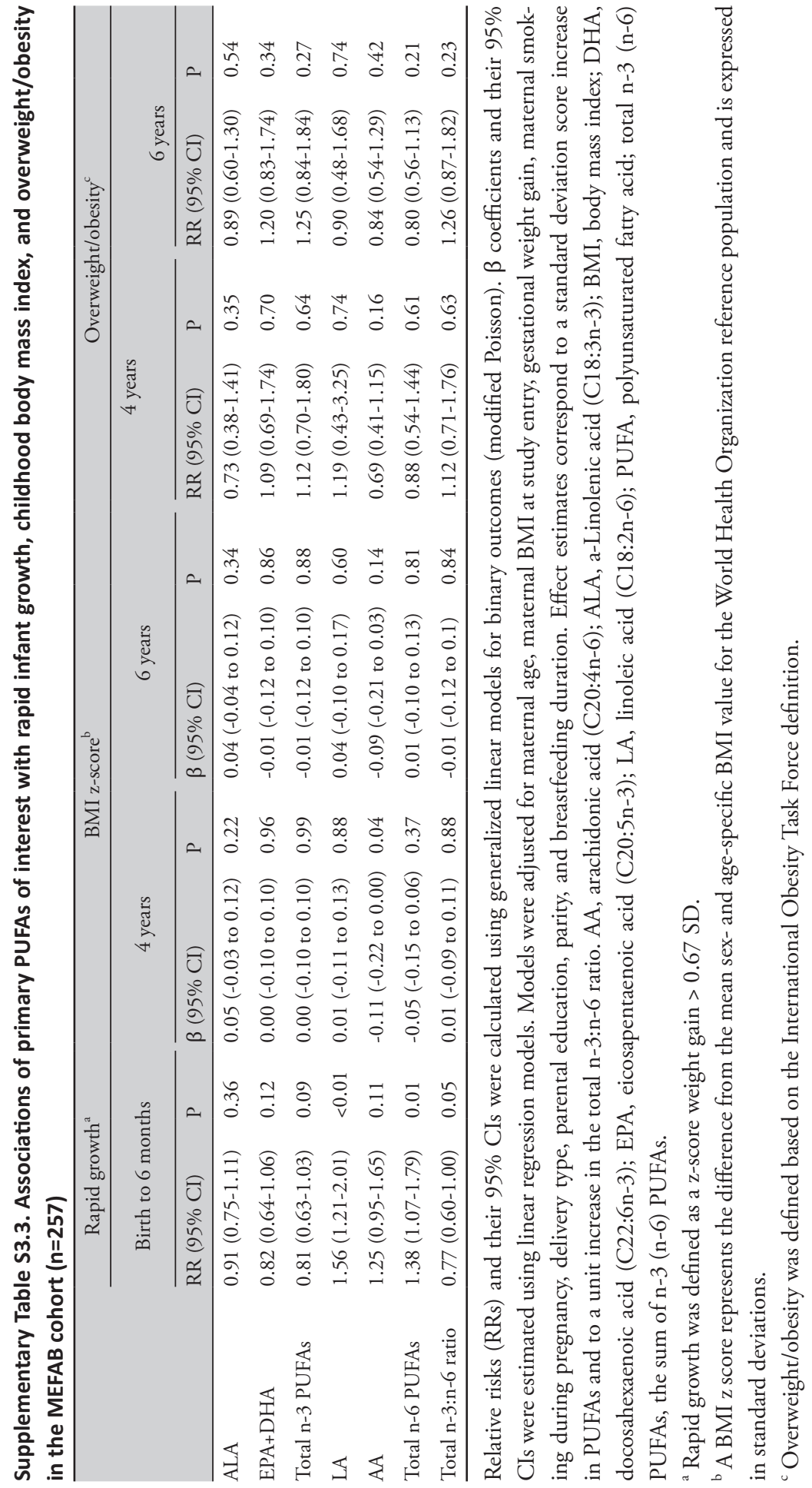




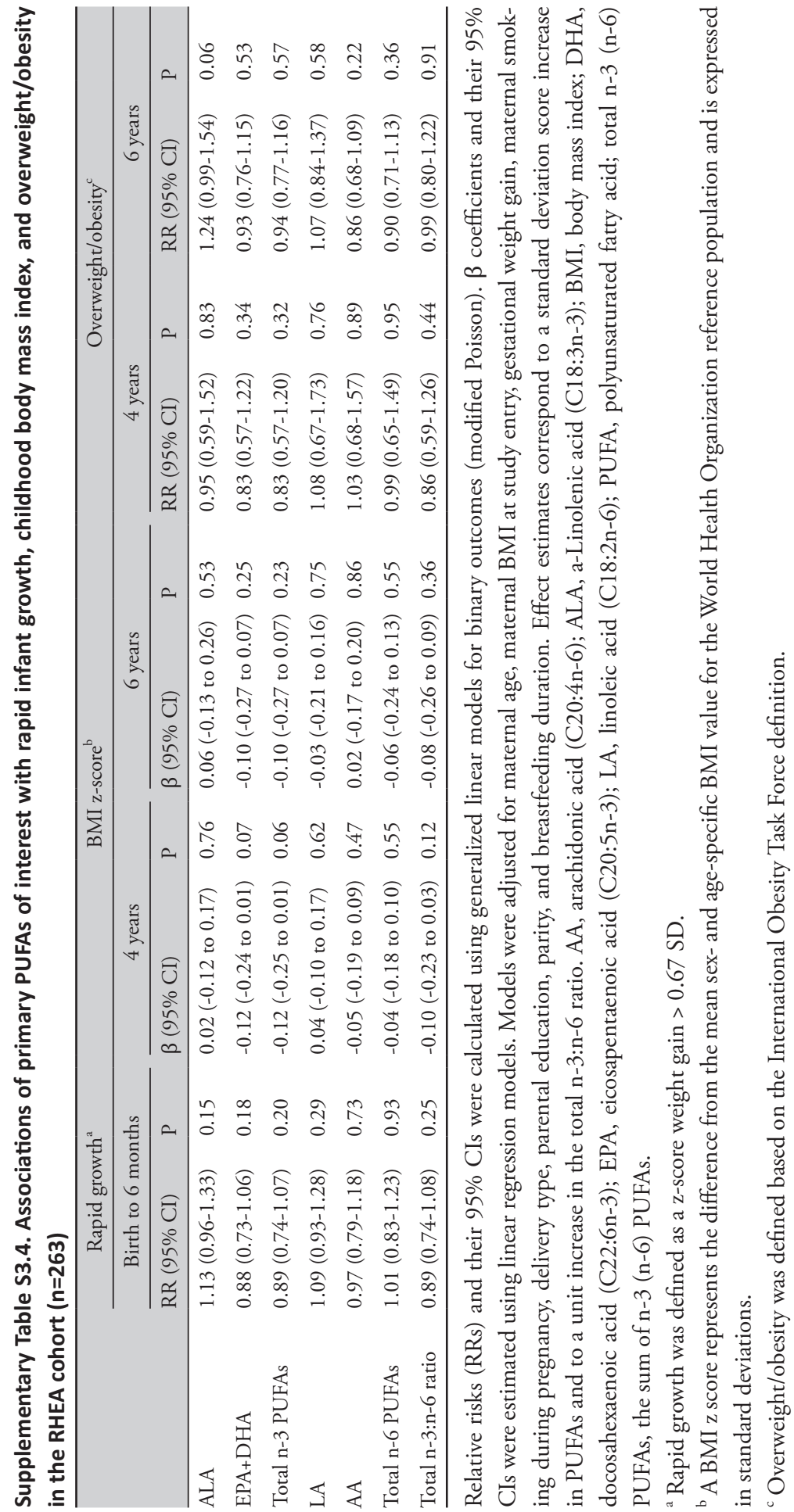




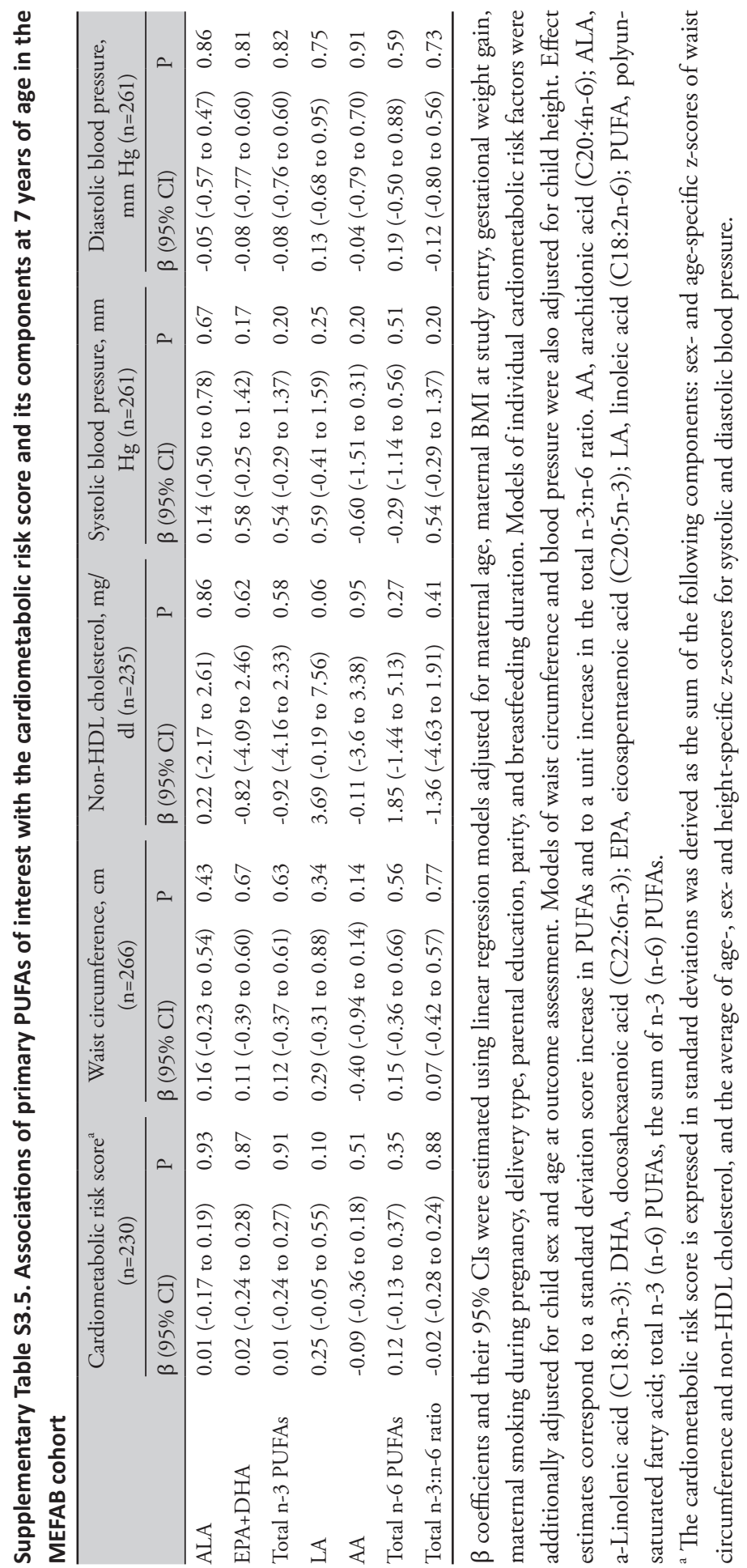




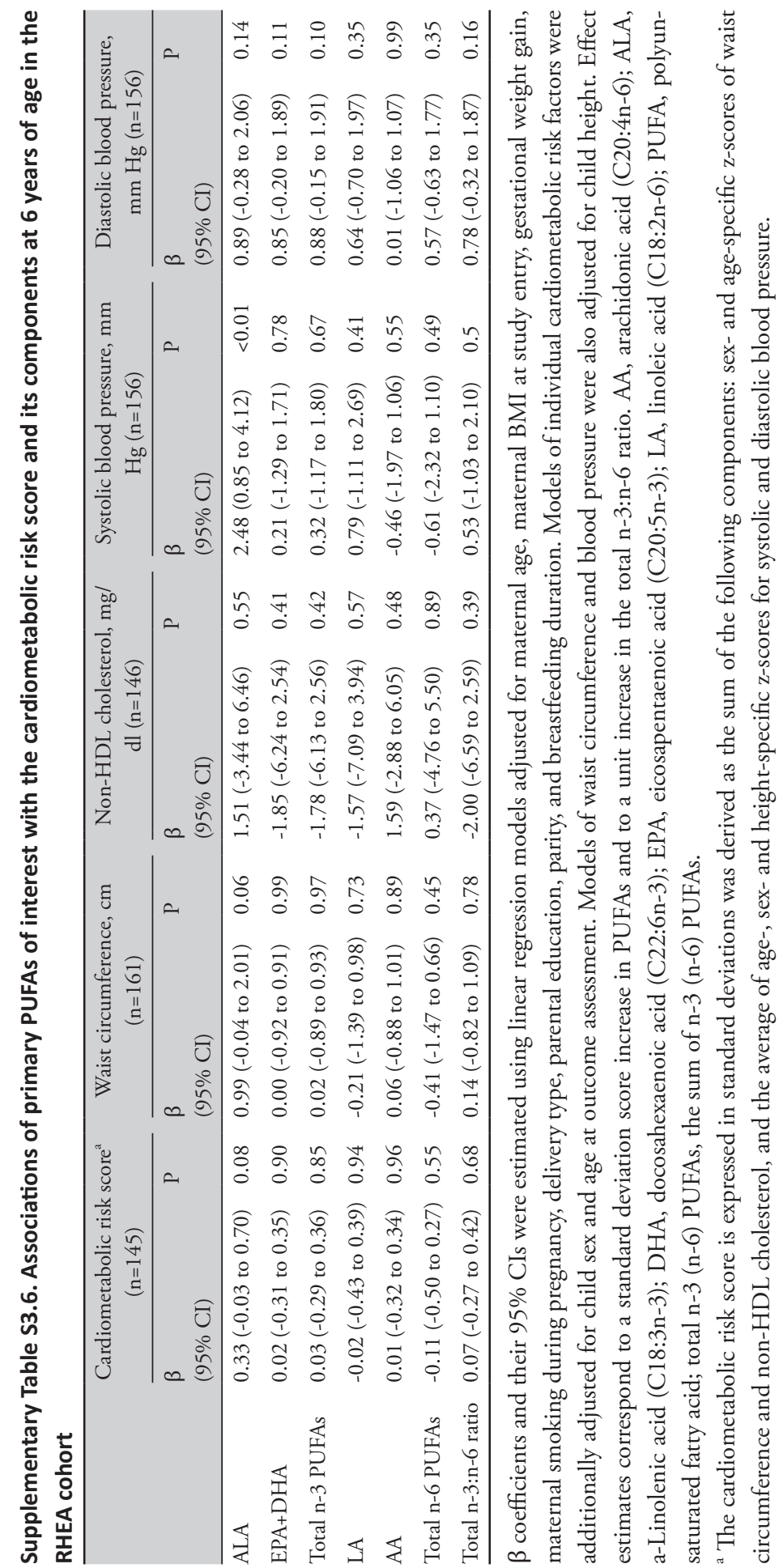




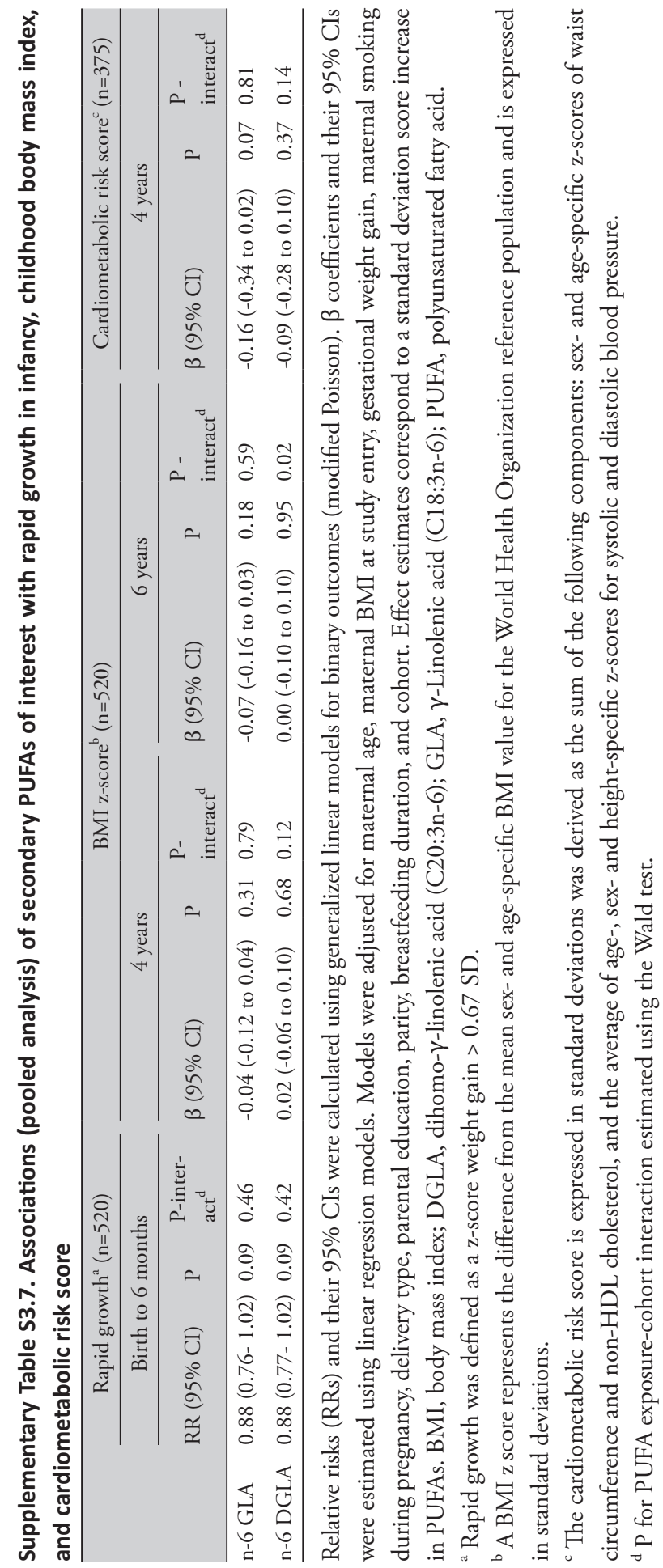




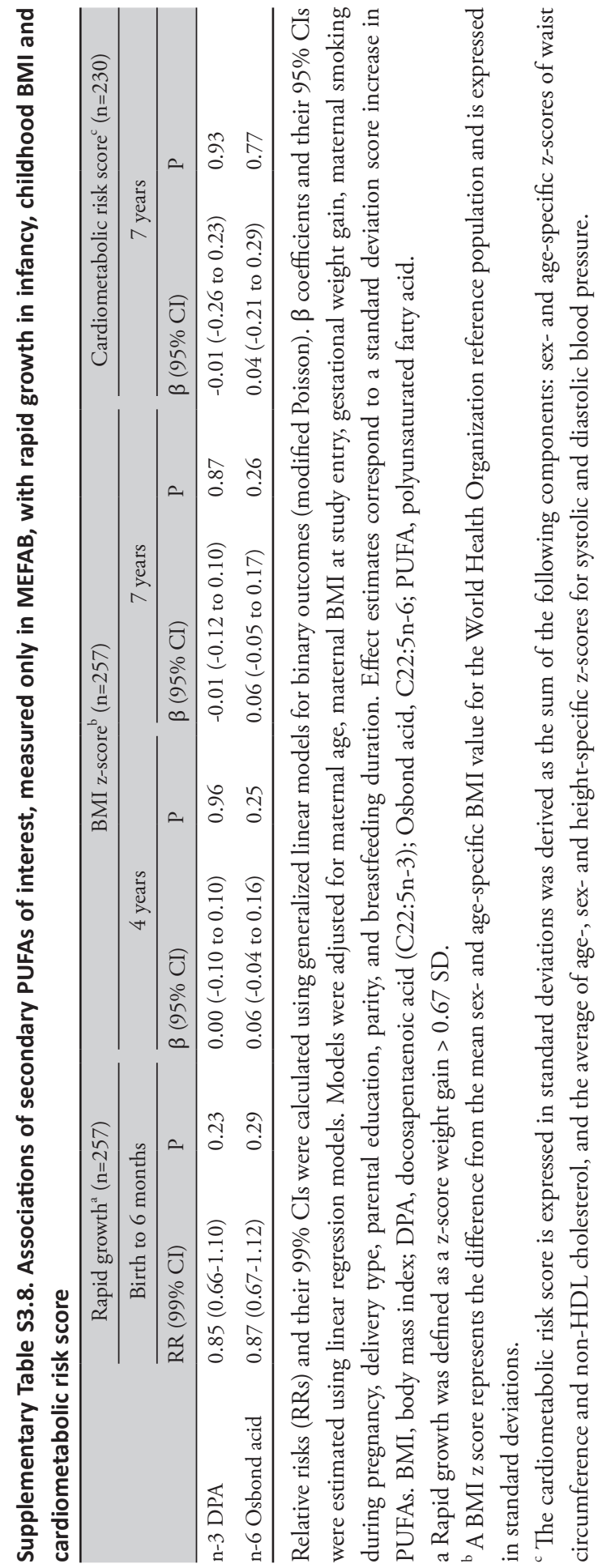


Supplementary Table S3.9. Associations of primary PUFAs of interest with cardiometabolic risk score including HOMA-IR at 7 years of age in the MEFAB birth cohort $(n=226)$

\begin{tabular}{lcc}
\hline & \multicolumn{2}{c}{ Cardiometabolic risk score $^{\mathrm{a}}$} \\
\cline { 2 - 3 } ALA & $\beta(95 \%$ CI $)$ & P \\
EPA+DHA & $-0.03(-0.25$ to 0.19$)$ & 0.80 \\
Total n-3 PUFAs & $0.00(-0.32$ to 0.33$)$ & 0.98 \\
LA & $0.00(-0.33$ to 0.32$)$ & 0.98 \\
AA & $0.22(-0.16$ to 0.59$)$ & 0.26 \\
Total n-6 PUFAs & $-0.01(-0.35$ to 0.33$)$ & 0.47 \\
Total n-3:n-6 ratio & $0.12(-0.20$ to 0.43$)$ & 0.82 \\
\hline
\end{tabular}

$\beta$ coefficients and their 95\% CIs were estimated using linear regression models adjusted for maternal age, maternal BMI at study entry, gestational weight gain, maternal smoking during pregnancy, delivery type, parental education, parity, and breastfeeding duration. Effect estimates correspond to a standard deviation score increase in PUFAs and to a unit increase in the total n-3:n-6 ratio. AA, arachidonic acid (C20:4n-6); ALA, a-Linolenic acid (C18:3n-3); DHA, docosahexaenoic acid (C22:6n-3); EPA, eicosapentaenoic acid (C20:5n-3); HOMA-IR, homeostasis model assessment of insulin resistance; LA, linoleic acid (C18:2n-6); PUFA, polyunsaturated fatty acid; total n-3 (n-6) PUFAs, the sum of n-3 (n-6) PUFAs.

${ }^{a}$ The cardiometabolic risk score (expressed in standard deviations) was derived as the sum of the following components: sex- and age-specific z-scores of waist circumference, non-HDL cholesterol and HOMA-IR, and the average of age-, sex- and height-specific z-scores for systolic and diastolic blood pressure. 
Supplementary Table S3.10. Associations (pooled analysis) of primary cord blood phospholipid PUFAs of interest with rapid body mass index growth in infancy $(n=520)$

\begin{tabular}{|c|c|c|c|}
\hline & \multicolumn{3}{|c|}{ Rapid BMI growth ${ }^{2}$} \\
\hline & \multicolumn{3}{|c|}{ Birth to 6 months } \\
\hline & RR (95\% CI) & $\mathrm{P}$ & P-interact ${ }^{\mathrm{b}}$ \\
\hline ALA & $1.02(0.92-1.14)$ & 0.70 & 0.28 \\
\hline $\mathrm{EPA}+\mathrm{DHA}$ & $0.92(0.80-1.07)$ & 0.28 & 0.51 \\
\hline Total n-3 PUFAs & $0.92(0.80-1.06)$ & 0.24 & 0.60 \\
\hline LA & $1.10(0.96-1.25)$ & 0.17 & 0.47 \\
\hline AA & $1.10(0.95-1.26)$ & 0.20 & 0.01 \\
\hline Total n-6 PUFAs & $1.06(0.92-1.23)$ & 0.40 & 0.06 \\
\hline Total n-3:n-6 ratio & $0.93(0.80-1.07)$ & 0.29 & 0.94 \\
\hline
\end{tabular}

Relative risks (RRs) and their 95\% CIs were calculated using generalized linear models for binary outcomes (modified Poisson). Models were adjusted for maternal age, maternal BMI at study entry, gestational weight gain, maternal smoking during pregnancy, delivery type, parental education, parity, breastfeeding duration, and cohort. Effect estimates correspond to a standard deviation score increase in PUFAs and to a unit increase in the total n-3:n-6 ratio. AA, arachidonic acid (C20:4n-6); ALA, a-Linolenic acid (C18:3n-3); BMI, body mass index; DHA, docosahexaenoic acid (C22:6n-3); EPA, eicosapentaenoic acid (C20:5n-3); LA, linoleic acid (C18:2n-6); PUFA, polyunsaturated fatty acid; total n-3 (n-6) PUFAs, the sum of n-3 (n-6) PUFAs; WHO, World Health Organization.

${ }^{a}$ Rapid BMI growth was defined as a BMI z-scores gain $>0.67$. In total, 159 children ( $/ \mathrm{N}=69 / 257$ in MEFAB and 90/263 in RHEA) were rapid BMI growers in infancy.

${ }^{\mathrm{b}} \mathrm{P}$ for PUFA exposure-cohort interaction estimated using the Wald test. 
Supplementary Table S3.11. Associations (pooled analysis) of primary cord blood phospholipid PUFAs of interest with childhood overweight/obesity defined based on the WHO definition $(n=520)$

\begin{tabular}{|c|c|c|c|c|c|c|}
\hline & \multicolumn{6}{|c|}{ Overweight/obesity $^{a}$} \\
\hline & \multicolumn{3}{|c|}{4 years } & \multicolumn{3}{|c|}{6 years } \\
\hline & $\mathrm{RR}(95 \% \mathrm{CI})$ & $P$ & P-interact ${ }^{b}$ & RR (95\% CI) & $\mathrm{P}$ & P-interact ${ }^{b}$ \\
\hline ALA & $0.89(0.49-1.63)$ & 0.71 & 0.94 & $1.11(1.00-1.23)$ & 0.05 & 0.61 \\
\hline $\mathrm{EPA}+\mathrm{DHA}$ & $1.23(0.79-1.91)$ & 0.35 & 0.93 & $1.01(0.87-1.17)$ & 0.89 & 0.19 \\
\hline Total n-3 PUFAs & $1.22(0.79-1.88)$ & 0.38 & 0.96 & $1.02(0.88-1.18)$ & 0.80 & 0.14 \\
\hline LA & $0.94(0.37-2.34)$ & 0.89 & 0.58 & $0.94(0.79-1.13)$ & 0.51 & 0.29 \\
\hline AA & $1.26(0.71-2.26)$ & 0.43 & 0.18 & $0.90(0.78-1.05)$ & 0.18 & 0.70 \\
\hline Total n-6 PUFAs & $1.11(0.65-1.89)$ & 0.71 & 0.53 & $0.90(0.78-1.04)$ & 0.15 & 0.86 \\
\hline Total $n-3: n-6$ ratio & $1.19(0.75-1.87)$ & 0.46 & 0.97 & $1.05(0.90-1.21)$ & 0.55 & 0.23 \\
\hline
\end{tabular}

Relative risks (RRs) and their 95\% CIs were calculated using generalized linear models for binary outcomes (modified Poisson). Models were adjusted for maternal age, maternal BMI at study entry, gestational weight gain, maternal smoking during pregnancy, delivery type, parental education, parity, breastfeeding duration, and cohort. Effect estimates correspond to a standard deviation score increase in PUFAs and to a unit increase in the total n-3:n-6 ratio. AA, arachidonic acid (C20:4n-6); ALA, a-Linolenic acid (C18:3n-3); BMI, body mass index; DHA, docosahexaenoic acid (C22:6n-3); EPA, eicosapentaenoic acid (C20:5n-3); LA, linoleic acid (C18:2n-6); PUFA, polyunsaturated fatty acid; total n-3 (n-6) PUFAs, the sum of n-3 (n-6) PUFAs; WHO, World Health Organization.

${ }^{a}$ Overweight/obesity was defined based on the WHO definition. In total, 24 ( $\mathrm{n} / \mathrm{N}=5 / 257$ in MEFAB and 19/263 in RHEA) and 141 (n/N = 39/257 in MEFAB and 102/263 in RHEA) children were classified as overweight or obese according to the WHO definition.

${ }^{\mathrm{b}} \mathrm{P}$ for PUFA exposure-cohort interaction estimated using the Wald test. 
Mothers enrolled during pregnancy

$(\mathbf{N}=\mathbf{1 3 3 4})$

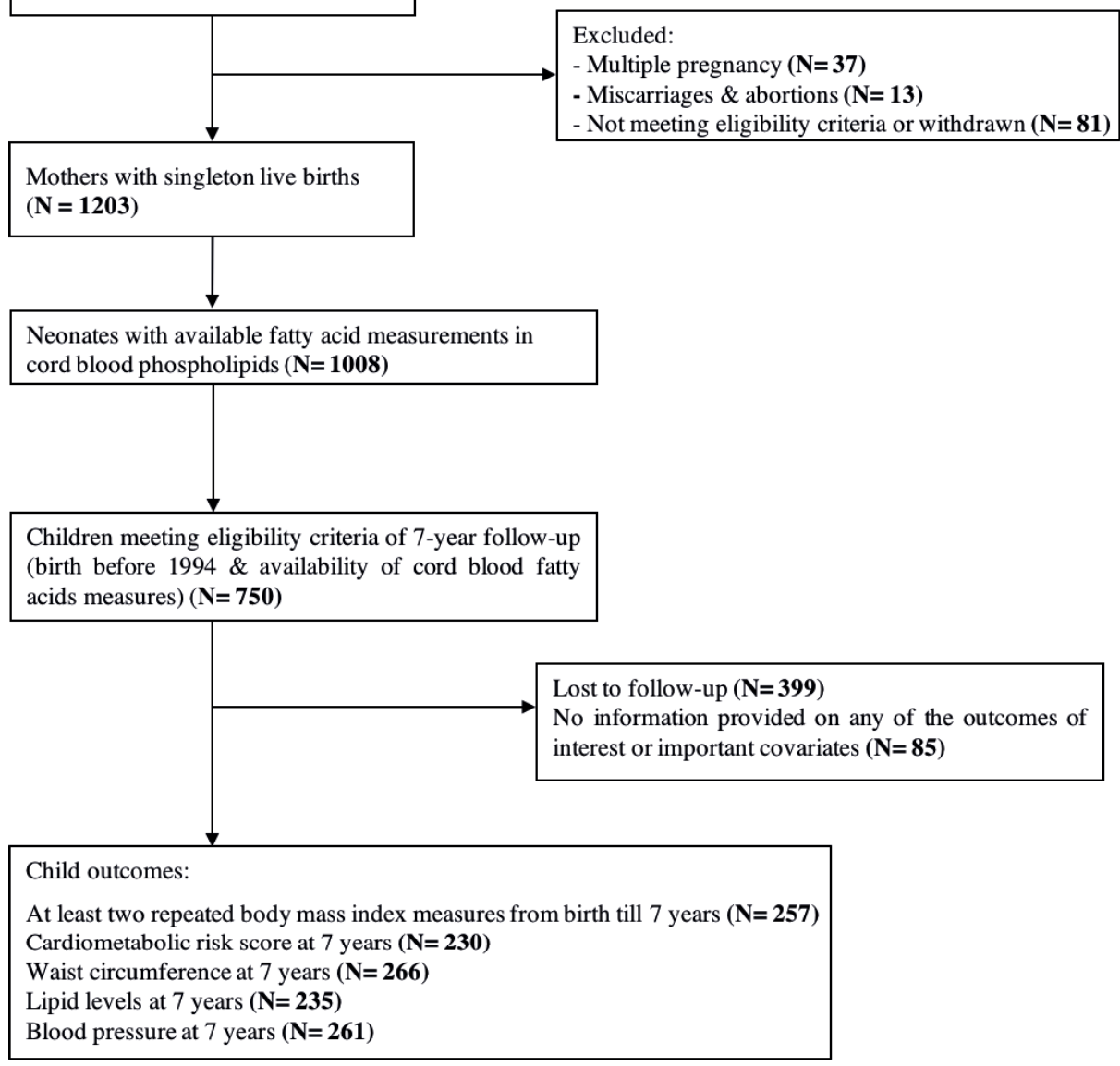

Supplementary Figure S3.1. Flow chart of the MEFAB participants. 


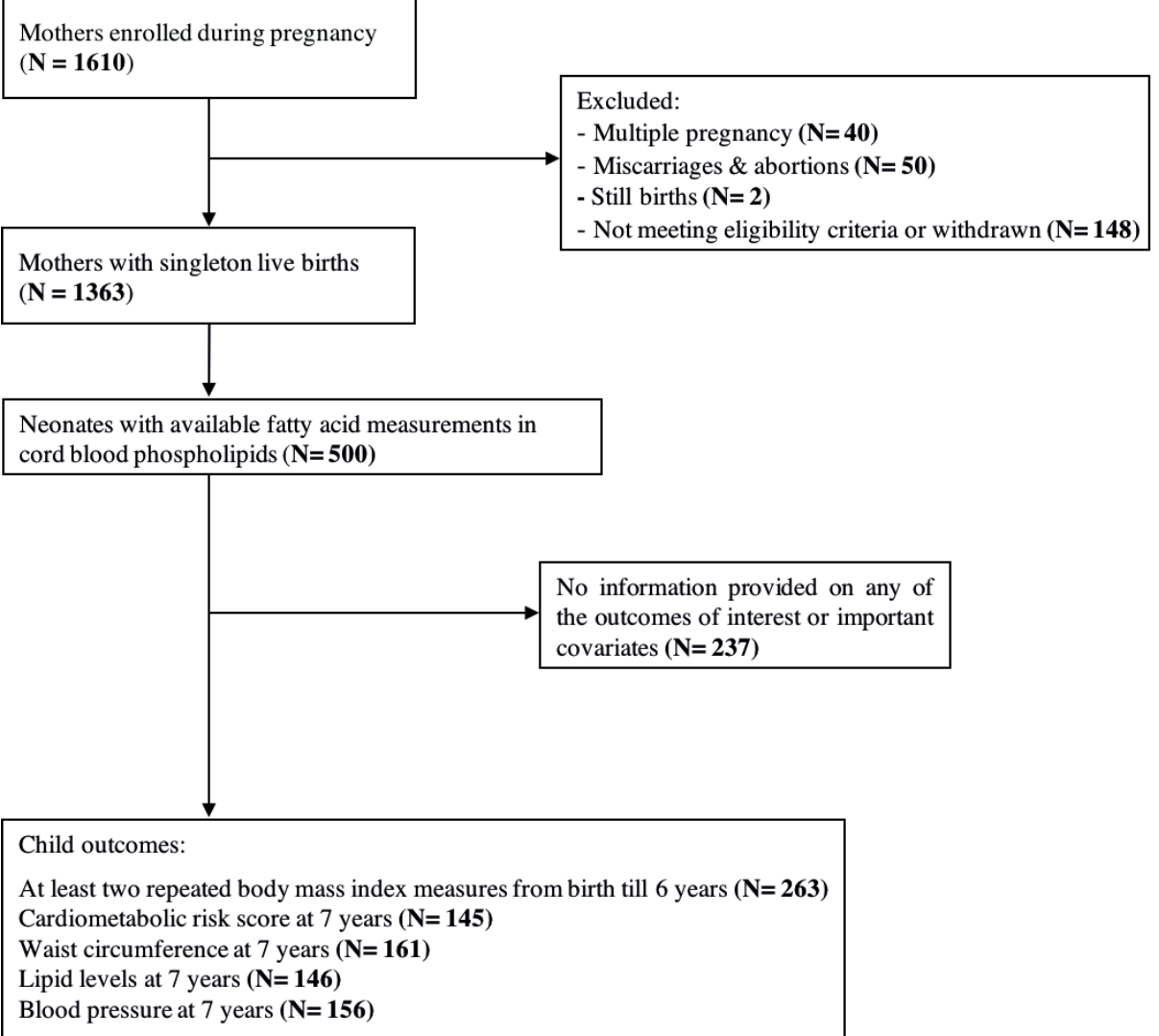

Supplementary Figure S3.2. Flow chart of the RHEA participants. 


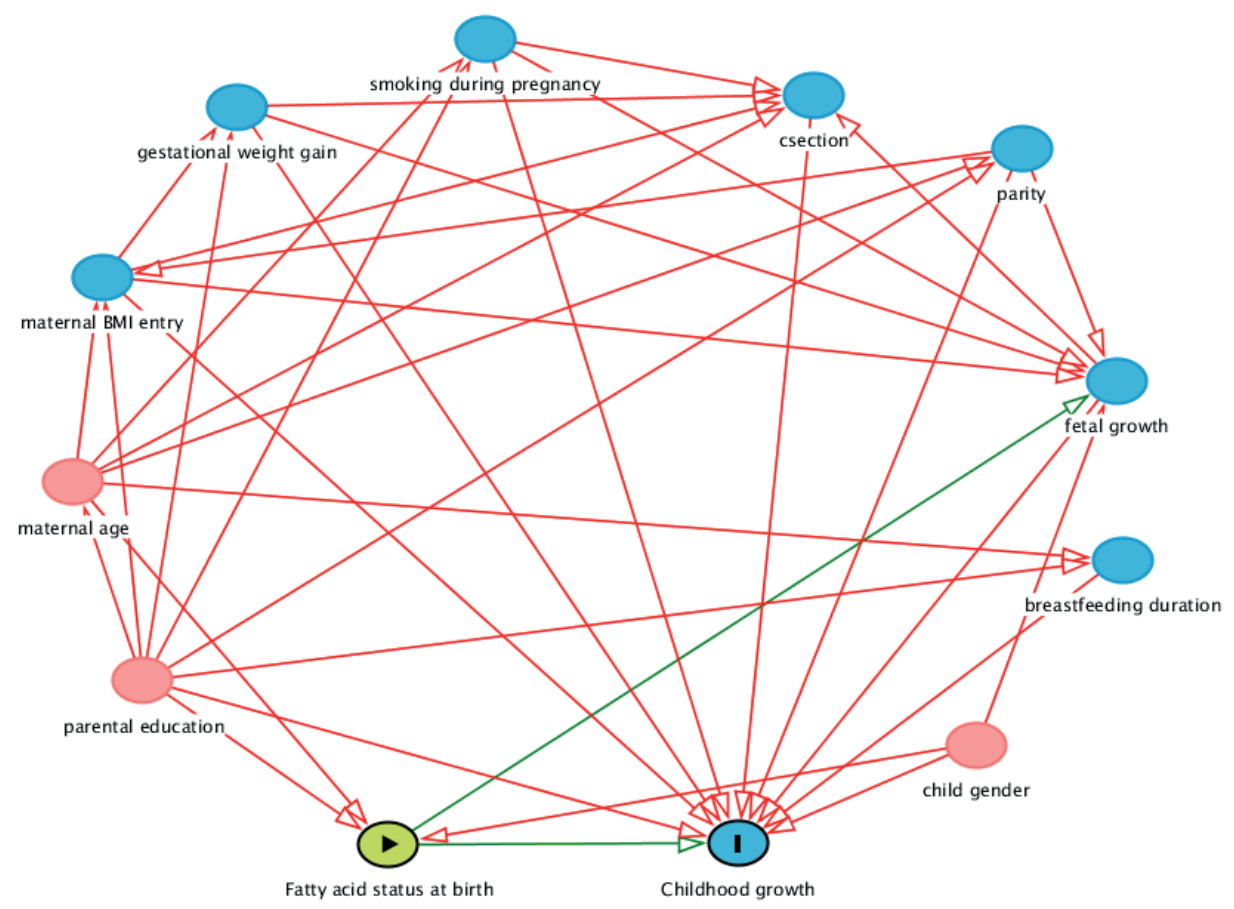

Supplementary Figure S3.3. Directed acyclic graph for the association of fatty acid status at birth with childhood growth. 


\section{Supplementary References}

1. Al MDM, Vanhouwelingen AC, Kester ADM, et al. Maternal Essential Fatty-Acid Patterns during Normal-Pregnancy and Their Relationship to the Neonatal Essential Fatty-Acid Status. Br $J$ Nutr 1995;74:55-68.

2. Hoving EB, Jansen G, Volmer M, et al. Profiling of plasma cholesterol ester and triglyceride fatty acids as their methyl esters by capillary gas chromatography, preceded by a rapid aminopropyl-silica column chromatographic separation of lipid classes. J Chromatogr 1988;434:395-409.

3. Glaser C, Demmelmair H, Koletzko B. High-throughput analysis of total plasma fatty acid composition with direct in situ transesterification. PLoS One 2010;5:e12045.

4. Hodson L, Skeaff CM, Fielding BA. Fatty acid composition of adipose tissue and blood in humans and its use as a biomarker of dietary intake. Prog Lipid Res 2008;47:348-80.

5. Stark KD, Van Elswyk ME, Higgins MR, et al. Global survey of the omega-3 fatty acids, docosahexaenoic acid and eicosapentaenoic acid in the blood stream of healthy adults. Prog Lipid Res 2016;63:132-52.

6. Royston P, Wright EM. A method for estimating age-specific reference intervals ("normal ranges") based on fractional polynomials and exponential transformation. J R Statist Soc 1998;161:79-101.

7. Long J, Ryoo J. Using fractional polynomials to model non-linear trends in longitudinal data. Br J Math Stat Psychol 2010;63:177-203.

8. Johnson W, Balakrishna N, Griffiths PL. Modeling physical growth using mixed effects models. Am J Phys Anthropol 2013;150:58-67.

9. Plat J, Mensink RP. Vegetable oil based versus wood based stanol ester mixtures: effects on serum lipids and hemostatic factors in non-hypercholesterolemic subjects. Atherosclerosis 2000;148:101-12.

10. Laboratory Methods Committee of the Lipid Research Clinics Program. Cholesterol and triglyceride concentrations in serum/plasma pairs. Clin Chem 1977;23:60-3.

11. Ruige JB, Dekker JM, Nijpels G, et al. Hyperproinsulinaemia in impaired glucose tolerance is associated with a delayed insulin response to glucose. Diabetologia 1999;42:177-80.

12. Matthews DR, Hosker JP, Rudenski AS, et al. Homeostasis model assessment: insulin resistance and beta-cell function from fasting plasma glucose and insulin concentrations in man. Diabetologia 1985;28:412-9.

13. Expert Panel on Integrated Guidelines for Cardiovascular Health and Risk Reduction in Children and Adolescents; National Heart Lung and Blood Institute. Expert panel on integrated guidelines for cardiovascular health and risk reduction in children and adolescents: summary report. Pediatrics 2011;128 Suppl 5:S213-S56. 



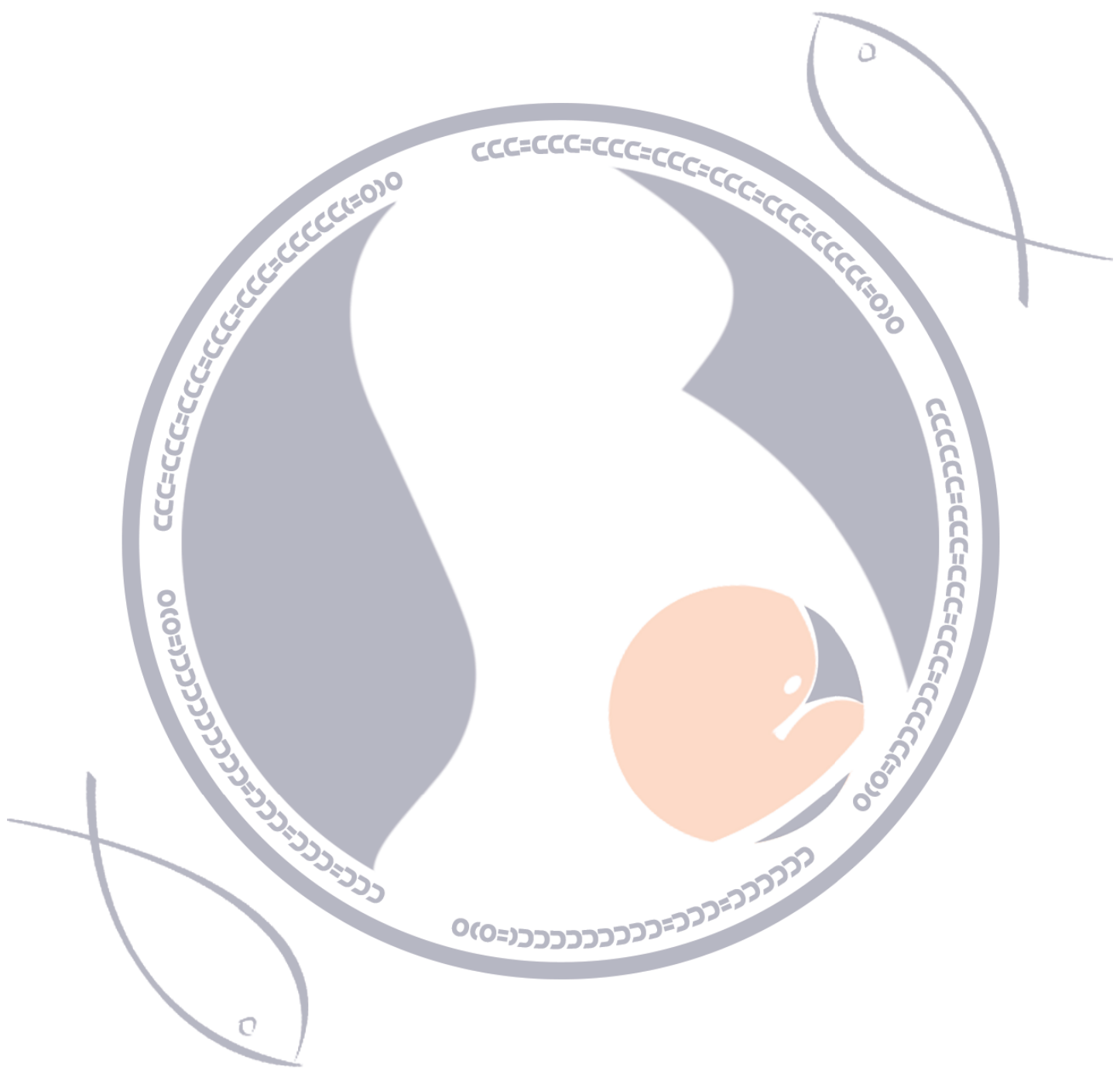




\section{CHAPTER 4}

\section{Polyunsaturated fatty acid levels at birth and child-to-adult growth: results from the MEFAB cohort}

N Stratakis, M Gielen, K Margetaki, RW Godschalk, I van der Wurff, S Rouschop, A Ibrahim, E Antoniou, L Chatzi, RHM de Groot, MP Zeegers

Adapted from Prostaglandins Leukot Essent Fatty Acids 2017 Nov;126:72-78 


\section{ABSTRACT}

Background: Prenatal exposure to polyunsaturated fatty acids (PUFAs) may influence childhood growth. However, available evidence mostly derived from short-term studies is inconsistent.

Objective: To assess whether fetal PUFA exposure is associated with height and body mass index (BMI), a common measure of adiposity, from 6 months to 23 years of age.

Methods: In the MEFAB cohort, we assessed cord blood phospholipid n-3 and n-6 PUFA levels, reflecting fetal exposure in late pregnancy. For $250(45.2 \%$ females) participants, we collected a total of 1770 ( $n=802$ for females) repeated growth measurements from infancy to young adulthood. We examined sexspecific associations of PUFAs with height and BMI at different developmental ages (infant: 6 months; toddler: 2 years; pre-schooler: 4 years; school-aged child: 7 years; adolescent: 12 years; and young adult: 23 years) using fractional polynomial mixed models adjusted for important covariates.

Results: Higher $n-3$ PUFA levels were associated with higher infant length in males ( $\beta=0.44 \mathrm{~cm}$ [95\% CI 0.07 to 0.82 ] per $1-S D$ increase in total $\mathrm{n}-3$ PUFA concentration), whereas, for females, higher $n-6$ PUFA concentrations were associated with lower length in infancy $(\beta=-0.69 \mathrm{~cm}$ [95\% CI -1.08 to -0.30 ] per 1-SD increase in total n-6 PUFAs). A higher ratio of $n-3$ to $n-6$ PUFAs was associated with higher infant length in both sexes $(\beta=0.40 \mathrm{~cm}$ [95\% CI 0.01 to 0.78 ] and $0.42 \mathrm{~cm}$ [ $95 \% \mathrm{CI} 0.05$ to 0.79$]$ per 1 -unit increase in the ratio for males and females, respectively). These associations were not detectable later in childhood and young adulthood. No associations with BMI were found at any time point examined.

Conclusions: Our findings suggest a small, sex-specific influence of PUFA status at birth on length in infancy, but this does not persist in later life up to young adulthood. PUFA status at birth does not seem to affect BMI from infancy till young adulthood. 


\section{INTRODUCTION}

Height and body mass index (BMI), a measure of body weight relative to height, are recognized as important markers of health. Accumulating evidence suggests that lower stature and higher BMI in both children and adults are associated with higher risk of later cardiovascular disease. ${ }^{1-3}$ Therefore, gaining knowledge about modifiable factors that may influence growth is highly relevant for the prevention of future disease risk.

Fetal life is a critical period of susceptibility during which a nutritional stressor can permanently alter body physiology and metabolism, the consequences of which are often observed much later in life. ${ }^{4}$ In this context, there has been an increasing interest in the potential programming effect of prenatal exposure to polyunsaturated fatty acids (PUFAs).

Animal and in vitro studies have suggested that n-3 PUFAs can inhibit inflammation, promote osteoblastogenesis, and decrease adipose tissue deposition, while those of the n- 6 family seem to exhibit opposite effects. ${ }^{5-7}$ Human studies assessing childhood height in relation to prenatal PUFA exposure have mostly focused on n-3 long-chain PUFA supplementation, and have not shown a clear and consistent effect. ${ }^{8-11}$ Cohort studies have also provided little evidence to confirm an association of prenatal PUFA status with later BMI. ${ }^{12-14}$ Likewise, evidence from trials assessing childhood BMI does not support or refute an effect of maternal fish oil supplementation. ${ }^{15}$

Limitations of prior studies not allowing conclusions to be drawn include the relatively short duration of follow-up and inconsistencies in timing and definition of exposure. Moreover, the effects of prenatal PUFA exposures have been traditionally examined at one time point in childhood, which may only partially capture their impact on growth. It is well established that individuals experience different rates of growth over time. Examining the long-term effects of early-life exposures at different ages, preferably on the child to adult transition, is important for more accurate identification of modifiable risk factors and for understanding when these factors exhibit their maximum influence. Hence, we used long-term longitudinal data from the Maastricht Essential Fatty Acid Birth (MEFAB) cohort to investigate the associations of cord blood phospholipid PUFA levels, reflecting fetal exposure in late pregnancy, with height and BMI at different developmental ages: infant, 6 months; toddler, 2 years; pre-schooler, 4 years; school-aged child, 7 years; adolescent, 12 years; and young adult, 23 years. 


\section{METHODS}

\section{Study population}

The MEFAB (Maastricht Essential Fatty Acid Birth, www.mefab.org) study prospectively examines a population-based sample of pregnant women and their children in the province of Limburg, the Netherlands. Pregnant women attending three maternity clinics for their first antenatal visit between 1989 and 1995 were invited to participate in the study. To be included in the study, women had to have a gestational age of less than 16 weeks, diastolic blood pressure of less than $90 \mathrm{mmHg}$, and no cardiovascular, neurological, renal, or metabolic disorders. A total of 1203 singletons were followed up until delivery. Face-to-face structured questionnaires along with self-administered questionnaires and medical records were used to obtain information on several sociodemographic characteristics in pregnancy. For the offspring's follow-up, we followed mother-offspring pairs at three waves (wave I: $n=296$, mean age $( \pm S D) 7.3 \pm 0.3$ years; wave II: $n=98$; mean age $12.8 \pm 1.1$ years; and wave III: $n=320$; mean age $23.1 \pm 1.5$ years). Details regarding the survey design and sampling frame have been previously described. ${ }^{16}$ In this analysis, we included 250 study subjects ( $45.2 \%$ females) with complete information on exposure and important covariates, contributing a total of 1770 ( $\mathrm{n}=802$ for females) observations from early childhood till young adulthood. The study was approved by the Medical Ethics Committee of the University Hospital Maastricht and the University of Maastricht, and participants provided written, informed consent for each follow-up evaluation.

Supplementary Table S4.1 shows that, compared to non-participants, those included in the present analysis had mothers with a lower BMI at study entry (mean BMI $23.5 \mathrm{vs} 24.2 \mathrm{~kg} / \mathrm{m}^{2}$ ) and higher age at delivery (mean age $30.2 \mathrm{vs} 29.1$ years), but did not differ in gestational weight gain, birth weight, gestational age, area-based socioeconomic status at birth, as well as in fatty acid exposure levels.

\section{Polyunsaturated fatty acid analysis}

Blood samples from the umbilical vein of infants were collected immediately after delivery. Methods of fatty acid analysis and the phospholipid PUFAs identified in MEFAB have been described in detail elsewhere. ${ }^{17}$

For the present analysis, our primary exposures of interest were the dietary essential n-3 PUFA precursor $\alpha$-linolenic acid (ALA, C18:3n-3), the sum of its major biologically active metabolic products eicosapentaenoic acid (EPA, C20:5n-3) and docosahexaenoic acid (DHA, C22:6n-3), and the sum of total n-3 fatty acids assayed; the dietary essential n-6 PUFA precursor, linoleic acid (LA, C18:2n-6), its major metabolic product arachidonic acid (AA, C20:4n-6), and the sum of total n- 6 fatty acids assayed; and the total n-3:n-6 PUFA ratio. 
Secondary exposures of interest were intermediate metabolic products in the biosynthetic pathway of PUFAs that have been previously associated with pregnancy outcomes and child growth including: the n-3 docosapentaenoic acid (DPA, C22:5n-3), and the $n-6 \gamma$-linolenic acid (GLA, C18:3n-6), dihomo- $\gamma$-linolenic acid (DGLA, C20:3n-6), and osbond acid (C22:5n-6). ${ }^{18,19}$ Individual fatty acid measurements were expressed as weight percentage of total fatty acids measured (wt $\%)$. To enable comparison with other studies, we constructed standardized values (z-scores) for PUFA concentrations.

\section{Child growth measures}

Data on length/height and weight were collected at 9 different time points from birth till young adulthood. Information on birth weight and length was obtained via medical records. At follow-up wave I, we collected data on length/height and weight measurements from 6 months up to 5 years with one-year interval from paediatrician records, which form part of standard child care in the Netherlands, and measured children's weight and height at age 7 using standard procedures. ${ }^{19}$ At age 12 (wave II), we measured adolescent weight and height following standard methodology. ${ }^{20}$ At the 23 -year follow-up evaluation (wave III), we collected selfreported information on weight and height using questionnaires. We excluded five implausible height/weight measurements above or below the age- and sex-specific mean $+/-4$ standard deviations. We also excluded children with only two growth measurements available so as to achieve sufficient stability of growth trajectory models and increase precision in our effect estimates. ${ }^{21}$ After these exclusions, an average of 7 repeated measurements (interquartile range: 1 for males, and 2 for females) were available for both BMI, calculated as weight in kilograms divided by height in meters squared, and height per offspring (either male or female).

We analysed untransformed growth measures, rather than age-standardized metrics (i.e z-scores), because they yield estimates that are more precise and sensitive to factors altering change when modelling growth trajectories; z-scores represent cross-sectional deviations from norms, thereby, removing some of the longitudinal change in growth patterns within individuals. ${ }^{22,23}$

\section{Statistical analysis}

We modelled height and BMI growth trajectories from birth through young adulthood using multi-level models (two levels: measurement occasion and individual) with fractional polynomials of age to identify the best-fitting curves. A description of the methodology followed is presented in the Supplementary Material. Given 
that males and females follow a different growth pattern and exhibit differences in fatty acid metabolism, ${ }^{24}$ all analyses were stratified by sex.

We examined associations between cord blood PUFA concentrations and growth measures with mixed linear regression models. We assessed possible non-linear relations by fitting and comparing linear and lowess curves. No severe departures from linearity were detected.

Following a directed acyclic graph approach (Supplementary Figure S4.1), we selected the following covariates for model adjustment: maternal age at delivery (years), maternal BMI at study entry (kilograms/meters squared), gestational weight gain (kilograms), maternal smoking during pregnancy (yes, no), maternal alcohol consumption during pregnancy (yes, no), parity (primiparous, multiparous), breastfeeding status (yes, no) and parental education (primary, secondary, or tertiary according to the highest completed education level of either parent). ${ }^{25}$ In our models, we did not include gestational age, as it might be in the intermediate pathway between prenatal PUFA levels and later growth. ${ }^{26,27}$

We present age-specific associations of PUFAs with growth measures at different developmental stages (infant: 6 months, toddler: 2 years, pre-schooler: 4 years, school-aged child: 7 years, adolescent: 12 years, and young adult: 23 years), estimated using cross-products of PUFAs with the polynomial age terms as fixed effects. As estimates obtained based on fractional polynomial models are not precise at the tails, ${ }^{21}$ we do not present estimates for ages 0 or more than 23 years to avoid misleading conclusions. Results from the MEFAB cohort on cord blood PUFAs and birth outcomes have been published elsewhere. ${ }^{28}$

As a sensitivity analysis, we re-run our models for primary PUFA exposures with further adjustment for gestational age (measured in weeks). We also repeated analyses after excluding children with impaired fetal growth (birth weight for gestational age $<10^{\text {th }}$ percentile). Finally, we assessed associations with BMI measures available in young adulthood following further adjustment for fish oil supplementation (yes, no), education level (primary, secondary, or tertiary) ${ }^{25}$ and alcohol consumption (glasses per week) measured at the age of outcome assessment. Statistical significance for all estimates was set at the 5\% level. We performed analyses with Stata version 13.0 (StataCorp). 


\section{RESULTS}

\section{Participant characteristics}

Basic characteristics of the study population are presented in Table 4.1. Participating mothers had a mean age (SD) of 30.2 (4.0) years at study entry, and more than half of the mothers did not breastfeed their infants. About $40 \%$ of parents had medium educational level. Mean (SD) cord blood levels of n-3 and n-6 PUFAs for boys were $6.79(1.58)$ and $32.26(1.67)$ wt \%, respectively; the corresponding values for girls were 6.92 (1.65) and 32.03 (1.61) wt \% (Table 4.2).

Table 4.1. Sociodemographic characteristics in males $(n=137)$ and females $(n=113)$ from the MEFAB cohort

\begin{tabular}{|c|c|c|}
\hline & Males & Females \\
\hline & Mean \pm SD or $\mathrm{n}(\%)$ & Mean \pm SD or $n(\%)$ \\
\hline \multicolumn{3}{|l|}{ Parental characteristics } \\
\hline Maternal age at birth (years) & $30.6 \pm 4.4$ & $29.7 \pm 3.5$ \\
\hline Maternal BMI at study entry $\left(\mathrm{kg} / \mathrm{m}^{2}\right)$ & $23.9 \pm 3.8$ & $23.1 \pm 3.4$ \\
\hline Underweight $\left(<18 \mathrm{~kg} / \mathrm{m}^{2}\right)$ & $2(1.5)$ & $2(1.8)$ \\
\hline Normal weight $\left(18-25 \mathrm{~kg} / \mathrm{m}^{2}\right)$ & $96(70.1)$ & $83(73.4)$ \\
\hline Overweight $\left(\geq 25 \mathrm{~kg} / \mathrm{m}^{2}\right)$ & $39(28.5)$ & $28(24.8)$ \\
\hline Gestational weight gain $(\mathrm{kg})$ & $11.2 \pm 3.9$ & $11.0 \pm 4.1$ \\
\hline \multicolumn{3}{|l|}{ Maternal smoking in pregnancy (\%) } \\
\hline No & $107(78.1)$ & $87(77.0)$ \\
\hline Yes & $30(21.9)$ & $26(23.0)$ \\
\hline \multicolumn{3}{|l|}{ Alcohol intake in pregnancy (\%) } \\
\hline No & $130(94.9)$ & $108(95.6)$ \\
\hline Yes & $7(5.1)$ & $5(4.4)$ \\
\hline \multicolumn{3}{|l|}{ Parity (\%) } \\
\hline Primiparous & $96(70.1)$ & $80(70.8)$ \\
\hline Multiparous & $41(29.9)$ & $33(29.2)$ \\
\hline \multicolumn{3}{|l|}{ Parental education (\%) } \\
\hline Low & $29(21.2)$ & $16(14.2)$ \\
\hline Middle & $59(43.1)$ & $44(38.9)$ \\
\hline High & $49(35.7)$ & $53(46.9)$ \\
\hline \multicolumn{3}{|l|}{ Offspring characteristics } \\
\hline Birth weight (gr) & $3335.2 \pm 499.5$ & $3265.0 \pm 452.6$ \\
\hline Gestational age (weeks) & $39.7 \pm 1.8$ & $40.1 \pm 1.3$ \\
\hline \multicolumn{3}{|l|}{ Breastfeeding (\%) } \\
\hline Never & $69(50.4)$ & $61(54.0)$ \\
\hline Ever & $68(49.6)$ & $52(46.0)$ \\
\hline
\end{tabular}


Table 4.2. Distribution of cord blood phospholipid PUFA levels in males $(n=137)$ and females $(n=113)$ from the MEFAB cohort

\begin{tabular}{lcc}
\hline & Males & Females \\
\cline { 2 - 3 } & Mean \pm SD & Mean \pm SD \\
\hline Total n-3 PUFAs (wt \%) & $6.79 \pm 1.58$ & $6.92 \pm 1.65$ \\
ALA (wt \%) & $0.06 \pm 0.14$ & $0.04 \pm 0.06$ \\
DPA (wt\%) & $0.45 \pm 0.18$ & $0.47 \pm 0.18$ \\
EPA (wt \%) & $0.22 \pm 0.11$ & $0.24 \pm 0.13$ \\
DHA (wt \%) & $5.97 \pm 1.40$ & $6.09 \pm 1.44$ \\
EPA+DHA (wt \%) & $6.19 \pm 1.43$ & $6.32 \pm 1.48$ \\
Total n-6 PUFAs (wt \%) & $32.26 \pm 1.67$ & $32.03 \pm 1.61$ \\
LA (wt \%) & $7.87 \pm 1.37$ & $7.56 \pm 1.15$ \\
GLA (wt \%) & $0.05 \pm 0.03$ & $0.05 \pm 0.04$ \\
DGLA (wt \%) & $5.18 \pm 0.87$ & $5.17 \pm 0.90$ \\
AA (wt \%) & $16.47 \pm 1.54$ & $16.67 \pm 1.53$ \\
Osbond acid (wt\%) & $0.85 \pm 0.28$ & $0.80 \pm 0.27$ \\
Total n-3:n-6 PUFA ratio & $0.21 \pm 0.05$ & $0.22 \pm 0.06$ \\
\hline
\end{tabular}

AA, arachidonic acid (C20:4n-6); ALA, a-linolenic acid (C18:3n-3); DGLA, dihomo- $\gamma$-linolenic acid (C20:3n-6); DHA, docosahexaenoic acid (C22:6n-3); DPA, docosapentaenoic acid (C22:5n-3); EPA, eicosapentaenoic acid (C20:5n-3); GLA, $\gamma$-Linolenic acid (C18:3n-6); LA, linoleic acid (C18:2n-6); Osbond acid, C22:5n-6; PUFA, polyunsaturated fatty acid; total n-3 (n-6) PUFAs, the sum of n-3 (n-6) PUFAs present in the chromatogram; wt \%, weight percentage of total fatty acids present in the chromatogram.

At the age of 6 months, mean (SD) length was $67.6(2.6) \mathrm{cm}$ for boys and 65.5 (3.0) $\mathrm{cm}$ for girls, while for BMI, boys had a mean (SD) of $17.0(1.3) \mathrm{kg} / \mathrm{m}^{2}$, and girls had a mean (SD) of $16.6(1.3) \mathrm{kg} / \mathrm{m}^{2}$ (Supplementary Table S4.2). At the age of 23 years, mean (SD) height for men and women was $182.2(8.2)$ and 167.0 (8.0) $\mathrm{cm}$, respectively; the corresponding values for BMI were 23.2 (3.8) and $23.3(3.8) \mathrm{kg} / \mathrm{m}^{2}$ (Supplementary Table S4.2). Figure 4.1 demonstrates the height and BMI trajectories from 6 months up to 23 years of age in our study sample. Repeated height and BMI measures throughout the study period were strongly correlated within individuals (Supplementary Tables S4.3 and S4.4). 
A

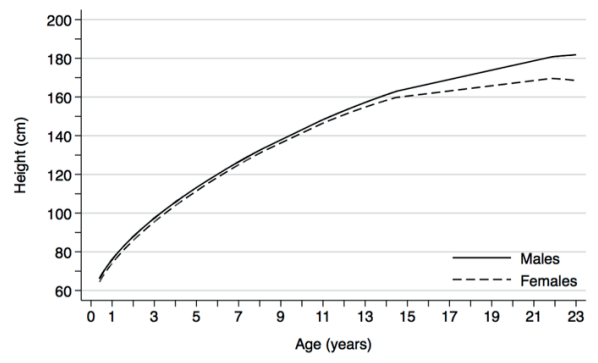

B

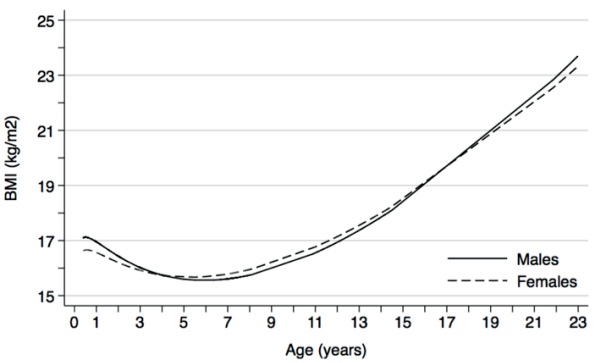

Figure 4.1. Height (A and BMI (B) trajectories from 6 months to 23 years of age in males $(n=137)$ and females $(n=113)$ from the MEFAB cohort.

\section{Cord blood PUFAs and height}

Among male participants, we found that both higher total n-3 PUFA levels and a higher $n-3: n-6$ ratio were associated with higher length at 6 months of age; $\beta=$ $0.44 \mathrm{~cm}$ [95\% CI 0.07 to 0.82 ] per 1-SD increase in total n-3 PUFA concentration, and $0.40 \mathrm{~cm}$ [95\% CI 0.01 to 0.78$]$ per 1-unit increase in the $\mathrm{n}-3: \mathrm{n}-6$ ratio (Table 4.3). When we examined individual PUFAs in males, we also found an association of $\mathrm{n}-3 \mathrm{EPA}+\mathrm{DHA}$ levels with higher infant length $(\beta=0.44 \mathrm{~cm}[95 \%$ CI 0.06 to 0.82 ] per 1 -SD increase) (Table 4.3). Among female participants, we observed that higher total $n-6$ PUFA concentrations were associated with lower length in infancy $(\beta=-0.69 \mathrm{~cm}[95 \% \mathrm{CI}-1.08$ to -0.30$]$ per $1-\mathrm{SD}$ increase in total n-6 PUFAs), whereas a higher $\mathrm{n}-3: \mathrm{n} 6$ ratio was associated with higher infant length ( $\beta=0.42 \mathrm{~cm}[95 \%$ CI 0.05 to 0.79$]$ per 1-unit increase). We also saw an inverse association of the n-6 LA with female length in infancy $(\beta=-0.73 \mathrm{~cm}$ [95\% CI -1.23 to -0.22$]$ per 1-SD increase) (Table 4.3). No other associations between cord blood PUFAs and height in mid-childhood and young adulthood were observed for both males and females.

\section{Cord blood PUFAs and BMI}

Among males, we did not find any association of cord blood PUFAs with BMI from 6 months to 23 years of age (Table 4.4). In females, we also found no evidence for an association of PUFA status with BMI at any time point examined (Table 4.4). 


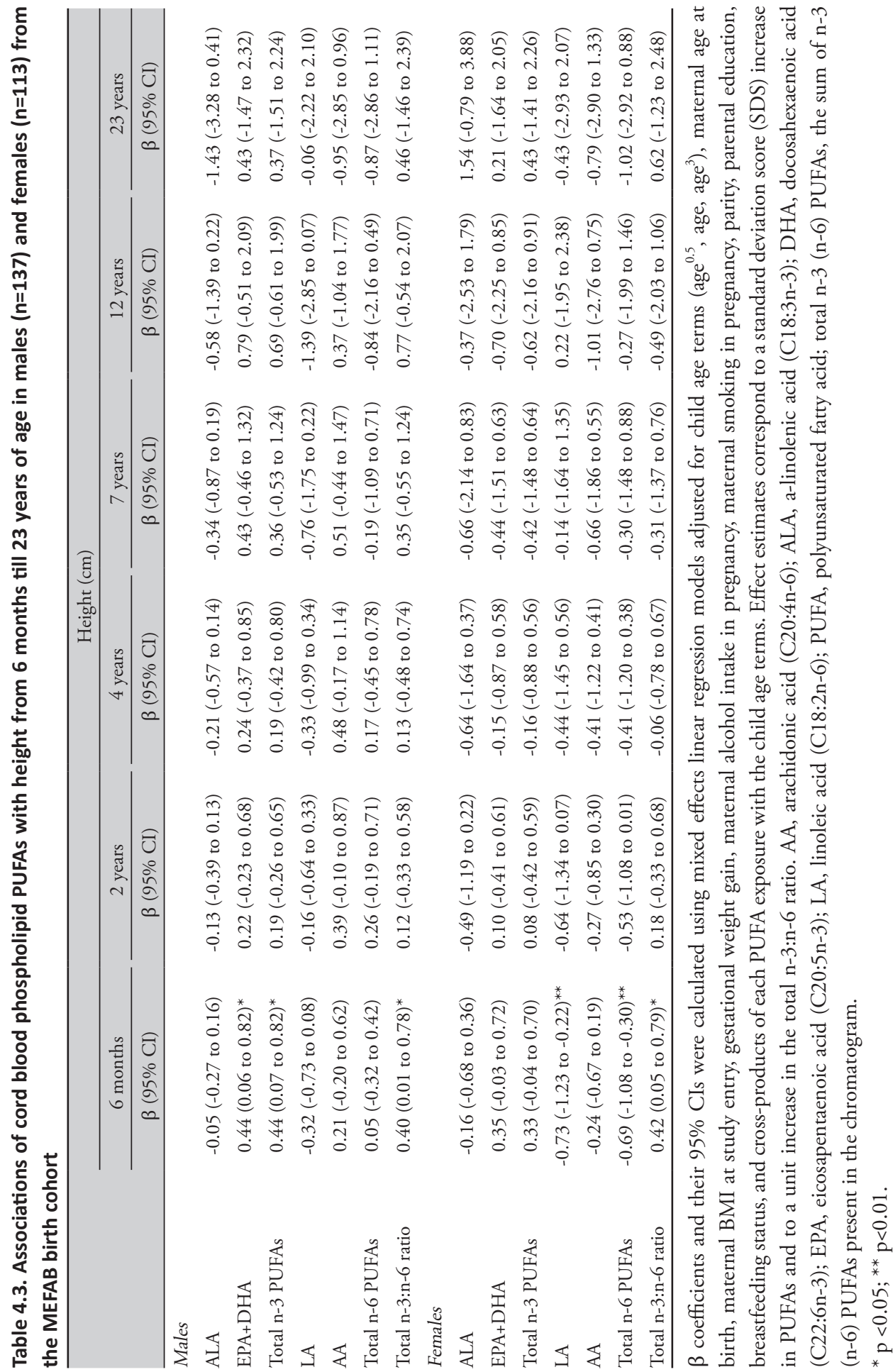




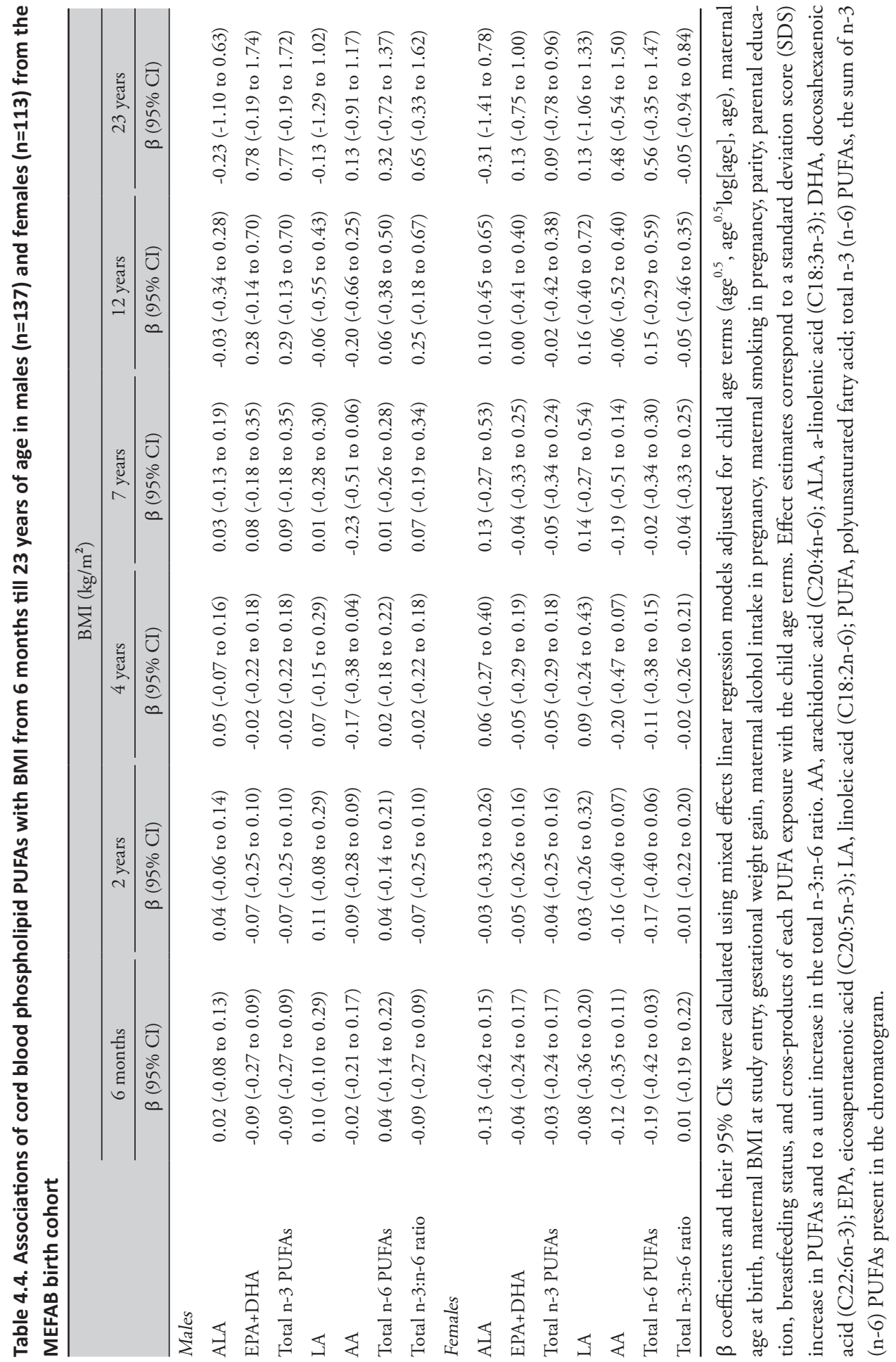




\section{Secondary and sensitivity analyses}

Among males, higher n-3 DPA levels in cord blood were associated with a higher length at 6 months, while higher n- 6 GLA concentrations were associated with lower length/height at 6 months and 2 years of age (Supplementary Table S4.5). We also found an association of higher $\mathrm{n}-6$ osbond acid levels with higher height among male adolescents at 12 years, but no other relations were found for PUFAs from early childhood onwards in either sex (Supplementary Table S4.5). No associations between secondary PUFAs of interest and BMI were found at any time point (Supplementary Table S4.6).

When we included gestational age in the models of height, we saw that effect estimates for n-3 PUFAs and infant length were largely attenuated (Supplementary Table S4.7). Associations between PUFAs and BMI measures remained of similar magnitude following additional adjustment for gestational age (Supplementary Table S4.8). Removal of children with impaired fetal growth did not alter the results (data not shown). Additionally, effect estimates for BMI in young adulthood did not substantially change when we further adjusted for fish oil supplementation, educational level, and alcohol consumption assessed at the age of outcome assessment (Supplementary Table S4.9).

\section{DISCUSSION}

In this analysis of the MEFAB cohort, we examined associations of cord blood PUFA levels with height and BMI measures from childhood to young adulthood. To our knowledge, this is the first study to examine child-to-adult growth in relation to early PUFA status. We measured cord blood phospholipid PUFA levels that constitute good surrogates of fetal exposure in late pregnancy, as they reflect maternal dietary intake of the last $2-4$ weeks, ${ }^{29}$ and the efficiency of placental transfer. ${ }^{30}$ We found that higher n-3 PUFA concentrations in cord blood were associated with increased infant length among males, while, for females, an association of the opposite direction was observed for n-6 PUFAs. A higher ratio of n-3 to n-6 PUFAs was associated with higher infant length in both sexes. The magnitude of these associations was small, corresponding to $0.4-0.7-\mathrm{cm}$ changes per SD increase in PUFA concentrations. The associations with infant length seemed to be transient, as they not did not persist later in childhood and in young adulthood. No associations of cord blood PUFAs with BMI from infancy to young adulthood were observed in either sex. 


\section{Interpretation of main findings}

We found sex-specific associations between PUFA levels at birth and infant height. Indeed, a systematic review has highlighted the importance of conducting sex stratification when examining the health effects of prenatal PUFAs. ${ }^{31}$ The placenta tissue carrying the fetal genome and sex appears as a promising candidate to be involved in mediating sex-specific effects. ${ }^{32}$ A human study has recently demonstrated that placentas show sexually dimorphic gene expression and responsiveness to maternal n-3 long-chain PUFA intervention in mid- and late pregnancy. ${ }^{33}$ Genes located on sex chromosomes can contribute to differential gene expression between male and female somatic tissues, and sex steroid hormones are thought to provide the initiation of this sex-specific responsiveness. ${ }^{33}$

A number of studies have previously assessed the effect of prenatal PUFA exposure on later height. An analysis of the Southampton Women's Survey reported a trend towards a positive relationship between maternal n-3 PUFA concentration in pregnancy and childhood height at 4 and 6 years. ${ }^{34}$ In a Mexican trial, offspring of primiparous women supplemented with DHA in pregnancy were taller at 18 months than those in the placebo group. ${ }^{8}$ In contrast, three small trials found no effect of n-3 long-chain PUFA supplementation in pregnancy on child height up to 2.5 years of age. ${ }^{9-11}$ Heterogeneity in previous findings may at least partly be explained by differences in the timing and definition of exposure, sample sizes and statistical analysis. Moreover, none of the previous studies stratified by sex. In our study, we found that higher levels of total n-3 PUFAs, EPA+DHA and DPA, and lower levels of n-6 GLA at birth were associated with a small increase in infant length in males. We also observed an association of $n-6$ osbond acid with higher height in male adolescents, but no associations were found for other PUFAs from early childhood onwards. Hence, we treat this result with caution, as we cannot rule out the possibility that the observed association is due to chance. Among females, higher levels of total n-6 PUFAs and LA were associated with a small decrease in infant length. The associations of PUFAs with infant length did not persist through young adulthood.

The observed effect estimates for n-3 PUFAs and infant length were attenuated when we adjusted for gestational age, suggesting that the potential benefit of $n-3$ PUFA exposure could be attributed to its influence on the length of gestation. Lower gestational age has been proposed to alter the endocrine regulation of postnatal growth, and preterm individuals have been shown to be shorter than term controls. ${ }^{27} \mathrm{n}-3$ PUFAs might increase pregnancy duration by reducing inflammation, inhibiting the activity of eicosanoid promoters of the parturition process, and increasing the activity of eicosanoids with myometrial relaxant properties. ${ }^{35,36}$ n-6 PUFAs are generally considered as pro-inflammatory, and in high amounts, 
they might adversely influence the intrauterine environment and lead to suboptimal fetal development, ${ }^{37}$ which in turn may translate to altered height growth. ${ }^{38}$ Another plausible explanation for the observed associations of PUFAs with height might relate to their effects on bone per se; n-6 PUFAs have been suggested to decrease osteoblastogenesis and inhibit bone formation, while n-3 PUFAs seem to have an opposite effect. ${ }^{7}$ Such effects may lead to altered bone growth, and thus, impact height. In line with our findings for strongest associations of fetal PUFA exposure with length in infancy, twin studies suggest that infancy is the most sensitive period regarding environmental influences on height variation. ${ }^{40}$ BMI is a widely-used surrogate measure of adiposity status. ${ }^{41}$ In our study, we did not find any association of cord blood PUFA levels with BMI from infancy through young adulthood. These findings are in line with those of trials showing no effect of maternal n-3 long-chain PUFA supplementation on offspring BMI up to 21 years of age. ${ }^{15,42}$ Previous results from cohort studies with shorter follow-up periods in Europe and the US have also provided little evidence to substantiate that a modification of prenatal PUFA status can affect later adiposity. ${ }^{12-14}$

\section{Methodological considerations}

Strengths of our study include the population-based prospective design, detailed information on PUFA levels, and repeated growth measures over a large time period that provide more definitive evaluation of within-person change across time and increase statistical power. ${ }^{43}$

A limitation of this study is attrition raising the likelihood of selection bias. Our study population had somewhat lower maternal BMI values at study entry compared to those lost to follow-up. Assuming that children of mothers with a higher BMI are more likely to be overweight, our estimates may be underestimated. Nevertheless, our study sample and those excluded did not substantially differ in many baseline variables including parental educational level and socioeconomic status, as well as in exposure levels. Although we controlled for a range of lifestyle and demographic characteristics that are associated with growth, we acknowledge that residual confounding from unmeasured covariates such as diet and physical activity patterns is still possible. Information on growth measures in young adulthood was self-reported, which might have led to misclassification. However, this potential misclassification is likely to be non-differential (ie, not to relate to cord blood PUFAs). Moreover, self-reported growth measures in young adulthood were significantly positively correlated with those in childhood. We assessed many PUFA exposures and child outcomes, raising concern about multiple testing. To our opinion, an application of Bonferroni-type corrections for multiple comparisons might be inappropriate in this case given that the outcomes examined are 
intercorrelated. ${ }^{44}$ Finally, we studied a population of relatively healthy participants from a high-income country. We cannot exclude the possibility that the small effect estimates for infant length and the lack of associations with growth outcomes at the other developmental ages cannot at least be partly explained by the fact that our study sample exhibited in general a healthy growth, thus having low variation in growth patterns, especially for height.

\section{Conclusion}

In the Dutch population-based MEFAB cohort, we found small, sex-specific associations of cord blood PUFA concentrations with length in infancy, corresponding to a $0.4-0.7-\mathrm{cm}$ change per SD increase in PUFA levels. The associations with infant length seemed to be transient, as they not did not persist later in childhood and in young adulthood. No associations of cord blood PUFAs with BMI from infancy till young adulthood were observed in either sex. Further studies, especially in low-income populations, are needed to disentangle the role of prenatal PUFAs in postnatal growth. 


\section{REFERENCES}

1. Paajanen TA, Oksala NK, Kuukasjarvi P, et al. Short stature is associated with coronary heart disease: a systematic review of the literature and a meta-analysis. Eur Heart J 2010;31:1802-9.

2. Owen CG, Whincup PH, Orfei L, et al. Is body mass index before middle age related to coronary heart disease risk in later life? Evidence from observational studies. Int J Obes (Lond) 2009;33:866-77.

3. Silventoinen K, Baker JL, Sorensen TI. Growth in height in childhood and risk of coronary heart disease in adult men and women. PLoS One 2012;7:e30476.

4. Symonds ME, Sebert SP, Hyatt MA, et al. Nutritional programming of the metabolic syndrome. Nat Rev Endocrinol 2009;5:604-10.

5. Ailhaud G, Guesnet P, Cunnane SC. An emerging risk factor for obesity: does disequilibrium of polyunsaturated fatty acid metabolism contribute to excessive adipose tissue development? Br J Nutr 2008;100:461-70.

6. Kalupahana NS, Claycombe KJ, Moustaid-Moussa N. (n-3) Fatty acids alleviate adipose tissue inflammation and insulin resistance: mechanistic insights. Adv Nutr 2011;2:304-16.

7. Kruger MC, Coetzee M, Haag M, et al. Long-chain polyunsaturated fatty acids: selected mechanisms of action on bone. Prog Lipid Res 2010;49:438-49.

8. Stein AD, Wang M, Martorell R, et al. Growth to age 18 months following prenatal supplementation with docosahexaenoic acid differs by maternal gravidity in Mexico. J Nutr 2011;141:316-20.

9. Much D, Brunner S, Vollhardt C, et al. Effect of dietary intervention to reduce the n- $6 / n-3$ fatty acid ratio on maternal and fetal fatty acid profile and its relation to offspring growth and body composition at 1 year of age. Eur J Clin Nutr 2013;67:282-8.

10. Dunstan JA, Simmer K, Dixon G, et al. Cognitive assessment of children at age 2(1/2) years after maternal fish oil supplementation in pregnancy: a randomised controlled trial. Arch Dis Child Fetal Neonatal Ed 2008;93:F45-50.

11. Innis SM, Friesen RW. Essential n-3 fatty acids in pregnant women and early visual acuity maturation in term infants. Am J Clin Nutr 2008;87:548-57.

12. Hauner H, Brunner S. Early fatty acid exposure and later obesity risk. Curr Opin Clin Nutr Metab Care 2015;18:113-7.

13. Vidakovic AJ, Gishti O, Voortman T, et al. Maternal plasma PUFA concentrations during pregnancy and childhood adiposity: the Generation R Study. Am J Clin Nutr 2016;103:1017-25.

14. Hakola L, Takkinen HM, Niinisto S, et al. Maternal fatty acid intake during pregnancy and the development of childhood overweight: a birth cohort study. Pediatr Obes 2016 Jul 05. doi: 10.1111/ijpo.12170. [Epub ahead of print]

15. Stratakis N, Gielen M, Chatzi L, et al. Effect of maternal n-3 long-chain polyunsaturated fatty acid supplementation during pregnancy and/or lactation on adiposity in childhood: a systematic review and meta-analysis of randomized controlled trials. Eur J Clin Nutr 2014;68:1277-87.

16. van der Wurff ISM, de Groot RHM, Stratakis N, et al. Maastricht essential fatty acid birth cohort. Lipid Technology 2015;27:59-62.

17. Al MDM, Vanhouwelingen AC, Kester ADM, et al. Maternal Essential Fatty-Acid Patterns during Normal-Pregnancy and Their Relationship to the Neonatal Essential Fatty-Acid Status. Br J Nutr 1995;74:55-68.

18. Hornstra G. Essential fatty acids during pregnancy. Impact on mother and child. Nestle Nutr Workshop Ser Pediatr Program 2005;55:83-96; discussion -100. 
19. de Vries PS, Gielen M, Rizopoulos D, et al. Association between polyunsaturated fatty acid concentrations in maternal plasma phospholipids during pregnancy and offspring adiposity at age 7: the MEFAB cohort. Prostaglandins Leukot Essent Fatty Acids 2014;91:81-5.

20. Vogels N, Posthumus DL, Mariman EC, et al. Determinants of overweight in a cohort of Dutch children. Am J Clin Nutr 2006;84:717-24.

21. Bornhorst C, Siani A, Russo P, et al. Early Life Factors and Inter-Country Heterogeneity in BMI Growth Trajectories of European Children: The IDEFICS Study. PLoS One 2016;11:e0149268.

22. Berkey CS, Colditz GA. Adiposity in adolescents: change in actual BMI works better than change in BMI z score for longitudinal studies. Ann Epidemiol 2007;17:44-50.

23. Cole TJ, Faith MS, Pietrobelli A, et al. What is the best measure of adiposity change in growing children: BMI, BMI \%, BMI z-score or BMI centile? Eur J Clin Nutr 2005;59:419-25.

24. Decsi T, Kennedy K. Sex-specific differences in essential fatty acid metabolism. Am J Clin Nutr 2011;94:1914S-9S.

25. Netherlands Institute for Public Health and the Environment. (RIVM). Indeling opleidingsniveau, in, 2008.

26. Reece MS, McGregor JA, Allen KG, et al. Maternal and perinatal long-chain fatty acids: possible roles in preterm birth. Am J Obstet Gynecol 1997;176:907-14.

27. Derraik JG, Lundgren M, Cutfield WS, et al. Association Between Preterm Birth and Lower Adult Height in Women. Am J Epidemiol 2017;185:48-53.

28. Rump P, Mensink RP, Kester AD, et al. Essential fatty acid composition of plasma phospholipids and birth weight: a study in term neonates. Am J Clin Nutr 2001;73:797-806.

29. Hodson L, Skeaff CM, Fielding BA. Fatty acid composition of adipose tissue and blood in humans and its use as a biomarker of dietary intake. Prog Lipid Res 2008;47:348-80.

30. Hornstra G. Essential fatty acids in mothers and their neonates. Am J Clin Nutr 2000;71:1262S-9S.

31. Decsi T, Kennedy K. Sex-specific differences in essential fatty acid metabolism. Am J Clin Nutr 2011;94:Suppl. 6, S1914-S9.

32. Sood R, Zehnder JL, Druzin ML, et al. Gene expression patterns in human placenta. Proc Natl Acad Sci US A 2006;103:5478-83.

33. Sedlmeier EM, Brunner S, Much D, et al. Human placental transcriptome shows sexually dimorphic gene expression and responsiveness to maternal dietary n-3 long-chain polyunsaturated fatty acid intervention during pregnancy. BMC Genomics 2014;15:941.

34. Moon RJ, Harvey NC, Robinson SM, et al. Maternal plasma polyunsaturated fatty acid status in late pregnancy is associated with offspring body composition in childhood. J Clin Endocrinol Metab 2013;98:299-307.

35. Olsen SF. Is supplementation with marine omega-3 fatty acids during pregnancy a useful tool in the prevention of preterm birth? Clin Obstet Gynecol 2004;47:768-74; discussion 881-2.

36. Hansen HS, Olsen SF. Dietary (n-3)-fatty acids, prostaglandins, and prolonged gestation in humans. Prog Clin Biol Res 1988;282:305-17.

37. van Eijsden M, Hornstra G, van der Wal MF, et al. Maternal n-3, n-6, and trans fatty acid profile early in pregnancy and term birth weight: a prospective cohort study. Am J Clin Nutr 2008;87:887-95.

38. Hindmarsh PC, Geary MP, Rodeck CH, et al. Factors predicting ante- and postnatal growth. Pediatr Res 2008;63:99-102.

39. Watkins BA, Lippman HE, Le Bouteiller L, et al. Bioactive fatty acids: role in bone biology and bone cell function. Prog Lipid Res 2001;40:125-48.

40. Jelenkovic A, Sund R, Hur YM, et al. Genetic and environmental influences on height from infancy to early adulthood: An individual-based pooled analysis of 45 twin cohorts. Sci Rep 2016;6:28496. 


\section{Chapter 4}

41. Boeke CE, Oken E, Kleinman KP, et al. Correlations among adiposity measures in school-aged children. BMC Pediatr 2013;13:99.

42. Blumfield ML. Can long-chain PUFA supplementation during pregnancy influence later obesity risk? Am J Clin Nutr 2016;103:1387-8.

43. Guo Y, Logan HL, Glueck DH, et al. Selecting a sample size for studies with repeated measures. BMC Med Res Methodol 2013;13:100.

44. Rothman KJ. No adjustments are needed for multiple comparisons. Epidemiology 1990;1:43-6. 


\section{Supplementary Material of Chapter 4}

Supplementary Text 4A: Modelling of height and BMI trajectories

Supplementary Table S4.1. Comparison of subject characteristics in MEFAB between those included and not included in the analysis

Supplementary Table S4.2. Distribution of length/height and body mass index measurements from 6 months till 23 years of age in MEFAB males $(n=137)$ and females $(\mathrm{n}=113)$ included in the analysis

Supplementary Table S4.3. Correlation coefficients among height measurements from 6 months till 23 years of age in males $(n=137)$ and females $(n=113)$ from the MEFAB birth cohort

Supplementary Table S4.4. Correlation coefficients among body mass index measurements from 6 months till 23 years of age in males $(n=137)$ and females $(\mathrm{n}=113)$ from the MEFAB birth cohort

Supplementary Table S4.5. Associations of secondary cord blood PUFAs of interest with height from 6 months till 23 years of age in males $(n=137)$ and females $(\mathrm{n}=113)$ from the MEFAB birth cohort

Supplementary Table S4.6. Associations of secondary cord blood PUFAs of interest with BMI from 6 months till 23 years of age in males $(n=137)$ and females $(n=113)$ from the MEFAB birth cohort

Supplementary Table S4.7. Associations of cord blood PUFAs with height from 6 months till 23 years of age in males $(n=137)$ and females $(n=113)$ from the MEFAB birth cohort after further adjusting for gestational age

Supplementary Table S4.8. Associations of cord blood phospholipid PUFAs with BMI from 6 months till 23 years of age in males $(n=137)$ and females $(n=113)$ from the MEFAB birth cohort after further adjusting for gestational age

Supplementary Table S4.9. Associations of cord blood PUFAs with BMI at 23 years of age in men $(n=81)$ and women $(n=117)$ from the MEFAB birth cohort after further adjustment for fish oil supplementation, alcohol intake, and educational level assessed at the age of outcome assessment

Supplementary Figure S4.1. Directed acyclic graph for the association of fatty acid status at birth with postnatal growth.

Supplementary References 


\section{Supplementary Text 4A: Modelling of height and BMI trajectories}

Growth follows a complex non-linear pattern. This non-linearity can be incorporated into multi-level models (two levels: measurement occasion and individual) by including non-linear age functions to find the best-fitting average growth trajectory in the study population. Such models allow for individual variation in growth curves, and can easily handle unbalanced data with different numbers of repeated measures per child. ${ }^{1}$ Moreover, these models allow for change in scale and variance of the growth measures over time. ${ }^{2}$

We modelled height and BMI growth trajectories from birth to 23 years of age. As age must be strictly positive when using fractional polynomials, a constant of 0.001 was added to age. A series of models for height (in $\mathrm{cm}$ ) and BMI (in $\mathrm{kg} / \mathrm{m}^{2}$ ) were run in which age was raised to a large number of combinations of powers out of the following: $-2,-1,-0.5,0$ (log function), $0.5,1,2$, or 3 in order to find a function of age that best describes each trajectory. ${ }^{3}$ All models were stratified by sex. We used the $-2 \log$ likelihood statistic and Bayesian Information Criterion (BIC) to select the model with the lowest deviance.

The best-fitting fractional polynomial model for each growth measure was fitted with an unstructured variance-covariance matrix for the random effects, ${ }^{1}$ and had the following form for male and female participants:

$B M I_{i, j}=\left(\beta_{0}+u_{i, 0}+\varepsilon_{i, j}\right)+\left(\beta_{1}+u_{i, 1}\right) a g e_{i, j}^{0.5}+\left(\beta_{2}\right) a g e_{i, j}^{0.5} \log \left(a g e_{i, j}\right)+\left(\beta_{3}+u_{i, 2}\right) a g e_{i, j}^{3}$

Height $_{i, j}=\left(\beta_{0}+u_{i, 0}+\varepsilon_{i, j}\right)+\left(\beta_{1}+u_{i, 1}\right) a g e_{i, j}+\left(\beta_{2}\right) a g e_{i, j}^{0.5}+\left(\beta_{3}+u_{i, 2}\right) a g e_{i, j}^{3}$

where $\beta_{0} \beta_{1}, \beta_{2}, \beta_{3}$ denote the fixed effects, ví, 0 , ví, 1 , ví,2 denote the random effects, and $\varepsilon$ í,j denotes the error term with $\mathrm{i}=1, \ldots$ n and $\mathrm{j}=1, \ldots$ ni (n: number of subjects; ni: number of measurements of individual i).

The tables 4A.1 and 4A.2 show that there were high levels of agreement between observed growth measurements and those predicted by the models. 
Table 4.A1. Agreement levels between observed and predicted measurements

\begin{tabular}{|c|c|c|c|c|c|c|c|c|c|c|c|}
\hline \multicolumn{6}{|c|}{ Males } & \multicolumn{6}{|c|}{ Females } \\
\hline \multicolumn{3}{|c|}{ Height } & \multicolumn{3}{|c|}{ BMI } & \multicolumn{3}{|c|}{ Height } & \multicolumn{3}{|c|}{ BMI } \\
\hline \multicolumn{3}{|c|}{$\underline{\text { Difference }}$} & \multicolumn{3}{|c|}{ Difference } & \multicolumn{3}{|c|}{$\underline{\text { Difference }}$} & \multicolumn{3}{|c|}{ Difference } \\
\hline rhoc & Mean & (SD) & rhoc & Mean & (SD) & rhoc & Mean & $(\mathrm{SD})$ & rhoc & Mean & (SD) \\
\hline 0.99 & 0.00 & $(1.56)$ & 0.94 & 0.00 & $(0.83)$ & 0.99 & 0.00 & $(1.70)$ & 0.95 & 0.00 & $(0.89)$ \\
\hline
\end{tabular}

rhoc, concordance correlation coefficient. Difference represents the average difference between observed and predicted values.

Table 4.A2. Distribution of observed and predicted sex-specific height and BMI values at specific time points

\begin{tabular}{|c|c|c|c|c|}
\hline & \multicolumn{4}{|c|}{ MEFAB } \\
\hline & \multicolumn{2}{|c|}{ Boys } & \multicolumn{2}{|c|}{ Girls } \\
\hline & Actual & Predicted & Actual & Predicted \\
\hline & Mean (SD) & Mean (SD) & Mean (SD) & Mean (SD) \\
\hline \multicolumn{5}{|l|}{ Height (cm) } \\
\hline $6 \pm 2$ months & $67.6(2.7)$ & $67.9(2.3)$ & $65.3(2.7)$ & $66.0(2.0)$ \\
\hline $24 \pm 6$ months & $88.8(3.6)$ & $88.1(2.9)$ & $87.6(3.7)$ & $86.8(2.8)$ \\
\hline $48 \pm 6$ months & $105.5(4.1)$ & $106.0(3.7)$ & $103.9(4.7)$ & $104.4(4.0)$ \\
\hline $84 \pm 6$ months & $127.2(5.0)$ & $127.9(5.3)$ & $125.5(5.8)$ & $126.3(5.8)$ \\
\hline $144 \pm 12$ months & $154.0(7.6)$ & $153.9(7.1)$ & $154.8(10.9)$ & $153.9(10.6)$ \\
\hline $277 \pm 12$ months & $181.6(7.2)$ & $181.7(6.9)$ & $166.2(8.1)$ & $166.7(8.1)$ \\
\hline \multicolumn{5}{|l|}{ BMI $\left(\mathrm{kg} / \mathrm{m}^{2}\right)$} \\
\hline $6 \pm 2$ months & $17.0(1.3)$ & $17.1(0.7)$ & $16.6(1.3)$ & $16.6(0.8)$ \\
\hline $24 \pm 6$ months & $16.3(1.2)$ & $16.4(0.8)$ & $16.1(1.4)$ & $16.2(1.0)$ \\
\hline $48 \pm 6$ months & $15.6(1.2)$ & $15.7(0.9)$ & $15.7(1.4)$ & $15.8(1.2)$ \\
\hline $84 \pm 6$ months & $15.4(1.7)$ & $15.6(1.4)$ & $15.6(1.6)$ & $15.8(1.4)$ \\
\hline $144 \pm 12$ months & $17.8(2.5)$ & $17.1(1.2)$ & $18.0(2.2)$ & $17.4(2.1)$ \\
\hline $277 \pm 12$ months & $23.0(4.3)$ & $23.2(3.9)$ & $22.4(3.2)$ & $22.6(2.8)$ \\
\hline
\end{tabular}


Supplementary Table S4.1. Comparison of subject characteristics in MEFAB between those included and not included in the analysis

\begin{tabular}{|c|c|c|c|}
\hline & $\begin{array}{l}\text { Subjects not included } \\
\qquad(\mathrm{n}=953)\end{array}$ & $\begin{array}{l}\text { Subjects } \\
\text { included } \\
(\mathrm{n}=250)\end{array}$ & \\
\hline & Mean (SD) or $\mathrm{n}(\%)$ & $\begin{array}{c}\text { Mean }(\mathrm{SD}) \text { or } \\
\mathrm{n}(\%)\end{array}$ & P for difference \\
\hline \multicolumn{4}{|l|}{ Parental characteristics } \\
\hline Maternal age at birth (years) & $29.1(4.3)$ & $30.2(4.0)$ & $<0.001$ \\
\hline Maternal BMI at study entry $(\mathrm{kg} / \mathrm{m} 2)$ & $24.2(4.3)$ & $23.5(3.7)$ & 0.02 \\
\hline Gestational weight gain $(\mathrm{kg})$ & $11.7(4.6)$ & $11.1(4.0)$ & 0.08 \\
\hline Maternal smoking in pregnancy (yes, \%) & $236(26.3)$ & $56(22.4)$ & 0.21 \\
\hline Alcohol intake in pregnancy (yes, \%) & $29(3.1)$ & $12(4.8)$ & 0.19 \\
\hline Parity (primiparous, \%) & $693(76.2)$ & $176(70.4)$ & 0.06 \\
\hline $\begin{array}{l}\text { Area-based socioeconomic status at birth } \\
(\text { high, \%)* }\end{array}$ & $268(38.4)$ & $78(35.1)$ & 0.08 \\
\hline \multicolumn{4}{|l|}{ Child characteristics } \\
\hline Sex (female, \%) & $423(44.8)$ & $113(45.2)$ & 0.91 \\
\hline Birth weight (gr) & $3272(519)$ & $3303(479)$ & 0.39 \\
\hline Gestational age (weeks) & $39.9(1.7)$ & $39.9(1.6)$ & 0.95 \\
\hline Cord blood n-3 PUFA levels (wt \%) & $6.9(1.6)$ & $6.8(1.6)$ & 0.57 \\
\hline Cord blood n-6 PUFA levels (wt \%) & $32.1(1.7)$ & $32.2(1.7)$ & 0.55 \\
\hline
\end{tabular}

Values are means (SD) or absolute numbers (percentages). P values for difference were estimated using Student's test for continuous variables and Pearson's $\chi 2$ test for categorical variables. PUFA, polyunsaturated fatty acid; wt \%, weight percentage of total fatty acids present in the chromatogram.

* Area-based socio-economic status at birth was determined based on the postal code of the place of residence. ${ }^{4}$ 
Supplementary Table S4.2. Distribution of length/height and body mass index measurements from 6 months till 23 years of age in MEFAB males $(n=137)$ and females $(n=113)$ included in the analysis

\begin{tabular}{|c|c|c|c|c|c|c|c|c|}
\hline & \multicolumn{4}{|c|}{ Height $(\mathrm{cm})$} & \multicolumn{4}{|c|}{ BMI $\left(\mathrm{kg} / \mathrm{m}^{2}\right)$} \\
\hline & \multicolumn{2}{|r|}{ Males } & \multicolumn{2}{|r|}{ Females } & \multicolumn{2}{|c|}{ Males } & \multicolumn{2}{|c|}{ Females } \\
\hline & $\mathrm{n}$ & Mean (SD) & $\mathrm{n}$ & Mean (SD) & $\mathrm{n}$ & Mean (SD) & $\mathrm{n}$ & $\begin{array}{c}\text { Mean } \\
(\mathrm{SD})\end{array}$ \\
\hline \multicolumn{9}{|c|}{ Mean age $(S D)$, years } \\
\hline $0.5(0.7)$ & 126 & $67.6(2.6)$ & 94 & $65.5(3.0)$ & 126 & $17.0(1.3)$ & 94 & $16.6(1.3)$ \\
\hline $2.0(0.2)$ & 124 & $88.7(3.5)$ & 100 & $87.2(5.0)$ & 123 & $16.3(1.2)$ & 99 & $16.3(1.9)$ \\
\hline $4.1(0.3)$ & 119 & $105.8(4.6)$ & 98 & $104.2(5.1)$ & 119 & $15.6(1.2)$ & 98 & $15.7(1.4)$ \\
\hline $7.3(0.3)$ & 137 & $127.2(4.9)$ & 113 & $126.1(5.8)$ & 137 & $15.5(1.7)$ & 113 & $15.8(1.7)$ \\
\hline $12.8(1.1)$ & 51 & $159.3(10.5)$ & 33 & $158.6(9.4)$ & 51 & $18.5(2.5)$ & 33 & $19.1(2.4)$ \\
\hline $23.8(1.1)$ & 45 & $182.2(8.2)$ & 59 & $167.0(8.0)$ & 45 & $23.2(3.8)$ & 59 & $23.3(3.8)$ \\
\hline
\end{tabular}


Supplementary Table S4.3. Correlation coefficients among height measurements from 6 months till 23 years of age in males $(n=137)$ and females $(n=113)$ from the MEFAB birth cohort

\begin{tabular}{|c|c|c|c|c|c|c|c|c|}
\hline Age & 6 months & 1 year & 2 years & 3 years & 4 years & 8 years & 12 years & 23 years \\
\hline \multicolumn{9}{|l|}{ Males } \\
\hline 6 months & 1.00 & & & & & & & \\
\hline 1 year & 0.59 & 1.00 & & & & & & \\
\hline $\mathrm{P}$-value & $<0.001$ & & & & & & & \\
\hline 2 years & 0.39 & 0.56 & 1.00 & & & & & \\
\hline P-value & $<0.001$ & $<0.001$ & & & & & & \\
\hline 3 years & 0.50 & 0.65 & 0.73 & 1.00 & & & & \\
\hline P-value & $<0.001$ & $<0.001$ & $<0.001$ & & & & & \\
\hline 4 years & 0.38 & 0.60 & 0.66 & 0.84 & 1.00 & & & \\
\hline P-value & $<0.001$ & $<0.001$ & $<0.001$ & $<0.001$ & & & & \\
\hline 8 years & 0.34 & 0.51 & 0.62 & 0.78 & 0.79 & 1.00 & & \\
\hline P-value & $<0.001$ & $<0.001$ & $<0.001$ & $<0.001$ & $<0.001$ & & & \\
\hline 12 years & -0.02 & 0.18 & 0.27 & 0.42 & 0.28 & 0.68 & 1.00 & \\
\hline P-value & 0.91 & 0.25 & 0.08 & 0.005 & 0.06 & $<0.001$ & & \\
\hline 23 years & 0.31 & 0.26 & 0.56 & 0.75 & 0.74 & 0.79 & 0.52 & 1.00 \\
\hline P-value & 0.05 & 0.10 & $<0.001$ & $<0.001$ & $<0.001$ & $<0.001$ & 0.01 & \\
\hline \multicolumn{9}{|l|}{ Females } \\
\hline 6 months & 1.00 & & & & & & & \\
\hline 1 year & 0.67 & 1.00 & & & & & & \\
\hline P-value & $<0.001$ & & & & & & & \\
\hline 2 years & 0.55 & 0.67 & 1.00 & & & & & \\
\hline P-value & $<0.001$ & $<0.001$ & & & & & & \\
\hline 3 years & 0.56 & 0.69 & 0.64 & 1.00 & & & & \\
\hline P-value & $<0.001$ & $<0.001$ & $<0.001$ & & & & & \\
\hline 4 years & 0.54 & 0.66 & 0.59 & 0.81 & 1.00 & & & \\
\hline P-value & $<0.001$ & $<0.001$ & $<0.001$ & $<0.001$ & & & & \\
\hline 8 years & 0.50 & 0.56 & 0.54 & 0.82 & 0.78 & 1.00 & & \\
\hline P-value & $<0.001$ & $<0.001$ & $<0.001$ & $<0.001$ & $<0.001$ & & & \\
\hline 12 years & 0.52 & 0.34 & 0.59 & 0.63 & 0.33 & 0.80 & 1.00 & \\
\hline P-value & 0.01 & 0.06 & $<0.001$ & $<0.001$ & 0.06 & $<0.001$ & & \\
\hline 23 years & 0.56 & 0.64 & 0.38 & 0.81 & 0.79 & 0.77 & 0.83 & 1.00 \\
\hline P-value & $<0.001$ & $<0.001$ & $<0.001$ & $<0.001$ & $<0.001$ & $<0.001$ & $<0.001$ & \\
\hline
\end{tabular}


Supplementary Table S4.4. Correlation coefficients among body mass index measurements from 6 months till 23 years of age in males $(n=137)$ and females $(n=113)$ from the MEFAB birth cohort

\begin{tabular}{|c|c|c|c|c|c|c|c|c|}
\hline Age & 6 months & 1 year & 2 years & 3 years & 4 years & 8 years & 12 years & 23 years \\
\hline \multicolumn{9}{|l|}{ Males } \\
\hline 6 months & 1.00 & & & & & & & \\
\hline 1 year & 0.50 & 1.00 & & & & & & \\
\hline P-value & $<0.001$ & & & & & & & \\
\hline 2 years & 0.34 & 0.62 & 1.00 & & & & & \\
\hline P-value & $<0.001$ & $<0.001$ & & & & & & \\
\hline 3 years & 0.25 & 0.57 & 0.67 & 1.00 & & & & \\
\hline P-value & 0.01 & $<0.001$ & $<0.001$ & & & & & \\
\hline 4 years & 0.18 & 0.45 & 0.55 & 0.65 & 1.00 & & & \\
\hline P-value & 0.06 & $<0.001$ & $<0.001$ & $<0.001$ & & & & \\
\hline 8 years & 0.16 & 0.39 & 0.63 & 0.56 & 0.71 & 1.00 & & \\
\hline P-value & 0.07 & $<0.001$ & $<0.001$ & $<0.001$ & $<0.001$ & & & \\
\hline 12 years & -0.01 & 0.06 & 0.42 & 0.36 & 0.49 & 0.81 & 1.00 & \\
\hline P-value & 0.96 & 0.71 & 0.005 & 0.02 & $<0.001$ & $<0.001$ & & \\
\hline 23 years & -0.27 & 0.09 & 0.34 & 0.18 & 0.34 & 0.44 & 0.51 & 1.00 \\
\hline $\mathrm{P}$-value & 0.09 & 0.60 & 0.03 & 0.29 & 0.03 & 0.003 & 0.01 & \\
\hline \multicolumn{9}{|l|}{ Females } \\
\hline 6 months & 1.00 & & & & & & & \\
\hline 1 year & 0.76 & 1.00 & & & & & & \\
\hline P-value & $<0.001$ & & & & & & & \\
\hline 2 years & 0.65 & 0.68 & 1.00 & & & & & \\
\hline P-value & $<0.001$ & $<0.001$ & & & & & & \\
\hline 3 years & 0.65 & 0.74 & 0.38 & 1.00 & & & & \\
\hline P-value & $<0.001$ & $<0.001$ & $<0.001$ & & & & & \\
\hline 4 years & 0.50 & 0.65 & 0.39 & 0.91 & 1.00 & & & \\
\hline P-value & $<0.001$ & $<0.001$ & $<0.001$ & $<0.001$ & & & & \\
\hline 8 years & 0.34 & 0.47 & 0.31 & 0.78 & 0.78 & 1.00 & & \\
\hline P-value & $<0.001$ & $<0.001$ & $<0.001$ & $<0.001$ & $<0.001$ & & & \\
\hline 12 years & 0.19 & 0.30 & 0.42 & 0.51 & 0.55 & 0.70 & 1.00 & \\
\hline P-value & 0.34 & 0.10 & 0.02 & 0.007 & 0.001 & $<0.001$ & & \\
\hline 23 years & -0.03 & 0.14 & 0.04 & 0.38 & 0.45 & 0.66 & 0.50 & 1.00 \\
\hline P-value & 0.82 & 0.33 & 0.79 & 0.007 & 0.002 & $<0.001$ & 0.02 & \\
\hline
\end{tabular}




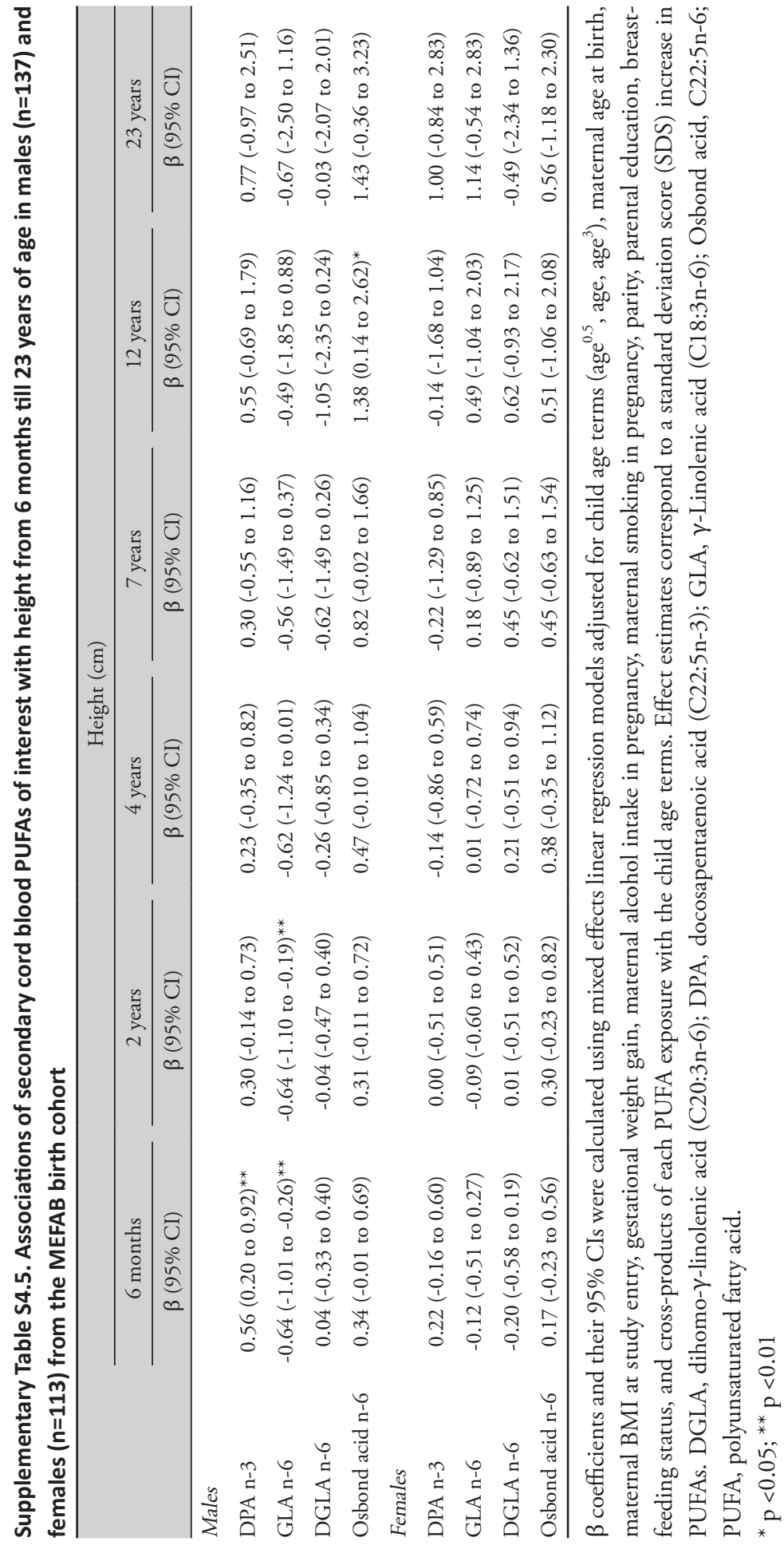




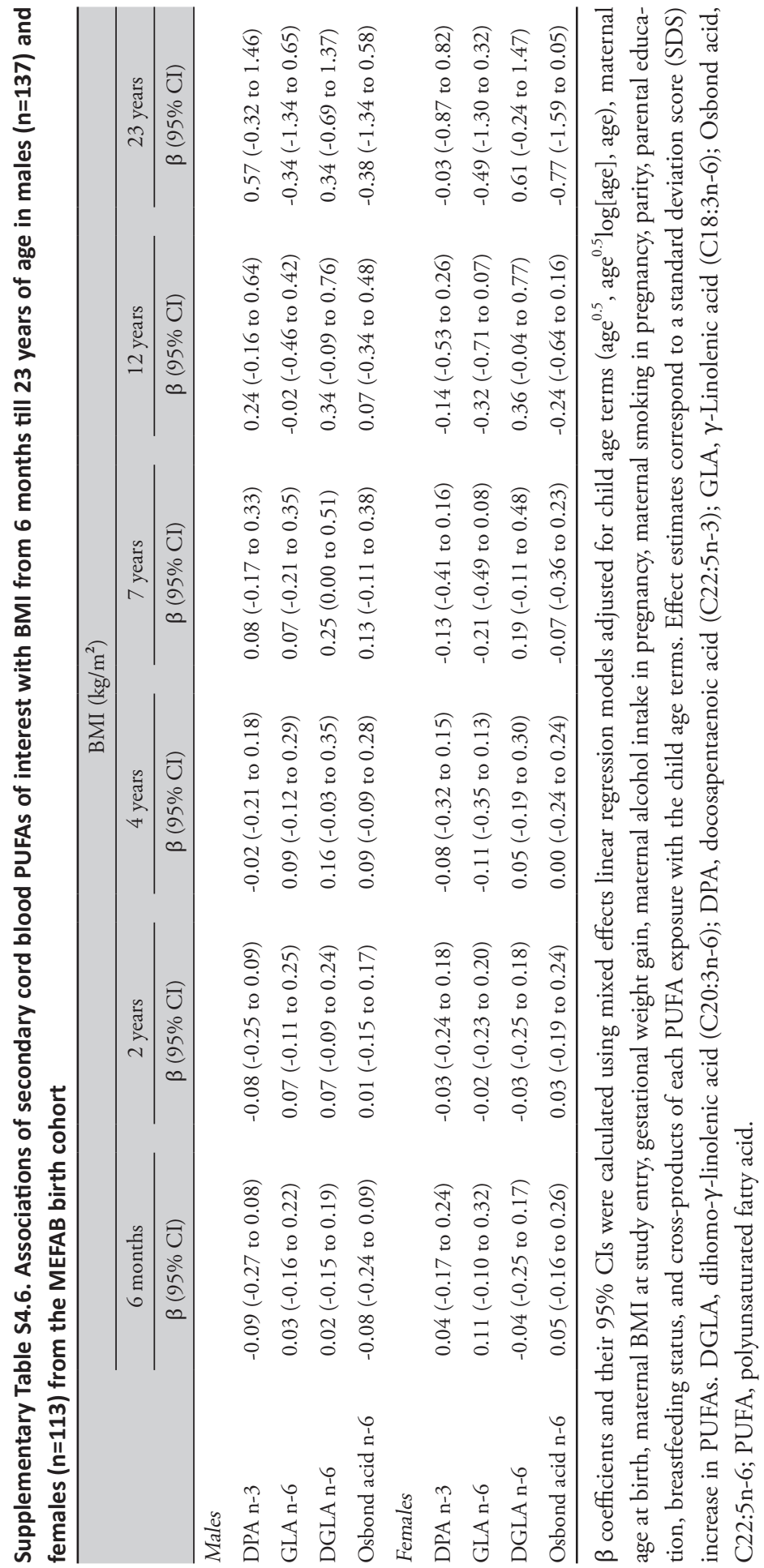




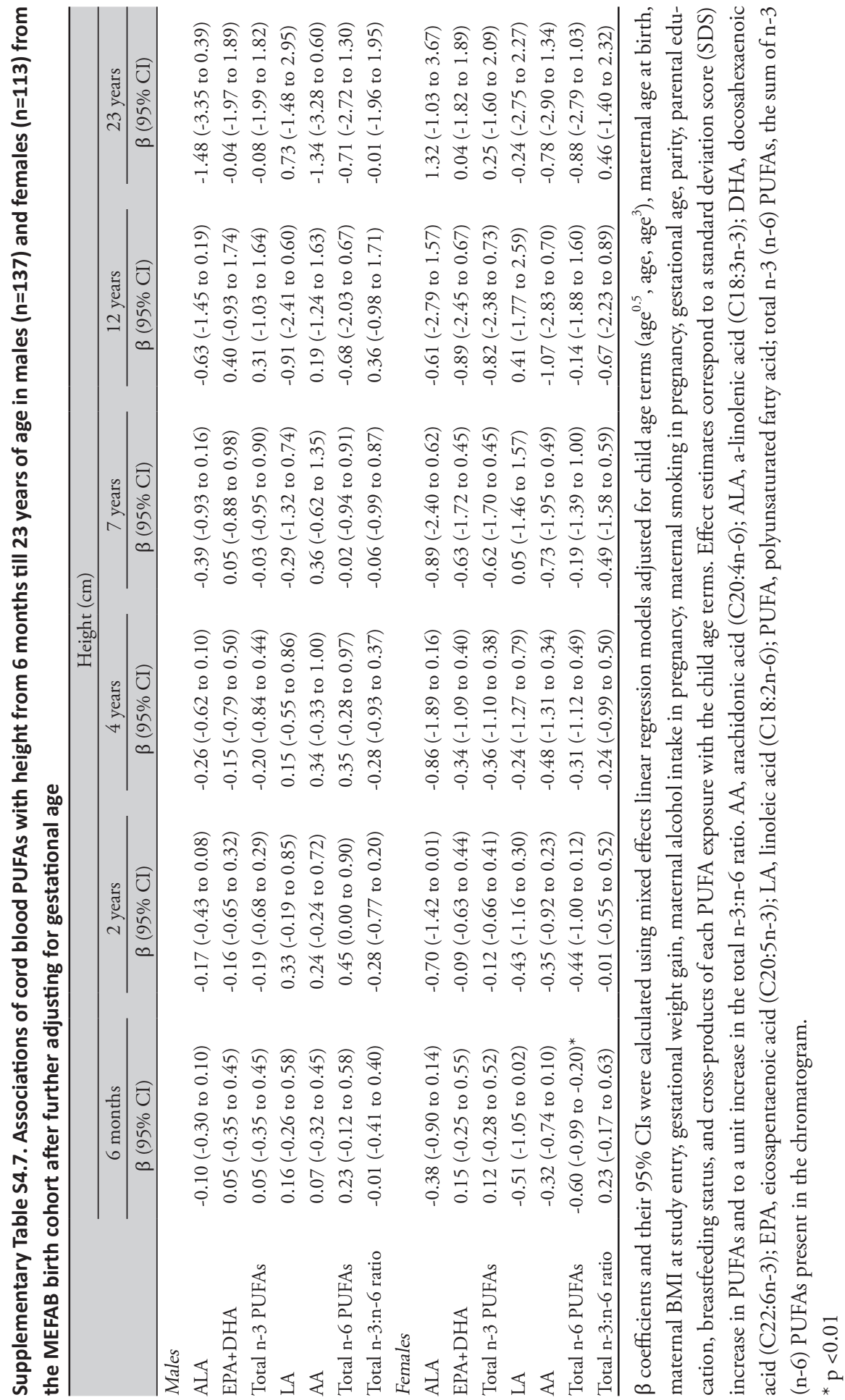




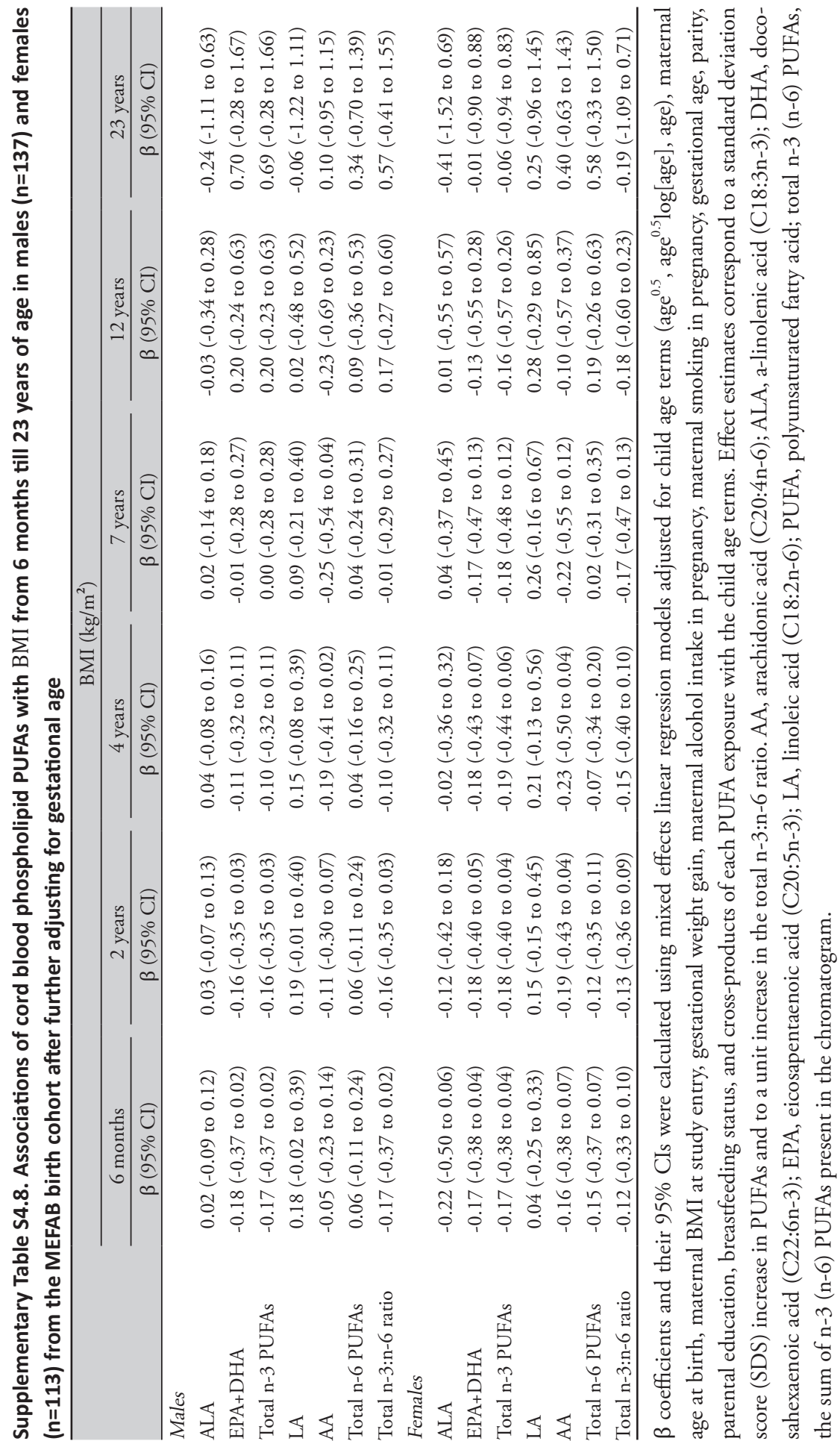


Supplementary Table S4.9. Associations of cord blood PUFAs with BMI at 23 years of age in men $(n=81)$ and women $(n=117)$ from the MEFAB birth cohort after further adjustment for fish oil supplementation, alcohol intake, and educational level assessed at the age of outcome assessment

\begin{tabular}{lcc}
\hline & \multicolumn{2}{c}{ BMI $\left(\mathrm{kg} / \mathrm{m}^{2}\right)$} \\
\cline { 2 - 3 } & $\beta(95 \% \mathrm{CI})$ & Women \\
\hline ALA & $-0.54(-1.61$ to 0.53$)$ & $\beta(95 \% \mathrm{CI})$ \\
EPA+DHA & $-0.03(-0.85$ to 0.78$)$ & $0.02(-0.64$ to 0.69$)$ \\
Total n-3 PUFAs & $-0.10(-0.90$ to 0.71$)$ & $0.54(-0.28$ to 1.37$)$ \\
LA & $-0.09(-1.07$ to 0.89$)$ & $0.52(-0.30$ to 1.33$)$ \\
AA & $0.33(-0.47$ to 1.13$)$ & $0.58(-0.45$ to 1.61$)$ \\
Total n-6 PUFAs & $-0.03(-0.85$ to 0.79$)$ & $0.18(-0.67$ to 1.02$)$ \\
Total n-3:n-6 ratio & $-0.06(-0.85$ to 0.74$)$ & $0.35(-0.47$ to 1.16$)$ \\
\hline
\end{tabular}

$\beta$ coefficients and their 95\% CIs were calculated using linear regression models adjusted for maternal BMI at study entry, gestational weight gain, maternal smoking during pregnancy, maternal age at birth, parity, parental education, breastfeeding, age, fish oil supplementation, alcohol intake, and educational level. Effect estimates correspond to a standard deviation score (SDS) increase in PUFAs and to a unit increase in the total n-3:n-6 ratio. AA, arachidonic acid (C20:4n-6); ALA, a-linolenic acid (C18:3n-3); DHA, docosahexaenoic acid (C22:6n-3); EPA, eicosapentaenoic acid (C20:5n-3); LA, linoleic acid (C18:2n-6); PUFA, polyunsaturated fatty acid; total n-3 (n-6) PUFAs, the sum of n-3 (n-6) PUFAs present in the chromatogram. 


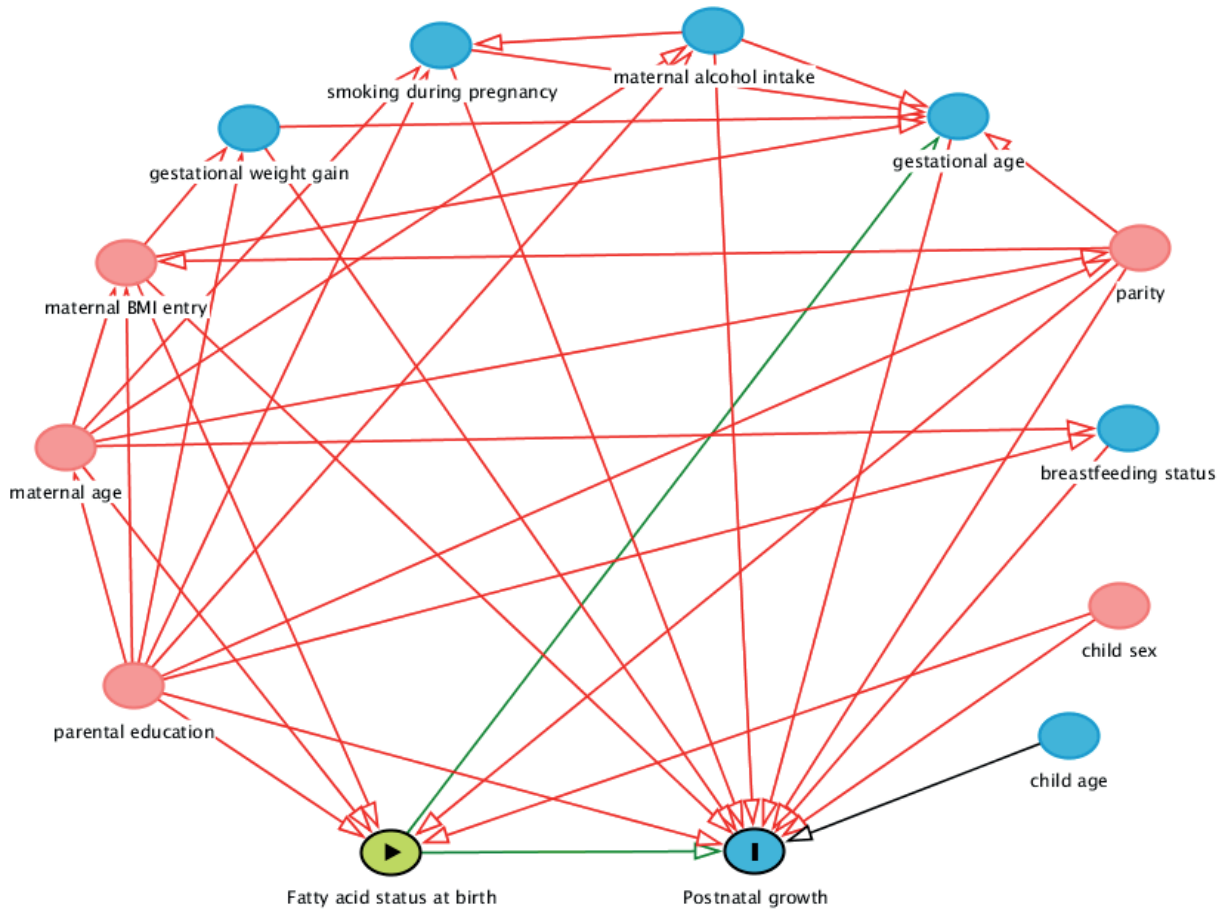

Supplementary Figure S4.1. Directed acyclic graph for the association of fatty acid status at birth with postnatal growth. 


\section{Supplementary References}

1. Johnson W, Balakrishna N, Griffiths PL. Modeling physical growth using mixed effects models. Am J Phys Anthropol 2013;150:58-67.

2. Graversen L, Howe LD, Sorensen TI, et al. Body mass index trajectories from 2 to 18 years - exploring differences between European cohorts. Pediatr Obes 2017;12:102-9.

3. Royston P, Wright EM. A method for estimating age-specific reference intervals ("normal ranges") based on fractional polynomials and exponential transformation. J R Statist Soc 1998;161:79-101.

4. Veldwijk J, Scholtens S, Hornstra G, et al. Body mass index and cognitive ability of young children. Obes Facts 2011;4:264-9. 



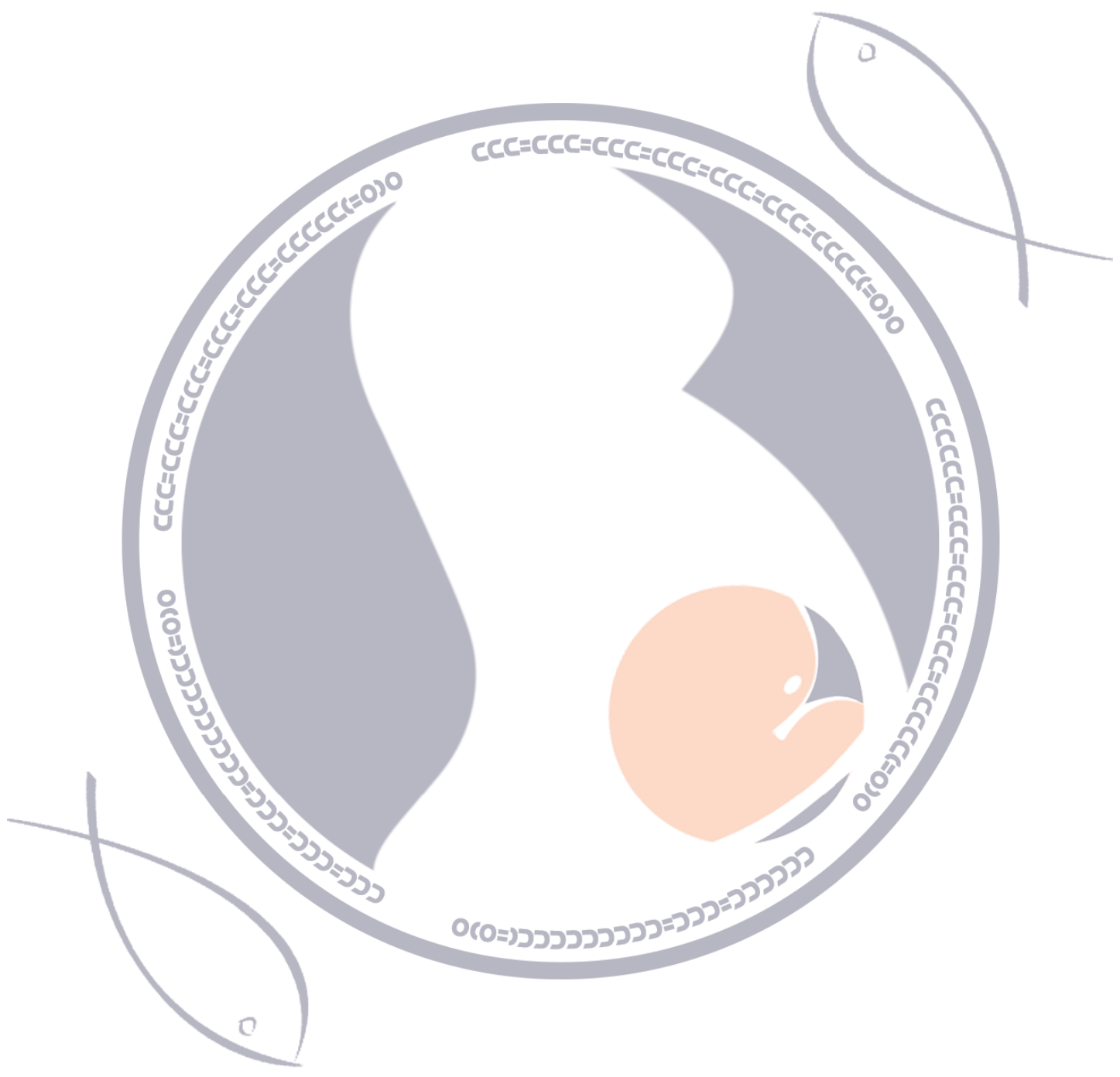




\section{CHAPTER 5}

Polyunsaturated fatty acid status at birth and allergy-related phenotypes in childhood: a pooled analysis of the MEFAB and RHEA birth cohorts

N Stratakis, M Gielen, K Margetaki, RHM de Groot, M Apostolaki,

G Chalkiadaki, M Vafeiadi, V Leventakou, RW Godschalk, M Kogevinas, EG Stephanou, MP Zeegers*, L Chatzi*

${ }^{*}$ shared senior co-authorship

Br J Nutr (in press) 


\section{ABSTRACT}

Background: Lower prenatal exposure to $n-3$ polyunsaturated fatty acids (PUFAs) relative to n-6 PUFAs has been hypothesized to influence allergy development, but evidence remains largely inconsistent.

Objective: To assess whether fetal PUFA exposure is associated with wheeze, asthma, rhinitis, and eczema in mid-childhood.

Methods: In the Dutch MEFAB ( $n=293)$ and Greek RHEA ( $n=213)$ birth cohorts, we measured cord blood phospholipid n-3 and n-6 PUFAs levels, reflecting fetal exposure in late pregnancy. Information on wheeze, asthma, rhinitis, and eczema prevalence at age 6-7 years was collected using validated questionnaires. Multivariable generalized linear regression models were used to examine associations between PUFAs and child outcomes.

Results: In pooled analyses, higher levels of the n-3 long-chain eicosapentaenoic acid (EPA) and docosahexaenoic acid (DHA) and a higher total n-3:n-6 PUFA ratio were associated with lower risk of current wheeze $(\mathrm{RR}=0.61$ [95\% CI 0.45 0.82] per 1-SD increase in EPA+DHA, and 0.54 [95\% CI 0.39-0.75] per 1-unit increase in the $n-3: n-6$ ratio $)$ and reduced asthma risk $(R R=0.50$ [95\% CI 0.310.79] for EPA+DHA and 0.43 [95\% CI 0.26-0.70] for the n-3:n-6 ratio). No associations were observed for other allergy-related phenotypes. The results were similar across cohorts and remained robust in sensitivity analyses.

Conclusions: We found that higher EPA and DHA levels and a higher n-3:n-6 fatty acid ratio in cord blood were associated with lower risk of child wheeze and asthma. Our findings suggest that dietary interventions resulting in a marked increase in the n-3:n-6 PUFA ratio, and mainly in n-3 long-chain PUFA intake in late gestation may reduce the risk of asthma symptoms in mid-childhood. 


\section{INTRODUCTION}

Childhood asthma and other allergy-related diseases pose a substantial health burden in developed countries. ${ }^{1}$ Fetal life is a critical time period of susceptibility during which a nutritional stressor can affect the development and maturation of respiratory and immune systems, and thus, have irreversible influences on the development of allergy. ${ }^{2}$ In this context, it has been hypothesized that a lower intake of n-3 relative to n-6 PUFAs might play a role in the etiology of allergyrelated diseases. ${ }^{3}$ PUFAs of $n-6$ and $n-3$ families can be readily transferred across the placenta, ${ }^{4}$ and have been shown in animal and in vitro studies to exert immunomodulatory effects. ${ }^{5,6}$ Specifically, n-6 PUFAs have been suggested to promote inflammation and increase allergic disease activity, while n-3 PUFAs seem to have an opposite effect. ${ }^{5,6}$

However, reports from human studies do not conclusively support that an increase in n-3 PUFA status, a decrease in n-6 PUFA status, or both in the prenatal period is an effective strategy to prevent allergy-related symptoms in childhood. A 2016 systematic review and meta-analysis of prospective cohort studies assessing maternal fish or n-3 long-chain PUFA intake during pregnancy and of trials assessing fish oil supplementation reported that the hypothesis linking maternal n-3 PUFA intake to childhood allergic disease cannot unequivocally be confirmed or rejected. ${ }^{7}$ Two subsequent trials on fish oil supplementation also showed discrepant results, with reports of null ${ }^{8}$ or beneficial (ie, lower incidence) effects. ${ }^{9}$ Likewise, findings from studies assessing prenatal PUFA biomarker concentrations, which constitute accurate surrogates of fetal exposure, ${ }^{10}$ have produced mixed results. The British Southampton Women's Survey and ALSPAC studies reported lower rates of childhood wheeze symptoms with higher prenatal n-3 PUFA concentration ${ }^{11}$ or a lower n-6:n-3 fatty acid ratio. ${ }^{12}$ In contrast, the Dutch Generation R study showed maternal concentration of n-6 PUFAs, but not of n-3 PUFA levels, to be associated with decreased asthma risk in childhood, ${ }^{13}$ while other studies in the Netherlands, Germany, and Singapore found no associations between any PUFAs and child asthma or wheeze symptoms. ${ }^{14-16} \mathrm{~A}$ lower risk of childhood eczema was shown with increasing cord blood n-3 PUFA concentration in the Spanish INMA cohort. ${ }^{17}$ The Generation $\mathrm{R}$ found a higher eczema risk with higher maternal n- 6 PUFA concentration, ${ }^{13}$ whereas the Dutch KOALA study reported a lower risk with increasing n-6:n-3 fatty acid ratio. ${ }^{14}$ Other studies showed weak or null associations of prenatal PUFA concentrations with rates of childhood eczema. ${ }^{12,15,16}$ Only a few studies have specifically examined childhood rhinitis and found no associations with prenatal PUFA concentrations. ${ }^{14-16}$

Contradictory findings of prior studies may at least partly be explained by heterogeneity in exposure and health outcome definitions, and differences in adjustment 
for confounding variables. Moreover, most of the trials were prone to methodologic limitations, such as selective reporting and absence of blinding of participants or personnel. Given the substantial health burden of allergic diseases, it is important to better characterize the potential role that PUFAs may play in the development of asthma and other allergy-related diseases. Pooled analyses of diverse populations constitute an important step towards more generalizable conclusions. Hence, in the present study, we harmonized and pooled individual data of two cohorts from West and South Europe to assess whether n-3 and n-6 PUFAs concentrations in cord blood phospholipids, reflecting fetal exposure in late pregnancy, are associated with symptoms of wheeze, asthma, rhinitis and eczema in children aged 6-7 years.

\section{METHODS}

\section{Study population}

The MEFAB (Maastricht Essential Fatty Acid Birth, www.mefab.org) cohort $^{18}$ enrolled 1334 pregnant women at the time of their first antenatal visit in three maternity clinics in the province of Limburg, the Netherlands, between 1989 and 1995. Inclusion criteria for participation in the study were: gestational age $<16$ weeks, diastolic blood pressure $<90 \mathrm{mmHg}$, and no cardiovascular, neurological, renal, or metabolic disorder. A total of 1203 women with singleton pregnancies were followed till delivery, and 1008 them provided umbilical cord blood samples. Between 1997 and 2000, when children were seven years of age (mean age 7.3 (SD 0.3) years) we conducted a follow-up evaluation; eligibility criteria included childbirth date before 1994, singleton pregnancy, and availability of cord blood fatty acid measurements. Among 750 eligible children, 306 participated in the follow-up study, during which information on allergy-disease symptoms was obtained for 293 of them. A flow chart of the MEFAB participants is shown in Supplementary Figure S5.1.

The RHEA (Mother-Child) Study in Crete (Greece, www.rhea.gr) ${ }^{19}$ enrolled pregnant women during 2007-2008 at the time of the first major ultrasound examination at 10-13 weeks of gestation if they were: resident at the prefecture of Heraklion, $>16$ years of ageand without communication handicaps. During the study period 1610 pregnant women agreed to participate and 1363 singleton pregnancies were followed-up until delivery. A random subgroup of 500 umbilical cord blood samples was essayed for fatty acid levels in RHEA. For the child's follow-up, the mothers were contacted at 9 months (n 1223), 4 years (n 873), and 6 years of age (n 608); sample sizes correspond to singleton children. In the present analysis, we included 228 children aged 9 months (mean age 9.1 (SD 1.9) months), 222 aged 4 years (4.2 (0.2) years), and 213 aged 6 years (6.6 (0.3) years) 
with available information on fatty acid exposure and allergy-related symptoms. A flow chart of the RHEA participants is shown in Supplementary Figure S5.2. In both cohorts, participants' parents or legal guardians provided written informed consent. The study was conducted according to the guidelines laid down in the Declaration of Helsinki. All procedures involving human subjects were approved by the local authorized institutional review boards (Medical Ethics Committee of the University Hospital Maastricht and the University of Maastricht, the Netherlands, for the MEFAB cohort, and the review board of the University Hospital in Heraklion, Crete, Greece for the RHEA cohort).

\section{Polyunsaturated fatty acid analysis}

In the MEFAB birth cohort, blood samples from the umbilical vein of infants were collected immediately after delivery in EDTA-containing tubes. Plasma was separated from blood cells by centrifugation and stored in aliquots at $-80^{\circ} \mathrm{C}$ until analysis. Methods of fatty acid analysis and the phospholipid PUFAs identified in MEFAB have been described in detail elsewhere ${ }^{20}$.

In the RHEA birth cohort, umbilical cord blood samples were collected at delivery in $10-\mathrm{mL}$ BD gel separator vacutainers. Serum was separated within $2 \mathrm{~h}$ after collection using centrifugation and stored in $0.5-\mathrm{mL}$ aliquots at $-80^{\circ} \mathrm{C}$ until assayed. The analysis of phospholipid fatty acids, including PUFAs, was conducted in the Environmental Chemical Processes Laboratory (University of Crete, Greece) following a standard methodology, as described by Glaser et al. ${ }^{21}$ Briefly, a fatty acid methyl ester (FAME) sample was obtained using Direct In Situ Transesterification. The individual FAMEs were quantified by gas chromatography (GC) with flame ionization detection. GC analysis was carried out on a Shimadzu-2010 chromatograph equipped with AOC-20i auto-injector; Shimadzu Europe, Duisburg, Germany. Separation of serum phospholipid FAMEs was performed on a SP-2560 fused silica capillary column $(100 \mathrm{~m} \times 0.25 \mathrm{~mm}$ i.d., $0.2 \mu \mathrm{m}$ film thickness; Supelco, Bellefonte, PA, USA) using helium as the carrier gas at a constant flow of $1.1 \mathrm{ml} / \mathrm{min}$. The injector port was maintained at $250^{\circ} \mathrm{C}$ and $1.6 \mu \mathrm{L}$ sample volumes were injected at a split ratio of $1: 20$. The $\mathrm{GC}$ oven initial temperature was $130^{\circ} \mathrm{C}$; then increased at a rate of $4^{\circ} \mathrm{C} / \mathrm{min}$ to $170^{\circ} \mathrm{C}$ and then at a rate of $2^{\circ} \mathrm{C} /$ min to $210^{\circ} \mathrm{C}$ and retained for $20 \mathrm{~min}$. Detector temperature was kept at $260^{\circ} \mathrm{C}$. Individual FAME peaks were identified by comparison of their retention times with SUPELCO 37 Component FAME mix; Supelco Bellefonte, PA, USA. PUFA composition of plasma phospholipids is similar to that of serum phospholipids, and slight differences in the analytical methods used for fatty acid analysis in serum and plasma do not appear to affect the way that PUFA composition can be interpreted. ${ }^{22,23}$ 
For the present analysis, our primary exposures of interest were the dietary essential n-3 PUFA precursor, $\alpha$-linolenic acid (ALA, C18:3n-3), the sum of its major biologically active metabolic products eicosapentaenoic acid (EPA, C20:5n-3) and docosahexaenoic acid (DHA, C22:6n-3), ${ }^{24}$ and the sum of total n-3 fatty acids assayed in each cohort; the dietary essential n-6 PUFA precursor, linoleic acid (LA, C18:2n-6), its major metabolic product arachidonic acid (AA, C20:4n-6), ${ }^{25}$ and the sum of total n-6 fatty acids assayed; and the total n-3:n-6 PUFA ratio.

Secondary exposures of interest were intermediate metabolic products in the biosynthetic pathway of PUFAs that have been previously associated with pregnancy and child allergy-related outcomes including: the n-3 docosapentaenoic acid (DPA, C22:5n-3), and the n-6 $\gamma$-linolenic acid (GLA, C18:3n-6), dihomo- $\gamma$ linolenic acid (DGLA, C20:3n-6), and osbond acid (C22:5n-6). ${ }^{13,26,27}$ Individual fatty acid measurements were expressed as weight percentage of total fatty acids present in the chromatogram (wt $\%)$.

\section{Childhood allergy-related outcomes}

In both cohorts, information on wheeze, asthma, rhinitis, and eczema occurrence was obtained by questionnaires adapted from the International Study on Asthma and Allergy in Childhood (ISAAC) ${ }^{28}$ We defined current wheeze as the presence of any episode of wheezing or whistling in the chest in the past 12 months. Asthma ever was defined as ever-reported doctor diagnosis of asthma, while current asthma was defined as a positive answer to both ever-reported diagnosis of asthma and presence of wheezing or whistling in the chest in the past 12 months. ${ }^{29}$ We defined current rhinitis as presence of sneezing or a runny or blocked nose in the last 12 months without common cold or flu. ${ }^{30}$ We defined current eczema as presence of an itchy rash in the last 12 months that affected any of the following places: the folds of the elbows, behind the knees, in front of the ankles, under the buttocks, or around the neck, ears or eyes. ${ }^{31}$ For the MEFAB cohort, we used available information on allergy-related outcomes at the age of 7 years. For the RHEA cohort, we used information on wheeze symptoms at three time points (at 9 months, 4 years, and 6 years), and information on asthma, rhinitis, and eczema at 6 years. Although in RHEA, there was available information on asthma, rhinitis and eczema at 4 years of age, we did not include these outcomes in the current analysis owning to very small numbers.

\section{Covariates}

We defined important confounders or effect modifiers as similarly as possible among the cohorts. Information on maternal weight and height at study entry which were used to calculate maternal body mass index (BMI; in $\mathrm{kg} / \mathrm{m}^{2}$ ), gesta- 
tional weight gain from study entry till birth (in $\mathrm{kg}$ ), maternal smoking during pregnancy (yes/no), maternal age at delivery (in years), delivery type (vaginal delivery or cesarean section), parity (primiparous or multiparous), birth weight (in g), gestational age (in weeks), and child sex (male or female) was collected by means of self-administered questionnaires or hospital records. Information on breastfeeding (months), number of siblings $(0,1,2$, or $\geq 3)$ parental education (cohort-specific definitions of low, medium, or high according to the highest completed education level of either parent), and history of parent atopy (presence of asthma, rhinitis or eczema to either one or both parents; yes/no) was obtained through self-administered questionnaires. At 6-7 years of age, child weight and height were measured according to standard procedures in both cohorts, and BMI (in $\mathrm{kg} / \mathrm{m}^{2}$ ) was calculated. In RHEA, we had available information on Mediterranean diet adherence in pregnancy calculated with an a priori defined score using a validated food frequency questionnaire. ${ }^{32}$ In MEFAB, we also collected fasting child blood samples at the age of outcome assessment and measured plasma levels of PUFA with standard procedures as described previously. ${ }^{33}$

\section{Statistical analysis}

Characteristics of the study population were determined using descriptive analysis. Differences in the baseline characteristics between children included the present analysis and those excluded were studied using Pearson's $\chi^{2}$ test for categorical variables and Student's t-test for continuous ones.

We calculated standardized $\mathrm{z}$ scores for concentrations of all PUFAs in each cohort to enable the comparison and pooling of cohort-specific effect estimates. We used multivariable generalized linear models for binary outcomes (with log link, Poisson distribution, and robust variance estimator $)^{34}$ to estimate relative risks (RRs) and 95\% confidence intervals (CIs) for the associations of each PUFA exposure with the outcomes of interest. We detected no departures from linearity in the exposure-outcome associations using a Wald test with the STATA command nlcheck. ${ }^{35}$ We conducted cohort-specific analyses. We also did pooled analyses by including a cohort indicator variable in the models and tested for heterogeneity in the associations between the two cohorts by examining the interaction between PUFA concentrations and cohort.

Following a Directed Acyclic Graph approach (Supplementary Figure S5.3), we selected the following variables for model adjustment: maternal age, maternal BMI at study entry, gestational weight gain, maternal smoking during pregnancy, parental education, parity, parent atopy, child sex and age at outcome assessment. We did not include birth weight or gestational age in our main models, as they 
might be in the intermediate pathway between prenatal PUFA levels and child allergy-related outcomes.

To further assess our research hypothesis, we conducted additional analyses utilizing different levels of available information within each cohort. First, we assessed the extent to which child plasma PUFA levels measured at the age of outcome assessment could explain the observed associations by further adjusting for them in the models for MEFAB and evaluating if they changed the effect estimates. Second, we conducted further adjustment for Mediterranean diet adherence in pregnancy in the RHEA models. Third, we examined associations between the primary PUFAs of interest and current wheeze symptoms at 9 months and 4 years of age in the RHEA birth cohort.

To increase sample size (precision) and reduce bias due to missing data, we imputed missing covariate data. Using multiple imputations with chained equations (MICE), we generated 50 imputed data sets for each cohort. ${ }^{36,37}$ The imputation model included exposures, outcomes, confounders, as well as additional auxiliary variables such as maternal origin, mode of delivery, pregnancy outcomes, breastfeeding duration, sibship size, and child BMI. In analytic models, we combined estimates from the imputed data sets with the use of Rubin's rules. ${ }^{36}$

Sensitivity analyses were performed to assess the robustness of our results. We repeated the analyses by including only those participants with no missing covariate data (complete case analysis). We made further adjustment for BMI at the age of outcome assessment to assess the extent to which child adiposity could influence the observed associations. We also repeated the analyses after including birth weight and gestational age in the multivariable models. Finally, we examined effect measure modification by child sex (boy vs girl) ${ }^{38}$ and parent atopy (yes vs no) $)^{39,40}$ in the associations of the total n-3:n-6 PUFA ratio with child outcomes by assessing both multiplicative and additive interaction measures between the potential effect modifier (one at a time) and the exposure. ${ }^{41,42}$ Statistical significance for effect modification was defined as $\mathrm{P}<0.10$.

Our study including 506 participants had $80 \%$ power at a $5 \%$ significance level to detect an effect size of as small as $48 \%$ for risk estimates of current asthma (the outcome with the lowest prevalence in the pooled dataset; 4.6\%) per SD increase in fatty acid concentrations, which is similar to those reported in previous studies. ${ }^{13-15}$ Power analysis was conducted using GPower version 3.1. ${ }^{43}$ All other analyses were conducted with STATA version 13.0 (StataCorp). 


\section{RESULTS}

\section{Participant characteristics}

In the pooled dataset, mothers had a mean (SD) age at delivery of 29.8 (4.5) years. Thirty-seven per cent of all parents included in this analysis had a high education level. The total ratio of boys to girls was 1.29. Participants' characteristics of the separate cohorts are presented in Table 5.1. In nonresponse analysis, we saw that compared with children without follow-up measurements, those with follow-up data had mothers who were more likely to be older at delivery and multiparous in MEFAB, while in RHEA, children with follow-up data were more likely to be males (Supplementary Table S5.1).

Table 5.1. Parental and child characteristics in MEFAB and RHEA birth cohorts

\begin{tabular}{|c|c|c|}
\hline & MEFAB (n 293) & RHEA (n 213) \\
\hline & Mean (SD) or n (\%) & Mean $(\mathrm{SD})$ or $\mathrm{n}(\%)$ \\
\hline \multicolumn{3}{|l|}{ Parental characteristics } \\
\hline Maternal age at birth (years) & $29.8(4.2)$ & $29.8(4.8)$ \\
\hline Maternal BMI at study entry $\left(\mathrm{kg} / \mathrm{m}^{2}\right)$ & $23.6(3.9)$ & $25.7(5.8)$ \\
\hline Missing (\%) & $21(7.2)$ & $14(6.6)$ \\
\hline Gestational weight gain (kg) & $11.1(4.0)$ & $13.8(6.1)$ \\
\hline Missing (\%) & $9(3.1)$ & $32(15.2)$ \\
\hline Maternal smoking in pregnancy, yes (\%) & $67(22.9)$ & $29(13.6)$ \\
\hline Missing (\%) & $5(1.7)$ & $2(0.9)$ \\
\hline \multicolumn{3}{|l|}{ Parental education (\%) } \\
\hline Low & $69(23.6)$ & $18(8.5)$ \\
\hline Middle & $121(41.3)$ & $103(48.4)$ \\
\hline High & $96(32.8)$ & $91(42.7)$ \\
\hline Missing & $7(2.4)$ & $1(0.5)$ \\
\hline Parity, primiparous (\%) & $206(70.3)$ & $97(45.5)$ \\
\hline Parental atopy, yes (\%) & $128(43.7)$ & $95(44.6)$ \\
\hline Missing & $7(2.4)$ & $13(6.1)$ \\
\hline \multicolumn{3}{|l|}{ Child characteristics } \\
\hline Sex, female (\%) & $131(44.7)$ & $90(42.3)$ \\
\hline Birth weight (grams) & $3296(516)$ & $3191(434)$ \\
\hline Gestational age (weeks) & $39.9(1.6)$ & $38.2(1.5)$ \\
\hline Breastfeeding duration (months) & $2.2(3.4)$ & $4.0(4.1)$ \\
\hline Age at follow-up (years) & $7.3(0.3)$ & $6.6(0.3)$ \\
\hline Body mass index at follow-up $\left(\mathrm{kg} / \mathrm{m}^{2}\right)$ & $15.6(1.8)$ & $17.1(2.6)$ \\
\hline Missing (\%) & $5(1.7)$ & - \\
\hline
\end{tabular}


Table 5.1. Parental and child characteristics in MEFAB and RHEA birth cohorts (continued)

\begin{tabular}{lcc}
\hline & MEFAB (n 293) & RHEA (n 213) \\
\cline { 2 - 3 } & Mean (SD) or n (\%) & Mean (SD) or n (\%) \\
\hline Child outcomes at 6-7 years & & $21(9.9)$ \\
Current wheeze $^{\mathrm{a}}$, yes (\%) & $21(7.2)$ & $9(4.2)$ \\
Current asthma $^{\mathrm{b}}$, yes (\%) & $14(4.8)$ & $23(10.8)$ \\
Asthma ever $^{\mathrm{c}}$, yes (\%) & $25(8.5)$ & $17(8.0)$ \\
Current rhinitis $^{\mathrm{d}}$, yes (\%) & $51(17.4)$ & $20(9.4)$ \\
Current eczema $^{\mathrm{e}}$, yes (\%) & $42(14.3)$ & \\
\hline
\end{tabular}

${ }^{\text {a }}$ Current wheeze was defined as presence of wheezing or whistling in the chest in the last 12 months.

${ }^{\mathrm{b}}$ Current asthma was defined as a positive answer to both ever-reported doctor diagnosis of asthma and presence of wheezing or whistling in the chest in the last 12 months.

${ }^{c}$ Asthma ever was defined as ever-reported doctor diagnosis of asthma.

${ }^{\mathrm{d}}$ Current rhinitis was defined as presence of sneezing or a runny or blocked nose in the last 12 months without common cold or flu.

${ }^{\mathrm{e}}$ Current eczema was defined presence of an itchy rash in the last 12 months that affected any of the following places: the folds of the elbows, behind the knees, in front of the ankles, under the buttocks, or around the neck, ears or eyes

Table 5.2 shows the distribution of the cord blood phospholipid PUFAs measured in each cohort. Mean (SD) concentrations of $n-3$ and n-6 PUFAs in MEFAB were 6.89 (1.59) and $32.16(1.69) \mathrm{wt} \%$, respectively; the corresponding values for RHEA were 5.38 (1.12) and $32.06(2.35) \mathrm{wt} \%$.

Table 5.2. Distribution of cord blood phospholipid PUFA (polyunsaturated fatty acid) levels in MEFAB and RHEA birth cohorts

\begin{tabular}{lcc}
\hline & MEFAB (n 293) & RHEA (n 213) \\
\cline { 2 - 3 } & Mean (SD) & Mean (SD) \\
\hline Total n-3 PUFAs (wt \%) & $6.89(1.59)$ & $5.38(1.12)$ \\
ALA (wt \%) & $0.05(0.11)$ & $0.08(0.05)$ \\
DPA (wt \%) & $0.45(0.17)$ & - \\
EPA + DHA (wt \%) & $6.29(1.44)$ & $5.28(1.10)$ \\
Total n-6 PUFAs (wt \%) & $32.16(1.69)$ & $32.06(2.35)$ \\
LA (wt \%) & $7.70(1.30)$ & $10.41(1.95)$ \\
GLA (wt \%) & $0.05(0.04)$ & $0.37(0.11)$ \\
DGLA (wt \%) & $5.14(0.89)$ & $3.91(0.71)$ \\
AA (wt \%) & $16.63(1.6)$ & $17.04(2.42)$ \\
Osbond acid (wt \%) & $0.83(0.27)$ & - \\
Total n-3:n-6 PUFA ratio & $0.22(0.06)$ & $0.17(0.04)$ \\
\hline
\end{tabular}

AA, arachidonic acid (C20:4n-6); ALA, a-Linolenic acid (C18:3n-3); DGLA, dihomo- $\gamma$-linolenic acid (C20:3n-6); DHA, docosahexaenoic acid (C22:6n-3); DPA, docosapentaenoic acid (C22:5n-3); EPA, eicosapentaenoic acid (C20:5n-3); DHA, docosahexaenoic acid (C22:6n-3); GLA, $\gamma$-Linolenic acid (C18:3n-6); LA, linoleic acid (C18:2n-6); Osbond acid, C22:5n-6; total n-3 (n-6) PUFAs, the sum of $\mathrm{n}-3$ (n-6) PUFAs present in the chromatogram; wt \%, weight percentage of total fatty acids measured. 


\section{Primary/pooled analyses}

Interaction $\mathrm{P}$ values between PUFA concentrations and cohort were indicative of no significant heterogeneity between cohorts for most exposure-outcome associations, although the magnitude and precision of some effect estimates differed slightly (Table 5.3 and Supplementary Tables S5.2 and S5.3).

In pooled analyses, we found that higher total n-3 PUFA levels were associated with a lower risk of current wheeze, while an inverse association was observed for total n-6 PUFAs (pooled RR= 0.60 [95\% CI 0.45-0.81], and 1.54 [95\% CI 1.09-2.17] per SD increase in n-3 and n-6 PUFAs, respectively). Accordingly, a higher ratio of total n-3 to $\mathrm{n}-6$ fatty acids at birth was associated with a lower risk of current wheeze (pooled $\mathrm{RR}=0.54$ [95\% CI 0.39-0.75] per unit increase) (Table 5.3). When we examined current asthma, we found a lower risk with higher $n-3$ PUFA levels (pooled $\mathrm{RR}=0.49$ [95\% CI 0.30-0.75]) and a higher $\mathrm{n}-3: \mathrm{n}-6$ fatty acid ratio (pooled $\mathrm{RR}=0.43$ [95\% CI 0.26-0.70]) (Table 5.3). Associations for ever-reported doctor diagnosis of asthma were in the same direction and magnitude (Supplementary Table S5.2). No associations were found for total n-6 PUFA, total n-3 PUFA levels, and the n-3:n-6 ratio with current rhinitis and eczema symptoms at 6-7 years (Supplementary Table S5.3).

In individual fatty acid analyses, we found EPA+DHA levels to be associated with decreased risk of both current wheeze and current asthma (pooled RR= $0.61[95 \%$ CI $0.45-0.82$ ], and 0.50 [95\% CI 0.31-0.79] per 1-SD increase in EPA+DHA, respectively) (Table 5.3). Similarly, a protective association was observed between $\mathrm{EPA}+\mathrm{DHA}$ and risk of ever asthma (Supplementary Table S5.2). No associations with any of the outcomes examined were found for the n-3 ALA, and the n-6 LA and AA in the pooled dataset (Table 3 and online supplementary Tables S2 and S3). Results from the crude models adjusted only for age and sex did not materially differ from those of the main adjusted models (Supplementary Table S5.4).

\section{Secondary analyses}

In secondary analyses, we found no associations of the n-3 DPA and n-6 GLA, DGLA and osbond acid with allergy-related outcomes (Supplementary Tables S5.5 and S5.6). Additionally, inclusion of child plasma PUFA levels at the age of outcome assessment in MEFAB models did not modify the direction and size of the effect estimates (data not shown). In RHEA, results remained similar when we further adjusted for maternal Mediterranean diet adherence in pregnancy (data not shown). We found no associations with current wheeze symptoms in the other time points in RHEA, though a trend towards a reduction in wheeze risk with higher levels of n-3 PUFAs, and especially EPA+DHA, and a higher n-3:n-6 ratio was observed with increasing child age from 9 months to 6 years (Figure 5.1 and Supplementary Table S5.7). 


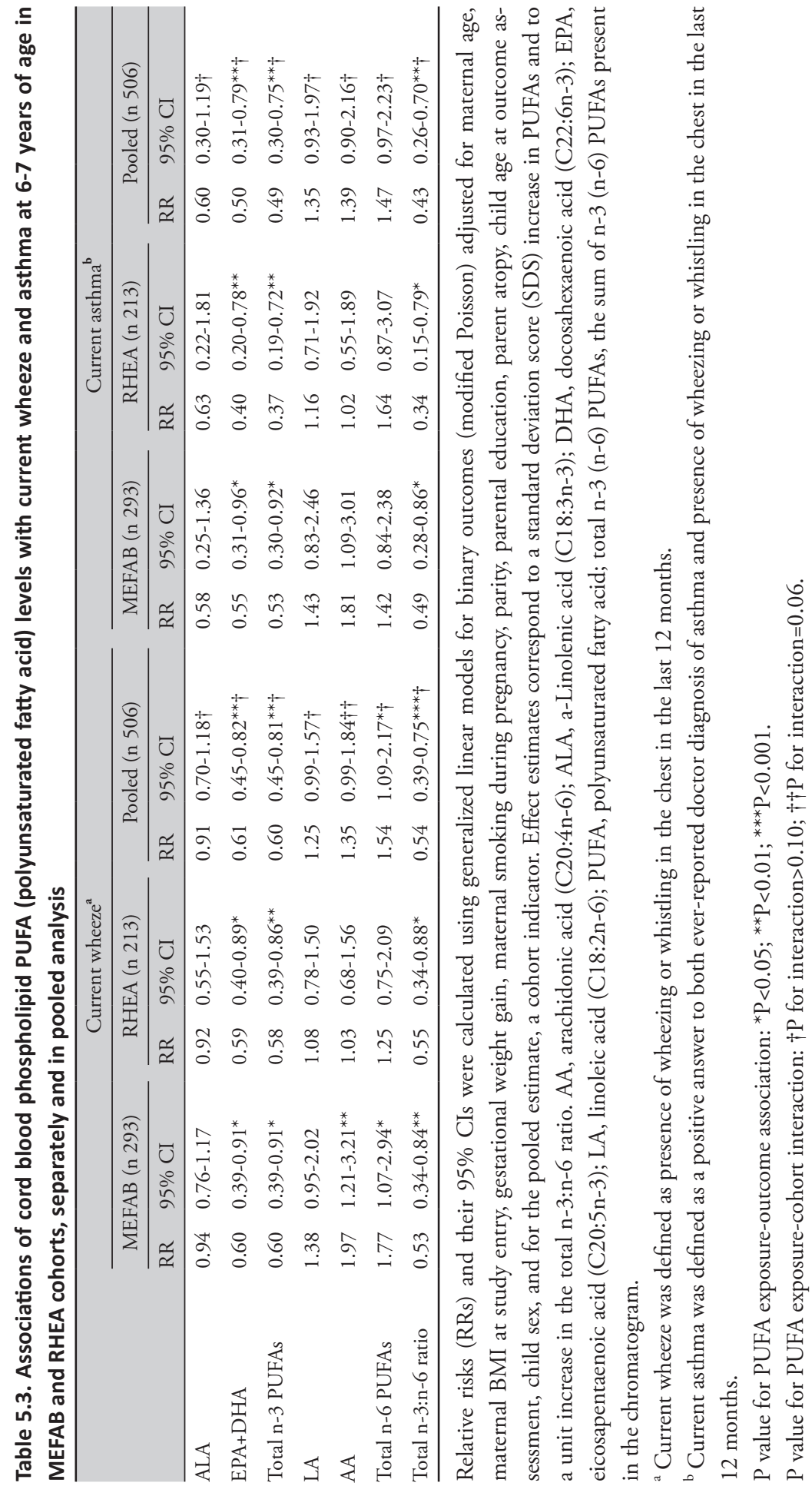




\section{Sensitivity analyses}

The effect estimates did not materially change in complete case analysis or when additional adjustment was made for child BMI (data not shown). Effects estimates also remained similar after including birthweight and gestational age in multivariable models (Supplementary Table S5.8). We found no evidence of effect measure modification by parent atopy for any of the outcomes of interest (Supplementary Table S5.9). There was some evidence of effect measure modification by female sex on the multiplicative scale for the association between the n-3:n-6 PUFA ratio and current rhinitis symptoms (pooled $\mathrm{RR}=0.57$ [95\% CI 0.32-1.01]; P=0.06) (Supplementary Table S5.10). However, no effect modification by child sex on either multiplicative or additive scale was observed for any of the other outcomes of interest (Supplementary Table S5.10).
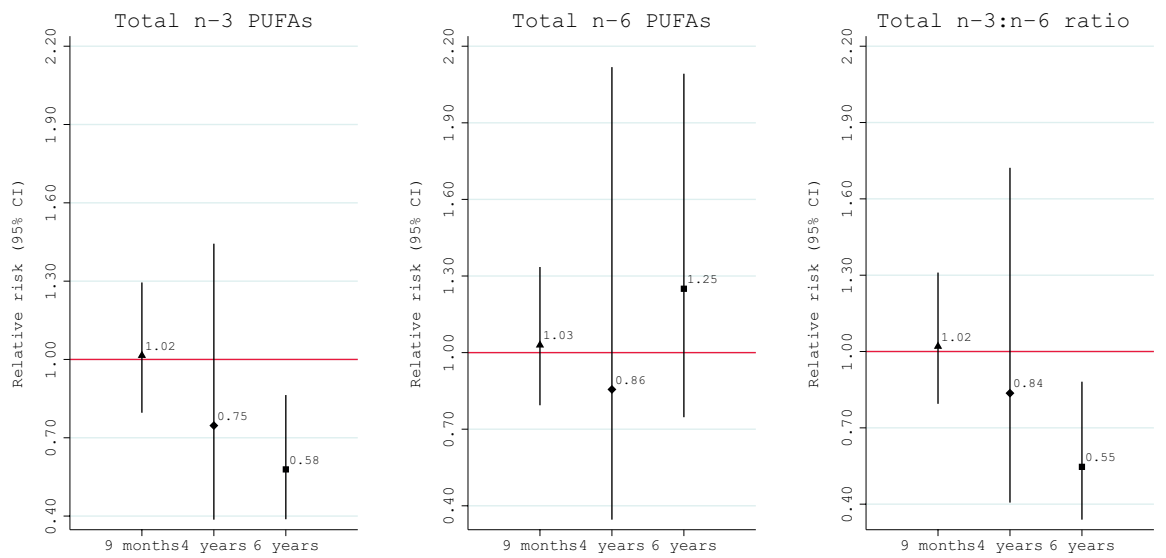

Figure 5.1. Associations of cord blood phospholipid PUFA (polyunsaturated fatty acid) levels with current wheeze symptoms at 9 months, 4 years, and 6 years of age in the RHEA cohort. Values are relative risks and their $\mathbf{9 5 \%} \mathrm{Cls}$ calculated using generalized linear models for binary outcomes (modified Poisson). Models were adjusted for maternal age, maternal BMI at study entry, gestational weight gain, maternal smoking during pregnancy, parity, parental education, parent atopy, child age at outcome assessment, and sex. Effect estimates correspond to a standard deviation score (SDS) increase in total n-3 (n-6) PUFAs and to a unit increase in the total n-3:n-6 ratio. Current wheeze was defined as presence of wheezing or whistling in the chest since birth at 9 months of age $(n / N=62 / 228)$ and in the last 12 months at 4 years $(n / N=10 / 222)$ and 6 years of age $(n / N=21 / 213)$. PUFA, polyunsaturated fatty acid; total $n-3(n-6)$ PUFAs, the sum of $n-3(n-6)$ PUFAs present in the chromatogram. Shapes represent the effect estimate of each time point; vertical lines denote $95 \% \mathrm{Cls}$. 


\section{DISCUSSION}

In this analysis of prospective data of two birth cohorts from West and South Europe with different sociodemographic characteristics, we found that higher concentrations of n-3 PUFAs, and especially of EPA and DHA, in cord blood were associated with reduced risk of current wheeze symptoms and asthma at 6-7 years of age. Higher cord blood n-6 PUFA concentrations were associated with a higher risk of current wheeze. We also observed a reduced risk of both current wheeze and asthma with increasing n-3 to n-6 fatty acid ratio. The results were fairly homogeneous across the cohorts after controlling for a variety of confounders, and remained robust in the pooled and sensitivity analyses.

We measured cord blood phospholipid PUFA concentrations, which reflect recent maternal dietary intake at a time frame of $2-4$ weeks, ${ }^{22}$ as well as the level of fetal demand, and selectivity and efficiency of placental transfer. ${ }^{4}$ Hence, they constitute good surrogates of fetal exposure in late pregnancy. The timing and biomarker used for assessing fetal exposure in our study are of high relevance as they coincide with the period during which there is an exponential increase in the rate of fetal fat accretion. ${ }^{4}$

Fetal life is a critical time period of susceptibility during which nutritional exposures could have irreversible long-term influences on airway and immune function. ${ }^{44}$ The n-6 and n-3 fatty acids have been proposed to exert immune-regulatory effects through involvement in several pathways. Animal and in vitro studies have shown that n-6 PUFAs, as substrate for eicosanoid synthesis, can promote inflammation, favor T-helper cell type 2-driven immune responses, and enhance the synthesis of immunoglobulin E antibodies. ${ }^{6}$ On the contrary, n-3 PUFAs have been suggested to exert a protective effect by competing with the $n-6$ fatty acids for incorporation into cell membranes and for metabolism by enzymes of eicosanoid synthesis. ${ }^{6}$ Additionally, n-3 long-chain PUFAs have been shown in vitro to alter the expression of inflammatory genes by modifying transcription factor activation, inhibit $\mathrm{T}$ cell signaling through alteration of membrane lipid rafts, as well as to give rise to a family of anti-inflammatory mediators termed resolvins. ${ }^{45}$

Reports from human trials cannot unequivocally confirm or reject a beneficial effect of n-3 EPA and DHA supplementation in fetal life on allergy in childhood. ${ }^{7,8,46}$ Findings from longitudinal cohort studies on prenatal PUFA exposure and childhood asthma or other allergy symptoms have also been mixed, with reports of null, ${ }^{13,15,16}$ beneficial (i.e. lower incidence), ${ }^{11,12,17,47,48}$ or even unfavorable $^{14}$ associations with higher n-3 PUFAs or decreasing n-6 to n-3 fatty acid ratio. Inconsistencies in findings may partly arise from exposure profile heterogeneity (i.e. categorization and timing of exposure), and differences in outcome definition and adjustment for confounding variables. In the present study, we used 
two independent cohorts for replication, and we followed a centralized statistical analysis following a consensus protocol with harmonized information on exposure variables, potential confounders, and child allergy-related symptoms. We found broadly similar associations across the different cohorts, though there was some evidence for between-cohort heterogeneity for the risk estimates of current wheeze in relation to $\mathrm{n}-6 \mathrm{AA}$, for ever asthma in relation to $\mathrm{n}-3 \mathrm{ALA}$, and for risk estimates of the n-6 GLA and DGLA. Such heterogeneity might have occurred due to variability in the analytical methods used for cord blood fatty acid analysis and/ or due to differences in confounder patterns (eg, differences in diet or lifestyle) between participating cohorts.

The EPA+DHA status in the Netherlands and Greece is characterized as very low-to-low, which is similar to most European and US populations following a Westernized diet. ${ }^{23}$ We found that $1 \mathrm{SD}$ increase in cord blood concentrations of n-3 PUFAs, and especially of EPA and DHA, was associated with lower risk for childhood symptoms of wheeze and asthma. An inverse association with childhood wheeze and asthma risk was also observed for the n-3:n-6 PUFA ratio, which was driven to a great extent by $\mathrm{n}-3$ long-chain PUFAs. We did not have information on dietary intake in late pregnancy so as to disentangle the amount of dietary PUFAs needed in order to achieve an SD change in cord blood longchain PUFA concentrations. This change is feasible and likely reflects a significant dietary modification. ${ }^{49}$ Thus, a sizeable effect on wheeze and asthma symptoms could be achieved from dietary interventions leading to a marked increase in the n-3:n-6 PUFA ratio, and mainly in the intake of n-3 long-chain PUFAs in late gestation. Indeed, in trials, a relation between fish-oil supplementation and effect becomes apparent at high doses, which are expected to significantly increase blood PUFA concentrations. In two trials conducted in Denmark, where population background levels of n-3 long-chain PUFAs are higher compared to most European populations, ${ }^{23}$ supplementation with high doses of fish oil in late gestation (providing $2.4 \mathrm{gr}$ EPA+DHA/day from week 24 of pregnancy onwards ${ }^{9}$ or $2.7 \mathrm{gr} /$ day from week 30 of pregnancy onwards $s^{50}$ ) resulted in significant increases ( $>1 \mathrm{SD}$ ) in blood n-3 long-chain PUFA concentrations and lower risk of offspring wheeze and asthma compared to placebo. In terms of safety, high doses of n-3 long-chain PUFAs up to $2.7 \mathrm{gr} /$ day in pregnancy through fish oil supplementation have not been associated with serious adverse effects, such as bleeding complications. ${ }^{51}$

Our findings are partly in line with the initial Black and Sharpe hypothesis suggesting that the balance between n-3 and n-6 PUFAs might have implications in the development of asthma and other allergy-related symptoms. ${ }^{3}$ Despite the unifying hypotheses that have been developed to explain the common co-existence of allergic disease symptoms in a child, ${ }^{52}$ asthma, rhinitis, and eczema represent 
different disease-entities with different underlying mechanisms. ${ }^{53-55}$ In the present study, we observed associations only for symptoms of wheeze and asthma, suggesting a specific effect of prenatal PUFAs on airway development and function. Further observational and experimental studies using spirometry and markers of airway inflammation are needed for replication and to explore the long-term effects of prenatal PUFA exposure.

A recent systematic review highlighted the importance of planning a subgroup analysis by sex in studies assessing the health effects of perinatal PUFAs. ${ }^{56}$ Sexual dimorphism in fatty acid metabolism has been suggested to exist owing to sex differences in rates of $\beta$-oxidation and fat mobilisation, and possible influences of sex hormones on the enzymatic synthesis of long-chain fatty acids. ${ }^{56,57}$ A previous analysis in the Generation $\mathrm{R}$ reported no effect measure modification in the association between maternal PUFA concentrations with childhood atopic diseases by offspring sex. ${ }^{13}$ In our study, we found some evidence for effect measure modification by female sex on the multiplicative scale for the association between the ratio of total n-3 to n-6 PUFAs at birth with rhinitis symptoms in childhood. However, we did not observe any effect modification by sex for the other outcomes of interest. Thus, we treat this result with caution, as we cannot rule out the possibility that the observed effect modification for rhinitis is due to chance.

As in all observational studies, there is the possibility of unmeasured residual confounding. Although, in our analyses, we took into account a variety of maternal and child lifestyle characteristics, including Mediterranean diet adherence during pregnancy $^{32}$ and child biomarker levels of PUFAs, we cannot exclude the possibility that other differences in diet or lifestyle factors might have influenced the observed findings. However, we did not see strong evidence for sociodemographic confounding in our analyses. Similar to most cohort studies, a limitation of the present study is attrition. It is possible that loss to follow-up could have introduced bias. Nevertheless, many sociodemographic characteristics, and the cord blood PUFA concentrations were similar in the study sample and in those excluded. Although assessment of several PUFA exposures and child outcomes might raise concern about multiple testing, in our opinion, an application of Bonferroni-type corrections for multiple comparisons might be inappropriate in this case given that the examined outcomes (eg, wheeze and asthma) are intercorrelated. ${ }^{58}$ Child allergic-disease symptoms were ascertained by symptom-based questionnaires, which could lead to outcome misclassification. However, both participating cohorts used questionnaires adapted from the ISAAC study. ${ }^{28}$ Moreover, to enhance asthma and eczema outcome accuracy, we used composite definitions that require many conditions to be met. 
In this pooled analysis of two cohorts from West and South Europe, we found that high concentrations of n-3 PUFAs, particularly EPA and DHA, and a high $\mathrm{n}-3: \mathrm{n}-6$ ratio in cord blood were associated with reduced risk of current wheeze and asthma in mid-childhood. Our findings suggest that dietary interventions resulting in a marked increase in the dietary n-3:n-6 PUFA ratio, and mainly in the intake of $n-3$ long-chain PUFAs in late gestation are likely to reduce the risk of asthma symptoms in mid-childhood. 


\section{REFERENCES}

1. Asher MI, Montefort S, Bjorksten B, et al. Worldwide time trends in the prevalence of symptoms of asthma, allergic rhinoconjunctivitis, and eczema in childhood: ISAAC Phases One and Three repeat multicountry cross-sectional surveys. Lancet 2006;368:733-43.

2. Devereux G. The increase in the prevalence of asthma and allergy: food for thought. Nat Rev Immunol 2006;6:869-74.

3. Black PN, Sharpe S. Dietary fat and asthma: is there a connection? The European respiratory journal 1997;10:6-12.

4. Haggarty P. Fatty acid supply to the human fetus. Annu Rev Nutr 2010;30:237-55.

5. Prescott SL, Dunstan JA. Prenatal fatty acid status and immune development: the pathways and the evidence. Lipids 2007;42:801-10.

6. Calder PC, Kremmyda LS, Vlachava M, Noakes PS, Miles EA. Is there a role for fatty acids in early life programming of the immune system? Proc Nutr Soc 2010;69:373-80.

7. Best KP, Gold M, Kennedy D, Martin J, Makrides M. Omega-3 long-chain PUFA intake during pregnancy and allergic disease outcomes in the offspring: a systematic review and meta-analysis of observational studies and randomized controlled trials. Am J Clin Nutr 2016;103:128-43.

8. Best KP, Sullivan T, Palmer D, et al. Prenatal Fish Oil Supplementation and Allergy: 6-Year Follow-up of a Randomized Controlled Trial. Pediatrics 2016;137: pii: e20154443.

9. Bisgaard H, Stokholm J, Chawes BL, et al. Fish Oil-Derived Fatty Acids in Pregnancy and Wheeze and Asthma in Offspring. N Engl J Med 2016;375:2530-9.

10. Hornstra G. Essential fatty acids in mothers and their neonates. Am J Clin Nutr 2000;71:1262S-9S.

11. Pike KC, Calder PC, Inskip HM, et al. Maternal Plasma Phosphatidylcholine Fatty Acids and Atopy and Wheeze in the Offspring at Age of 6 Years. Clin Dev Immunol 2012;2012:474613.

12. Newson RB, Shaheen SO, Henderson AJ, Emmett PM, Sherriff A, Calder PC. Umbilical cord and maternal blood red cell fatty acids and early childhood wheezing and eczema. J Allergy Clin Immunol 2004;114:531-7.

13. Rucci E, den Dekker HT, de Jongste JC, et al. Maternal fatty acid levels during pregnancy, childhood lung function and atopic diseases. The Generation R Study. Clin Exp Allergy 2016;46:461-71.

14. Notenboom ML, Mommers M, Jansen EH, Penders J, Thijs C. Maternal fatty acid status in pregnancy and childhood atopic manifestations: KOALA Birth Cohort Study. Clin Exp Allergy 2011;41:407-16.

15. Standl M, Demmelmair H, Koletzko B, Heinrich J. Cord blood LC-PUFA composition and allergic diseases during the first 10 yr. Results from the LISAplus study. Pediatr Allergy Immunol 2014;25:34450 .

16. Yu YM, Chan YH, Calder PC, et al. Maternal PUFA status and offspring allergic diseases up to the age of 18 months. Br J Nutr 2015;113:975-83.

17. Montes R, Chisaguano AM, Castellote AI, Morales E, Sunyer J, Lopez-Sabater MC. Fatty-acid composition of maternal and umbilical cord plasma and early childhood atopic eczema in a Spanish cohort. Eur J Clin Nutr 2013;67:658-63.

18. van der Wurff ISM, de Groot RHM, Stratakis N, Gielen M, Hornstra G, Zeegers M. Maastricht essential fatty acid birth cohort. Lipid Technol 2015;27:59-62.

19. Chatzi L, Plana E, Daraki V, et al. Metabolic syndrome in early pregnancy and risk of preterm birth. Am J Epidemiol 2009;170:829-36.

20. Al MDM, Vanhouwelingen AC, Kester ADM, Hasaart THM, Dejong AEP, Hornstra G. Maternal Essential Fatty-Acid Patterns during Normal-Pregnancy and Their Relationship to the Neonatal Essential Fatty-Acid Status. Br J Nutr 1995;74:55-68. 
21. Glaser C, Demmelmair H, Koletzko B. High-throughput analysis of total plasma fatty acid composition with direct in situ transesterification. PLoS One 2010;5:e12045.

22. Hodson L, Skeaff CM, Fielding BA. Fatty acid composition of adipose tissue and blood in humans and its use as a biomarker of dietary intake. Prog Lipid Res 2008;47:348-80.

23. Stark KD, Van Elswyk ME, Higgins MR, Weatherford CA, Salem N, Jr. Global survey of the omega-3 fatty acids, docosahexaenoic acid and eicosapentaenoic acid in the blood stream of healthy adults. Prog Lipid Res 2016;63:132-52.

24. Calder PC. Mechanisms of Action of (n-3) Fatty Acids. J Nutr 2012;142:592s-9s.

25. Schmitz G, Ecker J. The opposing effects of n-3 and n-6 fatty acids. Prog Lipid Res 2008;47:147-55.

26. Hornstra G. Essential fatty acids during pregnancy. Impact on mother and child. Nestle Nutr Workshop Ser Pediatr Program 2005;55:83-96; discussion -100.

27. de Vries PS, Gielen M, Rizopoulos D, et al. Association between polyunsaturated fatty acid concentrations in maternal plasma phospholipids during pregnancy and offspring adiposity at age 7: the MEFAB cohort. Prostaglandins Leukot Essent Fatty Acids 2014;91:81-5.

28. Asher MI, Keil U, Anderson HR, et al. International Study of Asthma and Allergies in Childhood (ISAAC): rationale and methods. The European respiratory journal 1995;8:483-91.

29. Pearce N, Ait-Khaled N, Beasley R, et al. Worldwide trends in the prevalence of asthma symptoms: phase III of the International Study of Asthma and Allergies in Childhood (ISAAC). Thorax 2007;62:758-66.

30. Bjorksten B, Clayton T, Ellwood P, Stewart A, Strachan D, Group IPIS. Worldwide time trends for symptoms of rhinitis and conjunctivitis: Phase III of the International Study of Asthma and Allergies in Childhood. Pediatr Allergy Immunol 2008;19:110-24.

31. Pinart M, Benet M, Annesi-Maesano I, et al. Comorbidity of eczema, rhinitis, and asthma in IgEsensitised and non-IgE-sensitised children in MeDALL: a population-based cohort study. The Lancet Respiratory medicine 2014;2:131-40.

32. Chatzi L, Torrent M, Romieu I, et al. Mediterranean diet in pregnancy is protective for wheeze and atopy in childhood. Thorax 2008;63:507-13.

33. Rump P, Popp-Snijders C, Heine RJ, Hornstra G. Components of the insulin resistance syndrome in seven-year-old children: relations with birth weight and the polyunsaturated fatty acid content of umbilical cord plasma phospholipids. Diabetologia 2002;45:349-55.

34. Zou G. A modified poisson regression approach to prospective studies with binary data. American journal of epidemiology 2004;159:702-6.

35. Ben J. NLCHECK: Stata module to check linearity assumption after model estimation. Statistical Software Components S456968. Boston College Department of Economics 2008.

36. Rubin DB. Multiple Imputation for Nonresponse in Surveys. Hoboken, NJ: Wiley-Interscience; 2004.

37. White IR, Royston P, Wood AM. Multiple imputation using chained equations: Issues and guidance for practice. Stat Med 2011;30:377-99.

38. Almqvist C, Worm M, Leynaert B, working group of GALENWPG. Impact of gender on asthma in childhood and adolescence: a GA2LEN review. Allergy 2008;63:47-57.

39. Nurmatov U, Nwaru BI, Devereux G, Sheikh A. Confounding and effect modification in studies of diet and childhood asthma and allergies. Allergy 2012;67:1041-59.

40. Duchen K, Bjorksten B. Polyunsaturated n-3 fatty acids and the development of atopic disease. Lipids 2001;36:1033-42.

41. Knol MJ, van der Tweel I, Grobbee DE, Numans ME, Geerlings MI. Estimating interaction on an additive scale between continuous determinants in a logistic regression model. Int $J$ Epidemiol 2007;36:1111-8.

42. VanderWeele TJ, Knol MJ. A Tutorial on Interaction. Epidemiol Methods 2014;3:33-72. 
43. Faul F, Erdfelder E, Buchner A, Lang AG. Statistical power analyses using G*Power 3.1: tests for correlation and regression analyses. Behav Res Methods 2009;41:1149-60.

44. Devereux G. Early life events in asthma-diet. Pediatr Pulmonol 2007;42:663-73.

45. Calder PC, Krauss-Etschmann S, de Jong EC, et al. Early nutrition and immunity - progress and perspectives. Br J Nutr 2006;96:774-90.

46. Ramaswami R, Serhan CN, Levy BD, Makrides M. Fish Oil Supplementation in Pregnancy. N Engl J Med 2016;375:2599-601.

47. Lumia M, Luukkainen P, Tapanainen H, et al. Dietary fatty acid composition during pregnancy and the risk of asthma in the offspring. Pediatr Allergy Immunol 2011;22:827-35.

48. Miyake Y, Sasaki S, Tanaka K, Ohfuji S, Hirota Y. Maternal fat consumption during pregnancy and risk of wheeze and eczema in Japanese infants aged 16-24 months: the Osaka Maternal and Child Health Study. Thorax 2009;64:815-21.

49. Miles EA, Noakes PS, Kremmyda LS, et al. The Salmon in Pregnancy Study: study design, subject characteristics, maternal fish and marine n-3 fatty acid intake, and marine n-3 fatty acid status in maternal and umbilical cord blood. Am J Clin Nutr 2011;94:1986S-92S.

50. Olsen SF, Osterdal ML, Salvig JD, et al. Fish oil intake compared with olive oil intake in late pregnancy and asthma in the offspring: 16 y of registry-based follow-up from a randomized controlled trial. Am J Clin Nutr 2008;88:167-75.

51. Makrides M, Duley L, Olsen SF. Marine oil, and other prostaglandin precursor, supplementation for pregnancy uncomplicated by pre-eclampsia or intrauterine growth restriction. Cochrane Database Syst Rev 2006:CD003402.

52. Spergel JM, Paller AS. Atopic dermatitis and the atopic march. J Allergy Clin Immunol 2003;112:Suppl. 6, S118-S27.

53. Martinez FD, Vercelli D. Asthma. Lancet 2013;382:1360-72.

54. Bieber T. Atopic dermatitis. N Engl J Med 2008;358:1483-94.

55. Skoner DP. Allergic rhinitis: definition, epidemiology, pathophysiology, detection, and diagnosis. J Allergy Clin Immunol 2001;108:Suppl. 1, S2-S8.

56. Decsi T, Kennedy K. Sex-specific differences in essential fatty acid metabolism. Am J Clin Nutr 2011;94:Suppl. 6, S1914-S9.

57. Childs CE, Romeu-Nadal M, Burdge GC, Calder PC. Gender differences in the n-3 fatty acid content of tissues. Proc Nutr Soc 2008;67:19-27.

58. Rothman KJ. No adjustments are needed for multiple comparisons. Epidemiology 1990;1:43-6. 


\section{Supplementary Material of Chapter 5}

Supplementary Table S5.1. Comparison of subject characteristics in MEFAB and RHEA between those included and not included in the main analyses

Supplementary Table S5.2. Associations of primary PUFAs (polyunsaturated fatty acids) of interest with asthma ever at 6-7 years of age in MEFAB and RHEA cohorts, separately and in pooled analysis

Supplementary Table S5.3. Associations of primary PUFAs (polyunsaturated fatty acids) of interest with current rhinitis and eczema at 6-7 years of age in MEFAB and RHEA cohorts, separately and in pooled analysis

Supplementary Table S5.4. Associations (pooled analysis; n 506) of primary PUFAs (polyunsaturated fatty acids) of interest with allergy-related phenotypes at 6-7 years of age after adjusting only for child sex and age

Supplementary Table S5.5. Associations of secondary PUFAs (polyunsaturated fatty acids) of interest with current wheeze and asthma at 6-7 years of age in MEFAB and RHEA cohorts, separately and in pooled analysis

Supplementary Table S5.6. Associations of secondary PUFAs (polyunsaturated fatty acids) of interest with current rhinitis and eczema at 6-7 years of age in MEFAB and RHEA cohorts, separately and in pooled analysis

Supplementary Table S5.7. Associations of primary PUFAs (polyunsaturated fatty acids) of interest with current wheeze symptoms at 9 months and 4 years of age in the RHEA cohort (n 213)

Supplementary Table S5.8. Associations (pooled analysis; n 506) of primary PUFAs (polyunsaturated fatty acids) of interest with allergic disease-related phenotypes at 6-7 years of age after including birth weight and gestational age in multivariable models

Supplementary Table S5.9. Assessment of effect measure modification by parent atopy in the association of the total n-3:n-6 PUFA ratio with current symptoms of asthma, rhinitis, and eczema at 6-7 years in the pooled dataset

Supplementary Table S5.10. Assessment of effect measure modification by sex in the association of the total n-3:n-6 PUFA ratio with current symptoms of asthma, rhinitis, and eczema at 6-7 years in the pooled dataset

Supplementary Figure S5.1. Flow chart of the MEFAB participants 
Supplementary Figure S5.2. Flow chart of the RHEA participants

Supplementary Figure S5.3. Directed acyclic graph for the association of fatty acid status at birth with allergic disease-related symptoms in childhood.

Text accompanying Supplementary Figure S5.3

Supplementary References 


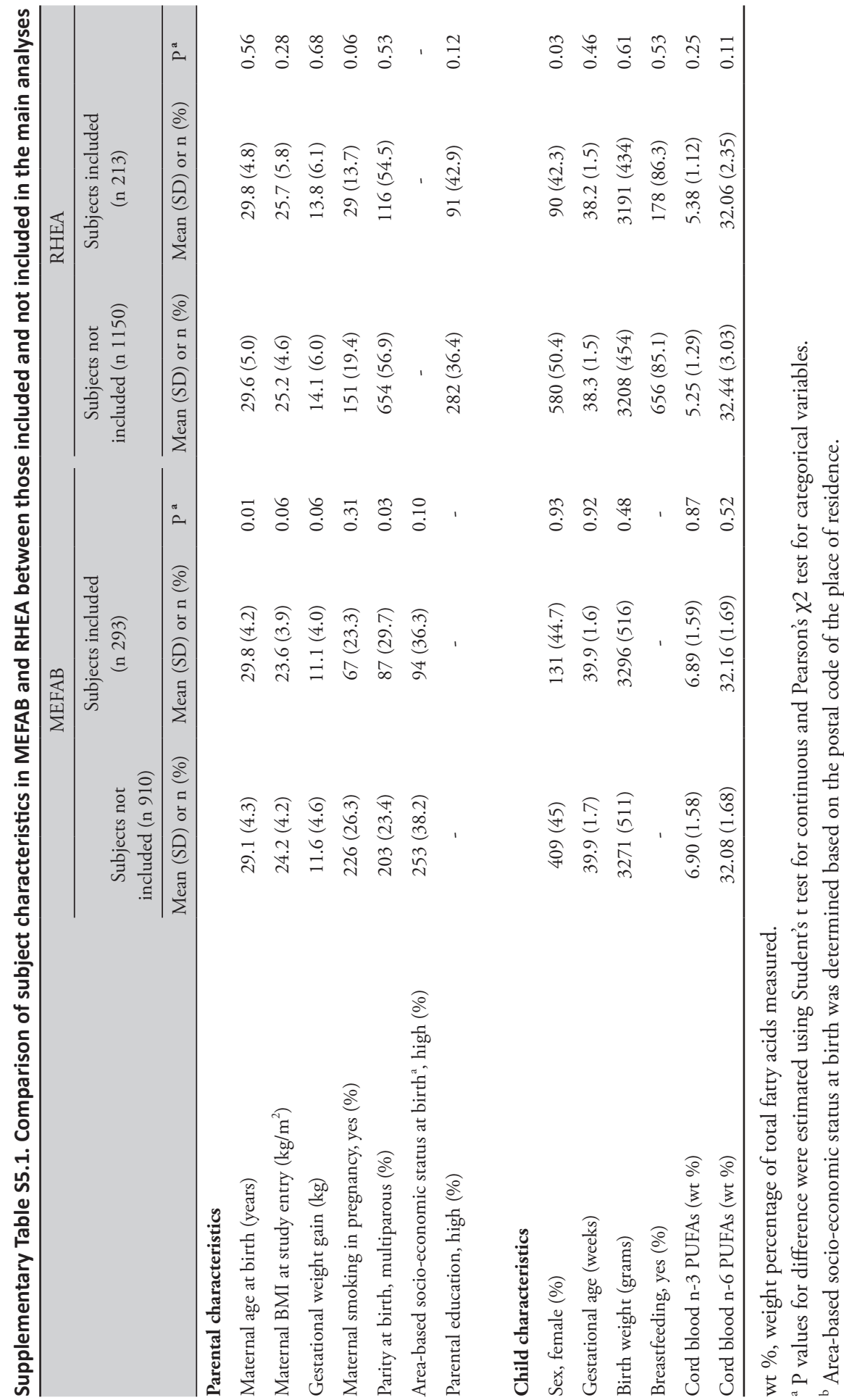


Supplementary Table S5.2. Associations of primary PUFAs (polyunsaturated fatty acids) of interest with asthma ever at 6-7 years of age in MEFAB and RHEA cohorts, separately and in pooled analysis

\begin{tabular}{|c|c|c|c|c|c|c|}
\hline & \multicolumn{6}{|c|}{ Asthma ever ${ }^{a}$} \\
\hline & \multicolumn{2}{|c|}{ MEFAB (n 293) } & \multicolumn{2}{|c|}{ RHEA (n 213) } & \multicolumn{2}{|c|}{ Pooled (n 506) } \\
\hline & $\mathrm{RR}$ & $95 \%$ CI & $\mathrm{RR}$ & $95 \% \mathrm{CI}$ & $\mathrm{RR}$ & $95 \% \mathrm{CI}$ \\
\hline ALA & 0.47 & $0.24-0.93^{*}$ & 1.02 & $0.66-1.58$ & 0.79 & $0.57-1.09 \dagger \dagger$ \\
\hline $\mathrm{EPA}+\mathrm{DHA}$ & 0.67 & $0.46-0.98^{*}$ & 0.59 & $0.39-0.89^{*}$ & 0.65 & $0.49-0.86^{* *} \dagger$ \\
\hline Total n-3 PUFAs & 0.66 & $0.46-0.96^{*}$ & 0.60 & $0.40-0.92^{*}$ & 0.65 & $0.49-0.86^{* * \dagger}$ \\
\hline LA & 1.48 & $1.01-2.17^{*}$ & 0.90 & $0.56-1.44$ & 1.17 & $0.90-1.52 \dagger$ \\
\hline $\mathrm{AA}$ & 1.28 & $0.85-1.93$ & 0.89 & $0.57-1.41$ & 1.06 & $0.78-1.43 \dagger$ \\
\hline Total n-6 PUFAs & 1.27 & $0.86-1.87$ & 0.79 & $0.52-1.19$ & 1.02 & $0.77-1.36 \dagger$ \\
\hline Total n-3:n-6 ratio & 0.64 & $0.44-0.93^{*}$ & 0.70 & $0.44-1.11$ & 0.67 & $0.49-0.90^{* * \dagger}$ \\
\hline
\end{tabular}

Relative risks (RRs) and their 95\% CIs were calculated using generalized linear models for binary outcomes (modified Poisson) adjusted for maternal age, maternal BMI at study entry, gestational weight gain, maternal smoking during pregnancy, parity, parental education, parent atopy, child age at outcome assessment, child sex, and for the pooled estimate, a cohort indicator. Effect estimates correspond to a standard deviation score (SDS) increase in PUFAs and to a unit increase in the total n-3:n-6 ratio. AA, arachidonic acid (C20:4n-6); ALA, a-Linolenic acid (C18:3n-3); DHA, docosahexaenoic acid (C22:6n-3); EPA, eicosapentaenoic acid (C20:5n-3); LA, linoleic acid (C18:2n-6); total n-3 (n-6) PUFAs, the sum of $n-3(n-6)$ PUFAs present in the chromatogram.

${ }^{a}$ Asthma ever was defined as ever-reported doctor diagnosis of asthma.

P value for PUFA exposure-outcome association: ${ }^{*} \mathrm{P}<0.05$; ${ }^{* *} \mathrm{P}<0.01$.

$\mathrm{P}$ value for PUFA exposure-cohort interaction: $\uparrow \mathrm{P}$ for interaction $>0.10$; $\dagger \dagger \mathrm{P}$ for interaction $=0.07$. 


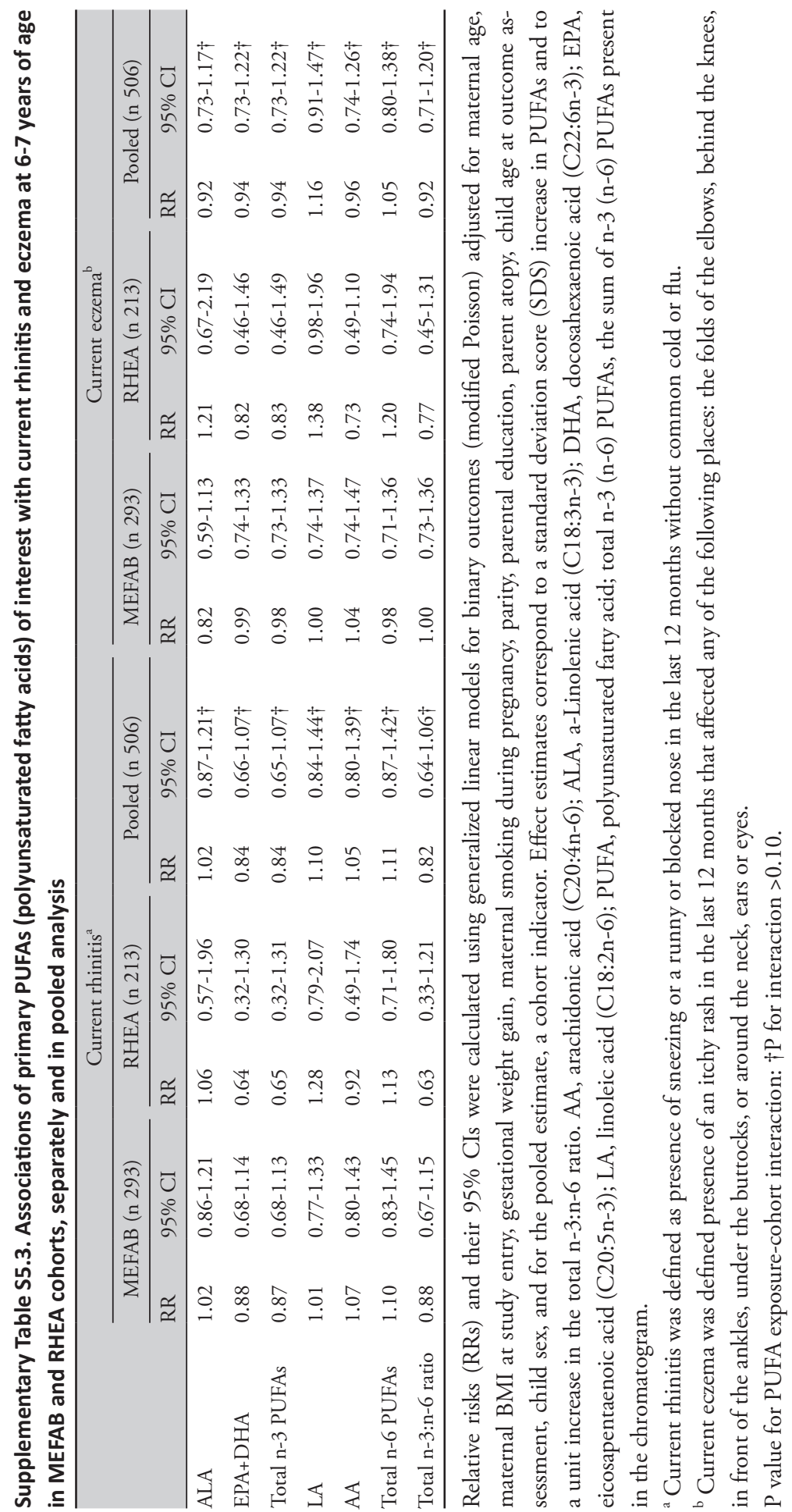


Supplementary Table S5.4. Associations (pooled analysis; $\mathbf{n}$ 506) of primary PUFAs (polyunsaturated fatty acids) of interest with allergy-related phenotypes at 6-7 years of age after adjusting only for child sex and age

\begin{tabular}{|c|c|c|c|c|c|c|c|c|}
\hline & \multicolumn{2}{|c|}{ Current wheeze $^{a}$} & \multicolumn{2}{|c|}{ Current asthma ${ }^{\mathbf{b}}$} & \multicolumn{2}{|c|}{ Current rhinitis $^{c}$} & \multicolumn{2}{|c|}{ Current eczema $^{\mathrm{d}}$} \\
\hline & $\mathrm{RR}$ & $95 \% \mathrm{CI}$ & RR & $95 \% \mathrm{CI}$ & RR & $95 \% \mathrm{CI}$ & RR & $95 \% \mathrm{CI}$ \\
\hline ALA & 0.91 & $0.69-1.20 \dagger$ & 0.61 & $0.32-1.17 \dagger$ & 1.02 & $0.85-1.22 \dagger$ & 0.92 & $0.73-1.16 \dagger$ \\
\hline $\mathrm{EPA}+\mathrm{DHA}$ & 0.59 & $0.44-0.79^{* * *} \dagger$ & 0.51 & $0.32-0.79^{* *} \dagger$ & 0.86 & $0.68-1.09 \dagger$ & 0.98 & $0.77-1.25 t$ \\
\hline Total n-3 PUFAs & 0.58 & $0.43-0.78^{* * *} \dagger$ & 0.49 & $0.31-0.76^{* *} \dagger$ & 0.86 & $0.68-1.09 \dagger$ & 0.98 & $0.76-1.26 t$ \\
\hline LA & 1.32 & $1.08-1.61^{* *} \dagger$ & 1.40 & $1.01-1.94^{*} \dagger$ & 1.10 & $0.85-1.42 \dagger$ & 1.10 & $0.87-1.40 \dagger$ \\
\hline AA & 1.33 & $0.99-1.80 \dagger \dagger$ & 1.27 & $0.84-1.93 \dagger$ & 1.06 & $0.82-1.38 \dagger$ & 0.95 & $0.73-1.23 \dagger$ \\
\hline Total n-6 PUFAs & 1.62 & $1.16-2.27^{* * \dagger}$ & 1.45 & $0.98-2.16 \dagger$ & 1.12 & $0.88-1.41 \dagger$ & 1.03 & $0.80-1.34 \dagger$ \\
\hline Total n-3:n-6 ratio & 0.52 & $0.38-0.72^{* * *} \dagger$ & 0.44 & $0.27-0.70^{* *} \dagger$ & 0.85 & $0.66-1.08 \dagger$ & 0.96 & $0.75-1.24 \dagger$ \\
\hline
\end{tabular}

Relative risks (RRs) and their 95\% CIs were calculated using generalized linear models for binary outcomes (modified Poisson) adjusted for child age at outcome assessment, child sex, and a cohort indicator. Effect estimates correspond to a standard deviation score (SDS) increase in PUFAs and to a unit increase in the total n-3:n-6 ratio. AA, arachidonic acid (C20:4n-6); ALA, a-Linolenic acid (C18:3n-3); DHA, docosahexaenoic acid (C22:6n-3); EPA, eicosapentaenoic acid (C20:5n-3); LA, linoleic acid (C18:2n-6); total n-3 (n-6) PUFAs, the sum of n-3 (n-6) PUFAs present in the chromatogram.

${ }^{a}$ Current wheeze was defined as presence of wheezing or whistling in the chest in the last 12 months.

${ }^{\mathbf{b}}$ Current asthma was defined as a positive answer to both ever-reported doctor diagnosis of asthma and presence of wheezing or whistling in the chest in the last 12 months.

${ }^{c}$ Current rhinitis was defined as presence of sneezing or a runny or blocked nose in the last 12 months without common cold or flu.

${ }^{d}$ Current eczema was defined presence of an itchy rash in the last 12 months that affected any of the following places: the folds of the elbows, behind the knees, in front of the ankles, under the buttocks, or around the neck, ears or eyes.

$\mathrm{P}$ value for PUFA exposure-outcome association: ${ }^{*} \mathrm{P}<0.05$; ${ }^{* *} \mathrm{P}<0.01$; ${ }^{* *} \mathrm{P}<0.001$.

$P$ value for PUFA exposure-cohort interaction: $\dagger P$ for interaction $>0.10$; $\dagger \dagger P$ for interaction $<0.05$. 


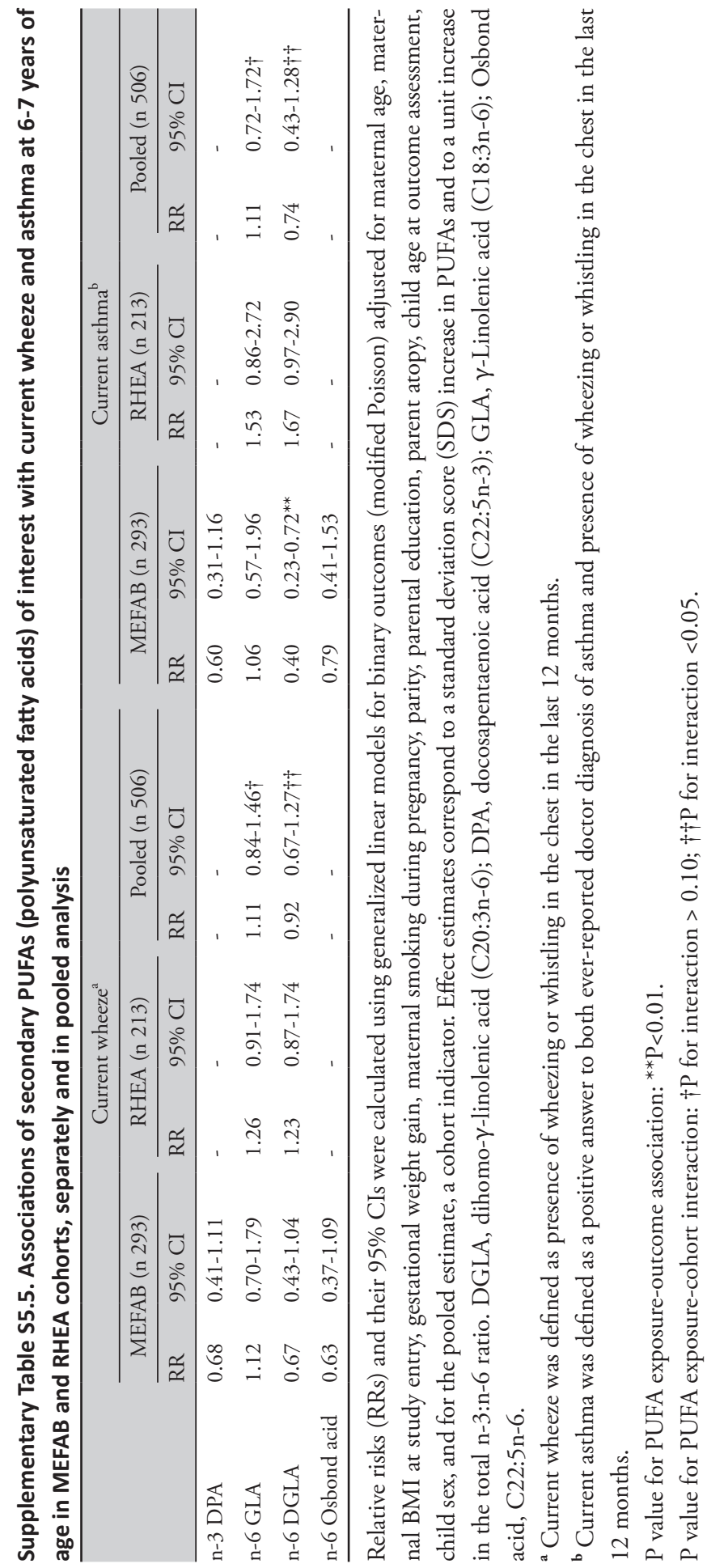




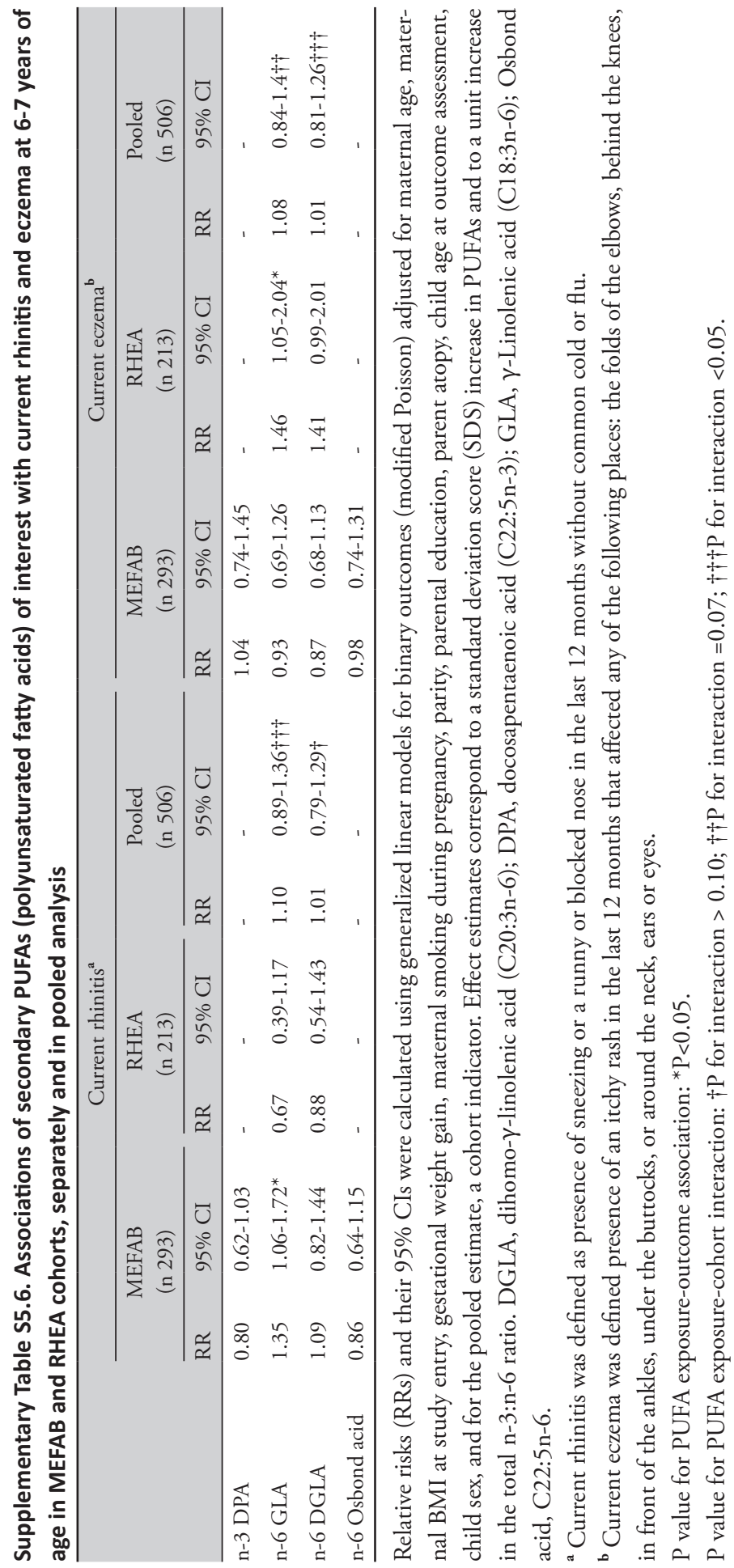


Supplementary Table S5.7. Associations of primary PUFAs (polyunsaturated fatty acids) of interest with current wheeze symptoms at 9 months and 4 years of age in the RHEA cohort (n 213)

\begin{tabular}{|c|c|c|}
\hline & \multicolumn{2}{|c|}{ Current wheeze ${ }^{a}$} \\
\hline & 9 months (n 228) & 4 years (n 222) \\
\hline & $\mathrm{RR}(95 \% \mathrm{CI})$ & RR $(95 \%$ CI) \\
\hline$\overline{A L A}$ & $0.99(0.76-1.28)$ & $1.57(0.68-3.60)$ \\
\hline $\mathrm{EPA}+\mathrm{DHA}$ & $1.00(0.79-1.28)$ & $0.74(0.38-1.44)$ \\
\hline Total n-3 PUFAs & $1.02(0.80-1.29)$ & $0.75(0.39-1.44)$ \\
\hline LA & $0.90(0.71-1.15)$ & $1.57(0.98-2.53)$ \\
\hline AA & $1.08(0.86-1.35)$ & $0.54(0.29-1.01)$ \\
\hline Total n-6 PUFAS & $1.03(0.79-1.34)$ & $0.86(0.35-2.11)$ \\
\hline Total n-3:n-6 ratio & $1.02(0.79-1.31)$ & $0.84(0.41-1.72)$ \\
\hline
\end{tabular}

Relative risks (RRs) and their 95\% CIs were calculated using generalized linear models for binary outcomes (modified Poisson) adjusted for maternal age, maternal BMI at study entry, gestational weight gain, maternal smoking during pregnancy, parity, parental education, parent atopy, child age at outcome assessment, and sex. Effect estimates correspond to a standard deviation score (SDS) increase in PUFAs and to a unit increase in the total n-3:n-6 ratio. AA, arachidonic acid (C20:4n-6); ALA, a-Linolenic acid (C18:3n-3); DHA, docosahexaenoic acid (C22:6n-3); EPA, eicosapentaenoic acid (C20:5n-3); LA, linoleic acid (C18:2n-6); total n-3 (n-6) PUFAs, the sum of n-3 (n-6) PUFAs present in the chromatogram.

${ }^{a}$ Current wheeze was defined as presence of wheezing or whistling in the chest since birth at 9 months of age $(n / N=62 / 228)$ and in the last 12 months at 4 years of age $(n / N=10 / 222)$. 


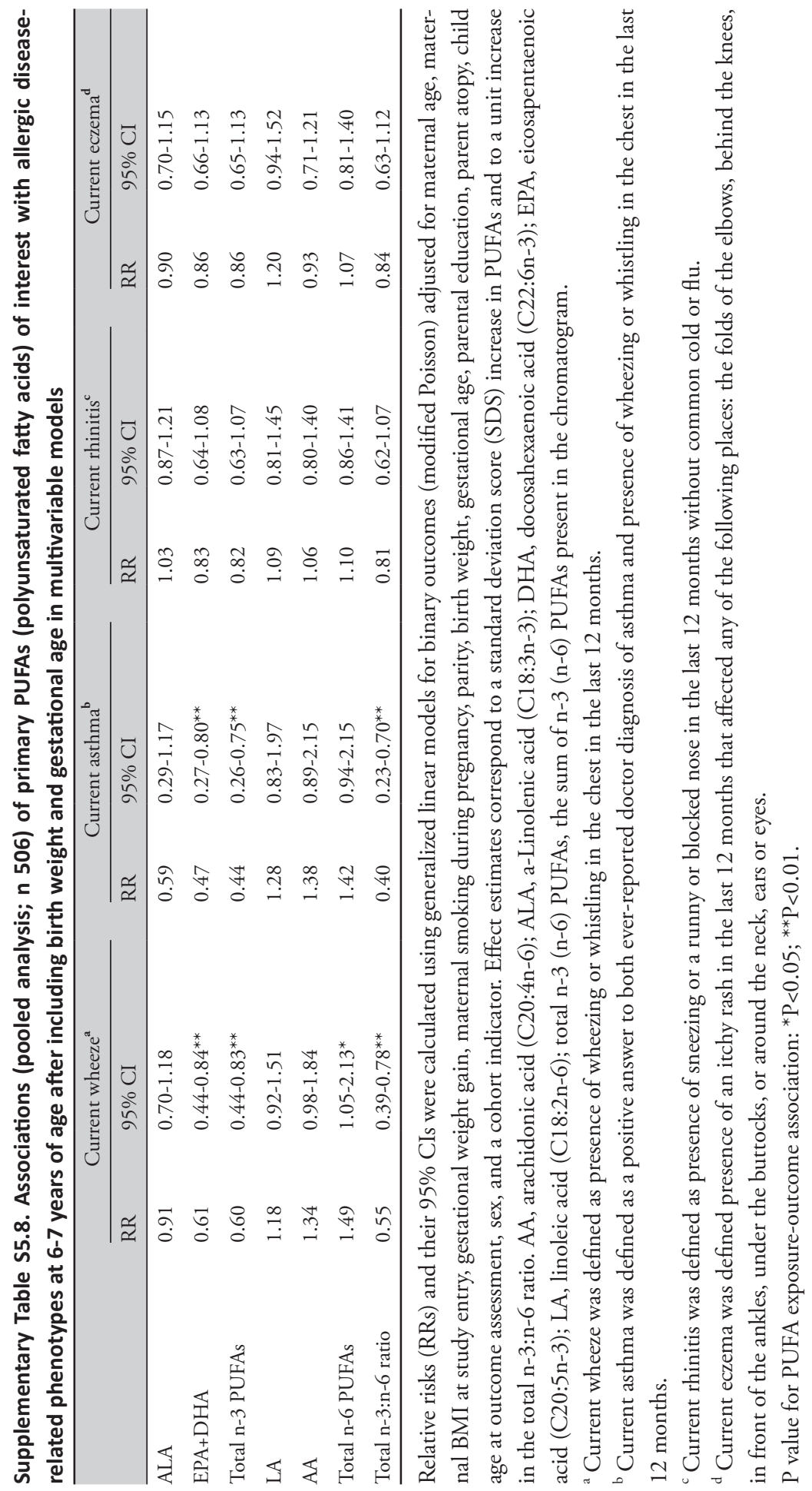


Supplementary Table S5.9. Assessment of effect measure modification by parent atopy in the association of the total n-3:n-6 PUFA ratio with current symptoms of asthma, rhinitis, and eczema at 6-7 years in the pooled dataset

\begin{tabular}{|c|c|c|c|c|c|c|c|c|c|c|}
\hline & \multirow[b]{2}{*}{$\mathrm{n}$} & \multicolumn{3}{|c|}{ Current asthma } & \multicolumn{3}{|c|}{ Current rhinitis } & \multicolumn{3}{|c|}{ Current eczema } \\
\hline & & $\mathrm{RR}$ & $95 \% \mathrm{CI}$ & $\mathrm{P}$ value & $\mathrm{RR}$ & $95 \% \mathrm{CI}$ & P value & $\mathrm{RR}$ & $95 \% \mathrm{CI}$ & $P$ value \\
\hline Parent atopy, no & 283 & 0.48 & $0.28,0.82$ & 0.01 & 0.84 & $0.59,1.21$ & 0.36 & 0.90 & $0.58,1.38$ & 0.63 \\
\hline Parent atopy, yes & 223 & 0.38 & $0.17,0.83$ & 0.02 & 0.82 & $0.59,1.15$ & 0.25 & 0.97 & $0.69,1.36$ & 0.86 \\
\hline
\end{tabular}

Measure of effect modification on multiplicative scale:

For current asthma: $\mathrm{RR}=0.94(95 \%$ CI $0.38,2.34) ; \mathrm{P}=0.90$

For current rhinitis: $\mathrm{RR}=0.92(95 \% \mathrm{CI} 0.56,1.51) ; \mathrm{P}=0.74$

For current eczema: $\mathrm{RR}=1.02(95 \%$ CI $0.60,1.73) ; \mathrm{P}=0.94$

Measure of effect modification on additive scale:

For current asthma: RERI $=-0.31(95 \% \mathrm{CI}-1.87,1.26) ; \mathrm{P}=0.70$

For current rhinitis: RERI $=0.04(95 \% \mathrm{CI}-0.88,0.95) ; \mathrm{P}=0.94$

For current eczema: RERI $=0.21(95 \%$ CI $-1.04,1.46) ; \mathrm{P}=0.74$

Effect estimates were adjusted for maternal age, maternal BMI at study entry, gestational weight gain, maternal smoking during pregnancy, parity, parental education, child age at outcome assessment, child sex, and a cohort indicator. 
Supplementary Table S5.10. Assessment of effect measure modification by sex in the association of the total n-3:n-6 PUFA ratio with current symptoms of asthma, rhinitis, and eczema at 6-7 years in the pooled dataset

\begin{tabular}{|c|c|c|c|c|c|c|c|c|c|c|}
\hline & \multirow[b]{2}{*}{$\mathrm{n}$} & \multicolumn{3}{|c|}{ Current asthma } & \multicolumn{3}{|c|}{ Current rhinitis } & \multicolumn{3}{|c|}{ Current eczema } \\
\hline & & $\mathrm{RR}$ & $95 \% \mathrm{CI}$ & P value & $\mathrm{RR}$ & $95 \% \mathrm{CI}$ & $P$ value & $\mathrm{RR}$ & $95 \% \mathrm{CI}$ & $P$ value \\
\hline Child sex, male & 285 & 0.47 & $0.27,0.80$ & $<0.01$ & 1.00 & $0.72,1.39$ & 0.98 & 0.95 & $0.67,1.35$ & 0.77 \\
\hline Child sex, female & 221 & 0.27 & $0.17,0.43$ & $<0.001$ & 0.61 & $0.37,1.01$ & 0.06 & 0.84 & $0.53,1.34$ & 0.47 \\
\hline
\end{tabular}

Measure of effect modification on multiplicative scale:

For current asthma: $\mathrm{RR}=0.80$ (95\% CI 0.38, 1.69); $\mathrm{P}=0.56$

For current rhinitis: $\mathrm{RR}=0.57(95 \% \mathrm{CI} 0.32,1.01) ; \mathrm{P}=0.06$

For current eczema: $\mathrm{RR}=0.88$ (95\% CI 0.52, 1.49); $\mathrm{P}=0.63$

Measure of effect modification on additive scale:

For current asthma: RERI $=0.58(95 \%$ CI $-0.09,1.26) ; \mathrm{P}=0.09$

For current rhinitis: RERI $=-1.83(95 \% \mathrm{CI}-5.60,1.94) ; \mathrm{P}=0.34$

For current eczema: RERI $=-0.41(95 \%$ CI $-1.89,1.07) ; \mathrm{P}=0.59$

Effect estimates were adjusted for maternal age, maternal BMI at study entry, gestational weight gain, maternal smoking during pregnancy, parent atopy, parity, parental education, child age at outcome assessment, and a cohort indicator. 


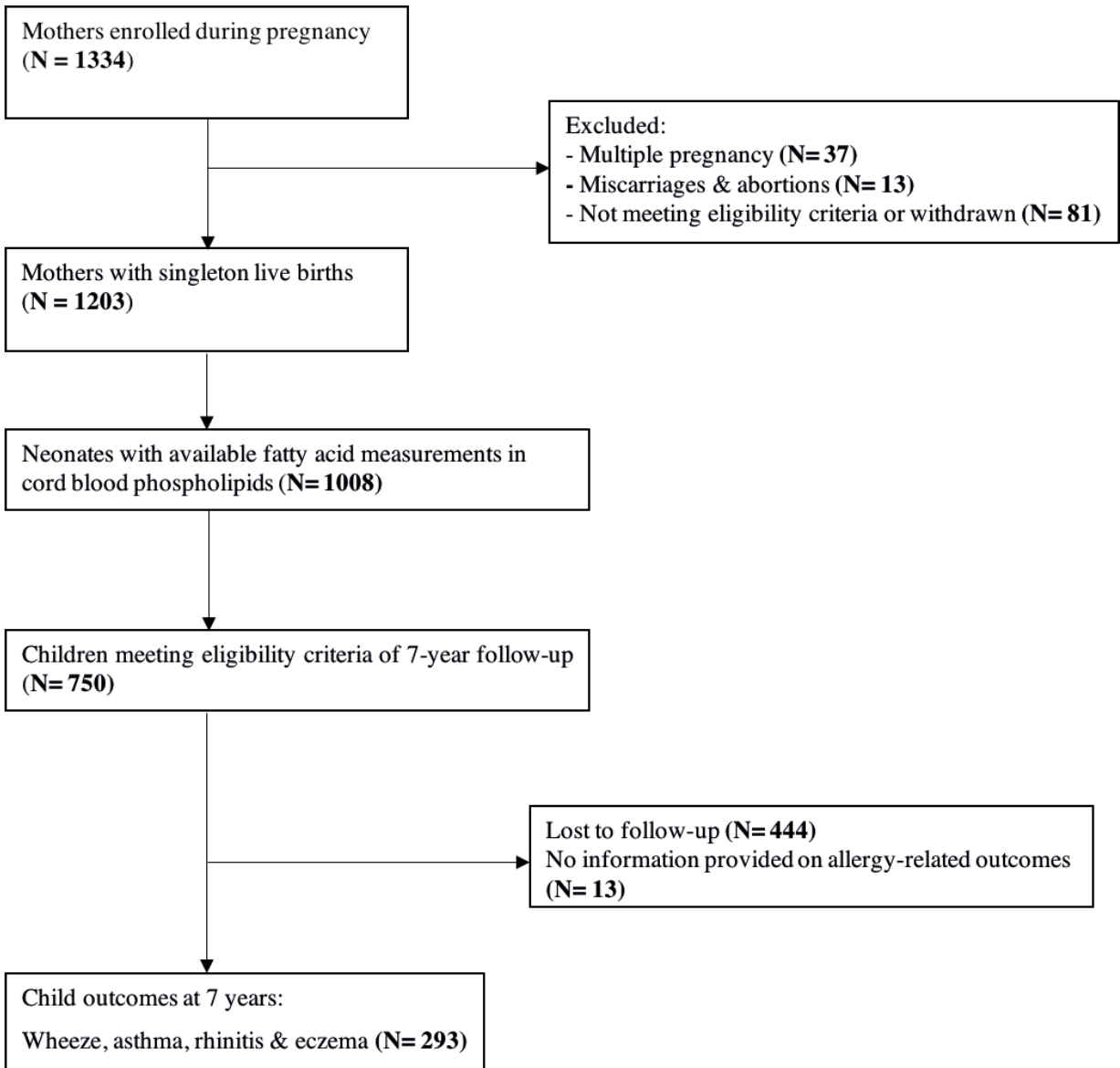

Supplementary Figure S5.1. Flow chart of the MEFAB participants 
Mothers recruited during pregnancy $(\mathbf{N}=\mathbf{1 6 1 0})$

Excluded:

- Multiple pregnancy $(\mathbf{N}=40)$

- Miscarriages \& abortions $(\mathbf{N}=\mathbf{5 0})$

- Still births $(\mathbf{N}=2)$

- Moved to another location or withdrawn $(N=148)$

Mothers with singleton live births $(\mathbf{N}=\mathbf{1 3 6 3})$

Neonates with available fatty acid measurements in cord blood phospholipids $(\mathbf{N}=\mathbf{5 0 0})$

Child outcomes:

Wheeze at 9 months $(\mathbf{N}=\mathbf{2 2 8})$

Wheeze at 4 years $(\mathbf{N}=\mathbf{2 2 2})$

Wheeze, asthma, rhinitis \& eczema at 6 years $(\mathbf{N}=\mathbf{2 1 3})$

\section{Supplementary Figure S5.2. Flow chart of the RHEA participants}




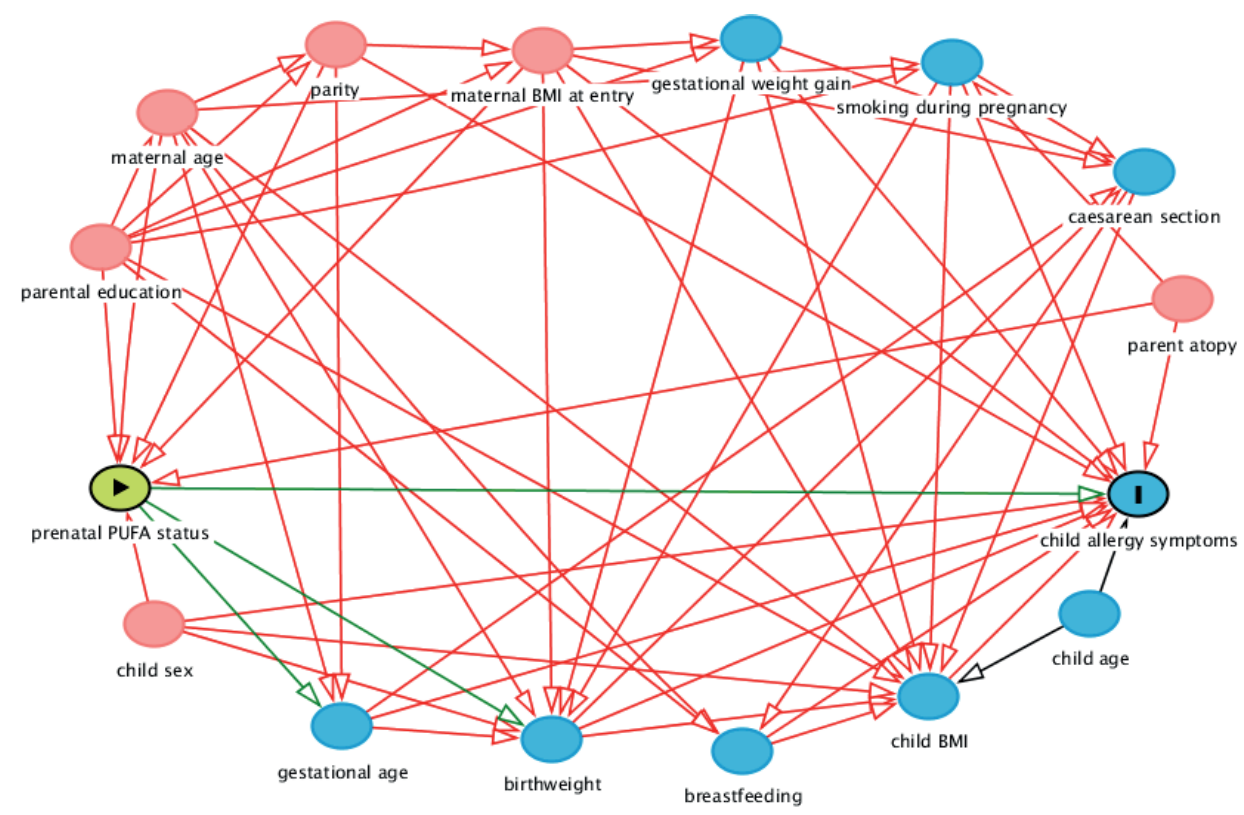

Supplementary Figure S5.3. Directed acyclic graph for the association of fatty acid status at birth with allergic disease-related symptoms in childhood. 


\section{Text accompanying Supplementary Figure S5.3}

In our models, selection of covariates for adjustment was based on a directed acyclic graph (DAG) approach. A DAG is a graphical tool that enables the visualization of the associations among the exposure of interest, the outcome(s) being studied, and all other related characteristics. It represents a conceptual model, and provides a structured way to present an overview of the research question and its context, therefore aiding in identifying the presence of confounding and ways to resolve it. ${ }^{1}$ Below, we use some illustrative examples for the reader who has not previous experience with DAGs. For those who are more interested in the concept of DAGs, there are some excellent reviews on this topic. ${ }^{1-3}$

Arrows in DAGs represent direct effects of one factor on another, either protective or harmful. Moreover, arrows should follow time order, or else the diagram contradicts the basic principle that causes must precede their effects. The arrows and their direction are drawn based on prior knowledge. For instance, in Supplemental Figure S5.3, parental education, as a proxy of SES status, can affect both prenatal fatty acid exposure ${ }^{4,5}$ and childhood allergic disease symptoms, ${ }^{6}$ so the arrows point away from parental education towards fatty acid status at birth as well as towards child allergic disease symptoms. The path from fatty acid status at birth towards child allergy via parental education is called a backdoor path because it starts with an arrowhead towards fatty acid status at birth, the exposure. The presence of a backdoor path in DAG identifies the presence of confounding. The path from prenatal fatty acids via gestational age to child allergy is not a backdoor path, as the first arrow points away from the exposure. There is good evidence to suggest that high exposure to n-3 long-chain PUFAs even in the last weeks of gestation increases the duration of gestation. ${ }^{7,8}$ Hence, gestational age is not a cause of prenatal PUFA exposure, but prenatal PUFAs can be regarded as a cause of gestational age. Additionally, the arrow from gestational age to child allergy indicates that gestational age predicts child allergy symptoms; an association that is supported by good epidemiological evidence. ${ }^{9}$ Gestational age is therefore in the causal pathway between fatty acid status and child allergy.

In our study, the DAG was drawn using DAGitty (www.dagitty.net), ${ }^{10}$ a user friendly software that allows the instant identification of minimum but sufficient sets of factors to control for in the analysis in order to resolve confounding. The adjustment set in our study included the following covariates: maternal age, maternal BMI at study entry, gestational weight gain, maternal smoking during pregnancy, parental education, parity, parent atopy, child age and sex. Through this adjustment set, the backdoor paths from fatty acid status at birth towards child allergy are blocked. 
Below, we present references for the assumed relationships in our DAG (Supplementary Figure S5.3):

\begin{tabular}{|c|c|}
\hline Parental education & $\begin{array}{l}\rightarrow \text { prenatal PUFA status: }{ }^{4,5} \\
\rightarrow \text { child allergy-related symptoms: } \\
\rightarrow \text { smoking during pregnancy: }:^{11} \\
\rightarrow \text { child BMI: }:^{12}\end{array}$ \\
\hline Maternal age & $\begin{array}{l}\rightarrow \text { smoking during pregnancy: }{ }^{11} \\
\rightarrow \text { child BMI: }{ }^{13} \\
\rightarrow \text { gestational age/birthweight: }{ }^{14}\end{array}$ \\
\hline Maternal BMI & $\begin{array}{l}\rightarrow \text { prenatal PUFA status: }{ }^{15} \\
\rightarrow \text { gestational age/birthweight: }{ }^{16} \\
\rightarrow \text { child BMI: }^{17} \\
\rightarrow{\text { child allergy-related symptoms: }{ }^{18}}^{18}\end{array}$ \\
\hline Gestational weight gain & $\begin{array}{l}\rightarrow \text { gestational age/birthweight: }{ }^{16} \\
\rightarrow \text { child BMI: }^{19} \\
\rightarrow{\text { child allergy-related symptoms: }{ }^{18}}^{18}\end{array}$ \\
\hline Smoking during pregnancy & $\begin{array}{l}\rightarrow \text { birthweight/gestational age: }:^{20,21} \\
\rightarrow \text { child BMI: }{ }^{22} \\
\rightarrow \text { child allergy-related symptoms: }:^{23}\end{array}$ \\
\hline Parity & $\begin{array}{l}\rightarrow \text { prenatal PUFA status: }{ }^{24} \\
\rightarrow \text { child allergy-related symptoms: }{ }^{25,26}\end{array}$ \\
\hline Parent atopy & $\begin{array}{l}\rightarrow \text { prenatal PUFA status: }:^{27} \\
\rightarrow \text { child allergy-related symptoms: }{ }^{28}\end{array}$ \\
\hline Prenatal PUFA status & $\rightarrow$ birthweight/gestational age: 8,29 \\
\hline Child sex & $\begin{array}{l}\rightarrow \text { prenatal PUFA status: }{ }^{30} \\
\rightarrow \text { allergy-related symptoms: }{ }^{31}\end{array}$ \\
\hline Gestational age/birth weight & $\rightarrow$ child allergy-related symptoms: ${ }^{32,33}$ \\
\hline Child BMI & $\rightarrow$ allergy-related symptoms: ${ }^{34}$ \\
\hline
\end{tabular}




\section{Supplementary References}

1. Shrier I, Platt RW. Reducing bias through directed acyclic graphs. BMC Med Res Methodol 2008;8:70.

2. Greenland S, Pearl J, Robins JM. Causal diagrams for epidemiologic research. Epidemiology 1999;10:3748.

3. Glymour MM, Greenland S. Causal Diagrams. In: Modern Epidemiology Vol 3. Lippencott-Raven Publishers: Philadelphia; 2008:183-209.

4. Wennberg M, Tornevi A, Johansson I, Hornell A, Norberg M, Bergdahl IA. Diet and lifestyle factors associated with fish consumption in men and women: a study of whether gender differences can result in gender-specific confounding. Nutr J 2012;11:101.

5. Steenweg-de Graaff JC, Tiemeier H, Basten MG, et al. Maternal LC-PUFA status during pregnancy and child problem behavior: the Generation R Study. Pediatr Res 2015;77:489-97.

6. Gehring U, Pattenden S, Slachtova H, et al. Parental education and children's respiratory and allergic symptoms in the Pollution and the Young (PATY) study. The European respiratory journal 2006;27:95107.

7. Olsen SF, Hansen HS, Sorensen TI, et al. Intake of marine fat, rich in (n-3)-polyunsaturated fatty acids, may increase birthweight by prolonging gestation. Lancet 1986;2:367-9.

8. Olsen SF, Hansen HS, Sommer S, et al. Gestational age in relation to marine n-3 fatty acids in maternal erythrocytes: a study of women in the Faroe Islands and Denmark. American journal of obstetrics and gynecology 1991;164:1203-9.

9. Sonnenschein-van der Voort AM, Arends LR, de Jongste JC, et al. Preterm birth, infant weight gain, and childhood asthma risk: a meta-analysis of 147,000 European children. J Allergy Clin Immunol 2014;133:1317-29.

10. Textor J, Hardt J, Knuppel S. DAGitty: a graphical tool for analyzing causal diagrams. Epidemiology 2011;22:745.

11. Vardavas CI, Patelarou E, Chatzi L, et al. Factors associated with active smoking, quitting, and secondhand smoke exposure among pregnant women in Greece. J Epidemiol 2010;20:355-62.

12. Muthuri SK, Onywera VO, Tremblay MS, et al. Relationships between Parental Education and Overweight with Childhood Overweight and Physical Activity in 9-11 Year Old Children: Results from a 12-Country Study. PLoS One 2016;11:e0147746.

13. Savage T, Derraik JG, Miles HL, Mouat F, Hofman PL, Cutfield WS. Increasing maternal age is associated with taller stature and reduced abdominal fat in their children. PLoS One 2013;8:e58869.

14. Lawlor DA, Mortensen L, Andersen AM. Mechanisms underlying the associations of maternal age with adverse perinatal outcomes: a sibling study of 264695 Danish women and their firstborn offspring. Int J Epidemiol 2011;40:1205-14.

15. Vidakovic AJ, Jaddoe VW, Gishti O, et al. Body mass index, gestational weight gain and fatty acid concentrations during pregnancy: the Generation R Study. Eur J Epidemiol 2015;30:1175-85.

16. Nohr EA, Vaeth M, Baker JL, Sorensen T, Olsen J, Rasmussen KM. Combined associations of prepregnancy body mass index and gestational weight gain with the outcome of pregnancy. Am J Clin Nutr 2008;87:1750-9.

17. Yu Z, Han S, Zhu J, Sun X, Ji C, Guo X. Pre-pregnancy body mass index in relation to infant birth weight and offspring overweight/obesity: a systematic review and meta-analysis. PLoS One 2013;8:e61627.

18. Forno E, Young OM, Kumar R, Simhan H, Celedon JC. Maternal obesity in pregnancy, gestational weight gain, and risk of childhood asthma. Pediatrics 2014;134:e535-46. 
19. Mourtakos SP, Tambalis KD, Panagiotakos DB, et al. Maternal lifestyle characteristics during pregnancy, and the risk of obesity in the offspring: a study of 5,125 children. BMC Pregnancy Childbirth 2015;15:66.

20. Kayemba-Kay's S, Geary MP, Pringle J, Rodeck CH, Kingdom JC, Hindmarsh PC. Gender, smoking during pregnancy and gestational age influence cord leptin concentrations in newborn infants. Eur $J$ Endocrinol 2008;159:217-24.

21. Li CQ, Windsor RA, Perkins L, Goldenberg RL, Lowe JB. The impact on infant birth weight and gestational age of cotinine-validated smoking reduction during pregnancy. JAMA 1993;269:1519-24.

22. von Kries R, Toschke AM, Koletzko B, Slikker W, Jr. Maternal smoking during pregnancy and childhood obesity. Am J Epidemiol 2002;156:954-61.

23. Gilliland FD, Li YF, Peters JM. Effects of maternal smoking during pregnancy and environmental tobacco smoke on asthma and wheezing in children. Am J Respir Crit Care Med 2001;163:429-36.

24. Hornstra G. Essential fatty acids in mothers and their neonates. Am J Clin Nutr 2000;71:1262S-9S.

25. Ball TM, Castro-Rodriguez JA, Griffith KA, Holberg CJ, Martinez FD, Wright AL. Siblings, day-care attendance, and the risk of asthma and wheezing during childhood. N Engl J Med 2000;343:538-43.

26. Bernsen RM, de Jongste JC, van der Wouden JC. Birth order and sibship size as independent risk factors for asthma, allergy, and eczema. Pediatr Allergy Immunol 2003;14:464-9.

27. Duchen K, Bjorksten B. Polyunsaturated n-3 fatty acids and the development of atopic disease. Lipids 2001;36:1033-42.

28. Alford SH, Zoratti E, Peterson EL, Maliarik M, Ownby DR, Johnson CC. Parental history of atopic disease: disease pattern and risk of pediatric atopy in offspring. J Allergy Clin Immunol 2004;114:104650.

29. Grootendorst-van Mil NH, Tiemeier H, Steenweg-de Graaff J, et al. Maternal plasma n-3 and n-6 polyunsaturated fatty acids during pregnancy and features of fetal health: Fetal growth velocity, birth weight and duration of pregnancy. Clin Nutr 2017.

30. Decsi T, Kennedy K. Sex-specific differences in essential fatty acid metabolism. Am J Clin Nutr 2011;94:Suppl. 6, S1914-S9.

31. Almqvist C, Worm M, Leynaert B, working group of GALENWPG. Impact of gender on asthma in childhood and adolescence: a GA2LEN review. Allergy 2008;63:47-57.

32. Mebrahtu TF, Feltbower RG, Greenwood DC, Parslow RC. Birth weight and childhood wheezing disorders: a systematic review and meta-analysis. J Epidemiol Community Health 2015;69:500-8.

33. Been JV, Lugtenberg MJ, Smets E, et al. Preterm birth and childhood wheezing disorders: a systematic review and meta-analysis. PLoS Med 2014;11:e1001596.

34. Gilliland FD, Berhane K, Islam T, et al. Obesity and the risk of newly diagnosed asthma in school-age children. Am J Epidemiol 2003;158:406-15. 


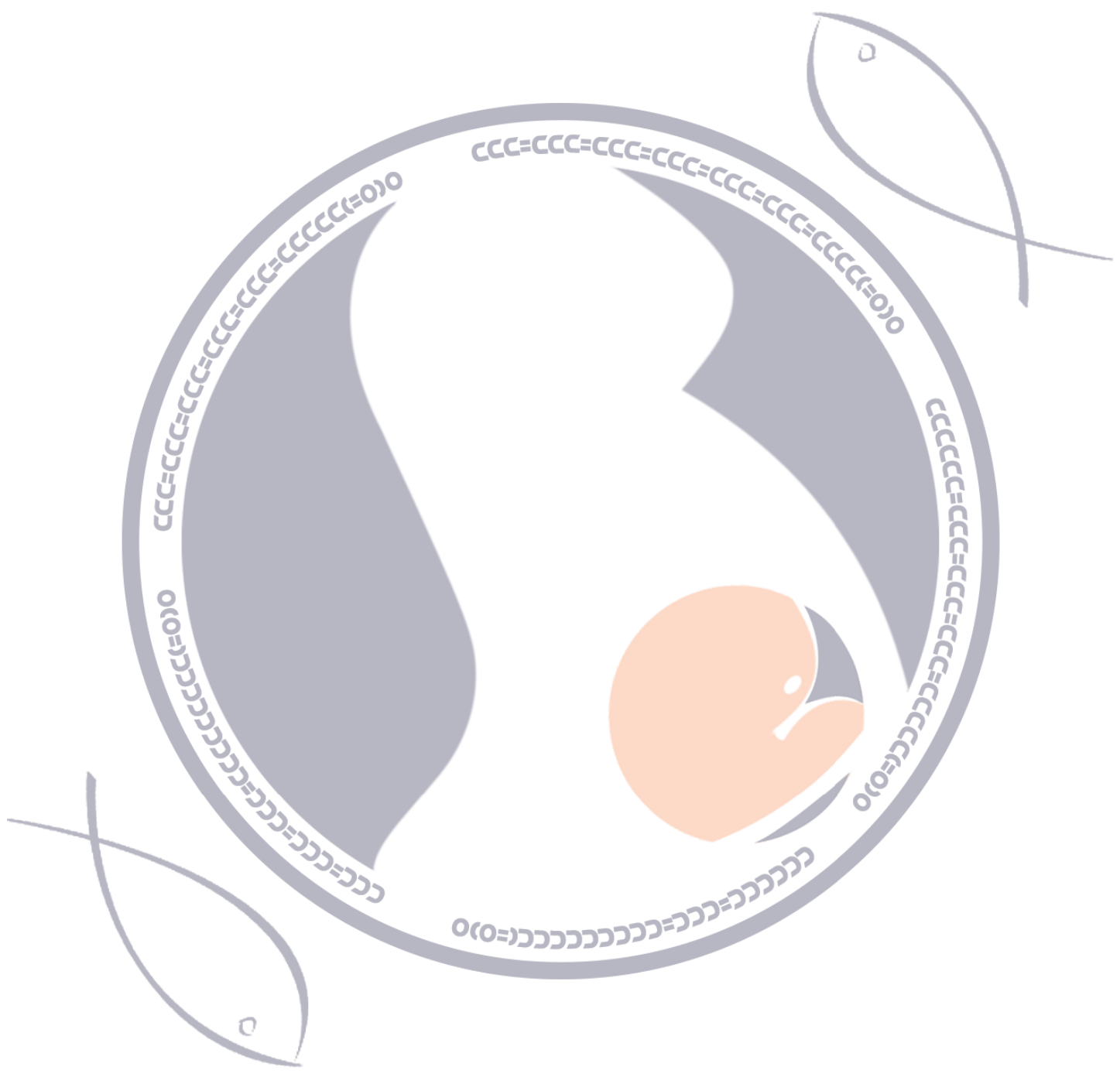




\title{
CHAPTER 6
}

\section{Maternal fish intake during pregnancy and effects on the offspring}

\author{
L Chatzi, N Stratakis
}

Adapted from R Rajendram, VR Preedy, VB Patel (Eds.). Diet, Nutrition, and Fetal Programming. Humana Press. 2017. 


\section{ABSTRACT}

Fish is generally considered an integral component of a healthy diet. However, it is a complex exposure. Fish is the primary dietary source of n-3 long-chain polyunsaturated fatty acids and a rich source of protein, selenium, iodine, and vitamin $\mathrm{D}$, but is also a major source of exposure to methylmercury and other environmental pollutants. In utero exposure to nutrients and toxicants found in the same fish might act on the exact same end points at an opposite direction. In the present chapter, we summarize findings from observational studies and randomized clinical trials on the association of fish consumption during pregnancy with fetal growth and preterm birth, childhood obesity, neurodevelopment, and allergic diseases. Overall, evidence on the association of maternal fish consumption during pregnancy with child health outcomes has been largely inconsistent. Further studies using biomarker information on both the amounts of fatty acids and environmental chemicals contained within fish are essential for refining estimates of the influence of prenatal fish intake on child growth and development. 


\section{INTRODUCTION}

Intrauterine life is a critical period of developmental plasticity. A nutritional stress or stimulus encountered during this period could elicit permanent alterations in body physiology and metabolism that have important long-term consequences for later health and disease susceptibility. ${ }^{1}$ In this context, fish constitutes a complex exposure. It is a rich source of protein, selenium, iodine, and vitamin D, and the primary dietary source of the n-3 long-chain polyunsaturated fatty acids (LCPUFAs), including eicosapentaenoic acid (EPA) and docosahexaenoic acid (DHA), which are considered beneficial for growth and development. ${ }^{2}$ In contrast, fish is also a well-known route of exposure to pollutants such as methylmercury ( $\mathrm{MeHg}$ ), polychlorinated biphenyls (PCBs), and dioxins, which may adversely affect child development. $^{3}$

Hence, the effect of fish intake by pregnant women remains an important issue, especially in populations that consume fish frequently. In June 2014, the US Food and Drug Administration (FDA) and Environmental Protection Agency updated their advice on fish consumption for women of childbearing age, encouraging women who are pregnant, breastfeeding, or likely to become pregnant to consume more fish, but no more than 3 servings per week to limit fetal exposure to $\mathrm{MeHg}$. ${ }^{4}$ The European Food Safety Authority (EFSA) has also reported recently that the benefits from fish consumption of up to 3-4 servings per week during pregnancy could outweigh the risks associated with $\mathrm{MeHg}$ exposure. ${ }^{5}$ Fish advisories have focused so far on potential neurocognitive harms from $\mathrm{MeHg}$ exposure, but have not considered other childhood outcomes including growth and asthma occurrence. Furthermore, pregnant women are faced with conflicting messages about the health effects of fish consumption, which result in confusion concerning the place of fish in a healthy prenatal diet.

We aimed in this chapter to examine the association of fish intake during pregnancy with offspring health outcomes, including fetal growth and preterm birth, childhood obesity, neurodevelopment, and allergic diseases.

\section{FISH, FETAL GROWTH, AND PRETERM BIRTH}

The failure of the fetus to reach its full growth potential is an important predictor of short and long-term health. Growth-restricted fetuses are at increased risk of infant mortality and morbidity, ${ }^{6}$ and have a higher risk of developing chronic diseases in adulthood, such type 2 diabetes, and coronary heart disease, ${ }^{7,8}$ Anthropometric measurements at birth (ie, birth weight, birth length, and head circumference) and the index small-for-gestational age (SGA; defined as a neonate whose birth size is below the tenth percentile for a given reference growth chart 
for sex and gestational age) are widely used to assess fetal growth. Preterm birth, defined as a gestational age of less than 37 completed weeks, is an established risk factor for later morbidity and mortality.

Early observations showing that gestation length and birth weight are increased among populations with a high habitual fish intake ${ }^{10}$ generated a lot of interest in the scientific literature regarding the association of fish intake during pregnancy with fetal growth. It has been hypothesized that the n-3 LCUFAs found in fish can prolong gestation by decreasing the production of eicosanoids that play a role in the initiation of delivery and increasing the production for prostacyclins $\left(\mathrm{PGI}_{2}\right.$ and $\mathrm{PGI}_{3}$ ) that exert myometrial relaxant properties. ${ }^{11} \mathrm{n}-3$ LCPUFAs can also increase fetal growth rate by raising the prostacyclin to thromboxane ratio and reducing blood viscosity, thereby enhancing placental blood flow. ${ }^{12}$ However, reports from human trials have not shown a clear and consistent benefit of fish oil supplementation in birth weight and duration of gestation. ${ }^{13,14}$

To date, several observational cohort studies have been conducted to assess the association of prenatal fish intake with measures of fetal growth and have produced puzzling results (Table 6.1). In the British Avon longitudinal Study of Parents and Children (ALSPAC) of 11585 mother and child pairs, women consuming rarely fish in late pregnancy had an increased risk of intrauterine growth retardation (defined as birth weight adjusted for sex and gestational age below the 10th percentile) compared with those with a mean consumption of 4 portions per week. ${ }^{15}$ Similarly, the Danish Aaarhus Birth cohort involving 8729 mother-child pairs showed that low consumption of fish in early pregnancy was a strong risk factor for low birth weight and preterm delivery, with the strongest associations being observed at a daily intake of less than $15 \mathrm{~g}$ of fish. ${ }^{16}$ In seemingly contrast to these results, Oken et al ${ }^{17}$ using data from 2109 mother-child pairs of the US Project Viva birth cohort, reported an inverse association of first-trimester seafood consumption with birth weight and fetal growth, while no effect was found on the length of gestation or risk of preterm birth. 


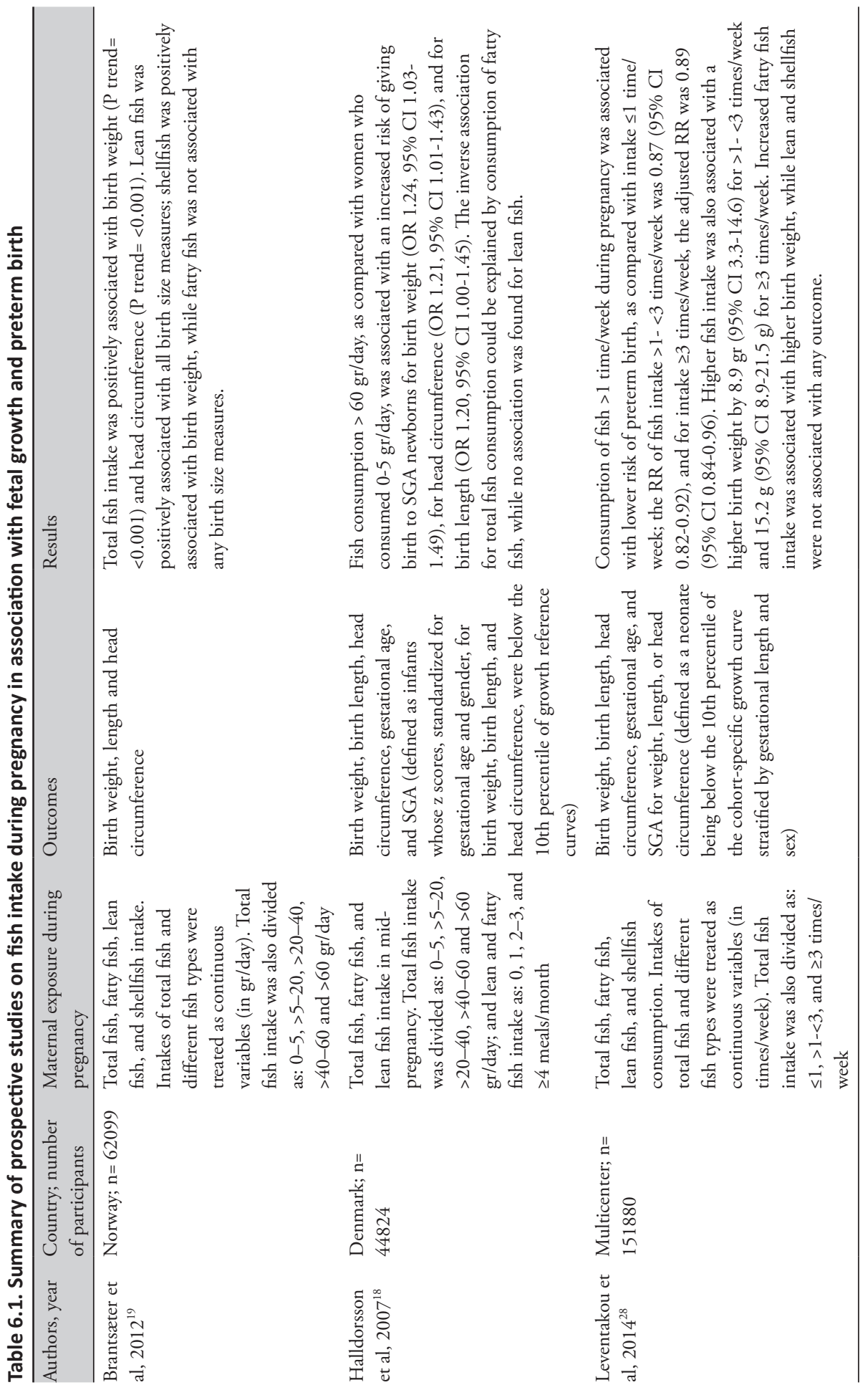




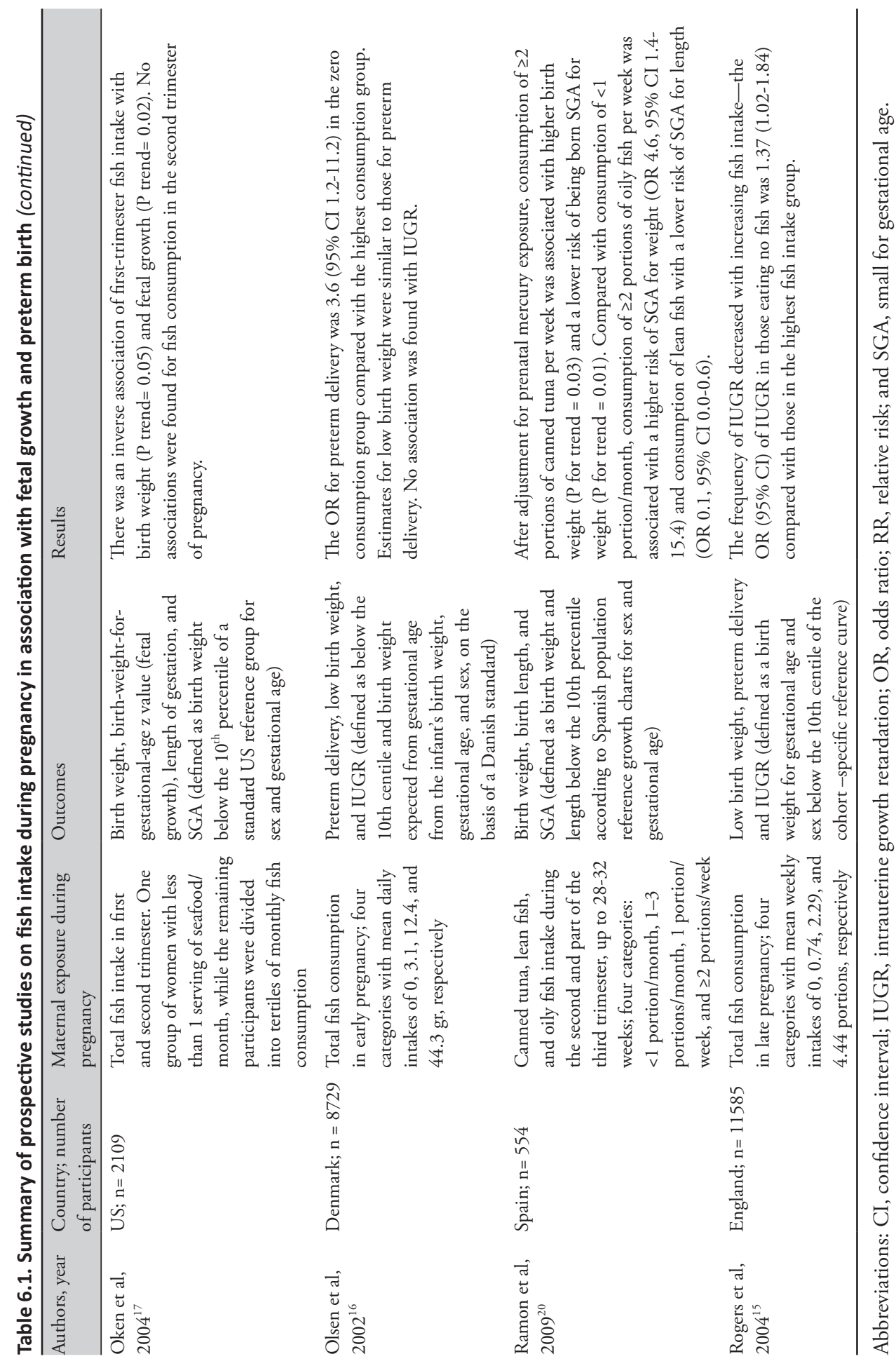


In the Danish National Birth Cohort (DNBC) including 44824 mother-child pairs, Halldorsson et $\mathrm{al}^{18}$ examined the separate effects of the types of fish consumed in mid-pregnancy and showed that fatty fish intake more than four times per month was associated with a higher risk of giving birth to children who were SGA, while no association was found for lean fish. By way of contrast, Brantsæter et $\mathrm{al}^{19}$ in the Norwegian Mother and Child Cohort Study (MoBa) including 62099 mother-child pairs, showed that increasing mid-pregnancy seafood consumption was associated with increased birth weight and head circumference. This positive association was mainly driven by consumption of lean fish, while fatty fish was not associated with any birth size measures. ${ }^{19}$ Ramon et $\mathrm{al}^{20}$ used data from a Spanish cohort of 554 mother-child pairs to assess associations of the type of fish consumed, cord blood mercury levels, and birth outcomes. As anticipated, higher fish consumption in pregnancy was associated with higher cord blood mercury levels. The authors showed that after adjusting for prenatal exposure to mercury, weekly consumption of more than 2 portions of lean fish and canned tuna consumption was associated with a lower risk of being born SGA, while large oily fish consumption was associated with a higher risk. ${ }^{20}$ The differential influence by different types of seafood on fetal growth might be indirect evidence of harmful contaminants found in fish. ${ }^{21} \mathrm{MeHg}$ can inhibit the antioxidant systems and stimulate the production of free radicals, which in turn can adversely affect fetal growth. ${ }^{20}$ Many studies, but not all, have reported associations of higher prenatal concentrations of mercury with reduced birth weight and an increased risk of SGA (reviewed in ${ }^{22}$ ). Additionally, persistent organic pollutants commonly found in fish, such as PCBs, can exert endocrine-disrupting properties and affect fetal growth through effects on sex steroid and thyroid hormone function. ${ }^{23}$ In the DNBC cohort, intake of fatty fish was associated with levels of PCBs in maternal plasma, and exposure to these pollutants was found to be inversely associated with low birth weight. ${ }^{24}$ Several other studies, ${ }^{23,25}$ but not all, ${ }^{26,27}$ have also reported an association between exposure to persistent organic pollutants and low birth weight.

Because the balance between the potential beneficial effect of n-3 LCPUFAs and deleterious effect of contaminants in fish intake is determined by the relative exposure, results may differ across populations consuming different types of seafood. Direct comparison between individual studies is also complicated by small sample sizes, exposure misclassification, exposure profile heterogeneity, or differences in adjustment for confounding variables.

In an effort to assess the strength and consistency of the association of fish intake during pregnancy with fetal growth, a Europe-wide study harmonized and pooled individual data from 151880 mother-child pairs participating in 19 birth cohort 
studies. ${ }^{28}$ The study showed that, compared to fish consumption of less than 1 time per week, moderate intake of more than 1 time per week was associated with a lower risk of preterm birth, and a small but significant increase in birth weight (Figure 6.1). The most pronounced effect on birth weight was observed for fatty fish types, while no association was observed with lean fish or shellfish intake. Notably, the protective effect of fish intake on preterm birth was shown only in the categorical analysis and not in the continuous analysis, suggesting that for very high amounts of fish intake, the protective effect is attenuated (U-shaped association). ${ }^{28}$

Further analyses using biomarker information on both the amounts of fatty acids and environmental chemicals contained within fish will be helpful for refining estimates of the influence of prenatal fish intake on fetal growth.

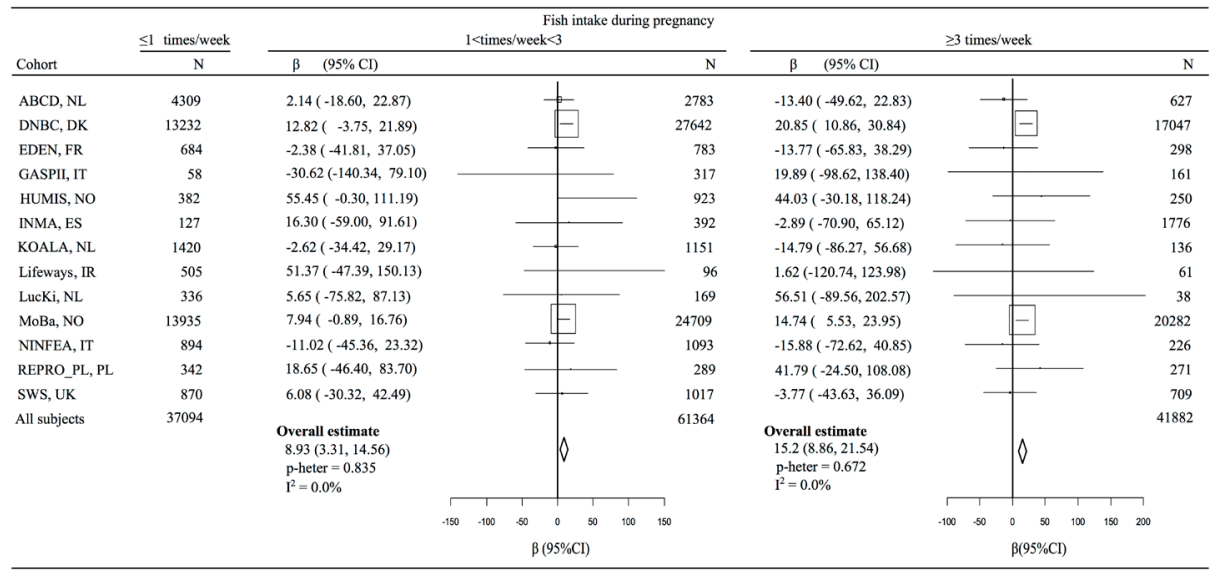

Figure 6.1. Fish intake during pregnancy and birth weight: a meta-analysis of 19 European birth cohort studies. $\beta$ coefficients $(95 \% \mathrm{Cls}$ ) by cohort were obtained by using linear regression models adjusted for maternal age, pre-pregnancy BMI, maternal height, education level, smoking during pregnancy, parity, infant sex, gestational age, and gestational age squared. Reference category was $\leq 1$ time/week. Overall estimates were obtained by using a random- or fixed-effects meta-analysis. p-heter values were estimated by using Cochran's $Q$ test. Reproduced with permission from Am J Clin Nutr 2014;99:506-516, American Society for Nutrition.

\section{FISH AND CHILDHOOD OBESITY}

Childhood overweight and obesity is considered a major public health issue. ${ }^{29}$ Evidence suggests that a high body mass index (BMI), used as a surrogate measure of excess adiposity, in childhood tends to track into adulthood. ${ }^{30}$ It has been argued that efforts to prevent obesity should begin early in life and even before birth. ${ }^{31}$ 
Intrauterine life is a critical period, during which the proliferation of mesenchymal precursor cells and their differentiation into adipocytes are highly sensitive to alterations of the nutritional environment. ${ }^{32}$ Evidence from in vitro and animal studies suggests that early exposure to n-3 LCPUFAs have the potential to reduce adipose tissue deposition by inhibiting adipocyte formation. ${ }^{33}$

Few human trials have been conducted to date to assess the effect of fish oil supplementation in early life on body composition later in life and have shown limited support for a benefit. ${ }^{34}$ Likewise, birth cohort studies on early n-3 LCPUFA exposure and later adiposity have produced discrepant results. ${ }^{35,36}$ Table 6.2 provides an overview of prospective studies assessing fish intake during pregnancy and child somatic growth. In the Project Viva cohort, Donahue et $\mathrm{al}^{35}$ demonstrated that higher mid-pregnancy fish intake was associated with lower odds of obesity at age 3. In contrast, the Dutch Prevention and Incidence of Asthma and Mite Allergy (PIAMA) birth cohort study failed to find an effect of maternal fish consumption on child BMI values from birth up to 14 years of age. ${ }^{37}$ Reasons for the divergent results may be small sample sizes, exposure heterogeneity, or differences in adjustment.

Recently, we harmonized and pooled individual data of repeated follow-ups until the age of 6 years from 26184 pregnant women and their children participating in 15 European and US cohort studies to assess the strength and consistency of the associations of fish intake during pregnancy with BMI growth trajectories and the risk of childhood overweight and obesity. ${ }^{38}$ We found that children of mothers consuming fish more than 3 times a week during pregnancy exhibited consistently higher BMI values from infancy up to age 6 than did those of mothers with an intake of less than 1 time a week (Figure 6.2). High fish intake during pregnancy was also associated with an increased risk of rapid infant growth from birth to 2 years, and increased risk of offspring overweight or obesity at 4 years and 6 years of age. Results indicated a non-detrimental effect of fish consumption of more than 1 time but less than 3 times per week on childhood somatic growth. ${ }^{38}$ 


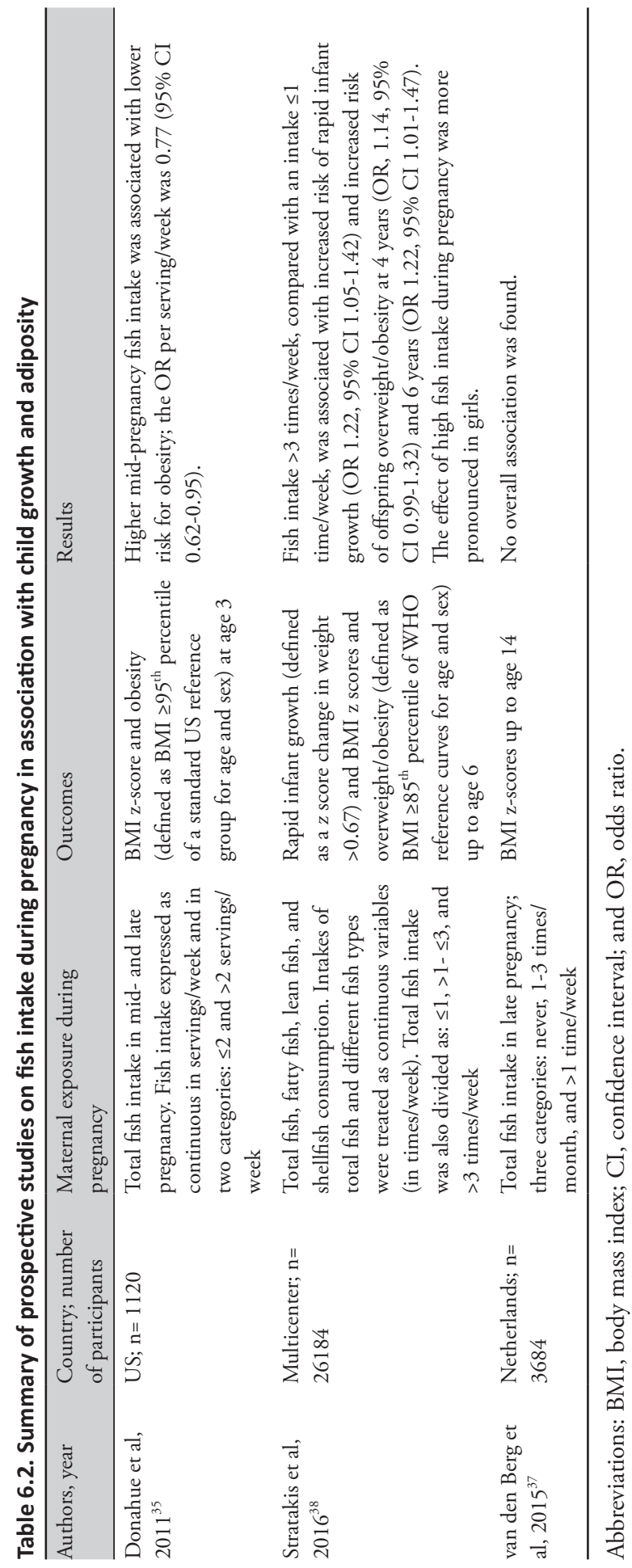




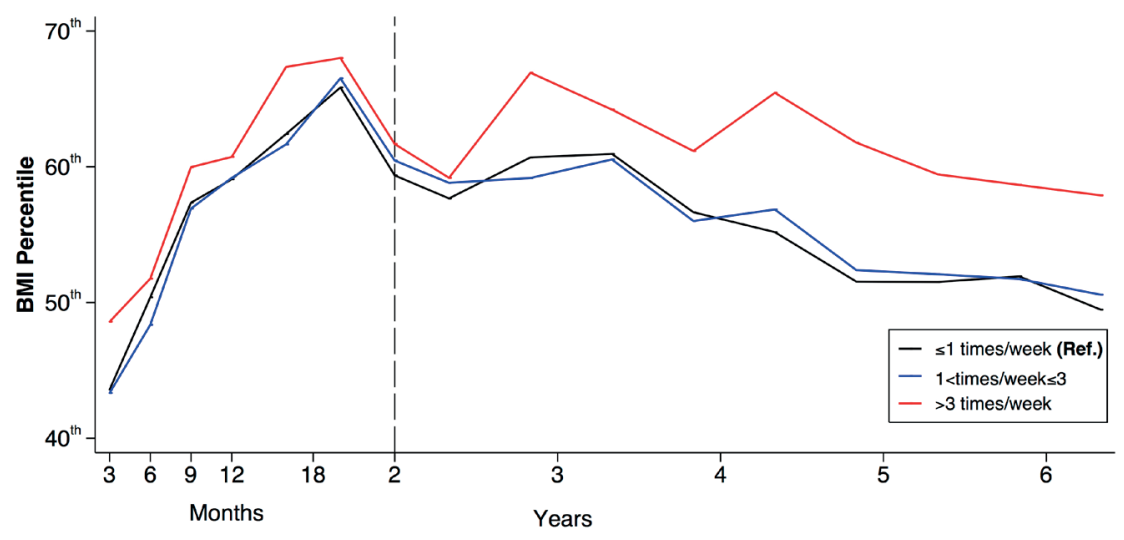

Child's age

Figure 6.2. Body mass index (BMI) percentile trajectories from 3 months to 6 years according to different levels of fish intake in pregnancy: a pooled analysis of 15 European and US birth cohort studies. BMI percentile values indicate the place of children in the corresponding growth chart of the WHO reference population, and were derived by using mixed-effects linear regression models fitted with: fish intake, an interaction term for fish intake and child age, maternal age, maternal education, pre-pregnancy BMI, smoking during pregnancy, and birth weight as fixed-effects parameters; random cohort and child intercepts; and a random slope for child age. Reproduced from JAMA Pediatr 2016 Apr;170(4):381-90.

Contamination by environmental pollutants in fish could provide an explanation for the association between high fish intake in pregnancy and increased childhood adiposity. Mixtures of organochlorine pesticides, PCBs, and dioxins found in fish have been shown to increase fat storage in cultured adipocytes, as well as weight gain in animals. ${ }^{39}$ It has been proposed that these toxicants may perturb signalling of several nuclear receptors, and through altered gene expression, influence adipocyte differentiation and fat metabolism. ${ }^{40}$ Many cohort studies, ${ }^{41}$ but not all, ${ }^{42}$ have shown that exposure to these pollutants during the intrauterine period is associated with an increased risk of childhood overweight or obesity.

Notably, none of the studies of maternal fish intake controlled for, or took into account, related exposure to persistent organic pollutants. Hence, the hypothesis that contaminants contained with fish may play a role in the influence of prenatal fish intake on later somatic growth remains speculative. Further studies that use direct measurements of fat mass and incorporate biomarker information on both the amounts of fatty acids and environmental chemicals potentially contained within fish will contribute to a clearer picture of the influence of fish intake during pregnancy on child growth. 


\section{FISH AND NEURODEVELOPMENT IN CHILDHOOD}

The effect of prenatal nutrition on later brain and cognitive development is receiving increasing attention. Most of the scientific literature regarding fish consumption during pregnancy and later neurodevelopment has placed emphasis on the opposing neurodevelopmental effects of $\mathrm{n}-3$ LCPUFAs and $\mathrm{MeHg}$ contained within fish.

n-3 LCPUFAs, especially DHA, are important components of neural membranes and have several influences on brain development. ${ }^{43}$ They seem to affect membrane fluidity and speed of signal transmission, gene expression, and neurogenesis. ${ }^{44}$ Accumulation of DHA in the human brain starts in utero, with marked deposition occurring in the later part of gestation when the brain undergoes a period of rapid growth. ${ }^{45}$ Hence, it has been hypothesized that increasing n-3 LCPUFA intake during pregnancy can benefit neurodevelopment of the offspring. Systematic reviews of randomized controlled trials have concluded that the evidence does not support or refute the hypothesis that $\mathrm{n}-3$ LCPUFA supplementation in pregnancy improves child cognitive or visual development (reviewed in ${ }^{46,47}$ ).

Animal studies have shown that ingested $\mathrm{MeHg}$ can cross the placenta and blood-brain barrier, ${ }^{48}$ and exert neurotoxic effects by promoting the production of free radicals and inhibiting antioxidant mechanisms through binding to thiolcontaining molecules. ${ }^{49}$ Reports from prospective studies of prenatal mercury exposure and neurodevelopment in infants are mixed, whereas, for older children, there has been a more consistent association with adverse neurodevelopmental outcomes (reviewed in ${ }^{22}$ ).

In terms of fish consumption, a dilemma arises of whether the potential beneficial effects of n-3 LCPUFAs on child neurocognitive development can counteract or outweigh the neurotoxic effects of MeHg. Several observational cohort studies have attempted to understand this benefit-risk trade-off (Table 6.3).

Few studies have assessed the association of prenatal fish intake with cognitive development in infancy, and they all showed positive results. In the DNBC cohort, Oken at $\mathrm{a}^{50}$ showed that higher fish consumption in mid-pregnancy was associated with increased scores of motor, social and cognitive developmental milestones at 6 and 18 months of age. Similarly, the Project Viva cohort study found that greater fish consumption during the second trimester of pregnancy was associated with higher infant scores on the visual recognition memory test (an assessment of visual memory that is correlated with later IQ) at 6 months of age. ${ }^{51}$ Additional adjustment for mercury levels strengthened the beneficial association of fish consumption with infant cognition. ${ }^{51}$ In the ALSPAC cohort, Daniels et $\mathrm{al}^{52}$ showed that fish consumption of more than 1 serving per week in pregnancy had modest but significant improvements in developmental scores of the offspring for language and communication skills at 15 and 18 months of age. This association remained 


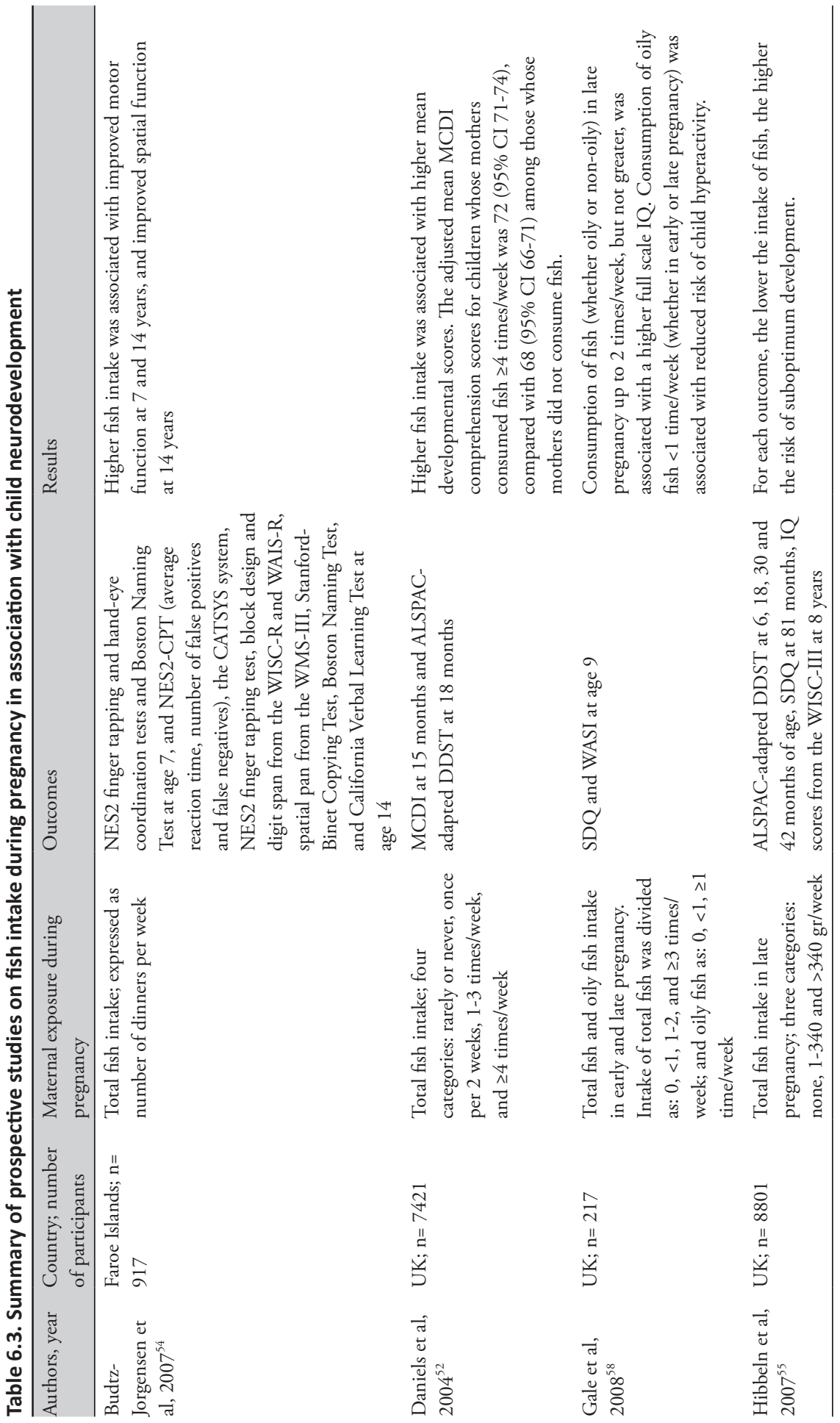




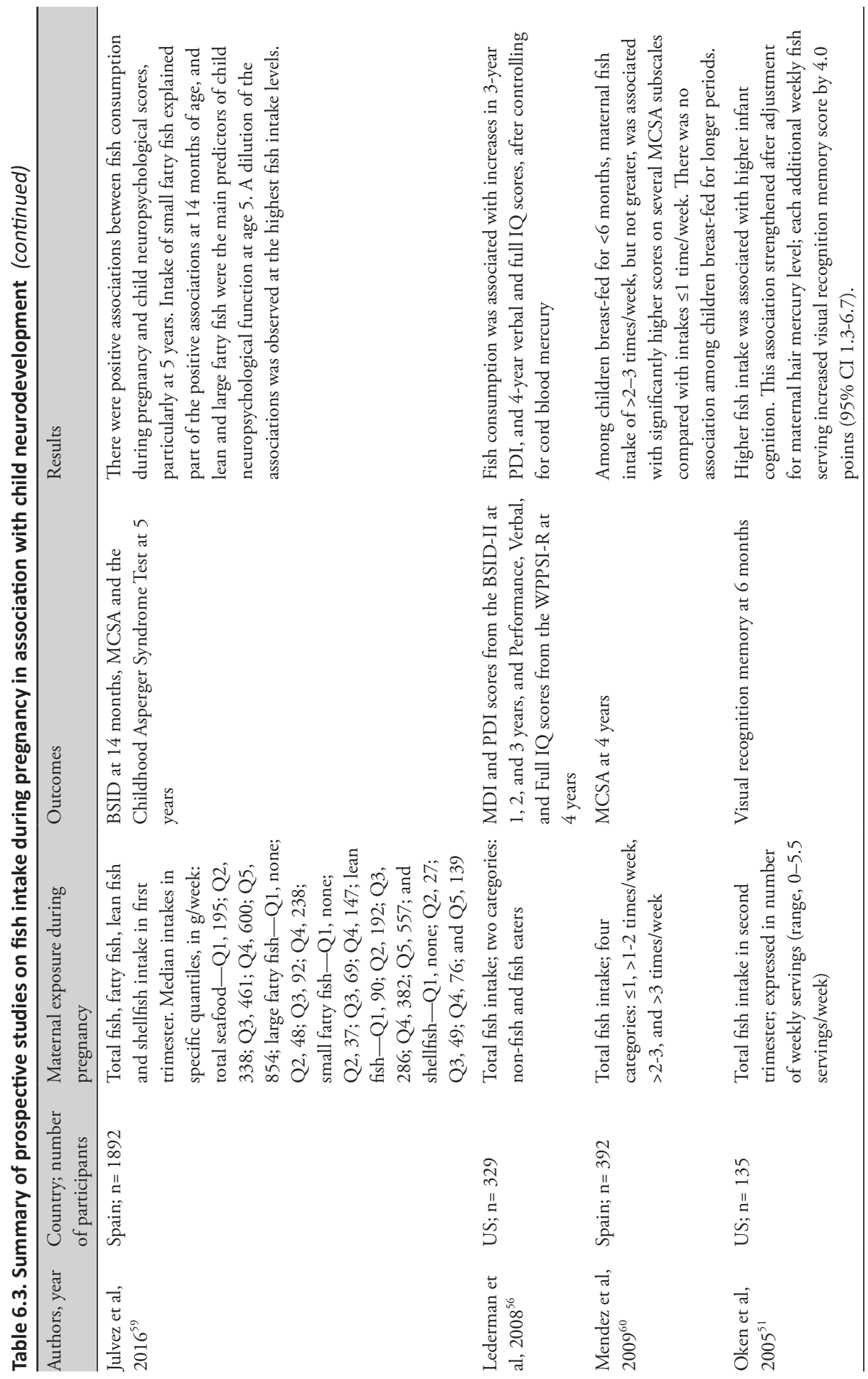




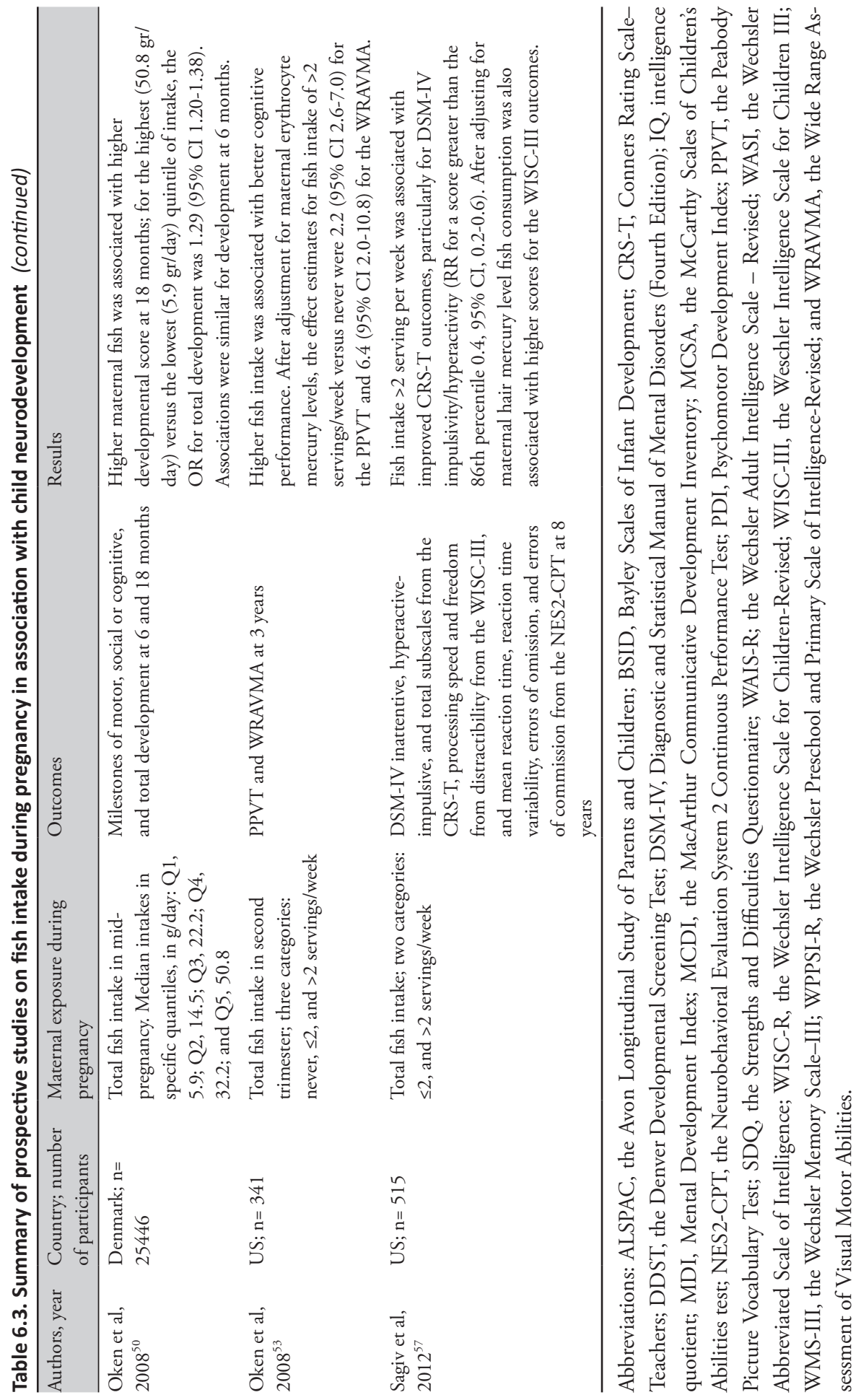


but was not strengthened when additional adjustment was made for cord blood mercury levels. ${ }^{52}$

Several studies have evaluated the effect of fish intake during pregnancy on various measures of cognitive performance in later childhood. Oken et al, ${ }^{53}$ in a follow-up of the Project Viva cohort at 3 years, showed that second-trimester fish intake of more than twice a week was associated with higher scores on tests of language and visual motor skills, with further adjustment for maternal erythrocyte mercury levels strengthening the estimates of the benefits. Similarly, four other studies reported an association of higher maternal fish intake with higher neurodevelopmental scores, ${ }^{54-56}$ and a lower risk for Attention-Deficit/Hyperactivity Disorderrelated behaviors in childhood. ${ }^{57}$ In three studies, moderate levels of fish intake during pregnancy were associated with improved child neurocognitive function, while a dilution of this benefit was observed at the highest intake levels ${ }^{58-60}$. To date, several observational cohort studies suggest that fish consumption during pregnancy can confer a neurodevelopmental benefit in the offspring. In some studies, there was a light attenuation of this benefit at the highest levels of fish consumption, which may be indicative of a counterbalancing association due to the potential harm of related contaminants. Findings from studies using biomarker information also indicate that women should avoid fish most highly contaminated with $\mathrm{MeHg}$ to gain the greatest possible benefit.

\section{FISH AND ALLERGIC DISEASES IN CHILDHOOD}

Black and Sharpe hypothesized that the observed increase in the prevalence of allergic disease in Western countries over the last decades has been preceded and then paralleled with a shift in the consumption of fatty acids towards a lower intake of $n-3$ LCPUFAs and fish and an increased intake of vegetable oils containing n- 6 fatty acids. ${ }^{61}$ In support of this hypothesis, animal and in vitro studies have shown that $\mathrm{n}-3$ LCPUFAs can exert anti-inflammatory properties and modulate immune responses. ${ }^{62}$. Intrauterine life is a critical period for the development of the immune system; hence, the role of prenatal n-3 LCPUFA and fish intakes in the etiology of allergic diseases has gained considerable interest. ${ }^{62}$ Many intervention studies, but not all, suggest a beneficial effect of fish oil supplementation during pregnancy on the incidence of allergic disease symptoms in childhood (reviewed in ${ }^{63}$ ). Similarly, findings from birth cohort studies assessing n-3 LCPUFA intake or biomarker levels in pregnancy and childhood allergic disease are discrepant, with reports of either beneficial (i.e. lower incidence) $)^{64,65}$ or null ${ }^{66,67}$ associations. Finding from prospective studies on the association of fish intake during pregnancy and the occurrence of eczema have been discrepant (Table 6.4). A Spanish 
study showed that increasing fish intake from once per week to 2.5 times per week in pregnancy was associated with a reduced risk of eczema among 12-month-yearold infants. ${ }^{68}$ Fish intake of more than 1-2 times per week in late pregnancy was inversely associated with parent reported, doctor diagnosed eczema at 2 years in a German study, ${ }^{69}$ and fish intake of once or more a week was associated with a decreased risk of doctor-confirmed eczema and currently treated eczema at 5 years in a UK study. ${ }^{70}$ In contrast to these results, studies conducted in Norway, ${ }^{71}$ France, ${ }^{72}$ Spain and Greece ${ }^{73}$ found no association of maternal fish intake with eczema occurrence. The Generation R study showed a harmful effect of first-trimester fatty fish and shellfish consumption (but not total or lean fish consumption) on eczema occurrence in the first 4 years of life. ${ }^{74}$ The authors speculated that potential toxicant contamination in these fish species could provide an explanation for their findings. $^{74}$

Three studies have assessed symptoms of childhood allergic rhinitis in association with fish intake in pregnancy (Table 6.4). One UK study found that higher maternal intake of oily fish intake was protective against hay fever at 5 years, ${ }^{70}$ while two studies conducted in Denmark ${ }^{75}$ and Finland ${ }^{76}$ found no association. Likewise, results from prospective studies assessing sensitization to inhalant and food allergens have been conflicting (Table 6.4). One Spanish study showed that an increase in maternal fish intake from once per week to 2.5 times per week during pregnancy was protective against sensitization to house dust mite at age 6 , while a study in Germany ${ }^{69}$ failed to find any effect. A French study showed that pre-parturition shellfish intake, but not total fish intake, at least once a month compared with a lower intake was associated with a higher risk of food allergy before age $2 .^{72}$

Several prospective studies have examined the association of fish intake during pregnancy with the occurrence of wheezing or asthma in childhood and have produced mixed results (Table 6.4). Fish intake of 2.5 times per week or more during pregnancy was associated with a reduced risk of wheezing in school-aged children in two separate Spanish studies. ${ }^{68,77}$ Similarly, the DNBC cohort study showed that children whose mothers never consumed fish in pregnancy were more likely to have a parent report of physician diagnosis of asthma at 18 months and clinically established asthma by the age of 7 years, as compared with those whose mothers consumed fish more than 2-3 times per week. ${ }^{75}$ In contrast, the Generation $\mathrm{R}$ study reported an association of first-trimester shellfish consumption, but not total, lean or fatty fish consumption, during pregnancy with wheezing occurrence by the age of 4 years. ${ }^{74}$ Other prospective studies conducted in the Netherlands, ${ }^{78}$ Finland, ${ }^{64,76}$ Norway, ${ }^{71}$ and France ${ }^{72}$ failed to find an association of prenatal fish consumption with the occurrence of wheeze or asthma in childhood. 


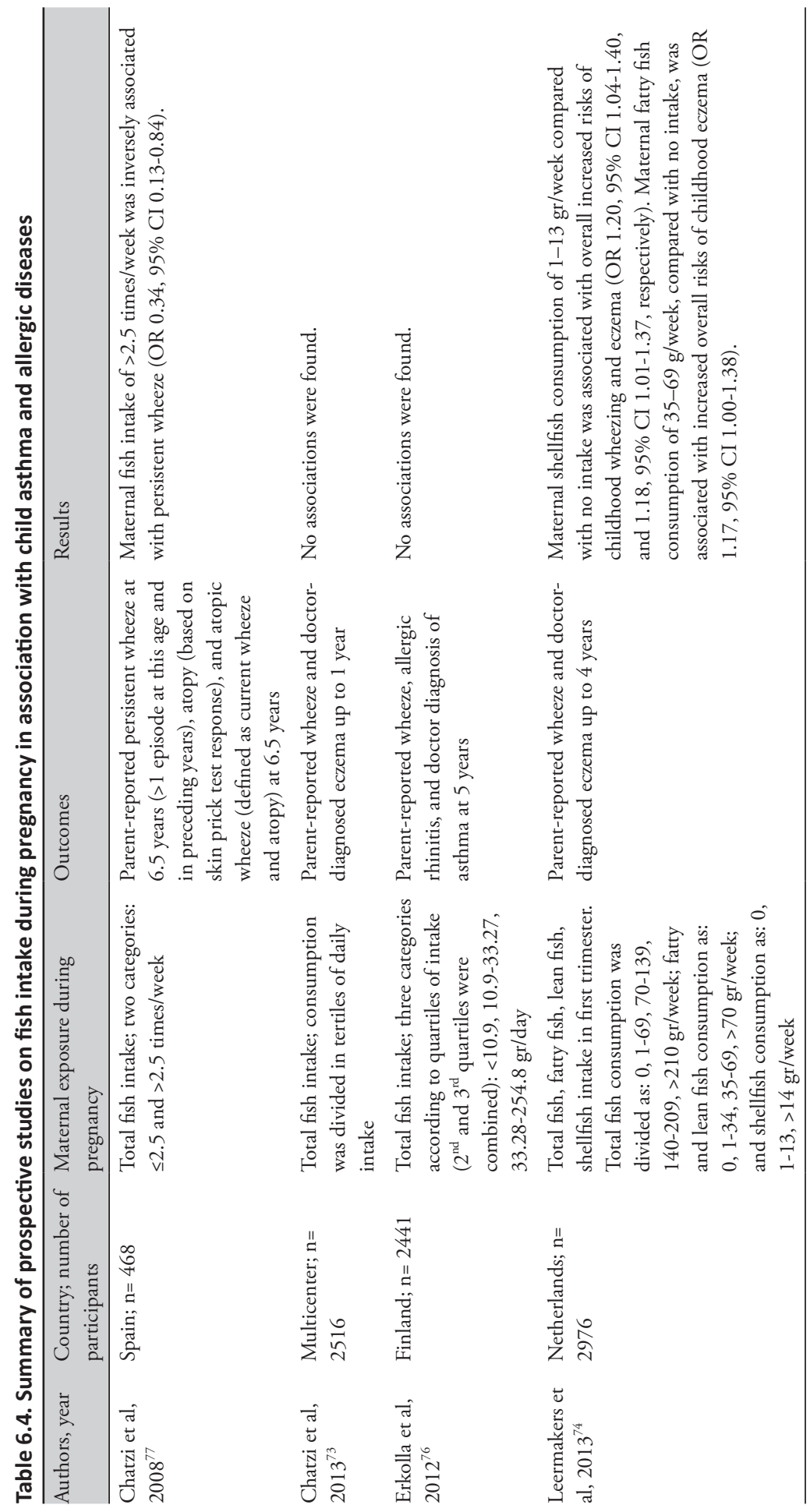




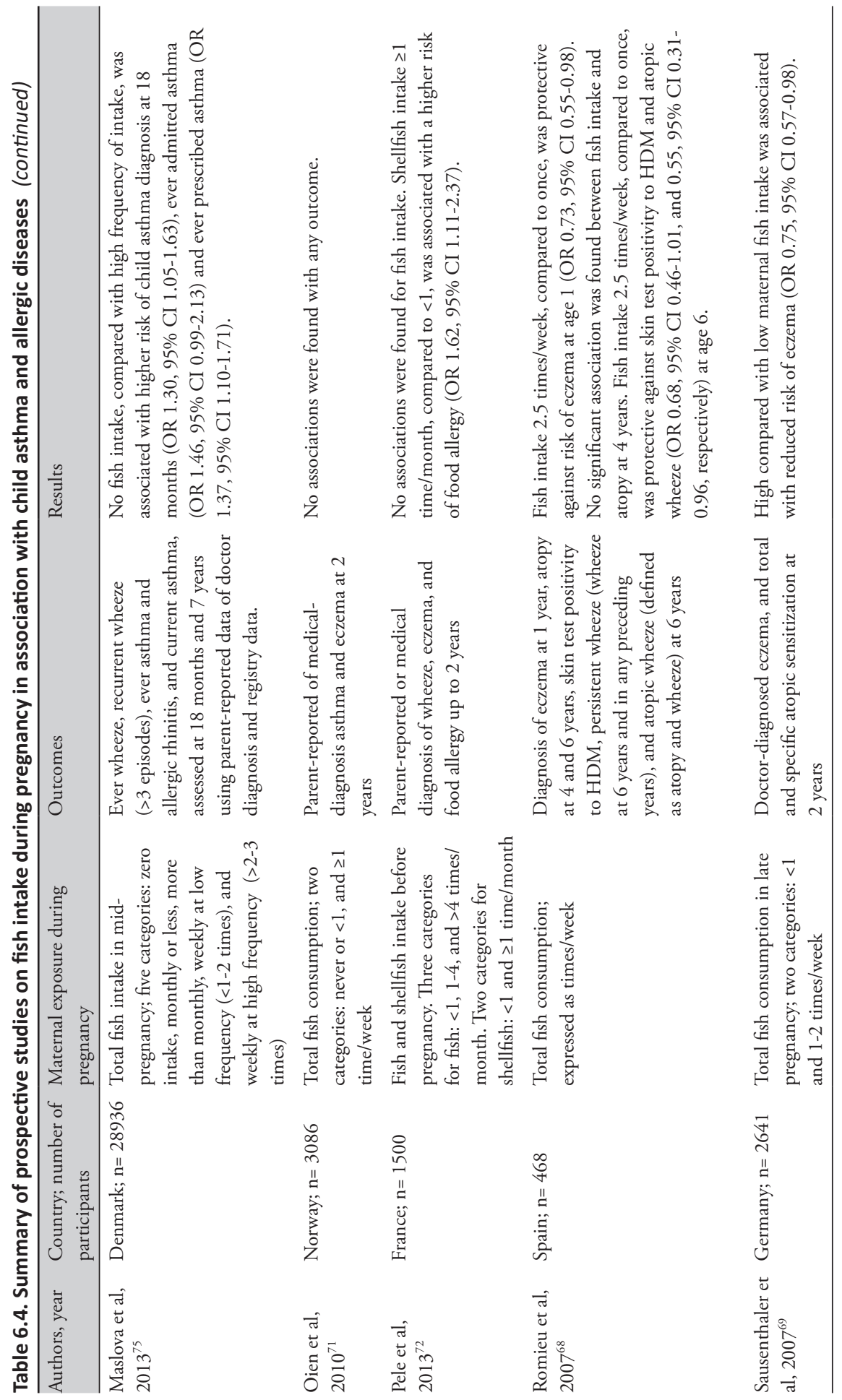




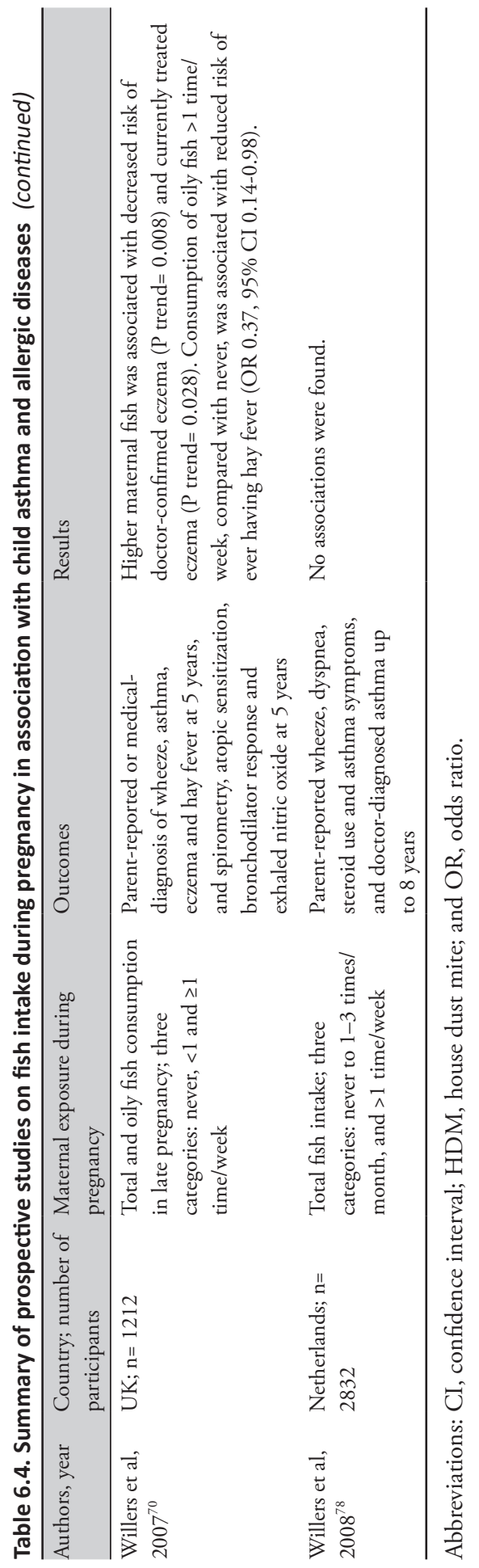


Inconsistencies in the results may be due to inadequate sample sizes, heterogeneity in exposure (eg, population level of fish consumption), exposure misclassification, or differences in adjustment for confounding variables. Most notably, none of the studies examining the impact of prenatal fish intake on allergic symptoms controlled for prenatal exposure to environmental pollutants. Emerging evidence suggests that persistent organic pollutants potentially contained within fish can exert adverse immunomodulatory effects and increase the risk of developing allergic disease symptoms. ${ }^{79,80}$

Currently, there is conflicting evidence on the association between maternal fish intake during pregnancy and childhood allergic diseases, with reports of beneficial, null or even harmful effects. Further large-scale epidemiological studies incorporating information on fish-related toxicant exposures are warranted before any conclusions can be drawn regarding the effects of prenatal fish intake on the developing respiratory and immune systems in children.

\section{CONCLUSION}

Fish provides n-3 LCPUFAs and other nutrients, but is also a common route of exposure to methylmercury and other pollutants. As a consequence, fish advisories generally suggest pregnant women to limit consumption up to 3-4 servings per week. Both nutrients and toxicants found in the same fish might act on the exact same end points at an opposite direction; it is therefore reasonable to presume that depending on the content of nutrients and pollutants, the health effect of a given fish type will vary. To date, several prospective studies have been conducted to assess the health effects of prenatal fish intake. Overall, the evidence on the association of maternal fish consumption during pregnancy with child health outcomes such as fetal growth, child neurodevelopment and the occurrence of allergic diseases has been largely inconsistent.

There is a need for well-designed intervention studies targeting maternal fish intake rather than supplement use, which may exert different mechanistic effects. To usefully inform policy, it is essential that future studies of maternal fish consumption assess and carefully account for fetal exposure to contaminants. 


\section{REFERENCES}

1. Gluckman PD, Hanson MA, Cooper C, et al. Effect of in utero and early-life conditions on adult health and disease. N Engl J Med 2008;359:61-73.

2. Gil A, Gil F. Fish, a Mediterranean source of n-3 PUFA: benefits do not justify limiting consumption. Br J Nutr 2015;113 Suppl 2:S58-67.

3. Food and Agriculture Organization of the United Nations (FAO) and World Health Organization (WHO). Joint FAO/WHO Expert Consultation on the Risks and Benefits of Fish Consumption FAO Fisheries and Aquaculture Report no. 978. Roma/Geneva: FAO/WHO.2010.

4. Fish: What Pregnant Women and Parents Should Know. Draft Updated Advice by FDA and EPA. June 2014. http://www.fda.gov/Food/FoodborneIllnessContaminants/Metals/ ucm393070.htm. (February 26 2016, date last accessed)

5. EFSA Scientific Committee. Statement on the benefits of fish/seafood consumption compared to the risks of methylmercury in fish/seafood. EFSA Journal 2015;13:3982.

6. McIntire DD, Bloom SL, Casey BM, et al. Birth weight in relation to morbidity and mortality among newborn infants. New England Journal of Medicine 1999;340:1234-8.

7. Barker DJP. Fetal Origins of Coronary Heart-Disease. British Medical Journal 1995;311:171-4.

8. Barker DJ, Hales CN, Fall CH, et al. Type 2 (non-insulin-dependent) diabetes mellitus, hypertension and hyperlipidaemia (syndrome X): relation to reduced fetal growth. Diabetologia 1993;36:62-7.

9. Beck S, Wojdyla D, Say L, et al. The worldwide incidence of preterm birth: a systematic review of maternal mortality and morbidity. Bull World Health Organ 2010;88:31-8.

10. Olsen SF, Hansen HS, Sorensen TI, et al. Intake of marine fat, rich in (n-3)-polyunsaturated fatty acids, may increase birthweight by prolonging gestation. Lancet 1986;2:367-9.

11. Hansen HS, Olsen SF. Dietary (n-3)-fatty acids, prostaglandins, and prolonged gestation in humans. Prog Clin Biol Res 1988;282:305-17.

12. Sorensen JD, Olsen SF, Pedersen AK, et al. Effects of fish oil supplementation in the third trimester of pregnancy on prostacyclin and thromboxane production. Am J Obstet Gynecol 1993;168:915-22.

13. Makrides M, Duley L, Olsen SF. Marine oil, and other prostaglandin precursor, supplementation for pregnancy uncomplicated by pre-eclampsia or intrauterine growth restriction. Cochrane Database Syst Rev 2006:CD003402.

14. Saccone G, Berghella V. Omega-3 long chain polyunsaturated fatty acids to prevent preterm birth: a systematic review and meta-analysis. Obstet Gynecol 2015;125:663-72.

15. Rogers I, Emmett P, Ness A, et al. Maternal fish intake in late pregnancy and the frequency of low birth weight and intrauterine growth retardation in a cohort of British infants. $J$ Epidemiol Community Health 2004;58:486-92.

16. Olsen SF, Secher NJ. Low consumption of seafood in early pregnancy as a risk factor for preterm delivery: prospective cohort study. BMJ 2002;324:447.

17. Oken E, Kleinman KP, Olsen SF, et al. Associations of seafood and elongated n-3 fatty acid intake with fetal growth and length of gestation: results from a US pregnancy cohort. Am J Epidemiol 2004; 160:774-83.

18. Halldorsson TI, Meltzer HM, Thorsdottir I, et al. Is high consumption of fatty fish during pregnancy a risk factor for fetal growth retardation? A study of 44,824 Danish pregnant women. Am J Epidemiol 2007;166:687-96.

19. Brantsaeter AL, Birgisdottir BE, Meltzer HM, et al. Maternal seafood consumption and infant birth weight, length and head circumference in the Norwegian Mother and Child Cohort Study. Br J Nutr 2012;107:436-44. 
20. Ramon R, Ballester F, Aguinagalde X, et al. Fish consumption during pregnancy, prenatal mercury exposure, and anthropometric measures at birth in a prospective mother-infant cohort study in Spain. Am J Clin Nutr 2009;90:1047-55.

21. Lee DH, Jacobs DR. Inconsistent epidemiological findings on fish consumption may be indirect evidence of harmful contaminants in fish. Journal of Epidemiology and Community Health 2010;64:190-2.

22. Karagas MR, Choi AL, Oken E, et al. Evidence on the human health effects of low-level methylmercury exposure. Environ Health Perspect 2012;120:799-806.

23. Vafeiadi M, Vrijheid M, Fthenou E, et al. Persistent organic pollutants exposure during pregnancy, maternal gestational weight gain, and birth outcomes in the mother-child cohort in Crete, Greece (RHEA study). Environ Int 2014;64:116-23.

24. Halldorsson TI, Thorsdottir I, Meltzer HM, et al. Linking exposure to polychlorinated biphenyls with fatty fish consumption and reduced fetal growth among Danish pregnant women: a cause for concern? Am J Epidemiol 2008;168:958-65.

25. Govarts E, Nieuwenhuijsen M, Schoeters G, et al. Birth weight and prenatal exposure to polychlorinated biphenyls (PCBs) and dichlorodiphenyldichloroethylene (DDE): a meta-analysis within 12 European Birth Cohorts. Environ Health Perspect 2012;120:162-70.

26. Farhang L, Weintraub JM, Petreas M, et al. Association of DDT and DDE with birth weight and length of gestation in the Child Health and Development Studies, 1959-1967. Am J Epidemiol 2005;162:71725.

27. Khanjani N, Sim MR. Maternal contamination with dichlorodiphenyltrichloroethane and reproductive outcomes in an Australian population. Environ Res 2006;101:373-9.

28. Leventakou V, Roumeliotaki T, Martinez D, et al. Fish intake during pregnancy, fetal growth, and gestational length in 19 European birth cohort studies. Am J Clin Nutr 2014;99:506-16.

29. Gluckman P, Nishtar S, Armstrong T. Ending childhood obesity: a multidimensional challenge. Lancet 2015;385:1048-50.

30. Singh AS, Mulder C, Twisk JW, et al. Tracking of childhood overweight into adulthood: a systematic review of the literature. Obes Rev 2008;9:474-88.

31. Gillman MW, Ludwig DS. How early should obesity prevention start? N Engl J Med 2013;369:2173-5.

32. Symonds ME, Mostyn A, Pearce S, et al. Endocrine and nutritional regulation of fetal adipose tissue development. J Endocrinol 2003;179:293-9.

33. Ailhaud G, Massiera F, Weill P, et al. Temporal changes in dietary fats: role of n-6 polyunsaturated fatty acids in excessive adipose tissue development and relationship to obesity. Prog Lipid Res 2006;45:20336.

34. Stratakis N, Gielen M, Chatzi L, et al. Effect of maternal n-3 long-chain polyunsaturated fatty acid supplementation during pregnancy and/or lactation on adiposity in childhood: a systematic review and meta-analysis of randomized controlled trials. Eur J Clin Nutr 2014;68:1277-87.

35. Donahue SMA, Rifas-Shiman SL, Gold DR, et al. Prenatal fatty acid status and child adiposity at age 3 y: results from a US pregnancy cohort. American Journal of Clinical Nutrition 2011;93:780-8.

36. Moon RJ, Harvey NC, Robinson SM, et al. Maternal Plasma Polyunsaturated Fatty Acid Status in Late Pregnancy Is Associated with Offspring Body Composition in Childhood. Journal of Clinical Endocrinology \& Metabolism 2013;98:299-307.

37. van den Berg SW, Wijga AH, van Rossem L, et al. Maternal fish consumption during pregnancy and BMI in children from birth up to age 14 years: the PIAMA cohort study. Eur J Nutr 2015.

38. Stratakis N, Roumeliotaki T, Oken E, et al. Fish Intake in Pregnancy and Child Growth: A Pooled Analysis of 15 European and US Birth Cohorts. JAMA Pediatr 2016. 
39. Ibrahim MM, Fjaere E, Lock EJ, et al. Chronic Consumption of Farmed Salmon Containing Persistent Organic Pollutants Causes Insulin Resistance and Obesity in Mice. Plos One 2011;6.

40. Grun F, Blumberg B. Endocrine disrupters as obesogens. Molecular and Cellular Endocrinology 2009;304:19-29.

41. Vafeiadi M, Georgiou V, Chalkiadaki G, et al. Association of Prenatal Exposure to Persistent Organic Pollutants with Obesity and Cardiometabolic Traits in Early Childhood: The Rhea Mother-Child Cohort (Crete, Greece). Environ Health Perspect 2015;123:1015-21.

42. Cupul-Uicab LA, Klebanoff MA, Brock JW, et al. Prenatal exposure to persistent organochlorines and childhood obesity in the US collaborative perinatal project. Environ Health Perspect 2013;121:1103-9.

43. Hadders-Algra M. Prenatal and early postnatal supplementation with long-chain polyunsaturated fatty acids: neurodevelopmental considerations. Am J Clin Nutr 2011;94:1874S-9S.

44. Uauy R, Mena P, Rojas C. Essential fatty acids in early life: structural and functional role. Proc Nutr Soc 2000;59:3-15.

45. Martinez M. Tissue levels of polyunsaturated fatty acids during early human development. J Pediatr 1992;120:S129-38.

46. Gould JF, Smithers LG, Makrides M. The effect of maternal omega-3 (n-3) LCPUFA supplementation during pregnancy on early childhood cognitive and visual development: a systematic review and metaanalysis of randomized controlled trials. Am J Clin Nutr 2013;97:531-44.

47. Campoy C, Escolano-Margarit MV, Anjos T, et al. Omega 3 fatty acids on child growth, visual acuity and neurodevelopment. Br J Nutr 2012;107 Suppl 2:S85-106.

48. Bridges CC, Zalups RK. Transport of inorganic mercury and methylmercury in target tissues and organs. J Toxicol Environ Health B Crit Rev 2010;13:385-410.

49. Fretham SJB, Caito S, Martinez-Finley EJ, et al. Mechanisms and modifiers of methylmercury-induced neurotoxicity. Toxicology Research 2012;1:32-8.

50. Oken E, Osterdal ML, Gillman MW, et al. Associations of maternal fish intake during pregnancy and breastfeeding duration with attainment of developmental milestones in early childhood: a study from the Danish National Birth Cohort. Am J Clin Nutr 2008;88:789-96.

51. Oken E, Wright RO, Kleinman KP, et al. Maternal fish consumption, hair mercury, and infant cognition in a US cohort. Environmental Health Perspectives 2005;113:1376-80.

52. Daniels JL, Longnecker MP, Rowland AS, et al. Fish intake during pregnancy and early cognitive development of offspring. Epidemiology 2004;15:394-402.

53. Oken E, Radesky JS, Wright RO, et al. Maternal fish intake during pregnancy, blood mercury levels, and child cognition at age 3 years in a US cohort. American Journal of Epidemiology 2008;167:1171-81.

54. Budtz-Jorgensen E, Grandjean P, Weihe P. Separation of risks and benefits of seafood intake. Environ Health Perspect 2007;115:323-7.

55. Hibbeln JR, Davis JM, Steer C, et al. Maternal seafood consumption in pregnancy and neurodevelopmental outcomes in childhood (ALSPAC study): an observational cohort study. Lancet 2007;369:57885.

56. Lederman SA, Jones RL, Caldwell KL, et al. Relation between cord blood mercury levels and early child development in a World Trade Center cohort. Environmental Health Perspectives 2008;116:1085-91.

57. Sagiv SK, Thurston SW, Bellinger DC, et al. Prenatal exposure to mercury and fish consumption during pregnancy and attention-deficit/hyperactivity disorder-related behavior in children. Arch Pediatr Adolesc Med 2012;166:1123-31.

58. Gale CR, Robinson SM, Godfrey KM, et al. Oily fish intake during pregnancy--association with lower hyperactivity but not with higher full-scale IQ in offspring. J Child Psychol Psychiatry 2008;49:1061-8. 
59. Julvez J, Mendez M, Fernandez-Barres S, et al. Maternal Consumption of Seafood in Pregnancy and Child Neuropsychological Development: A Longitudinal Study Based on a Population With High Consumption Levels. Am J Epidemiol 2016;183:169-82.

60. Mendez MA, Torrent M, Julvez J, et al. Maternal fish and other seafood intakes during pregnancy and child neurodevelopment at age 4 years. Public Health Nutr 2009;12:1702-10.

61. Black PN, Sharpe S. Dietary fat and asthma: is there a connection? Eur Respir J 1997;10:6-12.

62. Calder PC, Kremmyda LS, Vlachava M, et al. Is there a role for fatty acids in early life programming of the immune system? Proc Nutr Soc 2010;69:373-80.

63. Best KP, Gold M, Kennedy D, et al. Omega-3 long-chain PUFA intake during pregnancy and allergic disease outcomes in the offspring: a systematic review and meta-analysis of observational studies and randomized controlled trials. Am J Clin Nutr 2016;103:128-43.

64. Lumia M, Luukkainen P, Tapanainen $\mathrm{H}$, et al. Dietary fatty acid composition during pregnancy and the risk of asthma in the offspring. Pediatric Allergy and Immunology 2011;22:827-35.

65. Pike KC, Calder PC, Inskip HM, et al. Maternal Plasma Phosphatidylcholine Fatty Acids and Atopy and Wheeze in the Offspring at Age of 6 Years. Clinical \& Developmental Immunology 2012.

66. Newson RB, Shaheen SO, Henderson AJ, et al. Umbilical cord and maternal blood red cell fatty acids and early childhood wheezing and eczema. J Allergy Clin Immunol 2004;114:531-7.

67. Standl M, Demmelmair H, Koletzko B, et al. Cord blood LC-PUFA composition and allergic diseases during the first 10 yr. Results from the LISAplus study. Pediatr Allergy Immunol 2014;25:344-50.

68. Romieu I, Torrent M, Garcia-Esteban R, et al. Maternal fish intake during pregnancy and atopy and asthma in infancy. Clin Exp Allergy 2007;37:518-25.

69. Sausenthaler S, Koletzko S, Schaaf B, et al. Maternal diet during pregnancy in relation to eczema and allergic sensitization in the offspring at 2 y of age. American Journal of Clinical Nutrition 2007;85:5307.

70. Willers SM, Devereux G, Craig LC, et al. Maternal food consumption during pregnancy and asthma, respiratory and atopic symptoms in 5-year-old children. Thorax 2007;62:773-9.

71. Oien T, Storro O, Johnsen R. Do early intake of fish and fish oil protect against eczema and doctordiagnosed asthma at 2 years of age? A cohort study. J Epidemiol Community Health 2010;64:124-9.

72. Pele F, Bajeux E, Gendron H, et al. Maternal fish and shellfish consumption and wheeze, eczema and food allergy at age two: a prospective cohort study in Brittany, France. Environ Health 2013;12:102.

73. Chatzi L, Garcia R, Roumeliotaki T, et al. Mediterranean diet adherence during pregnancy and risk of wheeze and eczema in the first year of life: INMA (Spain) and RHEA (Greece) mother-child cohort studies. Br J Nutr 2013;110:2058-68.

74. Leermakers ET, Sonnenschein-van der Voort AM, Heppe DH, et al. Maternal fish consumption during pregnancy and risks of wheezing and eczema in childhood: the Generation R Study. Eur J Clin Nutr 2013;67:353-9.

75. Maslova E, Strom M, Oken E, et al. Fish intake during pregnancy and the risk of child asthma and allergic rhinitis - longitudinal evidence from the Danish National Birth Cohort. Br J Nutr 2013;110:1313-25.

76. Erkkola M, Nwaru BI, Kaila M, et al. Risk of asthma and allergic outcomes in the offspring in relation to maternal food consumption during pregnancy: a Finnish birth cohort study. Pediatr Allergy Immunol 2012;23:186-94.

77. Chatzi L, Torrent M, Romieu I, et al. Mediterranean diet in pregnancy is protective for wheeze and atopy in childhood. Thorax 2008;63:507-13.

78. Willers SM, Wijga AH, Brunekreef B, et al. Maternal food consumption during pregnancy and the longitudinal development of childhood asthma. Am J Respir Crit Care Med 2008;178:124-31. 


\section{Chapter 6}

79. Gascon M, Sunyer J, Casas M, et al. Prenatal exposure to DDE and PCB 153 and respiratory health in early childhood: a meta-analysis. Epidemiology 2014;25:544-53.

80. Hansen S, Strom M, Olsen SF, et al. Maternal concentrations of persistent organochlorine pollutants and the risk of asthma in offspring: results from a prospective cohort with 20 years of follow-up. Environ Health Perspect 2014;122:93-9. 



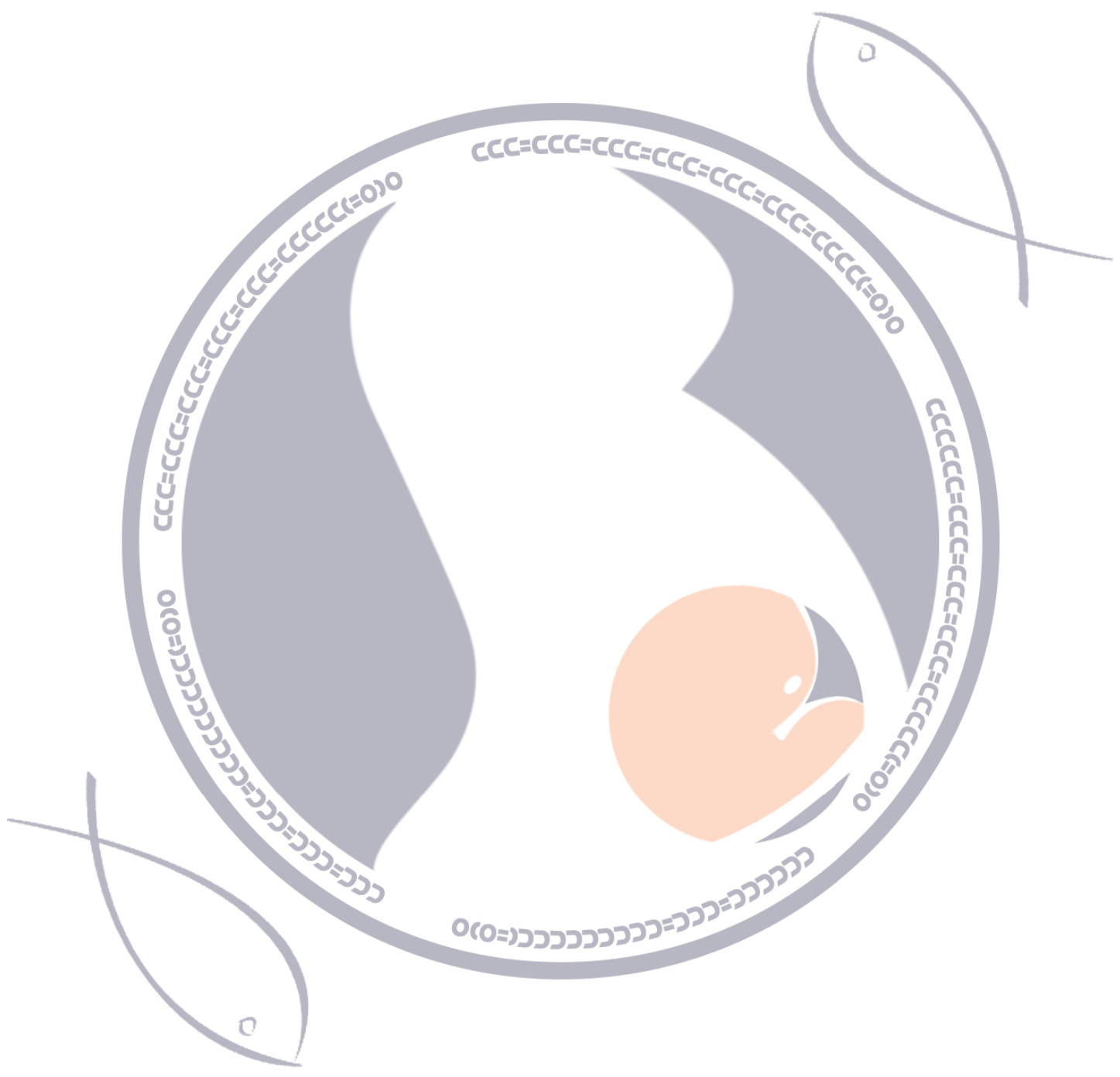




\section{CHAPTER 7}

\section{Fish intake in pregnancy and child growth: a pooled analysis of 15 European and US birth cohorts}

N Stratakis, T Roumeliotaki, E Oken, H Barros, M Basterrechea, MA Charles, M Eggesbø, F Forastiere, R Gaillard, U Gehring, E Govarts, W Hanke, B Heude, N Iszatt, VW Jaddoe, C Kelleher, M Mommers, M Murcia, A Oliveira, C Pizzi, K Polańska, D Porta, L Richiardi, SL Rifas-Shiman, G Schoeters, J Sunyer, C Thijs, K Viljoen, M Vrijheid, Tanja GM Vrijkotte, AH Wijga, MP Zeegers, M Kogevinas, L Chatzi

Adapted from JAMA Pediatr 2016 Apr;170(4):381-90

Fish intake during pregnancy and offspring adiposity-Reply

N Stratakis, MP Zeegers, L Chatzi

Adapted from JAMA Pediatr 2016 Aug;170(8):809-10 


\section{ABSTRACT}

Background: Maternal fish intake in pregnancy has been shown to influence fetal growth. The extent to which fish intake affects childhood growth and obesity remains unclear.

Objective: To examine whether fish intake in pregnancy is associated with offspring growth and the risk of childhood overweight and obesity.

Methods: We conducted a multicenter, population-based birth cohort study of singleton deliveries from 1996 to 2011 in Belgium, France, Greece, Ireland, Italy, the Netherlands, Norway, Poland, Portugal, Spain, and Massachusetts. A total of 26184 pregnant women and their children were followed up at 2-year intervals until the age of 6 years. We estimated offspring body mass index percentile trajectories from 3 months after birth to 6 years of age. We defined rapid infant growth as a weight gain $\mathrm{z}$ score greater than 0.67 from birth to 2 years and childhood overweight/obesity at 4 and 6 years as body mass index in the 85 th percentile or higher for age and sex. We calculated cohort-specific effect estimates and combined them by random-effects meta-analysis.

Results: The median fish intake during pregnancy ranged from 0.5 times/week in Belgium to 4.45 times/week in Spain. Women who ate fish more than 3 times/ week during pregnancy gave birth to offspring with higher body mass index values from infancy through middle childhood compared with women with lower fish intake (3 times/week or less). High fish intake during pregnancy ( $>3$ times/week) was associated with increased risk of rapid infant growth, with an adjusted odds ratio (aOR) of 1.22 [95\% CI 1.05-1.42] and increased risk of offspring overweight/obesity at 4 years $(\mathrm{aOR}=1.14$ [95\% CI $0.99-1.32])$ and 6 years $[\mathrm{aOR}=$ 1.22 (95\% CI 1.01-1.47]) compared with an intake of once per week or less. Interaction analysis showed that the effect of high fish intake during pregnancy on rapid infant growth was greater among girls ( $\mathrm{aOR}=1.31$ [95\% CI 1.08-1.59]) than among boys $(\mathrm{aOR}=1.11$ [95\% CI 0.92-1.34]; $\mathrm{P}=0.02$ for interaction).

Conclusions: High maternal fish intake during pregnancy was associated with increased risk of rapid growth in infancy and childhood obesity. Our findings are in line with the fish intake limit proposed by the US Food and Drug Administration and Environmental Protection Agency. 


\section{INTRODUCTION}

Early life is a critical period of developmental plasticity. A nutritional stressor or stimulus applied during critical periods of early development could permanently alter body physiology and metabolism, the consequences of which are often observed much later in life. ${ }^{1}$ Fish is the major dietary source of $\omega$-3 long-chain polyunsaturated fatty acids (LC-PUFAs), which are transferred across the placenta and may not only benefit offspring neurodevelopment but also influence adipose tissue development. ${ }^{2}$ However, fish is also a common source of human exposure to persistent organic pollutants, which may exert endocrine-disrupting properties and contribute to obesity development. ${ }^{3,4}$

In June 2014, the US Food and Drug Administration and Environmental Protection Agency updated their advice on fish consumption for women of childbearing age, encouraging women who are pregnant, breastfeeding, or likely to become pregnant to consume more fish, but no more than 3 servings/week to limit fetal exposure to methyl-mercury. ${ }^{5}$ Fish advisories have focused on neurocognitive harms from methyl-mercury exposure but, to our knowledge, have not considered other childhood outcomes including growth and childhood obesity, areas where evidence is limited.

In a large Europe-wide study (151880 mother-child pairs), ${ }^{6}$ we found that moderate fish intake during pregnancy was associated with a lower risk of preterm birth and a small but significant increase in birth weight. Few birth cohort studies have examined the association of fish intake in pregnancy with childhood adiposity and have yielded discrepant findings, with reports of either beneficial (ie, lower obesity) $)^{7}$ or null ${ }^{8}$ associations. Likewise, trials have not found a clear and consistent benefit of prenatal $\omega$-3 LC-PUFAs on obesity-related outcomes later in life. ${ }^{9}$ To our knowledge, there is no clear answer about the optimal amount and type of fish intake during pregnancy with regard to child growth and development.

In this study, we harmonized and pooled individual data of follow-ups 2-year intervals, until the age of 6 years, from 26184 pregnant women and their children participating in 15 European and US cohort studies to assess the strength and consistency of the associations of fish intake during pregnancy with body mass index (BMI) growth trajectories, calculated as weight in kilograms divided by height in meters squared, and the risk of childhood overweight and obesity. 


\section{METHODS}

\section{Study population}

European birth cohort studies participating in our previous analysis on fish intake in pregnancy and birth outcomes ${ }^{6}$ were invited to participate. From the 19 potentially eligible European cohorts, 14 cohorts provided relevant data for the present analysis. In addition, the Project Viva cohort from the US agreed to take part. All participating cohorts targeted the general population and, altogether, covered singleton deliveries from 1996 to 2011. All participating women provided informed consent for themselves and their children in the original cohort studies, and ethical approval was obtained from the local authorized institutional review boards. The Amsterdam Born Children and Their Development Study was approved by the Central Committee on Research Involving Human Subjects in The Netherlands, the medical ethics review committees of the participating hospitals, and the Registration Committee of the Municipality of Amsterdam. The Étude des Déterminants Pré et Postnatals du Développement et de la Santé de l'Enfant Study was approved by the Ethics Committee of Bicêtre Hospital and the National Commission on Data Processing and Liberties. The Flemish Center of Expertise on Environment and Health Study was approved by the Ethical Committee of the University of Antwerp and the Ghent University. The Genetica e Ambiente: Studio Prospettico dell'Infanzia in Italia Study was approved by the Ethics Committee of the Università Cattolica del Sacro Cuore in Rome. The Generation R Study was approved by by the Medical Ethical Committee of the Erasmus Medical Center in Rotterdam. The Generation XXI Study was approved by the Portuguese Data Protection Authority (Comissão Nacional de Protecção de Dados). The Norwegian Human Milk Study was approved by the Regional Ethics Committee for Medical Research in Norway and the Norwegian Data Inspectorate. The Infancia y Medio Ambiente Study was approved by the hospital ethics committees of each participating Spanish region. The Kind, Ouders en gezondheid: Aandacht voor Leefstijl en Aanleg Study was approved by the medical ethics committee of the Maastricht University/University Hospital of Maastricht. The Life-ways Cross Generation Study was approved by the ethics committees in the National University of Ireland, Galway; The Coombe Women's Hospital, Dublin; University College Hospital, Galway; and The Irish College of General Practitioners. The Nascita e INFanzia: gli Effetti dell'Ambiente Study was approved by the Ethical Committee of the San Giovanni Battista Hospital and CTO/CRF/Maria Adelaide Hospital of Turin. The Prevention and Incidence of Asthma and Mite Allergy Study by the the Medical Ethics Committees of the participating institutes the Dutch Central Committee on Research involving Human Subjects. The Project Viva Study by the human participants' committees of Harvard Pilgrim Health 
Care, Brigham, and Women's Hospital and Beth Israel Deaconess Medical Center. The Polish Mother and Child Cohort Study by the Ethical Committee of the Nofer Institute of Occupational Medicine in Lodz. The Mother Child Cohort Study in Crete by the Ethical Committee of the University Hospital of Heraklion A data-transfer agreement document was signed by each cohort study, and anonymized datasets were transferred to the University of Crete for analysis. Characteristics of the participating cohorts are shown in Supplementary Table S7.1 and Supplementary Figure S7.1.

\section{Fish intake during pregnancy}

The exposure of interest was the frequency (times/week) of total fish, fatty fish, lean fish, and seafood (other than fish) intake during pregnancy derived from cohortspecific food frequency questionnaires or questionnaires specifically designed to assess fish intake during pregnancy (Supplementary Methods and Supplementary Table S7.1).

To align our analysis with the recent US advice on fish intake during pregnancy, and examine a potential dose-response relationship, we categorized total fish intake into the following groups; low: $\leq 1$ time/week, moderate: $>1$ but $\leq 3$ times/week, and high: $>3$ times/week. Seven cohorts (the Polish Mother and Child Cohort Study, Flemish Center of Expertise on Environment and Health, Generation R Study, Generation XXI, Lifeways Cross Generation, Prevention and Incidence of Asthma and Mite Allergy, and the Mother Child Cohort in Crete) had less than $5 \%$ or 50 participants in at least 1 category and therefore were not included in the categorical dose-response analysis in an attempt to reduce the likelihood of conducting a type II error.

\section{Child growth and adiposity measures}

Cohorts provided information on child weight and height up to a maximum follow-up of 6 years, obtained from clinical examinations, medical records, or parental-completed questionnaires. Permissible intervals around the nominal ages were within 3 months for the first 2 years and within 6 months onwards. The time points of interest in childhood were the ages of 2 years, 4 years, and 6 years to reflect different developmental ages (toddler, preschooler, and school-aged child, respectively). Three cohorts (Infancia y Medio Ambiente, Mother Child Cohort in Crete, and the Polish Mother and Child Cohort Study) did not provide growth data at the age of 6 years owing to their relatively recent recruitment period. For each cohort, we constructed sex- and age-specific weight and height growth curves using mixed-effects linear regression models with fractional polynomials of age, including a random intercept for child and random age slopes (Supplementary 
Methods). Actual and predicted measurements were compared to assess model fit, and high levels of agreement were found (Supplementary Table S7.2). Predicted values were then used to calculate sex-and age-specific BMI $z$ scores and BMI percentile values based on the 2006 World Health Organization child growth standards for children 5 years and younger ${ }^{10}$ and 2007 World Health Organization growth references for children and adolescents aged 5 to 19 years (Supplementary Methods). ${ }^{11}$

We defined rapid infant growth from birth to 2 years as a $z$ score change in weight of greater than 0.67 and used the $z$ score change of 0.67 or less as the comparison. ${ }^{12,13}$ We analyzed child BMI $z$ score as a continuous outcome at 2 years, 4 years, and 6 years of age and in categories of overweight and obese (BMI $\geq 85$ th percentile for age and sex) at 4 and 6 years, compared with BMI below the 85 th percentile. $^{14}$

\section{Covariates}

Potential confounding variables were defined as similarly as possible among the cohorts. Information on maternal prepregnancy BMI (in weight in kilograms divided by height in meters squared), maternal smoking during pregnancy (yes or no), gestational weight gain (in kilograms), maternal age at delivery (in years), birth weight (in grams), and child sex (male or female) was collected by means of interviews or self-administered questionnaires, ad hoc measurements, birth records, or medical registries. Information on breastfeeding duration (in months) and maternal education (cohort-specific definitions of low, medium, or high) was obtained through interviews or self-administered questionnaires.

\section{Statistical analysis}

\section{Cohort-specific analyses}

We used linear regression models to examine the association of fish intake during pregnancy with BMI $z$ scores in childhood and logistic regression models for rapid infant growth and childhood overweight/obesity. To select the confounders for adjustment in multivariable models, we used a directed acyclic graph approach based on prior knowledge about parental and child covariates that may be related to child adiposity and/or fish intake in pregnancy. ${ }^{15}$ We constructed the graph using DAGitty version 2.1 (DAGitty) ${ }^{16}$ to identify minimally sufficient adjustment sets of covariates and chose the set on which we had the best available information (Supplementary Figure S7.2). We included the following variables in multivariable models: maternal education, maternal prepregnancy BMI, maternal smoking during pregnancy, maternal age at delivery, and birth weight. For the 
Lifeways Cross Generation cohort, the adjusted models did not include the full list of confounders owing to missing information on prepregnancy BMI.

\section{Meta-analysis and pooled analysis}

We combined cohort-specific effect estimates for each outcome of interest using random-effects meta-analysis in which the weight assigned to each study was based on both the within- and between-study variability. Heterogeneity among cohort-specific estimates was assessed with the $\chi^{2}$ test from the Cochran Q and $I^{2}$ statistic. ${ }^{17}$ To compare growth trajectories based on child BMI percentile values by category of maternal fish intake in pregnancy, we used a mixed-effects linear regression model fitted with random cohort and child intercepts and a random slope for child age. To take into account the potential age-varying effect of maternal fish intake, we added an interaction term for child age and fish intake as a fixed effect.

\section{Sensitivity analyses}

We performed several sensitivity analyses. First, we included all cohorts in the categorical dose-response analysis to examine whether the exclusion of cohorts with less than $5 \%$ or 50 participants in at least 1 fish intake category influenced the results. Second, we made further adjustment for gestational weight gain for cohorts with available information. Third, we calculated pooled-effect estimates for child outcomes using mixed-effects regression models with random intercepts for cohort and geographical location according to the United Nations' classification (Eastern Europe, Southern Europe, Western Europe, Northern Europe, and Northern America). Fourth, we calculated pooled-effect estimates using mixedeffects regression models with random intercepts for cohort and recruitment period (recruitment before and after 2004-2005). Fifth, we repeated analyses after removing birth weight from all multivariable models. Sixth, we examined the associations of fish intake variables with childhood obesity (BMI $\geq 95$ th percentile for age and sex). Finally, we assessed whether the effect estimates for childhood outcomes varied by child sex (boy vs girl), prepregnancy BMI ( $\geq 25$ vs $<25$ ), maternal smoking during pregnancy (yes vs no), timing of dietary assessment (first, second, or third trimester in pregnancy), low birth weight ( $<2500$ vs $\geq 2500 \mathrm{~g}$ ), and breastfeeding duration ( $>3$ vs $\leq 3$ months) by introducing interaction terms (one at a time). We performed analyses with STATA version 13 (StataCorp) and $\mathrm{R}$ version R3.1 (R Foundation). 


\section{RESULTS}

\section{Characteristics of the participants}

The total study population consisted of 26184 mothers and their offspring with follow-up from birth to 6 years of age. Participants' characteristics are presented in the Supplementary Tables S7.3 to S7.6 Mothers were predominantly older than 29 years, nonsmokers, had a normal prepregnancy BMI, and breastfed their children for more than 3 months. The ratio of boys to girls was 1.03 (Supplementary Table S7.3).

The median fish intake during pregnancy varied between study areas and ranged from 0.5 times/week in Belgium (Flemish Center of Expertise on Environment and Health) to 4.45 times/week in Spain (Infancia y Medio Ambiente) (Figure 7.1 and Supplementary Table S7.4). Compared with low and moderate fish consumers ( $\leq 3$ times/week), women with high fish intake ( $>3$ times/week) during pregnancy had a higher age at delivery in most of the cohorts (6 of 8 cohorts) and were less likely to have smoked during pregnancy. We did not observe any other clear pattern of difference for other sociodemographic characteristics (Supplementary Table S7.5).

In total, 8215 children (31.0\%) were rapid growers from birth to 2 years of age, with the prevalence across cohorts ranging from 17.3\% (Norwegian Human Milk Study, Norway) to $56.0 \%$ (Mother Child Cohort in Crete, Greece). Four thousand nine hundred eighty-seven (19.4\%) and 3476 children (15.2\%) were classified as overweight or obese at ages 4 and 6 years, respectively, while the cohort-specific prevalence ranged from 9.5\% (Kind, Ouders, en Gezondheid: Aandacht voor Leefstijl en Aanleg Birth Cohort Study, Netherlands) to 55.8\% (Lifeways Cross Generation, Ireland) at ages 4 years and from 6.4\% (Kind, Ouders, en Gezondheid: Aandacht voor Leefstijl en Aanleg Birth Cohort Study) to 36.7\% (Generation XXI, Portugal) at age 6 years (Supplementary Table S7.6). 


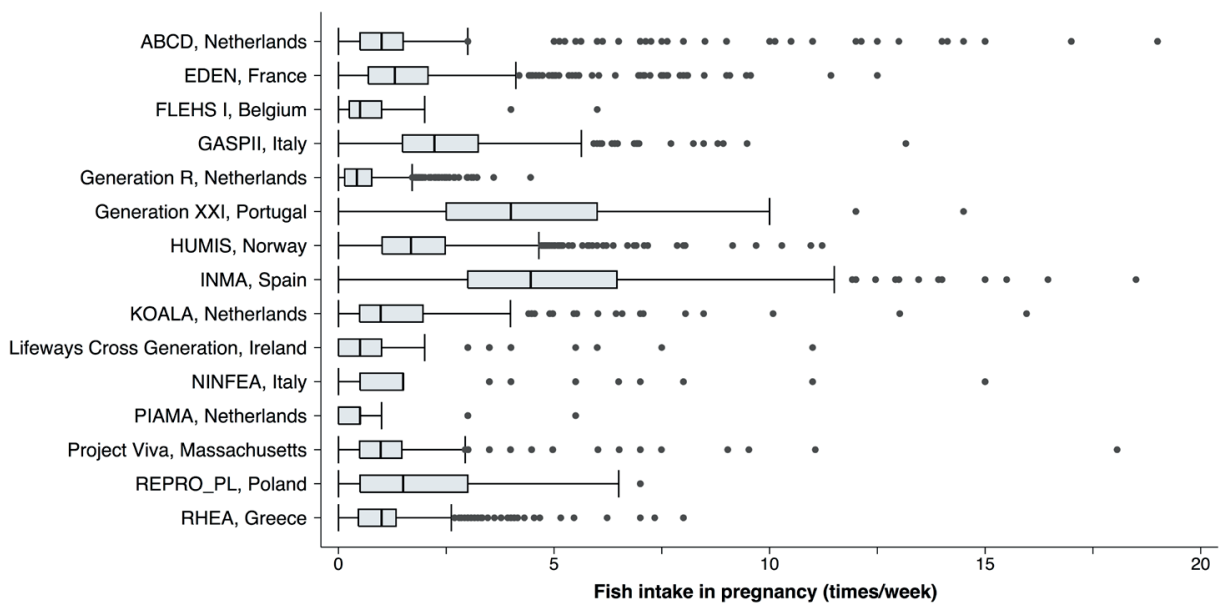

Figure 7.1. Frequency of fish intake in pregnancy (times/week) in participating cohorts. The line within the box marks the median; the boundaries of the box indicate the 25th and 75th percentiles; horizontal bars denote the variability outside the upper and lower quartiles (ie, within $\mathbf{1 . 5}$ IQR of the lower and upper quartiles); and circles represent outliers.

\section{Fish Intake during pregnancy and offspring BMI}

Women who ate fish more than 3 times/week during pregnancy gave birth to offspring with higher BMI values at 2 years, 4 years, and 6 years of age compared with women who rarely ate fish $(\leq 1$ time/week) (Table 7.1$)$. High fish intake during pregnancy was also associated with an increased risk of rapid infant growth from birth to 2 years (adjusted odds ratio $[\mathrm{aOR}]=1.22$ [95\% CI 1.05-1.42]) and increased risk of offspring overweight/obesity at 4 years $(\mathrm{aOR}=1.14[95 \% \mathrm{CI}$ $0.99-1.32])$ and 6 years of age $(\mathrm{aOR}=1.22$ [95\% CI 1.01-1.47]) (Table 7.2 and Figure 7.2). We did not find evidence for an association of moderate fish intake ( $>1$ but $\leq 3$ times/week) or consumption of different types of fish with child BMI and the risk of rapid infant growth and childhood overweight/obesity (Tables 7.1 and 7.2, Figure 7.2). The effect estimates from the pooled analyses were similar to those from random-effects meta-analyses (data not shown). 


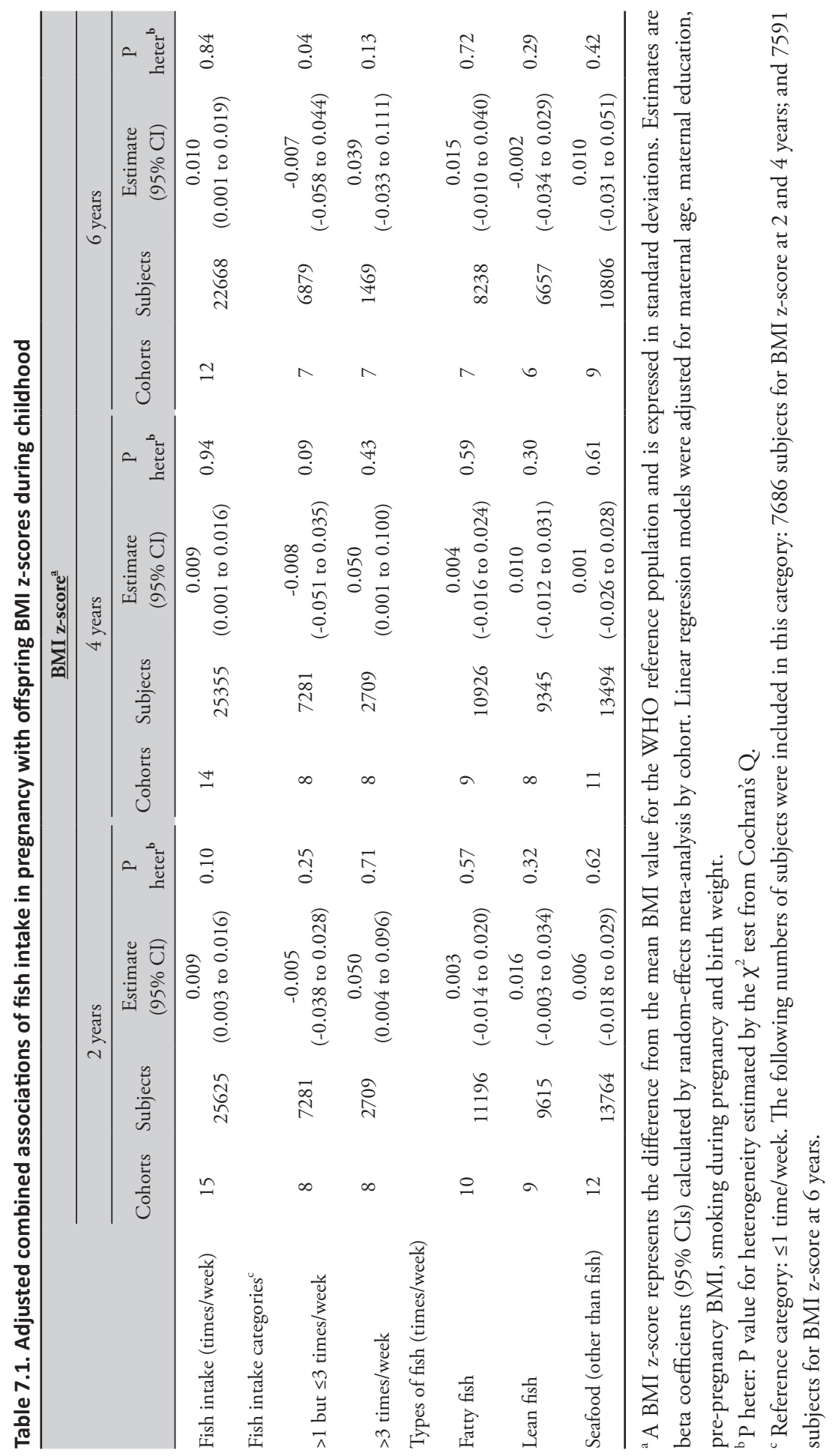




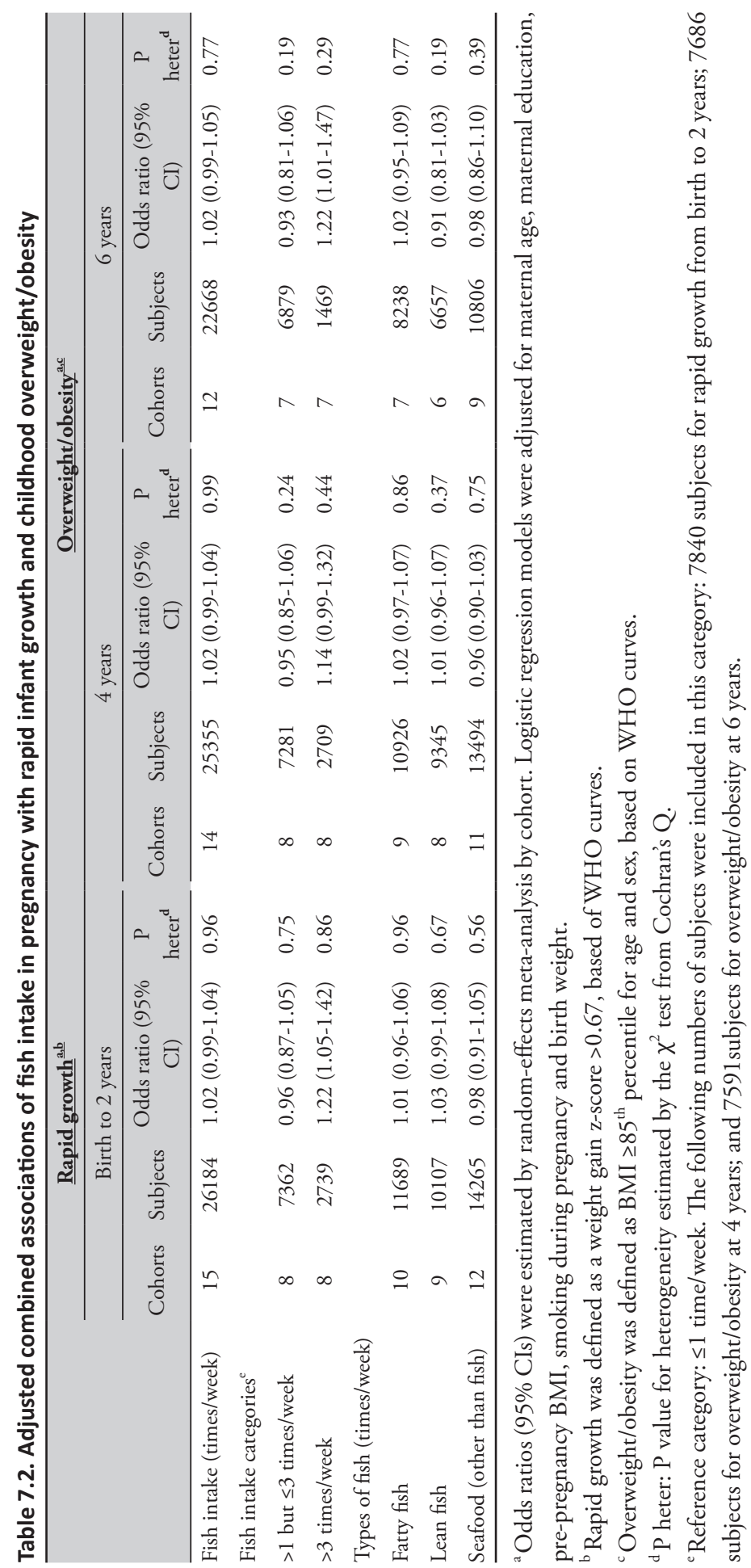




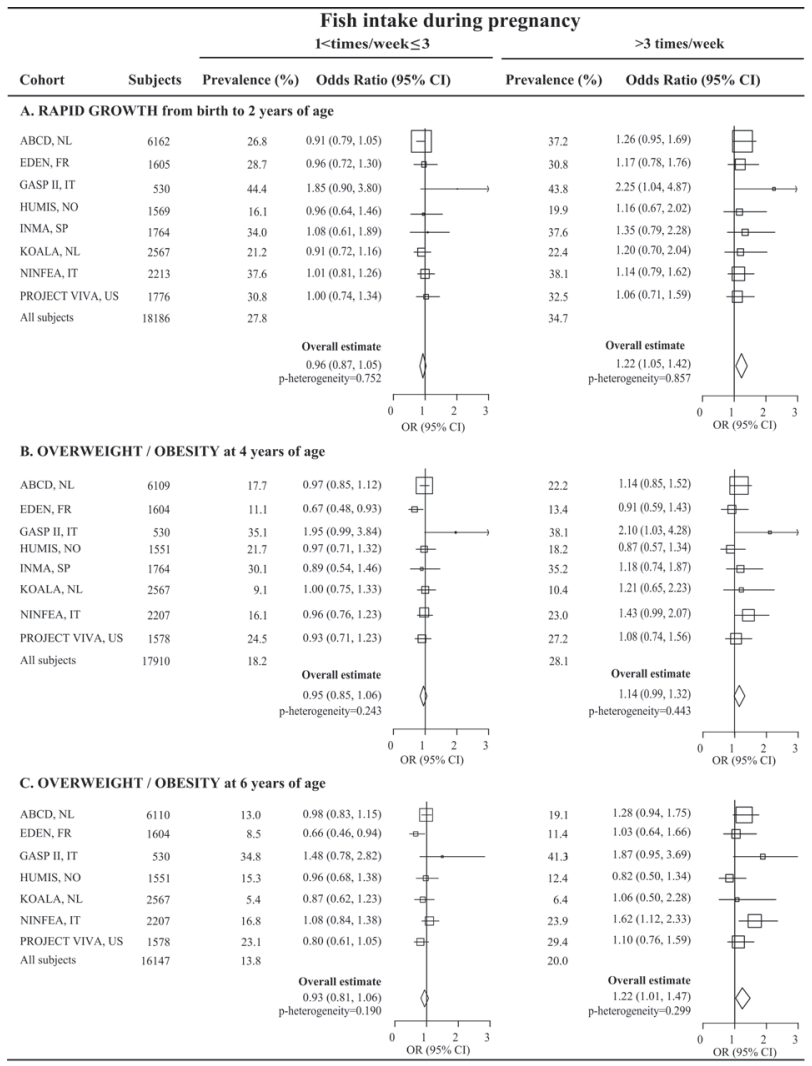

Figure 7.2. Adjusted associations of fish intake in pregnancy with rapid growth in infancy and childhood overweight/obesity. Rapid growth was defined as a weight gain z-score $>0.67$, based on WHO growth curves. Overweight/obesity was defined as $\mathrm{BMI} \geq 85^{\text {th }}$ percentile for age and sex, based on WHO growth curves. Cohort-specific prevalence (\%) of rapid growers in infancy and overweight/obese children for each fish intake category are presented. Odds ratios $195 \%$ Cls) by cohort were obtained by using logistic regression models adjusted for maternal age, maternal education, pre-pregnancy BMI, smoking during pregnancy and birth weight. Combined estimates were obtained by using a random-effects meta-analysis. The squares represent the point estimate of each study, whereas the size of the square is proportional to the weight assigned to each cohort based on both the within- and between-study variability; horizontal lines denote $\mathbf{9 5 \%} \mathrm{Cls}$; and diamonds represent overall estimates. Reference fish intake category was $\leq 1$ time/week.

Figure 7.3 depicts the modeled BMI percentile trajectories of children up to 6 years according to different levels of maternal fish consumption in pregnancy. Children of mothers with high fish intake exhibited consistently higher values than did those of mothers with low fish intake, and at 2.5 years onwards, the difference became more pronounced. Children of mothers with moderate fish intake during pregnancy had a similar trajectory to that of children whose mothers had 
low fish intake, and both followed the typical pattern of BMI growth, that is, a sharp increase in BMI during infancy followed by a decrease later in childhood.

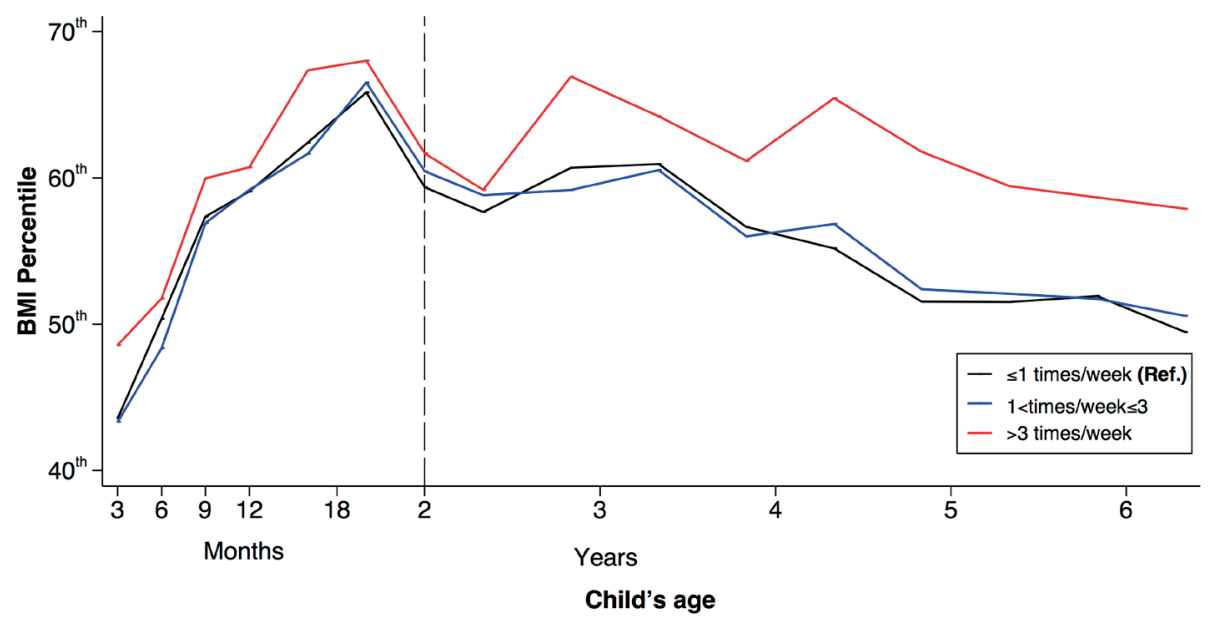

Figure 7.3. Body mass index (BMI) percentile trajectories from 3 months to 6 years of age according to different levels of fish intake in pregnancy. BMI percentile values indicate the place of children in the corresponding growth chart of the WHO reference population (Supplementary Methods), and were derived by using mixed-effects linear regression models fitted with: fish intake, an interaction term for fish intake and child age, maternal age, maternal education, pre-pregnancy BMI, smoking during pregnancy, and birth weight as fixed-effects parameters; random cohort and child intercepts; and a random slope for child age.

\section{Sensitivity analyses}

Inclusion of all cohorts in the categorical dose-response analysis did not materially change the reported effect estimates (Supplementary Tables S7.7 and S7.8). The associations of high fish intake with increased risk of childhood overweight and obesity remained significant and of similar magnitude when additional adjustment was made for gestational weight gain when we took into account the geographical location or the recruitment period of each cohort (data not shown). Additionally, exclusion of birth weight from the multivariate models did not materially change the effect estimates (Supplementary Tables S7.9 and S7.10). When we examined only childhood obesity as an outcome, the association had the same direction as when examining both overweight and obesity, but confidence intervals were wider, possibly because of the small number of obese children (Supplementary Table S7.11).

The magnitude of the fish intake effect on rapid infant growth and childhood overweight/obesity was greater in girls than in boys (Supplementary Table S7.12 
and Supplementary Figure S7.3). There was no evidence of effect modification by prepregnancy BMI, smoking during pregnancy, timing of dietary assessment, low birth weight, and breastfeeding duration ( $\mathrm{P}$ for interaction $>0.10$ for all).

\section{DISCUSSION}

We found that fish intake of more than 3 times/week in pregnancy was associated with higher offspring BMI and greater risk of rapid infant growth and childhood overweight/obesity. To our knowledge, this is the only multicenter study on the long-term effect of maternal fish intake in pregnancy on offspring growth and adiposity. The use of birth cohorts in several locations in Europe and the United States with varying fish intake levels and the absence of heterogeneity between individual cohort effect estimates provide evidence to support the robustness and generalizability of our findings.

Childhood overweight and obesity is considered a major public health issue. ${ }^{18}$ Rapid weight gain in infancy has consistently been associated with a subsequent elevated risk of obesity in childhood and later in adulthood. ${ }^{12,19}$ Intrauterine life is a sensitive period, during which the capacity for proliferation of mesenchymal precursor cells and their differentiation into adipocytes is very high. ${ }^{1,2}$ Fish is the primary dietary source of $\omega-3$ LC-PUFAs. The evidence for a programming effect of $\omega$-3 fatty acids in fetal or early life on later body composition mainly stems from cell culture and animal studies. ${ }^{20}$ Reports from human trials have shown limited support for a beneficial effect of $\omega$-3 LC-PUFAs supplementation during early life on body composition later in life. ${ }^{9,21}$ Findings from longitudinal birth cohort studies on fish or $\omega$-3 LC-PUFAs during pregnancy and childhood adiposity are also discrepant, with reports of either beneficial (ie, lower adiposity) ${ }^{7}$ or null ${ }^{8,22-25}$ associations. These inconsistencies may be caused by inadequate sample sizes, exposure profile heterogeneity, or differences in adjustment for confounding variables. Our findings underscore scientific gaps in the experimental evidence, specifically, the lack of studies involving healthy populations and interventions targeting fish intake rather than supplement use, which may exert different effects. Contamination by environmental pollutants in fish could provide an explanation for the observed association between high fish intake in pregnancy and increased childhood adiposity. In humans, fish consumption is a major source of exposure to endocrine disrupting chemicals. ${ }^{26,27}$ Mixtures of persistent organic pollutants found in fish have been shown to increase fat storage in cultured adipocytes, as well as weight gain in animals. ${ }^{28}$ It has been proposed that these toxicants may perturb signaling of several nuclear receptors, and through altered gene expression, influence adipocyte differentiation and fat metabolism. ${ }^{3,4}$ 
Although we collected information on the consumption of different fish types, we did not have enough data to distinguish between big and small species, cooking procedures, and water source of fish (ie, river or sea), which would be relevant with respect to toxicant exposure. Moreover, in the absence of information regarding levels of persistent organic pollutants across participating cohorts, our hypothesis that fish-associated contaminant exposure may play a role in the observed associations remains speculative. Further analyses incorporating biomarker information on both the amounts of fatty acids and environmental chemicals contained within fish will be helpful for refining estimates of the influence of prenatal fish intake on child growth.

We observed that the effect of fish intake during pregnancy on offspring obesity outcomes was more pronounced in girls than in boys. The placenta tissue carrying the fetal genome and sex appears as promising candidate to be involved in mediating sex-specific functions and programming effects. ${ }^{29}$ Animal studies suggest that maternal diet may induce sexually dimorphic responsiveness in placental gene expression and greater sensitivity of females to the maternal environment, especially in the biological process of cell cycle. ${ }^{30,31}$

Although we did not see strong evidence for sociodemographic confounding in this analysis, the possibility of unmeasured residual confounding may still remain. We had no data available to adjust for energy intake or dietary patterns during pregnancy; however, adjustment for gestational weight gain, as a good proxy for total energy intake in pregnancy, ${ }^{32,33}$ did not appreciably change the effect estimates. Moreover, although we examined breastfeeding duration as a potential confounder or effect modifier, other differences in child diet or lifestyle factors (eg, physical activity patterns) might have influenced the observed findings. The direction of confounding was differentially distributed across participating cohorts, reflecting that the study design by itself may control to some extent for unmeasured confounding.

Strengths of our study include the large sample size, the centralized data analysis following a consensus protocol, the standardized exposure definition, and the harmonized information about child outcomes and potential confounders.

As in most studies assessing the health effects of diet, we used self-reported dietary information; hence, some information bias might have occurred. However, in most cohorts, fish intake was assessed using detailed food frequency questionnaires that were developed and validated for use in pregnancy, and all data were collected before birth and before growth patterns were known. We used BMI as our main outcome, a measure that incorporates both lean and fat mass; however, a high BMI has been suggested as a sensitive marker for excess adiposity. ${ }^{34}$ Further analyses using direct measurements of fat mass and body fat distribution to as- 
sess the relationship between fish intake and childhood obesity are an important consideration for future studies.

This large, multicenter study indicates that fish intake of more than 3 times/week in pregnancy is associated with increased risk of rapid growth in infancy and increased adiposity in childhood. Our findings are in line with the fish intake limit for pregnancy proposed by the US Food and Drug Administration and Environmental Protection Agency.

\section{ACKNOWLEDMENTS}

This work was supported by the European Community's Seventh Framework Program [EU- FP7- HEALTH-2009-single-stage- 241604]. Details of funding per cohort are available in the published article: JAMA Pediatr 2016 Apr;170(4):38190 . 


\section{REFERENCES}

1. Symonds ME, Sebert SP, Hyatt MA, et al. Nutritional programming of the metabolic syndrome. Nat Rev Endocrinol 2009;5:604-10.

2. Ailhaud G, Guesnet P, Cunnane SC. An emerging risk factor for obesity: does disequilibrium of polyunsaturated fatty acid metabolism contribute to excessive adipose tissue development? Br $J$ Nutr 2008;100:461-70.

3. Casals-Casas C, Desvergne B. Endocrine disruptors: from endocrine to metabolic disruption. Annu Rev Physiol 2011;73:135-62.

4. Grun F, Blumberg B. Endocrine disrupters as obesogens. Molecular \& Cellular Endocrinology 2009;304:19-29.

5. Fish: What Pregnant Women and Parents Should Know. Draft Updated Advice by FDA and EPA. June 2014. http://www.fda.gov/Food/FoodborneIllnessContaminants/Metals/ucm393070.htm. (August 4 2015, date last accessed)

6. Leventakou V, Roumeliotaki T, Martinez D, et al. Fish intake during pregnancy, fetal growth, and gestational length in 19 European birth cohort studies. American Journal of Clinical Nutrition 2014;99:50616.

7. Donahue SMA, Rifas-Shiman SL, Gold DR, et al. Prenatal fatty acid status and child adiposity at age 3 y: results from a US pregnancy cohort. American Journal of Clinical Nutrition 2011;93:780-8.

8. van den Berg SW, Wijga AH, van Rossem L, et al. Maternal fish consumption during pregnancy and BMI in children from birth up to age 14 years: the PIAMA cohort study. Eur J Nutr 2015.

9. Stratakis N, Gielen M, Chatzi L, et al. Effect of maternal n-3 long-chain polyunsaturated fatty acid supplementation during pregnancy and/or lactation on adiposity in childhood: a systematic review and meta-analysis of randomized controlled trials. Eur J Clin Nutr 2014;68:1277-87.

10. WHO Multicentre Growth Reference Study Group. WHO Child Growth Standards: Length/heightfor-age, weight-for-age, weight-for-length, weight-for-height and body mass index-for-age: Methods and development. Geneva: World Health Organization. 2006.

11. de Onis M, Onyango AW, Borghi E, et al. Development of a WHO growth reference for school-aged children and adolescents. Bull World Health Organ 2007;85:660-7.

12. Monteiro PO, Victora CG. Rapid growth in infancy and childhood and obesity in later life--a systematic review. Obes Rev 2005;6:143-54.

13. Karaolis-Danckert N, Buyken AE, Bolzenius K, et al. Rapid growth among term children whose birth weight was appropriate for gestational age has a longer lasting effect on body fat percentage than on body mass index. Am J Clin Nutr 2006;84:1449-55.

14. Barlow SE. Expert committee recommendations regarding the prevention, assessment, and treatment of child and adolescent overweight and obesity: Summary report. Pediatrics 2007;120 Suppl 4:S164-S92.

15. Howards PP, Schisterman EF, Poole C, et al. "Toward a clearer definition of confounding” revisited with directed acyclic graphs. Am J Epidemiol 2012;176:506-11.

16. Textor J, Hardt J, Knuppel S. DAGitty: a graphical tool for analyzing causal diagrams. Epidemiology 2011;22:745.

17. Higgins JP, Thompson SG. Quantifying heterogeneity in a meta-analysis. Stat Med 2002;21:1539-58.

18. Gluckman P, Nishtar S, Armstrong T. Ending childhood obesity: a multidimensional challenge. Lancet 2015;385:1048-50.

19. Stettler N. Nature and strength of epidemiological evidence for origins of childhood and adulthood obesity in the first year of life. Int J Obes (Lond) 2007;31:1035-43. 
20. Massiera F, Saint-Marc P, Seydoux J, et al. Arachidonic acid and prostacyclin signaling promote adipose tissue development: a human health concern? J Lipid Res 2003;44:271-9.

21. Gonzalez-Casanova I, Stein AD, Hao W, et al. Prenatal Supplementation with Docosahexaenoic Acid Has No Effect on Growth through 60 Months of Age. J Nutr 2015;145:1330-4.

22. Moon RJ, Harvey NC, Robinson SM, et al. Maternal Plasma Polyunsaturated Fatty Acid Status in Late Pregnancy Is Associated with Offspring Body Composition in Childhood. J Clin Endocrinol Metab 2013;98:299-307.

23. Rytter D, Bech BH, Halldorsson T, et al. No association between the intake of marine n-3 PUFA during the second trimester of pregnancy and factors associated with cardiometabolic risk in the 20-year-old offspring. Br J Nutr 2013;110:2037-46.

24. Standl M, Thiering E, Demmelmair H, et al. Age-dependent effects of cord blood long-chain PUFA composition on BMI during the first 10 years of life. Br J Nutr 2014;13:1-8.

25. de Vries PS, Gielen M, Rizopoulos D, et al. Association between polyunsaturated fatty acid concentrations in maternal plasma phospholipids during pregnancy and offspring adiposity at age $7:$ the MEFAB cohort. Prostaglandins Leukot Essent Fatty Acids 2014;91:81-5.

26. Turyk ME, Bhavsar SP, Bowerman W, et al. Risks and benefits of consumption of Great Lakes fish. Environ Health Perspect 2012;120:11-8.

27. Mozaffarian D, Rimm EB. Fish intake, contaminants, and human health: evaluating the risks and the benefits. JAMA 2006;296:1885-99.

28. Ibrahim MM, Fjaere E, Lock EJ, et al. Chronic consumption of farmed salmon containing persistent organic pollutants causes insulin resistance and obesity in mice. PLoS One 2011;6:e25170.

29. Sood R, Zehnder JL, Druzin ML, et al. Gene expression patterns in human placenta. Proc Natl Acad Sci U S A 2006;103:5478-83.

30. Gabory A, Ferry L, Fajardy I, et al. Maternal diets trigger sex-specific divergent trajectories of gene expression and epigenetic systems in mouse placenta. PLoS One 2012;7:e47986.

31. Tarrade A, Panchenko P, Junien C, et al. Placental contribution to nutritional programming of health and diseases: epigenetics and sexual dimorphism. J Exp Biol 2015;218:50-8.

32. Streuling I, Beyerlein A, Rosenfeld E, et al. Weight gain and dietary intake during pregnancy in industrialized countries--a systematic review of observational studies. J Perinat Med 2011;39:123-9.

33. Stuebe AM, Oken E, Gillman MW. Associations of diet and physical activity during pregnancy with risk for excessive gestational weight gain. Am J Obstet Gynecol 2009;201:58 e1-8.

34. Boeke CE, Oken E, Kleinman KP, et al. Correlations among adiposity measures in school-aged children. BMC Pediatr 2013;13:99. 


\section{Supplementary Material of Chapter 7}

Supplementary Methods

Fish classification

Modeling of weight and height growth trajectories

Use of WHO growth charts

Supplementary Table S7.1. Description of participating cohorts

Supplementary Table S7.2. Comparison of prediction concordance from different fractional polynomial powers for sex-specific weight and BMI in participating cohorts

Supplementary Table S7.3. Distribution of maternal and child characteristics in participating cohorts

Supplementary Table S7.4. Distribution of fish and seafood intake in pregnancy in participating cohorts

Supplementary Table S7.5. Distribution of maternal and child characteristics in participating cohorts according to different levels of fish intake in pregnancy

Supplementary Table S7.6. Distribution of growth outcomes during childhood in participating cohorts

Supplementary Table S7.7. Adjusted combined associations of different levels of fish intake in pregnancy with offspring BMI z-scores during childhood after including all participating cohorts

Supplementary Table S7.8. Adjusted combined associations of different levels of fish intake in pregnancy with rapid infant growth and childhood overweight/ obesity after including all participating cohorts

Supplementary Table S7.9. Adjusted combined associations (pooled analysis) of fish intake in pregnancy with offspring BMI z-scores during childhood after removing birth weight from the multivariable models

Supplementary Table S7.10. Adjusted combined associations (pooled analysis) of fish intake in pregnancy with rapid infant growth and childhood overweight/ obesity after removing birth weight from the multivariable models 
Supplementary Table S7.11. Adjusted combined associations (pooled analysis) of fish intake in pregnancy with childhood obesity

Supplementary Table S7.12. Adjusted combined associations (pooled analysis) of fish intake in pregnancy with rapid infant growth and childhood overweight/ obesity by sex

Supplementary Figure S7.1. Location of birth cohorts

Supplementary Figure S7.2. Directed acyclic graph for assessing the association between maternal fish intake in pregnancy and child adiposity.

Supplementary Figure S7.3. Adjusted associations of different fish intake levels in pregnancy with rapid growth in infancy and childhood overweight/obesity by sex Supplementary References 


\section{Supplementary Methods}

\section{Fish classification}

Salmon, herring, mackerel, trout, sardines, Greenland halibut, anchovy, gurnard, and tuna were classified as fatty fishes. Cod, pollack, plaice, flounder, garfish, and similar species were classified as lean fishes.

\section{Modeling of weight and height growth trajectories}

Cohorts provided information on child weight and height up to a maximum follow-up to 6 years. In more detail, one cohort (REPRO_PL) provided information on child weight and height up to the age of 2 years, two cohorts (INMA, RHEA) up to the age of 4 years, and 12 cohorts (ABCD, EDEN, FLEHS I, GASPII, Generation R, Generation XXI, HUMIS, KOALA, Lifeways Cross Generation, NINFEA, PIAMA, and Project Viva) up to the age of 6 years. Each dataset was checked for completeness and consistency, and a total of 31 observations were excluded due to implausible values on postnatal growth measurements according to the World Health Organization (WHO) growth charts. ${ }^{1,2}$

Patterns of growth across childhood follow a complex pattern (growth is nonlinear). We used a two-step approach to estimate growth curves for participating cohorts. First we identified for each cohort the best fitting fractional polynomials of age and constructed sex-and age- specific weight and height growth curves. ${ }^{3}$ Briefly, a series of models were run for each cohort in which age was raised to a large number of combinations of powers (each of the following single powers, plus each combination of two powers: $-2,-1,-0.5,0,0.5,1,2,3$, where a power of zero is the log function), resulting in a wide range of possible weight and height curves. ${ }^{4}$ Then we used mixed-effects linear regression models with the previously identified fractional polynomials of age, including a random intercept for child and random age slopes. Such models allow for individual variation in growth curves within each cohort, and use all available data from all the eligible children under a missing at random assumption. ${ }^{5}$ Only subjects with at least two measurements from birth to 6 years were included. The number of available measurements per child ranged from 2 to 44 for weight [median and interquartile range (IQR), 6 and 8 ] and 2 to 33 for height (median and IQR, 5 and 6). Predicted weight and height values within each cohort were estimated for exact age of 2, 4 and 6 years, but not beyond the age for which information on actual measurements was not available. 


\section{Use of WHO growth charts}

In the present study, we used the WHO growth charts to monitor child growth. ${ }^{1,2}$ These charts are growth standards based on data collected from selected communities worldwide. Children included in our analysis had collectively higher BMI after the first six months of life compared to the WHO reference population, falling into the higher region of the growth chart (Figure 3). Similar growth patterns have already been described following the use of WHO standards in different westernized countries. ${ }^{6-8}$

The WHO charts are intended to reflect how children should grow when properly fed and cared for, rather than merely describing how children grow, as it is the case for national growth references. Given that we included data from various countries in Europe and the US, the use of WHO standards allow for growth assessment of children independent of ethnicity and socioeconomic status, thus, permitting international comparisons. These charts have been adopted in a growing number of countries in Europe and other parts of the world, ${ }^{9}$ and endorsed by international bodies such as the United Nations Standing Committee on Nutrition $^{10}$ and International Pediatric Association. ${ }^{11}$ 


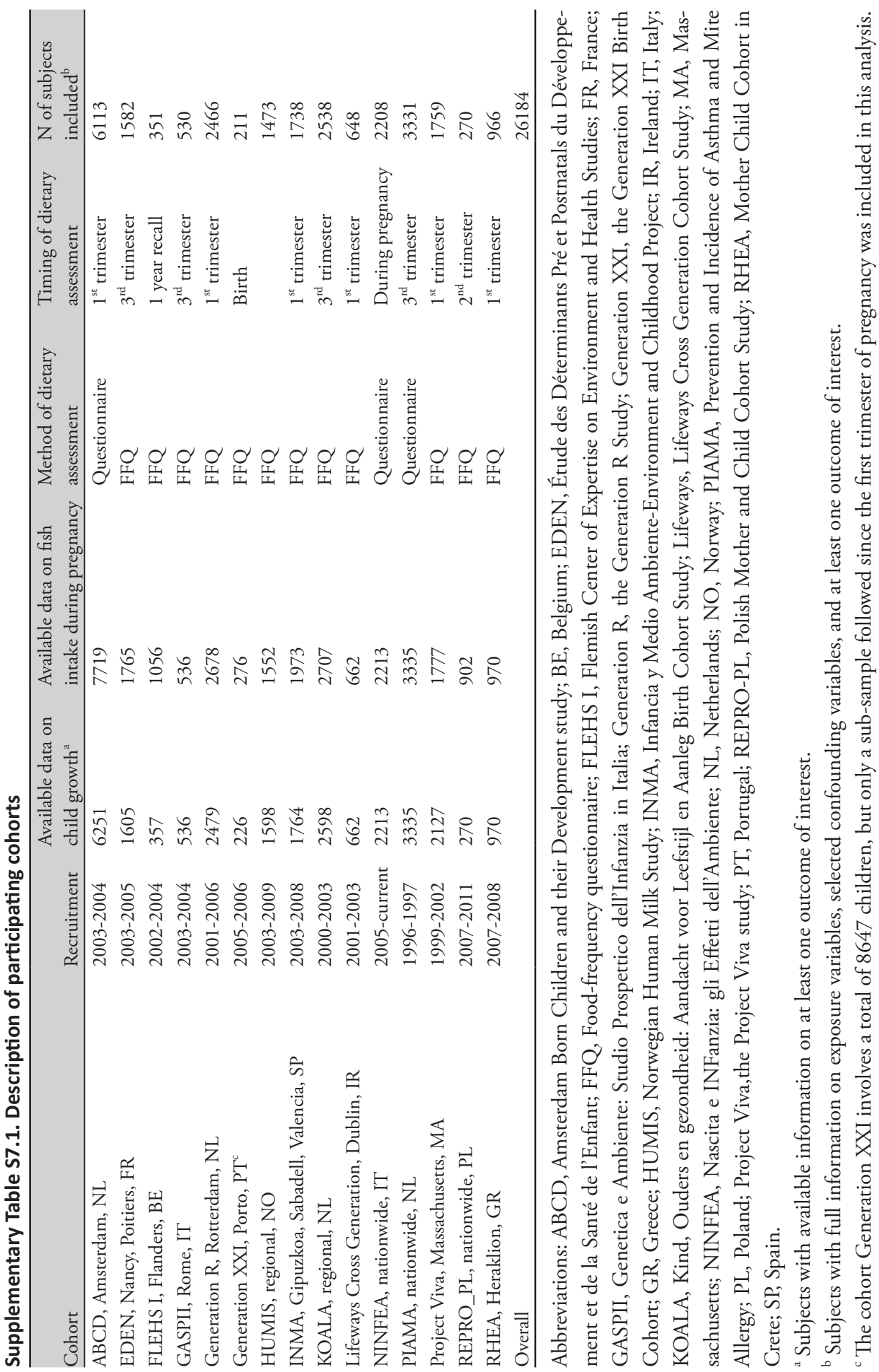




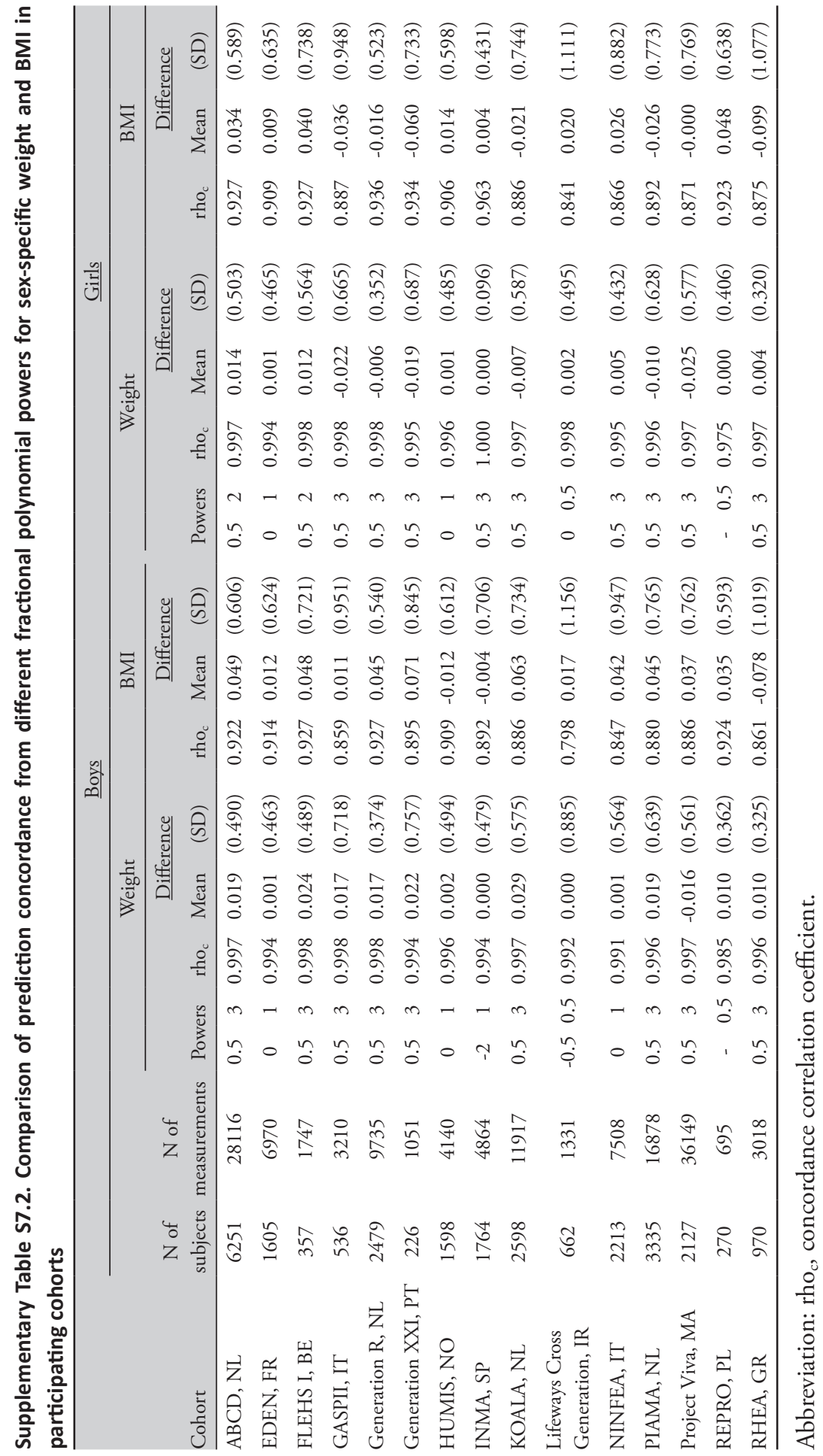




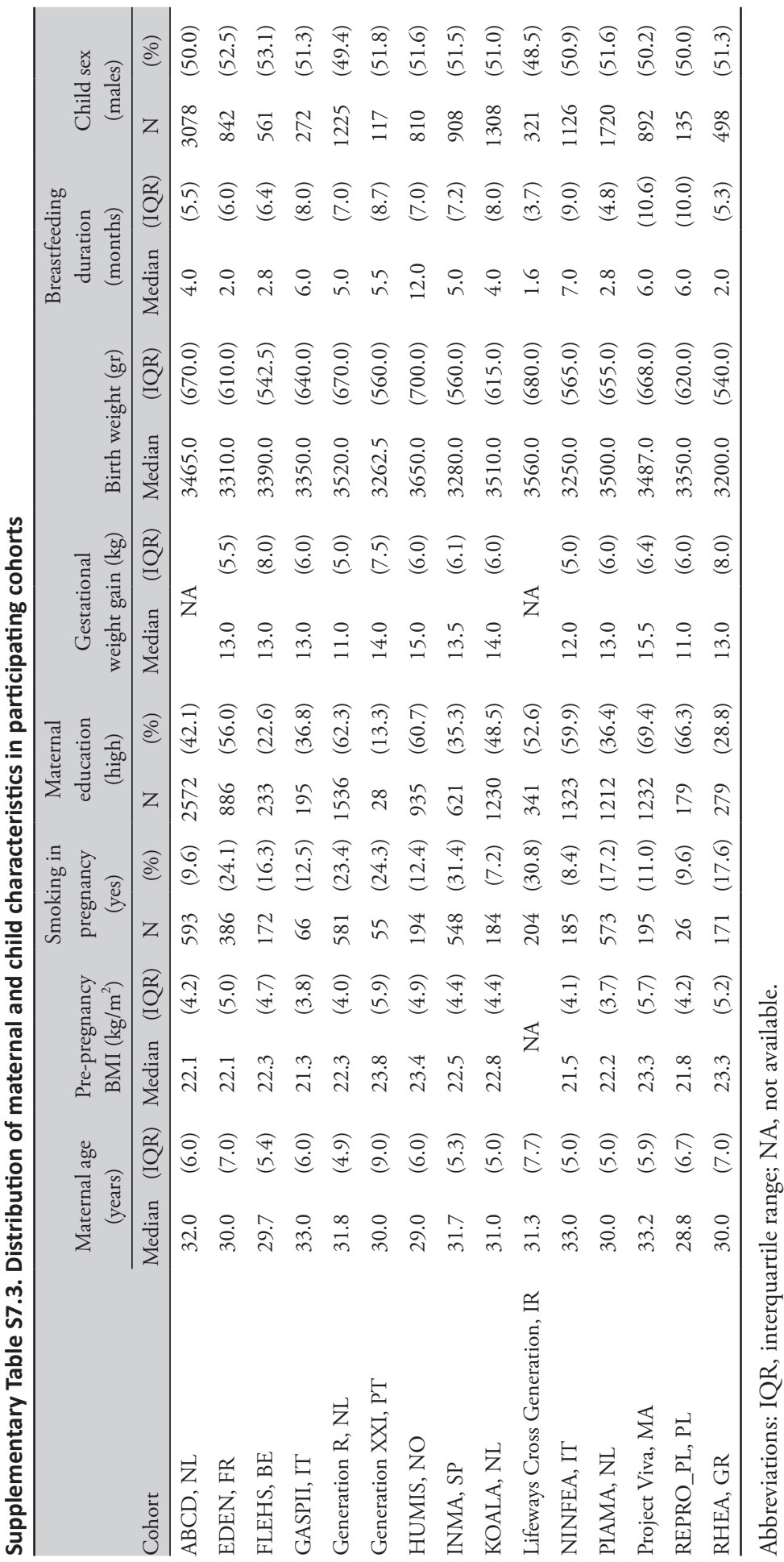


Supplementary Table S7.4. Distribution of fish and seafood intake in pregnancy in participating cohorts

\begin{tabular}{lcccc}
\hline & Total fish & Fatty fish & Lean fish & Seafood (other than fish) \\
\cline { 2 - 5 } Cohort & (times/week) & (times/week) & (times/week) & (times/week) \\
\cline { 2 - 4 } Median (IQR) & Median (IQR) & Median (IQR) & Median (IQR) \\
\hline GBCD, Netherlands & $1.0(1.0)$ & NA & NA & NA \\
Generation R, Netherlands & $0.42(0.63)$ & NA & NA & NA \\
KOALA, Netherlands & $0.98(1.47)$ & $0.49(0.49)$ & $0.98(0.49)$ & $0.0(0.0)$ \\
PIAMA, Netherlands & $0.5(0.5)$ & NA & NA & NA \\
NINFEA, Italy & $1.5(1.0)$ & NA & NA & $0.0(0.5)$ \\
GASPII, Italy & $2.22(1.75)$ & $1.0(0.92)$ & $0.5(0.69)$ & $0.37(0.59)$ \\
EDEN, France & $1.30(1.38)$ & $0.15(0.46)$ & NA & $0.0(0.1)$ \\
FLEHS I, Belgium & $0.50(0.75)$ & NA & NA & $0.25(0.25)$ \\
Generation XXI, Portugal & $4.0(3.5)$ & $1.0(2.5)$ & $2.0(2.5)$ & $0.5(1.0)$ \\
HUMIS, Norway & $1.68(1.46)$ & $0.37(0.61)$ & $1.19(1.23)$ & $0.15(0.25)$ \\
INMA, Spain & $4.45(3.45)$ & $0.91(1.45)$ & $3.45(3.0)$ & $0.91(1.0)$ \\
Lifeways Cross Generation, & $0.5(1.0)$ & $0.0(1.0)$ & $0.5(0.5)$ & $0.0(0.0)$ \\
Ireland & $1.5(2.5)$ & $1.5(2.0)$ & $0.0(0.5)$ & $0.0(0.23)$ \\
Project Viva, Massachusetts & $0.98(0.98)$ & $0.49(0.98)$ & $0.49(0.49)$ & $0.49(0.49)$ \\
REPRO_PL, Poland & $1.0(0.88)$ & $0.46(1.0)$ & $0.30(0.61)$ & $0.0)$ \\
RHEA, Greece & & & & 0.0 \\
\hline
\end{tabular}

Abbreviations: IQR, interquartile range; NA, not available. 


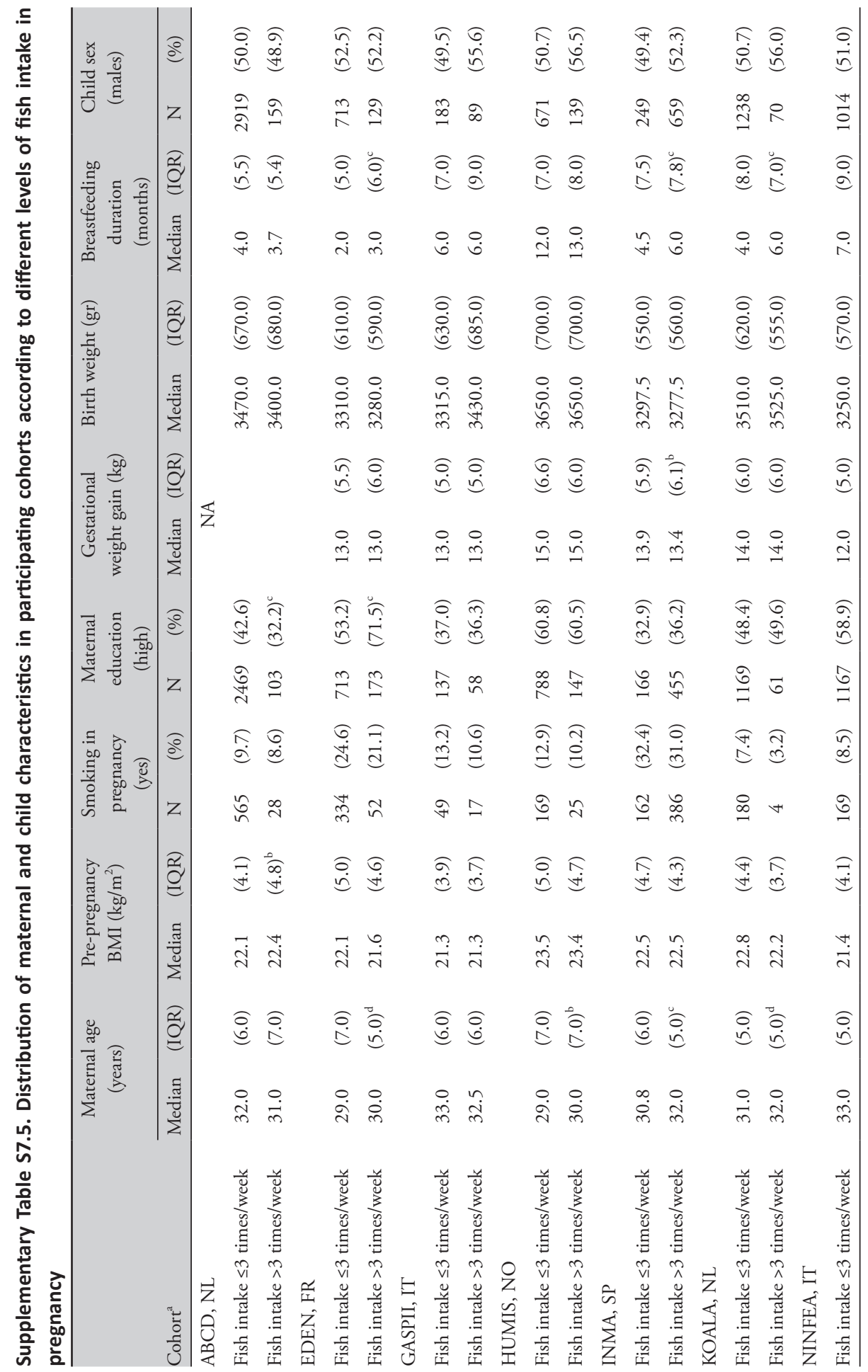




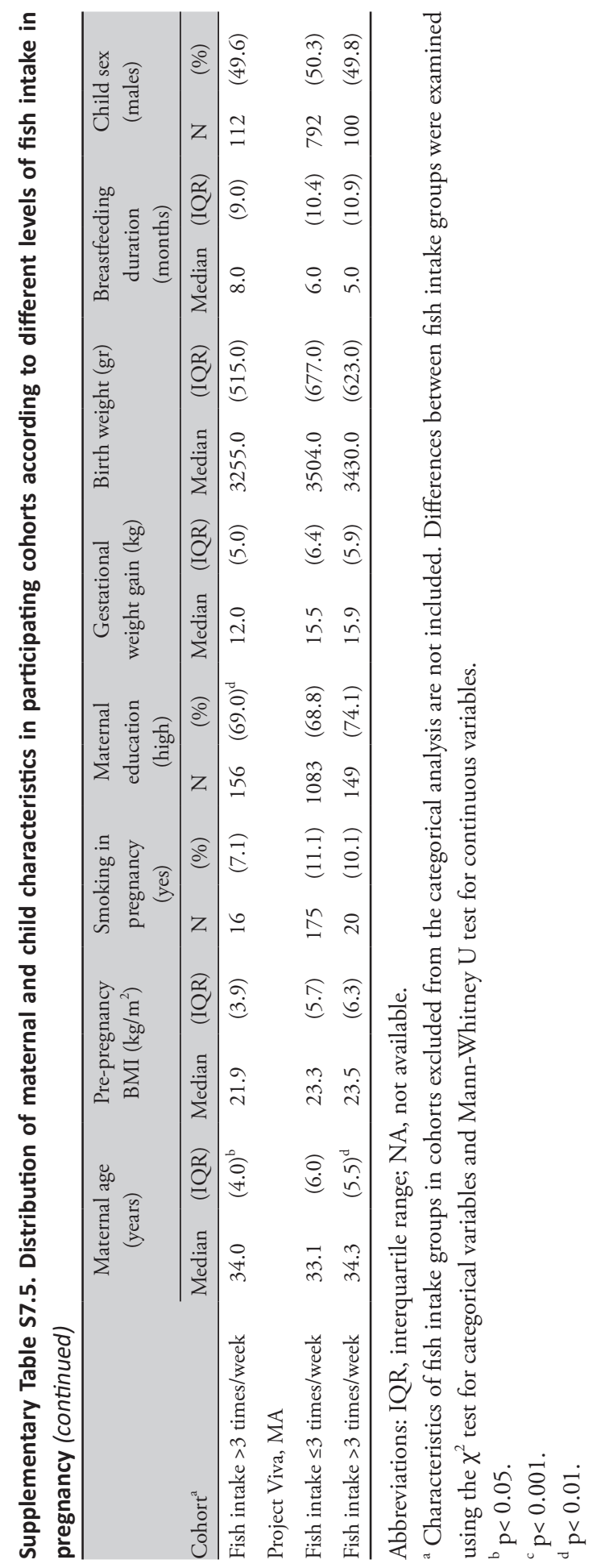




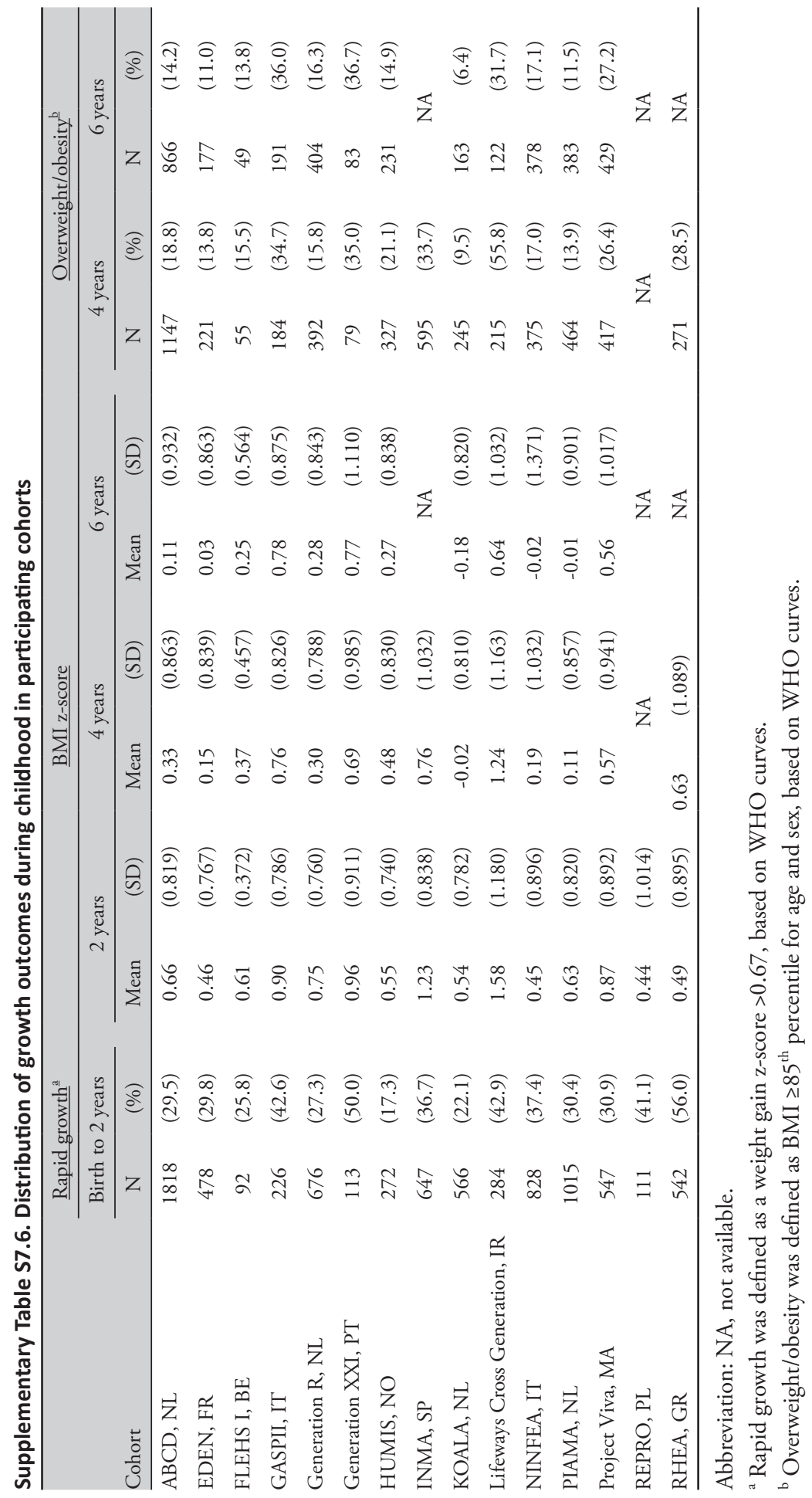




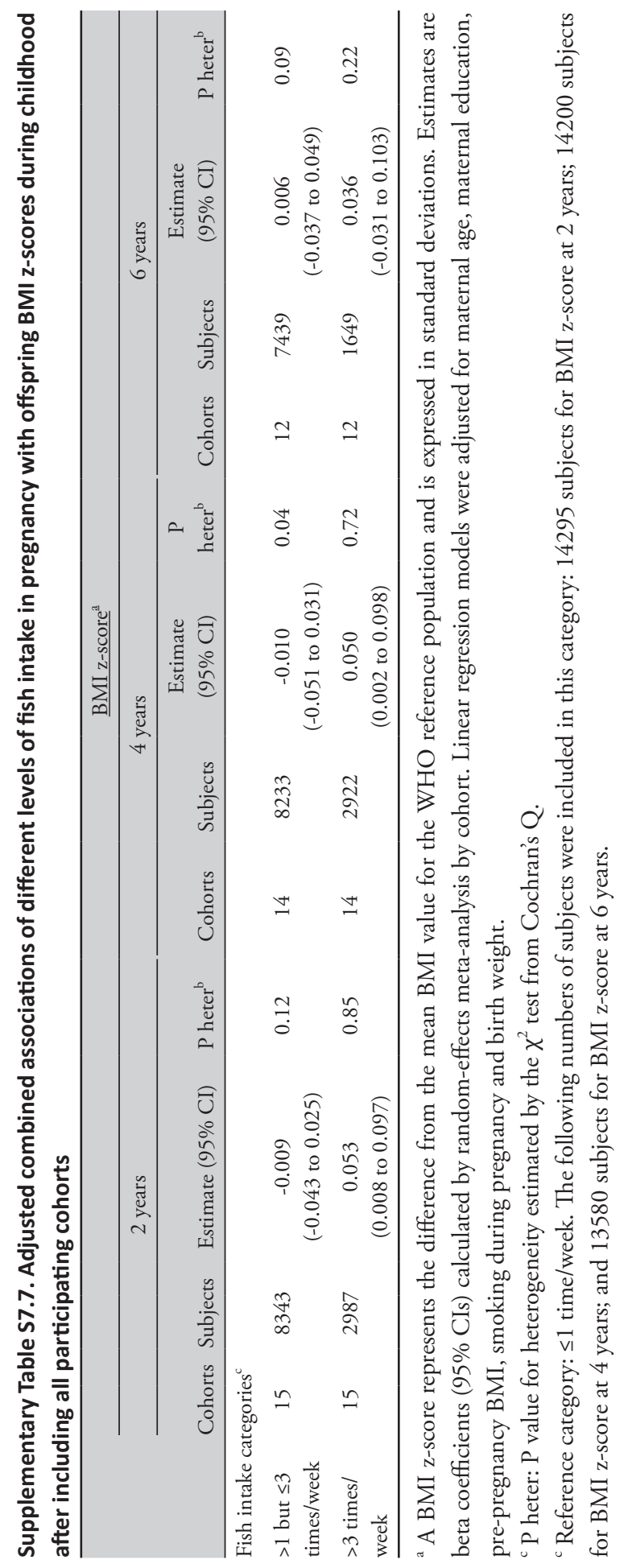




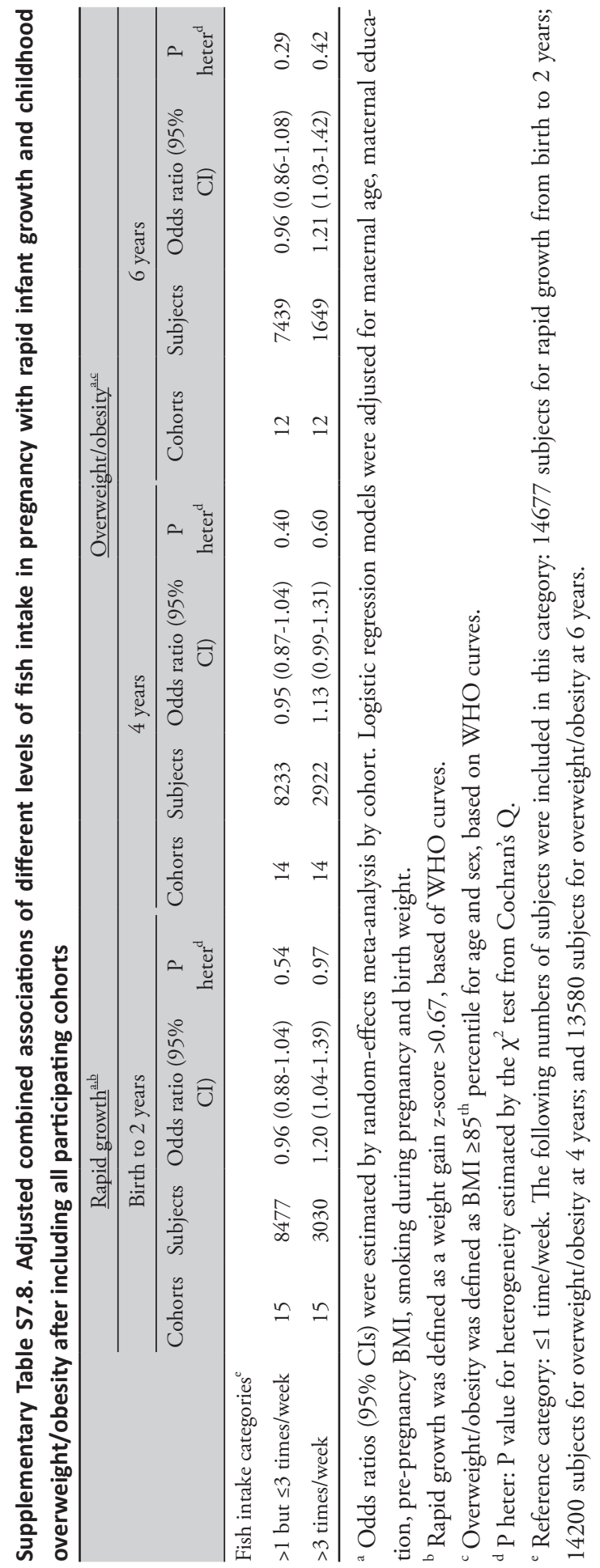


Supplementary Table S7.9. Adjusted combined associations (pooled analysis) of fish intake in pregnancy with offspring BMI z-scores during childhood after removing birth weight from the multivariable models

\begin{tabular}{|c|c|c|c|}
\hline & \multicolumn{3}{|c|}{ BMI z-score ${ }^{a}$} \\
\hline & 2 years & 4 years & 6 years \\
\hline & Estimate $(95 \% \mathrm{CI})$ & Estimate (95\% CI) & Estimate $(95 \% \mathrm{CI})$ \\
\hline Fish intake (times/week) & $\begin{array}{c}0.009 \\
(0.003 \text { to } 0.016)\end{array}$ & $\begin{array}{c}0.009 \\
(0.001 \text { to } 0.016)\end{array}$ & $\begin{array}{c}0.011 \\
(0.002 \text { to } 0.020)\end{array}$ \\
\hline \multicolumn{4}{|l|}{ Fish intake categories ${ }^{\mathrm{b}}$} \\
\hline$>1$ but $\leq 3$ times/week & $\begin{array}{c}-0.002 \\
(-0.029 \text { to } 0.025)\end{array}$ & $\begin{array}{c}0.0002 \\
(-0.0291 \text { to } 0.0295)\end{array}$ & $\begin{array}{c}0.007 \\
(-0.026 \text { to } 0.039)\end{array}$ \\
\hline$>3$ times/week & $\begin{array}{c}0.070 \\
(0.028 \text { to } 0.113)\end{array}$ & $\begin{array}{c}0.080 \\
(0.034 \text { to } 0.126)\end{array}$ & $\begin{array}{c}0.068 \\
(0.012 \text { to } 0.123)\end{array}$ \\
\hline \multicolumn{4}{|l|}{ Types of fish (times/week) } \\
\hline Fatty fish & $\begin{array}{c}0.001 \\
(-0.016 \text { to } 0.017)\end{array}$ & $\begin{array}{c}-0.0004 \\
(-0.020 \text { to } 0.019)\end{array}$ & $\begin{array}{c}0.014 \\
(-0.011 \text { to } 0.038)\end{array}$ \\
\hline Lean fish & $\begin{array}{c}0.023 \\
(0.009 \text { to } 0.037)\end{array}$ & $\begin{array}{c}0.015 \\
(-0.0004 \text { to } 0.031)\end{array}$ & $\begin{array}{c}0.001 \\
(-0.027 \text { to } 0.028)\end{array}$ \\
\hline Seafood (other than fish) & $\begin{array}{c}-0.003 \\
(-0.027 \text { to } 0.020)\end{array}$ & $\begin{array}{c}-0.013 \\
(-0.039 \text { to } 0.013)\end{array}$ & $\begin{array}{c}0.003 \\
(-0.037 \text { to } 0.043)\end{array}$ \\
\hline
\end{tabular}

${ }^{\mathrm{a}} \mathrm{A} \mathrm{BMI} \mathrm{z}$-score represents the difference from the mean BMI value for the WHO reference population and is expressed in standard deviations. Pooled estimates are beta coefficients ( $95 \%$ CIs) calculated by mixed-effects linear regression models with a random cohort intercept adjusted for maternal age, maternal education, pre-pregnancy BMI, and smoking during pregnancy.

${ }^{\mathrm{b}}$ Reference category: $\leq 1$ time/week. 
Supplementary Table S7.10. Adjusted combined associations (pooled analysis) of fish intake in pregnancy with rapid infant growth and childhood overweight/obesity after removing birth weight from the multivariable models

\begin{tabular}{|c|c|c|c|}
\hline & \multirow{2}{*}{$\begin{array}{l}\text { Rapid growth }^{\mathrm{a}, \mathrm{b}} \\
\text { Birth to } 2 \text { years }\end{array}$} & \multicolumn{2}{|c|}{$\underline{\text { Overweight/obesity }}^{\mathrm{a}^{\mathrm{a}, \mathrm{c}}}$} \\
\hline & & 4 years & 6 years \\
\hline & Odds ratio $(95 \% \mathrm{CI})$ & Odds ratio $(95 \% \mathrm{CI})$ & Odds ratio $(95 \% \mathrm{CI})$ \\
\hline Fish intake (times/week) & $1.02(1.00-1.04)$ & $1.02(1.00-1.04)$ & $1.03(1.00-1.05)$ \\
\hline \multicolumn{4}{|l|}{ Fish intake categories $^{\mathrm{d}}$} \\
\hline$>1$ but $\leq 3$ times/week & $0.96(0.90-1.04)$ & $0.96(0.88-1.05)$ & $0.94(0.85-1.04)$ \\
\hline$>3$ times/week & $1.18(1.05-1.32)$ & $1.17(1.03-1.33)$ & $1.23(1.05-1.43)$ \\
\hline \multicolumn{4}{|l|}{ Types of fish (times/week) } \\
\hline Fatty fish & $1.01(0.96-1.05)$ & $1.01(0.96-1.06)$ & $1.01(0.93-1.08)$ \\
\hline Lean fish & $1.04(1.00-1.08)$ & $1.03(0.99-1.07)$ & $0.94(0.85-1.02)$ \\
\hline Seafood (other than fish) & $1.01(0.95-1.08)$ & $0.95(0.88-1.01)$ & $0.97(0.87-1.08)$ \\
\hline
\end{tabular}

${ }^{\text {a }}$ Pooled odds ratios $(95 \% \mathrm{CIs}$ ) were estimated by using mixed-effects logistic regression models with a random cohort intercept adjusted for maternal age, maternal education, pre-pregnancy BMI, and smoking during pregnancy.

${ }^{\mathrm{b}}$ Rapid growth was defined as a weight gain z-score $>0.67$, based of WHO curves.

${ }^{\mathrm{c}}$ Overweight/obesity was defined as BMI $\geq 85^{\text {th }}$ percentile for age and sex, based on WHO curves.

${ }^{\mathrm{d}}$ Reference category: $\leq 1$ time/week. 


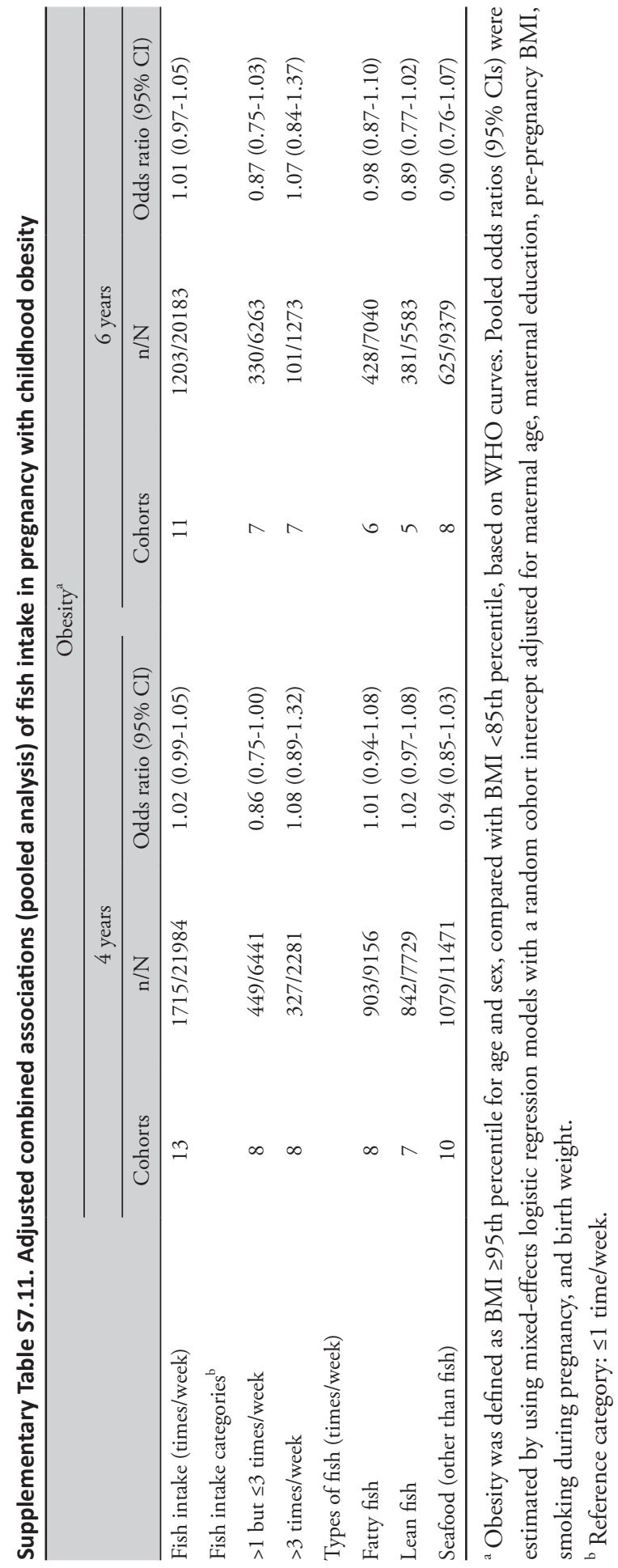




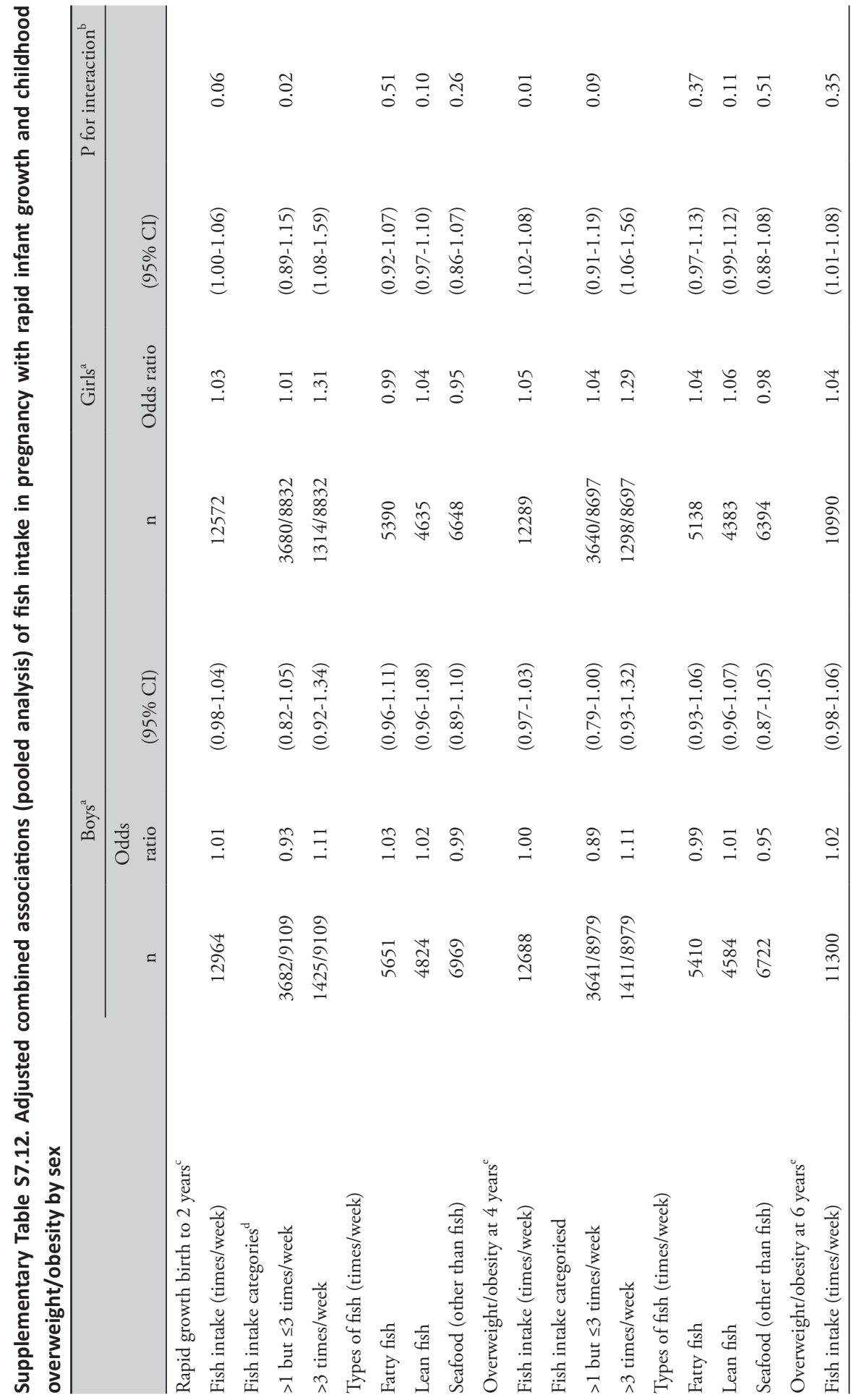




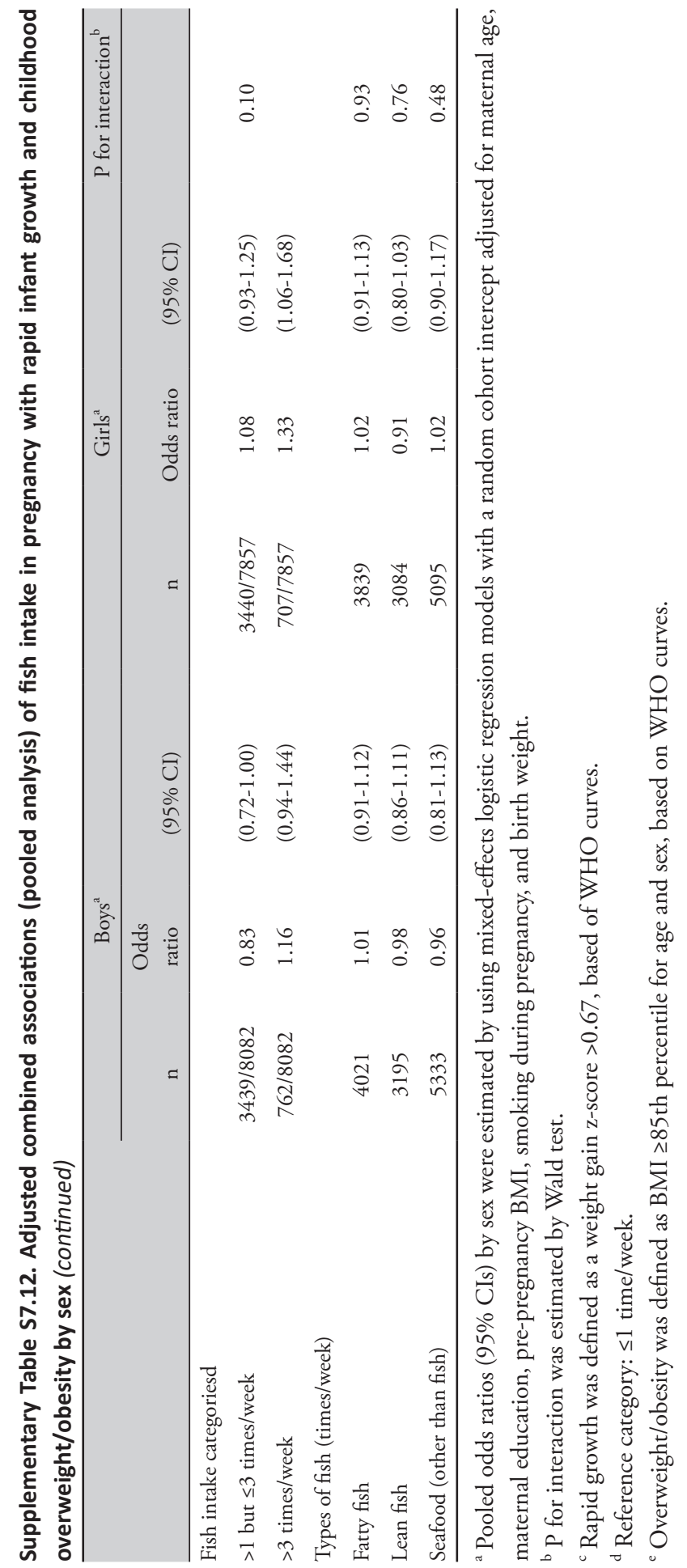




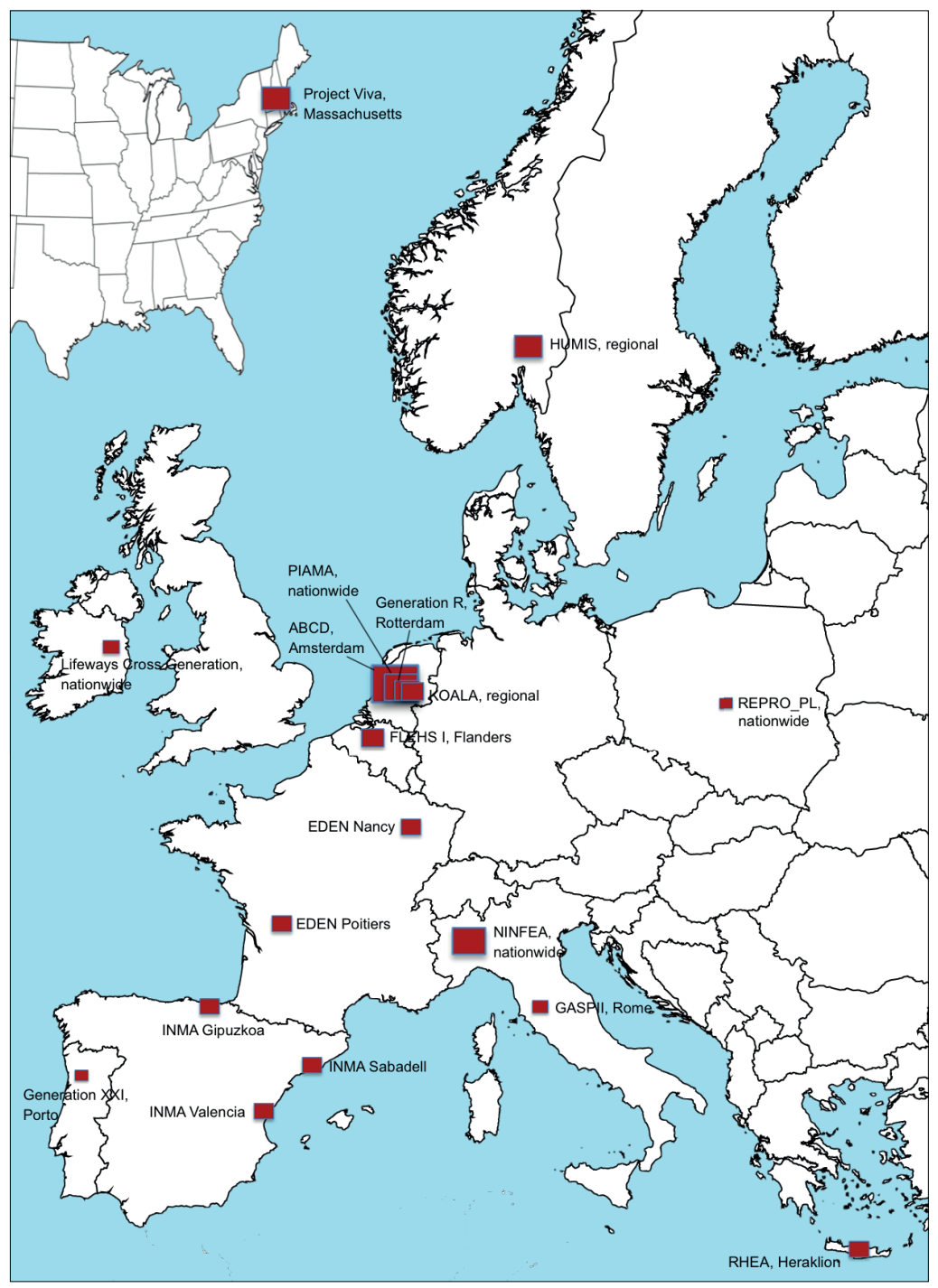

Supplementary Figure S7.1. Location of birth cohorts. The size of the square is indicative of the cohort size. ABCD, Amsterdam Born Children and their Development study; EDEN, Étude des Déterminants Pré et Postnatals du Développement et de la Santé de l'Enfant; FLEHS I, Flemish Center of Expertise on Environment and Health Studies; GASPII, Genetica e Ambiente: Studio Prospettico dell'Infanzia in Italia; Generation R, the Generation R Study; Generation XXI, the Generation XXI Birth Cohort; HUMIS, Norwegian Human Milk Study; INMA, Infancia y Medio Ambiente-Environment and Childhood Project; KOALA, Kind, Ouders en gezondheid: Aandacht voor Leefstijl en Aanleg Birth Cohort Study; Lifeways, Lifeways Cross Generation Cohort Study; NINFEA, Nascita e INFanzia: gli Effetti dell'Ambiente; PIAMA, Prevention and Incidence of Asthma and Mite Allergy; Project Viva,the Project Viva study; REPRO-PL, Polish Mother and Child Cohort Study; RHEA, Mother Child Cohort in Crete. 


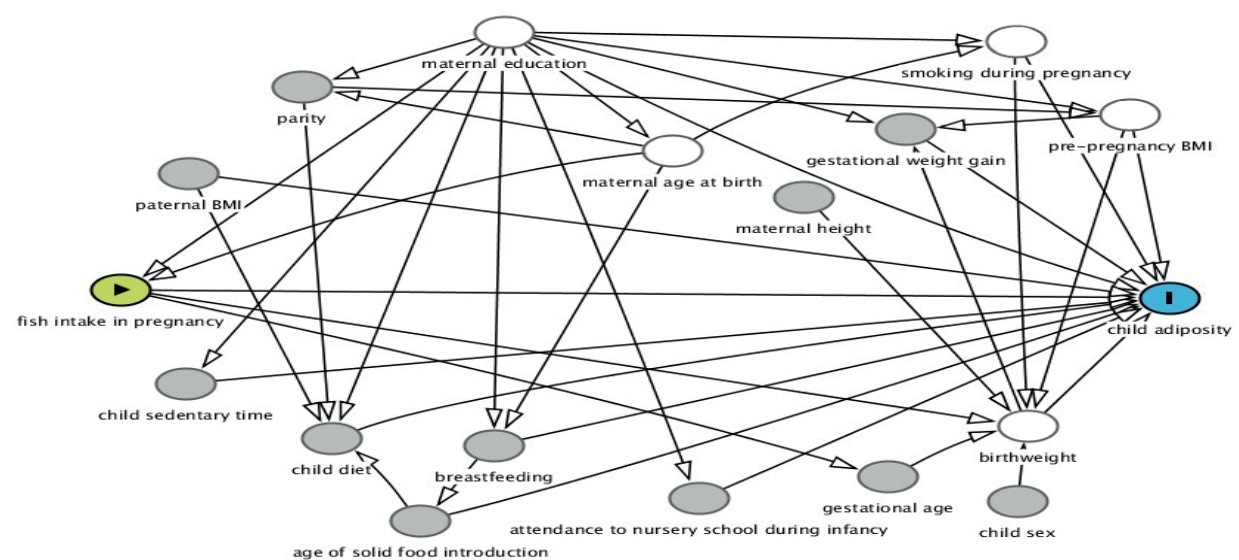

Supplementary Figure S7.2. Directed acyclic graph for assessing the association between maternal fish intake in pregnancy and child adiposity. Green node, exposure of interest; blue node: outcome of interest; and white nodes: the adjustment set used in the study. 


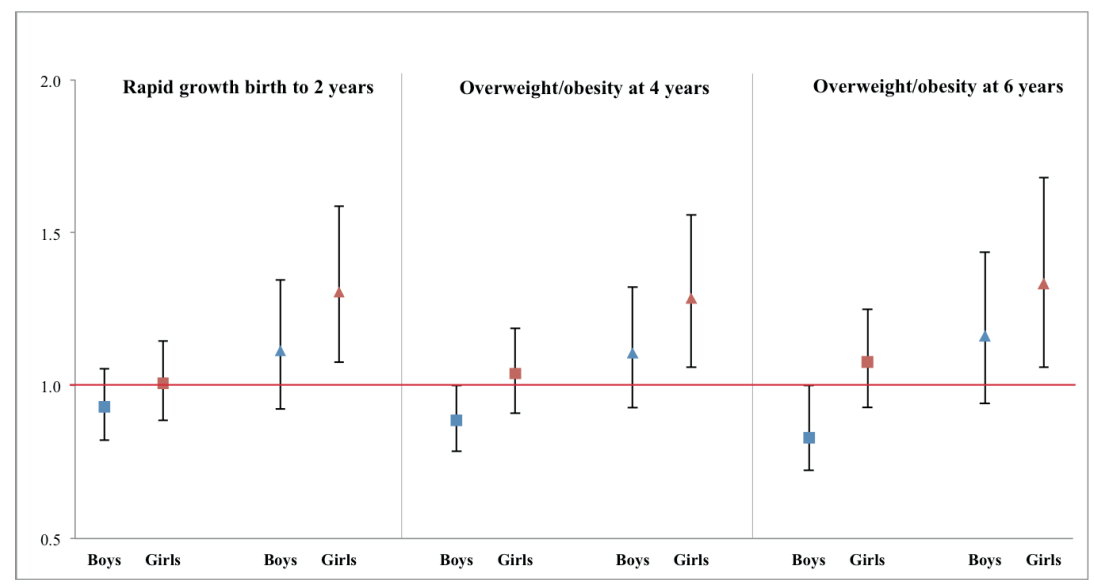

Supplementary Figure S7.3. Adjusted associations of different fish intake levels in pregnancy with rapid growth in infancy and childhood overweight/obesity by sex. Squares denote odds ratios for maternal fish intake of $>1$ but $\leq 3$ times/week; triangles denote odds ratios for maternal fish intake of $>3$ times/week; vertical bars indicate $95 \%$ Cls. Pooled odds ratios $(95 \% \mathrm{Cls}$ ) by sex were estimated by using mixed-effects logistic regression models with a random cohort intercept adjusted for maternal age, maternal education, pre-pregnancy BMI, smoking during pregnancy, and birth weight. Rapid growth was defined as a weight gain z-score $>0.67$, based on WHO curves. Overweight/obesity was defined as $\mathrm{BMI} \geq 85^{\text {th }}$ percentile for age and sex, based on WHO growth curves. Reference fish intake category was $\leq 1$ time/week. 


\section{Supplementary References}

1. WHO Multicentre Growth Reference Study Group. WHO Child Growth Standards: Length/heightfor-age, weight-for-age, weight-for-length, weight-for-height and body mass index-for-age: Methods and development. Geneva: World Health Organization. 2006.

2. de Onis M, Onyango AW, Borghi E, et al. Development of a WHO growth reference for school-aged children and adolescents. Bull World Health Organ 2007;85:660-7.

3. Royston P, Wright EM. A method for estimating age-specific reference intervals ("normal ranges") based on fractional polynomials and exponential transformation. J R Statist Soc 1998;161:79-101.

4. Long J, Ryoo J. Using fractional polynomials to model non-linear trends in longitudinal data. Br J Math Stat Psychol 2010;63:177-203.

5. Johnson W, Balakrishna N, Griffiths PL. Modeling physical growth using mixed effects models. Am J Phys Anthropol 2013;150:58-67.

6. Wright C, Lakshman R, Emmett P, et al. Implications of adopting the WHO 2006 Child Growth Standard in the UK: two prospective cohort studies. Arch Dis Child 2008;93:566-9.

7. Juliusson PB, Roelants M, Hoppenbrouwers K, et al. Growth of Belgian and Norwegian children compared to the WHO growth standards: prevalence below -2 and above +2 SD and the effect of breastfeeding. Arch Dis Child 2011;96:916-21.

8. Rolland-Cachera MF, Peneau S. Assessment of growth: variations according to references and growth parameters used. Am J Clin Nutr 2011;94:1794S-8S.

9. Turck D, Michaelsen KF, Shamir R, et al. World Health Organization 2006 child growth standards and 2007 growth reference charts: A discussion paper by the committee on Nutrition of the European Society for Pediatric Gastroenterology, Hepatology, and Nutrition. J Pediatr Gastroenterol Nutr 2013;57:258-64.

10. UN Standing Committee on Nutrition (SCN) Endorses the New WHO Growth Standards for Infants and Young Children. http://www.who.int/childgrowth/endorsement_scn.pdf (August 4 2015, date last accessed)

11. International Pediatric Association Endorsement. The New WHO Growth Standards for Infants and Young Children. http://www.who.int/childgrowth/Endorsement_IPA.pdf. (August 4 2015, date last accessed) 


\section{Fish intake during pregnancy and offspring adiposity-Reply}

In Reply- We are delighted that our article has generated interest in the ways that fish intake during pregnancy could affect the risk of childhood obesity. We welcome the Letters to the Editor appearing in this issue of JAMA Pediatrics highlighting vitamin $\mathrm{D}$ and tributyltin as nutritional and environmental constituents in fish.

Hyde et al suggest that vitamin D contained in fish could play a role in the association between fish intake during pregnancy and childhood obesity. Maternal serum 25-hydroxyvitamin $\mathrm{D}$, the major circulating form of vitamin $\mathrm{D}$, diffuses freely across the placenta, and fetal exposure depends solely on maternal concentrations. There is indeed increasing evidence that vitamin D status in pregnancy may influence normal fetal growth and development; ${ }^{1}$ however, the association with child adiposity remains uncertain. ${ }^{2-5}$ Vitamin $\mathrm{D}$ is obtained primarily via exposure to UV-B radiation in sunlight and to a limited extent from dietary sources. However, the type of fish consumed is an important predictor of dietary vitamin $\mathrm{D}$ intake. Fatty fish, in contrast to lean fish, is rich both in vitamin $\mathrm{D}$ and $\omega-3$ long-chain polyunsaturated fatty acids but is also a common route of exposure to persistent organic pollutants owing to their lipophilic characteristics. In our analysis, we did not find evidence for an association of fatty fish intake with childhood overweight and obesity. A possible explanation is the high content of fatty fish in beneficial nutrients, which may outweigh or mask the potential negative effects of pollutants. Another explanation might be the relatively low fatty-fish consumption levels across cohorts (median, 0.49 times/week; IQR, 0.00-0.98), which may have undermined the possibility to detect any association. Given the high prevalence of vitamin D deficiency and increasing rates of childhood obesity, further studies are needed to study the effect of serum 25-hydroxyvitamin D concentration in pregnancy on childhood obesity risk, and experimental studies are required to elucidate potential mechanisms.

Dr Bao highlighted the potential of exposure to the organotin tributyltin as a factor contributing to the association of high fish intake in pregnancy with childhood obesity. Animal studies suggest that prenatal exposure to tributyltin results in increased lipid accumulation in adipose tissue and liver, and thus increases susceptibility to obesity. ${ }^{6}$ Studies that have directly measured organotin compounds in human tissue and blood samples are very limited. Given that exposures to pollutants are correlated owing to common sources (eg, fish), most human populations are exposed to mixtures of endocrine-disrupting chemicals rather than to a single pollutant. With an increasing number of chemicals now proposed as suspected obesogens, there is a need to identify those most relevant for human obesity risk. 
To date, there is no clear answer about the optimal amount and type of fish intake during pregnancy in regards to child growth and development. Next steps should focus on the difficult task of elucidating which individual nutrients or environmental chemicals or combination of factors contained in fish explain the effect of fish intake during pregnancy on child health outcomes. Further analyses incorporating biomarker information on both nutrients and toxicants contained within fish will be helpful for informing dietary recommendations for pregnant women. 


\section{In Reply-References}

1. Theodoratou E, Tzoulaki I, Zgaga L, et al. Vitamin D and multiple health outcomes: umbrella review of systematic reviews and meta-analyses of observational studies and randomised trials. BMJ 2014;348:g2035.

2. Crozier SR, Harvey NC, Inskip HM, et al. Maternal vitamin D status in pregnancy is associated with adiposity in the offspring: findings from the Southampton Women's Survey. Am J Clin Nutr 2012;96:5763.

3. Morales E, Rodriguez A, Valvi D, et al. Deficit of vitamin D in pregnancy and growth and overweight in the offspring. Int J Obes (Lond) 2015;39:61-8.

4. Eckhardt CL, Gernand AD, Roth DE, et al. Maternal vitamin D status and infant anthropometry in a US multi-centre cohort study. Ann Hum Biol 2015;42:215-22.

5. Jensen CB, Gamborg M, Berentzen TL, et al. Prenatal exposure to vitamin-D from fortified margarine and milk and body size at age 7 years. Eur J Clin Nutr 2015;69:1169-75.

6. Grun F, Blumberg B. Endocrine disrupters as obesogens. Mol Cell Endocrinol 2009;304:19-29. 


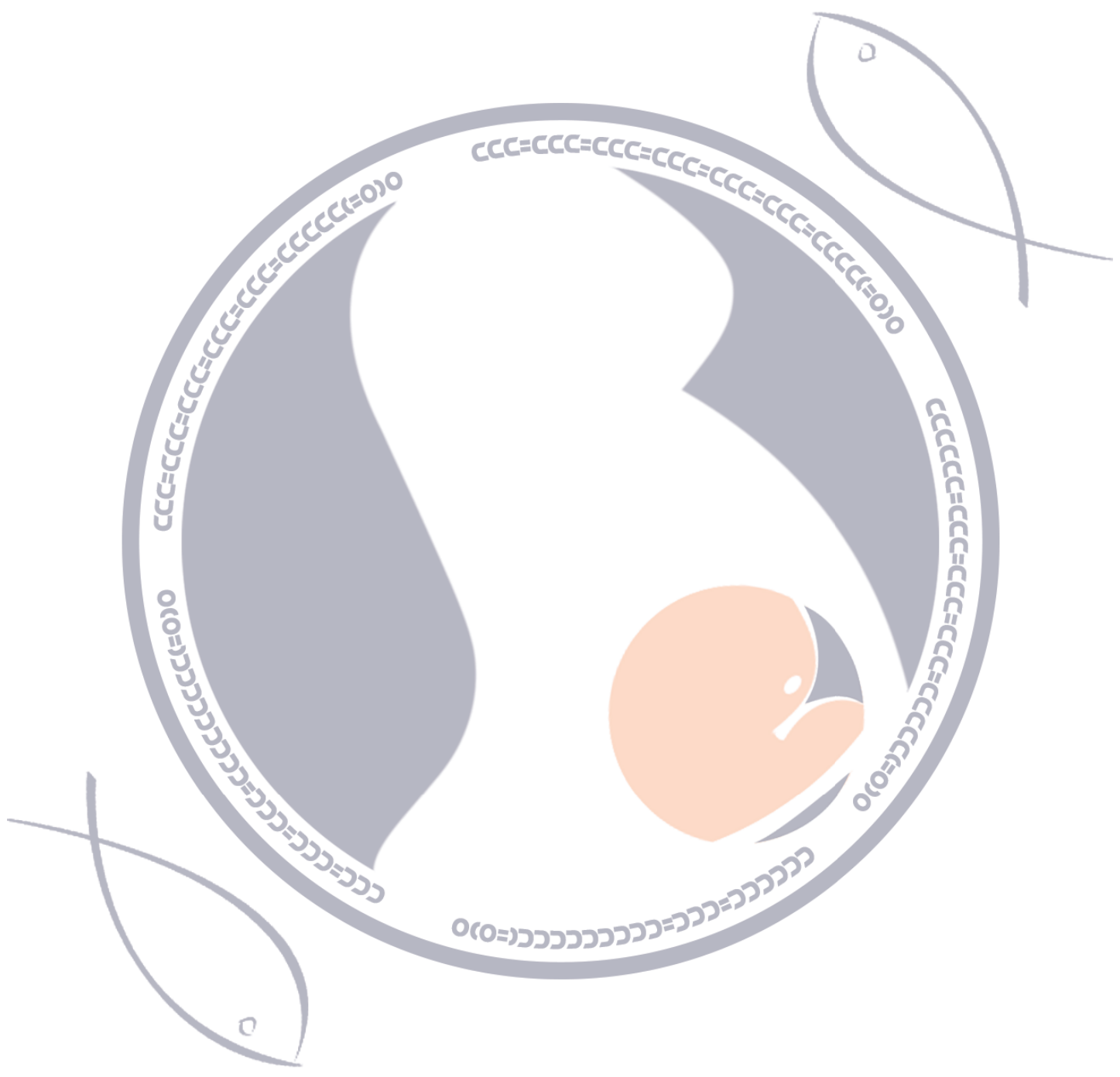




\section{CHAPTER 8}

\section{Fish and seafood consumption during pregnancy and the risk of asthma and allergic rhinitis in childhood: a pooled analysis of 18 European and US birth cohorts}

N Stratakis, T Roumeliotaki, E Oken, F Ballester, H Barros, M Basterrechea, $S$ Cordier, RHM de Groot, HT den Dekker, L Duijts, M Eggesbø, MP Fantini, F Forastiere, U Gehring, M Gielen, D Gori, E Govarts, HM Inskip, N Iszatt, M Jansen, C Kelleher, J Mehegan, C Moltó-Puigmartí, M Mommers, A Oliveira, SF Olsen, F Pelé, C Pizzi, D Porta, L Richiardi, SL Rifas-Shiman, SM Robinson, G Schoeters, M Strøm, J Sunyer, C Thijs, M Vrijheid, Tanja GM Vrijkotte, AH Wijga, MP Zeegers, M Kogevinas, L Chatzi

Adapted from Int J Epidemiol 2017 Oct 1;46(5):1465-1477. 


\section{ABSTRACT}

Background: It has been suggested that prenatal exposure to n-3 long-chain fatty acids protects against asthma and other allergy-related diseases later in childhood. The extent to which fish intake in pregnancy protects against child asthma and rhinitis symptoms remains unclear. We aimed to assess whether fish and seafood consumption in pregnancy is associated with childhood wheeze, asthma, and allergic rhinitis.

Methods: We pooled individual data from 60774 mother-child pairs participating in 18 European and US birth cohort studies. Information on wheeze, asthma and allergic rhinitis prevalence was collected using validated questionnaires. The time periods of interest were: infancy (0-2 years), preschool age (3-4 years), and school age (5-8 years). We used multivariable generalized models to assess associations of fish and seafood (other than fish) consumption during pregnancy with child respiratory outcomes in cohort-specific analyses, with subsequent random-effects meta-analyses.

Results: The median fish consumption during pregnancy ranged from 0.44 times/ week in the Netherlands to 4.46 times/week in Spain. Maternal fish intake during pregnancy was not associated with offspring wheeze symptoms in any age group nor with the risk of child asthma (adjusted meta-analysis relative risk [RR] per 1 -time/week = 1.01 [95\% CI 0.97-1.05]) and allergic rhinitis at school age $(\mathrm{RR}=1.01$ [95\% CI 0.99-1.03]). These results were consistently found in further analyses by type of fish and seafood consumption, and sensitivity analyses.

Conclusion: We found no evidence supporting a protective association of fish and seafood consumption during pregnancy with offspring symptoms of wheeze, asthma and allergic rhinitis from infancy to mid-childhood. 


\section{INTRODUCTION}

Asthma and allergic rhinitis are two of the most common chronic diseases in childhood, and their prevalence rates have increased over the past decades. ${ }^{1}$ Emerging evidence suggests that a nutritional stressor or stimulus applied in intrauterine and early postnatal life can affect the development and maturation of respiratory and immune systems. ${ }^{2}$ In this context, there has been a strong debate about the role of fish intake during pregnancy in the prevention of child asthma and other allergic diseases. Fish, and especially oily fish, is a rich source of n-3 long-chain fatty acids, which have been suggested to decrease the risk of allergic disease by exerting antiinflammatory properties and modulating immune responses. ${ }^{3}$ However, it is also a major source of human exposure to harmful environmental contaminants, such as methylmercury, polychlorinated biphenyls and dioxins. ${ }^{4}$ Findings from birth cohort studies on the association of fish intake during pregnancy with offspring allergy-related disease symptoms are inconsistent, with reports of beneficial (ie, lower incidence), ${ }^{5-8}$ null ${ }^{9-16}$ or even harmful effects. ${ }^{17}$ Likewise, prospective studies assessing $n-3$ fatty acid intake ${ }^{18}$ or biomarker levels in pregnancy ${ }^{19-25}$ and trials of $\mathrm{n}-3$ long-chain fatty acid supplementation in pregnancy ${ }^{18,26,27}$ have yielded divergent results.

Most of prior studies relied on relatively small sample sizes with a short duration of follow up, and findings from one study were not replicated across populations with different fish consumption patterns. In the present analysis, we addressed these limitations by harmonizing and pooling for the first time individual data from a large network of cohorts in Europe and the US, involving 60774 pregnant women and their children with repeated follow-ups until the age of 8 years, to assess the strength and consistency of the association of fish and seafood consumption during pregnancy with the risk of childhood wheeze, asthma and allergic rhinitis.

\section{METHODS}

\section{Study population}

European birth cohort studies participating in our previous analysis on fish intake in pregnancy and birth outcomes ${ }^{28}$ were invited to participate if information on symptoms of asthma and rhinitis in childhood was available. From the 19 potentially eligible cohorts, 16 cohorts provided relevant data for the present analysis: ABCD (Netherlands); DNBC (Denmark); FLEHS I (Belgium); GASPII (Italy); Generation R study (Netherlands); Generation XXI study (Portugal); HUMIS (Norway); INMA (Spain); KOALA (Netherlands); Lifeways Cross Generation (Ireland); LucKi (Netherlands); NINFEA (Italy); PELAGIE (France); PIAMA 
(Netherlands); RHEA (Greece); and SWS (UK). In addition, the Project Viva cohort from Massachusetts and the Bologna Birth Cohort agreed to take part in the current analysis. All participating cohorts covered deliveries from 1996 to 2011. In all cohorts, participants' parents or legal guardians provided written informed consent, and ethical approval was obtained from the local authorized institutional review boards. A data-transfer agreement document was signed by each cohort study, and anonymized datasets were transferred to the University of Crete for analysis.

Overall, the study population included 60774 mother-child pairs with information on fish intake during pregnancy, selected confounding variables, and at least one of the health outcomes studied. Characteristics of the participating cohorts are shown in the Supplementary Table S8.1 and Supplementary Figure S8.1.

\section{Fish and seafood consumption during pregnancy}

The exposure of interest was the frequency (times/week) of total fish, fatty fish, lean fish, and seafood (other than fish) intake during pregnancy derived from cohort-specific food frequency questionnaires or questionnaires specifically designed to assess fish intake during pregnancy (Supplementary Table S8.1). Salmon, herring, mackerel, trout, sardines, Greenland halibut, anchovy, gurnard, and tuna were classified as fatty fishes, whereas cod, pollack, plaice, flounder, garfish, and similar species were classified as lean fishes. To align our analysis with the recent advice from the United States Food and Drug Administration and Environmental Protection Agency ${ }^{29}$ on fish intake during pregnancy, and examine a potential dose-response relationship, we categorized total fish intake into the following categories; low: $\leq 1$ time/week, moderate: $>1$ but $\leq 3$ times/week, and high: $>3$ times/week.

\section{Symptoms of asthma and rhinitis in childhood}

Participating cohorts provided information on wheeze, asthma and allergic rhinitis occurrence, mostly obtained by questionnaires adapted from the International Study on Asthma and Allergy in Childhood (ISAAC), ${ }^{30}$ up to a maximum follow-up of 8 years. Cohort-specific information on outcome collection is shown in Supplementary Table S8.2. We defined wheeze in infancy as the presence of any episode of wheezing or whistling in the chest during the first 2 years of life, while wheeze in preschool and school age children was defined as the presence of wheezing or whistling in the chest in the past 12 months reported at the age of 3-4 years and 5-8 years, respectively, depending on the cohort-specific time points of assessment. Persistent wheeze at preschool and school age was defined as presence of wheeze both in infancy and the respective time period examined. ${ }^{31}$ 
We defined asthma at preschool and school age as satisfying at least two of the three following criteria for each time period: (1) ever-reported doctor diagnosis of asthma, (2) presence of wheezing or whistling in the chest in the past 12 months, or (3) asthma medication in the past 12 months at the age of 3-4 years and 5-8 years, respectively. ${ }^{32}$ Allergic rhinitis at school age was defined as ever-reported diagnosis of allergic rhinitis or hay fever at the age of 5-8 years. If participants among the cohorts had repeatedly collected data within the same period, we used data collected at the oldest age.

\section{Covariates}

Important covariates or effect modifiers were defined as similarly as possible among the cohorts. Information on maternal pre-pregnancy body mass index (BMI; in $\mathrm{kg} / \mathrm{m}^{2}$ ), maternal smoking during pregnancy (yes/no), gestational weight gain (in $\mathrm{kg}$ ), maternal age at delivery (in years), birth weight (in grams), gestational age (in weeks), and child sex (male or female) was collected by means of interviews or self-administered questionnaires, ad hoc measurements, birth records, or medical registries. Information on parity (primiparous or multiparous), breastfeeding (yes/ no), maternal education (cohort-specific definitions of low, medium, or high), attendance at nursery school during infancy (yes/no), exposure to mold or dampness at home during infancy (yes/no), and history of parent asthma or hay fever (yes/no) was obtained through interviews or self-administered questionnaires.

\section{Statistical analysis}

We used multivariable generalized linear models for binary outcomes (modified Poisson $^{33}$ ) to estimate relative risks (RRs) and their $95 \%$ confidence intervals (CIs) for the associations of maternal fish and seafood consumption during pregnancy with offspring wheeze, asthma and allergic rhinitis in childhood. Selection of suitable covariates for model adjustment was based on prior knowledge and a Directed Acyclic Graph (DAG) approach (Supplementary Figure S8.2). The set of variables identified by the DAG were maternal education, maternal smoking during pregnancy, parity, breastfeeding, and parent asthma or hay fever (maternal allergy for PELAGIE). This list of variables plus maternal age and child sex, which are considered important determinants of child health outcomes, were the covariates included in multivariable models. For the cohort Generation XXI, the adjusted models did not include parent history of asthma or hay fever, owing to a large proportion of missing information on this variable. Further adjustment for maternal pre-pregnancy BMI, attendance at nursery school during infancy, and exposure to mold or dampness at home during infancy did not materially change estimates for fish intake but reduced substantially the sample size, thus, we did 
not to include these factors in our final models. ${ }^{34}$ Additionally, we did not include birth weight or gestational age in our main models, as we assumed that they are potential mediators in the pathway between fish intake during pregnancy and childhood respiratory health outcomes. ${ }^{28,35}$

We analyzed associations by undertaking cohort-specific analyses with subsequent random-effects meta-analysis. We assessed heterogeneity between cohort-specific effect estimates with the $\mathrm{I}^{2}$ statistic and $\chi^{2}$ test from Cochran's $\mathrm{Q}^{36}{ }^{36} \mathrm{We}$ also did pooled analysis including all participating cohorts by using mixed models with a random cohort intercept.

We performed several sensitivity analyses. First, we assessed the robustness of the results by repeating the meta-analyses while excluding one cohort at a time. Second, we examined the potential effect of the geographical location of participating cohorts, based on the United Nations' classification (Southern Europe, Western Europe, Northern Europe, and Northern America), by conducting meta-regression analyses. Third, we made further adjustment for gestational weight gain, used as a proxy for energy intake during pregnancy, for cohorts with available information. Fourth, we included birth weight and gestational age in the multivariable models to account for potential mediating effect of fetal growth. Finally, we assessed whether the effect estimates for wheeze in infancy and asthma in childhood varied by parent asthma or hay fever (yes vs no), and child sex (boy vs girl) by introducing interaction terms (one at a time), and conducting stratified analyses. ${ }^{34}$ We performed analyses with STATA version 13 (StataCorp) and R version R3.1 (R Foundation).

\section{RESULTS}

Participants' characteristics are shown in Supplementary Tables S8.3 to S8.6. Mothers had a median age of 30.4 years, were predominantly nonsmokers, gave birth mainly to normal weight neonates, and most of them breastfed their children. The ratio of boys to girls was 1.04 (Supplementary Table S8.3). The median fish consumption during pregnancy varied between cohorts and ranged from 0.44 times/week in the Generation R Study to 4.46 times/week in INMA (Figure 8.1 and Supplementary Table S8.4). The highest median seafood intake was also reported in INMA (0.92 times/week) (Supplementary Table S8.4). We observed no differences in fish consumption levels between mothers with and without asthma or hay fever across cohorts (Supplementary Table S8.5). 


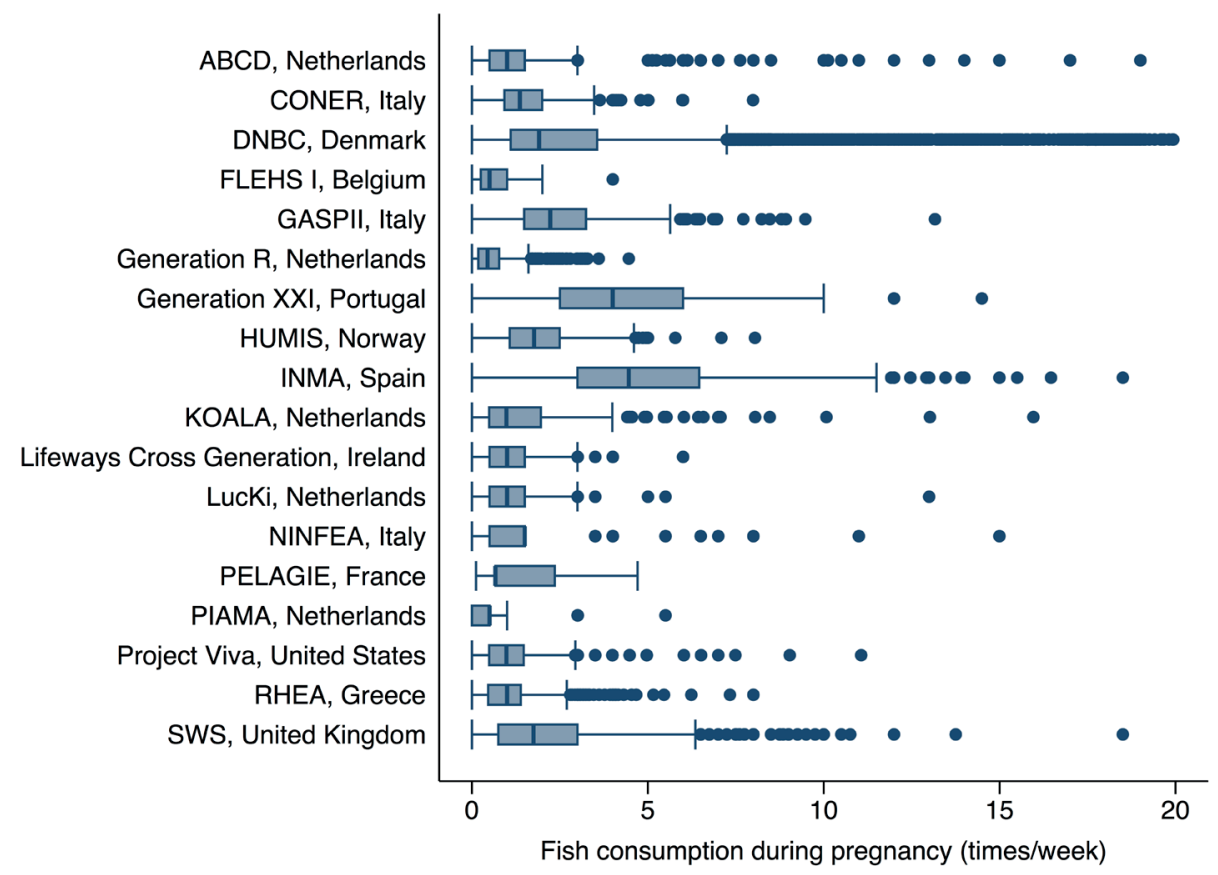

Figure 8.1. Frequency of fish consumption during pregnancy (times/week) in participating cohorts. The line within the box marks the median; the boundaries of the box indicate the 25th and 75th percentiles; horizontal bars denote the variability outside the upper and lower quartiles (ie, within 1.5 IQR of the lower and upper quartiles); and circles represent outliers.

In total, 17518 (29.2\%), 1949 (15.4\%), and 3050 (13.1\%) children had wheeze in infancy ( $0-2$ years), at preschool (3-4 years) and school age (5-8 years), respectively (Supplementary Table S8.6). Nine hundred twenty-one (9.2\%) and 2479 $(10.5 \%)$ children had asthma at preschool and school age, respectively, while 1914 (5.4\%) children had allergic rhinitis at school age (Supplementary Table S8.6).

Maternal fish intake during pregnancy was not associated with offspring wheeze symptoms in any age group (Figure 8.2 and Table 8.1). Also, no association was observed for persistent wheeze at preschool or school age (Supplementary Table S8.7). When we examined the risk of asthma at either preschool or school age, we observed no association with fish intake during pregnancy (adjusted meta-analysis RR per 1-time/week= 1.02 [95\% CI 0.97-1.07] and 1.01 [95\% CI 0.97-1.05], respectively; Figure 8.3 and Table 8.2). Similarly, fish intake was not associated with the diagnosis of allergic rhinitis among school-age children (Table 8.2). 


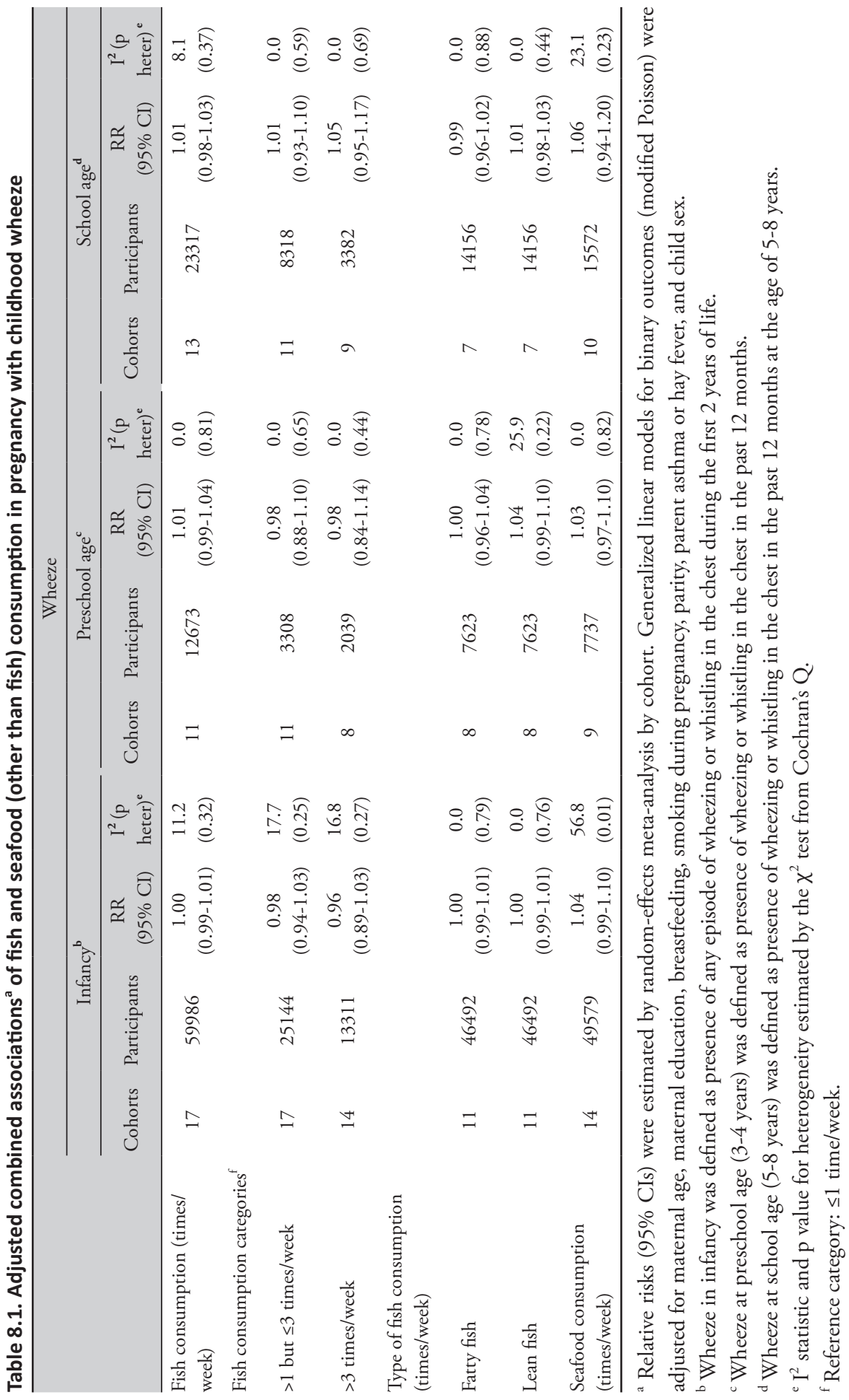


Further analysis by type of fish and seafood (other than fish) consumption did not modify the observed associations (Tables 8.1 and 8.2). Crude and adjusted estimates did not differ substantially (data not shown). Heterogeneity between cohort-specific risk estimates was low for most exposure-outcome associations (Tables 8.1 and 8.2; Supplementary Table S8.7), and the combined effect estimates from random-effect meta-analyses were broadly similar to those from the pooled analysis (Supplementary Tables S8.8 and S8.9).

In sensitivity analyses, omitting one cohort at a time did not materially change the results (Supplementary Tables S8.10 and S8.11). The risk estimates also remained similar when we took into account the geographical location of participating cohorts in meta-regression analyses, and when additional adjustment was made for gestational weight gain, or birth weight and gestational age in multivariable models (data not shown). In stratified analyses, we found that fish intake in pregnancy was associated with a lower risk of asthma in preschool children with no history of parental asthma or hay fever (RR per 1 time/week= 0.91 [95\% CI 0.860.96]), but not in those of parents having either of these conditions (1.04 [95\% CI 0.98-1.10]; P for interaction= 0.03; Supplementary Table S8.12). However, this interaction with parental history of asthma or hay fever was not observed for associations with wheeze, or with asthma and allergic rhinitis at school age. Fish intake during pregnancy tended to be more protective against asthma in school-age boys (RR per 1 time/week= 0.99 [95\% CI 0.99-1.00]) but not in girls (1.01 [95\% CI 0.99-1.03]; P for interaction= 0.03), but associations were small in magnitude and not clinically relevant (Supplementary Table S8.12). 


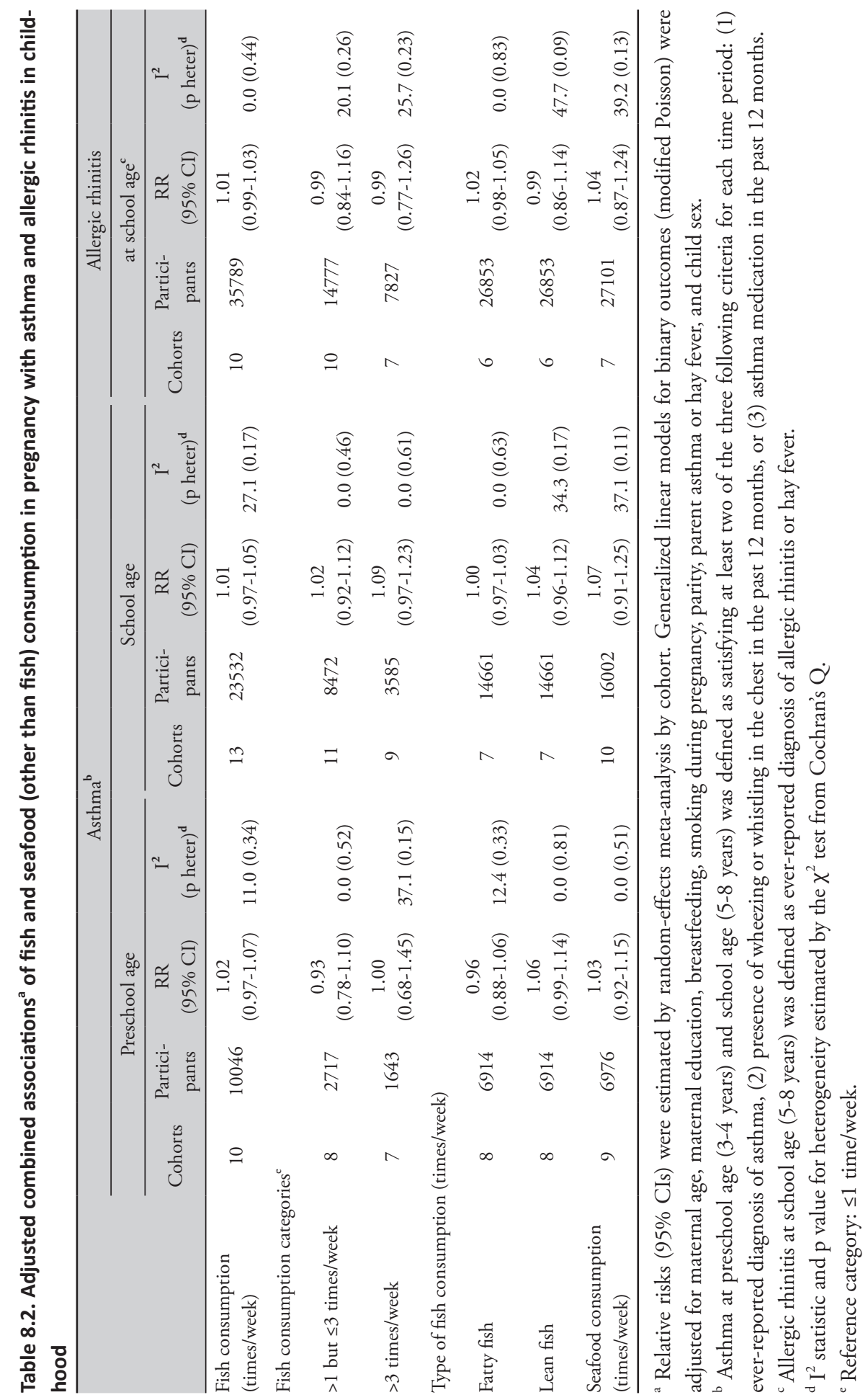




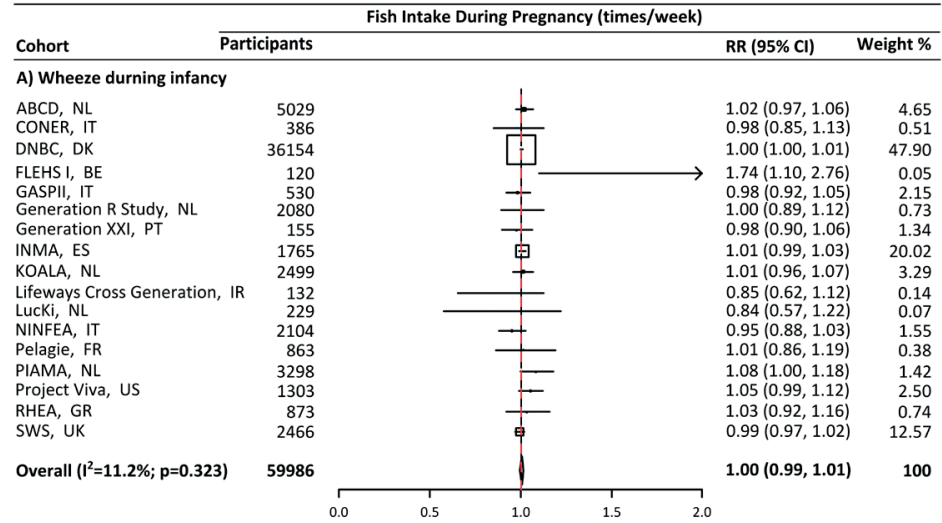

B) Wheeze at preschool age

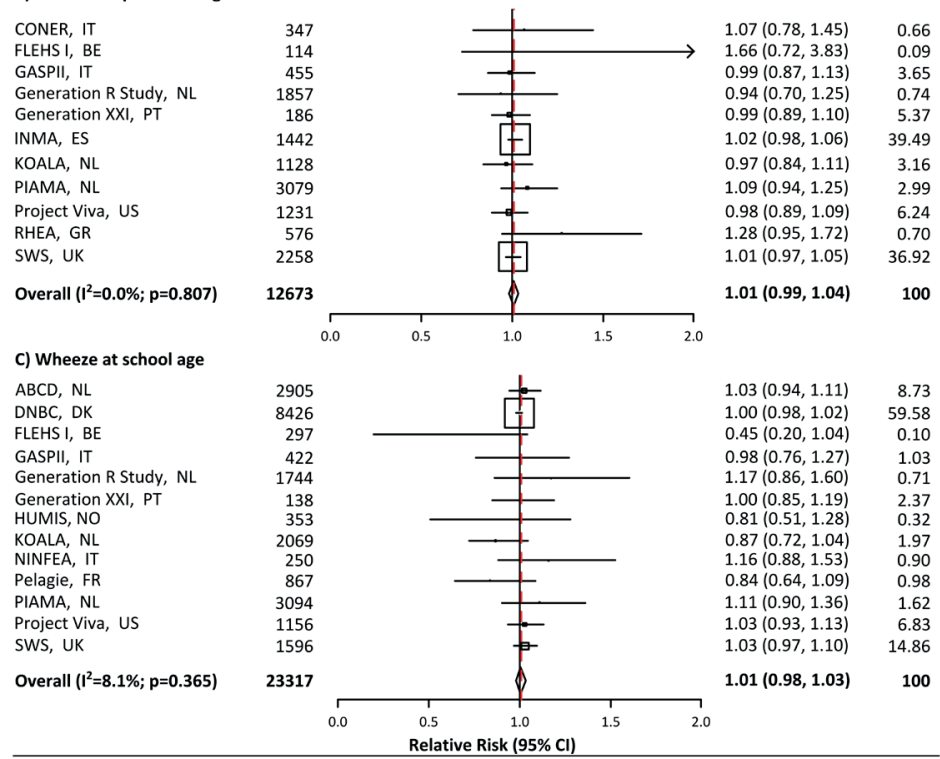

Figure 8.2. Adjusted associations of fish consumption during pregnancy (times/week) with offspring wheeze in infancy, preschool age, and school age. Wheeze in infancy was defined as presence of any episode of wheezing or whistling in the chest during the first 2 years of life. Wheeze at preschool age was defined as presence of wheezing or whistling in the chest in the past 12 months at the age of 3-4 years. Wheeze at school age was defined as presence of wheezing or whistling in the chest in the past 12 months at the age of 5-8 years. RRs (95\% Cls) by cohort were obtained by using generalized linear models for binary outcomes (modified Poisson) adjusted for maternal age, maternal education, breastfeeding, smoking during pregnancy, parity, parent asthma or hay fever, and child sex. Combined estimates were obtained by using a random-effects meta-analysis. The names of the cohorts and number of participants per each cohort are shown on the left. Cohort-specific RRs (95\% Cls), and their weights in meta-analysis are shown on the right. Squares represent the point estimate of each cohort, while the size of the square is proportional to the weight with which each cohort contributed to the overall RR; horizontal lines denote $95 \% \mathrm{Cls}$; and diamonds represent overall estimates. RR= relative risk. 


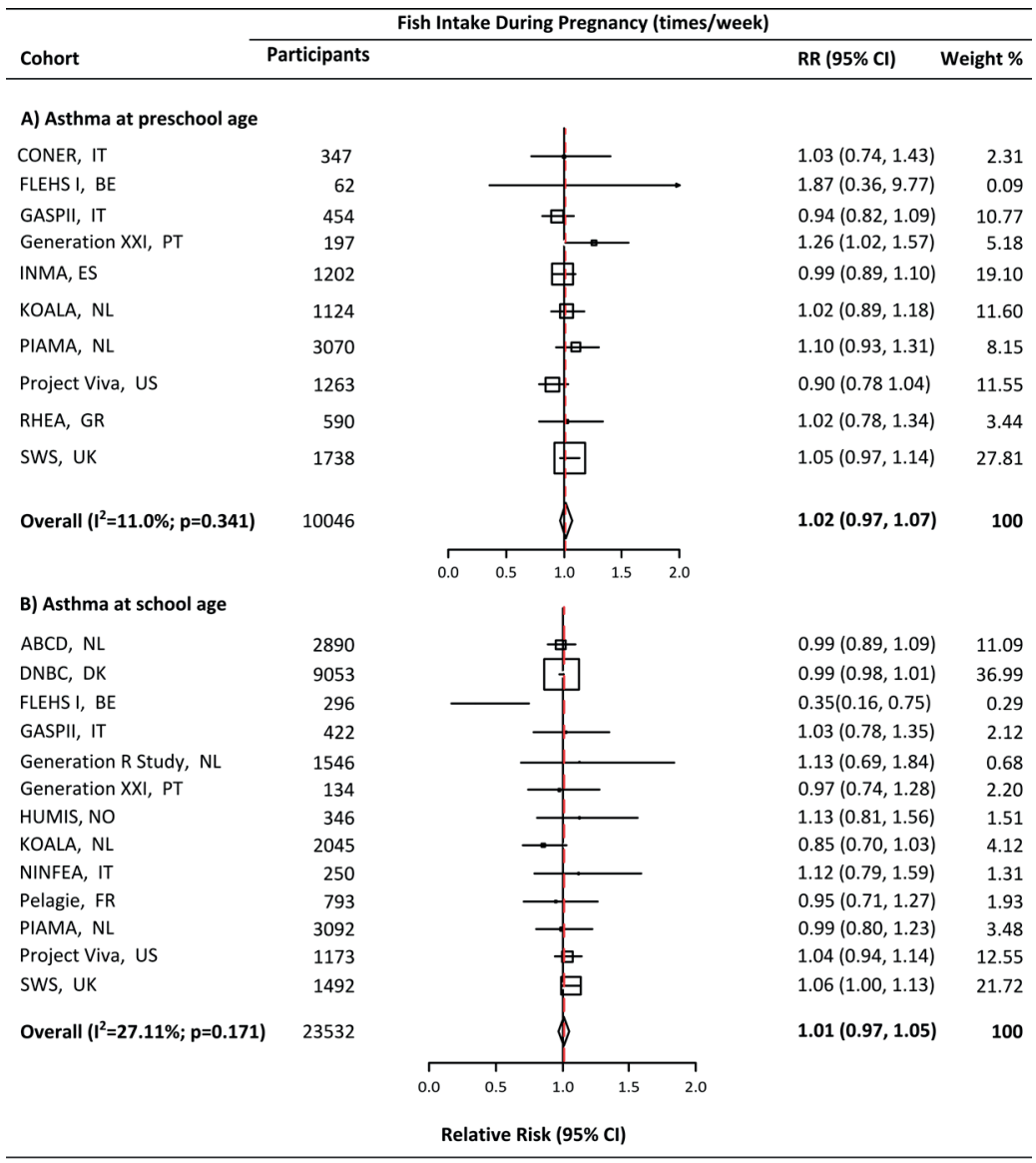

Figure 8.3. Adjusted associations of fish consumption during pregnancy (times/week) with offspring asthma at preschool age and school age. Asthma at preschool age (3-4 years) and school age (5-8 years) was defined as satisfying at least two of the three following criteria for each time period: (1) ever-reported diagnosis of asthma, (2) presence of wheezing or whistling in the chest in the past 12 months, or (3) asthma medication in the past 12 months. RRs (95\% Cls) by cohort were obtained by using generalized linear models for binary outcomes (modified Poisson) adjusted for maternal age, maternal education, breastfeeding, smoking during pregnancy, parity, parent asthma or hay fever, and child sex. Combined estimates were obtained by using a random-effects meta-analysis. The names of the cohorts and number of participants per each cohort are shown on the left. Cohort-specific RRs ( $95 \% \mathrm{Cls})$, and their weights in meta-analysis are shown on the right. Squares represent the point estimate of each cohort, while the size of the square is proportional to the weight with which each cohort contributed to the overall RR; horizontal lines denote $95 \% \mathrm{Cls}$; and diamonds represent overall estimates. RR= relative risk. 


\section{DISCUSSION}

In this study of more than 60000 mother child pairs from several locations in Europe and the United States with repeated follow-up data from birth up to 8 years, we found no protective association between fish and seafood consumption during pregnancy and risk of childhood wheeze, asthma and allergic rhinitis. The present study is a major extension of previous collaborative work examining fish intake during pregnancy and child health. ${ }^{28,37}$

Fetal life is a critical time period for airway and immune development during which dietary exposures could have irreversible long-term influences on the development of asthma and other allergy-related diseases. ${ }^{38}$ The n-3 long-chain fatty acids contained within fish can be transferred across the placenta and have been proposed to exert a protective effect through involvement in several immune regulatory pathways. Animal and in vitro studies have shown n-3 fatty acids to alter the expression of inflammatory genes by modifying transcription factor activation, inhibit $\mathrm{T}$ cell signaling through alteration of membrane lipid rafts, decrease the production of proinflammatory eicosanoids, cytokines, and reactive oxygen species, as well as to give rise to a family of anti-inflammatory mediators termed resolvins (reviewed in ${ }^{39}$ ). A beneficial effect of $n-3$ long-chain fatty supplementation in fetal or early life on childhood allergic disease symptoms has been shown in many, but not all, randomized trials. ${ }^{18,26,27,40}$ A relation between supplementation dose and effect becomes more apparent in the very low end of the exposure spectrum of the n-3 long-chain fatty acids. Although fish and seafood is the major source of preformed n-3 long-chain fatty acids in the diet in most populations worldwide, maternal status is also determined by endogenous conversion from dietary alpha-linolenic acid from plant sources. A minority of the population has a genetically determined low endogenous conversion rate, and hence a higher dependence of preformed n-3 long-chain fatty acid intake from the diet. ${ }^{41}$ Therefore, our results do not exclude the possibility of a protective effect of fish intake against asthma and allergy in vulnerable subgroups.

Several birth cohort studies have examined the association of prenatal fish intake with asthma and rhinitis symptoms in childhood and have produced mixed results. High fish intake during pregnancy was associated with a lower risk of wheeze up to the age of 6.5 years in the INMA-Menorca cohort, ${ }^{5,7}$ while the DNBC study showed a protective effect of fish intake on ever asthma at the age of 7 years by using hospital admission and prescription registry data. ${ }^{6}$ Other prospective studies conducted in the Netherlands, ${ }^{12}$ Finland, ${ }^{11,16}$ Norway, ${ }^{14}$ Spain and Greece, ${ }^{13}$ France $^{15}$, and Japan ${ }^{9,10}$ did not find an association of prenatal fish consumption with wheeze or asthma occurrence in childhood. Only few previous studies have assessed symptoms of childhood allergic rhinitis in association with 
fish intake in pregnancy; one UK study found that higher maternal intake of oily fish was protective against hay fever at 5 years, ${ }^{8}$ whereas the DNBC study ${ }^{6}$ and a Finnish study ${ }^{11}$ found no association with allergic rhinitis up to the age of 7 years. Heterogeneity in previous findings may arise from differences in sample sizes, exposure assessment, health outcome definition, and adjustment for confounding variables. In the present study, we had a large sample size, and harmonized information on exposure variables, potential confounders, and child asthma and rhinitis symptoms. We examined child outcomes at different time points from early infancy to mid-childhood, and found little evidence for an association with fish intake during pregnancy.

Fish is generally considered an integral component of a healthy diet. However, it is a complex nutritional exposure. Fish is a good source of beneficial nutrients, including $\mathrm{n}-3$ long-chain fatty acids, but is also a common route of exposure to persistent organic pollutants, such as polychlorinated biphenyls and dioxins. ${ }^{4}$ There is emerging evidence suggesting that prenatal exposure to these chemicals can exert adverse immunomodulatory effects and increase the risk of developing allergic disease symptoms in childhood. ${ }^{42,43}$ Hence, it is possible that pollutants bioaccumulating in fish counterbalance the effects of beneficial nutrients, moving the association between fish intake and child respiratory health towards the null. We did not have the possibility to collect information on amounts of nutrients and environmental chemicals contained in fish. Although we collected information on different fish types (fatty fish, lean fish, shellfish), we did not have enough data to distinguish between consumption of big and small species and fish origin, which would be relevant with respect to toxicant exposure. Further analyses using biomarker information on both nutrients and toxicants in fish are required to examine their interplay, as well as to disentangle potential opposite effect on child outcomes.

Genetic predisposition plays a major role in asthma. ${ }^{44,45}$ A recent systematic review concluded that family history of allergy should be considered as an important effect modifier when studying the association of diet with the development of childhood asthma or allergies. ${ }^{34}$ It has been proposed that atopic heredity is associated with abnormalities in fatty acid metabolism, ${ }^{46}$ and this might affect body utilization of the fatty acids contained in fish. Prior studies examining a potential modifying effect of maternal history of asthma or atopy on prenatal fish intake have produced inconsistent results. ${ }^{47,48}$ In our study, we found that, among children of parents with no history of asthma or hay fever, prenatal fish intake was associated with lower asthma risk at preschool age, but not later in childhood, or with wheeze or allergic rhinitis. Thus, we treat this result with caution, as we cannot rule out the possibility that the observed association is due to chance. Further 
prospective studies incorporating genetic analysis are needed to evaluate whether genetic predisposition can modify the effect of fish intake during pregnancy on child allergy symptoms.

As in any observational study, there is the possibility of unmeasured residual confounding accounting for at least part of the observed findings. We had no data available to adjust for energy intake or dietary patterns during pregnancy; however, adjustment for gestational weight gain, as a good proxy for total energy intake in pregnancy, ${ }^{49,50} \mathrm{did}$ not appreciably change the effect estimates. Moreover, although we examined breastfeeding as a potential confounder, other differences in child diet or lifestyle factors might have influenced the observed findings. However, the absence of important heterogeneity across cohorts and the similar effect estimates observed in the meta-analysis and the pooled analysis provide evidence in support of more robust and generalized conclusions.

Similar to most studies assessing the health effects of diet, we used self-reported dietary information, and we cannot rule out the possibility of exposure measurement error, especially in the absence of biomarkers of fish intake. However, most cohorts assessed fish intake using food frequency questionnaires that were developed and validated for use in pregnancy. Additionally, previous analyses in many participating cohorts have shown fish intake, as assessed by questionnaires, to positively correlate with exposure to $\mathrm{n}-3$ long-chain fatty acids or environmental pollutants commonly found in fish. ${ }^{51-57}$ Any measurement error in the assessment of fish intake itself is likely to be non-differential with respect to the outcomes of interest, given that fish intake levels did not differ between mothers with and without asthma or hay fever across the cohorts, and dietary data were collected before child respiratory outcomes were known. Child asthma and allergic rhinitis symptoms were ascertained by symptom-based questionnaires, which could lead to outcome misclassification. However, most participating cohorts used questionnaires adapted from the ISAAC study. ${ }^{30}$ To enhance asthma outcome accuracy, we used a definition developed by a panel of experts within the Mechanisms of the Development of Allergy consortium ${ }^{32}$ that requires at least two conditions to be met. We assessed several asthma-related phenotypes, raising concern about multiple testing. However, an application of correction to take into account multiple comparisons would be inappropriate given that we studied outcomes that are highly correlated. ${ }^{58}$

In conclusion, this large multicohort study found no evidence supporting a protective association between fish intake during pregnancy and offspring symptoms of wheeze, asthma and allergic rhinitis from infancy to mid-childhood. 


\section{ACKNOWLEDGMENTS}

This work was supported by the European Community's Seventh Framework Program [EU- FP7- HEALTH-2009-single-stage- 241604]. Details of funding per cohort are available in the published article: Int J Epidemiol 2017 Feb 27. doi: 10.1093/ije/dyx007. [Epub ahead of print] 


\section{REFERENCES}

1. Bousquet J, Khaltaev N (eds). Global surveillance, prevention and control of chronic respiratory diseases: a comprehensive approach. 2007. World Health Organization.

2. Devereux G. The increase in the prevalence of asthma and allergy: food for thought. Nat Rev Immunol 2006;6:869-74.

3. Calder PC, Kremmyda LS, Vlachava M, et al. Is there a role for fatty acids in early life programming of the immune system? Proc Nutr Soc 2010;69:373-80.

4. Mozaffarian D, Rimm EB. Fish intake, contaminants, and human health: evaluating the risks and the benefits. JAMA 2006;296:1885-99.

5. Romieu I, Torrent M, Garcia-Esteban R, et al. Maternal fish intake during pregnancy and atopy and asthma in infancy. Clin Exp Allergy 2007;37:518-25.

6. Maslova E, Strøm M, Oken E, et al. Fish intake during pregnancy and the risk of child asthma and allergic rhinitis - longitudinal evidence from the Danish National Birth Cohort. Br J Nutr 2013;110:1313-25.

7. Chatzi L, Torrent M, Romieu I, et al. Mediterranean diet in pregnancy is protective for wheeze and atopy in childhood. Thorax 2008;63:507-13.

8. Willers SM, Devereux G, Craig LC, et al. Maternal food consumption during pregnancy and asthma, respiratory and atopic symptoms in 5-year-old children. Thorax 2007;62:773-9.

9. Miyake $\mathrm{Y}$, Tanaka K, Okubo $\mathrm{H}$, et al. Maternal fat intake during pregnancy and wheeze and eczema in Japanese infants: the Kyushu Okinawa Maternal and Child Health Study. Ann Epidemiol 2013;23:67480 .

10. Miyake Y, Sasaki S, Tanaka K, et al. Maternal fat consumption during pregnancy and risk of wheeze and eczema in Japanese infants aged 16-24 months: the Osaka Maternal and Child Health Study. Thorax 2009;64:815-21.

11. Erkkola M, Nwaru BI, Kaila M, et al. Risk of asthma and allergic outcomes in the offspring in relation to maternal food consumption during pregnancy: a Finnish birth cohort study. Pediatr Allergy Immunol 2012;23:186-94.

12. Willers SM, Wijga AH, Brunekreef B, et al. Maternal food consumption during pregnancy and the longitudinal development of childhood asthma. Am J Respir Crit Care Med 2008;178:124-31.

13. Chatzi L, Garcia R, Roumeliotaki T, et al. Mediterranean diet adherence during pregnancy and risk of wheeze and eczema in the first year of life: INMA (Spain) and RHEA (Greece) mother-child cohort studies. Br J Nutr 2013;110:2058-68.

14. Oien T, Storro O, Johnsen R. Do early intake of fish and fish oil protect against eczema and doctordiagnosed asthma at 2 years of age? A cohort study. J Epidemiol Community Health 2010;64:124-9.

15. Pele F, Bajeux E, Gendron H, et al. Maternal fish and shellfish consumption and wheeze, eczema and food allergy at age two: a prospective cohort study in Brittany, France. Environ Health 2013;12:102.

16. Lumia M, Luukkainen P, Tapanainen H, et al. Dietary fatty acid composition during pregnancy and the risk of asthma in the offspring. Pediatr Allergy Immunol 2011;22:827-35.

17. Leermakers ET, Sonnenschein-van der Voort AM, Heppe DH, et al. Maternal fish consumption during pregnancy and risks of wheezing and eczema in childhood: the Generation R Study. Eur J Clin Nutr 2013;67:353-9.

18. Best KP, Gold M, Kennedy D, et al. Omega-3 long-chain PUFA intake during pregnancy and allergic disease outcomes in the offspring: a systematic review and meta-analysis of observational studies and randomized controlled trials. Am J Clin Nutr 2016;103:128-43.

19. Rucci E, den Dekker HT, de Jongste JC, et al. Maternal fatty acid levels during pregnancy, childhood lung function and atopic diseases. The Generation R Study. Clin Exp Allergy 2016;46:461-71. 
20. Yu YM, Chan YH, Calder PC, et al. Maternal PUFA status and offspring allergic diseases up to the age of 18 months. Br J Nutr 2015;113:975-83.

21. Barman M, Johansson S, Hesselmar B, et al. High levels of both n-3 and n-6 long-chain polyunsaturated fatty acids in cord serum phospholipids predict allergy development. PLoS One 2013;8:e67920.

22. Notenboom ML, Mommers M, Jansen EH, et al. Maternal fatty acid status in pregnancy and childhood atopic manifestations: KOALA Birth Cohort Study. Clin Exp Allergy 2011;41:407-16.

23. Pike KC, Calder PC, Inskip HM, et al. Maternal plasma phosphatidylcholine fatty acids and atopy and wheeze in the offspring at age of 6 years. Clin Dev Immunol 2012;2012:474613.

24. Newson RB, Shaheen SO, Henderson AJ, et al. Umbilical cord and maternal blood red cell fatty acids and early childhood wheezing and eczema. J Allergy Clin Immunol 2004;114:531-7.

25. Standl M, Demmelmair H, Koletzko B, et al. Cord blood LC-PUFA composition and allergic diseases during the first 10 yr. Results from the LISAplus study. Pediatr Allergy Immunol 2014;25:344-50.

26. Best KP, Sullivan T, Palmer D, et al. Prenatal fish oil supplementation and allergy: 6-year follow-up of a randomized controlled trial. Pediatrics 2016;137:e20154443.

27. Hansen S, Strom M, Maslova E, et al. Fish oil supplementation during pregnancy and allergic respiratory disease in the adult offspring. J Allergy Clin Immunol 2016 Apr 25. doi: 10.1016/j.jaci.2016.02.042. [Epub ahead of print]

28. Leventakou V, Roumeliotaki T, Martinez D, et al. Fish intake during pregnancy, fetal growth, and gestational length in 19 European birth cohort studies. Am J Clin Nutr 2014;99:506-16.

29. Fish: What Pregnant Women and Parents Should Know. Draft Updated Advice by FDA and EPA. June 2014. http://www.fda.gov/Food/FoodborneIllnessContaminants/Metals/ucm393070.htm (8 December 2016, date last accessed)

30. Asher MI, Keil U, Anderson HR, et al. International Study of Asthma and Allergies in Childhood (ISAAC): rationale and methods. Eur Respir J 1995;8:483-91.

31. Martinez FD, Wright AL, Taussig LM, et al. Asthma and wheezing in the first six years of life. The Group Health Medical Associates. N Engl J Med 1995;332:133-8.

32. Pinart M, Benet M, Annesi-Maesano I, et al. Comorbidity of eczema, rhinitis, and asthma in IgEsensitised and non-IgE-sensitised children in MeDALL: a population-based cohort study. Lancet Respir Med 2014;2:131-40.

33. Zou G. A modified poisson regression approach to prospective studies with binary data. Am J Epidemiol 2004;159:702-6.

34. Nurmatov U, Nwaru BI, Devereux G, et al. Confounding and effect modification in studies of diet and childhood asthma and allergies. Allergy 2012;67:1041-59.

35. den Dekker HT, Sonnenschein-van der Voort AM, de Jongste JC, et al. Early growth characteristics and the risk of reduced lung function and asthma: A meta-analysis of 25,000 children. J Allergy Clin Immunol 2016;137:1026-35.

36. Higgins JP, Thompson SG. Quantifying heterogeneity in a meta-analysis. Stat Med 2002;21:1539-58.

37. Stratakis N, Roumeliotaki T, Oken E, et al. Fish Intake in Pregnancy and Child Growth: A Pooled Analysis of 15 European and US Birth Cohorts. JAMA Pediatr 2016;170:381-90.

38. Devereux G. Early life events in asthma-diet. Pediatr Pulmonol 2007;42:663-73.

39. Calder PC, Krauss-Etschmann S, de Jong EC, et al. Early nutrition and immunity - progress and perspectives. Br J Nutr 2006;96:774-90.

40. Gunaratne AW, Makrides M, Collins CT. Maternal prenatal and/or postnatal n-3 long chain polyunsaturated fatty acids (LCPUFA) supplementation for preventing allergies in early childhood. Cochrane Database Syst Rev 2015:CD010085. 
41. Minihane AM. Impact of Genotype on EPA and DHA Status and Responsiveness to Increased Intakes. Nutrients 2016;8:123.

42. Gascon M, Sunyer J, Casas M, et al. Prenatal exposure to DDE and PCB 153 and respiratory health in early childhood: a meta-analysis. Epidemiology 2014;25:544-53.

43. Hansen S, Strom M, Olsen SF, et al. Maternal concentrations of persistent organochlorine pollutants and the risk of asthma in offspring: results from a prospective cohort with 20 years of follow-up. Environ Health Perspect 2014;122:93-9.

44. Litonjua AA, Carey VJ, Burge HA, et al. Parental history and the risk for childhood asthma - Does mother confer more risk than father? Am J Respir Crit Care Med 1998;158:176-81.

45. Bjerg A, Hedman L, Perzanowski MS, et al. Family history of asthma and atopy: in-depth analyses of the impact on asthma and wheeze in 7- to 8-year-old children. Pediatrics 2007;120:741-8.

46. Duchen K, Bjorksten B. Polyunsaturated n-3 fatty acids and the development of atopic disease. Lipids 2001;36:1033-42.

47. Salam MT, Li YF, Langholz B, et al. Maternal fish consumption during pregnancy and risk of early childhood asthma. J Asthma 2005;42:513-8.

48. Calvani M, Alessandri C, Sopo SM, et al. Consumption of fish, butter and margarine during pregnancy and development of allergic sensitizations in the offspring: role of maternal atopy. Pediatr Allergy Immunol 2006;17:94-102.

49. Streuling I, Beyerlein A, Rosenfeld E, et al. Weight gain and dietary intake during pregnancy in industrialized countries--a systematic review of observational studies. J Perinat Med 2011;39:123-9.

50. Stuebe AM, Oken E, Gillman MW. Associations of diet and physical activity during pregnancy with risk for excessive gestational weight gain. Am J Obstet Gynecol 2009;201:58 e1-8.

51. Papadopoulou E, Vafeiadi M, Agramunt S, et al. Maternal diet, prenatal exposure to dioxins and other persistent organic pollutants and anogenital distance in children. Sci Total Environ 2013;461-462:2229.

52. van den Berg SW, Wijga AH, van Rossem L, et al. Maternal fish consumption during pregnancy and BMI in children from birth up to age 14 years: the PIAMA cohort study. Eur J Nutr 2016;55:799-808.

53. Bilau M, Matthys C, Baeyens W, et al. Dietary exposure to dioxin-like compounds in three age groups: results from the Flemish environment and health study. Chemosphere 2008;70:584-92.

54. Halldorsson TI, Thorsdottir I, Meltzer HM, et al. Linking exposure to polychlorinated biphenyls with fatty fish consumption and reduced fetal growth among Danish pregnant women: a cause for concern? Am J Epidemiol 2008;168:958-65.

55. Molto-Puigmarti C, Plat J, Mensink RP, et al. FADS1 FADS2 gene variants modify the association between fish intake and the docosahexaenoic acid proportions in human milk. Am J Clin Nutr 2010;91:1368-76.

56. Ramon R, Murcia M, Aguinagalde X, et al. Prenatal mercury exposure in a multicenter cohort study in Spain. Environ Int 2011;37:597-604.

57. Donahue SMA, Rifas-Shiman SL, Gold DR, et al. Prenatal fatty acid status and child adiposity at age 3 y: results from a US pregnancy cohort. Am J Clin Nutr 2011;93:780-8.

58. Rothman KJ. No adjustments are needed for multiple comparisons. Epidemiology 1990;1:43-6. 


\section{Supplementary Material of Chapter 8}

Supplementary Table S8.1. Description of participating cohorts

Supplementary Table S8.2. Data collection on respiratory outcomes in the participating cohorts

Supplementary Table S8.3. Description of maternal and child characteristics in participating cohorts

Supplementary Table S8.4. Distribution of fish and seafood consumption in participating cohorts

Supplementary Table S8.5. Distribution of fish consumption levels among mothers with and without asthma or hayfever in participating cohorts

Supplementary Table S8.6. Distribution of respiratory outcomes in participating cohorts

Supplementary Table S8.7. Adjusted combined associations ${ }^{a}$ of fish and seafood (other than fish) consumption in pregnancy with childhood persistent wheeze

Supplementary Table S8.8. Adjusted combined associations (pooled analysis) ${ }^{a}$ of fish and seafood (other than fish) consumption in pregnancy with childhood wheeze

Supplementary Table S8.9. Adjusted combined associations (pooled analysis) ${ }^{a}$ of fish and seafood (other than fish) consumption in pregnancy with asthma and allergic rhinitis in childhood

Supplementary Table S8.10. Adjusted combined association ${ }^{\text {a }}$ of fish consumption during pregnancy (times/week) with childhood wheeze after omitting one cohort at a time

Supplementary Table S8.11. Adjusted combined association ${ }^{a}$ of fish consumption during pregnancy (times/week) with asthma and allergic rhinitis in childhood after omitting one cohort at a time

Supplementary Table S8.12. Adjusted combined associations (pooled analysis) ${ }^{a}$ of fish consumption in pregnancy (times/week) with wheeze in infancy and asthma in childhood by parent asthma or hay fever, and child sex

Supplementary Figure S8.1. Location of participating birth cohorts..

Supplementary Figure S8.2. Directed acyclic graph for the association of fish consumption during pregnancy with child asthma or rhinitis symptoms. 


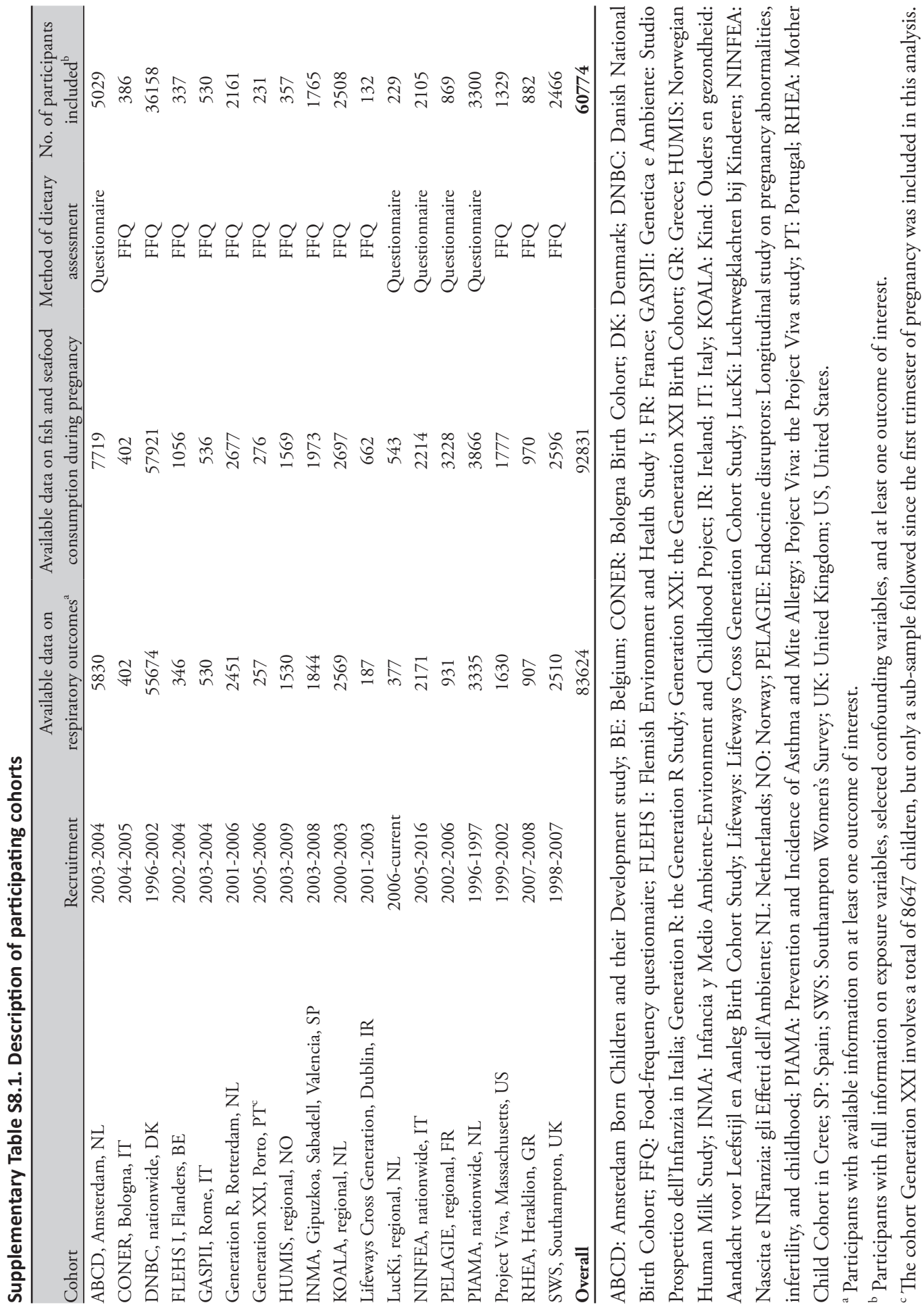




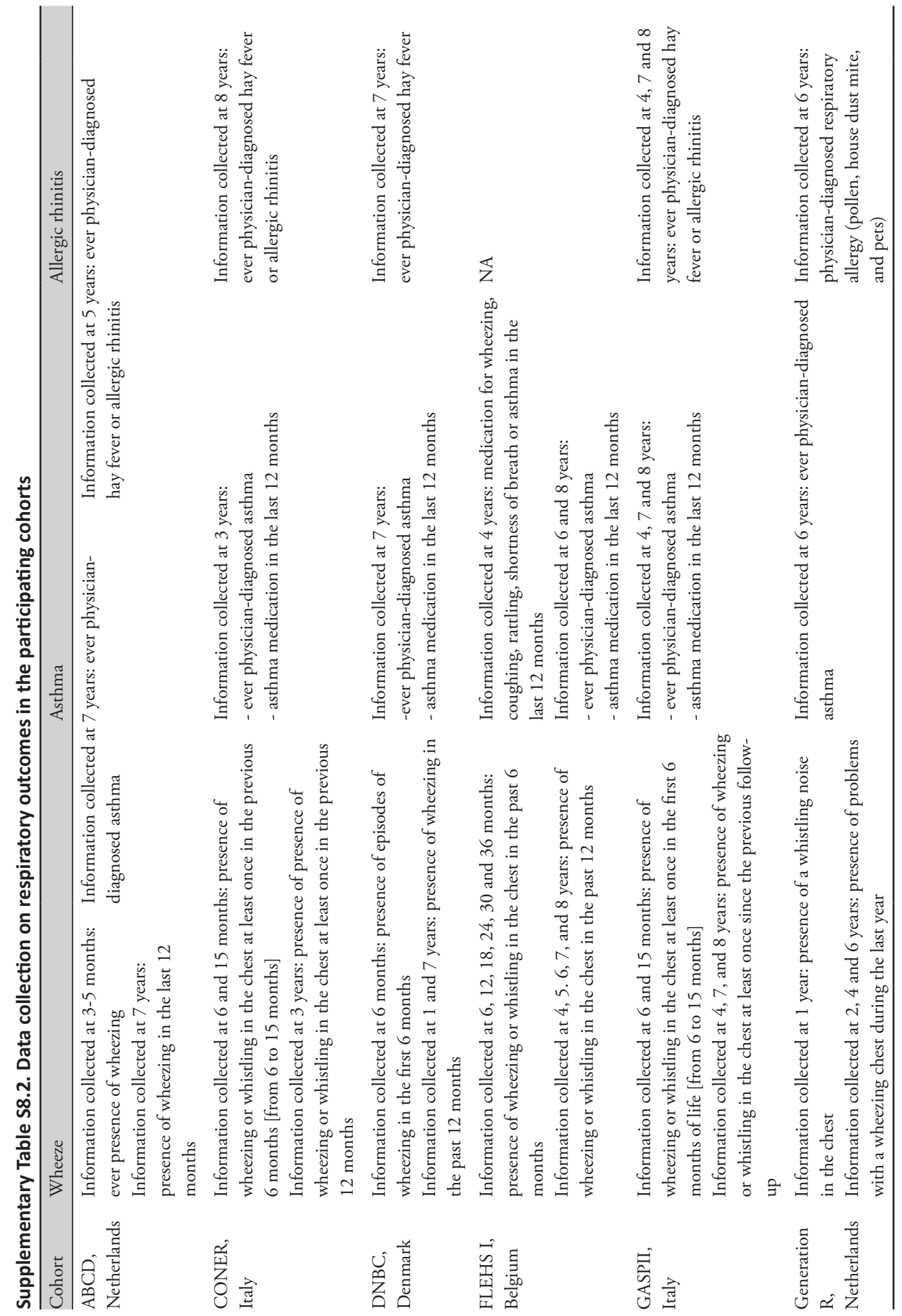




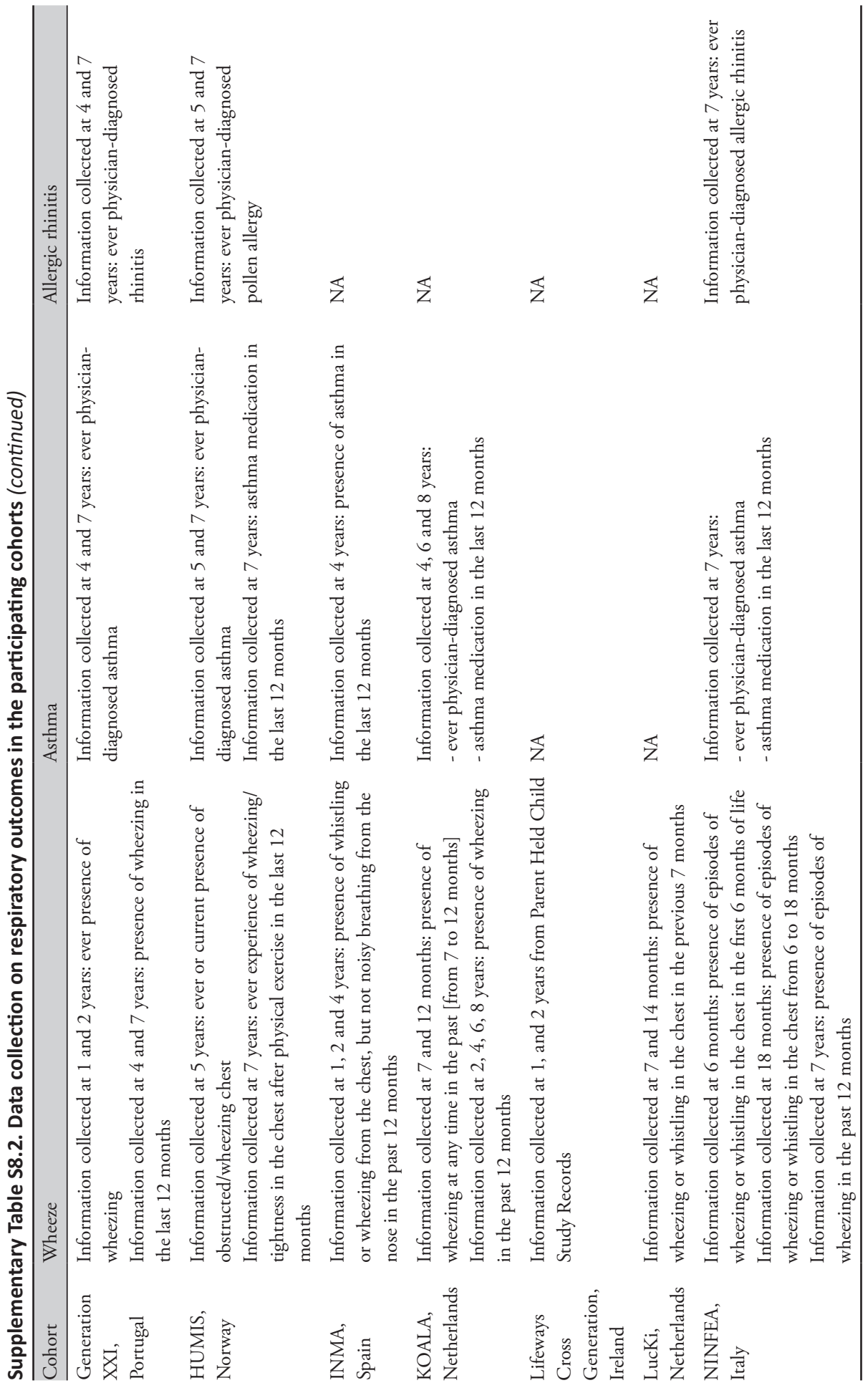




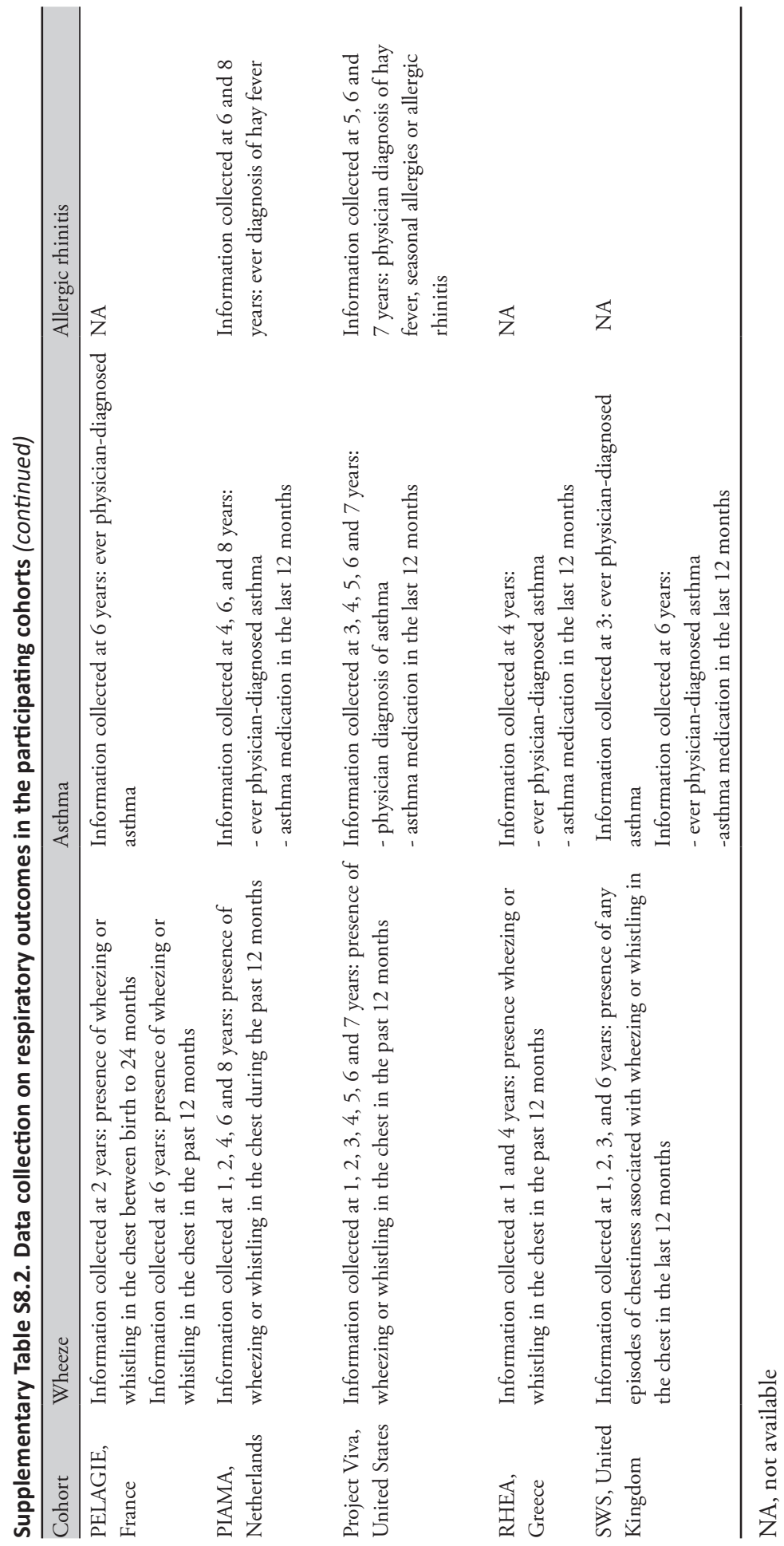




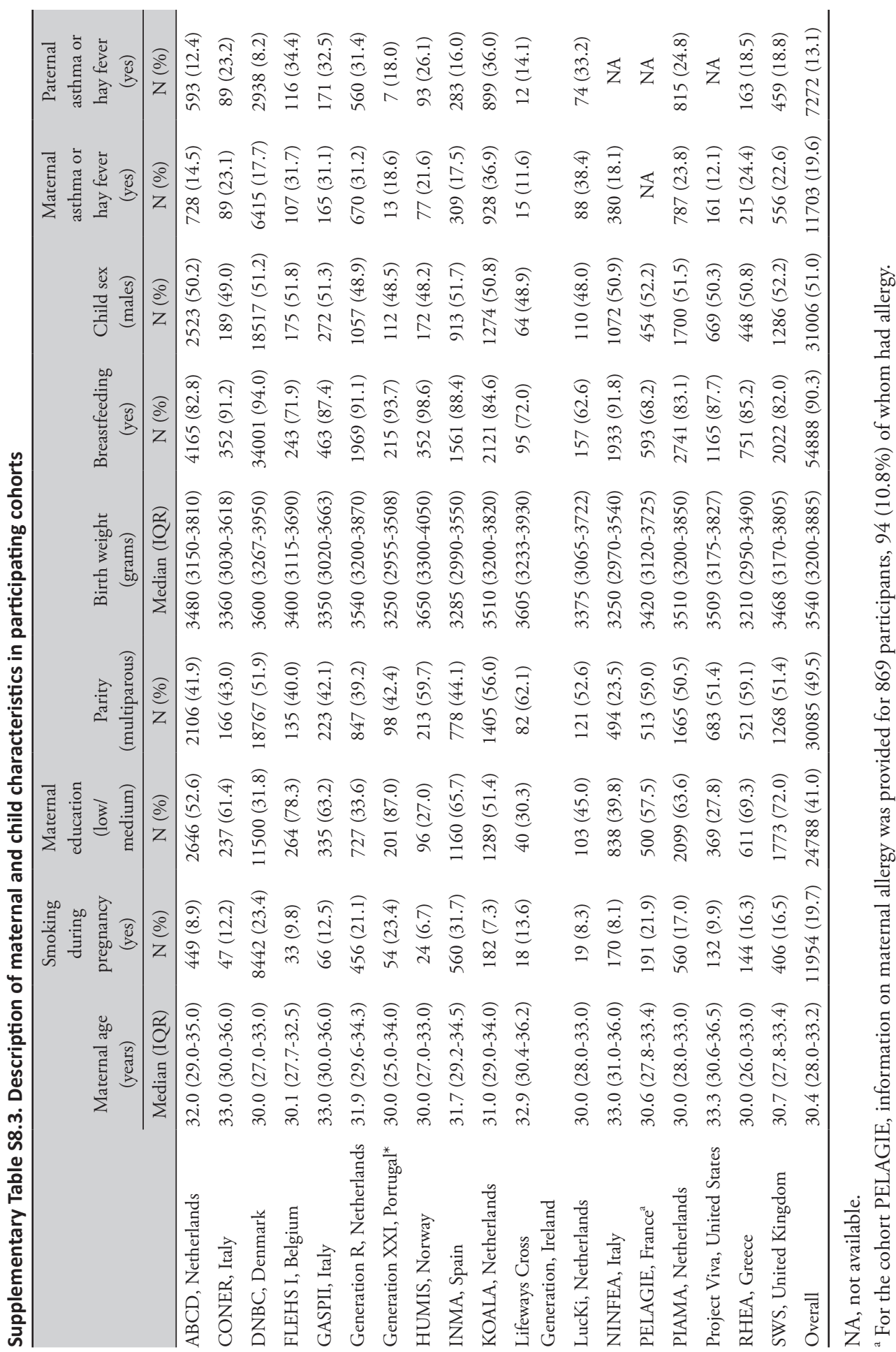




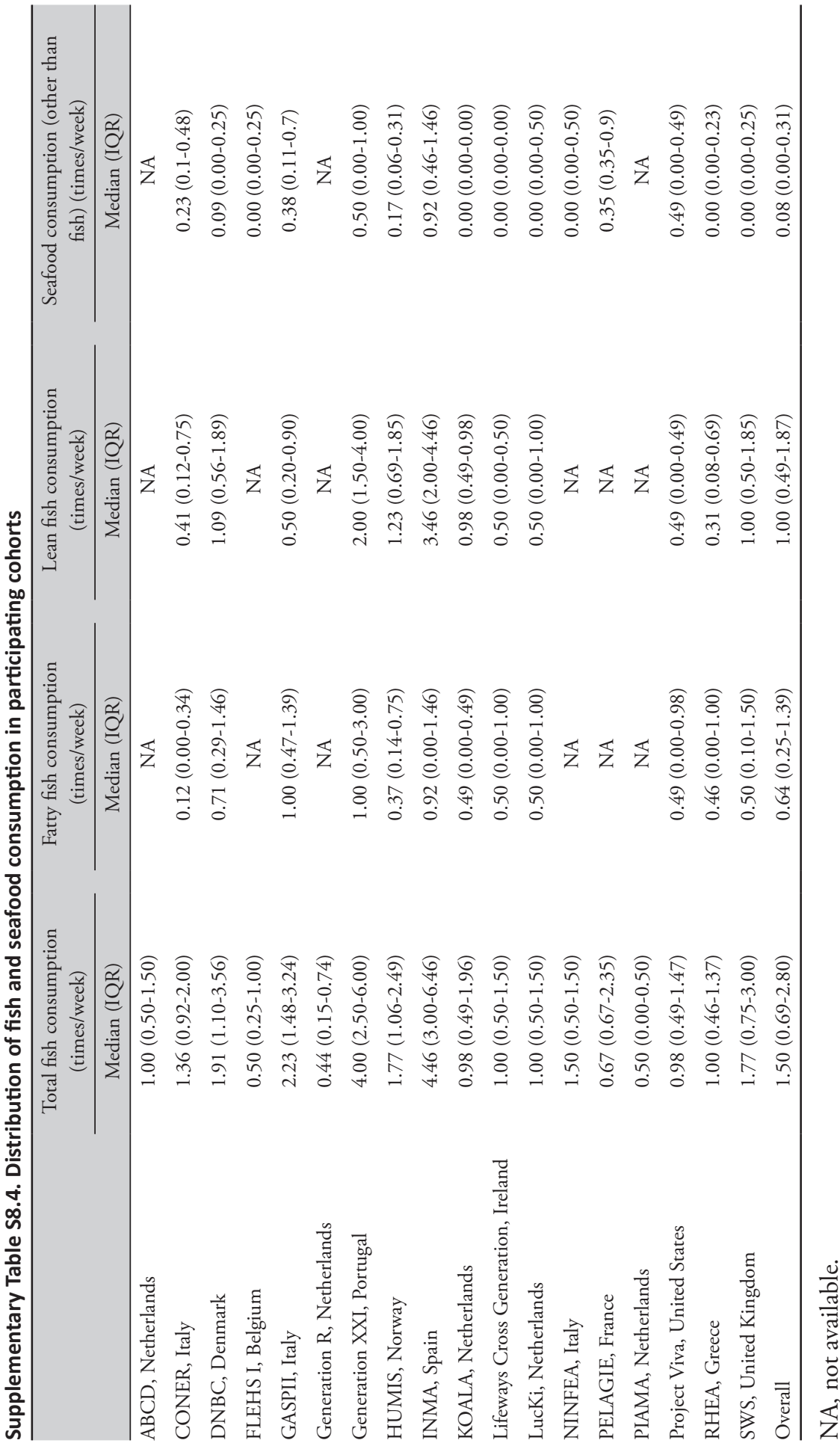


Supplementary Table S8.5. Distribution of fish consumption levels among mothers with and without asthma or hayfever in participating cohorts

\begin{tabular}{|c|c|c|c|}
\hline & $\begin{array}{l}\text { Mothers without asthma or } \\
\text { hayfever }\end{array}$ & Mothers with asthma or hayfever & $\begin{array}{l}\text { P value for } \\
\text { difference }^{a}\end{array}$ \\
\hline & $\begin{array}{c}\text { Total fish consumption in } \\
\text { times/week }\end{array}$ & $\begin{array}{l}\text { Total fish consumption in times/ } \\
\text { week }\end{array}$ & \\
\hline & Median (IQR) & Median (IQR) & \\
\hline ABCD, Netherlands & $1.00(0.50-1.50)$ & $1.00(0.50-1.62)$ & 0.36 \\
\hline CONER, Italy & $1.40(0.93-2.00)$ & $1.18(0.66-1.88)$ & 0.06 \\
\hline DNBC, Denmark & $1.91(1.11-3.57)$ & $1.88(1.07-3.54)$ & 0.04 \\
\hline FLEHS I, Belgium & $0.50(0.25-1.00)$ & $0.50(0.25-1.00)$ & 0.54 \\
\hline GASPII, Italy & $2.19(1.47-3.18)$ & $2.29(1.53-3.37)$ & 0.72 \\
\hline Generation R, Netherlands & $0.43(0.15-0.77)$ & $0.44(0.15-0.80)$ & 0.20 \\
\hline Generation XXI, Portugal & $4.50(3.00-6.00)$ & $3.00(2.00-6.50)$ & 0.35 \\
\hline HUMIS, Norway & $1.79(1.02-2.51)$ & $1.67(1.14-2.25)$ & 0.52 \\
\hline INMA, Spain & $4.46(3.00-6.46)$ & $4.46(3.00-6.89)$ & 0.79 \\
\hline KOALA, Netherlands & $0.98(0.49-1.96)$ & $1.47(0.49-1.96)$ & 0.17 \\
\hline $\begin{array}{l}\text { Lifeways Cross Generation, } \\
\text { Ireland }\end{array}$ & $1.00(0.50-1.50)$ & $1.00(0.50-1.00)$ & 0.96 \\
\hline LucKi, Netherlands & $1.00(0.50-2.00)$ & $1.00(0.50-1.62)$ & 0.82 \\
\hline NINFEA, Italy & $1.50(0.50-1.50)$ & $1.50(0.50-1.50)$ & 0.38 \\
\hline PELAGIE, France & $0.67(0.67-2.35)$ & $0.67(0.67-2.35)$ & 0.10 \\
\hline PIAMA, Netherlands & $0.50(0.00-0.50)$ & $0.50(0.00-1.00)$ & 0.10 \\
\hline Project Viva, United States & $0.98(0.49-1.47)$ & $0.98(0.49-1.47)$ & 0.28 \\
\hline RHEA, Greece & $1.00(0.46-1.34)$ & $1.00(0.46-1.46)$ & 0.23 \\
\hline SWS, United Kingdom & $1.75(0.75-3.00)$ & $1.85(0.75-3.00)$ & 0.69 \\
\hline
\end{tabular}

${ }^{a} \mathrm{P}$ values for difference were estimated using the Mann-Whitney $\mathrm{U}$ test. 


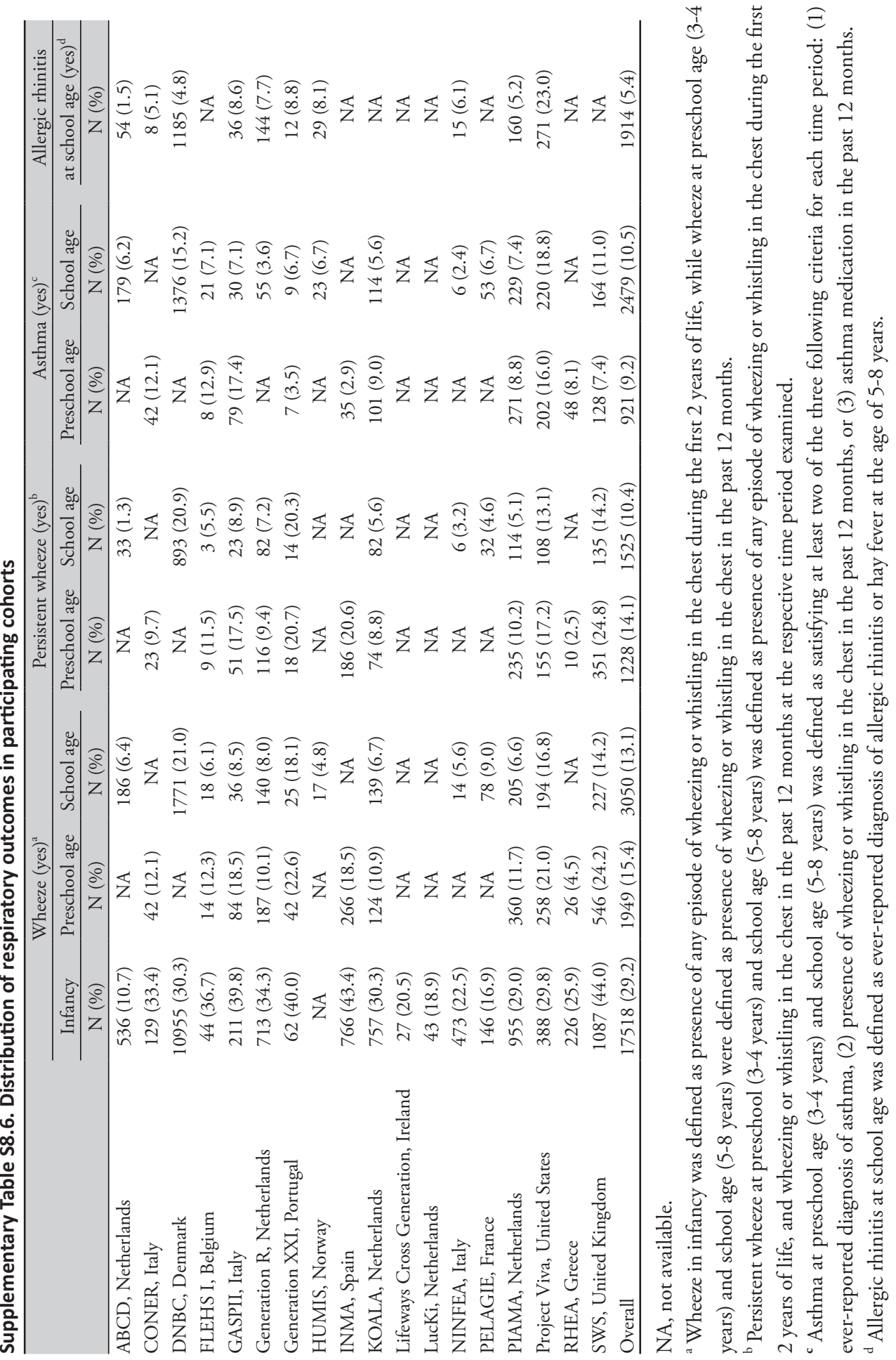




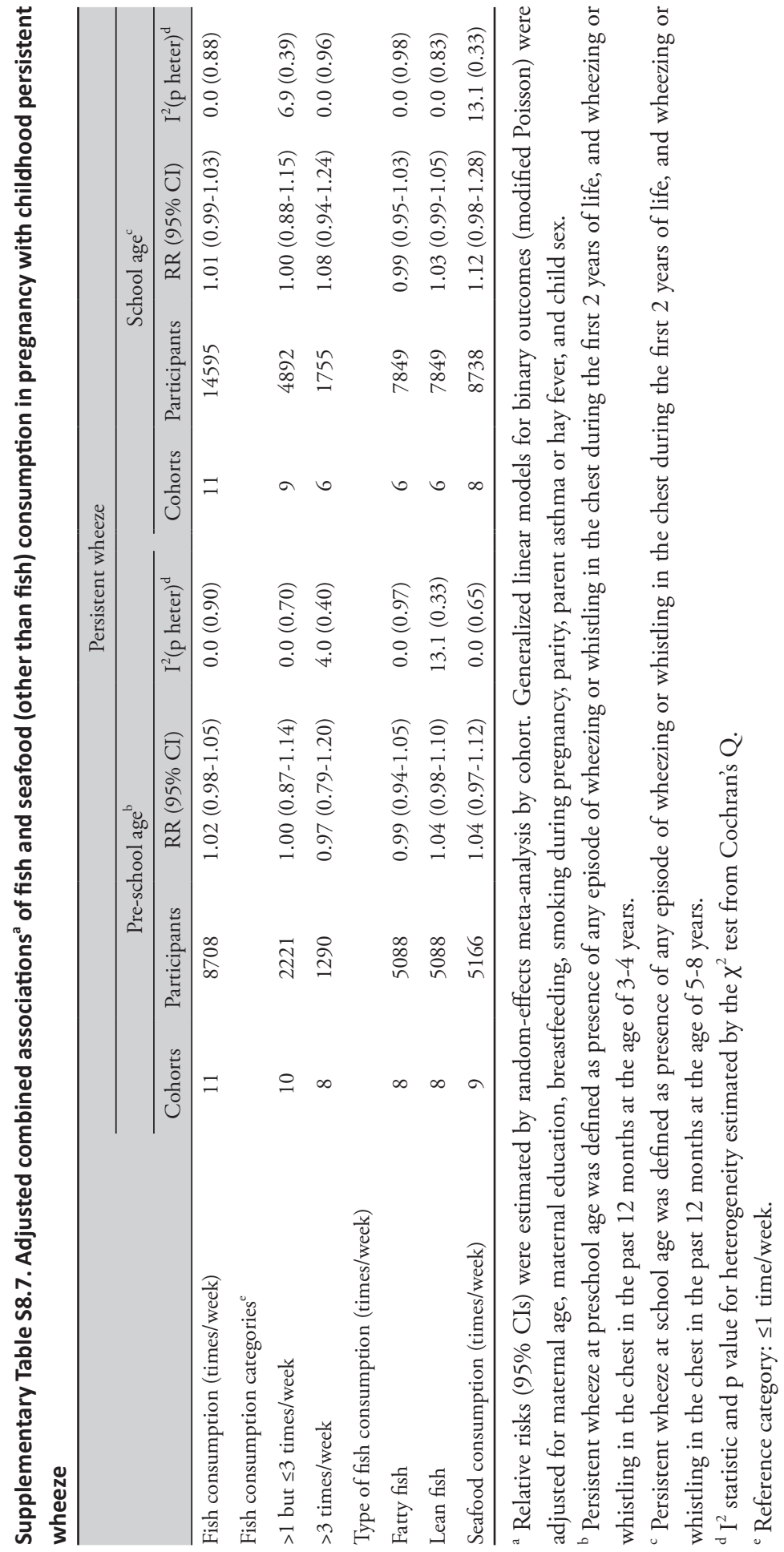




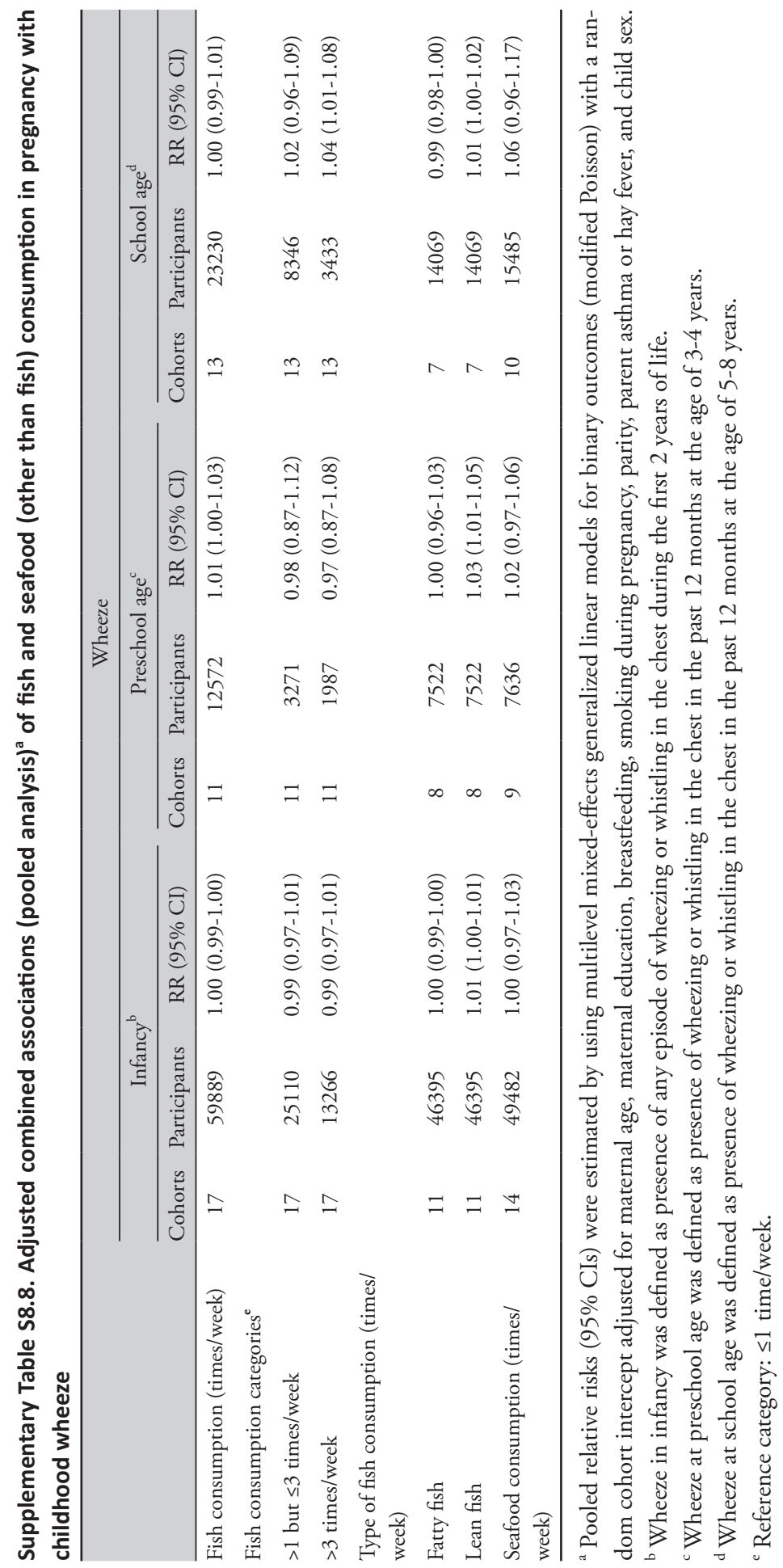




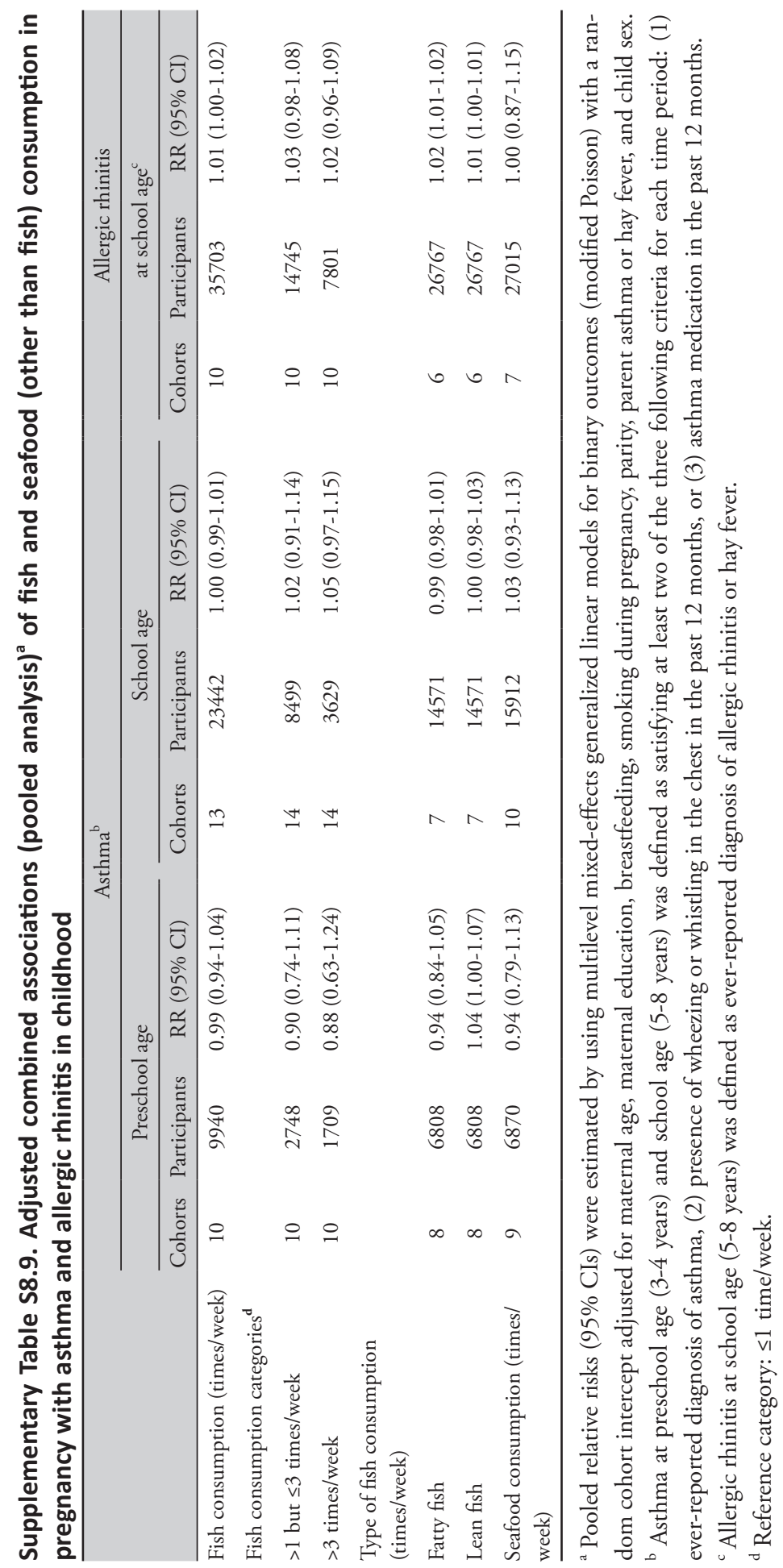


Supplementary Table S8.10. Adjusted combined association ${ }^{a}$ of fish consumption during pregnancy (times/week) with childhood wheeze after omitting one cohort at a time

\begin{tabular}{|c|c|c|c|}
\hline & \multicolumn{3}{|c|}{ Wheeze } \\
\hline & Infancy $^{\mathrm{b}}$ & Preschool age ${ }^{c}$ & School age $^{d}$ \\
\hline & $\mathrm{RR}(95 \% \mathrm{CI})$ & $\mathrm{RR}(95 \% \mathrm{CI})$ & $\mathrm{RR}(95 \% \mathrm{CI})$ \\
\hline Overall & $1.00(0.99-1.01)$ & $1.01(0.99-1.04)$ & $1.01(0.98-1.03)$ \\
\hline \multicolumn{4}{|l|}{ Cohort omitted } \\
\hline $\mathrm{ABCD}, \mathrm{NL}$ & $1.00(0.99-1.01)$ & NA & $1.00(0.97-1.04)$ \\
\hline CONER, IT & $1.00(0.99-1.01)$ & $1.01(0.99-1.04)$ & NA \\
\hline DNBC, DK & $1.00(0.99-1.02)$ & NA & $1.01(0.97-1.06)$ \\
\hline FLEHS I, BE & $1.00(1.00-1.01)$ & $1.01(0.99-1.04)$ & $1.00(0.99-1.02)$ \\
\hline GASPII, IT & $1.00(0.99-1.01)$ & $1.01(0.99-1.04)$ & $1.01(0.98-1.04)$ \\
\hline Generation R Study, NL & $1.00(0.99-1.01)$ & $1.01(0.99-1.04)$ & $1.00(0.98-1.03)$ \\
\hline Generation XXI, PT & $1.00(0.99-1.01)$ & $1.01(0.99-1.04)$ & $1.01(0.98-1.04)$ \\
\hline HUMIS, NO & NA & NA & $1.01(0.98-1.03)$ \\
\hline INMA, ES & $1.00(0.99-1.02)$ & $1.01(0.97-1.04)$ & NA \\
\hline KOALA, NL & $1.00(0.99-1.01)$ & $1.01(0.99-1.04)$ & $1.00(0.99-1.02)$ \\
\hline Lifeways Cross Generation, IR & $1.00(0.99-1.01)$ & NA & NA \\
\hline LucKi, NL & $1.00(0.99-1.01)$ & NA & NA \\
\hline NINFEA, IT & $1.00(0.99-1.01)$ & NA & $1.00(0.98-1.03)$ \\
\hline PELAGIE, FR & $1.00(0.99-1.01)$ & NA & $1.00(0.98-1.02)$ \\
\hline PIAMA, NL & $1.00(1.00-1.01)$ & $1.01(0.98-1.03)$ & $1.00(0.98-1.03)$ \\
\hline Project Viva, US & $1.00(0.99-1.01)$ & $1.01(0.99-1.04)$ & $1.01(0.97-1.04)$ \\
\hline RHEA, GR & $1.00(0.99-1.01)$ & $1.01(0.98-1.03)$ & NA \\
\hline SWS, UK & $1.01(0.99-1.02)$ & $1.01(0.98-1.05)$ & $1.00(0.97-1.04)$ \\
\hline
\end{tabular}

${ }^{a}$ All effect estimates correspond to an increase of 1 time/week in fish consumption in pregnancy. Relative risks (95\% CIs) were estimated by random-effects meta-analysis by cohort. Generalized linear models for binary outcomes (modified Poisson) were adjusted for maternal age, maternal education, breastfeeding, smoking during pregnancy, parity, parent asthma or hay fever, and child sex.

${ }^{\mathbf{b}}$ Wheeze in infancy was defined as presence of any episode of wheezing or whistling in the chest during the first 2 years of life.

${ }^{\mathrm{c}}$ Wheeze at preschool age was defined as presence of wheezing or whistling in the chest in the past 12 months at the age of 3-4 years.

${ }^{d}$ Wheeze at school age was defined as presence of wheezing or whistling in the chest in the past 12 months at the age of 5-8 years. 
Supplementary Table S8.11. Adjusted combined association ${ }^{a}$ of fish consumption during pregnancy (times/week) with asthma and allergic rhinitis in childhood after omitting one cohort at a time

\begin{tabular}{|c|c|c|c|}
\hline & \multicolumn{2}{|c|}{ Asthma $^{\mathrm{b}}$} & \multirow{2}{*}{$\begin{array}{l}\text { Allergic rhinitis } \\
\text { at school age }\end{array}$} \\
\hline & Preschool age & School age & \\
\hline & $\mathrm{RR}(95 \% \mathrm{CI})$ & $\mathrm{RR}(95 \% \mathrm{CI})$ & RR $(95 \% \mathrm{CI})$ \\
\hline Overall & $1.02(0.97-1.07)$ & $1.01(0.97-1.05)$ & $1.01(0.99-1.03)$ \\
\hline \multicolumn{4}{|l|}{ Cohort omitted } \\
\hline $\mathrm{ABCD}, \mathrm{NL}$ & NA & $1.01(0.96-1.06)$ & $1.02(0.98-1.06)$ \\
\hline CONER, IT & $1.02(0.96-1.08)$ & NA & $1.01(0.97-1.05)$ \\
\hline DNBC, DK & NA & $1.01(0.95-1.07)$ & $1.02(0.95-1.09)$ \\
\hline FLEHS I, BE & $1.02(0.97-1.07)$ & $1.00(0.98-1.02)$ & NA \\
\hline GASPII, IT & $1.03(0.97-1.08)$ & $1.01(0.96-1.05)$ & $1.01(0.99-1.03)$ \\
\hline Generation R Study, NL & NA & $1.01(0.96-1.05)$ & $1.01(0.99-1.03)$ \\
\hline Generation XXI, PT & $1.01(0.96-1.06)$ & $1.01(0.96-1.05)$ & $1.01(0.97-1.05)$ \\
\hline HUMIS, NO & NA & $1.01(0.96-1.05)$ & $1.01(0.98-1.05)$ \\
\hline INMA, ES & $1.03(0.96-1.09)$ & NA & NA \\
\hline KOALA, NL & $1.02(0.96-1.08)$ & $1.01(0.98-1.05)$ & NA \\
\hline Lifeways Cross Generation, IR & NA & NA & NA \\
\hline NINFEA, IT & NA & $1.01(0.96-1.05)$ & $1.01(0.99-1.03)$ \\
\hline PELAGIE, FR & NA & $1.01(0.97-1.05)$ & NA \\
\hline PIAMA, NL & $1.01(0.96-1.07)$ & $1.01(0.96-1.05)$ & $1.01(0.98-1.04)$ \\
\hline Project Viva, US & $1.03(0.99-1.09)$ & $1.00(0.96-1.05)$ & $1.01(0.96-1.06)$ \\
\hline RHEA, GR & $1.02(0.96-1.08)$ & NA & NA \\
\hline SWS, UK & $1.01(0.95-1.07)$ & $0.99(0.96-1.03)$ & NA \\
\hline
\end{tabular}

a All effect estimates correspond to an increase of 1 time/week in fish consumption in pregnancy. Relative risks (95\% CIs) were estimated by random-effects meta-analysis by cohort. Generalized linear models for binary outcomes (modified Poisson) were adjusted for maternal age, maternal education, breastfeeding, smoking during pregnancy, parity, parent asthma or hay fever, and child sex.

${ }^{\mathbf{b}}$ Asthma at preschool age (3-4 years) and school age (5-8 years) was defined as satisfying at least two of the three following criteria for each time period: (1) ever-reported diagnosis of asthma, (2) presence of wheezing or whistling in the chest in the past 12 months, or (3) asthma medication in the past 12 months.

${ }^{\mathrm{c}}$ Allergic rhinitis at school age (5-8 years) was defined as ever-reported diagnosis of allergic rhinitis or hay fever. 


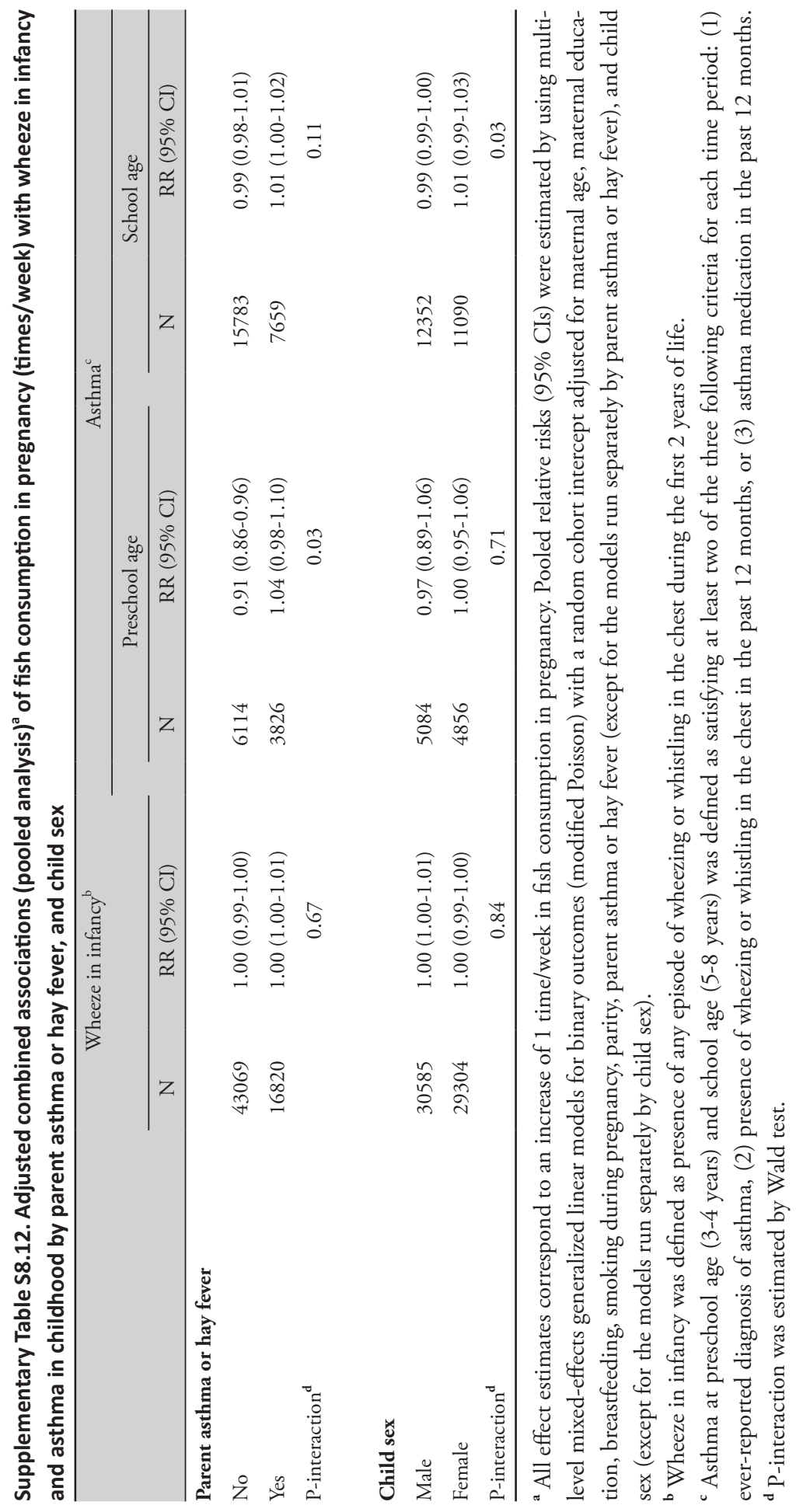




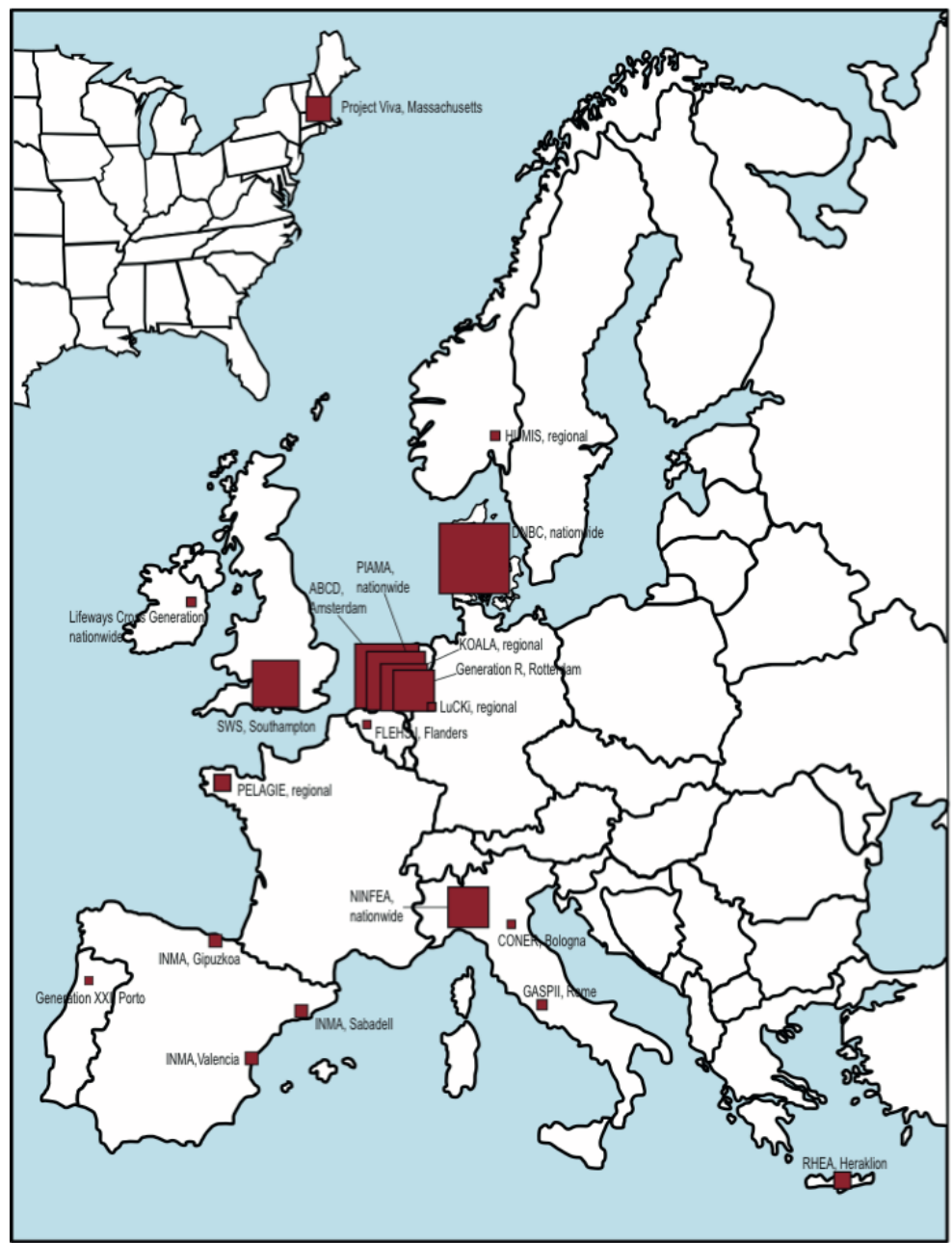

Supplementary Figure S8.1. Location of participating birth cohorts. The size of the square is indicative of the cohort size. ABCD: Amsterdam Born Children and their Development study; CONER: Bologna Birth Cohort; DNBC: Danish National Birth Cohort; FLEHS I: Flemish Environment and Health Study I; GASPII: Genetica e Ambiente: Studio Prospettico dell'Infanzia in Italia; Generation R: the Generation R Study; Generation XXI: the Generation XXI Birth Cohort; HUMIS: Norwegian Human Milk Study; INMA: Infancia y Medio Ambiente-Environment and Childhood Project; IR: Ireland; KOALA: Kind: Ouders en gezondheid: Aandacht voor Leefstijl en Aanleg Birth Cohort Study; Lifeways: Lifeways Cross Generation Cohort Study; LucKi: Luchtwegklachten bij Kinderen; NINFEA: Nascita e INFanzia: gli Effetti dell'Ambiente; PELAGIE: Endocrine disruptors: Longitudinal study on pregnancy abnormalities, infertility, and childhood; PIAMA: Prevention and Incidence of Asthma and Mite Allergy; Project Viva: the Project Viva study; RHEA: Mother Child Cohort in Crete; SWS: Southampton Women's Survey. 


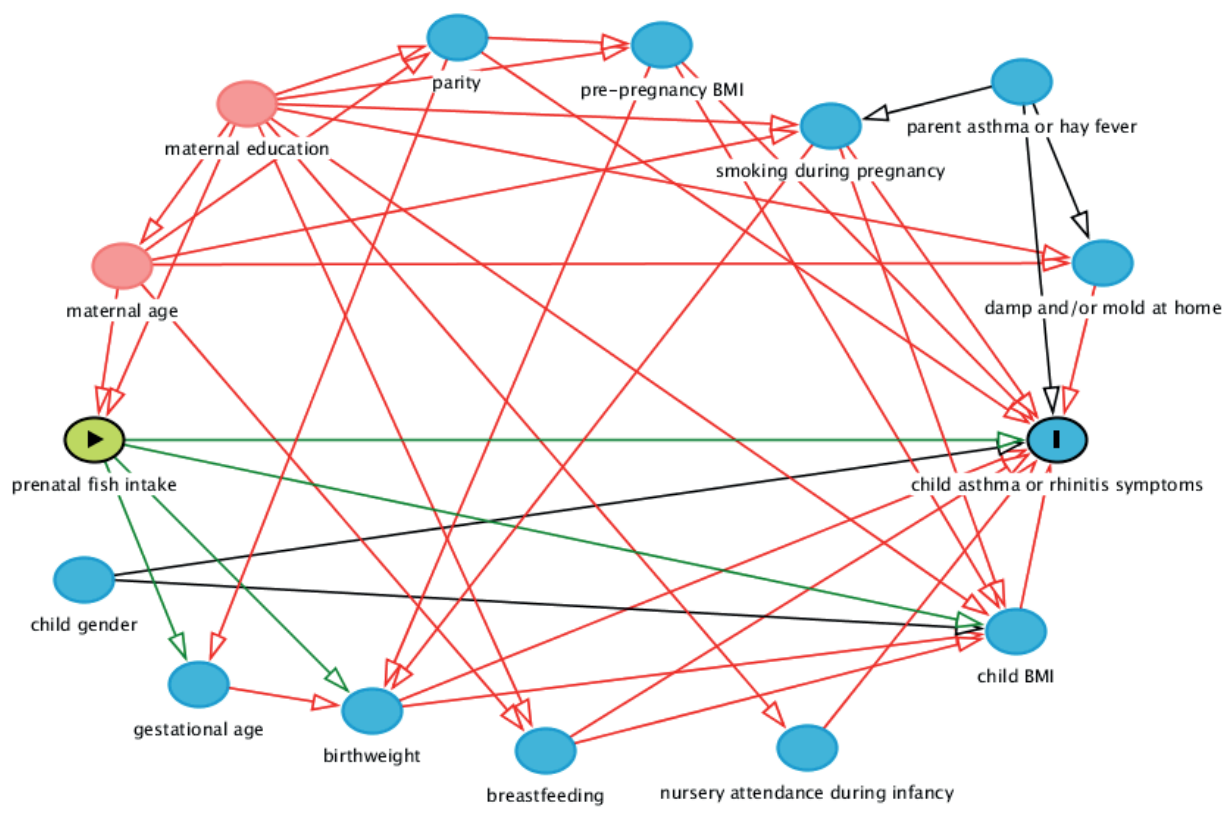

Supplementary Figure S8.2. Directed acyclic graph for the association of fish consumption during pregnancy with child asthma or rhinitis symptoms. 



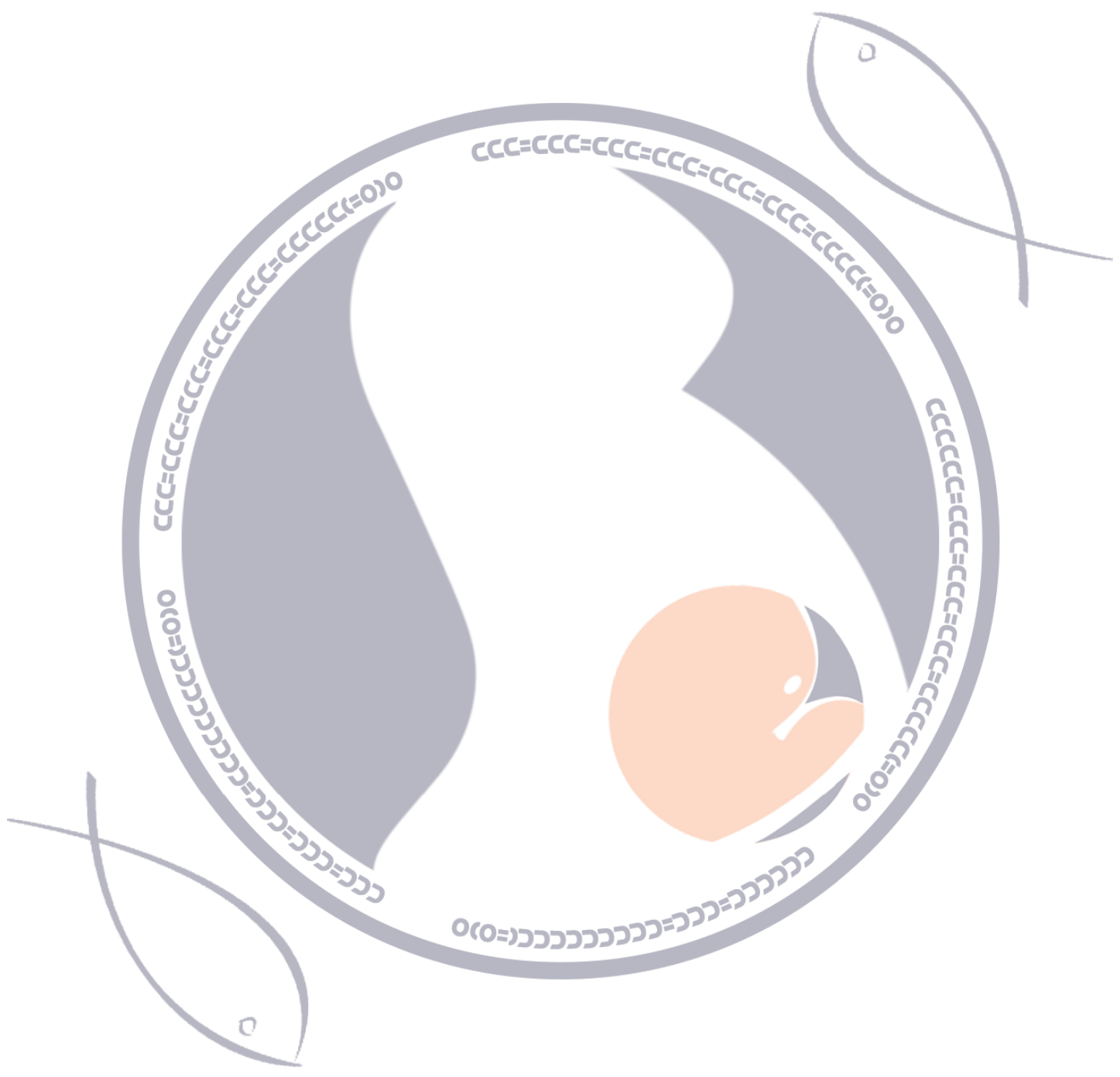




\section{CHAPTER 9}

General Discussion 



\section{INTRODUCTION}

Childhood obesity and allergy constitute two major public health threats in developed countries with serious lifelong consequences. ${ }^{1-3}$ Mounting evidence suggests that the environment experienced in utero contributes significantly to the genesis of these chronic diseases, a corpus of knowledge embodied in the "Developmental Origins of Health and Disease" concept. ${ }^{4}$

Fetal life is a critical period of developmental plasticity, as it is the time period when most organs and tissues are formed. A nutritional stimulus or stressor applied during this critical period can lead to physiologic and metabolic adaptations in the human body with long term consequences. ${ }^{5}$ Hence, pregnancy constitutes a time period of both great opportunity and considerable risk. Over the last years, there has been a considerable research effort in trying to identify key nutritional factors in the prenatal period that can be targeted for early preventive interventions. In this context, there has been particularly interest in the exposure to $n-3$ and n-6 PUFAs. Because humans cannot synthesize the parent PUFAs of the n-3 and n-6 family, and can only ineffectively synthesize their longer-chain, more biologically active fatty acid precursors, human status of PUFAs is mainly determined by dietary consumption. ${ }^{6,7}$ Consequently, the developing fetus depends on maternal PUFA supply to cover its needs, and is amenable to maternal dietary modification. ${ }^{8}$ Mechanistic studies have suggested that PUFAs of the $n-3$ and $n-6$ family can exert pleiotropic effects on body physiology and metabolism that are often in the opposite direction. ${ }^{9}$ For instance, n-3 PUFAs have been suggested to inhibit inflammation, promote osteoblastogenesis, and decrease adipose tissue deposition, while those of the n-6 family seem to exhibit opposite effects. ${ }^{10-12}$ The major aim of the thesis was to identify the association of PUFA status in the prenatal period with childhood growth, obesity and associated cardiometabolic risk, and allergy-like symptoms. We also examined prenatal fish intake as an exposure of interest, since fish is the primary source of $n-3$ long-chain PUFAs in the human diet. The main results and limitations of the studies presented in this thesis have been discussed in the previous chapters. This chapter provides a general overview and interpretation of the main findings of this thesis, considers general methodological issues applied in our studies, and suggests directions for future research. 


\section{INTERPRETATION OF MAIN FINDINGS}

\section{Child growth and obesity}

Childhood height and adiposity status are important markers of health. Accumulating evidence suggests that lower stature and higher values of BMI, which is a common measure of adiposity, are associated with higher adult risk of cardiovascular disease. ${ }^{13-15}$ Moreover, rapid weight gain in infancy, which reflects primarily a gain in fat, has consistently been associated with a subsequent elevated risk of obesity both in childhood and in adulthood. ${ }^{16}$

In this thesis, we first conducted a systematic review and meta-analysis of trials to evaluate whether supplementation with n-3 long-chain PUFAs in the prenatal and early postnatal period exerts a beneficial effect on later adiposity status (Chapter 2). To take into account growth and maturation during childhood, we assessed each adiposity measure according to the following age categories: preschool children ( $0-5$ years), school-aged children (6-12 years), and adolescents ( $\geq 13$ years). Owing to the limited available data, we pooled study results relating to BMI only for preschool and school-aged children. Additionally, it was not possible to combine study results relating to the other adiposity measures including waist circumference and sum of skinfolds. Overall, we found no effect of supplementation on BMI in preschool (0-5 years) and school-aged (6-12 years) children. There was also no evidence to support a benefit of $\mathrm{n}-3$ long-chain PUFA supplementation in other adiposity measures in individual studies. However, the trials included in the systematic review were prone to methodological limitations, such as small sample sizes and selective attrition, thereby not allowing firm conclusions to be made.

Next, we pooled and harmonized individual data from mother-offspring pairs in the MEFAB and RHEA birth cohorts with repeated offspring follow-ups until the age of 6-7 years to assess whether cord blood phospholipid PUFA levels are associated with rapid weight gain in infancy, childhood BMI, and the risk of childhood overweight including obesity (Chapter 3). Cord blood phospholipid PUFA levels have been shown to strongly correlate with those in maternal circulation and be influenced by maternal dietary intake. ${ }^{17-20}$ Moreover, they reflect the selectivity and efficiency of placental transfer. ${ }^{21}$ Given their relatively high turnover rates, PUFAs in the phospholipid fraction reflect recent exposure, specifically at a time frame of 2-4 weeks. ${ }^{22}$ For these reasons, cord blood phospholipid concentrations of PUFAs can be considered as good surrogates for fetal exposure in late gestation. In our study, we used overweight including obesity as outcome to retain statistical power in the analyses owing to the small number of children classified as obese. A large body of research has shown that being overweight or obese in childhood increases substantially the risk for obesity and cardiovascular disease in adult- 
hood. ${ }^{23-25}$ Results from our pooled analysis do not support an association between fetal PUFA exposure in late pregnancy with rapid infant weight gain, childhood BMI, or the risk of overweight including obesity. Previous findings from birth cohort studies assessing prenatal PUFA exposure have yielded largely inconsistent results (reviewed in Chapter 1). These inconsistencies may partly arise from exposure profile heterogeneity (ie, definition and timing of exposure) and differences in adjustment for covariates. Moreover, findings from any of the previous studies were not replicated across populations with different characteristics and behaviors. Our study addresses these issues by including two cohorts from West and South Europe with different sociodemographic characteristics and by following a centralized statistical analysis with harmonized information on exposure variables, potential confounders, and child outcomes.

It is well established that individuals experience different rates of growth over time- some individuals have a low growth rate in early childhood but experience accelerated growth later in life. Examining the long-term effects of early-life exposures at different ages, preferably on the child to adult transition, is important for accurate identification of modifiable risk factors and for understanding when these factors exhibit their maximum influence. Hence, as part of this thesis, we planned and carried out a follow-up study of the MEFAB cohort when the offspring had reached young adulthood.

We used the long-term longitudinal data available within MEFAB to investigate the associations of cord blood phospholipid PUFA levels with height and BMI at different developmental ages: infant, 6 months; toddler, 2 years; pre-schooler, 4 years; school-aged child, 7 years; adolescent, 12 years; and young adult, 23 years (Chapter 4). To date, this is the first study to examine child-to-adult growth in relation to prenatal n-3 and n-6 PUFA exposure. We found that higher n-3 PUFA levels were associated with higher infant length among males, whereas, for females, an association of similar magnitude but of the opposite direction was observed for n-6 PUFA concentrations. The observed associations were of small magnitude and not clinically relevant, corresponding to $0.4-0.7-\mathrm{cm}$ changes in infant length per SD increase in PUFA concentrations. The associations with infant length seemed to be transient, as they did not persist later in childhood and young adulthood. Previous studies assessing childhood height have followed participants for a short period of time and have mostly focused on $\mathrm{n}-3$ long-chain PUFA supplementation. ${ }^{26-29}$ Moreover, they have not examined sex-specific effects and have shown inconsistent findings. A growing body of evidence highlights the importance of assessing effect modification by sex in the associations of prenatal PUFA exposure with later health outcomes owing to differential effects of sex steroid hormones on fatty acid metabolism and responsiveness to PUFAs. ${ }^{30,31}$ 
In our long-term study, we saw no associations of PUFAs with BMI from infancy till young adulthood. These results extend and expand our previous findings from the meta-analysis of trials on n-3 long-chain PUFA supplementation (Chapter 2), and the pooled analysis of the MEFAB and RHEA cohorts (Chapter 3).

Fish is the main dietary source of $\mathrm{n}-3$ long-chain PUFAs, but is also a common route of human exposure to persistent organic pollutants that can act as endocrine disruptors. ${ }^{32,33}$ This complexity of fish as a nutritional exposure has resulted in confusion over its place in a healthy prenatal diet. ${ }^{34}$ We carried out a review of cohort studies and found largely inconsistent results on the association of maternal fish intake during pregnancy with childhood obesity outcomes (Chapter 5). These inconsistencies might at least partly arise due to heterogeneity in exposure definition, and differences in covariate adjustment. Given that the effect of fish intake by pregnant women remains an important issue, especially in populations that consume fish frequently, it is important to disentangle the association of maternal fish consumption during pregnancy with child health.

Hence, we harmonized and pooled individual data of mother-offspring pairs from 15 cohorts with repeated offspring follow-up evaluations up to 6 years of age to assess the strength and consistency of the associations of fish intake during pregnancy with rapid growth in infancy, childhood BMI, and the risk of childhood overweight including obesity (Chapter 7). We found that women with high fish consumption of $>3$ times/week during pregnancy gave birth to offspring with higher body mass index values from infancy through middle childhood compared with women with lower fish intake ( $\leq 1$ time/week). Offspring of women with high fish consumption in pregnancy had higher odds of having a rapid weight gain in infancy $(\mathrm{OR}=1.22$ [95\% CI 1.05-1.42]) and being overweight or obese at 4 years $(\mathrm{OR}=1.14$ [95\% CI 0.99-1.32]) and 6 years $(\mathrm{OR}=1.22[95 \% \mathrm{CI}$ 1.01-1.47]) compared with those whose mothers were rare fish consumers. The use of birth cohorts in several locations in Europe and the United States with varying fish intake levels and the absence of important heterogeneity between individual cohort effect estimates provide evidence to support the robustness of our findings. Contamination by pollutants in fish could provide an explanation for the observed associations. Mixtures of persistent organic pollutants potentially found in fish, such as polychlorinated biphenyls (PCBs) and pesticides, have been shown to increase adipogenesis and fat deposition in cultured adipocytes and animal models, ${ }^{35}$ as well as obesity risk in children. ${ }^{36}$ However, we did not have available information on biomarkers of pollutants across participating cohorts, hence, the hypothesis of pollutant-driven effects of maternal fish intake remains speculative. Finally, we observed that the association of high fish intake during pregnancy with offspring obesity outcomes was more pronounced in girls than in 
boys. The placenta tissue carrying the fetal genome and sex appears as a promising candidate to be involved in mediating sex-specific functions and programming effects. ${ }^{37}$ Animal studies suggest that maternal dietary exposures may induce sexually dimorphic responsiveness in placental gene expression, especially in the biological process of the cell cycle. ${ }^{38}$

In summary, we found that cord blood PUFA levels were not associated with obesity-related outcomes including rapid weight gain in the first months of life, and BMI till young adulthood. A small, not clinically relevant association was observed between PUFA status at birth and length in infancy, and this did not persist in later life up to young adulthood. High maternal fish intake of more than 3 times/week during pregnancy was associated with increased risk of rapid weight gain in infancy and of overweight and obesity in mid-childhood.

\section{Child cardiometabolic risk}

Studies have shown that obesity, and especially central obesity, is associated with the co-occurrence of multiple cardiometabolic risk factors including high blood pressure and dyslipidemia. ${ }^{39-41}$ Evidence from pathological and imaging studies also suggest that the clustering of multiple cardiometabolic risk factors in children is highly predictive of the extent of adult atherosclerosis. ${ }^{42,43}$

Few studies have previously examined the association of prenatal PUFA exposure with childhood cardiometabolic traits other than adiposity, and have reported largely mixed results. ${ }^{44-48}$ Consequently, a recent systematic review underlined the importance of further studies in order to clarify the role of prenatal PUFAs in childhood cardiometabolic health. ${ }^{49}$

Hence, as part of our pooled analysis in MEFAB and RHEA cohorts on cord blood PUFAs and childhood obesity (Chapter 3), we also assessed associations with an aggregate cardiometabolic risk score comprised of measures of waist circumference (a measure of central adiposity), non-HDL cholesterol, and blood pressure, as well as with the individual cardiometabolic factors at age 6-7 years. We found no associations of cord blood PUFA levels with the clustered cardiometabolic risk score and its individual components.

\section{Child allergy-related phenotypes}

Allergic diseases result in significant disruption of life, and constitute a major cause of hospitalizations among children. ${ }^{50,51}$ It has been hypothesized that a shift in PUFA composition of Western diets towards an increased intake of vegetable oils containing n-6 fatty acids, and lower n-3 PUFA and fish consumption has contributed to the observed increase in the prevalence of allergic diseases over the last years. ${ }^{52}$ 
In support of this hypothesis, animal and in vitro studies have shown n-3 PUFAs to inhibit inflammatory responses and reduce allergic disease activity, while $n-6$ PUFAs seem to have an opposite effect. ${ }^{53,54}$ However, findings from cohort studies on the association of prenatal exposure to PUFAs with childhood asthma and other allergy-related symptoms such as eczema and rhinitis have been largely inconsistent (reviewed in Chapter 1). These inconsistencies may partly arise from exposure profile heterogeneity, and differences in health outcome definition and adjustment for confounding variables. Given the substantial health burden of allergic diseases, it is important to better characterize the potential role of PUFAs in the development of asthma and other allergy-related diseases.

Hence, we harmonized and pooled individual mother-offspring data from the MEFAB and RHEA cohort to assess whether $n-3$ and $n-6$ PUFAs concentrations in cord blood phospholipids are associated with symptoms of wheeze, asthma, rhinitis and eczema in children aged 6-7 years (Chapter 5). We found that higher n-3 PUFA levels, and especially EPA and DHA, were associated with reduced risk of current wheeze and asthma ( $\mathrm{RR}=0.61$ [95\% CI 0.45-0.82] and 0.50 [95\% CI 0.31-0.79] per 1-SD increase in EPA+DHA concentration, respectively). Higher cord blood n-6 PUFA levels were associated with a higher risk of current wheeze $(\mathrm{RR}=1.54$ [95\% CI 1.09-2.17] per 1-SD increase in total $\mathrm{n}-6$ PUFAs). We also observed a reduced risk of both current wheeze and asthma with increasing $\mathrm{n}-3$ to $\mathrm{n}-6$ fatty acid ratio $(\mathrm{RR}=0.54$ [95\% CI $0.39-0.75]$ and 0.43 [95\% CI $0.26-$ 0.70] per 1-unit increase, respectively). No associations were observed for rhinitis and eczema. These findings suggest a specific effect of prenatal PUFAs on airway development and function.

As part of this thesis, we also assessed the association of fish intake during pregnancy with childhood allergy-related symptoms. We summarized prior findings from cohort studies and found mixed results (Chapter 6). Next, we harmonized and pooled individual data of mother-offspring pairs from 18 European and US cohorts with repeated offspring follow-ups until the age of 8 years to assess the strength and consistency of the association of fish consumption during pregnancy with the risk of childhood wheeze, asthma and allergic rhinitis. Maternal fish intake during pregnancy was not associated with offspring wheeze symptoms in infancy ( $0-2$ years), preschool (3-4 years), or school-age (5-8 years). When we examined the risk of asthma at either preschool or school age, we also observed no associations with maternal fish intake. Similarly, fish intake was not associated with the risk of allergic rhinitis among school-age children. Heterogeneity across cohort-specific effect estimates was low for most exposure-outcome associations. It is possible that persistent organic pollutants bio-accumulating in fish counterbalance or mask the effects of beneficial nutrients such as n-3 long chain PUFAs, 
moving the association of fish intake with child respiratory health towards the null. Indeed, there is emerging evidence to suggest that prenatal exposure to PCBs and pesticides can exert adverse immunomodulatory effects and increase the risk of developing allergic disease symptoms in childhood. ${ }^{556}$ In this international study, we did not have the possibility to collect biomarker information on both nutrients and toxicants in fish so as to examine their interplay, as well as to disentangle potential opposite effect on child outcomes.

In summary, we found that high EPA and DHA levels and a high n-3:n-6 fatty acid ratio in cord blood were associated with decreased risk of wheeze and asthma in mid-childhood. No associations were observed between PUFA status at birth and mid-childhood risk of eczema and rhinitis. We also found no evidence supporting a protective association of fish consumption during pregnancy with offspring respiratory symptoms including wheeze, asthma, and allergic rhinitis in early and mid-childhood.

\section{METHODOLOGICAL CONSIDERATIONS}

Apart from our meta-analysis of supplementation trials, studies presented in this thesis were population-based, prospective birth cohort studies. Specific methodological considerations have been discussed in previous chapters. Here, we will discuss some general methodological issues in observational research that are applied to our studies, namely confounding, selection bias, outcome and exposure misclassification, and causality.

\section{Confounding}

A confounder is an extraneous factor that is related to both the exposure and the outcome under study, but it is not located in the causal pathway. If it is not taken properly into account, confounding can lead to biased effect estimates. Hence, it is considered an important threat to validity of observational research. ${ }^{57}$

Traditional approaches to control for confounding include either inclusion of a priori defined potential confounders in the analytical model or inclusion of each covariate that satisfies the criteria of association with both the exposure and outcome, and changes the effect estimate when included in the model. However, these traditional approaches may introduce problems such as collinearity, decreased precision of effect estimates due to unnecessary adjustment, or overadjustment bias resulting by adjusting for a descending proxy of an intermediate variable on a causal path from exposure to outcome. ${ }^{58,59}$

To select the confounders for adjustment in our studies, we used a directed acyclic graph (DAG) approach based on prior knowledge about the relationships among 
parental and child covariates. The use of DAGs allows for a better, overall insight in the assumed relationships among the covariates, and overcomes problems related to the traditional approaches. ${ }^{58,60}$ Moreover, they allow for identification of a minimum but sufficient set of factors to control for in the analysis in order to resolve confounding. ${ }^{60,61}$

In our analyses, we adjusted for a range of sociodemographic characteristics. Similar to all observational studies, residual confounding - that is, the confounding left after controlling for the available confounders- cannot be ruled out. ${ }^{57}$ Nevertheless, in most of our studies, we conducted pooled analyses of cohorts from different parts of Europe with similar data but potentially very different confounding patterns, and we did not find evidence of important heterogeneity between cohort-specific effect estimates. This reduces the likelihood that residual confounding has affected markedly the results.

\section{Selection bias}

Selection bias exists when the association between the exposure and outcome of interest is different in study participants and those who were in the target population but did not participate. Such bias can result from procedures used for study selection and from factors that influence study participation. ${ }^{57}$

Selection bias at entry constitutes a threat to generalizability of study results, however, it is difficult to quantify. The studies presented this thesis were populationbased. In general, among participating cohorts, there was a higher participation at study entry of populations with relatively high education levels, and an underpresentation of ethnic minority groups, which are generally characterized by low socio-economic status and unhealthy lifestyle habits. ${ }^{62-65}$ Hence, our results may not be generalizable to more socioeconomically disadvantaged populations.

Another potential source of selection bias is selective attrition, which can occur when the association of the exposure with the outcome of interest is different between participants and those lost to follow-up. ${ }^{66}$ In our studies involving MEFAB and RHEA children, loss to follow up evaluations at 6-7 years of age (for both MEFAB and RHEA) and at 23 years of age (for MEFAB) was high, rising to more than $50 \%$. In non-response analyses, we found that, compared to non-participants at the 6-7-year follow-up, those included in our studies had somewhat higher cord blood n-3 PUFA levels in RHEA but not in MEFAB, and did not substantially differ in main sociodemographic characteristics such as maternal BMI at study entry, gestational weight gain, birthweight, gestational age, parental education or socio-economic status. This lowers the likelihood of selective participation of a healthier population, and thus, of selective attrition affecting our results on the associations of prenatal PUFA levels with mid-childhood 
allergy-related phenotypes and obesity. At the 23-year follow up of the MEFAB cohort, we observed that participants and those not participating did not differ in exposure levels, however, participants had lower maternal BMI values at study entry. Assuming that offspring of mothers with a higher BMI are more likely to be overweight, our effect estimates on the association of prenatal PUFAs with adult BMI may be biased towards the null. Finally, in the pooled analyses of fish intake during pregnancy with childhood growth and respiratory symptoms, we saw that, compared to non-participants, participating mothers had higher age at delivery in 10 out of 18 cohorts, and were more frequently higher educated in 9 out of 18 cohorts. Assuming that a higher educated population is likely to have a healthier lifestyle and constitute a relatively healthier population, our effect estimates on fish intake may be underestimated.

\section{Outcome and exposure misclassification}

Misclassification, otherwise known as information bias, usually occurs in the data collection stage, and refers to a distortion of the estimated effect due to an error in the measurement of exposure and/or outcome under study. ${ }^{57}$ Misclassification can be classified into two types: non-differential (random) and differential (random). Non-differential misclassification can occur when the error in classification of exposure or outcome is random. Differential misclassification occurs when the degree or direction of misclassification of exposure depends on outcome status, or when misclassification of the health outcome depends on exposure status. Nondifferential misclassification will usually bias effect estimates towards the null, whereas differential misclassification can bias effect estimates either towards or away from the null. ${ }^{57}$

In this thesis, information on exposure was based on biomarker levels (for assessment of PUFA status) or validated food frequency questionnaires (for assessment of fish intake), and collected before assessment of the outcomes. Moreover, participating cohort studies in this thesis had multiple research objectives during data collection, assessing multiple outcomes and exposures. Mother-child pairs included in our analyses were unlikely to be aware of the specific research questions addressed in the studies of this thesis. Hence, differential exposure misclassification is less likely to have occurred. However, we cannot exclude the possibility of non-differential exposure misclassification, especially for the assessment of fish intake.

In our studies of postnatal growth, anthropometric measurements in childhood were assessed using medical records or by trained research assistants following standardized procedures. Moreover, when measurements collected, there was 
no knowledge of the specific research questions addressed in this thesis. Hence, differential misclassification of anthropometric measurements in childhood is unlikely. Information on growth measures in young adulthood among MEFAB participants was self-reported, which might have led to misclassification. This potential misclassification is likely to be non-differential (ie, not to relate to cord blood PUFAs). Finally, childhood allergy-related symptoms in our studies were ascertained using parent-completed questionnaires. Underreporting or overreporting of symptoms that might depend on parental history of atopy could have occurred, leading to misclassification of diagnosis. We did not observe any differences in exposure levels (prenatal PUFAs or fish intake) between mothers with and without asthma or hay fever across the cohorts. Moreover, for asthma and eczema, we used composite definitions requiring at least two conditions to be met in order to enhance outcome accuracy.

Overall, differential misclassification is less likely to have affected the results of the studies presented in this thesis. Nevertheless, we cannot exclude the possibility of non-differential misclassification for both the exposures and outcomes under study.

\section{Causality}

In this thesis, we assessed associations rather than causal effects due to the observational design of the included studies. All the above-mentioned methodological issues that apply to observational research, and, thus, to our studies constitute important threats in terms of establishing cause-and-effect relationships. Judgment of a causal relationship for a specific research question can be tough. Few rules apply for implying causal relationships, though criteria proposed by Hill have received the most attention. ${ }^{67,68}$ These criteria include temporality, observation of a biological gradient, biologically plausibility, coherence with current knowledge of the biology, magnitude and consistency of the observed associations, experimental evidence, specificity, and analogy with comparable exposures. ${ }^{67}$

Temporality indicates that the exposure must precedes the health outcome in time, and is considered an ironclad criterion for causal inference. Our studies fulfil this criterion, as they had a prospective design where exposures were measured before the occurrence of the health outcomes of interest. According to Hill, evidence of a biological gradient also provides support for a causal association. Others have been less demanding, requiring only a particular shape of relationship (not necessarily linear) that is in line with a substantive theory. ${ }^{69}$ In our studies, we observed dose-response associations of cord blood n-3 PUFA levels with the risk of mid-childhood asthma. For maternal fish intake during pregnancy, an association 
with higher rapid infant weight gain and childhood risk of overweight/obesity was observed for high intake levels above 3 times/week, which is in line with dietary guidelines. It is possible that high fish intake reflects high fetal exposure to pollutants that have been suggested to increase adipogenesis. Fish consumption levels were relatively low among most participating cohorts, and, thus, there was low variability. This has undermined the possibility to examine a dose-response relationship for higher intake levels, and construct additional categories of fish consumption. For all observed associations in our studies, plausible underlying mechanisms, coherent with knowledge obtained using animal and in vitro models, have been proposed, as described in previous chapters.

The magnitude and consistency of exposure-outcome associations are also considered important for establishing causality. Inconclusive results from trials on the association of prenatal fish oil supplementation with childhood allergy, and the absence of trials on dietary fish intake during pregnancy and childhood growth argue against current inferences for causal associations. Previous cohort studies on prenatal PUFA status or fish intake and child health have also yielded inconsistent results (reviewed in previous chapters), possibly due to exposure heterogeneity, inconsistencies in outcome definition, or differences in adjustment for covariates. In this thesis, we tried to address this by conducting pooled analyses of separate cohorts from different parts of Europe with harmonized information on exposures, outcomes, and important confounders. In general, we observed consistent associations with no evidence of important heterogeneity between cohort-specific estimates. Although the magnitude of effect sizes reported in this thesis were generally small (eg, fish consumption $>3$ times/week during pregnancy was associated with $20 \%$ excess risk of being overweight or obese in childhood), they are of clinical relevance given that we studied widespread nutritional exposures and adverse child health outcomes having reached epidemic proportions in developed countries. Finally, the specificity and analogy criteria of Hill have been proposed to be less useful, as most exposures usually lead to many outcomes, and reasoning by analogy can be dependent on the inventive imagination of researchers. ${ }^{57,68}$

Overall, the studies presented in this thesis have methodological limitations related to their observational design. Interpretation of epidemiological results should always be made in the context of all other available evidence. Our findings fulfil some, but not all, of the Hill criteria for causal inference. Further large-scale studies among populations with different confounding and correlation structures in the data are needed for a more definitive assessment of the causality of associations observed in this thesis. 


\section{FUTURE PERSPECTIVES}

Human genetic variation that accounts for differential response to dietary exposures has received increased attention in terms of disease prevention. ${ }^{70}$ Recent studies have uncovered population-related genetic variation in the endogenous metabolism of PUFAs. Among related gene variants, the fatty acid desaturase gene (FADS) cluster has received most attention. ${ }^{71,72}$ Within this cluster, FADS1 and FADS2 genes encode for the $\delta-5$ and $\delta-6$ desaturases, which are considered the rate-limiting steps in the PUFA biosynthetic pathway. ${ }^{71}$ Genetic association studies of single nucleotide polymorphisms (SNPs) in the FADS1 and FADS2 genes in pregnant women have shown that, independent of dietary effects, the minor alleles were associated with reduced amounts of long-chain PUFA both in maternal and cord blood. ${ }^{73,74}$ Hence, genetic variation affects PUFA exposure levels in the developing fetus and, thereby, might modify the association of prenatal PUFAs with later health outcomes. In our studies, we did not have information on SNPs in genes encoding for enzymes involved in fatty acid metabolism so as to examine how prenatal PUFAs interact with genetic variants. A recent trial of fish oil supplementation during pregnancy demonstrated that the supplementation effect on childhood asthma risk was strongest in the children of mothers carrying the minor G allele of the FADS2 variant rs $1535 .{ }^{75}$ Hence, a modulation of prenatal PUFA status by FADS genotypes may be of major relevance for child health outcomes, and explain some of the contradictory findings among studies. Future studies on the relation between prenatal PUFA exposure and child health outcomes should aim at inclusion of genetic analyses to evaluate FADS genotype-dependent associations.

Moreover, epigenetic regulation of gene expression is a promising area of research in this field. ${ }^{76}$ Epigenetic mechanisms, such as DNA methylation, are commonly defined as mechanisms of regulating gene activity independently of the underlying DNA sequence, and are known to play a central role in fetal development, allowing cells, tissues and systems to adopt varying phenotypes despite a common genotype. ${ }^{77,78}$ Epigenetic alteration often precede the disease phenotype, making them important diagnostic tools for disease risk. ${ }^{79}$ Emerging evidence from animal models suggests that PUFAs may influence global DNA methylation patterns because of their role in one-carbon metabolism. ${ }^{80,81}$ Recently, a randomised trial showed that DHA supplementation in pregnancy was associated with 21 differentially DNA methylated regions of the genome in blood cells of children at birth. ${ }^{82}$ Notably, some of these regions, including RAETIL and LTB, have been related to immune function. ${ }^{82}$ There is still relatively little human data on the magnitude and extent of epigenetic changes impacted by maternal diet during pregnancy. Further studies examining methylation changes in response to prenatal 
dietary exposures including PUFAs and how these changes play a role in later disease phenotypes would provide valuable insight into underlying mechanisms and further support for causal inferences.

Randomized controlled trials are considered an important epidemiological study design for establishing causality. Most prior trials on fish oil supplementation and childhood growth, obesity, and allergy-symptoms are limited by small sizes and high attrition rates, and have yielded largely inconsistent results (reviewed in previous chapters). Further large-scale trials with long-term follow-ups are needed in order to establish whether prenatal PUFAs affect child health outcomes. With respect to fish intake, it might be unethical to randomize pregnant women to higher or lower levels of fish intake than those currently proposed by fish consumption advisories (2-4 times/week during pregnancy). ${ }^{83,84}$ Future studies should assess the impact of intervention promoting recommended fish intake levels on various health outcomes. Such studies might provide additional evidence for causal associations. Importantly, they might strengthen the evidence base of current fish consumption advisories, the establishment of which was based mainly on potential neurocognitive harms associated with fish-related methylmercury exposure. $^{83,84}$

In our international studies on prenatal fish intake, we did not have the possibility to incorporate biomarker information on nutrients and environmental chemicals contained in fish so as to examine their interplay. Further studies should focus on the difficult task of elucidating which nutrients or environmental chemicals or combination of factors within fish could explain the effect of fish intake during pregnancy. Such studies will be helpful for informing dietary recommendations for pregnant women.

\section{LIFE COURSE EPIDEMIOLOGY}

A life course approach to epidemiology refers to the study of the long-term effects on later health or disease risk of physical or social exposures during gestation, childhood, adolescence, young adulthood, and later adult life. ${ }^{85}$ It aims to identify environmental factors acting across an individual's life course to influence health and disease. Although the life course perspective has a long history in sociological and psychological sciences, ${ }^{86,87}$ epidemiologists have just recently started to adopt a life course approach in understanding disease aetiology ${ }^{88}$

The "developmental origins of health and disease" paradigm provided a stimulus for broader thinking about a range of influences acting at critical developmental periods early in life, in addition to conventional adult risk factors, to affect later health. ${ }^{89}$ This paradigm provided the catalyst for a life course approach in epide- 
miology. Life course epidemiology tries to bridge different life stages, and is built on the premise that environmental factors operate throughout life independently, cumulatively, and interactively to influence health and disease in adult life. ${ }^{89}$ The importance of the life course as a framework in disease causation is increasingly being recognized, ${ }^{90}$ and has been incorporated into many policy documents about lifelong health and ageing. ${ }^{91,92}$

Adopting a life course approach to chronic disease epidemiology is challenging. Fundamentally, investigating life course processes for chronic diseases such as obesity and asthma requires measuring data at multiple time points throughout the life span. Moreover, collection of adequate exposure measures, such as biomarker levels, at multiple points is quite expensive to perform, but essential in order to understand how specific exposures may influence the development of chronic diseases through the timing of their action and/or through their accumulation.

Further analyses in the MEFAB and RHEA birth cohorts supplemented with additional follow up evaluations will allow us to study how exposure to PUFAs may operate independently at specific time windows or in a cumulative fashion throughout the life course to alter chronic disease risk.

\section{CONCLUSION}

In the present thesis, we followed a population-based approach and studied the associations of prenatal exposure to PUFAs and maternal fish intake during pregnancy with major health outcomes in the postnatal period. We found sexspecific, not clinically relevant associations of cord blood PUFA levels with length in infancy, and these did not persist later in life up to young adulthood. PUFAs were not associated with obesity-related outcomes including rapid infant weight gain, BMI, and cardiometabolic risk. In contrast, higher levels of EPA and DHA and a higher ratio of total n-3 to n-6 PUFAs in cord blood were associated with lower risk of childhood asthma symptoms. We also found that high maternal fish consumption above current recommended intakes (3 times/week) was associated with increased risk of rapid infant weight gain and childhood overweight including obesity, while no association was observed with childhood respiratory symptoms. To conclude, our findings suggest that a modification of prenatal PUFA status towards higher levels of n-3 long-chain PUFAs in late gestation might assist in the prevention of childhood asthma, but not in tackling the obesity epidemic in children. Pregnant women should adhere to current fish consumption guidelines, and not exceed recommended intakes as a means of improving n-3 PUFA status. Future studies are needed to establish causality and to examine the interplay between fatty acids and pollutants found in fish. 


\section{REFERENCES}

1. The Global Asthma Report 2014. Auckland, New Zealand: Global Asthma Network.

2. Han JC, Lawlor DA, Kimm SY. Childhood obesity. Lancet 2010;375:1737-48.

3. Meltzer EO. Quality of life in adults and children with allergic rhinitis. J Allergy Clin Immunol 2001;108:S45-53.

4. Gluckman PD, Hanson MA, Cooper C, et al. Effect of in utero and early-life conditions on adult health and disease. N Engl J Med 2008;359:61-73.

5. Hanson MA, Gluckman PD. Early developmental conditioning of later health and disease: physiology or pathophysiology? Physiol Rev 2014;94:1027-76.

6. Brenna JT, Salem N, Jr. Workshop proceedings: DHA as a required nutrient. Prostaglandins Leukot Essent Fatty Acids 2009;81:97.

7. Heird WC, Lapillonne A. The role of essential fatty acids in development. Annu Rev Nutr 2005;25:54971.

8. Hornstra G. Essential fatty acids in mothers and their neonates. Am J Clin Nutr 2000;71:1262S-9S.

9. Schmitz G, Ecker J. The opposing effects of n-3 and n-6 fatty acids. Prog Lipid Res 2008;47:147-55.

10. Ailhaud G, Guesnet P, Cunnane SC. An emerging risk factor for obesity: does disequilibrium of polyunsaturated fatty acid metabolism contribute to excessive adipose tissue development? $\mathrm{Br} J$ Nutr 2008;100:461-70.

11. Kalupahana NS, Claycombe KJ, Moustaid-Moussa N. (n-3) Fatty acids alleviate adipose tissue inflammation and insulin resistance: mechanistic insights. Adv Nutr 2011;2:304-16.

12. Kruger MC, Coetzee M, Haag M, et al. Long-chain polyunsaturated fatty acids: selected mechanisms of action on bone. Prog Lipid Res 2010;49:438-49.

13. Paajanen TA, Oksala NK, Kuukasjarvi P, et al. Short stature is associated with coronary heart disease: a systematic review of the literature and a meta-analysis. Eur Heart J 2010;31:1802-9.

14. Owen CG, Whincup PH, Orfei L, et al. Is body mass index before middle age related to coronary heart disease risk in later life? Evidence from observational studies. Int J Obes (Lond) 2009;33:866-77.

15. Silventoinen K, Baker JL, Sorensen TI. Growth in height in childhood and risk of coronary heart disease in adult men and women. PLoS One 2012;7:e30476.

16. Gillman MW. The first months of life: a critical period for development of obesity. Am J Clin Nutr 2008;87:1587-9.

17. Al MD, van Houwelingen AC, Kester AD, et al. Maternal essential fatty acid patterns during normal pregnancy and their relationship to the neonatal essential fatty acid status. Br J Nutr 1995;74:55-68.

18. Al MD, Hornstra G, van der Schouw YT, et al. Biochemical EFA status of mothers and their neonates after normal pregnancy. Early Hum Dev 1990;24:239-48.

19. Al MD, Badart-Smook A, von Houwelingen AC, et al. Fat intake of women during normal pregnancy: relationship with maternal and neonatal essential fatty acid status. J Am Coll Nutr 1996;15:49-55.

20. van Houwelingen AC, Sorensen JD, Hornstra G, et al. Essential fatty acid status in neonates after fish-oil supplementation during late pregnancy. Br J Nutr 1995;74:723-31.

21. Duttaroy AK. Transport of fatty acids across the human placenta: a review. Prog Lipid Res 2009;48:5261.

22. Hodson L, Skeaff CM, Fielding BA. Fatty acid composition of adipose tissue and blood in humans and its use as a biomarker of dietary intake. Prog Lipid Res 2008;47:348-80.

23. Whitaker RC, Wright JA, Pepe MS, et al. Predicting obesity in young adulthood from childhood and parental obesity. N Engl J Med 1997;337:869-73. 
24. Baker JL, Olsen LW, Sorensen TI. Childhood body-mass index and the risk of coronary heart disease in adulthood. N Engl J Med 2007;357:2329-37.

25. Gunnell DJ, Frankel SJ, Nanchahal K, et al. Childhood obesity and adult cardiovascular mortality: a 57-y follow-up study based on the Boyd Orr cohort. Am J Clin Nutr 1998;67:1111-8.

26. Stein AD, Wang M, Martorell R, et al. Growth to age 18 months following prenatal supplementation with docosahexaenoic acid differs by maternal gravidity in Mexico. J Nutr 2011;141:316-20.

27. Much D, Brunner S, Vollhardt C, et al. Effect of dietary intervention to reduce the n-6/n-3 fatty acid ratio on maternal and fetal fatty acid profile and its relation to offspring growth and body composition at 1 year of age. Eur J Clin Nutr 2013;67:282-8.

28. Dunstan JA, Simmer K, Dixon G, et al. Cognitive assessment of children at age 2(1/2) years after maternal fish oil supplementation in pregnancy: a randomised controlled trial. Arch Dis Child Fetal Neonatal Ed 2008;93:F45-50.

29. Innis SM, Friesen RW. Essential n-3 fatty acids in pregnant women and early visual acuity maturation in term infants. Am J Clin Nutr 2008;87:548-57.

30. Decsi T, Kennedy K. Sex-specific differences in essential fatty acid metabolism. Am J Clin Nutr 2011;94:Suppl. 6, S1914-S9.

31. Sedlmeier EM, Brunner S, Much D, et al. Human placental transcriptome shows sexually dimorphic gene expression and responsiveness to maternal dietary n-3 long-chain polyunsaturated fatty acid intervention during pregnancy. BMC Genomics 2014;15:941.

32. Turyk ME, Bhavsar SP, Bowerman W, et al. Risks and benefits of consumption of Great Lakes fish. Environ Health Perspect 2012;120:11-8.

33. Mozaffarian D, Rimm EB. Fish intake, contaminants, and human health: evaluating the risks and the benefits. JAMA 2006;296:1885-99.

34. Bloomingdale A, Guthrie LB, Price S, et al. A qualitative study of fish consumption during pregnancy. Am J Clin Nutr 2010;92:1234-40.

35. Ibrahim MM, Fjaere E, Lock EJ, et al. Chronic consumption of farmed salmon containing persistent organic pollutants causes insulin resistance and obesity in mice. PLoS One 2011;6:e25170.

36. Vafeiadi M, Georgiou V, Chalkiadaki G, et al. Association of Prenatal Exposure to Persistent Organic Pollutants with Obesity and Cardiometabolic Traits in Early Childhood: The Rhea Mother-Child Cohort (Crete, Greece). Environ Health Perspect 2015;123:1015-21.

37. Sood R, Zehnder JL, Druzin ML, et al. Gene expression patterns in human placenta. Proc Natl Acad Sci U S A 2006;103:5478-83.

38. Gabory A, Ferry L, Fajardy I, et al. Maternal diets trigger sex-specific divergent trajectories of gene expression and epigenetic systems in mouse placenta. PLoS One 2012;7:e47986.

39. Taksali SE, Caprio S, Dziura J, et al. High visceral and low abdominal subcutaneous fat stores in the obese adolescent: a determinant of an adverse metabolic phenotype. Diabetes 2008;57:367-71.

40. Zimmet P, Alberti KG, Kaufman F, et al. The metabolic syndrome in children and adolescents - an IDF consensus report. Pediatr Diabetes 2007;8:299-306.

41. Katzmarzyk PT, Srinivasan SR, Chen W, et al. Body mass index, waist circumference, and clustering of cardiovascular disease risk factors in a biracial sample of children and adolescents. Pediatrics 2004;114:e198-205.

42. Berenson GS, Srinivasan SR, Bao W, et al. Association between multiple cardiovascular risk factors and atherosclerosis in children and young adults. The Bogalusa Heart Study. N Engl J Med 1998;338:16506. 
43. Juonala M, Jarvisalo MJ, Maki-Torkko N, et al. Risk factors identified in childhood and decreased carotid artery elasticity in adulthood: the Cardiovascular Risk in Young Finns Study. Circulation 2005;112:1486-93.

44. Rump P, Popp-Snijders C, Heine RJ, et al. Components of the insulin resistance syndrome in seven-yearold children: relations with birth weight and the polyunsaturated fatty acid content of umbilical cord plasma phospholipids. Diabetologia 2002;45:349-55.

45. Rytter D, Bech BH, Halldorsson T, et al. No association between the intake of marine n-3 PUFA during the second trimester of pregnancy and factors associated with cardiometabolic risk in the 20-year-old offspring. Br J Nutr 2013;110:2037-46.

46. Jochems SH, Gielen M, Rump P, et al. Potential programming of selected cardiometabolic risk factors at childhood by maternal polyunsaturated fatty acid availability in the MEFAB cohort. Prostaglandins Leukot Essent Fatty Acids 2015;100:21-7.

47. Vidakovic AJ, Jaddoe VW, Voortman T, et al. Maternal plasma polyunsaturated fatty acid levels during pregnancy and childhood lipid and insulin levels. Nutr Metab Cardiovasc Dis 2017;27:78-85.

48. Vidakovic AJ, Gishti O, Steenweg-de Graaff J, et al. Higher Maternal Plasma n-3 PUFA and Lower n-6 PUFA Concentrations in Pregnancy Are Associated with Lower Childhood Systolic Blood Pressure. J Nutr 2015;145:2362-8.

49. Voortman T, van den Hooven EH, Braun KV, et al. Effects of polyunsaturated fatty acid intake and status during pregnancy, lactation, and early childhood on cardiometabolic health: A systematic review. Prog Lipid Res 2015;59:67-87.

50. Bousquet J, Nikolai Khaltaev N. Global surveillance, prevention and control of chronic respiratory diseases : a comprehensive approach. Geneva, Switzerland: World Health Organization 2007.

51. Weiss KB, Sullivan SD. The health economics of asthma and rhinitis. I. Assessing the economic impact. J Allergy Clin Immunol 2001;107:3-8.

52. Black PN, Sharpe S. Dietary fat and asthma: is there a connection? Eur Respir J 1997;10:6-12.

53. Prescott SL, Dunstan JA. Prenatal fatty acid status and immune development: the pathways and the evidence. Lipids 2007;42:801-10.

54. Calder PC, Kremmyda LS, Vlachava M, et al. Is there a role for fatty acids in early life programming of the immune system? Proc Nutr Soc 2010;69:373-80.

55. Gascon M, Sunyer J, Casas M, et al. Prenatal exposure to DDE and PCB 153 and respiratory health in early childhood: a meta-analysis. Epidemiology 2014;25:544-53.

56. Hansen S, Strom M, Olsen SF, et al. Maternal concentrations of persistent organochlorine pollutants and the risk of asthma in offspring: results from a prospective cohort with 20 years of follow-up. Environ Health Perspect 2014;122:93-9.

57. Rothman KJ, Greenland S. Modern epidemiology. 2nd ed. Philadelphia: Lippincott Williams \& Wilkins. 1998.

58. Shrier I, Platt RW. Reducing bias through directed acyclic graphs. BMC Med Res Methodol 2008;8:70.

59. Schisterman EF, Cole SR, Platt RW. Overadjustment bias and unnecessary adjustment in epidemiologic studies. Epidemiology 2009;20:488-95.

60. Greenland S, Pearl J, Robins JM. Causal diagrams for epidemiologic research. Epidemiology 1999;10:3748.

61. Tu YK, Greenwood DC. Modern Methods for Epidemiology. London: Springer. 2012.

62. Guxens M, Ballester F, Espada M, et al. Cohort Profile: the INMA--INfancia y Medio Ambiente-(Environment and Childhood) Project. Int J Epidemiol 2012;41:930-40.

63. Oken E, Baccarelli AA, Gold DR, et al. Cohort profile: project viva. Int J Epidemiol 2015;44:37-48. 
64. van Eijsden M, Vrijkotte TG, Gemke RJ, et al. Cohort profile: the Amsterdam Born Children and their Development (ABCD) study. Int J Epidemiol 2011;40:1176-86.

65. Jaddoe VW, Mackenbach JP, Moll HA, et al. The Generation R Study: Design and cohort profile. Eur J Epidemiol 2006;21:475-84.

66. Gail MH, Benichou J. Encyclopedia of Epidemiologic Methods. Chichester: John Wiley \& Sons. 2000.

67. Hill AB. The Environment and Disease: Association or Causation? Proc R Soc Med 1965;58:295-300.

68. Grimes DA, Schulz KF. Bias and causal associations in observational research. Lancet 2002;359:248-52.

69. Cox DR, Wermuth N. Multivariate Dependencies Models, Analyses and Interpretation. London: Chapman and Hall. 1996.

70. Stover PJ. Influence of human genetic variation on nutritional requirements. Am $J$ Clin Nutr 2006;83:436S-42S.

71. Minihane AM. Impact of Genotype on EPA and DHA Status and Responsiveness to Increased Intakes. Nutrients 2016;8:123.

72. Schaeffer L, Gohlke H, Muller M, et al. Common genetic variants of the FADS1 FADS2 gene cluster and their reconstructed haplotypes are associated with the fatty acid composition in phospholipids. Hum Mol Genet 2006;15:1745-56.

73. Koletzko B, Lattka E, Zeilinger S, et al. Genetic variants of the fatty acid desaturase gene cluster predict amounts of red blood cell docosahexaenoic and other polyunsaturated fatty acids in pregnant women: findings from the Avon Longitudinal Study of Parents and Children. Am J Clin Nutr 2011;93:211-9.

74. Lattka E, Koletzko B, Zeilinger S, et al. Umbilical cord PUFA are determined by maternal and child fatty acid desaturase (FADS) genetic variants in the Avon Longitudinal Study of Parents and Children (ALSPAC). Br J Nutr 2013;109:1196-210.

75. Bisgaard H, Stokholm J, Chawes BL, et al. Fish Oil-Derived Fatty Acids in Pregnancy and Wheeze and Asthma in Offspring. N Engl J Med 2016;375:2530-9.

76. Burdge GC, Lillycrop KA. Nutrition, epigenetics, and developmental plasticity: implications for understanding human disease. Annu Rev Nutr 2010;30:315-39.

77. Bernstein BE, Meissner A, Lander ES. The mammalian epigenome. Cell 2007;128:669-81.

78. Portela A, Esteller M. Epigenetic modifications and human disease. Nat Biotechnol 2010;28:1057-68.

79. Kelly TK, De Carvalho DD, Jones PA. Epigenetic modifications as therapeutic targets. Nat Biotechnol 2010;28:1069-78.

80. Kulkarni A, Dangat K, Kale A, et al. Effects of altered maternal folic acid, vitamin B12 and docosahexaenoic acid on placental global DNA methylation patterns in Wistar rats. PLoS One 2011;6:e17706.

81. Egger G, Liang G, Aparicio A, et al. Epigenetics in human disease and prospects for epigenetic therapy. Nature 2004;429:457-63.

82. van Dijk SJ, Zhou J, Peters TJ, et al. Effect of prenatal DHA supplementation on the infant epigenome: results from a randomized controlled trial. Clin Epigenetics 2016;8:114.

83. EFSA Scientific Committee. Statement on the benefits of fish/seafood consumption compared to the risks of methylmercury in fish/seafood. EFSA Journal 2015;13:3982.

84. Cunningham E. Methylmercury and Seafood: What Are the Latest Guidelines? J Acad Nutr Diet 2017;117:824.

85. Ben-Shlomo Y, Kuh D. A life course approach to chronic disease epidemiology: conceptual models, empirical challenges and interdisciplinary perspectives. Int J Epidemiol 2002;31:285-93.

86. Elder GH. Time, human agency, and social change: perspectives on the life course. Soc Psychol Quart 1994;57:4-15.

87. Giele JZ, Elder GH. Methods of life course research: qualitiative and quantitative approaches. Thousand Oaks: Sage. 1998. 
88. Kuh D, Ben-Shlomo Y, Lynch J, et al. Life course epidemiology. J Epidemiol Community Health 2003; $57: 778-83$.

89. Lynch J, Smith GD. A life course approach to chronic disease epidemiology. Annu Rev Public Health 2005;26:1-35.

90. Aboderin I, Kalache A, Ben-Shlomo Y, et al. Life Course Perspectives on Coronary Heart Disease, Stroke and Diabetes: Key Issues and Implications for Policy and Research. Geneva: World Health Organization. 2002.

91. Government Office for Science. Foresight report: future of an ageing population. 2016. https://www. gov.uk/government/collections/future-of-ageing. (9 July 2017, date last accessed)

92. World Health Organization. World report on ageing and health. 2015. http://www.who.int/ageing/ events/world-report-2015-launch/en/. (9 July 2017, date last accessed) 


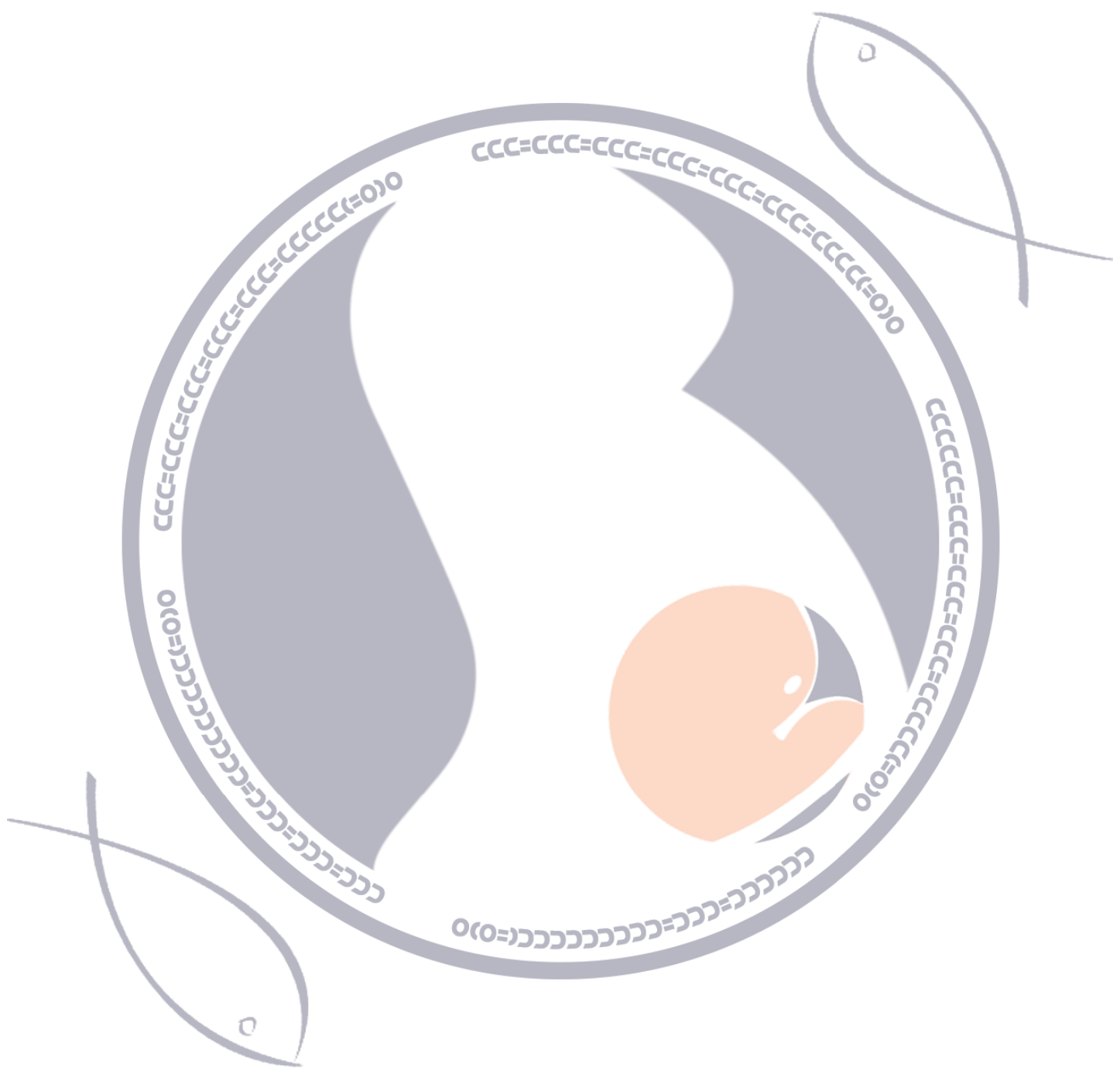


CHAPTER 10

Thesis summary

Samenvatting

Valorization addendum 



\section{Thesis summary}

Rates of childhood obesity and allergy are increasing at alarmingly across the globe, with severe long-term consequences. Hence, there is an urgent need to identify early-life modifiable determinants that can be targeted for preventive interventions. In this context, there has been an increasing interest in the potential role of prenatal polyunsaturated fatty acid (PUFA) status. Animal and in vitro studies have shown that $\mathrm{n}-3$ PUFAs can inhibit inflammation, and decrease adipose tissue deposition, while those of the $\mathrm{n}-6$ family have been suggested to exhibit opposite effects. However, findings from human studies on the association of prenatal PUFA status with childhood outcomes including obesity and allergic disease symptoms remain largely inconsistent. Likewise, prospective studies assessing prenatal fish intake, which constitutes the primary dietary source of n-3 long-chain fatty acids, have produced mixed results. Limitations of prior studies not allowing firm conclusions to be drawn include the relatively short duration of follow-up, heterogeneity in exposure and outcome definition, and lack of replicated findings across populations with different characteristics and behaviors. Studies in this thesis were designed to address these limitations, and to provide an overall picture on the role of prenatal PUFA exposures in later growth, cardiometabolic health and allergy-related phenotypes. In Chapter 1, we summarized the background and hypotheses of the studies included in this thesis.

Chapters 2 to 5 include our studies on PUFA exposures. Specifically, in Chapter 2, we conducted a systematic review and meta-analysis according to the Cochrane methodology and the PRISMA (Preferred Reporting Items for Systematic Reviews and Meta-Analyses) guidelines in order to evaluate whether maternal n-3 long-chain PUFA in pregnancy and/or lactation exerts a beneficial effect on adiposity status in childhood. Studies were eligible for inclusion if they were randomized controlled trials (RCTs) of supplementation with n-3 long-chain PUFAs, as compared with a control regimen, to pregnant and/or lactating women and reported data on BMI, body fat mass, waist circumference, and/or sum of skinfold thicknesses in childhood. A total of six RCTs (9 publications) involving 2847 participants were identified. Overall, we found no evidence to support that maternal supplementation exerts a favourable programming effect on childhood adiposity. However, most of the trials reviewed were prone to limitations including small sample sizes and selective attrition, thereby, limiting generalizability of findings. In Chapter 3, we pooled individual data of two birth cohorts from West (the Dutch MEFAB cohort) and South Europe (the Greek RHEA cohort) involving a total of 529 participants to assess whether n-3 and n-6 PUFAs concentrations in cord blood phospholipids, reflecting fetal exposure in late pregnancy, are associated with rapid infant growth, childhood obesity and cardiometabolic risk at 6-7 years of 
age. Such pooled analyses of diverse populations with different characteristics and behaviors constitute an important step towards more generalizable conclusions. We defined rapid infant growth as a weight gain z-score> 0.67 from birth to 6 months. We analysed body mass index (BMI) as continuous and in categories of overweight/obesity at 4 and 6 years. We also derived a mid-childhood cardiometabolic risk score as the sum of waist circumference, non-high-density lipoprotein cholesterol and blood pressure z-scores. Our results did not support an association of cord blood PUFA levels with obesity outcomes and cardiometabolic risk profile in childhood.

It is well established that individuals experience different rates of growth over time- some individuals have a low growth rate in early childhood but experience accelerated growth later in childhood. Examining the long-term effects of early-life exposures at different ages, preferably on the child to adult transition, is important for accurate identification of modifiable risk factors and for understanding when these factors exhibit their maximum influence. Hence, in Chapter 4, we used long-term longitudinal data of the MEFAB birth cohort to assess whether cord blood phospholipid PUFA levels are associated with height and body mass index (BMI) from 6 months to 23 years of age. For 250 (45.2\% females) participants, we collected a total of 1770 ( $n=802$ for females) repeated growth measurements from infancy to young adulthood. We found sex-specific, not clinically relevant associations of cord blood PUFA levels with infant length, and these associations did not persist later in childhood and young adulthood. No associations of cord blood PUFAs with BMI from infancy till young adulthood were observed in either sex. In Chapter 5, we studied mid-childhood symptoms of allergic disease in relation to prenatal PUFA status in a pooled analysis of the MEFAB and RHEA birth cohorts involving a total of 524 mother-child pairs. Information on wheeze, asthma, rhinitis, and eczema prevalence at age 6-7 years was collected using validated questionnaires. We found that higher EPA and DHA levels and a corresponding higher n-3:n-6 fatty acid ratio in cord blood were associated with lower risk of child wheeze and asthma.

In Chapters 6 to 8, the focus of the thesis is shifted to fish consumption in pregnancy. Fish is the major dietary source of $\mathrm{n}-3$ long-chain PUFAs, but is also a common route of human exposure to environmental pollutants. Thus, the effect of fish intake by pregnant women remains an important issue, especially in populations that consume fish frequently. In Chapter 6, we summarized prior findings on the association of fish consumption during pregnancy with child health outcomes including obesity and allergic diseases, and found largely inconsistent evidence. In utero exposure to fatty acids and toxicants found in the same fish might act on the exact same end points at an opposite direction, resulting 
in confusion concerning the place of fish in a healthy prenatal diet. Hence, we harmonized and pooled individual data of mother-child pairs participating in a large network of European and US cohort studies to assess the strength and consistency of the associations of fish intake during pregnancy with growth and the risk of asthma and allergic rhinitis in childhood. In Chapter 7, we analysed individual data of follow-ups 2-year intervals, until the age of 6 years, from 26184 pregnant women and their children participating in 15 European and US cohort studies. We found that women who ate fish more than 3 times/week during pregnancy gave birth to offspring with higher BMI values from infancy through middle childhood compared with women with lower fish intake (3 times/week or less). High maternal fish intake during pregnancy ( $>3$ times/week) was associated with increased risk of rapid growth in infancy and increased risk of childhood overweight/obesity up to 6 years of age, as compared with an intake of once per week or less. These findings are in line with the fish intake limit proposed by the US Food and Drug Administration and Environmental Protection Agency. In Chapter 8, we examined maternal fish intake during pregnancy in relation to childhood symptoms of wheeze, asthma and allergic rhinitis up to 8 years of age among 60774 mother-child pairs participating in 18 European and US birth cohort studies. We found no evidence supporting a protective association between maternal fish consumption and offspring symptoms of wheeze, asthma and allergic rhinitis from infancy to mid-childhood. Finally, Chapter 9 provides a general overview and interpretation of our study findings, and suggests directions for future research.

To conclude, findings from this thesis suggest that a modification of prenatal PUFA status in late gestation towards higher levels of n-3 long-chain PUFAs might assist in the prevention of childhood asthma, but not in tackling the obesity epidemic in children. Pregnant women should adhere to current fish consumption guidelines, and not exceed recommended intakes as a means of improving n-3 PUFA status. Further studies are needed to establish causality and to examine the interplay between fatty acids and pollutants found in fish. 


\section{Samenvatting}

Wereldwijd stijgt de aanwezigheid van obesitas en allergie bij kinderen in alarmerende mate, wat ernstige lange termijn problemen met zich mee brengt. Daarom is er een dringende behoefte om inzicht te krijgen in beïnvloedbare determinanten in het vroege leven, welke kunnen worden aangepakt met preventieve interventies. In deze context is er een groeiende interesse naar de mogelijke rol van de prenatale meervoudig onverzadigde vetzuur (MOV) status. Uit dierstudies en in vitro studies is gebleken dat omega-3 vetzuren inflammatie kunnen afremmen en de afzetting van vetweefsel kunnen beperken, terwijl omega- 6 vetzuren het omgekeerde effect lijken te hebben. De bevindingen uit humane studies over de associatie tussen prenatale meervoudig onverzadigde vetzuurstatus en kinderaandoeningen zoals obesitas en allergische symptomen zijn echter overwegend inconsistent. Prospectieve studies op het gebied van prenatale visinname, de voornaamste bron van omega-3 vetzuren in het dieet, laten ook gemengde resultaten zien. Tekortkomingen van eerdere studies waardoor er geen sterke conclusies getrokken kunnen worden zijn onder andere een korte duur van de studie, heterogeniteit in de blootstelling en de definitie van de uitkomst en een gebrek aan repliceerbare bevindingen over verschillende populaties met variatie in gedrag en andere kenmerken. De studies in dit proefschrift zijn opgezet om deze tekortkomingen aan te pakken, en om een compleet beeld te schetsen over de rol van prenatale meervoudig onverzadigde vetzuur blootstelling in groei in het latere leven, cardiometabole gezondheid en allergie gerelateerde fenotypes. In hoofdstuk 1 worden de achtergrond en hypothesen van de studies in het proefschrift beschreven.

Hoofdstukken 2 tot en met 5 beschrijven studies met betrekking tot de blootstelling aan meervoudig onverzadigde vetzuren. Specifiek beschrijft hoofdstuk 2 de door ons uitgevoerde systematische review en meta-analyse volgens de Cochrane methode en de PRISMA richtlijnen (Preferred Reporting Items for Systematic Reviews and Meta-Analyses) met als doel inzicht te krijgen in de vraag of blootstelling aan lange keten omega-3 vetzuren tijdens de zwangerschap en/of de periode van borstvoeden een voordelig effect heeft op de mate van vetzucht bij kinderen. Studies waren geschikt voor inclusie als het een randomised controlled trail (RCT) betrof over omega-3 vetzuur supplementatie in vergelijking met een controle groep bij zwangere of borstvoedende vrouwen waarvan informatie bekend was over BMI, vetmassa, taille omtrek, en/of huidplooimeting van het kind. Er werden zes RCTs geïncludeerd (beschreven in negen publicaties), met in totaal 2847 participanten. We hebben geen bewijs gevonden voor de bewering dat supplementatie bij de moeder een voordelig effect heeft op vetzucht bij het kind. Echter, de meeste geïncludeerde studies hadden limitaties zoals een kleine groep deelnemers en selectieve uitval van deelnemers, waardoor de bevindingen maar 
beperkt te generaliseren zijn. In hoofdstuk 3 hebben we individuele data van twee geboortecohorten samengevoegd, uit West (het Nederlandse Mefab cohort) en Zuid Europa (het Griekse RHEA cohort) waarin bij 529 participanten werd onderzocht of omega-3 en omega- 6 MOV concentratie in fosfolipiden uit het navelstrengbloed, waarmee blootstelling van de fetus tijdens de laatste fase van de zwangerschap wordt bepaald, is gerelateerd aan snelle groei van het kind, obesitas bij het kind, en risicofactoren voor hart- en vaatziekten bij 6 tot 7 -jarigen. Deze gepoolde analyse van verschillende populaties, met verschillende kenmerken en gedragingen zijn een belangrijke stap in de richting van een meer generaliseerbare conclusie. Snelle groei van het kind werd gedefinieerd als een stijging in gewicht met een z-score $>0.67$ in de eerst zes maanden van het leven. BMI (Body Mass Index) werd geanalyseerd als een continue variabele en in categorieën voor overgewicht en obesitas bij de leeftijd van vier tot zes jaar. Een cardiometabole risicoscore voor de midden-kindjaren is bepaald door de som van de z-scores van de tailleomtrek, niet hoge dichtheid lipoproteine cholesterol en bloeddruk. Onze bevindingen vonden geen steun voor de associatie tussen MOV in het navelstrengbloed en obesitas gerelateerde uitkomsten en cardiometabole risicoprofiel in de kindertijd. Het is reeds bekend dat individuen verschillende groeisnelheden ervaren gedurende het leven; sommige individuen hebben een lage groeisnelheid tijdens de vroege kindertijd, maar ervaren een versnelling van de groei tijdens de latere kindertijd. Onderzoek naar de lange termijn effecten van blootstelling tijdens het jonge leven op verschillende leeftijden, vooral in de overgang tussen de kindertijd en het volwassen leven, is van belang voor de accurate identificatie van wijzigbare risicofactoren en voor het begrip wanneer deze factoren de grootste invloed hebben. Daarom hebben we in hoofdstuk 4 gebruik gemaakt van de lange termijn longitudinale data uit het MEFAB geboorte cohort om onderzoek te doen naar de vraag of het niveau van MOV in fosfolipiden in het navelstrengbloed samenhangen met lengte en BMI vanaf zes maanden tot 23-jarige leeftijd. Van 250 participanten (45,2\% vrouwelijk) hebben we 1770 ( $\mathrm{n}=802$ voor vrouwen) herhaalde metingen met betrekking tot groei, tussen de kindertijd en de jong volwassen leeftijd geanalyseerd. We hebben geslachtsspecifieke, niet klinisch relevante, associaties gevonden tussen het MOV niveau in navelstrengbloed met lengte van het jonge kind, en deze associaties bleven niet bestaan in het latere leven en de vroege volwassenheid. Er werd geen samenhang gevonden tussen MOV in navelstrengbloed en BMI tijdens de kindertijd en de jong volwassen leeftijd, voor zowel mannen als vrouwen. In hoofdstuk 5 hebben we gekeken naar symptomen van allergie tijdens de midden-kindertijd in relatie tot prenatale MOV status in een gepoolde analysevan het MEFAB en het RHEA cohort waarbij 524 moeder-kind paren zijn onderzocht. Informatie over astma-, hooikoorts-, 
piepen- en eczeemprevalentie bij 6 a 7 jarigen was verzameld met een gevalideerde vragenlijst. Onze bevindingen lieten zien dat hogere EPA en DHA niveaus en een bijbehorende hogere omega-3:omega- 6 ratio in het navelstrengbloed samenhingen met een lager risico op piepen en astma voor het kind.

In de hoofdstukken $\mathbf{6}$ tot en met $\mathbf{8}$ is de focus van het proefschrift verlegd naar visinname tijdens de zwangerschap. Vis is de grootste voedingsbron van omega-3 lange keten MOV, het is echter ook een veelvoorkomende manier van blootstelling aan milieuverontreinigende stoffen. Vandaar dat het effect van visinname door zwangere vrouwen een belangrijk onderwerp blijft, vooral in populaties met een hoge visconsumptie. In hoofdstuk $\mathbf{6}$ hebben we de eerdere bevindingen samengevat over de associatie tussen visconsumptie tijdens de zwangerschap en gezondheidsuitkomsten bij het kind, zoals obesitas en allergische ziekten. Het bewijs hiervoor bleek grotendeels inconsistent. Blootstelling in de baarmoeder aan vetzuren en toxische stoffen afkomstig uit dezelfde vissen kunnen reageren op dezelfde eindpunten in een tegenovergestelde richting, waardoor er verwarring bestaat over de rol van vis in een gezond prenataal voedingspatroon. Daarom hebben we de individuele data van moeder-kind paren uit een groot netwerk van Europese en Amerikaanse cohort studies samengevoegd en gepoold, om de kracht en samenhang van de associaties tussen visinname tijdens de zwangerschap en het risico op astma en hooikoorts in de kindertijd vast te stellen. In hoofdstuk 7 hebben we individuele data van follow-upmetingen met intervallen van twee jaar geanalyseerd, tot de leeftijd van zes jaar, afkomstig van 26184 zwangere vrouwen en hun kinderen die deelnamen in 15 Europese en Amerikaanse cohortstudies. We hebben hierbij gevonden dat vrouwen die tijdens de zwangerschap meer dan drie keer per week vis aten kinderen kregen met een hoger BMI dat aanhield van de jonge tot de midden kindertijd, in vergelijking met vrouwen die minder vis aten ( $<3$ keer per week). Grote visinname van de moeder tijdens de zwangerschap hing samen met een hoger risico voor snelle groei in de kindertijd en een grotere kans op obesitas tot zesjarige leeftijd, in vergelijking met zwangere vrouwen die minder dan drie keer per week vis consumeren. Deze bevingingen komen ook overeen met de limieten voor visinname die zijn gesteld door de US Food and Drug Administration en de Environmental Protection Agency. In hoofdstuk 8 hebben we gekeken naar de relatie tussen maternale visinname tijdens de zwangerschap en symptomen van piepen, astma en hooikoorts bij het kind tot achtjarige leeftijd, onder 60774 moeder-kindparen die deelnamen in 18 Europese en Amerikaanse geboortecohorten. We hebben geen bewijs gevonden ter ondersteuning van een positieve associatie tussen maternale visconsumptie en symptomen van piepen, astma en hooikoorts bij het kind, tussen de jonge kindertijd en de middelste kin- 
derjaren. Hoofdstuk 9 biedt uiteindelijk een algemeen overzicht en interpretatie van onze bevindingen en geeft suggesties voor vervolgonderzoek.

Ter conclusie laten de bevindingen van dit proefschrift zien dat aanpassing van de prenatale MOV status in de late zwangerschap richting een hogere inname van omega-3 vetzuren mogelijk bijdragen in de preventie van astma bij kinderen, maar niet in het oplossen van de obesitas epidemie bij kinderen. Zwangere vrouwen zouden de huidige richtlijnen met betrekking tot visconsumptie moeten naleven en deze aanbeveling niet overstijgen met als doel de omega-3 MOV inname te verhogen. Vervolgstudies zijn nodig voor het vaststellen van causaliteit en om de interactie tussen vetzuren en verontreinigende stoffen uit vis te onderzoeken. 


\section{Valorization addendum}

Among the health issues that the youth of today is facing in developed countries, obesity and allergic disease are two of the most serious ones, with life-long consequences. Indeed, the European Commission classifies childhood obesity and allergy as two chronic conditions of major public health importance. ${ }^{1}$ It is estimated that around 50 million girls and 74 million boys worldwide are currently obese, while 213 million children are in the range of overweight, but below the obesity threshold. ${ }^{2}$ Moreover, according to the most recent estimates, approximately 1 in every 10 children aged 14 years or less worldwide suffer from asthma. ${ }^{3}$ Hence, there is an urgent need to identify modifiable determinants that can be targeted for preventive strategies. Such strategies are likely to bring substantial benefits over the whole lifespan (Figure 10.1).

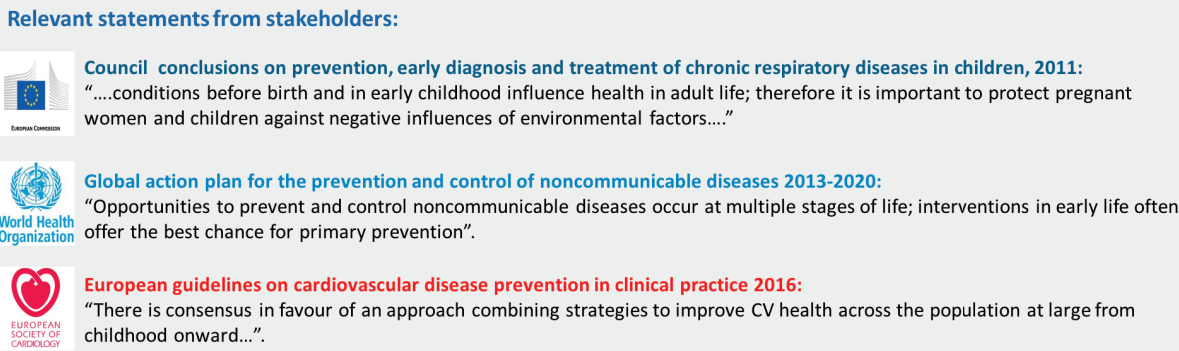

Figure 10.1. Stakeholder statements on the importance of noncommunicable disease prevention in early life.

Our work strengthens the evidence that nutrition during pregnancy, which is a highly modifiable lifestyle factor, can influence child obesity and asthma. We focused on prenatal polyunsaturated fatty acid concentrations and fish intake, for which previous research has yielded largely inconsistent results (see Chapter 1 ), thus limiting health risk assessments. Our work has relevance for a number of policy documents including the European food Action Plan 2015-2020 and the European policy framework Health $2020^{5}$, both of which underscore the importance of providing healthy food and nutrition across the lifecourse as a means for promoting population health. Moreover, it addresses the recent European Council's conclusions towards halting childhood obesity, which highlight the need for further research to understand the drivers of childhood overweight and obesity to explore approaches to a healthy diet across the life course. ${ }^{6}$

Potential end-users of our research can be identified at various ecological levels: individual level (e.g. parents, future parents), community level (e.g. physicians, 
local communities), national level (food industry, think-tanks, public health institutes), European and international level (e.g. patient organizations, food industry, EFSA, WHO, Schools for Public Health Europe).

Claims about the numerous potential health benefits of omega-3 fatty acids have contributed substantially to the increase of the global omega- 3 supplement market, with its value being estimated at around USD 33.04 billion in 2016. ${ }^{7}$ Our findings on n-3 long-chain PUFA supplementation in the perinatal period (Chapter 2) and those on cord blood PUFA biomarker levels (Chapters 3, 4, and 5), in addition to findings of other studies, may have implications for productive and commercial activities used by the food industry.

Nutrient-based studies using biomarker levels may provide more insight on mechanistic pathways through which food products containing numerous nutrients can affect health and development. In our studies on cord blood PUFA biomarker levels, we did not have information on prenatal dietary intake so as to unravel the amount of dietary PUFAs or PUFA-containing foods (see Chapter 1) needed in order to achieve a change in biomarker levels. Together with prior research findings, our results could jointly be used for formulating dietary recommendations for pregnant woman. Currently, a number of health or patient organization produce such recommendations, including but not limited to the World Health Organization (WHO), Health Council of the Netherlands, ${ }^{8}$ Greek Institute of Preventive Medicine, Environmental and Occupational Health (Prolepsis), ${ }^{9}$ U.S. Department of Health and Human Services and U.S. Department of Agriculture, ${ }^{10}$ and Public Health England. ${ }^{11}$

In 2015, the European Food Safety Authority (EFSA) in its statement on the benefits of fish/seafood consumption compared to the risks of methylmercury in fish/seafood concluded that pregnant women should consume up to 3-4 servings of fish per week. ${ }^{12}$ In January 2017, the US Food and Drug Administration (FDA) and Environmental Protection Agency (EPA) issued their final updated advice on fish consumption for women of childbearing age, encouraging women who are pregnant, breastfeeding, or likely to become pregnant to consume more fish, but no more than 3 servings/week. ${ }^{13}$ Notably, fish advisories have focused on the potential neurocognitive harms associated with exposure to methyl-mercury but have not considered other childhood outcomes, including obesity and asthma, areas where evidence is generally quite limited. Hence, our international studies on fish intake during pregnancy aimed to address this knowledge gap (Chapters 7 and 8). Our findings that fish intake of more than 3 times per week during pregnancy is associated with an increased risk of rapid infant growth and childhood overweight/obesity are in line with the limit proposed by FDA and EPA, but not with the EFSA conclusion. 
We used a wide range of tools to disseminate knowledge generated from this work (Figure 10.2). We published our work in leading international fully peer-reviewed journals and one book chapter to increase awareness and communicate our study findings within the scientific community. Our study results were also presented at the Dutch Epidemiological Society (WEON) conferences (2014, 2015, 2016), with an audience consisting mostly of researchers and clinicians. Moreover, we showed our results to The Obesity Society (TOS) Obesity Week (2015), the International Society for the Study of Fatty Acids and Lipids (ISSFAL) congress (2016) and the Euro Fed Lipid Congress (2016), in which food industry had also an active participation. Additionally, we developed the MEFAB birth cohort website (www. mefab.org), with links to specific pages for scientific and non-scientific audiences. An example of the latter is a page (www.mefab.org/wist-je-dat) that explains in lay language the health issues surrounding early-life nutrition and our most recent research findings. Furthermore, we developed a documentary about the paradigm of "developmental origins of health and disease" and the contribution of the MEFAB cohort in this research field (https://youtu.be/Bc6MEAULY08). Given that social media constitute an effective way of reaching the lives of many people and sharing knowledge nowadays, we created social media accounts (Facebook, twitter) for the MEFAB cohort, where we disseminated knowledge generated from this work in a simple and direct way. Furthermore, our findings on fish intake during pregnancy and childhood growth and obesity received international press coverage, being mentioned in prominent news media including but not limited to CNN (http://cnn.it/2hxe43l), Reuters (http://reut.rs/2jAPrqv), and TIME (http://ti.me/1PMES7S).

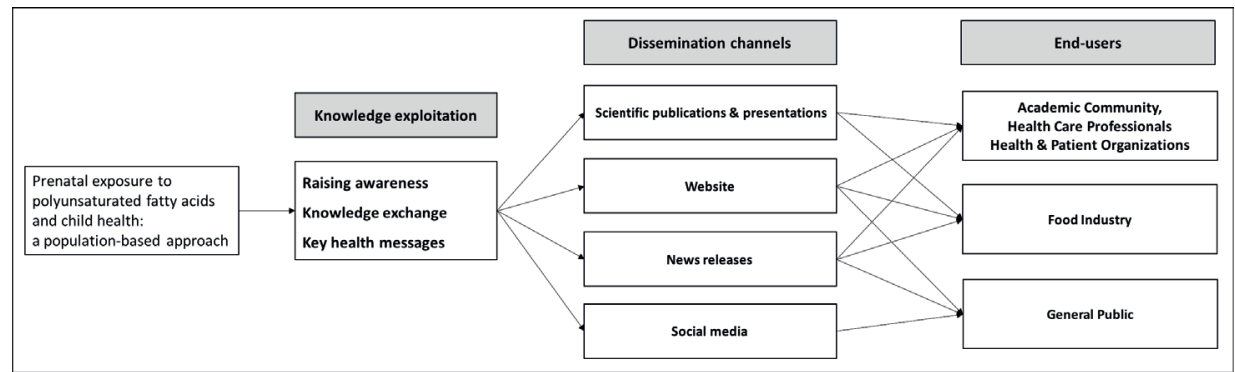

Figure 10.2. Dissemination channels used for our work and potential end-users.

To conclude, our work focused on childhood obesity and asthma, which constitute public health priorities, and therefore, has major potential for translational utilization. It examined the influence of two highly modifiable lifestyle factors (PUFA levels and fish intake) during the prenatal period, which is critical period 
of developmental plasticity. Evidence from this work are positioned to inform (future) parents for dietary advice, and better policy- and decision-making for public health interventions. Interventions specifically targeting this early-life period are considered to have a large and long-lasting impact across the life course, and thus, give substantial long-term social and economical returns on investment. 


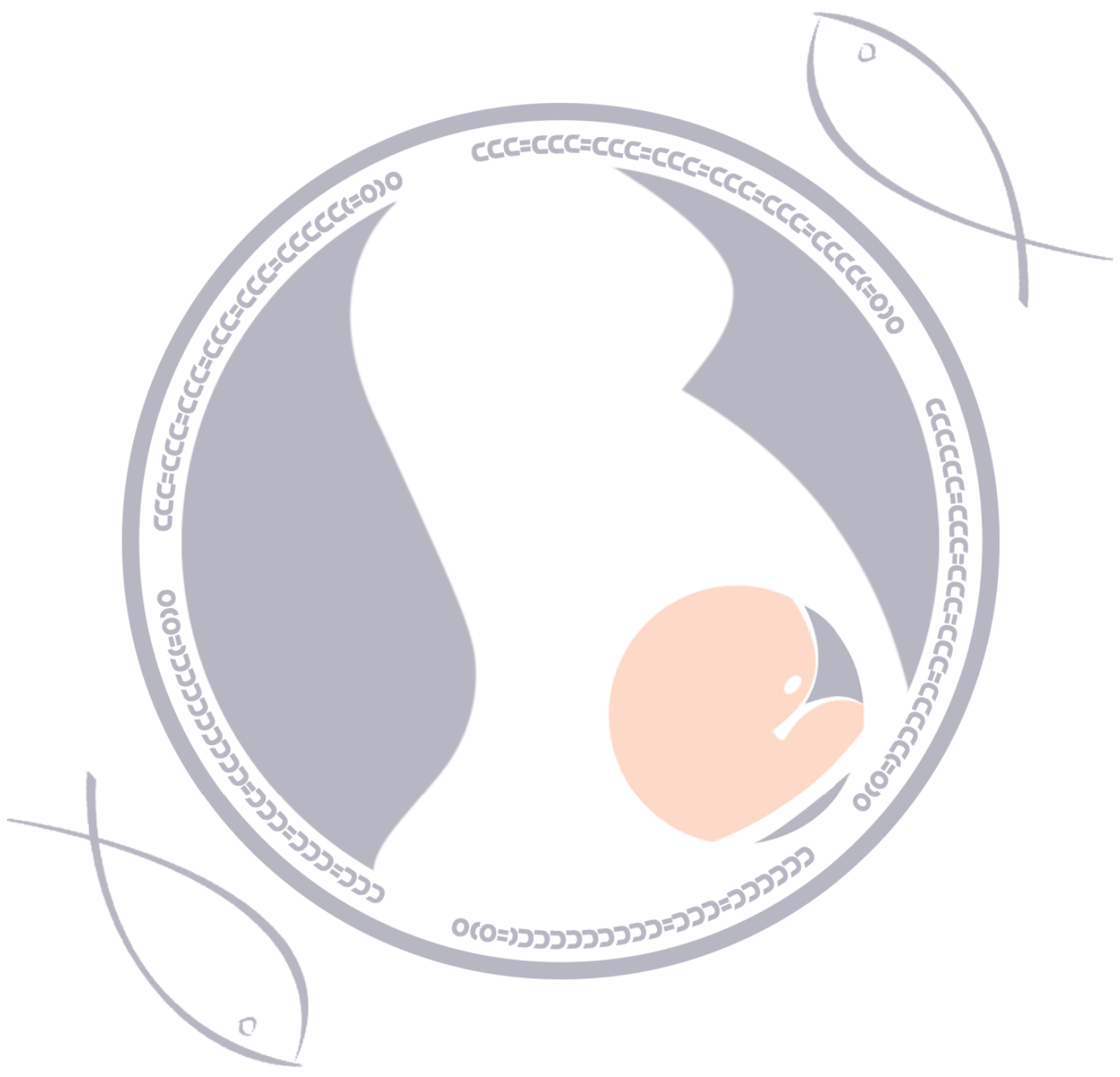




\section{CHAPTER 11}

List of publications-presentations

About the author

Dankwoord (words of gratitude) 



\section{Publications}

- Stratakis N, Roumeliotaki T, Oken E, Ballester F, Barros H, Basterrechea M, Cordier S, de Groot R, den Dekker HT, Duijts L, Eggesbø M, Pia Fantini M, Forastiere F, Gehring U, Gielen M, Gori D, Govarts E, Inskip HM, Iszatt N, Jansen M, Kelleher C, Mehegan J, Moltó-Puigmartí C, Mommers M, Oliveira A, Olsen SF, Pelé F, Pizzi C, Porta D, Richiardi L, Rifas-Shiman SL, Robinson SM, Schoeters G, Strøm M, Sunyer J, Thijs C, Vrijheid M, Vrijkotte TG, Wijga AH, Kogevinas M, Zeegers MP, Chatzi L. Fish and seafood consumption during pregnancy and the risk of asthma and allergic rhinitis in childhood: a pooled analysis of 18 European and US birth cohorts. Int J Epidemiol. 2017 Oct 1;46(5):1465-1477.

- Stratakis N, Roumeliotaki T, Oken E, Barros H, Basterrechea M, Charles MA, Eggesbø M, Forastiere F, Gaillard R, Gehring U, Govarts E, Hanke W, Heude B, Iszatt N, Jaddoe VW, Kelleher C, Mommers M, Murcia M, Oliveira A, Pizzi C, Polańska K, Porta D, Richiardi L, Rifas-Shiman SL, Schoeters G, Sunyer J, Thijs C, Viljoen K, Vrijheid M, Vrijkotte TG, Wijga AH, Zeegers MP, Kogevinas M, Chatzi L. Fish Intake in Pregnancy and Child Growth: A Pooled Analysis of 15 European and US Birth Cohorts. JAMA Pediatr. 2016 Apr;170(4):381-90.

- Stratakis N, MP Zeegers, L Chatzi. Fish Intake in Pregnancy and Offspring Adiposity-Reply. JAMA Pediatr. 2016 Aug 1;170(8):809-10.

- Arnaoutis G, Kavouras SA. Stratakis N, Likka M, Mitrakou A, Papamichael C, Sidossis LS, Stamatelopoulos K. The effect on hypodration on endothelial function in young healthy adults. Eur J Nutr. 2017 Apr;56(3):1211-1217.

- Stratakis N, Gielen M, Chatzi L, Zeegers MP. The effect of maternal supplementation with n-3 long-chain polyunsaturated fatty acids during pregnancy and/or lactation on child adiposity: a systematic review and meta-analysis of randomized controlled trials. Eur J Clin Nutr. 2014 Dec; 68(12): 1277-1287.

- Papadopoulou E, Stratakis N, Roumeliotaki T, Sarri K, Merlo DF, Kogevinas M, Chatzi L. The effect of high doses of folic acid and iron supplementation in early-to-mid pregnancy on prematurity and fetal growth retardation: the mother-child cohort study in Crete, Greece (Rhea study). Eur J Nutr. 2013 Feb; 52(1): 327-36.

- Chatzi L, Papadopoulou E, Koutra K, Roumeliotaki T, Georgiou V, Stratakis N, Lebentakou V, Karachaliou M, Vassilaki M, Kogevinas M. Effect of high doses of folic acid supplementation in early pregnancy on child neurodevelopment at 18 months of age: the mother-child cohort 'Rhea' Study in Crete, Greece. Public Health Nutr. 2012 Sep; 15(9): 1728-36. 
- Stratakis N, Gielen M, Margetaki M, de Groot RHM, Apostolaki M, Chalkiadaki G, Vafeiadi M, Leventakou V, Godschalk RW, Kogevinas M, Stephanou EG, Zeegers MP, Chatzi L. Polyunsaturated fatty acid status at birth and allergy-related phenotypes in childhood: a pooled analysis of the MEFAB and RHEA birth cohorts. Accepted Br J Nutr.

- Stratakis N, Gielen M, Margetaki M, Godschalk RW, van der Wurff I, Rouschop S, Ibrahim A, Antoniou E, Chatzi L, de Groot RHM, Zeegers MP. Polyunsaturated fatty acid status at birth and child-to-adult growth: results from the MEFAB cohort. Prostaglandins Leukot Essent Fatty Acids 2017 Nov; 126:72-78.

- Stratakis N, Gielen M, Margetaki M, de Groot RHM, Apostolaki M, Chalkiadaki G, Vafeiadi M, Leventakou V, Karachaliou M, Godschalk RW, Kogevinas M, Stephanou EG, Zeegers MP, Chatzi L. Polyunsaturated fatty acid status at birth, childhood growth and cardiometabolic risk: a pooled analysis of the MEFAB and RHEA cohorts. Provisionally accepted Eur J Clin Nutr.

\section{Book chapter}

- L Chatzi, N Stratakis. Maternal fish intake during pregnancy and effects on the offspring. R Rajendram, VR Preedy, VB Patel (Eds.). Diet, Nutrition, and Fetal Programming. Humana Press. 2017

\section{Oral presentations}

- WEON Conference (Dutch Epidemiological Society). The effect of maternal supplementation with n-3 long-chain polyunsaturated fatty acids during pregnancy and/or lactation on child adiposity: a systematic review and metaanalysis of randomized controlled trials. June 2014. Leiden, NL.

- $1^{\text {st }}$ Erasmus Nutrition Workshop (invited key-note speaker). Nutrition-specific and nutrition-sensitive health indicators in children. September 2015. Rotterdam, NL

- Bridge Birth Cohort Meeting (invited key-note speaker). Fish intake during pregnancy and child health: results of a collaborative analysis from EU birth cohorts. June 2017. Barcelona, SP.

- WEON Conference (Dutch Epidemiological Society). Polyunsaturated fatty acid status at birth and allergy-related phenotypes in childhood: a pooled analysis of the MEFAB and RHEA birth cohorts. June 2017. Antwerp, BE. 
- Dutch National Program of Lung Research conference (invited key-note speaker). Fish oil supplementation during pregnancy and child respiratory symptoms. June 2017. Amersfoort. NL

\section{Poster presentations}

- Stratakis N, Gielen M, Chatzi L, Zeegers MP. The effect of maternal supplementation with n-3 long-chain polyunsaturated fatty acids during pregnancy and/or lactation on child adiposity: a systematic review and meta-analysis of randomized controlled trials. Annual NUTRIM research symposium. December 2014 .

- Stratakis N, Roumeliotaki T, Oken E, Barros H, Basterrechea M, Charles MA, Eggesbø M, Forastiere F, Gaillard R, Gehring U, Govarts E, Hanke W, Heude B, Iszatt N, Jaddoe VW, Kelleher C, Mommers M, Murcia M, Oliveira A, Pizzi C, Polańska K, Porta D, Richiardi L, Rifas-Shiman SL, Schoeters G, Sunyer J, Thijs C, Viljoen K, Vrijheid M, Vrijkotte TG, Wijga AH, Zeegers MP, Kogevinas M, Chatzi L. Fish Intake in Pregnancy and Child Growth: A Pooled Analysis of 15 European and US Birth Cohorts. The Obesity Society (TOS) Obesity Week. November 2015. Los Angeles, US.

- Stratakis N, van der Wurff ISM, Gielen M, de Groot R, Ibrahim A, Godschalk R, Kremers S, Antoniou E, Zeegers MP. Follow-up of the MEFAB birth cohort from birth to adulthood. ISSFAL Congress. September 2016. Stellenbosch, South Africa.

- Stratakis N, van der Wurff ISM, Gielen M, de Groot R, Ibrahim A, Godschalk R, Kremers S, Antoniou E, Zeegers MP. Follow-up of the MEFAB birth cohort from birth to adulthood. $14^{\text {th }}$ Euro Fed Lipid Congress. September 2016. Ghent, BE.

- Stratakis N, Gielen M, Margetaki M, de Groot RHM, Apostolaki M, Chalkiadaki G, Vafeiadi M, Leventakou V, Godschalk RW, Kogevinas M, Stephanou EG, Zeegers MP, Chatzi L. Polyunsaturated fatty acid status at birth and allergy-related phenotypes in childhood: a pooled analysis of the MEFAB and RHEA birth cohorts. Annual NUTRIM research symposium. December 2017.

- Stratakis N, Gielen M, Margetaki M, de Groot RHM, Apostolaki M, Chalkiadaki G, Vafeiadi M, Leventakou V, Karachaliou M, Godschalk RW, Kogevinas M, Stephanou EG, Zeegers MP, Chatzi L. Polyunsaturated fatty acid status at birth, childhood growth and cardiometabolic risk: a pooled analysis of the MEFAB and RHEA cohorts. WEON Conference (Dutch Epidemiological Society). June 2017. Antwerp, BE. 


\section{About the author}

Nikos Stratakis was born in Heraklion, Crete, on May 12th 1986. In 2004, he graduated from the 2nd General Lyceum in Heraklion, and at the same year, he began the BSc programme in Dietetics and Nutritional Science at Harokopio University in Athens, Greece. In 2007, Nikos started his trip in Epidemiology and Birth Cohort Research in the Mother-Child "Rhea" cohort study at the Department of Social Medicine, University of Crete under the supervision of Dr. Leda Chatzi. He obtained his bachelor's degree in 2009. Subsequently, he worked as a clinical dietitian in a private practice and, at the same time, he continued his studies in the part-time MSc programme in Nutritional Medicine at the University of Surrey, UK. In 2013, he obtained his master's degree and won the award for outstanding student of the year. In September 2013, Nikos joined Maastricht University, where he started his $\mathrm{PhD}$ trajectory at the Section of Complex Genetics and Epidemiology (Supervisor: Prof. dr. MP Zeegers; Co-supervisors: Dr. Leda Chatzi, Prof. dr. RHM de Groot, and Dr. Marij Gielen). His work focused on the association of prenatal exposure to fatty acids and fish with major child health outcomes including obesity and asthma. The results of this work are presented in the present thesis. 


\section{Dankwoord (words of gratitude)}

One for all, and all for one. Alexandros Dumas: The three musketeers

This thesis would not have been possible without the overflowing support of my supervisors, co-authors, colleagues, friends, and family. I would like to express my sincere gratitude and appreciation to all of them.

Dear Prof.dr. M. Zeegers, dear Maurice. Thank you so much for all the support, advice, and guidance that you gave me and that you continue to give me. You are a great example demonstrating that authority and respect can easily be acquired through high context knowledge and positive attitude. Your will to help and share your knowledge is remarkable. I have learned a lot from you, and you have been inspiring me ever since the day we met.

Dear Dr. L Chatzi, dear Leda, next to you I grew not only as a researcher but also as a person. Thanks to you, I got introduced to epidemiology and birth cohort research, where I found my passion. You have always been present for me, providing support, encouragement, and guidance when needed. Words cannot express my gratitude for being able to work with you.

Dear Dr. M. Gielen, dear Marij, countless thanks for your contribution to this work. You were always prompt with your answers, comments, and suggestions. Your help and support were vital, and helped me to overcome small and big challenges throughout my $\mathrm{PhD}$ trajectory.

Dear Prof.dr. R. H. M de Groot, dear Renate, thank you for your time and effort that you put into this work. Your comments and remarks were valuable, and improved substantially the quality of this work.

Dear Prof.dr. P. C. Dagnelie, Prof.dr. A. Schols, Prof.dr. L. Zimmermann, Dr. L. Lauritzen, and Dr. V. Benetou, thank you for agreeing to read and evaluate my thesis. It is an honour to have you as members of the reading committee. I am also honoured to have Dr. T. Voortman as a member of the plenary committee for my $\mathrm{PhD}$ defence.

Dear Frits, Miriam, Bram, Kelly, Gerard, Anke, and Hanneke, it has been a great pleasure working with and learning from you. Work would not be as enjoyable as it is without you. Jullie zijn heel erg aardig. 
Dear Abdalla, I am happy that our paths crossed. It has been a huge support and a lot of fun having you around. Thanks for being such a great friend.

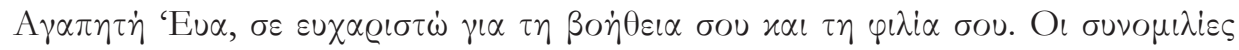

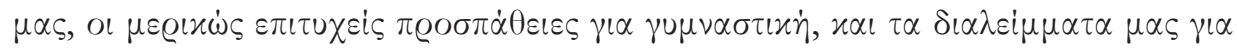

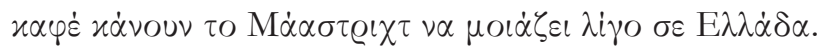

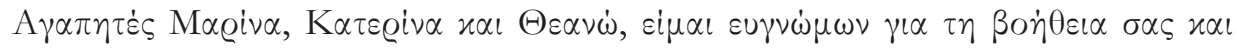

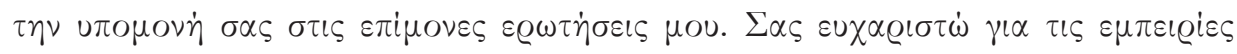

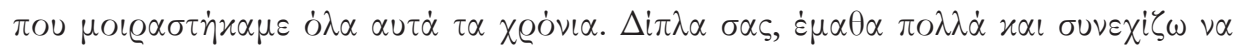
$\mu \alpha \theta \alpha i v \omega$.

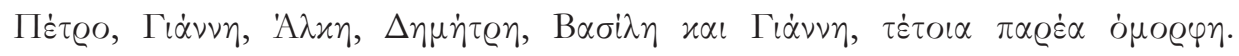

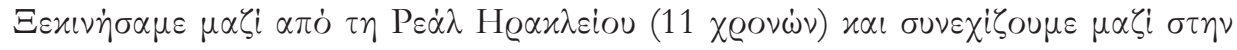

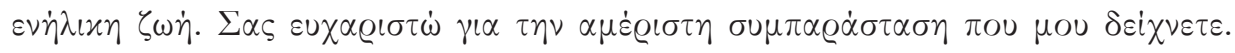

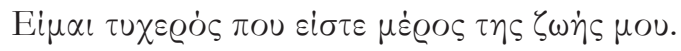

Dorikam, it has been quite a journey. During these years, we met, we fell in love, and we got engaged. It is difficult to imagine getting through this $\mathrm{PhD}$ without your support, loving words, and smile. You are an inspirational person and an incredible source of strength. Thank you for being so wonderfully you. Nagyon szeretlek!

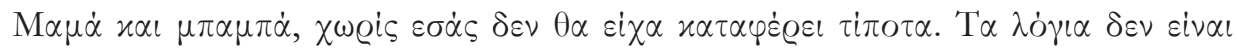

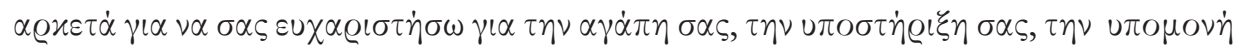

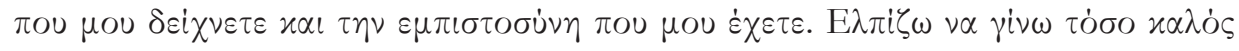

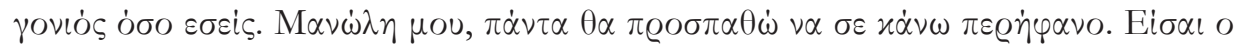

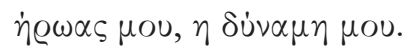


